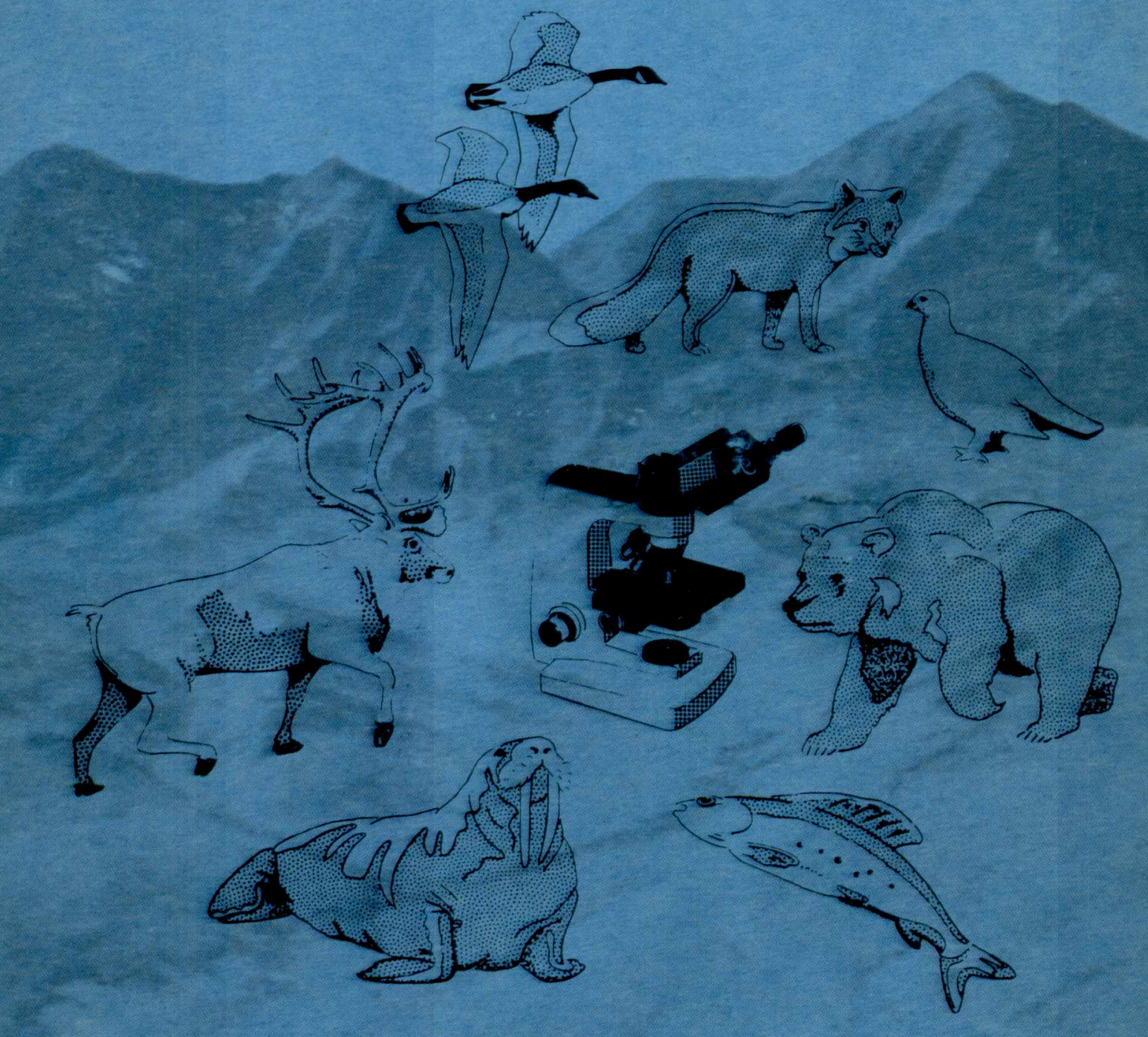

UNIVERSITY OF ALASKA

FAIRBANKS, ALASKA 


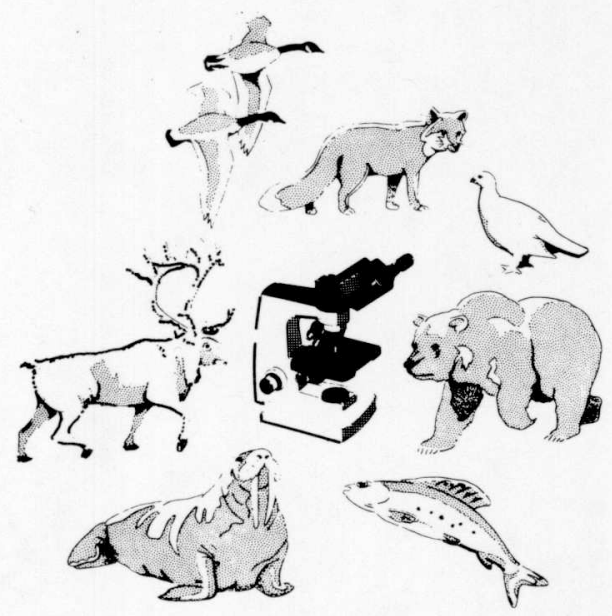

\section{ALASKAN WILDLIFE DISEASES}

\section{Edited by}

ROBERT A. DIETERICH, D.V.M.

Institute of Arctic Biology

University of Alaska

Fairbanks, Alaska 99701

1981 


\section{Dedication}

Dedicated to

Jim Dau

and

Terri Jordan

For their efforts which helped make this publication possible 


\section{Contributors}

Richard Barrett, B.S.

Biologist

P.0. Box 720

State Federal Cooperative Laboratory

Palmer, Alaska 99645

John Burke, Predoctoral Candidate

Fish Pathologist I

Alaska Department of Fish \& Game

Anchorage, Alaska 99502

Jim Dau, B.S.

Biologist

University of Alaska

Fairbanks, Alaska 99701

Al Didier, M.S.

Hatchery Manager

Snett isham Hatchery

Alaska Department of Fish \& Game 230 S. Franklin St.

Juneau, Alaska 99801

Robert A. Dieterich, D.V.M.

Professor of Veterinary Science

University of Alaska

Fairbanks, Alaska 99701

Jill E. Follett, B.S.

Microbiologist II

Alaska Department of Fish \& Game

333 Raspberry Rd.

Anchorage, Alaska 99502

Albert W. Franzmann, D.V.M., Ph.D. Director Moose Research Center Alaska Department of Fish \& Game Soldotna, Alaska 99669

Howard Gelberg, D.V.M., Ph.D.

Assistant Professor

University of 111 inois

Urbana, Illinois 61801

Roger S. Grischkowsky, Ph.D. Principal Pathologist

Alaska Department of Fish \& Game 333 Raspberry Rd.

Anchorage, Alaska

99502
Glenn E. Haas, Ph.D.

404 W. Santa Fe Ave.

\# 104

Flagstaff, Arizona

86001

A. Kent Hauck, M.S.

Fish Pathologist I

Alaska Department of Fish \& Game

333 Raspberry Rd.

Anchorage, Alaska 99502

Newton Kingston, Ph.D.

Professor of Parasitology

Box 3354

University of Wyoming

Laramie, Wyoming 82071

Jamie K. Morton, M.S.

Research Assistant

University of Alaska

Fairbanks, Alaska 99701

Richard A. Neve', Ph.D.

Professor of Marine Pathology and Toxicology

Institute of Marine Science

University of Alaska

Fairbanks, Alaska 99701

Lee Michael Philo, D.V.M.

Research Assistant

University of Alaska

Fairbanks, Alaska 99701

Donald G. Ritter, B.S.

Unit Chief

State of Alaska Virology Rabies Unit

Fairbanks, Alaska 99701

Joe Sullivan, Ph.D.

Fish Pathologist I

Alaska Department of Fish \& Game

333 Raspberry Rd.

Anchorage, Alaska 99502

Randall L. Zarnke, Ph.D.

Game Biologist

Alaska Department of Fish \& Game

Fairbanks, Alaska 99701 


\section{Acknowledgements}

This publication is the result of considerable effort on the part of authors, and many other people who did not contribute to it directly in the form of manuscripts. We would like to acknowledge this latter group of individuals for their investment in this compendium. The publication would have fallen far short of its goals without the contribution of: Dr. R. L. Rausch, who reviewed all manuscripts covering helminth parasites of mammals, and who generously drew upon his extensive parasitological experience in Alaska to provide many unpublished observations and invaluable comments; Dr. F. H. Fay, who reviewed Echinococcus, always found time to answer questions and provided many parasitological reprints that would have been otherwise unavailable; Dr. G. L. Swartz, who provided unpublished observations and color photographs regarding avian diseases; Dr. N. Wilson, who made helpful comments on certain ecto- parasites; the Fisheries Rehabilitation Enhancement and Development Division of the Alaska Department of Fish and Game for providing support during the preparation of the section on diseases of fish; Ms. Sue. Raich, who provided excellent life cycle diagrams, the cross-reference index and who offered many helpful editorial suggestions; Ms. Leslie Moorman, who provided the list of common and scientific names of susceptible species and assisted with the glossary, proofreading and the cross-reference; Ms. Kathy Allen, who assisted with the final draft output on the computer; Mr. Don Hartbauer and Ms. Vickie McGrane, who proofread the entire manuscript; and Ms. Terri Jordan, who verified references, made editorial suggestions, performed all computer-related operations, and who was largely responsible for meeting the publication deadline. To these people, we would like to express our sincere appreciation. 


\section{Preface}

This compendium of Alaskan wildlife diseases has been written to serve several purposes. It is an attempt to condense published information on diseases of wildlife species found in Alaska so that the wildlife student, the biologist, the wildlife manager and the wildlife disease investigator may easily obtain basic information. Hopefully, it defines the symptoms and lesions of each disease and helps clarify the significance of the condition in wildlife populations with special reference to Alaska. We use a broad definition of the word "disease" to include any condition in which bodily health is impaired. Such conditions as starvation, stress, accidental death, chemical and physical trauma play an all important role in the bodily health of our state's wildlife population. This compendium is not meant to serve as a publication of original results or observations, but many of the writers have included experimental results and comments that have not previously been reported. Readers should realize that this is not an attempt to cover all possible diseases of Alaskan wildlife, but rather to clarify and place in context the major disease entities.

Alaska is currently experiencing strong interest by a large number of residents in the development of a domestic animal industry. The interaction of wildlife and domestic animals has raised many questions as to the potential transmission of disease from 1 group to the other. This compendium will hopefully serve as an information source that presents the current "state of the art" relating to diseases of Alaskan wildlife to aid in the decision making process regarding domestic and wild animal interactions.

The expertise of the writers of Alaskan Wildlife Diseases covers a broad area of science including veterinary medicine, bacteriology, virology, parasitology, physiology, biochemistry and pathology. They each gave freely their time without compensation because of a basic desire to share their knowledge, observations and experiences. These writers are all professional wildlife disease investigators who have worked in Alaska. Their dealings with wildlife in Alaska total more than 70 years of experience. Hopefully, this joint effort will serve to bring people in this area of interest together so that they may each express their opinions and receive comments in a continuing effort to share and expand knowledge of wildlife disease.

We invite comments on this first edition of Alaskan Wildlife Diseases so that sometime in the future we may be able to smooth out our roughness, correct our errors, and improve what we hope is a worthwhile and useable publication. Perhaps this compendium will stimulate individuals with expertise concerning various wildlife diseases to volunteer additional material for a future edition.

ROBERT A. DIETERICH 


\section{CONTENTS}

PAGE

\section{Part I. Mammals}

CHAPTER 1. VIRAL DISEASES - Randall L. Zarnke, Chapter Editor..... 5

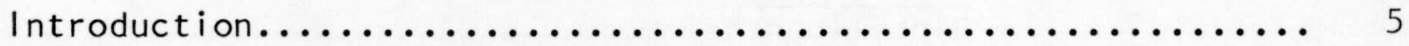

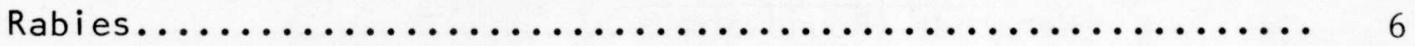

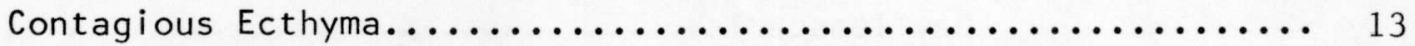

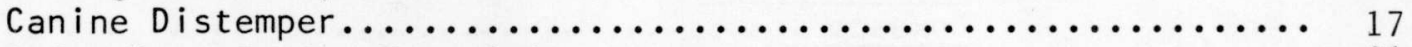

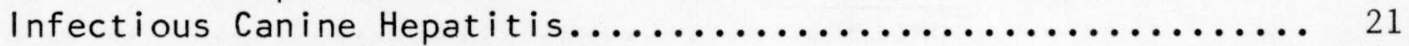

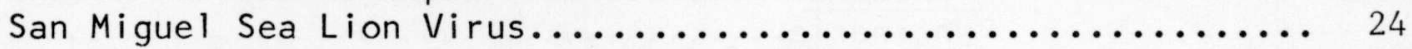

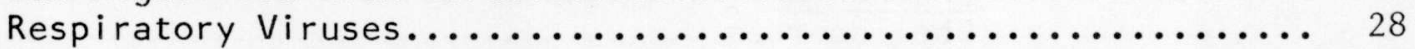

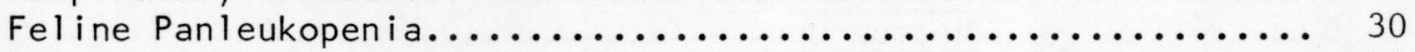

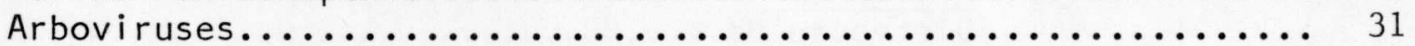

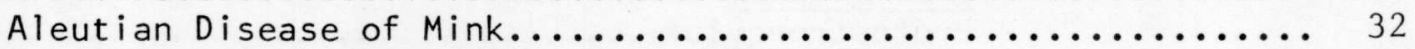

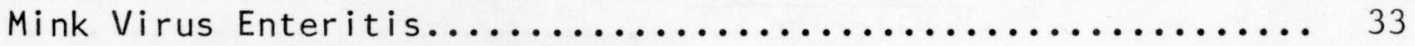

Transmissible Mink Encephalopathy..................... 34

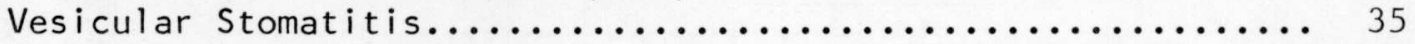

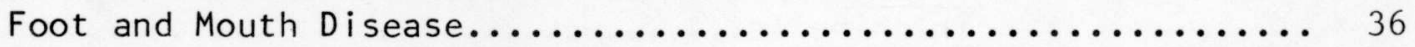

Epizootic Hemorrhagic Disease....................... 37

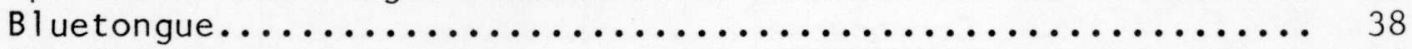

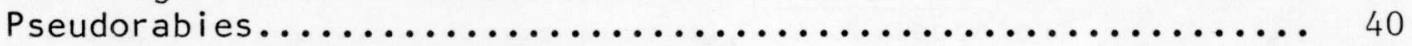

Lymphocytic Choriomeningitis...................... 41

CHAPTER 2. BACTERIAL DISEASES - Jamie Morton, Chapter Editor..... 45

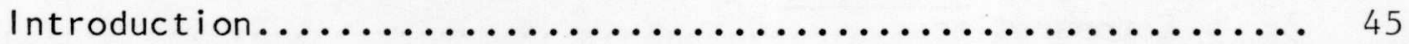

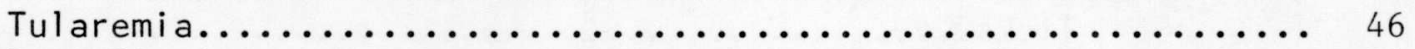

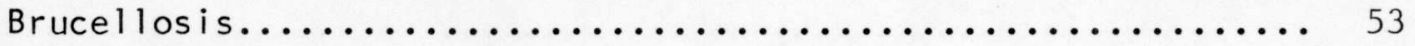

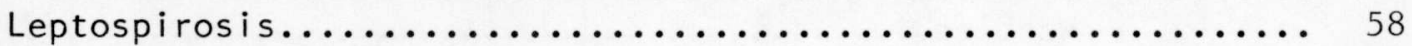

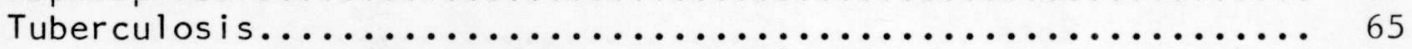

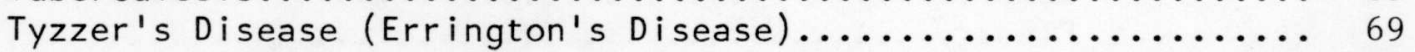

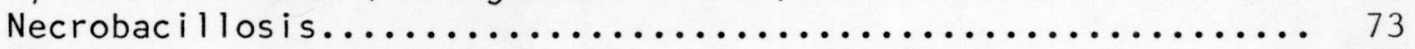

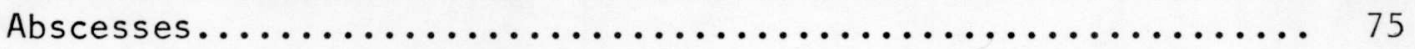

CHAPTER 3. PARASITIC DISEASES - R. Barrett \& J. Dau,

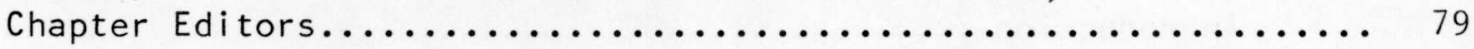

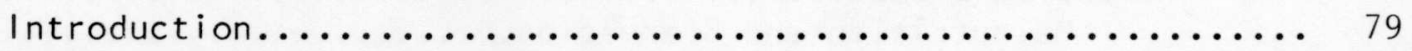

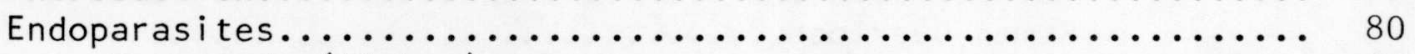

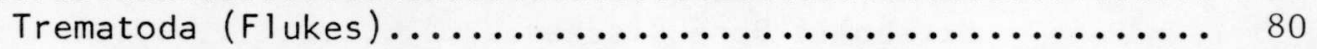

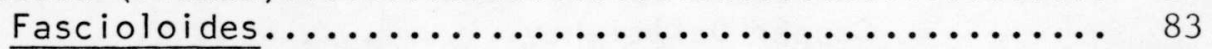

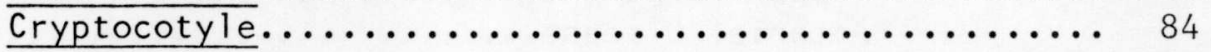

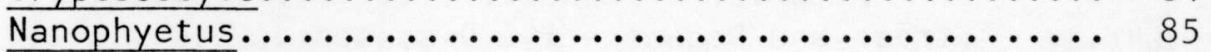

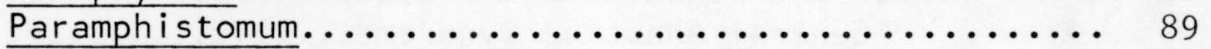

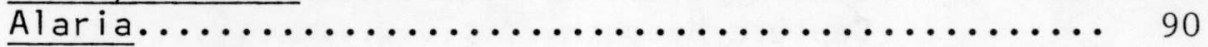




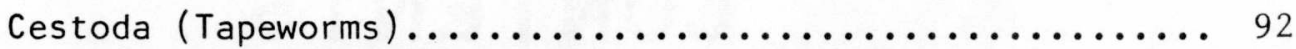

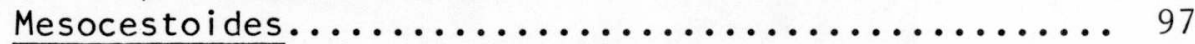

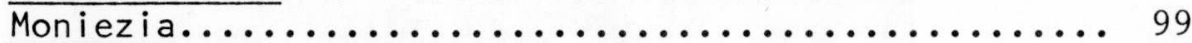

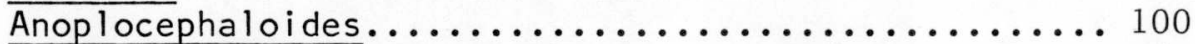

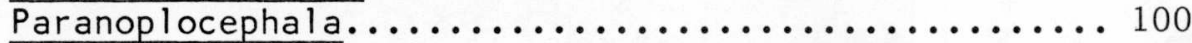

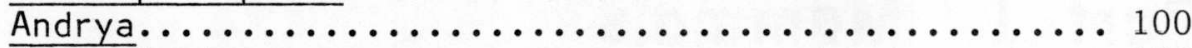

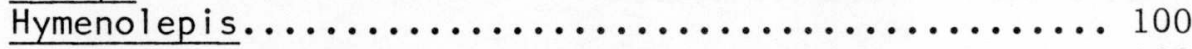

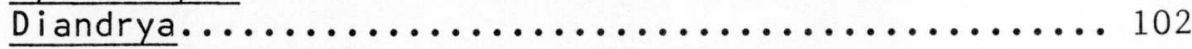

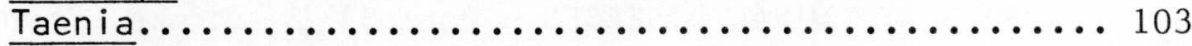

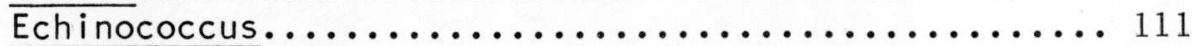

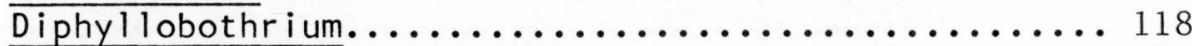

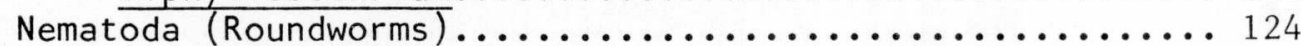

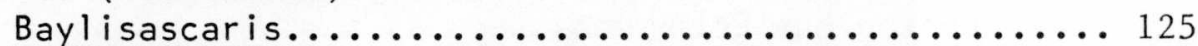

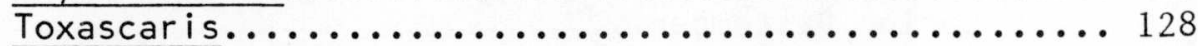

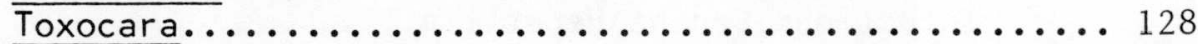

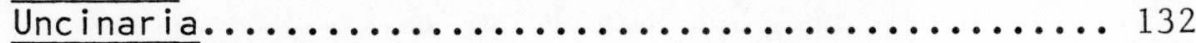

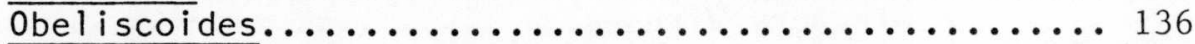

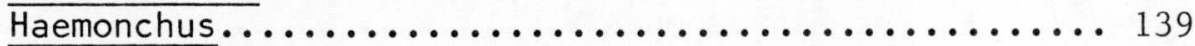

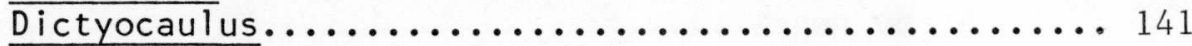

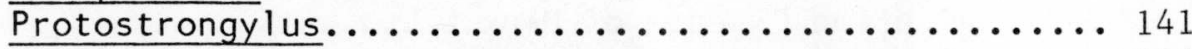

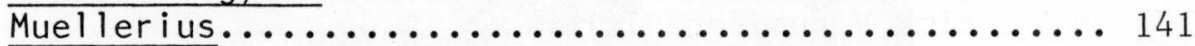

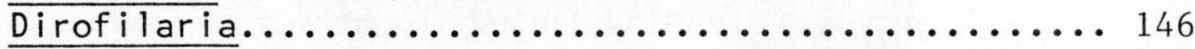

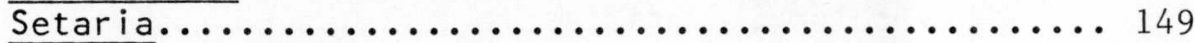

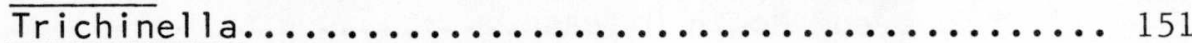

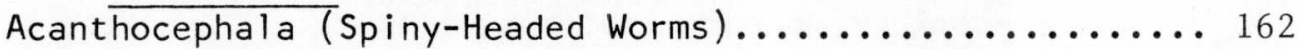

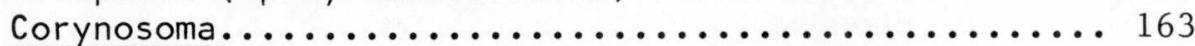

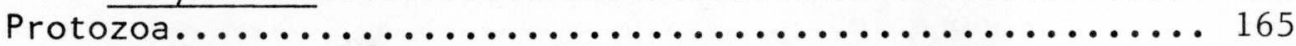

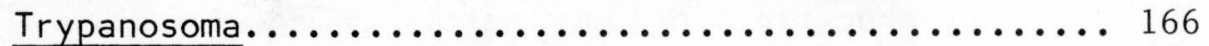

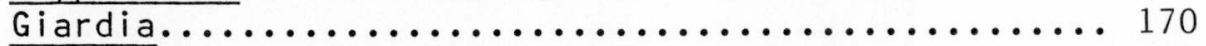

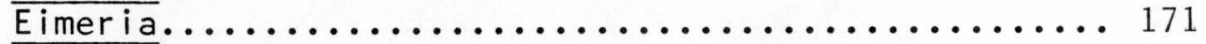

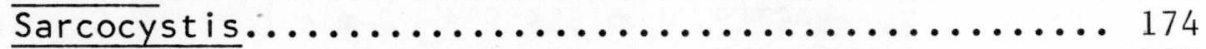

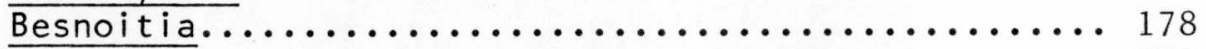

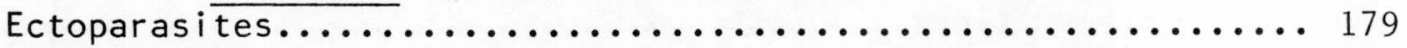

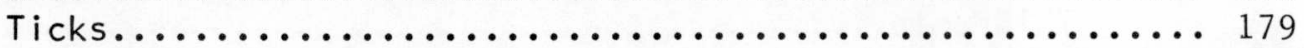

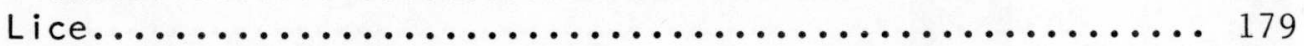

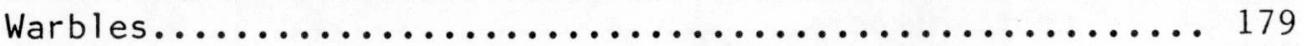

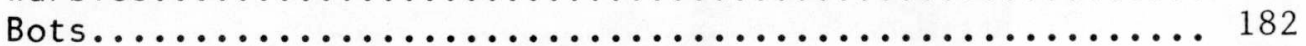

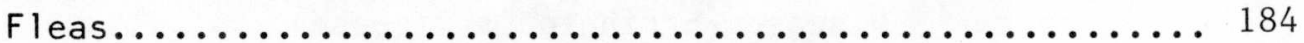

CHAPTER 4. NON-INFECTIOUS DISEASES - Albert W. Franzmann,

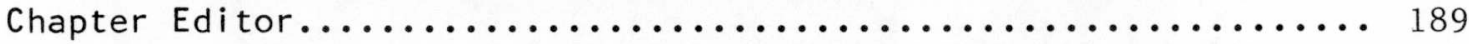

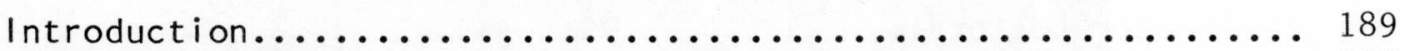

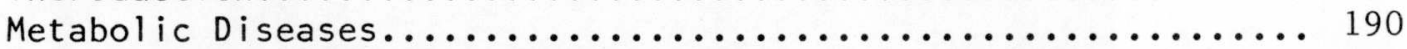

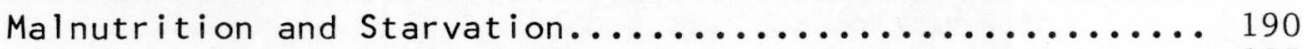

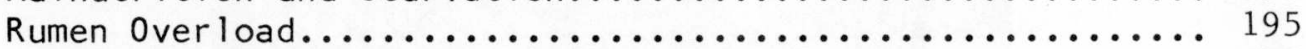

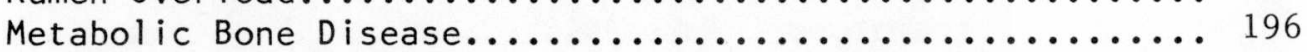

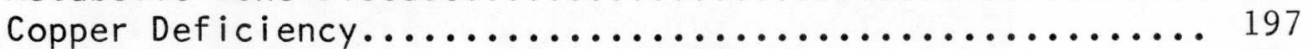

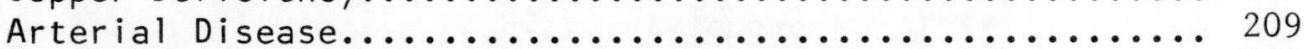

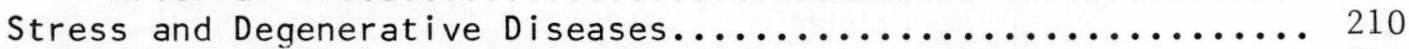

General Adaptive Syndrome...................... 210

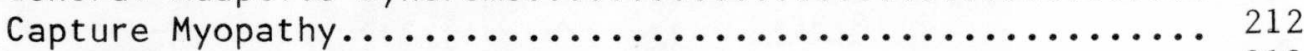

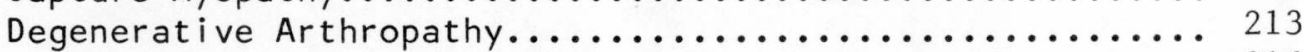

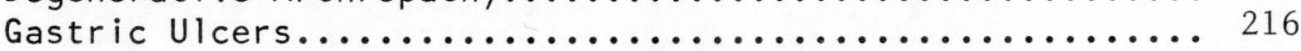


Diseases of Physical and Chemical Origin........................ 217

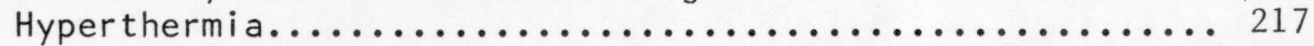

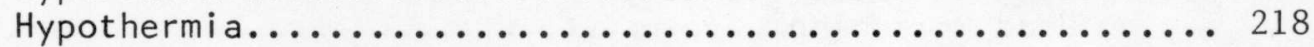

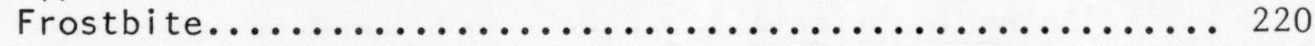

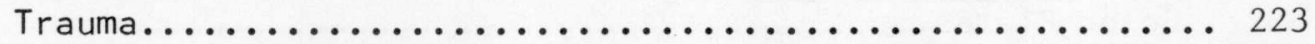

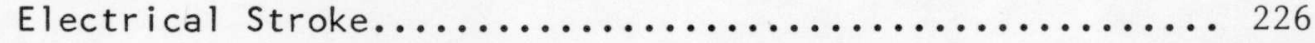

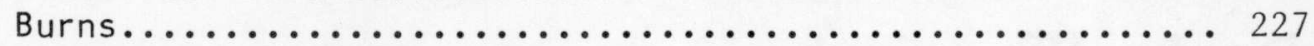

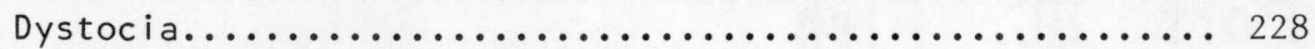

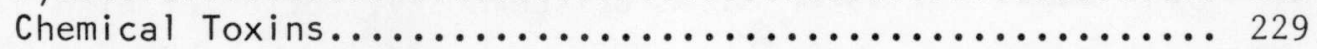

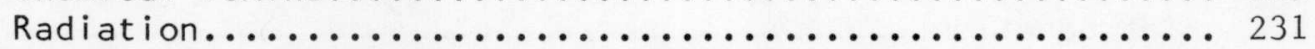

Congenital and Miscellaneous Non-Infectious Diseases.......... 234

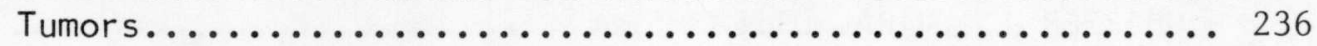

CHAPTER 5. MISCELLANEOUS DISEASES - Robert A. Dieterich,

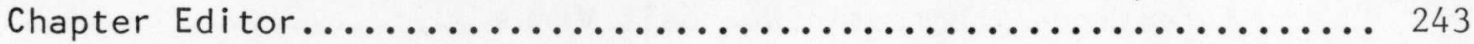

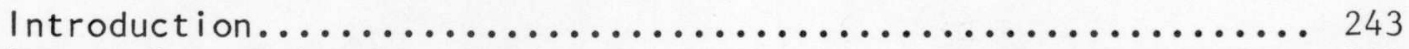

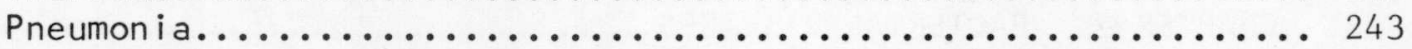

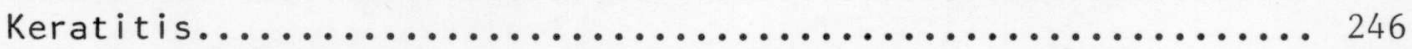

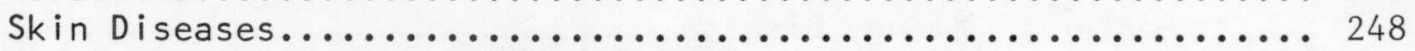

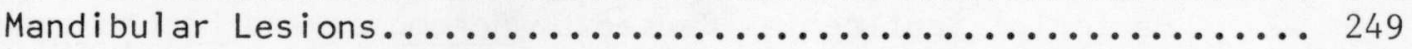

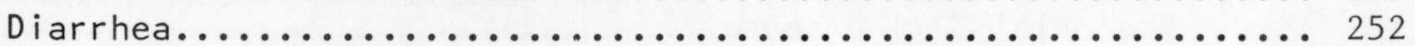

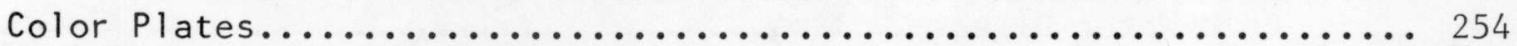

\section{Part II. Birds}

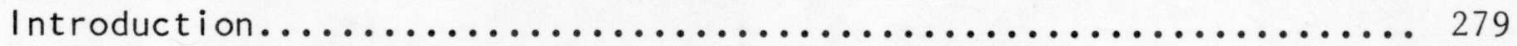

CHAPTER 1. VIRAL DISEASES - L. Michael Philo, Chapter Editor...... 281

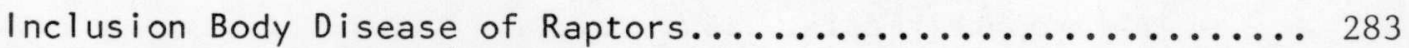

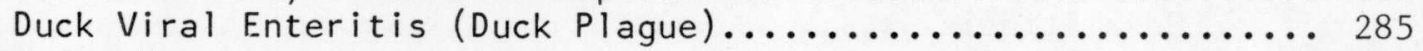

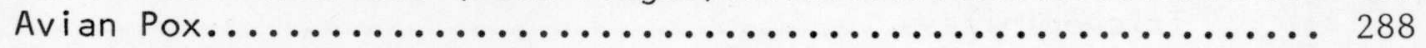

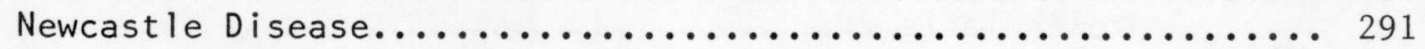

CHAPTER 2. BACTERIAL AND FUNGAL DISEASES - L. Michael Philo,

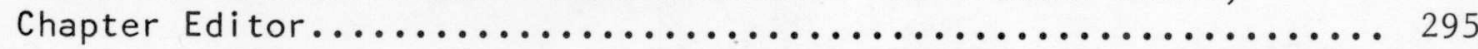

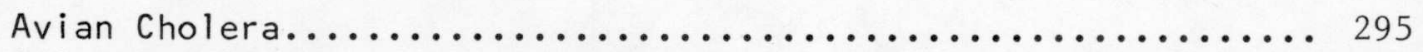

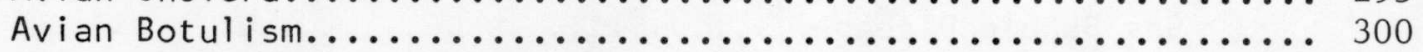

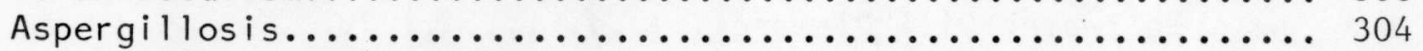

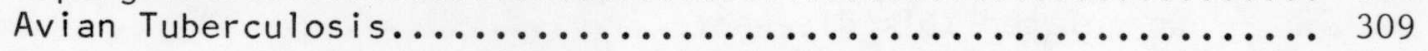

CHAPTER 3. PROTOZOAN BLOOD PARASITES - L. Michael Philo,

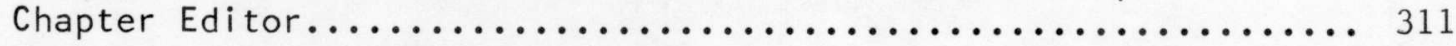

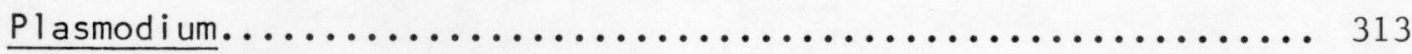

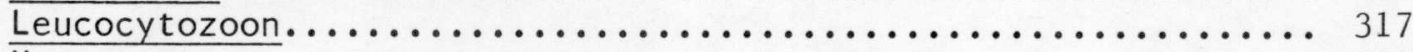

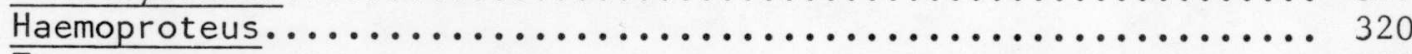

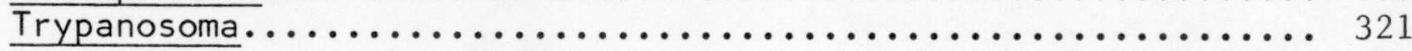


CHAPTER 4. MISCELLANEOUS CONDITIONS - Jim Dau, Chapter Editor.....

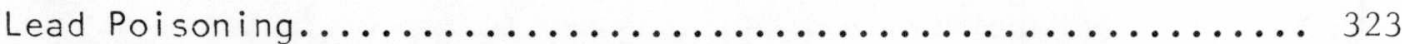

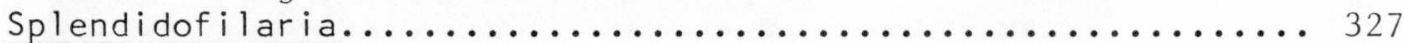

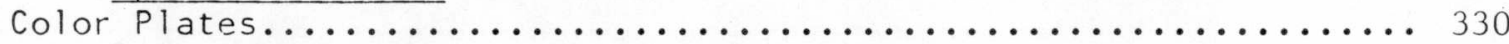

\section{Part II. Fish R. S. Grischkowsky, Editor of Fish Diseases}

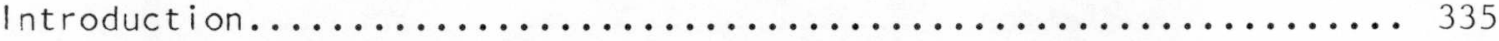

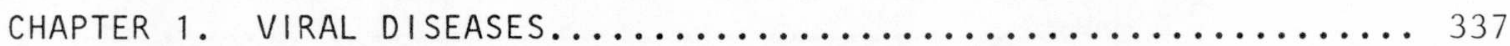

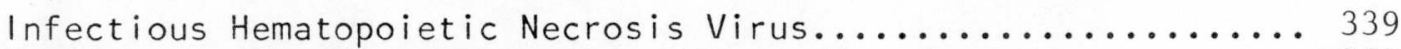

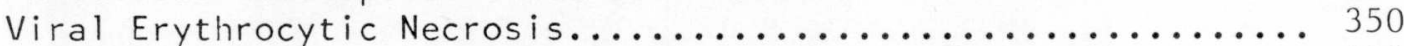

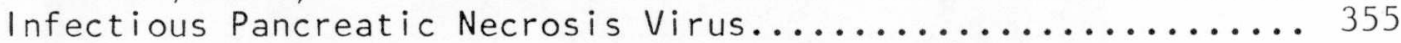

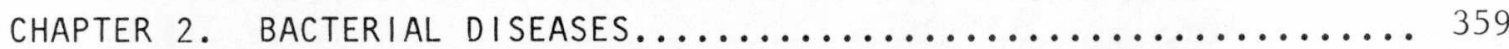

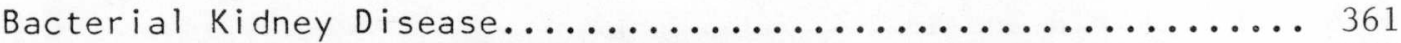

Bacterial Septicemias Caused by Aeromonas and Pseudomonas...... 368

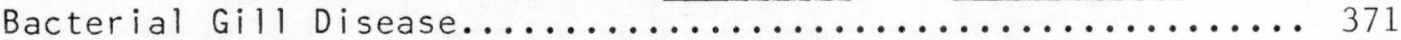

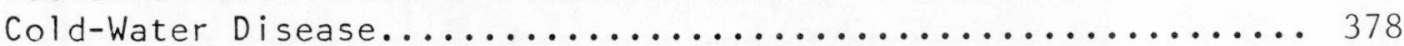

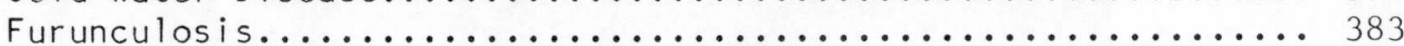

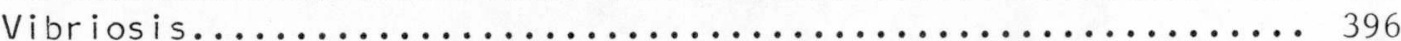

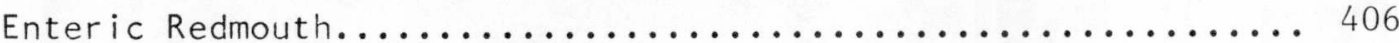

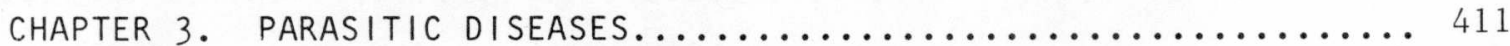

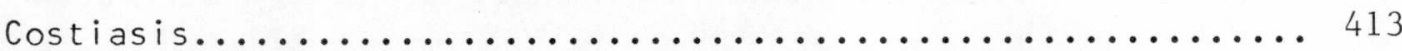

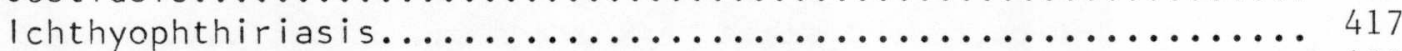

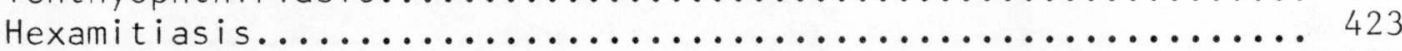

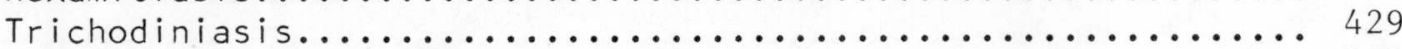

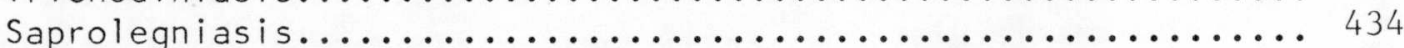

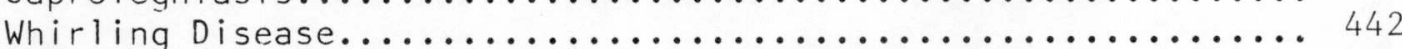

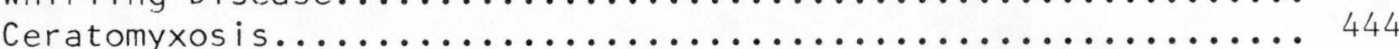

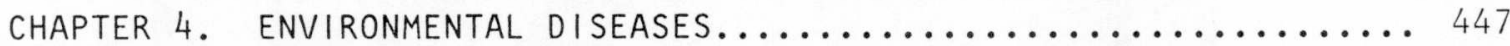

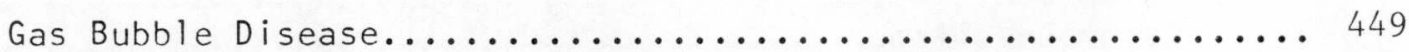

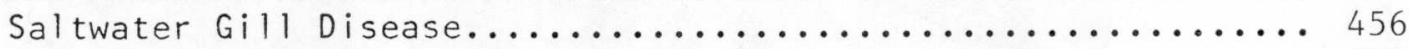

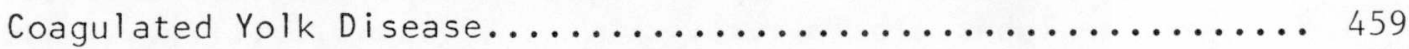

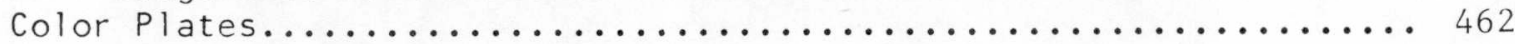


Part 五. Invertebrates R. Neve', Editor

Shellfish: Public Health Significance and Diseases........... 483

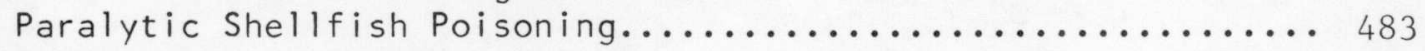

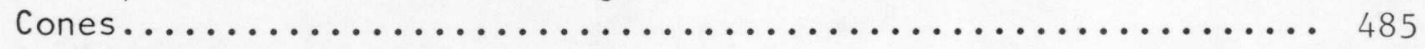

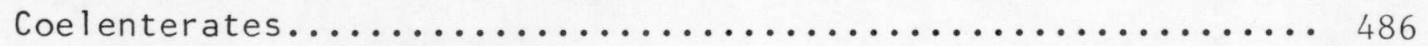

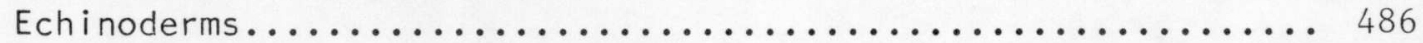

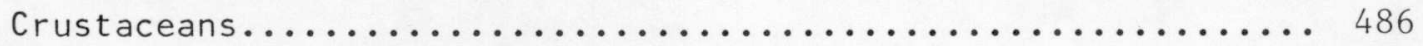

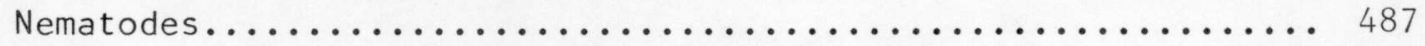

Appendix 1. Rabies Sample Collection.................... 489

Appendix 11. Specimen Collection and Preservation.............. 493

Appendix 111. Common and Scientific Names of Susceptible Species...... 501

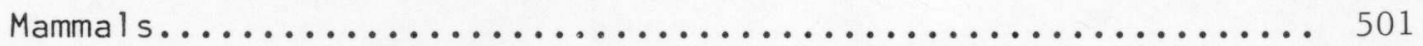

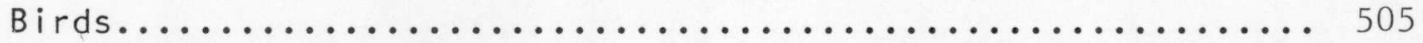

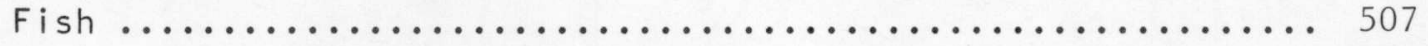

Appendix IV. Cross Reference.............................. 511

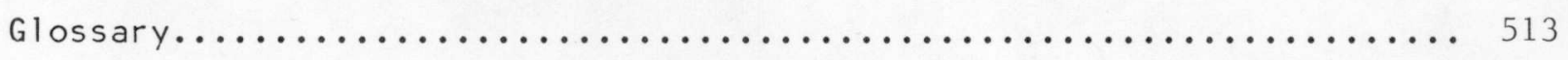


Part I

MAMMALS 
Chapter I.

\section{VIRAL DISEASES}




\title{
Introduction
}

\author{
Randall L. Zarnke
}

The significance of most wildlife diseases is hard to assess. In many cases, even such an elementary component of the epizootiology as the incidence of the disease in the host population(s) may be unknown. In cases where such information is available, we may attempt to evaluate the significance of the disease based upon it's impact on: (1) the wildlife resource (2) the domestic animal resource and (3) public health. Given these 3 parameters, certainly the most significant viral disease of wildlife in Alaska is rabies. Components of the epizootiology and control of rabies which lead to this ranking include: direct wildlife mortality (primarily foxes, Vulpes and Alopex spp.), domestic dog (Canis familiaris) and cat (Felis catus) mortality (and the at tempted prevention thereof), and human and public health considerations. The significance of the other diseases in the first portion of this section (contagious ecthyma, canine distemper, infectious canine hepatitis, San Miguel sea lion virus, respiratory viruses, feline panleukopenia and arboviruses) is not as clear. Based upon virus isolation and/or serologic techniques, we are aware of the presence of these agents in Alaska. In some cases (such as contagious ecthyma) there have been out- breaks of the disease which have prompted specific research projects that have provided necessary data to assess the significance of the disease. It appears that although ecthyma is present in muskox (Ovibos moschatus) and Dall sheep (Ovis dalii) populations, it is not a significant mortality factor except under unusual conditions. More commonly, however, little is known of the scope of diseases on a state-wide basis, or the impact of certain diseases on individuals or specific populations of wild animals. More research is needed before the significance of many diseases can be evaluated.

The diseases included in the second portion of this section (Aleu$t i a n$ diseases of mink, mink virus enteritis, transmissible mink encephalopathy, vesicular stomatitis, foot and mouth disease, epizootic hemorrhagic disease, bluetongue, pseudorabies and lymphocytic choriomeningitis) are not known to be present in Alaska at this time. They have been diagnosed in other parts of the world in species which are also found in Alaska. The possibility of transmission of these diseases to Alaska (however immediate or remote that possibility may be) warrants their inclusion. 


\title{
Rabies
}

\author{
D. Ritter
}

\section{History}

Rabies is one of the oldest diseases known to mankind. Sellers described what appears to be the first documentation of a quarantine in the Pre-Mosaic Eshnunna Code. This code predates the better known Code of Hammurabi of ancient Babylon of 2300 B.C. (Sellers 1954).

It is thought that rabies virus reported to be present in northern Asia, may have gained access to Alaska via the Bering Land Bridge 30,000 to 75,000 years ago (Williams 1949). Singleton pointed out that pre-European folklore of the Canadian Eskimo has many stories of a fox disease resembling rabies, which was transmitted to both the Eskimo and their dogs (Canis familiaris) (Singleton 1969). The first documentation of rabies in the arctic areas was during the 19th century when Nelson reported rabies, "a disease in sledge dogs of Greenland... to the Bering Sea" (Nelson 1887).

A disease of dogs resembling rabies was reported in 1859 and 1866 in northern Greenland. The causative agent was never isolated and clinical descriptions varied: "extreme exhaustion, hydrophobia, and weakness of the hindquarters... the affected dogs died without sign of excitation" (Wamberg 1960).

In 1907, Murie (1944) reported the outbreak of rabies in foxes (Vulpes vulpes) along the lower Kuskokwim River during the spring, "several" dogs bitten by the foxes died.

Ferenbaugh (1916) investigated a number of cases of hydrophobia that had occurred among foxes of the Yukon Delta during the spring of 1915. These foxes (referred to as "crazy foxes") came into the village, their mouths were hanging down and were dripping with foam and they attacked dogs. The dogs attacked by these foxes died 2 to 3 weeks later. Ferenbaugh also cited the first case of human rabies in Alaska in which a man was attacked by a sledge dog near Candle in February of 1914.

Epizootics also occurred in sledge dogs in the Canadian arctic, and were described by Elton from records supplied by the Hudson Bay Company in an effort by the company to ascertain the cause of the dog and fox disease. Elton described not only rabies, but also what later investigators felt to be canine distemper and infectious canine hepatitis (Elton 1931).

Williams was the first to demonstrate the presence of rabies virus in Alaska during the 1945-1947 epizootic. The disease was later found widely distributed over a major portion of Interior Alaska. Later, reports were received from along the Yukon, Kuskokwim, Porcupine and Black Rivers, at Salmon Fork, Old Crow, Central, Circle Hot Springs, Crooked Creek and Alatna. Subsequent spread westward occurred with reports from the Seward Peninsula and northward along the arctic coast to Kobuk during 1946-1947 (Williams 1949). There were 2 other human cases of rabies (Newcomb 1944).

Rausch summarized rabies distribution from 1949-1957. There was a residual rabies focus in the dogs residing in the Yukon River drainage as a result of the 1945-1947 epizootic. 
Prime areas of involvement were the western arctic coastal regions, and a focus within arctic fox (Alopex lagopus) at and around Nome during 1954. This epizootic then spread northward to Barrow. He also noted that in regions further south on the Alaska Peninsula, al though also treeless, rabies was infrequent. Rabies was present on the Peninsula but as sporadic cases. This sporadic prelude culminated in an epizootic which began in the upper Peninsula in February 1969 at Egegik (Rausch 1972). Subsequent reports of mixed red fox and dog rabies were received from Naknek, Pilot Point and thence southward to Cold Bay. During 1971, there were scattered reports of rabies in red fox over the entire Peninsula with a northern extension to Nondalton. There was 1 case of rabies in a land otter (Lutra canadensis), at Port Heiden and represents the 1 and, presently, only documented case in an Alaskan mustelid.

A rabies epizootic developed within the arctic fox population during 1972-1974, with reports from Barrow, Barter Island, Little Diomede, Point Hope, Nome, Deadhorse, Prudhoe Bay, Franklin Bluffs and Toolik Camp. As this arctic coast epizootic was declining, a number of red foxes and dogs were found rabid in the lower Kuskokwim drainage area centered around Bethel, and also at Shageluk on the Yukon River system. There was a single report of a positive red fox taken near the Air Force site at Takotna. As a result of the extensive outbreak at Bethel, a quarantine was declared March 1974 and remained in effect until July 1974.

Sporadic cases of rabies were reported from the Alaska Peninsula. During 1976, the epizootic expanded with many positive red foxes, i.e. 45 in 1976 versus 6 in 1975 (based upon virus isolation). A second quarantine was requested by the Bristol Bay Corporation.

Red fox constituted the majority of all rabid animals assayed during 1976 with $80 \%$; dogs $13 \%$; and a rare single case in cat (the second to date for Alaska) at Adak Island, an apparently introduced case. Conversely, the principal means of transmission to humans was the dog which was responsible for $87 \%$ of the human exposures. There were 70 human exposures: 65 were due to 7 dogs, 4 to 1 cat, (Felis catus) and 1 to a red fox.

The rabies epizootic peaked in April 1977 on the North Slope with virus isolation from 22 of 59 (37\%) arctic fox. Three wolves and 2 dogs were also positive. Ninety-three percent of the total positives were due to wild animals and $7 \%$ of the total were dogs; conversely, $11 \%$ of the human exposures were due to arctic fox and $89 \%$ of the exposures were due to dogs. The dogs were improperly vaccinated, not vaccinated at all, or vaccinated with an improper vaccine which induced rabies.

During the summer of 1977 , Chapman (1978) documented the rare occurrence of rabies in a wolf pack that he was observing. Prior to this epizootic in wolves, Rausch $(1972,1975)$ had documented 5 cases of rabies in Alaskan wolves. Two of the individuals from the 3 documented human cases of rabies in Alaska were bitten by wolves.

During 1978, there was a single positive red fox at Platinum and a caribou (Rangifer tarandus) south of Deadhorse. The observations of rabies in Rangifer is rare, with 2 cases in caribou (Barrow and Anak- 
tuvuk) and 1 in domestic reindeer (St. Lawrence Island). Sporadic cases of rabies occurred in dogs and arctic foxes on the North Slope. Alaska Department of $\mathrm{Fish}$ and Game wolf studies in northwestern Alaska revealed a 4-year-old male in poor nutritional condition. The wolf was rabid.

Through April of 1979, there have been positive arctic foxes at Barrow and Wainwright; 2 red foxes and 1 dog from Naknek and Egegik respectively. Public Health Service sanitarians and local people of the Bristol Bay community initiated a program of vaccination of dogs, elimination of stray dogs and reduction of carnivore attractants. Subsequent to this intense effort, there have not been any additional cases reported.

\section{Transmission}

Virus-laden saliva of a rabid animal may be introduced by a bite, scratch or other fresh break in the skin. Airborne spread to man has been demonstrated in caves near Frio, Texas but this is very rare. Roosting bats shed virus which was present in aerosols. Transmission from infected vampire bats to domestic animals is common in South and Central America, but in the United States the role of indigenous bats in transmission of rabies to other animals in the wild has not been established (Rausch 1977).

\section{Signs and Symptoms}

The incubation period in humans is usually 2 to 8 weeks and depends on the following: the extent of laceration, site of the wound, amount of virus introduced into the wound, absorption of viral material by pro- tective clothing, etc. In dogs and foxes the time ranges from 2 to 12 weeks. Exceptions in Alaska have been as long as 6 months and as short as 5 days.

Clinical manifestations in dogs, arctic fox and red fox consist of behavioral changes; i.e. they may appear to be tame or unafraid (so called "dumb rabies"), or they may become overly aggressive and attack dogs, man or any moving inanimate object (so called "furious" or mad rabies"). Pica, the eating of wood and stones, is sometimes encountered. Auto or self-mutilation is a rare occurrence in the fox, but neuritis is frequent, and the fox or dog will lick and chew on the affected part of the body. Other symptoms consist of voice modification, paralysis of the jaw muscles with loss of motor control of the lower jaw (hang-jaw), and salivation. These may be followed by lingual and facial paralysis. If the virus is introduced into an extremity, there may be a loss of motor control of the hindquarters which progresses until all 4 limbs are paralyzed. Death usually results from respiratory arrest.

\section{Pathogenesis}

Rabies virus enters somatic muscle tissue and absorption occurs. In experimental infections, Murphy noted that an early invasion of the site of entry takes place in striated muscle and upon the plasma membranes of muscle cells. The virus then advances to the peripheral and central nervous system (Murphy et al. 1973). Previous work had indicated virus presence in the peripheral nervous tissue as the only method of infection. Presently it appears that muscle is the likely source of virus for invasion of the peripheral ner- 
RABIES (RAGE, HYDROPHOBIA)

CAUSATIVE AGENT: Rabies Virus

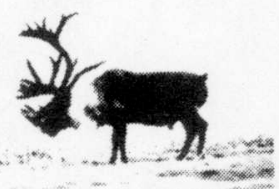

CAMIBCU ANE REINDEER

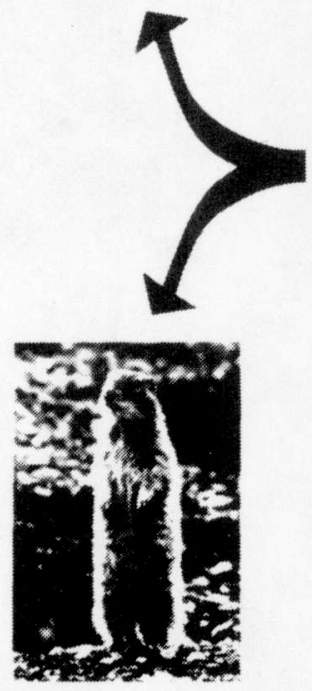

OTHER MAMMALS
THE PRINGIPAL RESERVOI OF RABIES VIRUS

IN ALASKA IS THE FOX

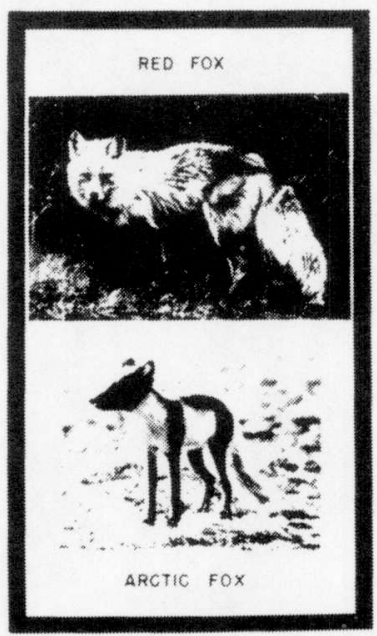

RABID ANIMALS ARE USUALLY CHARACTERIZED BY THEIR AND FEARLESSNESS

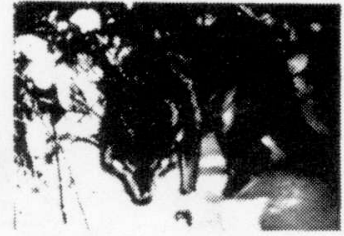

WOLF
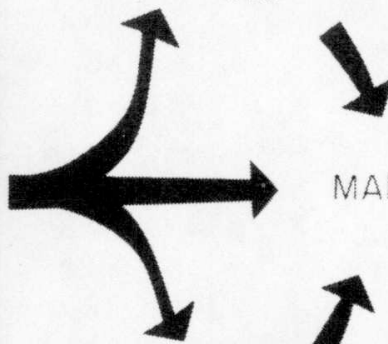

MAN

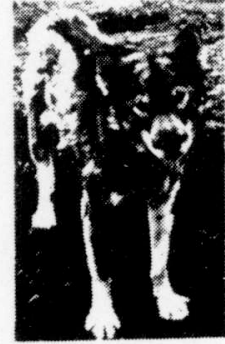

DOMESTIC MAMMALS DOG - CAT
OTMER MAMMALS EECON INFECTEO HEN BITIEA B A RABBC ANIMAOA ANC WA INFEC T ANE ANDTMER AN

ME VIRUS $Q$ TKANSUITTO VIA THE SALNA, AM IS INJECTED INTO TME OF THE RABID ANIMAL.

RABIES IS A MSEASE CO THE CENTRAL NERYOUS IN NOST NAMBALES 
vous system. Intramuscular harborage or extra-neural infection may account for long incubation periods with rabies, whereas relatively short incubation periods would indicate direct infection of peripheral nervous $t$ issue. The virus replicates in muscle, connective tissue, or in nerves at the site of exposure, and may remain undetectable within infected tissue for weeks or months before moving up the associated nerve.

There is presently no information available as to what form the virus assumes during these long periods nor its relation to adjacent tissue. This characteristic, i.e. viral infection of muscle tissue, is of importance in post exposure rabies prophylaxis, since efficacy of treatment depends on accessibility to the virus by the host's immune system before it enters the nervous system. Once in the nervous system, the virus is not affected by humoral antibody and is free to move either passively through associated spaces of the nerve or via interstitial nerve spaces. The spread of rabies infection within the central nervous system (CNS) appears to take place by several routes.

A. Active spread--viral multiplication in brain, spinal cord and cells of the CNS.

B. Passive spread--transport of the virus in the cerebral spinal fluid.

This multiple route provides more than 1 pathway to the brain, and $u 1-$ timately to the point of excretion for further passage to other hosts. The viral pathway via the trigeminal nerves that originate near vital centers provide a viral pathway to the salivary glands. As the virus makes this outward passage, the host becomes irritable and aggressive. The correlation of these 2 functions creates the precise conditions necessary for rabies infection to cycle successfully through the centuries, i.e. virus replication in the brain results in altered behavior, such as unprovoked attacks. Presence of the virus in saliva during this period of altered behavior, allows transmission to a new host. The many CNS pathways available for rabies virus makes it difficult to stop the infection once the virus has reached the brain or spinal cord. Antibody or other viral inhibiting agents presently available cannot stop the intracelluar virus replication without also stopping vital cell function, i.e. they are unable to kill the virus without killing the host cells as well. The only present control of the infection is while the virus is present and multiplying outside of the CNS. This may be accomplished by induction of interferon together with vaccination and administration of hyperimmune serum.

\section{Diagnosis} Prior to the availability of
biological, histochemical and
fluorescent antibody techniques, the
necropsied stomachs of animals
suspected of having rabies were
checked for wood, stones and inanimate objects. Later, nervous animals and introduced into laboratory animals (biological test). In 1903, Negri reported the unique presence of inclusion bodies in nervous material of rabid animals, and another diagnostic tool was obtained in the detection of rabies virus. This technique was widely used until the advent of fluorescent antibody methods by Coons in 1941. 
The use of direct fluorescent antibody technique as a diagnostic tool occurred during the 1960s. This technique, briefly, consists of chemically linking the fluorochrome (a compound capable of fluorescing when properly stimulated) to an antibody developed against rabies virus. Impression smears taken of the CNS are mixed with the fluorochrome. An ultraviolet source of light provides the energy to excite the fluorochrome. When viewed through a microscope, the complex between rabies virus and the antibody-fluorochrome emits light.

\section{Significance and Control}

Rabies is a viral disease which affects the central nervous system and can infect all warm-blooded animals including man. In Alaska, rabies is always present in a small number of arctic and red fox and periodically, as the fox population increases, rabies becomes widespread within coastal regions of northern and western Alaska and the Alaska Peninsula. The virus is most frequently transmitted through the bite of a rabid animal, but can also be transmitted through exposure of an open cut or scratch to virus-laden saliva. Once clinical symptoms appear, the disease is almost always fatal.

Rabies infections in arctic and red foxes are seasonal with higher frequencies during the months of November through March. Younger animals and male foxes, who range further for food, are most frequently infected. Periodic population increases in the numbers of foxes occur in 3 to 4 year cycles. Rabies may cause declines in fox populations.

The arctic fox is the enzootic host for rabies, and distribution is presently along the north and west coasts of Alaska. The red fox perpetuates rabies along the lower Kuskokwim River drainage, Bristol Bay region and along the Alaska Peninsula, southward to Cold Bay.

Control measures via population reduction, as employed in Europe and the United States, when applied to a land area the size of Alaska, would be a massive undertaking. Population reduction employs trapping, shooting, gassing and poison baits. To be effective, $65-75 \%$ of the animals must be removed in order to control the disease for more than 1 generation or season (Crandell 1975). Disease control via population reduction must also consider the obvious economic importance of the furbearer.

Control measures by use of a "wildlife" vaccine may in the future, offer an alternative. This technique employs a "bait vaccine." To be practical, the vaccine must be safe for all animals; it must provide immunological protection for the "target" animal; it must attract the fox; must be stable and potent while in the field locale; and must not cause disease in non-target species which accidentally consume the bait (Winkler and Baer 1976, Winkler et al. 1975, 1976). Vaccines of this type are presently undergoing extensive testing and have resulted in the infection of 1 microbiologist. If this type of vaccine, after extensive testing, is proven to be safe, it might provide a future answer to the control of fox rabies.

Control measures, which are presently available, reside in the care of domestic animals owned by man. Interruption of virus transmission from fox to dog and dog to man is possible by vaccination of the dog, elimination of stray animals, restraint of owned animals and the elimination of carnivore attractants. 
The State of Alaska, formerly the Alaska Division of Natural Resources, and presently the Alaska Division of Public Health, routinely utilize a modified live-virus vaccine, High Egg Passage (HEP) which is produced by Norden Laboratories (Endurall-R). This further attenuated vaccine has been found to be very potent and safe for dogs and cats. Vaccine of this type is very effective. It should be noted that dogs vaccinated before 6 to 6.5 months of age must be revaccinated at 12 months. Alternate vaccine choices where doubt is involved are the inactivated vaccines.

Where questions arise concerning proper vaccines, consult a licensed, practicing veterinarian, or refer to the National Association of State Public Health Veterinarians, Inc., Compendium of Animal Vaccine in the United States.

\section{References}

CIFUENTES, E., E. CALDERON and G. BIJLENGA. 1971. Rabies in a child diagnosed by a new intravitam method: the cornea test. J. Trop. Med. Hyg. 74:23-35.

CHAPMAN, R. G. 1978. Rabies:decimation of a wolf pack in Arctic Alaska. Science 201:365-367.

CRANDELL, R. A. 1965. Laboratory investigation of arctic strains of rabies virus. Acta Pathol. Microbiol. Scand. 63:587-596.

- 1975. Arctic fox rabies. History of Rabies. Vol. II G. M. BAER, ed. Academic Press, Inc., New York, NY.

DEAN, D. J. and M. K. ABELSETH. 1973. The fluorescent antibody test. pp. 73-84. In: Laboratory Techniques in Rabies. 3rd ed. M. M. KAPLAN and H. KOPROWSKI, ed. WHO, Geneva, Switzerland.
ELTON, C. 1931. Epidemics among sledge dogs in Canadian Arctic and their relation to disease in the arctic fox. Can. J. of Res. 58:673-692.

FERENBAUGH, T. L. 1916. A note concerning the occurrence of hydrophobia in the foxes of Alaska. Military Surgeon 38:656-657.

JENKINS, M. and K. WAMBERG. 1960. Rabies discovered in Greenland. J. Amer. Vet. Med. Assoc. 137:183.

LARGHI, 0. P., E. L. GONZALEZ and J. R. HELD. 1973. Evaluation of the corneal test as a laboratory method for rabies diagnosis. Appl. Micro. 25:187-189

MURPHY, F. A., S. P. BAUER, A. K. HARRISON and C. W. WASHINGTON. 1973. Comparative pathogenesis of rabies and rabies-like viruses, viral infection and transit from inoculation site to the central nervous system. Lab. Investigations 28:361-376.

MURIE, A. 1944. The wolves of Mt. McKinley. U.S. Dept. Interior, Nat1. Park Service, Fauna Ser. No. 5. $238 \mathrm{pp}$.

NELSON, E. W. 1887. Report upon natural history collections made in Alaska between the years 1877 and 1881. Arctic Ser. No. 3. U.S. Army Signal Serv. Washington, DC. 337 pp.

NEWCOMB, T/5. 1944. Wolf attack. A report. 183rd Station Hospital, APO No. 942, U.S. Army, 20 November 1943.

PLUMMER, P. J. G. 1947a. Preliminary note on arctic dog disease and its relationship to rabies. Canad. J. Comp. Med. $11: 154-160$.

- 1947b. Further note on arctic dog disease and its relationship to rabies. Canad. J. Comp. Med. 11:330-334.

RAMSDEN, R. 0 . and D. H. JOHNSTON. 1975. Studies on the oral infectivity of rabies in carnivora. J. Wildl. Dis. $11: 318-324$. 
RAUSCH, R. L. 1958. Some observations on rabies in Alaska with special reference to wild canidae. J. Wildl. Manage. 22:246-260.

1972. Observations on some natural focal zoonoses in Alaska. Arch. Envir. Health 25:246-252.

1975. Rabies in experimentally infected bears, Ursus spp. with epizootiologic notes. Zbl. Vet. Med. B. 22:420-437.

- 1977. Rabies in Alaska prevention and control: USDHEW, USPHS, Arctic Health Research Center, Report No. III. 22 pp.

SCHNEIDER, L. G. 1969. The cornea test; a new method for the intravitam diagnosis of rabies. Fed. Res. Inst. Animal Virus Dis. Tubingen, Germany 16:24-31.

SELLERS, T. F. 1954. Rabies. pp. 1106-1109. In: Principles of $\frac{\text { Internal }}{\text { HARRISON, ed. McGraw-Hill, }}$ New York, NY.

SIKES, R. K. 1968. Arctic rabies. Arch. Environ. Health 17:622-626.

SINGLETON, J. R. 1969. History of rabies in Canada. State Vet. J. 24:205- 209 .
SMITH, W. B., D. C. BLENDEN, TSU-HUEI FUH, L. HILER. 1972. Diagnos is of rabies by immunofluorescent staining of frozen section of skin. J. Amer. Vet. Med. Assoc. $161: 1495-1501$.

WAMBERG, K. $1960 . \quad$ Nord. Veterinaermed. 12:769.

WILLIAMS, R. B. 1949. Epizootic of rabies in interior Alaska 1945-47. Canad. J. Comp. Med. 13:136-143.

WINKLER, W. G. 1975. Fox rabies. pp. 3-22. In: The Natural History of Rabies. Vol. IT.G. M. BAER, ed. Academic Press, Inc., New York, NY.

WINKLER, W. G. and G. M. BAER. 1976. Rabies immunization of red foxes (Vulpes fulva) with vaccine in sausage baits. Amer. J. Epidem. $103: 408-415$.

WINKLER, W. G., R. G. MCLEAN and J. C. COWART. 1975. Vaccination of foxes against rabies using ingested baits. J. Wildl. Dis. $11: 382-388$.

WINKLER, W. G., J. H. SHADDOCK and L. W. WILLIAMS. 1976. Oral rabies vaccine: evaluation of its infectivity in three species of rodents. Amer. J. 


\title{
Contagious Ecthyma
}

\author{
Randall L. Zarnke
}

\section{Introduction}

Contagious ecthyma is caused by a virus which is a member of the paravaccinia subgroup of pox-viruses. Hosts known to be susceptible to infection under natural conditions include: domestic sheep (Ovis aries) (Erickson et al. 1974), domestic goats (Capra hircus) (Linnabary et al. 1976), Dall sheep (Ovis dalli), bighorn sheep (Ovis canadensis) (Blood 1971, Connell 1954, Samuel et al. 1975), mountain goat (Oreamnos americanus) (Carr 1968, Hebert et al. 1977, Samuel et al. 1975), chamois (Rupicapra rupicapra) (Daniel and Christie 1963), thar (Hemitragus jemlaicus) (Daniel and Christie 1963, Hebert et al. 1977), feral goats (Capra hircus) (Daniel and Christie 1963), muskox (Ovibos moschatus) (Bell 1931, Falk 1978, Palmer and Rouse 1963), domestic dog (Canis familiaris) (Wilkinson et al. 1970), domestic cat (Felis catus) (Grishaev and Shchepetova 1970), Merino sheep (Ovis aries) (Kumar et al. 1974), reindeer (Rangifer tarandus) (Samuel et al. 1975) and humans (Homo sapiens) (Beck and Taylor 1974, Carr 1968, Erickson et al. 1974, Falk 1978, Moore 1973). Moose (Alces alces) and caribou (Rangifer tarandus) have been experimentally infected (unpublished data, University of Alaska and Alaska Department of Fish and Game).

\section{History}

Infection of domestic sheep and goats by contagious ecthyma virus is common worldwide (Beck and Taylor 1974). Overt disease is most common and severe in younger animals (Ah- luwalia et al. 1974, Blood 1971, Falk 1978). Mortality as a result of infection is low unless complicated by secondary bacterial infection (Falke 1978). The disease was first diagnosed in wildlife by Connell in 1953 when lesions were observed on the lips and muzzles of bighorn sheep in western Canada (Connell 1964). Prior to this time, lesions resembling those of ecthyma were observed in captive muskox and Dall sheep in Alaska during 1930 (Bell 1931). There were no deaths. A lamb Dall sheep with similar lesions was found dead near Healy, Alaska in 1964 by personnel of the Alaska Department of Fish and Game. Whether the animal died of ecthyma infection is not known. Epizootics of the disease occurred among captive Dall sheep near Fairbanks and captive muskox at Fairbanks and Unalakleet in 1976 and 1977. Two Dall sheep lambs were euthanatized because of severe lesions. Two other lambs in the same pen had only minor lesions. No losses occurred in the muskox at Fairbanks. Six of 39 infected muskox died at Unalakleet.

An unconfirmed case of the disease was reported in a man in southeastern Alaska following a hunting expedition during which he dressed and skinned 2 mountain goats (Carr 1968).

\section{Transmission}

Contact with contaminated articles is believed to be the most common means by which ecthyma is transmitted (Falk 1978). Virus is often present in the scab-like lesions which are present late in the course of the disease. As the lesions heal, this 
scab material is shed. The virus is quite stable when thus protected, and can contaminate the surrounding area (food, pasture and structures such as trees or fences) (Falk 1978). The portal of entry for the virus may be any mucous membrane or abraded area and is most commonly the oral mucosa (Falke 1978). Virus can be transmitted from mother to offspring, or vice versa, during nursing.

\section{Signs, Symptoms and \\ Pathogenesis}

In most species, visible lesions follow this established sequence:

1. Macule (discolored spot), not elevated, usually 1-3 $\mathrm{mm}$ in diameter.

2. Papule (red bump), elevated, usually $1-5 \mathrm{~mm}$ in diameter.

3. Vesicle (blister), size varies.

4. Pustule (pus-filled blister), size varies.

5. Crust (scab), 1-100 mm, usually darkly pigmented.

Incubation period is 3 to 7 days in sheep (Falk 1978).

Because of the size and proliferative appearance, the most often recognized stage of the above sequence is the crust or scab. Lesions may occur on the lips, muzzle, udder, coronary band of the foot, or around the eyes (Blood 1971, Samuel et al. 1975). They may be present for 1 to 4 weeks and usually heal without leaving scars (Falk 1978). The affected area becomes sensitive to touch, and lesions in the area of the mouth may prevent an animal from eating (Samuel et al. 1975). This is especially serious in the young animals. Lesions on the feet may result in lameness (Blood 1971).

\section{Diagnosis}

Preliminary diagnosis is usually based upon gross observation of the scab-like lesions. Laboratory confirmation can be provided by:

1. Transmission of the disease to susceptible hosts (usually domestic sheep) (Connell 1954, Samuel et al. 1975).

2. Isolation of the virus in tissue culture systems (Falk 1978).

3. Electron microscopic observation of the virus in host tissue (Falke 1978, Harkness et al. 1977).

4. Histopathologic examination of affected tissue (Samuel et al. 1975).

5. Demonstration of a significant rise in serum antibody levels to ecthyma virus (Falk 1978).

Suggested specimen collection procedures for the field worker include: (1) freeze a portion of a lesion for transmission and/or isolation tests, and (2) preserve a portion of a lesion in $10 \%$ formal in for histopathologic investigation.

\section{Significance and Control}

High serum antibody prevalence in Dall sheep in the Dry Creek (near Healy) and Sheep Creek (near Tok) areas indicates that these populations have ample exposure to, and transmission of the disease. There was low prevalence in domestic sheep 
and goats along the Alaska Highway. These results fuel questions as to which species represent threatening reservoirs of infection for other species. Based upon presently available data, it is not possible to satisfactorily answer such questions.

Given the very low incidence of observations of diseased animals in the wild, it is tempting to assume that this disease is not a significant source of mortality in Dall sheep. On the other hand, the death of 2 captive Dall sheep lambs shows that mortality can occur. This latter occurrence may have been a result of the animals being held in captivity and being underweight at birth.

Serum samples from animals with a known history of CE exposure are only available for 5 Dall sheep. All of these animals had been infected approximately 10 months prior to the time of sampling. For 4 of the sheep, antibody titers had fallen to undetectable levels. The other however, remained high $(1: 256)$. Based upon these limited data, immunity in Dall sheep appears to be variable as is the case in domestic sheep.

An equally complicated situation may exist in relation to muskox. Of 5 wild muskox collected by sport hunters on Nunivak Island, none had serologic evidence of past infection with ecthyma. Of 9 animals held captive at Unalakleet, none showed serologic evidence of past infection, although 4 of the 9 were known to have been infected within 2 years prior to the time of sampling, 3 of these within 1 year. This suggests that immunity is short-lived in this species. There have also been 2 epizootics of the disease in a captive herd in Fairbanks. It does not seem likely that the outbreaks in captive animals (either Dall sheep or muskox) were a result of increased density. Both of these species are herd animals, and live in close proximity to other members of the herd. Such densities could be expected to exacerbate symptoms of the disease if density were the sole determining factor. Other predisposing factors may have been of greater importance than density. The 2 lamb Dall sheep which died in captivity at Fairbanks were underweight. Two normal weight lambs in the same pen had only minor lesions. The muskox facilities at Unalakleet were substandard. The pens were muddy and had areas of standing water. It may be hypothesized that normal, healthy animals in favorable surroundings experience only mild lesions when exposed to the disease. On the other hand, animals which have been subjected to influences which are in opposition to ideal husbandry practices (malnutrition, poor sanitary conditions) may experience more severe cases of the disease. Careful visual examination for signs of disease of captive animals is to be encouraged.

In order to determine the susceptibility of moose and caribou to the disease, a single individual of each species was inoculated with the virus. A domestic sheep served as a control. All 3 animals developed typical lesions and diagnostic levels of serum antibody. In lieu of data on an adequate number of experimental animals, the significance of these findings to the natural situation are unknown. Serologic surveys of these species in the wild and larger scale experiments will hopefully clarify the importance of CE in wild populations.

To reduce the possibility of transmission between domestic sheep 
or goats and mountain goats or Dall sheep, it is prudent to curtail the practice of allowing domestic species in high elevations or any area where contact with game species may occur.

The reported case of ecthyma in a hunter, derived from a mountain goat which he had shot, indicates that the disease does occur in these species in Alaska (Carr 1968). Because more extensive data are not available, any further comments are unwarranted other than to suggest that all persons who handle animals be aware of the possibility of contracting this and other diseases, and provide themselves with adequate protection (physical and/or immunological). Immunity in humans is believed to be long-lasting (Carr 1968).

In all cases of disease (whether human or animal), the only treatment is to prevent or combat secondary infection of the ecthyma lesions. Lesions usually heal without leaving a scar, and therefore traditionally it has been suggested not to lance the proliferative scabs (Beck and Taylor 1974). A recent article contradicts this belief, and suggests lancing of all lesions (Falk 1978).

\section{References}

AHLUWALIA, J. S., A. N. SINGH and B. K. SINHA. 1974. Age susceptibility of contagious pustular dermatitis in sheep. Ind. J. Anim. Res. 8:69-71.

BECK, C. C. and W. B. TAYLOR. 1974. Orf: it's awrful! Vet. Med. Sm. Anim. Clin. 69:1413-1417.

BELL, W. D. 1931. Experiments in re-establishing muskoxen in Alaska. J. Mammal. 12:292-297.
BLOOD, D. A. 1971. Contagious ecthyma in Rocky Mountain bighorn sheep. J. Wildl. Manage. $35: 270-275$.

CARR, R. W. 1968. A case of orf (ecthyma contagiosum; contagious postular dermatitis) contracted by a human from a wild Alaskan mountain goat. Alaska Med. 10:75-77.

CONNELL, R. 1954. Contagious ecthyma in Rocky Mountain bighorn sheep. Can. J. Comp. Med. $18: 59-62$.

DANIEL, M. J. and A. H. C. CHRISTIE. 1963. Untersuchungen uber krankheiten der gemse (Rupicpra rupicapra L.) und des thars (Hemitragus jemlaicus Smith) in den sudalpen von Neuseeland. Schweiz. Arch. Tierheilk. 105:399-411.

ERICKSON, G. A., E. A. CARBREY and G. A. GUSTAFSON. 1974. Diagnos is of contagious ecthyma: a disease of sheep and man. J. Am. Vet. Med. Assoc. 165:737-738.

1975. Generalized contagious ecthyma in a sheep rancher: diagnostic considerations. J. Am. Vet. Med. Assoc. 166:262-263.

FALK, E. S. 1978. Parapoxvirus infections of reindeer and muskox associated with unusual human infections. Brit. J. Derm. $99: 647-654$.

GRISHAEV, N. E. and N. I. SHCHEPETOVA. 1970. Biological test on cats for the diagnosis of ovine contagious ecthyma. Veterinariya, Moscow 10:112-113.

HARKNESS, J. W., A. C. SCOTT and C. N. HERBERT. 1977. Electron microscopy in the rapid diagnosis of orf. Brit. Vet. J. 133:81-87. HEBERT, D. M., W. M. SAMUEL and G. W. SMITH. 1977. Contagious ecthyma in mountain goat of British Columbia. J. Wildl. Dis. $13: 135-136$. 
KARSTAD, L. H. 1970. Contagious ecthyma. pp. 171-172. In: Infectious Diseases of Wild Mammals. J. W. DAVIS, L. H. KARSTAD and D. 0. TRAINER, eds. Iowa State Univ. Press, Ames, IA.

KATER, J. C. and N. F. HANSEN. 1962. Contagious ecthyma in wild thar in the South Island. N. Z. Vet. J. $10: 116-117$.

KUMAR, P. N., S. V. PACHALAG and S. K. CHATTOPADHYAY. 1974. A note on fatal contagious ecthyma in recently imported adult Russian Merino sheep. Ind. Vet. J. $51: 725-728$.

LINNABARY, R. D., H. S. POWELL, M. A. HOLSCHER and B. K. WALKER. 1976. Contagious ecthyma (orf) in a goat herd. Vet. Med. Sm. Anim. Clin. $71: 1261-1263$.
MOORE, R. M., Jr. 1973. Human orf in the United States, 1972. J. Inf. Dis. 127:731-732.

PALMER, L. J. and C. H. ROUSE. 1963. Muskoxen investigations in Alaska 1930-1935. Dept. Interior, Bur. Sport Fish. and Wildl.

SAMUEL, W. M., G. A. CHALMERS, J. G. STELFOX, A. LOEWAN and J. J. THOMSEN. 1975. Contagious ecthyma in bighorn sheep and mountain goat in western Canada. J. Wildl. Dis. 11:26-31.

WILKINSON, G. T., J. PRYDIE and J. SCARNELL. 1970. Possible "orf" (contagious pustular dermatitis, contagious ecthyma of sheep) infection in the dog. Vet. Rec. $87: 766-767$.

\title{
Canine Distemper
}

\author{
Randall L. Zarnke
}

\section{Introduction}

Canine distemper (CD) is an acute, febrile, highly contagious viral disease (Budd 1970). It may occur in members of the Canidae, Mustelidae, Procyonidae, and Viverridae (Gorham 1966). The causative agent of the disease is a myxovirus which is closely related to the viruses which cause measles and rinderpest (Budd 1970). In a population of susceptible hosts, the disease may exhibit both high morbidity and high mortality (Gorham 1966).

\section{History}

Based upon a layman's description of signs of the disease, canine distemper is believed to have been present in Europe since early times (Budd 1970). The disease is presently distributed worldwide in domestic dogs (Canis familiaris) (Budd 1970). The Alaska StateFederal Laboratory which is located in Palmer reported 122 cases of CD in domestic dogs during 1977, 119 cases during 1978 and 190 cases during 1979 (R. Barrett, pers. comm.). These 
diagnoses were based upon clinical signs of the disease and were in some cases substantiated by isolation of the CD virus. The disease was first described in the wolf (Canis lupus) in 1904, coyote (Canis latrans) in 1916, red fox (Vulpes fulva) in 1921, and arctic fox (Alopex lagopus) in 1945 (Budd 1970). Diagnosis based upon clinical signs revealed that $C D$ killed approximately one-half of 1000 dogs at Barrow in 1951-52 (Reinhard et al. 1955). Arctic foxes also died of CD during this epizootic (Rausch 1953). During the 1970s in Alaska, $C D$ has been diagnosed in 2 wolves from the Kenai National Moose Range, a red fox from the Solomon area, another red fox from Tetlin village, a domestic dog from Northway (D. Ritter, pers. comm.) and ranch fox from Fairbanks (R. Dieterich, pers. comm.). These diagnoses were based on fluorescent antibody tests and/or direct isolation of the CD virus. Tissue culture neutralization tests revealed $C D$ infection during the mid-1970s in $2 / 31$ wolves from the Tanana Flats area located south of Fairbanks, 2/16 wolves from the $\mathrm{Nel}$ china River basin, and $0 / 5$ wolves from the northwestern portion of Alaska (Ritter and Stephenson, ms. in prep.). Canine distemper neutralizing antibody was found in 2 of 86 wolves which were collected in the Mackenzie District, Northwest Territories, Canada (Choquette and Kuyt 1974). Three of five wolf pups held captive at Alaskaland in Fairbanks were clinically diagnosed as having died of CD in 1977 (R. Dieterich, pers. comm.).

\section{Transmission}

In an infected individual, the virus often localizes in mucous membranes of the gastrointestinal and/or urogenital tracts (Budd 1970). Nasal and conjunctival exudates of ten contain virus, whereas the virus has not been shown to be shed in urine or feces (Budd 1970). Natural transmission of the virus commonly occurs via direct contact or aerosols (Budd 1970). Transplacental transmission of the virus has been documented in domestic dogs (Krakowka et al. 1977). Increased fox population densities are believed to result in increased transmission of CD (Parker et al. 1961).

\section{Signs, Symptoms and \\ Pathogenesis}

The red fox is the only wild animal for which adequate information concerning signs, symptoms and pathogenesis is available. The following description is derived primarily from this species (Budd 1970). Unfortunately, signs, symptoms and pathogenesis are variable in the fox. Fever often begins as early as the third day after infection, lasts 1 to 3 days, and may exhibit a diphasic character. During the acute stage of the disease, an animal may exhibit photophobia, aimless wandering, loss of fear, thirst, listlessness, labored breathing (dyspnea) and loss of appetite (anorexia). Such signs may lead to a mistaken preliminary diagnosis of rabies. Other signs include redness (hyperemia) around the lips and eyes, pusfilled (purulent) discharge from the eyes and nose, diarrhea, cough and emaciation. The animal may succumb at the height of clinical signs. Another individual may appear to recover, only to die later with a sudden onset of convulsions. The course of the disease may be short and result in either recovery or death, or may be prolonged for months with periods of abatement followed by relapse. Although $C D$ causes no distinctive lesions, post mortem examination may reveal an enlarged spleen and/or congestion of the lungs (Anonymous 1973, Pinkerton et al. 
1945). Intramuscular, acidophilic inclusion bodies in the central nervous system (Gillespie and Rickard 1956) and demyel inating encephalitis (Pinkerton 1940) have been observed. Mortality rates for juveniles appear to be higher than those for adults (Gier and Ameel 1959).

\section{Diagnosis}

Perhaps the easiest and most reliable means of diagnosis is the fluorescent antibody test, which detects viral particles in tissue or blood (Anonymous 1973). Another effective technique is to test acute and convalescent sera by means of the neutralization (Appel and Robson 1973) or complement fixation tests (Anonymous 1973). A significant increase in antibody titer is considered diagnostic. Inclusion bodies may be observed in blood smears and epithelial cells from tongue and conjunctivae when stained with Schorr's hematoxilin and eosin (Budd 1970). Clinical signs may be helpful in diagnosis, but (as mentioned above) may be confused with those of other diseases.

Another diagnostic method involves maceration of lung and/or spleen tissue from the animal suspected of distemper, followed by inoculation of a susceptible ferret (Mustela putorius) with the homogenate. If after 30 days the ferret exhibits no gross or histologic signs of $C D$, the suspect animal may be declared free of the disease (Budd 1970).

\section{Treatment}

An effective vaccine is available for ranch mink (Mustela vison) (Budd 1970). The standard canine vaccine is safe and effective in protecting fox and wolves from CD. Hyperimmune canine antiserum is also available, but its effectiveness in combating the disease in wild species is unknown. In addition, both of these methods are of questionable value once clinical signs appear. For individual animals, one can only treat the symptoms of the disease, since (as is the situation with any viral disease) no drug is effective in killing the CD virus. Such treatment may include: (1) administration of antibiotics to limit secondary bacterial infection, (2) administration of electrolyte solutions to counteract fluid imbalance, (3) administration of antispasmodic drugs and/or dietary supplements to control nervous manifestations of the disease, and (4) provision of nursing care to minimize the animal's discomfort (Anonyinous 1973).

Effective treatment of $C D$ in wild populations is generally considered unfeasible. Possible means of controlling this disease in wild species include: (1) removal of carcasses of animals which have succumbed to $C D$ and which thus serve as sources of infection for other susceptible members of the population, (2) vaccination of susceptible domestic species which will decrease the number of susceptible hosts and (3) reduction of wildlife populations which also reduces the number of potential hosts (Budd 1970).

\section{$\underline{\text { Significance }}$}

Once infected, ranch-raised mink and fox experience mortality ranging from 20-90\% (Budd 1970). In captive ferrets, the disease is almost invariably fatal (Budd 1970). Ten of 10 captive coyote pups died of $C D$ (Gier and Ameel 1959). It is suspected that CD takes a high toll of pups in the wild (Gier et al. 1978). However, the outcome of natural infection in wild species is largely unknown (Trainer and Knowlton 1968). 


\section{References}

ANONYMOUS. 1973. Canine distemper. pp. 311-313. In: The Merck Veterinary Manual. $0 . H$. SIEGMUND, ed. $\overline{\text { Merck }}$ and Co. Inc., Rahway, NJ.

APPEL, M. and D. S. ROBSON. 1973. A microneutralization test for canine distemper virus. Am. J. Vet. Res. 34:1459-1463.

BUDD, J. 1970. Canine distemper. pp. 36-49. In: Infectious Diseases of Wild Mammals. J. W. DAVIS, L. H. KARSTAD and D. 0 . TRAINER, eds. lowa State Univ. Press, Ames, IA.

CHOQUETTE, L. P. E. and E. KUYT. 1974. Serological indication of canine distemper and of infectious canine hepatitis in wolves (Canis lupus) in northern Canada. J. Wildl. Dis. 10:321-324.

GIER, H. T. and 0. J. AMEEL. 1959. Parasites and diseases of Kansas coyotes. Kansas Agric. Exp. State Tech. Bull. 91. 35 pp.

GIER, H. T., S. M. KRUCKENBERG and R. J. MARLER. 1978. Parasites and diseases of coyotes. pp. 37-69. In: Coyotes: Biology, Behavior, and Management. M. BEKOFF, ed. Academic Press, NY.

GILLESPIE, J. H. and C. G. RICKARD. 1956. Encephalitis in dogs produced by distemper virus. Am. J. Vet. Res. 17:103-108.
GORHAM, J. R. 1966. The epizootiology of distemper. J. Am. Vet. Med. Assoc. 149:610-622.

KRAKOWKA, S., E. A. HOOVER, A. KOESTER and K. KETRING. 1977. Experimental and naturally occurring transplacental transmission of canine distemper virus. Am. J. Vet. Res. 38:919-922.

PARKER, R. L., V. J. CABASSO, D. J. Dean and E. L. CHEATUM. 1961. Serologic evidence of certain virus infections in wild animals. J. Am. Vet. Med. Assoc. $138: 437-440$.

PINKERTON, H. 1940. Immunological and histological studies on mink distemper. J. Am. Vet. Med. Assoc. $96: 347$.

PINKERTON, H., W. L. SMILEYAND and $W$. A. D. ANDERSON. 1945. Giant cell pneumonia. A lesion common to Hecht's disease, distemper, and measles. Am. J. Pathol. 21:1-6.

RAUSCH, R. L. 1953. On the status of some arctic mammals. Arctic $6: 91-148$.

REINHARD, K. R., R. L. RAUSCH and R. L. GRAY. 1955. Field investigations of prophylaxis against epizootic distemper in arctic sled dogs. pp. 223-277 In: Proc. Am. Vet. Med. Assoc., Minneapolis, MN.

TRAINER, D. 0 . and F. F. KNOWLTON. 1968. Serologic evidence of diseases in Texas coyotes. J. Wildl. Manage. 32:981-983. 


\title{
Infectious Canine Hepatitis
}

\author{
Randall L. Zarnke
}

Introduction

Infectious canine hepatitis ( $\mathrm{ICH}$ ) is a contagious disease which is of primary importance in members of the Canidae including the domestic dog (Canis familiaris) (Cabasso 1962), coyote (C. latrans) (Chaddock and Carlson $1 \overline{9} 50$, Green 1925, Parker et al. 1961), wolf (C. lupus) (Choquette and Kuyt 1974) and foxes (Vulpes fulva) (Cabasso 1962) and members of the Mustelidae including mink (Mustela vison) and ferrets (Mustela putorius) (Cabasso 1962). Infectious canine hepatitis has also been described (but not confirmed) in Ursidae (Camenzind 1978). The disease is caused by an adenovirus (Cabasso 1962). It is found worldwide in foxes and domestic dogs (Cabasso 1962, Love 1972). Animals of all ages are susceptible (Anonymous 1973). Symptoms range from a slight fever accompanied by congestion of the mucous membranes to severe depression and/or encephalitis (Anonymous 1973).

\section{History}

An epizootic with central nervous system signs and symptoms occurred in foxes in Europe during 1925 (Rubarth 1947). The disease was thus named fox encephalitis. Central nervous system signs of the disease are usually absent in the domestic dog (Cabasso 1970). In what has come to be considered a classic study and report, Rubarth described his 20 years of research on the natural occurrence of the disease in domestic dogs in Sweden (Siedentopf and CarIson 1949). These diseases (fox encephalitis and canine hepatitis) were originally considered to be separate entities, but subsequent studies revealed that the causative agent was the same in both cases (Trainer and Knowlton 1968).

The Alaska State-Federal Laboratory which is located in Palmer reported 2 cases of $\mathrm{ICH}$ in domestic dogs during 1977, 18 cases during 1978 and 29 cases during 1979 ( $R$. Barrett, pers. comm.). These diagnoses were based upon clinical signs of the disease, and were in some cases substantiated by isolation of the $\mathrm{ICH}$ virus. A recent serologic survey has provided evidence of $\mathrm{ICH}$ infection in 31 of 31 wolves from the Tanana Flats area south of Fairbanks, 16 of 16 from the Nelchina River basin, and 5 of 5 from northwestern Alaska (Ritter and Stephenson, ms. in prep.). The effect of $\mathrm{ICH}$ on the wolves is unknown.

\section{Transmission}

Infectious canine hepatitis is highly contagious (Cabasso 1970). In dogs, the virus is shed in all secretions and excretions during the acute stage of the disease (Anonymous 1973). The infection often localizes in the kidney and may become chronic, i.e. long-lasting (Marler et al. 1976). Infectious virus may be shed in the urine for many months (Anonymous 1973, Cabasso 1962, Gocke et al. 1970). Exposure of susceptible individuals usually occurs by ingestion (Anonymous 1973), or direct contact with contaminated exudates (Cabasso 1962, 1970) or contaminated fomites (Cabasso 1962, 1970). Airborne transmission is not believed to be important (Cabasso 1970). Transplacental transmission of $\mathrm{ICH}$ has been proposed (Marler et 
al. 1976). If transplacental transmission is indeed possible, a female with a chronic $\mathrm{ICH}$ infection could serve as a continual source of infection for subsequent litters (Marler et al. 1976).

Signs, Symptoms and

Pathogenes is

In foxes, the period between exposure and the onset of symptoms ranges from 2 to 6 days. The disease often has a sudden onset (Parker et al. 1961). Infected individuals exhibit loss of appetite, inflammation of oral and nasal mucous membranes, a period of hyperexcitement and occasional convulsions, prolonged clotting time of blood, a diphasic rise in temperature and mucous-filled or blood-filled feces. In nonfatal cases, opacity of the cornea of 1 or both eyes (sometimes referred to as "blue eyes") may occur. In fatal cases, the eyes usually remain clear and no loss of body weight occurs. This lack of symptoms reflects a rapid, fulminating course of the disease (Cabasso 1970).

In foxes, $\mathrm{ICH}$ infection probably occurs via the oropharynx. The virus spreads to the viscera and central nervous system via the blood. At necropsy, many organs exhibit general congestion (Cabasso 1970). Hemorrhages may be observed in the lining of the gastrointestinal tract, lymph nodes and around the eyes (Cabasso 1970). Necrosis of the liver results in various changes in color (Cabasso 1970, Gier et al. 1978). The liver may also appear swollen and have rounded edges (Cabasso 1970). Bloodstained fluid may be present in the peritoneal cavity (Cabasso 1962). The gall bladder may be thickened and swollen (Cabasso 1962).

\section{Diagnosis}

Diagnosis based upon signs and symptoms is difficult because clinical signs of $\mathrm{ICH}$ are variable and nonspecific. The most conclusive evidence is isolation of $\mathrm{ICH}$ virus from affected tissues. Serologic tests to detect evidence of past $\mathrm{ICH}$ infection are available. Also of value as diagnostic aids are: (1) the presence of intranuclear inclusion bodies in formalin-preserved liver cells, (2) increased clotting time and, (3) tests which determine the level of glutamic pyruvic transaminase in the serum (elevated levels indicate liver damage) (Cabasso 1970 ).

\section{Treatment}

Treatment of a large wildlife population would be virtually impossible. For individuals, treatment similar to that for domestic dogs may be effective:

1. Daily blood transfusions.

2. Daily intravenous administration of $5 \%$ dextrose in a balanced salt solution plus $5 \%$ protein hydrolysate.

3. Hyper-immune gamma-globulin (serum) as required.

4. Antibiotics to combat secondary bacterial infection.

5. Topical eye ointment for corneal opacity (Anonymous 1973).

Procedures 1 to 4 may be complicated by the increased clotting time of the blood. 
Immunity

For foxes and perhaps wolves, recovery from natural infection usually results in complete and longlasting immunity (Cabasso 1962, 1970, Choquette and Kuyt 1974). For these species, the wide dissemination of (and thus constant exposure to) $\mathrm{ICH}$ provides frequent "boosters" (Cabasso 1962). Pups may acquire passive immunity from passage of maternal antibody (Cabasso 1962). Virus may persist in (and presumably be shed by) animals which have recovered from acute infection and developed antibody (Cabasso 1962).

\section{Prophylaxis}

Vaccines are available for domestic dogs but are not recommended for use in foxes (Cabasso 1970). In dogs, passage of maternal antibody to pups may interfere with vaccination. For this reason, vaccination of pups prior to 9 weeks of age may not be effective (Cabasso 1970). Certain authorities recommend vaccination in order to ensure protection for those individuals which do not receive maternal antibody. If a vaccine is approved for wild canids, a similar recommendation will likely apply. In dogs, infectious virus has been shed following vaccination with attenuated live virus (Cabasso 1962). Hence, safer vaccines should be developed before being used in wild species.

\section{Significance}

Under natural conditions, the mortality rate for a fox population may range from 10-25\% (Cabasso 1962, 1970). Mortality rate of pups is generally greater than that for adults. In pups less than 6 months of age, mortality may reach $80 \%$. As host population levels increase, the chance of exposure also increases
(Cabasso 1970). There may be minimum host population level necessary for maintenance of the transmission of ICH (Love 1972). The effect of $\mathrm{ICH}$ on wild canid populations in Alaska is unknown. Humans are not susceptible to infection with $\mathrm{ICH}$ virus.

\section{References}

ANONYMOUS. 1973. Infectious canine hepatitis. pp. 313-315. In: The Merck Veterinary Manual. 0. $\mathrm{H}$. SIEGMUND, ed. Merck and Co. Inc., Rahway, NJ.

CABASSO, V. J. 1962. Infectious canine hepatitis virus. Ann. NY Acad. Sci. 101:498-514.

CABASSO, V. J. 1970. Infectious canine hapatitis. pp. 134-139. In: Infectious Diseases of Wild Mammals. J. W. DAVIS, L. H. $\overline{\text { KARSTAD }}$ and D. O. TRAINER, eds. lowa State Univ. Press, Ames, IA.

CAMENZIND, F. J. 1978. Behavioral ecology of coyotes on the National Elk Refuge, Jackson, Wyoming. pp. 267-293. In: Coyotes: Biology, Behavior, and Management. M. BEKOFF, ed. Academic Press, NY.

CHADDOCK, T. T. and W. E. CARLSON. 1950. Fox encephalitis (infectious canine hepatitis) in the dog. N. Am. Vet. 31:35-41.

CHOQUETTE, L. P. E. and E. KUYT. 1974. Serological indication of canine distemper and of infectious canine hepatitis in wolves (Canis lupus L.) in northern Canada. J. Wildl. Dis. 10:321-324.

GIER, H. T., S. M. KRUCKENBERG and R. J. MARLER. 1978. Parasites and diseases of coyotes. pp. 37-69. In: Coyotes: Biology, Behavior and Management. M. BEKOFF, ed. Academic Press, NY.

GOCKE, D. J., T. W. MORRIS and S. E. BRADLEY. 1970. J. Am. Vet. Med. Assoc. 156:1700-1705. 
GREEN, R. G. 1925. Distemper in the silver fox (Vulpes vulpes). Proc. Soc. Expt1. Biol. Med. 22:546-548. MARLER, R. J., S. M. KRUCKENBERG, J. E. COOK and C. M. O'KEEFE. 1976. Encephalitis in a confined coyote. J. Am. Vet. Med. Assoc. $169: 964-965$.

LOVE, D. N. 1972. Review of canine viral disease. Australia Vet. J. 48:567-570.

PARKER, R. L., V. J. CABASSO, D. J. and E. L. CHEATUM. 1961. Serologic evidence of certain virus infections in wild animals. J. Am. Vet. Med. Assoc. $138: 437-440$.
RUBARTH, S. 1947. An acute virus disease with liver lesions in dogs (hepatitus contagiosa canis). A pathologicoanatomical and etiological investigation. Acta Pathol. Microbiol. Scand., Suppl. 69.

SIEDENTOPF, H. A. and W. E. CARLSON. 1949. A comparative study of the fox encephalitis virus and the virus of infectious canine hepatitis. J. Am. Vet. Med. Assoc. 115:109-111.

TRAINER, D. O. and F. F. KNOWLTON. 1968. Serologic evidence of diseases in Texas coyotes. J. Wildl. Manage. 32:981-983.

\title{
San Miguel Sea Lion Virus
}

\author{
Howard Gelberg
}

\section{Introduction}

Abortions among the California sea lion (Zalophus californianus) population on San Miguel Island, California reaches epizootic proportions. In 1972, a virus was isolated from an affected sea lion (Gilmartin et al. 1976). Subsequently, similar viruses were isolated from northern fur seals (Callorhinus ursinus) and northern elephant seals (Mirounga angustirostris) (Smith et al. 1979, 1978b). Serologic surveys revealed the presence of antibody (suggesting prior infection) to these viruses in a variety of mammalian species including:. California sea lion (Zalophus californianus), northern fur seal (Callorhinus ursinus), Stellar sea lion (Eumetopias jubatus), northern elephant seal (Mirounga angustirostris), gray whale
(Eschrichtius robustus), sperm whale (Physeter catodon), fin whale (Balaenoptera physalus), Sei whale (Balaenoptera borealis), feral sheep (Ovis aries), feral donkey (Equis asinus), gray fox (Urocyon cinereoargenteus) and bison (Bison bison) (Akers et al. 1974, Madin et al. 1976, Prato et al. 1977, Smith and Akers 1976, Smith et al. 1979, Smith and Latham 1978).

\section{History}

The history of San Miguel sea lion virus (SMSV) begins not in 1972 with the discovery of the agent, but 40 years earlier in 1932, when a vesicle (blister) forming disease appeared in California swine. This disease was clinically similar to diseases produced by other vesicle causing 
viruses such as foot-and-mouth disease. Subsequent investigations revealed that this agent was a unique and previously undescribed virus and that the disease was specific for swine. It was named vesicular exanthema of swine (VES). The virus is highly contagious among pigs. The disease was confined to California for 20 years, but then spread to 43 states and the District of Columbia within 1 year.

The federal government embarked on an intense slaughter and disinfection program after the disease crossed state lines in 1952. By 1956, VES had been eradicated from the United States' swine population. Until the discovery of SMSV in 1972, it was believed that VES virus was unique in the history of veterinary virology in that it apparently arose de novo in an animal population and was subsequently eradicated through the efforts of humans (Madin 1975). However, characterization of the virus isolated from seal lions and later fur seals showed that it was identical to the "eradicated" agent which causes VES (Burroughs and Brown 1976). Lesions identical to those of VES were produced when SMSV was inoculated into swine. It is currently believed that the apparent de novo appearance of VESV in 1932 was in fact due to the feeding of raw marine-source garbage to swine. Enactment of federal and state garbage-cooking laws in 1956 prohibits this practice.

The naturally-occurring disease in wildlife is currently believed to be limited to sea lions and fur seals off the southern California coast and to fur seals on the Pribilof Islands, Alaska. In this latter closely monitored environment, the incidence of observed lesions in harvested animals varies between 1 and $2 \%$ annually (Sawyer 1976). The far ranging migrations of fur seals and other marine mammals pose a threat of disease spread on a global basis.

\section{Transmission}

Transmission of the disease among swine is by direct contact with viral contaminated foodstuffs and by horizontal (pig to pig) spread. The viruses have also been implicated in swine abortion indicating possible vertical (parent to offspring) spread.

Transmission of the naturallyoccurring disease in marine mammals is somewhat different than in swine in that direct inoculation of experimental animals does not consistently produce visible disease (Gelberg 1980).

It is currently hypothesized that natural infection of marine mammals occurs through a food-fish intermediary. Virus has been isolated from a shallow water fish, Girella nigricans (Smith and Latham 1978). The isolation of SMSV from mammalian species that also frequent shallow waters (both whales and seals), and a detection of SMSV antibody in these same species lends credence to such a theory. Additionally, virus has been isolated from the sea lion lung worm (Parafilaroides decorum) (Smith and Latham 1978) which has an intermediary stage in this fish. This parasite may also be important in disease transmission.

Transmission to terrestrial mammals presumably occurs at the landsea interface where scavengers and possible insect transmitters (vectors) come in contact with virus contaminated carcasses and animal wastes. Additionally, carcasses from the Pribilof Island fur seal harvest have been fed to mink and swine on ranches in Utah. Such operations are also capable of transmitting disease, 
and negate the natural geographical isolation of these species from one another.

\section{Signs, Symptoms and}

\section{Pathogenesis}

In swine, the natural and experimental disease is characterized by the appearance of vesicles on nonhaired portions of the skin and tongue (Madin 1975). This is accompanied by a febrile response (increased body temperature). After rupture of the vesicles at 24 to 48 hours post infection, the fever subsides. One to 2 days later, secondary vesicles appear again accompanied by fever, inappetence and difficulty in walking. Recovery is generally complete and prompt in the absence of bacterial contamination of the lesion. Occasionally, vesicles forming in the coronary bands (nailbeds) of the feet result in hoof wall separation. Such animals have a prolonged recovery. Major losses in swine are due to weight loss, poor feed efficiency, stillbirth, agalactia (lack of milk production) and abortions. The disease is not fatal.

The natural disease in otarid seals differs somewhat from that of swine in that vesicles appear only on non-haired regions of the flippers (color Fig. 5). Infected animals do not appear debilitated. Experimentally, lesions can be induced on the tongue following inoculation. The disease is milder in seals than in swine.

In phocid seals, viral isolations in naturally-occurring cases have not been accompanied by visible lesions. The virus has been isolated from orphaned pups and normal animals (Smith et al. 1978b, Smith and Skilling 1979). Experimentally, lesions can be produced in the tongue and skin. These are small, mild and selflimiting. Immunity is short lived.
The virus has not been isolated from cetaceans although neutralizing antibodies have been detected (Akers et al. 1974). Experimental work with these viruses has not been conducted on any whale species.

\section{Diagnosis}

In otarid seals, diagnosis is based primarily on the gross appearance of skin (flipper) lesions and should be verified by isolation of virus. Laboratory confirmation of virus (contained within intact vesicles) that has been frozen in dry ice is recommended. These samples should be shipped to the Plum Island Animal Disease Center of the U.S. Department of Agriculture in New York. Prior arrangements for packing, shipping, and receiving such specimens must be made through the state and/or federal veterinarian in charge who will obtain the necessary federal approval to receive, ship and examine the material. The virus should not be handled outside of the Plum Island Animal Disease Laboratory due to its exotic nature and potential danger for domestic livestock. Serologic diagnosis can also be performed at the same institution.

\section{Significance and Control}

San Miguel sea lion virus has been isolated from various pinnipeds on numerous occasions. The disease apparently does not influence population dynamics in these marine mammals. The discovery of the virus in association with aborting sea lions has not as yet been shown to have a cause and effect relationship (Gilmartin et al. 1976). Because of the difficulty of capture and the wide migrations of marine species, eradication of this disease agent is impractical. Since the virus has not 
been isolated from cetaceans, special effort should be made to identify the naturally occurring disease in these species. Additionally, the discovery of virus in fish opens new horizons in both virology and ecology.

Effort is being directed at preventing the spread of disease to domestic livestock populations. Such prevention primarily involves the physical separation of marine mammals and their carcasses from susceptible scavenging animals and domestic swine. Suspected vesicular disease outbreaks in swine should be promptly reported to state and federal authorities.

Antibody to these agents has been detected in the sera of laboratory workers. Direct evidence for disease production in man is lacking (Smith et al. 1978a). Apparently, SMSV and VES pose no public health threat.

\section{References}

AKERS, A. G., A. W. SMITH, A. B. LATHAM and H. M. S. WATKINS. 1974. Calicivirus antibodies in California gray whales and Steller sea lions. Archiv. Gestamte. Virusforsch. 46:175-177.

BURROUGHS, R. and R. BROWN. 1976. Physiochemical evidence for the reclassification of caliciviruses. J. Gen. Virol. 22:281-286.

GELBERG, H. 1980. Comparative Studies on the Pathology, Pathogenesis and Cross-Infectivity of Vesicular Exanthema of Swine Virus and San Miguel Sea Lion Virus in Swine and Seals. Ph.D. Thesis, Cornell Univ.

GILMARTIN, W. G., R. L. DELONG, A. W. SMITH, J. C. SWEENEY,, B. W. DELAPPE, R. W. RISEBOROUGH, L. GRINER, M. DAILEY and D. B. PEAKALL. 1976. Premature parturition in a California sea lion. J. Wildl. Dis. 12:104-115.
MADIN, S. H. 1975. Vesicular exanthema. pp. 286-307. In: Diseases of Swine. 4 th ed. H. W. DUNNE, ed. lowa State Univ. Press, Ames, IA.

MADIN, S. H., A. W. SMITH and T. G. AKERS. 1976. Current status of caliciviruses isolated from marine mammals and their relationship to caliciviruses of terrestrial animals. pp. 197-204. In: Wildlife Diseases. L. A. PAGE, ed. Plenum Press, New York, NY.

PRATO, C. M., T. G. AKERS and A. W. SMITH. 1977. Calicivirus antibodies in wild fox populations. J. Wildl. Dis. 13:448-450.

SAWYER, J. C. 1976. Vesicular exanthema of swine and San Miguel sea lion virus. J. Am. Vet. Med. Assoc. 169:707-709.

SMITH, A. W. and T. G. AKERS. 1976. Vesicular exanthema of swine. J. Am. Vet. Med. Assoc. 169:700-703.

SMITH, A. W., T. G. AKERS, A. B. LATHAM, D. E. SKILLING and H. L. BRAY. 1979. A new calicivirus isolated from a marine mammal. Archiv. Virol. 61:225-239.

SMITH, A. W., T. G. AKERS, C. M. PRATO and H. L. BRAY. 1976. Prevalence and distribution of four serotypes of San Miguel sea lion virus neutralizing antibody in wild animal populations. J. Wildl. Dis. 12:326-334.

SMITH, A. W. and A. B. LATHAM. 1978. Prevalence of vesicular exanthema of swine antibodies among feral mammals associated with the southern California coastal zone. Am. J. Vet. Res. 29:291-296.

SMITH, A. W., C. M. PRATO and D. E. SKILLING. 1978a. Calicivirus infecting monkeys and possibly man. Am. J. Vet. Res. 29:287-289.

SMITH, A. W., N. A. VEDROS, T. G. AKERS and W. G. GILMARTIN. 1978b. Hazards of disease transfer from marine mammals to land mammals: review and recent findings. J. Am. Vet. Med. Assoc. 17:1131-1133.

SMITH, A. W. and D. E. SKILLING. 1979. Viruses and virus diseases of marine mammals. J. Am. Vet. Med. Assoc. 175:918-920. 


\title{
Respiratory Viruses
}

\author{
Robert A. Dieterich
}

Introduction and History

A number of viruses which were originally isolated from cattle (Bos spp.) have been grouped together and called bovine respiratory disease viruses. This disease complex constitutes the most costly and troublesome disease problem in the cattle industry (Mohanty 1978). It has worldwide prevalence and many times is associated with other disease agents such as bacteria, mycoplasma, rickettsiae and chlamydiae.

Recently, a small herd of reindeer (Rangifer tarandus) held at Fairbanks were found to be positive, that is, to have serologic evidence of past exposure to 3 viruses belonging to the bovine respiratory disease group. Seven of 18 were positive for infectious bovine rhinotracheitis (IBR), 18 of 18 were positive for bovine virus diarrhea (BVD) and 1 of 18 was positive for parainfluenza type 3 $(\mathrm{PI}-3)$ (Univ. of Alaska, unpub. data). Subsequent tests performed on specimens from reindeer in the Nome area have yielded further serologic evidence of exposure to 1 or more of these viruses (Univ. of Alaska, unpub. data). A group of 18 reindeer located in a New York area zoo were tested in 1962 after having been in captivity for several years. No evidence of respiratory virus infection was found (Bolton and Murray $1964)$.

After the initial positive serologic results for respiratory viruses were found in reindeer, serologic evidence of exposure to BVD was found in Alaskan caribou (Rangifer tarandus), Dall sheep (Ovis dalli), moose (Alces alces) and additional reindeer (Univ. of Alaska, unpub. data). Evidence of exposure to PI-3 was found in caribou, Dall sheep, moose and in a limited number of reindeer (Univ. of Alaska, unpub. data). Members of a cattle herd in the Matanuska Valley of Alaska were found to have elevated titers to $\mathrm{PI}-3$ virus (Univ. of Alaska, unpub. data). Several reindeer from the Seward Peninsula and moose from the Kenai Peninsula were serologically positive for IBR (Univ. of Alaska, unpub. data).

Transmission and

Pathogenesis

By definition, the bovine respiratory disease viruses commonly cause respiratory tract infections. In cattle, they may also cause a number of other conditions including genital infection, diarrhea, conjunctivitis, abortion and encephalitis. These viruses are usually spread by aerosol droplets or by contact with infectious exudates from diseased animals.

Little is known about the effects of these viral agents in wildife. Under certain conditions they can be highly pathogenic. Ten Rocky Mountain bighorn sheep (Ovis canadensis) at the Sybille Big Game Research Unit near Laramie, Wyoming died from respiratory disease (Parks et al. 1972). Serologic titers to PI-3 were found to have increased markedly during their illness. Parainfluenza Type 3 virus was isolated from nasal washings from 3 of the 10 sheep tested during the course of the disease. Other investigators have 
found evidence of respiratory viruses in moose in Canada, mule deer (Odocoileus hemionus) in Colorado, pronghorns (Antilocapra americana) in Canada plus numerous other species in various parts of the world (Barrett and Chalmers 1975, Chow and Davis 1964, Parks and England 1974, Thorsen and Henderson 1971, Thorsen et al. 1977).

\section{Significance and Control}

It is now known that serologic evidence of IBR, BVD, and PI-3 viruses is present in many species of domestic and wild animals in Alaska. The significance of these findings is not known. In cattle, it is known that actual field cases of these diseases are normally a mixture of the virus and other agents of which the most common is Pasteurella spp. bacteria. Such mixed infections are often more severe than single virus experimental laboratory infections and present an identifiable clinical picture (Mohanty 1978). Before the causes of such symptoms were known, the disease was given the common name of "shipping fever." This same type of clinical pattern has been seen in the field in reindeer and caribou but not all of the organisms causing the syndrome have been isolated. The severity of disease in Alaskan wildlife caused by these viruses either in pure form or as mixed infections is unknown at this time. A preliminary experiment was recently carried out at the University of Alaska. Two reindeer yearlings and 1 bovine calf were exposed to BVD virus. Only minimal lesions and symptoms were observed under carefully controlled experimental conditions in isolation facilities. The bovine calf developed lesions within the nostrils and 1 reindeer had a nasal discharge. The questions of incidence, pathogenesis and transmission must be addressed in the future in order to assess the impact of these agents on wildlife in Alaska. As the domestic cattle and reindeer herds increase in Alaska, the interaction of these species with wildlife species will increase. The importance of such interaction in relation to the spread of the respiratory viruses remains to be determined.

Many of the bovine respiratory diseases can be controlled in cattle through the use of specially developed vaccines. The effectiveness of these vaccines in wildlife is not known other than from observations in zoo animals where there are apparently some beneficial results.

\section{References}

BARRETT, M. W. and G. A. CHALMERS. 1975. A serologic survey of pronghorns in Alberta and Saskatchewan, 1970 - 1972. J. Wildl. Dis. $11: 157-163$.

BOLTON, W. D. and R. W. MURRAY. 1964. An immunity study on a reindeer herd. Am. J. Vet. Res. 25:1783-1784.

CHOW, T. L. and R. W. DAVIS. 1964. The susceptibility of mule deer to infectious bovine rhinotracheitis. Am. J. Vet. Res. 25:518-519.

MOHANTY, S. B. 1978. Bovine respiratory viruses. pp. 83-110. In: Advances in Veterinary Science and Comparative Medicine. Vol. 22. C. A. BRANDLY, ed. Academic Press Inc., New York, NY. PARKS, J. B., G. POST, T. THORNE and P. NASH. 1972. Parainfluenza - 3 virus infection in Rocky Mountain bighorn sheep. J. Am. Vet. Med. Assoc. 161:669-672. 
PARKS, J. B. and J. J. ENGLAND. 1974. A serological survey for selected viral infections of Rocky Mountain bighorn sheep. J. Wildl. Dis. 10:107-110.

THORSEN, J. and J. P. HENDERSON. 1971. Survey for antibody to infectious bovine rhinotracheitis (IBR), bovine virus diarrhea (BVD) and Parainfluenza 3 (PI3) in moose sera. J. Wildl. Dis. 7:93-95.
THORSEN, J., L. KARSTAD, M. W. BARRETT and G. A. CHALMERS. 1977. Viruses isolated from captive and free-ranging wild ruminants in Alberta. J. Wildl. Dis. $13: 74-79$.

\title{
Feline Panleukopenia
}

\author{
Randall L. Zarnke
}

Feline panleukopenia (FP) is an acute viral enteritis of members of the Felidae, plus mink (Mustela vison) and raccoons (Procyon lotor). The disease was first described in 1925 in domestic cats (Felis catus) from England. It is believed to be distributed worldwide. There are several strains of the FP virus. There is a close morphologic, antigenic and epizootiologic relationship between FP, mink virus enteritis, and the parvovirus of canine which causes enteritis.

Feline panleukopenia is highly contagious. Experimentally, FP can be transmitted via the intramuscular, intravenous, intraperitoneal, subcutaneous, intracerebral, oral and intranasal routes. The virus is shed in all secretions and excretions of diseased animals. Transmission occurs via direct contact or via contaminated fomites.

Feline panleukopenia has a sudden onset. Signs of the disease include depression, loss of appetite, diphasic elevation in body temperature, vomiting and diarrhea. The latter 2 signs lead to a severe dehydration. Tenderness of the abdomen may be observed.

Common necropsy findings include an empty gastrointestinal tract, hemorrhage in the small intestine, swollen lymph nodes, and a fluid appearance of the marrow of long bones. Case fatality rates (for acute cases) may reach $90 \%$. This may vary depending on the virulence of the strain of FP virus involved in a particular outbreak.

Maternal antibody provides shortterm protection for the young. Recovery from clinical infection results in life-long protection.

Diagnosis may be based upon clinical signs of the disease, decreased white blood cell count, or a significant rise in antibody titer 2 to 3 weeks post infection. 
As with other viral diseases, there is no drug treatment which can safely and effectively eliminate the causative virus. Antibiotics may be helpful in preventing secondary bacterial infection. Additional supportive treatment which may help alleviate symptoms includes administration of electrolytic solutions to combat fluid loss and kaolin to counteract the diarrhea. Although vaccines are safe and effective in domestic species, there is no known means of control for FP in wildlife populations. The Alaska StateFederal Laboratory which is located in Palmer reported 25 cases of FP in domestic cats during 1977, 58 cases during 1978 and 35 cases during 1979 (R. Barrett, pers. comm.). These diagnoses were based upon clinical signs of the disease, and were in some cases substantiated by isolation of the FP virus. The significance of FP for Alaskan wildlife is unknown.

\section{References}

BITTLE, J. L. 1970. Feline panleukopenia. pp. 85-89. In: Infectious Diseases of Wild Mammals. J. W. DAVIS, L. H. KAR $\overline{S T A D}$ and D. 0. TRAINER, eds. I owa State Univ. Press, Ames, IA.

\title{
Arboviruses
}

\author{
Randall L. Zarnke
}

The term "arbovirus" refers to viruses which are transmitted by arthropods (ARthropod BOrne $=$ ARBO). By definition, these viruses must be able to: 1) infect and replicate in a vertebrate host, 2) infect and replicate in an arthropod vector, and 3) be transmitted from 1 vertebrate host to another by an infected arthropod. There has been substantial evidence of various arboviruses in arthropods and/or vertebrates (including humans) in Alaska and the neighboring Yukon and Northwest Territories of Canada. To date, there has been no evidence of clinical disease in any of the vertebrates. Such absence of overt disease is not the case in other areas of North America. For example, members of the California encephalitis virus complex (one of which, namely the snowshoe hare subtype, has been isolated from Alaskan mosquitoes) were implicated in 280 cases of illness in humans in the contiguous United States from 1964 through 1968. Encephalitis (inflammation of the brain and/or spinal cord) is not uncommon. This may be evidenced by abnormal behavior in animals such as trembling, staggering and/or loss of fear of humans. Two of the so-called equine encephalitides, namely St. Louis encephalitis and western quine encephalitis, are of potential threat to Alaskan vertebrates. Human and equine mortality as a result of infection by these viruses have periodically erupted in widespread epizootics. Fortunately for Alaska, these outbreaks have traditionally 
been limited to the contiguous United States and the lower half of the western Canadian provinces. It is presently believed that these viruses are carried long distances by migratory birds. Some of the avian species which have been implicated are common residents of Alaska during summer months. The possibility of these viruses infecting Alaskan wildlife cannot be ignored.

\section{$\underline{\text { References }}$}

CALISHER, C. H., H. S. LINDSEY, D. G. RITTER and K. M. SOMMERMAN. 1974. Northway virus: a new Bunyamwera group arbovirus from Alaska. Can. J. Microbiol. 20:219-223.
FELTZ, E. J., B. LIST-YOUNG, D. G. RITTER, P. HOLDEN, G. R. NOBLE and P. S. CLARK. 1972. California encephalitis virus: serological evidence of human infections in Alaska. Can. J. Microbiol. $18: 757-762$.

RITTER, D. G., C. H. CALISHER, D. J. MUTH, R. E. SHOPE, F. A. MURPHY and S. G. WHITFIELD. 1978. New Minto virus: A new rhabdovirus from ticks in Alaska. Can. J. Microbiol. 24:422-426.

RITTER, D. G. and E. T. FELTZ. 1974. On the natural occurrence of California encephalitis virus and other arboviruses in Alaska. Can. J. Microbiol. 20:1359-1366.

\title{
Aleutian Disease of Mink
}

\author{
Randall L. Zarnke
}

\begin{abstract}
Aleutian disease (AD) is a slowly progressive parvovirus infection of mink (Mustela vison). The disease is primarily of importance in ranchraised mink. Wild mink and perhaps other species are also susceptible. The disease has not been diagnosed in Alaska. The name of the disease is derived from the fact that the infection is invariably fatal in those mink which are homozygous for a particular gene, namely the "Aleutian" gene which determines blue fur color. All mink appear to be susceptible to the disease, but mortality rates are highest in the homozygous Aleutian animals. Stressful conditions such as handling or cold weather are often followed by large die-offs in infected populations.
\end{abstract}

Signs of the disease include loss of appetite, loss of weight, loss of strength and increased thirst. At necropsy, the following alterations may be observed: 1) spleen several times normal size with rounded edges, 2) white focal lesions in kidney (kidney may be enlarged and fluidfilled, or shrunken and granular depending on stage of disease), 3) liver 2-3 times normal size, white focal lesions may or may not be present and 4) lymph nodes several times normal size.

Virus is shed in urine, feces and saliva. Transmission is believed to occur via ingestion of materials contaminated by these excretions or via inhalation of aerosolized AD virus. 
As with other viral diseases, there are no drugs which can safely and effectively inactivate the virus once an animal becomes infected. Antibiotics may be administered to combat secondary bacterial infection.

The iodine agglutination test is an inexpensive, accurate and simple diagnostic test. Present recommendations call for sacrificing all mink which exhibit evidence of infection. By eliminating homozygous Aleutian mink from a ranch, a substantial decrease in the mortality due to $A D$ may be achieved.
Based upon present knowledge, the significance of $A D$ on wild mink populations is unknown.

\section{Reference}

GORHAM, J. R., J. B. HENSON, G. A. PADGETT and T. C. MCGUIRE. 1970. Aleutian Disease. pp. 140-152. In: $\quad \frac{\text { Infectious }}{\text { Diseases }}$ of $\frac{\text { Wild }}{\text { DAVIS }}$

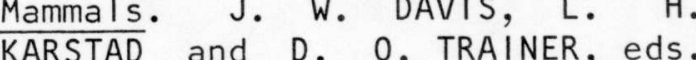
lowa State Univ. Press, Ames, IA.

\title{
Mink Virus Enteritis
}

\author{
Randall L. Zarnke
}

Mink virus enteritis is a highly contagious viral disease of mink (Mustela vison). The causative agent, signs of disease, and epizootiology are similar (if not identical) to those of feline panleukopenia. The disease was first described during a major epizootic at Fort William, Ontario, Canada during 1947. It has since occurred in all major mink raising areas of North America and northern Europe.

The disease exhibits a strong seasonality, with most outbreaks occurring from July through September. The disease is transmitted primarily via direct contact or fomites such as contaminated gloves of mink handlers. Neither food-borne nor aerosol transmission appear to be of major significance. Infection localizes in the gastrointestinal tract. Overt disease occurs in both kits and adults, although kits are more severely affected. Loss of appetite and profuse diarrhea are observed initially 4 to 7 days post infection. Sluggishness and vomiting may also be observed. Affected individuals usually either recover within 4 to 5 days of the onset of symptoms, or succumb.

At necropsy, necrosis of the intestinal epithelium is often observed, accompanied by shedding of the intestinal mucosa. Intestinal contents are quite fluid and may appear yellow or red in color. The spleen may be enlarged, dark and mottled. Lymph nodes are often enlarged and fluid-filled (edematous).

Maternal antibody protects newborn mink until at least 6 weeks of age. Safe and effective vaccines are available. The case fatality rate in an outbreak may range from $20-80 \%$. As with other viral diseases, there 
is no safe and effective drug treatment which can kill the virus. There have been no documented reports of the disease in Alaska. Its significance for Alaskan wildlife is unknown.

\section{Reference}

BURGER, D. and J. R. GORHAM. 1970. Mink virus enteritis. pp. 76-84. In: Infectious Diseases of Wild Mammals. J. W. DAVIS, L. H. $\overline{\text { KARSTAD }}$ and D. 0. TRAINER, eds. lowa State Univ. Press, Ames, IA.

\title{
Transmissable Mink Encephalopathy
}

\author{
Randall L. Zarnke
}

Transmissible mink encephalopathy (TME) is a disease of ranch mink (Mustela vison) which was first identified in Wisconsin during 1947. Transmissible mink encephalopathy has never been reported in wild mink. The causative agent is a member of a group known as "slow" viruses. These agents closely resemble more common viruses in certain characteristics. In other characteristics they are vastly different. Final characterization of these agents remains to be determined. There have been sporadic outbreaks of TME in certain locations of North America but it has never occurred in Alaska. Evidence suggests that in the initial outbreak the disease agent was introduced in the mink's diet, and that the oral route of transmission was most important. Incubation time is variable and may be as long as several months to a few years. Transmissible mink encephalopathy is a progressive, invariably fatal disease. It affects the central nervous system, where it causes a spongiform degeneration of the gray matter. As might be expected, one of the major signs of the disease is a change in behavior. This change may take any of several forms, such as increased aggression, timidity, friendliness, etc. There is often some locomotor dysfunction. Transmissible mink encephalopathy may be diagnosed histopathologically or via virus isolation. The latter is costly and time-consuming. As in other viral diseases, there is no drug treatment which is both safe and effective. Because of the lack of evidence of the presence of TME in Alaska, and the lack of evidence of TME infection in wild mink worldwide, it is felt that this disease poses little threat to wildlife in Alaska.

\section{Suggested References}

BURGER, D. and G. R. HARTSOUGH. 1965. Encephalopathy of mink. II. Experimental and natural transmission. J. Infect. Dis. $115: 393-399$.

HARTSOUGH, G. R. and D. BURGER. 1965. Encephalopathy of mink. I. Epizootiologic and clinical observations. J. Infect. Dis. $115: 387-392$. 


\title{
Vesicular Stomatitis
}

\author{
Randall L. Zarnke
}

\begin{abstract}
Vesicular stomatitis virus (VSV) is a rhabdovirus which can infect horses (Equus spp.), cattle (Bos spp.), domestic swine (Sus spp.), humans (Homo sapiens) and wild animals such as deer (Odocoileus spp.), raccoons (Procyon lotor) and bobcats (Lynx rufus). Vesicular stomatitis is found in North, Central and South America. There have been no reports of the disease in Alaska. The disease in ungulates is characterized by blisters (vesicles) on the mucous membranes of the mouth, muzzle, udder and on the coronary band of the hoof.
\end{abstract}

Traditionally, the disease has been maintained in a low-level (enzootic) manner interspersed with dramatic epizootics. During the enzootic periods, transmission occurs via direct contact or contaminated fomites. During epizootics, biting arthropods are believed to be the primary vectors of VSV. Neither the reservoir mammalian host, nor the means by which the virus over-winters are known.

In infected deer, a febrile response (fever) is observed 1 to 2 days post exposure. This is followed closely by the formation of blisters on the tongue, hard palate, teats and/or hooves. These lesions are quite painful and often interrupt normal feeding and movement. Thus, the primary detrimental effect of VSV to an individual animal is a general loss of health and vigor. The lesions heal within 10 days, and recovery is usually uneventful. As with other viral diseases, there is no drug which can safely and effectively kill the virus. Diagnosis may be accomplished via serologic tests, isolation of the causative virus or identification of the signs of the disease. Because signs and symptoms of VSV may be confused with those of foot and mouth disease, virus isolation is preferred.

Based upon the past absence of the disease in Alaska, VSV is not felt to be a major threat to wild animals in the state.

\section{$\underline{\text { Suggested }}$ Reference}

KARSTAD, L. H. 1970. Vesicular stomatitis. pp. 64-67. In: Infectious Diseases of Wild Mammals. J. W. DAVIS, L. H. KAR STAD and D. 0. TRAINER, eds. Iowa State Univ., Ames, IA. 


\title{
Foot and Mouth Disease
}

\author{
Randall L. Zarnke
}

Foot and mouth disease (FMD) is a viral disease of cloven-footed (artiodactyl) domestic and wild animals. Humans are also susceptible. Susceptible wildlife species in Alaska include blacktailed deer (Odocoileus hemionus), elk (Cervus elaphus), moose (Alces alces), caribou (Rangifer tarandus) and bison (Bison bison). The disease is characterized by blisters (vesicles) of the oral mucosa and on the coronary band of the hoof. The most common means of transmission of FMD virus is via the oral route on contaminated feed. Fomites and contaminated animal products are also important. The first sign of the disease is a high body temperature often accompanied by depression and loss of appetite. As the fever subsides, inflammation of the oral mucosa occurs. Blisters appear in and around the mouth and on the hooves. The blisters rupture and release virus-contaminated fluid within 2 days. Virus may also be found in urine, feces, saliva, milk and semen. Once an outbreak erupts, transmission to other susceptible animals is usually rapid. The lesions heal in 7 to 10 days. The lesions are quite painful and interfere with normal feeding and movement. Thus, the primary detrimental effect on an individual is a general loss of health and vigor. Convalesence may take up to 6 months.
Foot and mouth disease became established in cattle (Bos spp.), and in a deer herd in California during 1924. It was deemed necessary to kill over 22,000 deer in order to eliminate this reservoir of the virus.

As with other viral diseases, there is no drug treatment which can safely and effectively kill the FMD virus. Diagnosis may be accomplished by serologic tests, isolation of the virus, or by signs of the disease. Because of the possibility of confusing the signs of FMD with those of vesicular stomatitis and vesicular exanthema, isolation of the virus or serology is preferred.

Although FMD has never been diagnosed in Alaska, its highly contagious nature warrants continued surveillance, especially in view of the ever increasing cattle industry in the state.

\section{Suggested Reference}

FLETCH, A. L. 1970. Foot-and-mouthdisease. $\mathrm{pp}$. 68-75. In: Infectious Diseases of Wild Mammals. J. W. DAVIS, L. H. KARSTAD and D. 0. TRAINER, eds. Iowa State Univ. Press, Ames, IA. 


\title{
Epizootic Hemorrhagic Disease
}

\author{
Randall L. Zarnke
}

Epizootic hemorrhagic disease (EHD) is caused by an orbivirus (aka diplornavirus). The natural host range appears to be strictly limited to wild ruminants white-tailed deer (Odocoileus virginianus), mule deer ( 0 . hemionus) and pronghorn antelope (Antilocapra americana). The disease was first formally described when an outbreak occurred in New Jersey during 1955. Seven hundred white-tailed deer died during the outbreak. Documented epizootics have subsequently occurred in Michigan, South Dakota, Alberta, North Dakota and Wyoming. Suspected outbreaks have occurred in Missouri, Washington, British Columbia, Nebraska and lowa.

...outbreaks involving large numbers of deer--as in Michigan, New Jersey, and Alberta--...are single epizootics which do not reoccur. Die-offs involving small numbers of deer--as in South Dakota and Nebraska--occur almost annually, and the disease appears to be enzootic. All documented outbreaks of EHD have occurred during late summer and early fall (August-0ctober) and have ceased abruptly with the onset of frost [see reference].

The seasonality of the outbreaks and the long-term viremia ( 4 to 5 days) lend credence to the belief that EHD may be transmitted by arthropods. When inoculated via the subcutaneous, intramuscular or intravenous routes, a susceptible deer population may experience $80-90 \%$ mortality. When infected via the oral route, deer exhibit a marked antibody rise, and are subsequently resistant to intramuscular challenge. The period between infection and the onset of clinical symptoms usually lasts 5 to 10 days. Deer may have an elevated body temperature from day 3 to 5 post infection. Symptoms include loss of appetite, loss of fear of humans, loss of strength, excess salivation, rapid heart beat, and labored breathing. Hemorrhages may appear in orbital and/or oral mucosa. Blood may appear in saliva, feces, and/or urine. From 8 to 36 hours after the onset of symptoms, deer enter shock, become prostrate and then die. It is believed that deer which are exposed via the oral route or small rodents may serve as a reservoir wherein EHD is maintained. Epizootic hemorrhagic disease virus is transmitted to susceptible deer when an appropriate vector population reaches a threshold level of abundance and an epizootic occurs.

Petechial to massive hemorrhages may be found in many tissues and organs including heart, liver, spleen, kidney, lung and the gastrointestinal tract at necropsy. No visceral organs are exempt from hemorrhage. Hemorrhages arise as a result of a derangement of the clotting mechanism and a degeneration of the vessel walls. Clots may form inside the vessels. When all clotting factors are exhausted, blood leaks out of the damaged vessels. There may also be pleural edema, pulmonary edema, subcutaneous edema, intramuscular edema and an increase in the pericardial fluid.

Diagnosis may be based on case history, signs of the disease and the epizootic nature and seasonality of an outbreak. Because of the similarity of the lesions to those of 
bluetongue and malignant catarrhal fever, isolation and identification of the EHD virus is necesasary for a conclusive diagnosis.

It was originally thought that the case fatality rate was inevitably very high because of the epizootic nature of the disease. Recently, studies have revealed many deer with high levels of antibody, which indicates recovery following infection. These may be animals which were exposed orally, and suggests the possibility of an oral vaccine. At present, there is no method of treatment or control. Epizootic hemor- rhagic disease has not been diagnosed in Alaska, but its severity and importance in other areas of North America should be borne in mind by Alaska wildlife biologists.

\section{Reference}

TRAINER, D. 0 . and L. H. KARSTAD. 1970. Epizootic hemorrhagic disease. $\quad$ 50-54 In: Infectious Diseases of Wild Mammals. J. W. DAVIS, L. H. KARSTAD and D. 0. TRAINER, eds. I owa State Univ. Press, Ames, IA.

\title{
Bluetongue
}

\author{
Randall L. Zarnke
}

Bluetongue (BT) is an acute orbivirus (aka diplornavirus) disease of wild and domestic animals. The virus (of which there are several strains) is closely related, if not identical, to the virus of epizootic hemorrhagic disease. The disease is of primary importance in domestic sheep (Ovis aries). Domestic cattle (Bos taurus) are also susceptible, but the infection is usually inapparent. Bighorn sheep (Ovis canadensis) and white-tailed deer (Odocoileus virginianus) are also susceptible to the disease. The susceptibility of Alaskan wildlife species is unknown. Serologic surveys indicate that elk (Cervus elaphus) may be naturally exposed. Bluetongue virus was isolated from pronghorn antelope (Antilocapra americana) from Missouri, Nebraska and Wyoming during a 1978 epizootic (J. Morton, pers. comm.). There have been unsubstantiated reports of virus isolation from wild rodents.

The disease was first recorded in South Africa in 1876. It was not until 1948 that the first case was documented in the United States when it was found in a domestic sheep from Texas. As of 1968, the disease had been documented in 1 ivestock in 23 of the 48 contiguous United States.

Culicoides spp. gnats are biological vectors of the disease and serve as the major means of transmission. Disease incidence peaks in late summer and early autumn when the vector population is most abundant. Warm and wet weather favors increased Culicoides population levels and therefore results in an increase in disease transmission. The disease disappears with the onset of colder 
weather in an area. The disease has also been experimentally transmitted via the oral route.

Signs in white-tailed deer are similar to those in domestic sheep but vary between individuals. An increase in body temperature begins 4 to 6 days post infection (PI) and peaks at $106^{\circ} \mathrm{F} 6$ to 7 days PI. An increase in the respiration rate and loss of appetite parallel the increase in body temperature. The muzzle, lips and ears may appear reddened. Erosive lesions occur in the mouth accompanied by excess salivation and a swollen and discolored tongue ("bluetongue") which hangs from the side of the mouth. Occasionally, conjunctivitis may occur. A nasal exudate which forms a dried crust on the nose is found in all fatal cases. Bloody diarrhea is common in severe cases. In prolonged cases, hemorrhage of the coronary band of the hoof is common and results in lameness. Animals may exhibit photophobia and loss of fear. Death occurs 7 to 8 days PI.

Post mortem signs are quite variable. The lining of the stomachs may be reddened and hemorrhagic. Inflammation of the intestines is common. Liver, spleen and lymph nodes may be enlarged, congested and hemorrhagic. Kidneys may be reddened and fluid-filled. The heart muscle and some striated muscles may be hemorrhagic. Clots may be found inside blood vessels.
Both sheep and deer which have recovered from the disease are solidly resistant to reinfection by the same strain of virus. Therefore, vaccination of domestic species is feasible. Use of a polyvalent vaccine is recommended because of the existence of several strains of BT virus. Research has been conducted on the potential of vaccinating wild species. It is presently felt that large-scale vaccination programs are logistically unfeasible.

Diagnosis of BT may be based upon clinical signs of the disease. Confirmation via isolation of the virus is recommended. As with other viral diseases, there is no drug treatment which is safe and effective for killing the virus once infection occurs. Insect control and quarantine of diseased individuals may decrease the severity of an outbreak. Bluetongue has never been diagnosed in Alaska. Its potential significance in the state is unknown.

\section{Reference}

TRAINER, D. 0. 1970. Bluetongue. pp. 55-59. In: Infectious Disease of Wild Mammals. J. W. $\overline{\text { DAVIS, L. }} \frac{\text { H. KARSTAD }}{\text { and D. } 0 .}$ TRAINER, eds. Iowa State Univ. Press, Ames, IA. 


\title{
Pseudorabies
}

\author{
Randall L. Zarnke
}

Pseudorabies is an acute infectious disease of mammals caused by a herpesvirus. Domestic swine (Sus spp.) are the primary reservoir of the disease. Ranch mink (Mustela vison), ranch fox (Vulpes vulpes) and adult swine rarely die from the disease. In most other species and in young pigs the disease is often fatal. The disease has never been documented in wildlife in Alaska. The disease has been identified in other parts of the world in arctic fox (Alopex lagopus), red fox (Vulpes vulpes) and mink (Mustela vison) which are also common in Alaska.

The disease was first described in 1902 in Hungary in the ox (Bos taurus), dog (Canis familiaris) and cat (Felis catus). It has since been reported from North America, South America, Africa, Asia and many areas of Europe.

Infection may occur via abraded skin or intact nasal mucosa. The virus may also be transmitted venereally or via contaminated milk. In fur farm mink, the primary sign of disease is marked localized itching. This often leads to excess scratching, which may reach the stage of self-mutilation. Other symptoms may include loss of appetite, excess salivation, alternate excitement and depression, impaired equilibrium and spasms. The severity of symptoms in- creases with the length of the incubation period. Virus is often present in nasal discharges. It may also persist in a carcass for many days. As is the case with other viral diseases, there is no drug which can safely and effectively kill the virus.

Necropsy findings may include inflamed mucous membranes, hemorrhagic heart tissue and congested, fluidfilled or hemorrhagic lungs. None of these symptoms are considered to be diagnostic of pseudorabies infection.

Isolation of the causative virus is the only conclusive means of diagnosis. Nervous manifestations of the disease (especially self-mutilization in response to incessant itching) may be helpful in diagnosis.

Based upon the historical absence of any evidence of the disease in Alaska, pseudorabies is not felt to pose a major threat to wild animals in the state.

\section{Suggested Reference}

FRIEND, M. and D. O. TRAINER. 1970. Pseudorabies. pp. 90-96. In: Infectious Diseases of Wild Mammals. J. W. DAVIS, L. H. KAR STAD and D. 0. TRAINER, eds. Iowa State Univ., Press, Ames, IA. 


\title{
Lymphocytic Choriomeningitis
}

\author{
Randall L. Zarnke
}

Lymphocytic choriomeningitis (LCM) is a viral disease of mice (Mus spp.) and perhaps certain other rodents. In humans, it may cause a non-fatal meningitis. Transmission to humans is not common. The virus has been isolated from wild mice virtually worldwide. Although significant disease and mortality may result from experimental infection, it is believed that natural infection is asymptomatic and not harmful to mice. Transmission of LCM virus occurs via many routes. It is believed that in utero transmission is necessary for maintenance of the virus in nature. Multiplication and excretion of infectious virus often continues for the life of naturally- infected mice. Isolation of the virus is the preferred method of diagnosis. In order to prevent the possibility of
LCM transmission, humans should avoid handling mice. It is advisable to wear rubber gloves and to wash one's hands after handling mice. There is no drug treatment which can safely and effectively kill the virus. Due to the lack of evidence of LCM in Alaska, and because of the absence of symptoms in LCM-infected mice elsewhere, it is felt that LCM poses no threat to wildlife in Alaska.

\section{Suggested Reference}

HOTCHIN, J. E. and L. M. BENSON. 1970. Lymphocytic choriomeningitis. $\quad 153-165$ In: Infectious Diseases of Wild Mammals. J. W. DAVIS, L. H. KAR STAD and D. 0. TRAINER, eds. I owa State Univ. Press, Ames, IA. 
Chapter 2.

\section{BACTERIAL DISEASES}




\title{
Introduction
}

\author{
Jamie K. Morton
}

Bacteria are a normal part of our existence. Various species are found in the soil, air, fresh and saltwater and in and on our bodies. Essentially where there is life, there are bacteria. Many are necessary for normal functioning. For instance, bacteria are responsible for ruminant digestion, the production of certain foods (cheese, yogurt, pickles, vinegar, etc.), decay of organic matter, sewage processing, nitrogen fixation in the soil and production of silage.

Bacteria are single-celled organisms. Their nucleus is not separated from the cytoplasm by any membrane. They contain DNA but no individual chromosomes. Division is by binary fission. No membrane bound organelles such as mitochondria are found in the cytoplasm, and bacteria are capable of carrying out respiration, fermentation or photosynthesis by other means.

Shapes are round (cocci), cylindrical (rods) or helical (spirilla). Some are motile by means of 1 or more tail-like appendages called flagella. Certain bacteria produce endospores within the vegetative cell which are liberated when the vegetative part dies. Spores may be dormant for years and then germinate under favorable conditions. This survival tactic is used by the organisms of tetanus and botulism.

Gram staining a smear of bacteria on a microscope slide is a tool used to separate bacteria into 2 broad taxonomic groups. Differences are based on structural, chemical and physical properties. Those staining blue are called Gram positive, and those staining red are Gram negative.

Many organisms are part of the normal flora of the human or animal body and may be found on the skin, in the nose and throat, intestinal tract, urinary tract and genital tract. They are not normally present in blood or other tissues. Nondisease-causing bacteria are called non-pathogens, and those causing disease are called pathogens.

Skin and mucous membranes provide the first line of defense against pathogens. If the organisms break these barriers, the body's immune system must take over either by producing antibodies, engulfing the invader or producing toxic substances. Antibodies are found in the serum portion of the blood and may be detected by laboratory tests (serology). Immune responses are quite specific, and immunity to 1 disease does not protect against another.

To name a specific bacteria (or virus or parasite or other agent) as being the cause of a disease is usually an oversimplified approach. Physiology of the animal, environmental conditions, characteristics of the organism(s) and other factors can all interact to contribute to the disease state. 


\title{
Tularemia
}

\author{
Jamie K. Morton
}

$\underline{\text { Introduction }}$

Tularemia is a bacterial disease of animals and humans caused by Francisella tularensis. The organism is a short, non-motile, nonencapsulated, non-sporeforming, Gram negative rod. It usually takes 2 to 10 days to grow on laboratory media and will not grow at all without enrichment (Davis et al. 1974). Two subspecies of Francisella are now recognized based on acid production from sucrose, serologic tests and pathogenicity. Francisella tularensis var. tularensis is the more virulent form, and $\underline{F}$. tularensis var. palaertica, the usual rodent form, is the less virulent (Buchanan and Gibbons 1974).

Rabbits (Leporidae), hares (Lepus spp.), muskrats (Ondatra zibethica), voles (Microtus spp.) and numerous small rodents are the usual hosts. Infections have also been reported in fox (Vulpes vulpes and Alopex lagopus), coyotes (Canis latrans), beavers (Castor canadensis), bobcats (Felis rufus), dogs (Canis familiaris), cats (Felis catus), sheep (Ovis spp.), calves (Bos spp.),

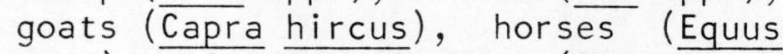
spp.), birds, mule deer (Odocoileus hemionus) and bears (Ursus spp.) (Binninger et al. 1980, Buchanan and Gibbons 1974, Emmons et al. 1976). The disease is now found in North America, Europe (except the British Isles, Spain and Portugal), the Soviet Union, Turkey and Japan. The organism is also found in natural waters (Buchanan and Gibbons 1974).

\section{History}

McCoy and Chapin first isolated the organism in 1912 from ground squirrels (Citellus spp.) in California following reports of a new "plague-like" disease. They called the organism Bacterium tularense after Tulare County, California, where it was found. About 2 years later, the same organism was isolated from cottontail rabbits (Sylvilagus floridanus) in southern Indiana, and the disease was described in humans. Cottontail and jack rabbits (Lepus spp.) have been recognized as important sources of human infections since before 1920 (Davis et al. 1974). Recently a human case associated with bears was reported (Center for Disease Control 1979).

Francisella tularensis was first recorded in Alaska in 1937 when it was isolated from a hare tick, Haemaphysalis leporispalustris, by Philip and Parker. Williams reported the first serologically confirmed human case of ulcero-glandular tularemia in Alaska in 1946 in a man from Northway in central Alaska who had trapped muskrats prior to his illness. Three cases acquired from snowshoe hares (Lepus americanus Erxleben) were reported in $\overline{1949,1950}$ and 1954 in white residents in central Alaska (Miller 1974). In August 1979 , a Fairbanks man was diagnosed as having tularemia after he became ill 3 days after dressing a snowshoe hare killed by his dog (Center for Disease Control 1979).

A serologic survey of 20 villages in central Alaska in 1954 revealed 4\% of 592 humans tested had tularemia titers greater than or equal to 40 . In 1960, 793 Eskimos and Indians in the National Guard representing villages on the offshore islands in the Bering Sea and along the coast from Bristol Bay to Point Barrow were serologically tested for tularemia. 
Seven percent of those tested from the offshore islands were positive while $39 \%$ of those from the Yukon River delta where there are numerous ponds and lakes with abundant aquatic mammals and wild fowl were positive. None of the preceding serologic reactors demonstrated clinical signs of the disease (Philip et al. 1962).

Rausch isolated F. tularensis from a tundra vole (Microtus oeconomus Pallus) from Ugashik Lake on the upper Alaska Peninsula in 1969 (Rausch et al. 1969). Eight isolates of the organism were recovered from snowshoe hares and ticks in 1971 and again in 1980 following significant mortalities in hares near Fairbanks (R. Zarnke, pers. comm.). Also in 1980,3 domestic dogs each having a history of bringing home hares were found serologically positive for tularemia, and others with similar clinical signs were treated by a Fairbanks veterinarian ( $L$. Winterer, pers. comm.). A wolverine (Gulo gulo) live-trapped near Driftwood, Alaska and a raven collected near Fairbanks, Alaska in 1980 were found to be serologically positive for tularemia with titers of $1: 400$ and $1: 40$ respectively ( $R$. Zarnke, pers. comm.).

\section{Transmission}

Tularemia is transmitted among animal populations by biting insects such as flies (Chrysops), ticks (Dermacentor spp. and Haemaphysal is leporispalustris) and a rabbit louse (Haemodipsus ventricosus) (Jawetz et al. 1976, Reilly 1970) (Fig. 1). The organism is transmitted in aquatic environments by rodents or other animals that die and contaminate the water (Fig. 2). Tularemia is trans- mitted to humans by many animals including rabbits, hares, muskrats, beavers, squirrels (Sciuridae) or biting insects including ticks, flies and mosquitoes. Man becomes infected by handling infected carcasses, ingesting contaminated water or improperly cooked meat, inhaling airborn organisms in dust or by being bitten by an animal whose mouthparts have been contaminated from eating another infected animal (Weaver and Hollis 1980) (Figs. 1 and 2 ).

\section{Signs, Symptoms and Pathogenesis}

After the causative organism gains access to the body or bloodstream, it moves from the vascular endothelium to the superficial lymphatics. Lymphadenitis or a transitory bacteremia follows, resulting in necrosis in the spleen, liver, lungs, lymph nodes and bone marrow. Alveolar epithelium, hepatic cells, and reticuloendothelial cells of the spleen and lymph nodes may be invaded. Bacterial and cellular debris enhance the production of thrombi which in turn leads to necrotic foci in the spleen, liver and lymph nodes. Clinical signs in animals are not always readily apparent. Moreover, infected wildlife species are usually not found until they are moribund or dead. Infected hares and rabbits have been observed to behave oddly, run slowly or to be easily captured. Infected rabbits do not raise their heads or carry their front feet well. They may rub their noses or forefeet into the ground and have recurrent tonic spasms with intermittent staggering. Infected mink and fox are reported to have anorexia and lassitude (Reilly 1970). Signs in domestic dogs include an elevated 


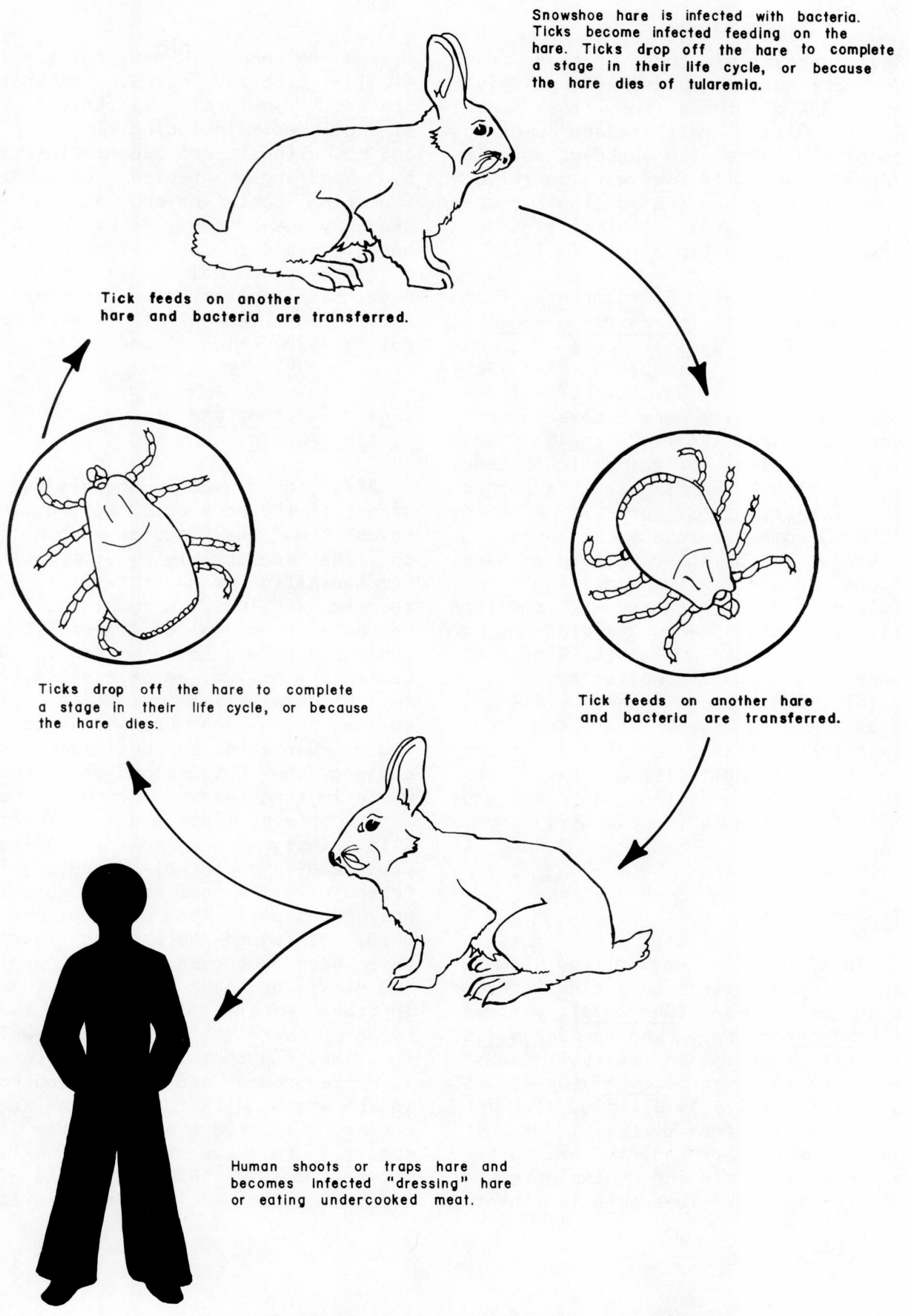

Figure I. Terrestrial Cycle of Tularemia. 


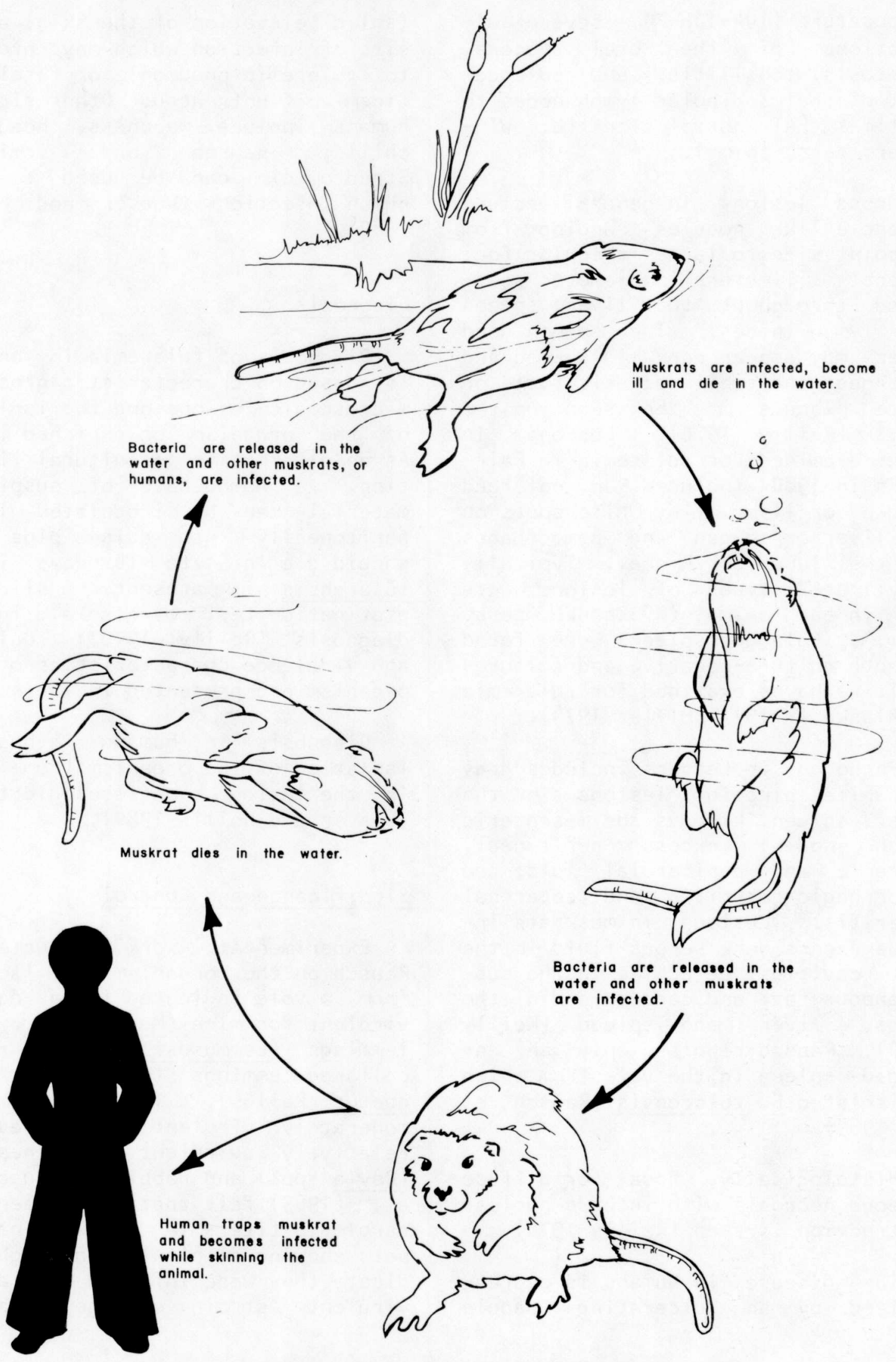

Figure 2. Aquatic Cycle of Tularemia. 
temperature $\left(104-106^{\circ} \mathrm{F}\right)$, severe $u l-$ cerations of the oral mucosa, halotosis, tonsilitis, and enlargement of the mandibular lymph nodes to 10 times their normal size (L. Winterer, pers. comm.).

Gross lesions in general include tubercle like nodules ranging from pinpoint size to large irregular foci several millimeters in diameter scattered throughout the liver, spleen and lymph nodes. The spleen and liver may appear dark bluish-red and enlarged, and small necrotic foci or white plaques may be seen on the lungs (Reilly 1970). Lesions in hares examined for tularemia in Fairbanks in 1980 included an enlarged spleen or liver, tiny white spots on the liver or spleen and hemorrhages in the lung or kidney. Typically only 1 or 2 types of lesions were seen in each animal (R. Zarnke, pers. comm.). Enlarged spleens were found in both culture-negative and culturepositive hares examined for tularemia in Alaska in 1971 (Miller 1974).

Pathology in beavers includes gray and white pinpoint lesions in the liver, spleen, kidneys and mesenteric lymph nodes; excess peritoneal, thoracic and pericardial fluid; and hemorrhagic gastritis and catarrhal enteritis. Lesions in muskrats include excess dark serous fluid in the body cavities; petechiae in the subcutaneous fat; and congestion in the lungs, liver and spleen (Reilly 1970). Rausch reported only an enlarged spleen in the vole from which he isolated $\underline{F}$. tularensis (Rausch et al. 1969).

Histologically, focal or diffuse caseous necrosis with intense nuclear destruction is seen (Reilly 1970).

The disease in humans is characterized by an ulcerating papule (solid elevation of the skin) at the site of infection which may progress to tularemic pneumonia or fatal septicemia if untreated. Other signs in humans include weakness, headache, chills, nausea and vomiting. Streptomycin can be used to treat human infections (Weaver and Hollis 1980).

\section{Diagnosis}

Diagnosis of tularemia in animals is based on characteristic gross and microscopic lesions and the isolation of the organism on enriched media. As an alternative to cultural isolation, a homogenate of suspicious material can be inoculated intraperitoneally into guinea pigs which should die in 5 to 10 days if $\mathbf{F}$. tularensis is present. A slide agglutination test may also aid in the diagnosis (Reilly 1970). Cultural and virulence characteristics of the organism are presented in Table 1.

Diagnosis in humans is based on isolation of the organism from fluid in the lesion or on serologic titers (Weaver and Hollis 1980).

\section{Significance and Control}

Experimental work conducted by Rausch on the organism he isolated from a vole indicated it was equally virulent for mice (Mus spp.), brown lemmings (Lemmus sibericus Kerr) and collared lemmings (Dicrostonyx torquatus Pallus). The organism was moderately virulent for voles but relatively avirulent for guinea pigs (Cavia spp.) and rabbits. Rausch et al. (1969) felt that the presence of serologic titers in Alaskan natives not showing clinical signs might indicate they were infected with a less virulent strain of the organism. 
Virulence studies conducted by Miller also indicated Rausch's vole isolate did not kill guinea pigs or hares, but that the organism isolated from hares and ticks was fatal for guinea pigs, hares, rabbits and mice. He concluded that there are 2 distinct strains of $F$. tularensis in Alaska: the virulent strain from hares and the avirulent strain from rodents (Miller 1974). The Alaskan vole isolate differs from $F$. tularensis var. palearctica in that the former is avirulent for rabbits and guinea pigs, while the latter is moderately virulent for rabbits and virulent for guinea pigs (Buchanan and Gibbons 1974, Rausch et al. 1969). Thus, the Alaskan hare isolate appears to be conspecific with F. tularensis var. tularensis, but the vole isolate does not seem to fit existing species descriptions.

Rabbits infected with virulent organisms rarely live more than 1 week and invariably die. There is no evidence that jack rabbits survive infection, and it may be the same for beavers and muskrats (Reilly 1970). Diagnosis of tularemia in hares and dogs near Fairbanks is significant not only for mortalities in the hare populations, but also for transmission to domestic animals and possibly man.

Human tularemia associated with hares in Alaska is well documented. Recent serologic evidence of the disease in black bears (Ursus americanus) in Idaho and an associated human infection indicate these and other species might pose a public health hazard in Alaska also.

Large-scale poisoning of voles and mice has been used in the Soviet Union and may be indicated in massive outbreaks, but it is doubtful the disease could be eradicated by these means. Control of interstate shipment of lagomorphs for food or stocking purposes might reduce the incidence of the disease in man. Hunters should be advised to wear gloves when handling hares, rabbits and bears, and to cook the meat thoroughly. In addition, natural waters in enzootic areas should not be drunk and ticks and fleas should be avoided (Reilly 1970).

\section{$\underline{\text { References }}$}

BINNINGER, C. E., J. J. BEECHAM, L. A. THOMAS and $L$. D. WINWARD. 1980. A serological survey for selected infectious diseases of black bears in Idaho. J. Wildl. Dis. $16: 423-430$.

BUCHANAN, R. E. and N. E. GIBBONS, eds. 1974. Bergey's Manual of Determinative Microbiology. Williams and Wilkins Co., Baltimore, MD. $1246 \mathrm{pp}$.

CENTER FOR DISEASE CONTROL. 1979. Morbidity and mortality weekly report. U.S. Dept. HEW, PHS. Nov. 9, 1979.

CENTER FOR DISEASE CONTROL. 1980. Morbidity and mortality weekly report. U.S. Dept. HEW, PHS. Feb. 8, 1980.

DAVIS, B. D., R. DULBECCO, H. N. EISEN, H. S. GINSBERG and W. B. WOOD, eds. 1974. Microbiology. Harper and Row, Hagerstown, MD. $1562 \mathrm{pp}$.

EMMONS, R. W., J. RUSKIN, M. L. BISSET, D. A. UYEDA, R. M. WOOD and C. L. LEAR. 1976. Tularemia in a mule deer. J. Wildl. Dis. $12: 459-463$.

JAWETZ, E., J. L. MELNICK and E. A. ADELBERG. 1976. Medical Microbiology. Land Medical Publications, Los Altos, CA. 542 pp. 
TABLE 1

Comparison of various species of Francisella.

\begin{tabular}{|c|c|c|c|c|c|c|}
\hline Species & $\begin{array}{l}\text { Acid from } \\
\text { Sucrose }\end{array}$ & $\begin{array}{l}\text { Utilizes } \\
\text { Glycerol }\end{array}$ & $\begin{array}{c}\text { Citrulline } \\
\text { ureidose }\end{array}$ & Rabbits & $\begin{array}{c}\text { Pathogenic for } \\
\text { White mice }\end{array}$ & Guinea pigs \\
\hline
\end{tabular}

\section{F. tularensis}

var. tularensis 1

$+$

$+$

$+$

$+$

$+$

F. tularensis var. palaearctica2

$-$

$-$

Less

Less

More

F. novicida

Alaskan vole isolate

Alaskan hare isolate

1Reported only in North America 20ld World species 
MILLER, L. G. 1974. Further studies on tularemia in Alaska: virulence and biochemical characteristics of indigenous strains. Can. J. Microbiol. 20:1585-1590.

PHILIP, R. M., B. HUNTLEY, D. B. LACKMAN and G. W. COMSTOCK. 1962. Serologic and skin test evidence of tularemia infection among Alaskan Eskimos, Indians and Aleuts. J. Infec. Dis. $110: 220-230$.

RAUSCH, R., B. E. HUNTLEY and J. G. BRIDGES. 1969. Notes on Pastuerella tularensis isolated from a vole, Microtus oeconomus Pallas, in Alaska. Can. J. Microbiol. 15:47-55.
REILLY, J. R. 1970. Tularemia. pp. 175-199. In: Infectious Diseases of Wild Mammals. J. W. DAVIS, L. H. KARSTAD and D. O. TRAINER, eds. lowa State Univ. Press, Ames, IA. WEAVER, R. E. and D. G. HOLLIS. 1980. Gram negative fermentative bacteria and Francisella tularensis. pp. 242-262. In: Manual of Clinical Microbiology. E. H. LENNETTE, A. BALOWS, W. H. HOUSLER, JR. and J. P. TRUANT, eds. American Society for Microbiology, Washington, DC.

\title{
Brucellosis
}

\author{
Robert A. Dieterich
}

\section{Introduction}

Brucellosis is a bacterial disease caused by a small, Gram negative nonsporeforming coccobacillus. Several different species of this organism are recognized including Brucella abortus, B. suis, B. melitensis, B. canis, B. neotomae, $\underline{B}$. ovis and $\underline{B}$. suis type 4. The disease has several synonyms of which Bang's disease, contagious abortion and undulant fever (in humans) are the more common. Brucellosis occurs in domestic cattle (Bos spp.), sheep (Ovis spp.), goats (Capra spp.), swine (Sus spp.), horses (Equus spp.) and dogs (Canis familiaris). It also infects a variety of wild animals such as elk (Cervus elaphus), bison (Bison bison), caribou and reindeer (Rangifer tarandus), wolves (Canis lupus), red and arctic fox (Vulpes vulpes and Alopex lagopus) and bears (Ursus spp.) (Neilland 1970). In Alaska, the disease is of primary concern in reindeer and caribou which are infected by the species $B$. suis type 4. It has been suggested that this species of Brucella should actually be named B. rangiferi in reference to its usual host.

\section{History}

The disease organism was first isolated from humans dead of "gastric fever" in 1887 by David Bruce, whose name is the basis for the term brucellosis. In 1897, Frederick Bang isolated and identified Brucella abortus from aborted bovine fetuses. As time passed, more was learned of the organism, and it was found in the 
wide range of hosts mentioned earlier. Important sources of infection for humans were found to be milk, aborted fetuses or slaughtered cattle carcasses. Symptoms in humans include malaise, fever, weakness, aches, sweats, gastrointestinal and nervous disturbances, hepatitis and osteomyelitis.

Brucella has been known to infect reindeer and caribou in the Soviet Union for a number of years (Davidov 1974, Gorban 1977). The disease was identified in Alaska in humans with 49 cases being recorded between 1939 and 1953. During that period, it was believed that these cases were attributable to unpasteurized milk or contact with cattle or swine. However, epidemiologic evidence suggested that caribou might have been the source of infection in some cases. Brucella was isolated from caribou in Alaska in 1963, which established the actual source of most recent infections (Huntley et al. 1963). It is not known whether the disease was introduced into Alaska with the importation of reindeer from Siberia in the late 1800 s or if it has been present since prehistoric times. The species of Brucella isolated from caribou was found to be identical to human isolates obtained from several Native patients in rural Alaska (Meyer 1966). In 1964, approximately $20 \%$ of the residents of Fort Yukon and Arctic Village had positive titers for brucellosis as determined by the rapid slide test. In a serologic study of 7 villages from 1962-1964, $11 \%$ of 763 individuals tested had evidence of past Brucella infection (Brody et al. 1966). It was also reported that 8 clinical cases occurred from 1962-1964 in Eskimos having frequent contact with reindeer or caribou in northern Alaska. No cases were diagnosed at that time among individuals living outside that area. Seventeen cases of brucellosis in humans were reported in Alaska between 1966 and 1975. Authors point out that there is little doubt that many cases do not come to the attention of medical personnel. Physicians in Alaskan bush communities are frequently transient and many cases of brucellosis may go undiagnosed because medical personnel are not alerted to the multiple symptoms of the disease.

Following the detection of brucellosis in Alaskan reindeer and caribou, evidence of the disease was also found in Alaskan grizzly bears (Ursus arctos), wolves, red foxes, sled dogs and arctic ground squirrels (Spermophilus parryii) that come into contact or feed upon tissues of reindeer and caribou (Neiland 1970, 1975). Serologic testing of caribou and reindeer for brucellosis was carried out during the early 1960s and 1970s (Neiland et al. 1968). The highest prevalence of animals having a positive blood test at various times was $30 \%$ for the Arctic Caribou Herd, $6.5 \%$ for the Nelchina Caribou Herd and up to $15 \%$ for some of the reindeer herds on the Seward Peninsula. Recently, renewed testing of reindeer on the Seward Peninsula indicates that the disease has spread into herds located on the northern portion with incidence of serologically positive animals reaching $20 \%$ Lesions and symptoms of brucellosis are commonly seen in these infected reindeer herds when the animals are closely observed.

Brucellosis is also recognized as a health problem in elk and bison herds in North America. The disease is currently under study in these populations by several different investigative teams (Thorne and Morton 1978, Thorne et al. 1978). 
Transmission and

\section{Pathogenesis}

Brucella have an affinity for the male and female reproductive organs, and the major impact on herd health occurs because of abortion and sterility. Brucellosis causes abortion, retained placentae and impaired health in female reindeer and caribou. In males, infection of the seminal vesicles, ampullae, testicles and epididymides is seen. In both males and females there can be swelling of the joints with associated lameness (Zhigunov 1961). It is believed that the primary spread of the disease is by contact with infective uterine discharges following abortion. Abortion in reindeer appears to occur 1 to 2 months before normal calving time in early May (Rausch 1978). Calves may also be born alive but weak and die within a few days. Other calves born to infected females can survive, but remain infected as carriers of the disease. In domestic cattle, females commonly abort when initially infected. They may abort the next year, but thereafter they can produce viable calves. This same pattern appears to be true for reindeer, but osteoarthritis, abscesses, and other chronic symptoms of the disease appear as the infection progresses. The exact pathogenesis and impact of brucellosis on reindeer and caribou herds in Alaska has yet to be determined. The role of male animals in the sexual transmission of the disease is not fully understood.

It should be stressed that the primary population impact of brucellosis is reduced reproductive success. Clinically visible symptoms in reindeer or caribou herds represent only a small portion of the overall effect of the disease. When a herd containing infected reindeer or caribou is observed, one can commonly see lame animals. Infected individuals will most often have enlarged carpal (knee) or fetlock (hoof) joints and will only use the affected limb when being chased. These animals will be particularly lame when they first move after resting. Careful examination of males will reveal enlarged testicles, some as large as $12-20 \mathrm{~cm}$ in diameter. Others may only have a swollen epididymis which can only be detected by palpation or necropsy.

Abscesses containing an odorless, thick, light-green purulent material are found in Brucella infected reindeer and caribou. These are most commonly located in the mammary gland but can be found in the reproductive organs, liver, kidney, abdominal cavity or as subcutaneous enlargements.

\section{Diagnosis}

Diagnosis of brucellosis in wildlife can be made by serologic testing or by actual isolation of the causative organism (B. suis type 4 in Alaska) in the Taboratory. In reindeer, it has been found that the commonly used field tests (rapid card test and standard plate test) will not identify all chronically infected carrier animals. These tests do accurately identify acutely infected animals. Research is underway at the University of Alaska to determine which type of serologic test will identify chronically infected animals, and at this time the complement fixation test is the most promising. Unfortunately, this is a complex procedure and can not presently be performed in the field.

The actual isolation of the organism in the laboratory constitutes 
a positive diagnosis, but this procedure depends on providing the laboratory with suitable tissues for culture. Tissues (lesions, lymph nodes, organs, bone marrow) must be submitted within a few hours of aseptic collection, or can be frozen immediately and submitted at a later time. Isolation of the organism is dependent on the proper collection of infected tissues. Brucella infected tissues may not be visibly altered. Handling diseased animals should be done with extreme care using aseptic procedures to reduce the possibility of human infection.

\section{Significance and Control}

The importance of brucellosis in reindeer appears to be substantial. In December 1979, the Reindeer Herders Association named the control of brucellosis and warble fly infestation as its highest research priorities.

It should be emphasized that brucellosis in reindeer and caribou is a disease of low mortality for adult animals and of moderate morbidity. That is, it causes relatively few deaths in adults as a direct result but does infect many animals chronically. Of course, it does cause death of unborn young and greater loss through predation and weather related mortality because of debilitation. Therefore, the occasional observer of a herd will not be impressed with the impact of the disease because they will not see animals lying about dead nor many obviously ill. The actual impact will be seen in herd reproductive success which is difficult to assess in wild populations. Other factors such as climate, nutrition, predators, etc. all affect population numbers, but it is difficult to as- sess the influence of each factor in groups of animals that are only occasionally observed. In domestic animals that are herded closely or confined to pens, the owner will easily notice a retained placenta, dead calf or more importantly, a cow that is barren.

It is not known if the predators of reindeer and caribou serve as end hosts for the disease, or if they are capable of spreading the disease back to uninfected reindeer or caribou. Also unknown are the effects of the disease on the predator itself or its reproductive success. Grizzly bears harbor the disease for a prolonged period after being experimentally infected (pers. obs.).

Brucellosis has rarely been reported in moose (Alces alces) even in areas of overlap with reindeer or caribou range. Moose are highly susceptible to Brucella, and it has been postulated that infected moose may die in a short period of time and thus, are removed from any population being sampled (Corner and Connell 1958, Jellison et al. 1953). Further research is needed before any conclusion can be reached.

Brucellosis is a zoonotic disease, that is, a disease that can spread from animals to man. Some Alaskans that handle reindeer or caribou tissues are infected each year. The number of infections is not great and the degree of illness varies between individuals. Some humans with serologic evidence of brucellosis report few or no symptoms. Others are severely ill and require hospitalization. If brucellosis is recognized early, it can be treated successfully with antibiotics. The chronic cases are more difficult to cure. Brucella organisms rarely infect muscle tissue, so most meat from 
infected animals is safe to eat. Reproductive organs, internal organs, lymph nodes and bone marrow should be handled with care, preferably with protective gloves. Thorough cooking kills the organism but freezing does not. Extreme care should be exercised in handling any fetal membranes or aborted tissues.

Two methods of control are currently being developed for reindeer. First, with the aid of an accurate blood test, infected animals could be detected and subsequently removed from the herd. This removal would have to be linked to the economics of herding because many animals would be involved. However, it is difficult if not unfeasible to round up all the reindeer at 1 time for testing. Therefore, the possibility would exist of reinfection of "clean" herds by non-tested reindeer or by infected migratory caribou or predators. Therefore, development of a suitable vaccine which would protect the animals from infection and thus prevent abortion is a major long-term goal (Dieterich et al. 1980). Such a vaccine would not only increase herd productivity, but would also reduce the main source of transmission. Costs associated with the destruction of infected animals would be greatly reduced. Information gained from reindeer control programs may eventually lead to attempts to control brucellosis in other wildlife species.

Newly developed drug delivery methods such as the Ballistic Implant System could make vaccination or treatment of some free-ranging species possible. This system is based on an air powered rifle that delivers a biodegradable implant pellet containing a vaccine or drug, plus a dye marker ball that stains and identifies animals as they are treated.

\section{References}

BRODY, J. A., B. HUNTLEY, T. M. OVERFIELD and J. MAYNARD. 1966. Studies of human brucellosis in Alaska. J. Inf. Dis. 116:263-269. CORNER, A. H. and R. CONNELL. 1958. Brucellosis in bison, elk and moose in Elk Island National Park, Alberta, Canada. Can. J. Comp. Med. 22:9-20.

DAVIDOV, N. N. 1974. On the epizootiology of brucellosis in domestic reindeer in Yakutia. Proceedings of the Fifth All-Union Symposium on Biological Problems in the North, 1972, Magadan. pp. 229-231.

DIETERICH, R. A., J. K. DIETERICH and B. L. DEYOE. 1980. Observations on reindeer vaccinated with Brucella melitensis strain $\mathrm{H}-38$ vaccine and challenged with Brucella suis type 4. Second International Reindeer/Caribou Symposium. In press.

GORBAN, L. V. 1977. Natural foci of brucellosis in the far north. Microbiol. Epidemiol. Immunobiol. V8.

HUNTLEY, B. E., R. N. PHILIP and J. E. MAYNARD. 1963. Survey of brucellosis in Alaska. J. Inf. Dis. 112:100-106.

JELLISON, W. L., C. W. FISHEL and E. L. CHEATUM. 1953. Brucellosis in a moose, Alces americanus. J. Wildl. Manage. $17: \overline{217-218 .}$

MEYER, M. E. 1966. Identification and virulence studies of Brucella strains isolated from Eskimos and reindeer in Alaska, Canada and Russia. Am. J. Vet. Res. 37:353-358.

NEILAND, K. A., J. A. KING, B. E. HUNTLEY and R. E. SKOOG. 1968. The diseases and parasites of Alaskan wildlife populations, Part I. Some observations on brucellosis in caribou. J. Wildl. Dis. $4: 27-36$. 
NEILAND, K. A. 1970. Rangiferine brucellosis in Alaskan canids. J. Wildl. Dis. 6:136-139.

- 1975. Further observations in rangiferine brucellosis in Alaskan carnivores. J. Wildl. Dis. $11: 45-52$.

RAUSCH, R. L. 1978. Brucellosis in reindeer, Rangifer tarandus L., inoculated experimentally with Brucella suis type 4. Can.J. Microbiol. 24:129-135.

THORNE, E. T. and J. K. MORTON. 1978. Brucellosis in elk. I. Serologic and bacteriologic survey in Wyoming. J. Wildl. Dis. $14: 74-81$.
THORNE, E. T., J. K. MORTON, F. M. BLUNT and H. A. DAWSON. 1978. Brucellosis in elk. II. Clinical effects and means of transmission as determined through artificial infections. J. Wildl. Dis. $14: 280-291$.

WITTER, J. and D. C. O'MEARA. 1970. Brucellosis. pp. 249-255. In: Infectious Diseases of Wild Mammals. J.W. DAVIS, L. H. KAR $\overline{S T A D}$ and D. 0. TRAINER, eds. I owa State University Press, Ames, IA.

ZHIGUNOV, P. S., ed. 1961. Reindeer Husbandry. Izdatel'stvo Sel'skokhozyaistvennoi Literatury, Zhurnalov i Plakatov, Moskow, USSR. $348 \mathrm{pp}$.

\title{
Leptospirosis
}

\author{
Jamie K. Morton
}

\section{Introduction}

Leptospirosis is caused by a group of antigenically distinct serologic groups or serotypes of the genus Leptospira. Two complexes are currently recognized: L. interrogans, the parasitic or pathogenic group; and $\underline{L}$. biflexa, the free-living or water forms (Buchanan and Gibbons 1974). Nomenclature in the older literature actually refers to genus and serotype rather than to genus and species. Leptospira interrogans serovar icterohaemorrhagiae is most commonly found in rats (Rattus spp.), L. interrogans serovar canicola in dogs (Canis familiaris), and $\underline{L}$. interrogans serovar pomona in swiñe (Sus spp.) and cattle (Bos spp.).

The leptospires are extremely small, coiled, motile spirochete bacteria. They are easily killed by heat, drying and most disinfectants. Leptospires do not survive in alkaline or acidic extremes and do best in the $\mathrm{pH}$ range of 6.0 to 8.0 (Alexander 1970).

Numerous species have been described as susceptible, but those indigenous or related to species found in Alaska include raccoon (Procyon lotor), beaver (Castor canadensis), red fox (Vulpes vulpes), muskrat (Ondatra zibethica), bobcat (Lynx rufus), woodchuck (Marmota monax) and voles (Microtus spp.) (Roth 1970). Leptospira interrogans serovars ballum and grippotyphosa were isolated from muskrats in central Illinois in 1972 (Paul et al. 1972). Leptospira interrogans serovar ballum was isolated from a vole on the Kenai peninsula in 1966 (Woods 1974). Serologic evidence of leptospirosis in Alaska has been 
detected in a wolf (Canis lupus) ( $L$. Schultz, pers. comm.). It is likely that other species yet unreported are also susceptible.

California sea lions (Zalophus californianus californianus) (Scheffer 1958) and northern fur seals (Callorhinus ursinus) are susceptible to leptospirosis and may be infected with serotypes canicola, icterohemorrhagiae, autumnalis or pomona, the latter being the most predominant.

Leptospirosis in terrestrial wildlife is important primarily because of the potential of serving as a reservoir for human infections and domestic animals. In marine mammals it may also cause mortality of adults and pups, and may be a contributing factor or causative agent of abortion.

Because manifestations of the disease in Alaska differ between terrestrial wildlife and marine mammals, the following discussion will be divided into two parts.

Terrestrial Wildlife

\section{History}

Noguchi ascribed the name Leptospira to the spirochete group of bacteria obtained from Japanese workers in 1917 (Roth 1970). By 1954 over 50 serotypes had been named, and there are currently over 130 serotypes 1 isted in Bergey's Manual of Determinative Bacteriology (Buchanan and Gibbons 1974). The organism was first cultured from cattle in the $1940 \mathrm{~s}$, and most of the knowledge of the disease in wildlife has been gained since the 1950s. Most of the leptospirosis research in wild species has been done east of the Mississippi River. Infections in domestic and wild animals are reported worldwide (Roth 1970).

\section{Transmission}

Leptospire bacteria tend to localize in the kidneys and may be excreted in the urine. Excretion of the organisms usually appears 10 to 12 days post infection and may last from 1 day to 2 years. Mice and rats excrete leptospires for the remainder of their lives following infection. Leptospires may persist for weeks in moist, neutral or slightly alkaline soil (Gillespie et al. 1957). Animals and humans may be infected by contact with or ingestion of contaminated water or food (when moist). People are commonly exposed by swimming in water contaminated with fecal matter. Organisms may also enter the host via the mucous membranes of the eye, nose, mouth and abraded skin (Alston and Broon 1958, Busch 1970, Diesch et al. 1970).

\section{Signs, Symptoms and Pathogenesis}

Effects of leptospirosis range from fatal to inapparent depending on the host and serotype involved. Signs usually occur during the second week of infection and include lack of appetite (anorexia), weakness, anemia, jaundice (icterus), fever and inflammation of the kidney (nephritis). The fever in experimentally infected guinea pigs lasts 3 to 5 days followed by jaundice, hemorrhages in the skin, subcutaneous tissues and muscles and death within 2 weeks. Naturally infected rats have a life-long chronic infection affecting the kidneys. The disease may cause abortion in cattle. Fatal cases are characterized by hemoglobin in the urine (hemoglobinuria), ic- 
terus, interstitial nephritis, liver inflammation (hepatitis) and hemorrhages (Alexander 1970). Kidney lesions highly suggestive of leptospirosis in opossums (Didelphis virginiana), and raccoons have included scarring and degeneration of the kidney of glomeruli (Diesch et al. 1970).

In limited experiments conducted in white-tailed deer (Odocoileus virginianus), symptoms have ranged from inapparent to a febrile response to abortion. The pathogenesis in wild animals is not well determined (Roth 1970).

\section{Diagnosis}

Although difficult, the organisms can be demonstrated in the urine and tissues by darkfield microscopy, silver stains (Warthin-Starry) or fluorescent antibody procedures. Identification of serotypes by these methods is not possible (Alexander 1970).

The microscopic agglutination test using living or formalin-killed organisms as antigen is a standard diagnostic test and is highly serotype specific. The macroscopic agglutination test is easier to perform, but is not as sensitive in other than current or recent infections; thus, its application for serologic surveys is limited (Alexander 1970).

Isolation of the organism gives the most conclusive results but the culturing is very difficult. Blood and urine are frequently cultured in live animals, but the choice of tissue type of body fluid for culture is also related to the stage of the disease. Blood should be cultured during the first week of disease, kidneys in chronic infections and liver, spleen and kidney on post mortem examination. Urine is valuable after the first week of infection. All tissues should be cultured as soon as possible after death as leptospires are quite fragile and do not survive the changes of post mortem autolysis. Commercially available media such as Fletcher's semi-solid may be employed. Undiluted and a 1:10 dilution of urine may also be cultured in a media with added 5-fluorouracil to inhibit contaminants. Growth may not be detected for several days.

Discrepancies between serologic and bacteriologic tests have been reported. However, antibodies are not expected to be detected during the first week of infection. Diesch et al. (1970) reported that the macroscopic agglutination test was more reliable for detecting L. ballum in opossums, but that the microscopic agglutination test was more valuable for detecting ㄴ. grippotyphosa in raccoons. He concluded that there may also be insufficient reaction of the particular serotype in the host and the antigen being used, and that the antibody-antigen reaction may not be the same for all hosts anyway.

\section{Significance and Control}

The major role of leptospirosis in wildlife appears to lie in their role as reservoirs and vectors to domestic animals and humans (Diesch et al. 1970). No cases of human leptospirosis were reported in Alaska from 1969-1978 (Center for Disease Control 1978), but a serologic titer was detected on 1 human in 1979 ( $B$. Edmundson, pers. comm.).

Kingscote reported serologic evidence of predominantly $\underline{L}$. inter- 
rogans serovars pomona and grippotyphosa in a white-tailed deer in Ontario, but concluded the disease had not depressed the deer population (Kingscote 1969). Shotts and Hayes (1970) detected antibodies in whitetailed deer in the southeastern United States, but concluded the natural infections were subclinical and of little or no consequence to the deer.

Immunity varies with the serotype and the host species. Human leptospirosis can be treated with streptomycin, penicillin and tetracyclines.

Control in terrestrial wildlife is considered generally unfeasible due to the multiplicity of serotypes, the broad range of susceptible hosts, and the extended carrier state of the disease (Roth 1970).

\section{Marine Mammals}

\section{History}

In the fall of 1947 an epizootic of unknown cause occurred among California sea lions (Northway 1972, Vedros et al. 1971). An outbreak of abortions was observed in sea 1 ions in California in the Channel Island rookeries in the spring of 1970. That summer and fall, stranded sea 1 ions numbered about 4 times what was usually observed. of 315 stranded on the California coast, 125 were dead, and 5 dead ones were observed among 230 animals sick or stranded on the Oregon coast. Leptospirosis was diagnosed as a contributing factor in that epizootic (Vedros et al. 1971). In 1972 leptospirosis was reported in northern fur seals. "Multiple hemorrhagic perinatal complex"' is now associated with acute leptospirosis of the newborn (Smith et al. 1977).
Transmission

Leptospirosis is transmitted among marine mammals by direct contact with the organism shed by infected animals. Transmission among marine mammals may be enhanced by their thigmotactic nature, i.e. the use of certain "hauling" areas are contaminated by infected urine and placental remnants. Leptospires then penetrate abrasions and wounds of susceptible animals (Mcllihattan et al. 1971).

It is felt that the low numbers of prepelagic pups with leptospirosis antibody titers indicates that the primary source of infection is probably in the ocean environments. The low titers that are present in the pups are probably due to passive maternal antibodies. Transmission may occur in the rookeries though to a lesser extent (Smith et al. 1977). It is also hypothesized that the occurrence of leptospirosis may be at least partially correlated to environmental pollutant concentrations (Gilmartin et al. 1976, Vedros et al. 1971).

\section{Signs, Symptoms and}

\section{Pathogenesis}

Infected pre-term sea lion pups display an incomplete or complete lack of fur, near lack of motor activity and shallow breathing (Gilmartin et al. 1976). Adults have been observed to have general lethargy, a disinclination to use their hind limbs, difficulty moving about, dehydration manifested by extreme thirst and the use of fresh water sloughs, a febrile response and respiratory congestion (Smith et al. 1974b, Vedros et al. 1971). 
Most consistently reported observations include a chronic interstitial nephritis (Gilmartin et al. 1976, Mclllhattan et al. 1971, Smith et al. 1977, Vedros et al. 1971), subcapsular hemorrhage of the kidney, liver and lymph nodes (Gilmartin et al. 1976, Smith et al. 1974a, 1974b, 1977, Vedros et al. 1971) and modification of the pericardial fluid (Mcllihattan et al. 1971, Northway 1972, Smith et al. 1974b, Vedros et al. 1971). Other lesions observed include increased size (hypertrophy) of the adrenal medulla (Gilmartin et al. 1976); fluid (edema) around the bronchioles of the lung ( $G$ ilmartin et al. 1976); congested spleen (blood accumulation in the vessels) with central abscesses (Gilmartin et al. 1976); mild bronchopneumonia (involving the bronchi) (Gilmartin et al. 1976, Mclllhattan et al. 1971); friable (easily broken) liver (Gilmartin et al. 1976, Smith et al. 1974b); blood in the anterior eye chamber (Smith et al. 1974b, 1977); hemorrhages in the dividing portion (septum) of the thymus, mucous membranes (mucosa) of the colon and iris (Smith et al. 1974b); congestion of the brain and adrenal glands (Smith et al. 1974b, 1977); depletion of cellular components in the lymph nodes and bone marrow (Smith et al. 1974a); thick b!ack bile in the gall bladder; enteritis (Vedros et al. 1971); round, black areas 0.4-0.5 mm in diameter in the mucosa of the midportion of the stomach (Mclllhattan et al. 1971); focal inflammation of the tongue (glossitis) (Smith et al. 1977); focal death (necrosis) and scarring of the placenta (Smith et al. 1977); and small nodules of macrophages (granulomas) in the connective tissue of the outer layer of the eyeball (sclera) (Smith et al. 1977).
Cellular poisons (cytotoxins) produced by the organisms may be responsible for the loss of vascular integrity leading to hemorrhage. Cytotoxins may also cross placental membranes to affect the pups (Smith et al. 1974a).

\section{Diagnosis}

Serologic testing is the primary diagnostic tool. Both micro- and macroagglutination tests are used, the microagglutination test being more sensitive. Organisms can be demonstrated in urine and tissues by darkfield microscopy, silver stains (Warthin-Starry) or fluorescent antibody procedures (Alexander 1970).

Culture techniques using guinea pig inoculation or selective media can be used (Smith et al. 1974b). Smith et al. (1974a) reported that isolation of leptospires from pups was inconsistent and felt that the lesions were probably caused by cytotoxins transferred from the mothers to pups in utero.

\section{Significance and Control}

The prevalence of Leptospira agglutinating antibodies among northern fur seals sampled in the Bering Sea from 1972 to 1975 was about 10\% in pelagic females and bachelor bulls and $2 \%$ in pre-pelagic 4-month-old pups (Smith et al. 1977). Premature birth (parturition) has been reported to account for $20 \%$ of California sea lion pup mortality on San Miguel Island, California (Gilmartin et al. 1976).

It is felt that the significance of leptospirosis is influenced by environmental pollutant levels such as PCB's (polychlorinated biphenyl 
compounds) or DDT ( $p, p$ - DDE and $p, p$ - DDD). Tissue concentrations of pollutants has been found to be 2.4 to 8 times higher in aborting females than in full-term females with normal pups (Gilmartin et al. 1976). These pollutants may be directly responsible for abortions, or they may cause immunosuppression resulting in increased susceptibility to disease (De Long et al. 1973, Vos and Deroij 1972).

Strandings and mortalities reported by Vedros in 1971 were confined to subadult males 2 to 8 years old. Vedros suggested that this may have reflected a herd immunity (or susceptibility) and may have been density related (Vedros et al. 1971).

Smith (1977) proposed that fur seals prior to their first pelagic cycle were most susceptible and suggested vaccination of the newborn on the rookeries (Smith et al. 1977).

Marine mammal leptospirosis constitutes a potential public health hazard. Dead animals washed ashore are often pillaged for teeth, skulls, etc. Alaska coastal areas rely heavily on marine mammals for subsistence and economic purposes.

\section{References}

ALEXANDER, A. D. 1970. Leptospira. pp. 244-256. In: Manual of Clinical Microbiology. I. E. BLAIR, E. H. TENNETTE and J. P. TRUANT, eds. Am. Soc. Microbiol., Bethesda, MD. $970 \mathrm{pp}$.

ALSTON, J. M. and J. C. BROON. 1958. Leptospirosis in Man and Animals. E \& S Livingstone, Ltd. London, ENG. $367 \mathrm{pp}$.

BUCHANAN, R. E. and N. E. GIBBONS, eds. 1974. Bergey's Manual of Determinative Bacteriology. 8 th ed. Williams and Wilkins Co., Baltimore, MD. $1246 \mathrm{pp}$.
BUSCH, L. A. 1970. Epizootiology and epidemiology of leptospirosis. J. Wildl. Dis. 6:273-274.

CENTER FOR DISEASE CONTROL. 1978. Leptospirosis Surveillance, Annual Summary. U.S. Dept. HEW, PHS (1969-1977).

DE LONG, R. L., W. G. GILMARTIN and J. G. SIMPSON. 1973. Premature births in California sea lions: Association with organochlorine pollutant residue levels. Science 181:1168-1170. .

DIESCH, S. L., W. F. MCCULLOCH, L. J. BRAUN and J. R. DAVIS. 1970. Detection and ecology of leptospirosis in lowa wildlife. J. Wildl. Dis. 6:275-278.

GILLESPIE, R. W. H., S. G. KENZY, L. M. RINGEN and $F$. $K$. BRACKEN. 1957. Studies on bovine leptospirosis. III. Isolation of Leptospira pomona from surface waters. Am. J. Vet. Res. $18: 76-80$.

GILMARTIN, W. G., R. L. DE LONG, A. W. SMITH, J. C. SWEENY, B. W. DE LAPP, R. W. RISEBROUGH, M. D. DAILY and D. B. PEAKALL. 1976. Premature parturition in the California sea lion. J. Wildl. Dis. 12:104-115.

KINGSCOTE, B. F. 1969. Leptospirosis survey in a whitetailed deer herd in Ontario. J. Wildl. Dis. 5:68-72.

McGOWAN, J. E., L. KARSTAD and N. A. FISH. 1963. Leptospirosis in Ontario Cervidae, isolation of Leptospira pomona from a deer fetus. Trans. 28th North Am. Wildl. Nat. Res. Conf. pp. 199-206.

MCILLHATTAN, T. J., J. W. MARTIN, R. J. WAGNER and J. O. IVERSON. 1971. Isolation of Leptospira pomona from a naturally infected California sea lion, Sonoma County, California. J. Wildl. Dis. 7:195-197. 
NORTHWAY, R. B. 1972. Leptospirosis in a California sea lion (Zalophus californianus). Vet. Med./Sm. Animal Clin. 67:138\&141.

PAUL, J. R., L. E. HANSON, P. R. SCHNURRENBERGER and R. J. MARTIN. 1972. Leptospira interrogans serotypes ballum and grippotyphosa isolated from the muskrat. J. Wildl. Dis. 8:54-56.

ROTH, E. E. 1970. Leptospirosis. pp. 293-303. In: Infectious Diseases of Wild Mammals. J.W. DAVIS, L. H. KARSTAD, D. 0. TRAINER, eds. lowa State Univ. Press, Ames, IA.

SCHEFFER, B. V. 1958. Seals, Sea Lions, and Walruses. Stanford Univ. Press, Palo Alto, CA. 179 pp.

SHOTTS, E. B. and F. A. HAYES. 1970. Leptospiral antibodies in whitetailed deer of southeastern United States. J. Wildl. Dis. 6:295-298.

SMITH, A. W., R. J. BROWN, D . E. SKILLING and R. L. DE LONG. 1974a. Leptospira pomona and reproductive failure in California sea lions. J. Am. Vet. Med. Assn. 165(11): 996-998.
SMITH, A. W., C. M. PRATO, W. G. GILMARTIN, R. J. BROWN and M. C. KEYES. 1974b. A preliminary report on potentially pathogenic microbial agents recently isolated from pinnipeds. J. Wildl. Dis. $10: 54-59$.

SMITH, A. W., R. J. BROWN, D. E. SKILLING, H. L. BRAY and M. C. KEYES. 1977. Naturally-occurring leptospirosis in northern fur seals (Callorhinus ursinus). J. Wildl. Dis. 13:144-148.

VEDROS, N. A., A. W. SMITH, J. SCHONEWALD, G. MIGAKI and R. C. HUBBARD. $1971 . \quad$ Leptospirosis epizootic among California sea lions. Science 172:1250-1251.

VOS, J. G. and T. DEROIJ. 1972. Immunosuppressive activity of polychlorinated biphenyl preparation on the humoral immune response in guinea pigs. Tox. and Appl. Pharm. 21:549-555.

WOODS, F. N. 1974. Leptospira interrogans in the Ballum serogroup from a vole Microtus oeconomous (Pallas) in Alaska. J. Wildl. Dis. 10:325-326. 


\title{
Tuberculosis
}

\author{
Jamie K. Morton
}

\section{Introduction}

Tuberculosis is a chronic infectious disease caused by acid-fast, non-motile, non-sporeforming bacteria of the genus Mycobacterium (Hagan and Bruner 1961, Tarshis 1966, Winkler and Gale 1970). Three species are most frequently encountered: Mycobacterium tuberculosis, M. bovis and $M$. avium. The organism is moderátely resistant to heat, fairly resistant to dessication and is rapidly destroyed by direct sunlight (Hagan and Bruner 1961).

Each species may be pathogenic for several hosts. Mycobacterium tuberculosis typically infects man, but also produces the disease in other primates, dogs (Canis familiaris), guinea pigs (Cavia spp.) and hamsters (Cricetus spp.). Experimentally, it is usually non-pathogenic for rabbits (Sylvilagus spp.), cats (Felis catus), goats (Capra spp.), bovine animals (Bos spp.) or domestic fowl (Tetraonidae). Mycobacterium bovis, originally isolated from cattle, also causes tuberculosis in wild and domestic ruminants, man and other primates, dogs, cats, swine. (Sus spp.), parrots (Psittaciformes), birds of prey (Falconiformes), rabbits, guinea pigs, mice (Mus spp.), hamsters and rats (Rat $\overline{t u s}$ spp.). It is not pathogenic for most fowl. Mycobacterium avium is widely distributed as the causal agent of tuberculosis in birds but can also be pathogenic for cattle, swine or other animals. It has been implicated in human tuberculosis in a few recorded cases. Mycobacterium microti causes generalized tuberculosis in voles (Microtus spp.). Mycobacterium marinum causes a tuberculosis-like disease in fish and a chronic skin lesion known as "swimming pool granuloma" in humans (Wolinsky 1973).

The geographic distribution of tuberculosis is worldwide and the host range is unlimited. Incidence of the disease in man and animals is a function of close intra- and interspecific association and contact. It has long been recognized as a problem in exotic animals in zoos and zoological gardens. Susceptible species indigenous to Alaska include voles, mink (Mustela vison), otters (Lutra canadensis), beavers (Castor canadensis), deer (Odocoileus spp.) and birds (Winkler and Gale 1970). Tuberculosis in humans remains a problem in Alaskan bush communities.

\section{History}

Records of tuberculosis in man date back to 2000 B.C. and the disease spread with civilization. Romans probably carried the disease to Great Britain. Europeans may have brought the disease to the New World, but there is evidence that the disease may have already existed among the American Indians. Historical dissemination of the disease in animals parallels that in humans. It was first reported in wild animals by Koch in 1884, and was reported in white-tailed deer (Odocoileus virginianus) in 1934 (Winkler and Gale 1970).

\section{Transmission}

Methods by which the organisms are shed from the infected host depend on the location of the tubercle lesions 
within the host. If lesions are in the lungs, organisms may be shed with exhaled air or sputum, or they may be swallowed and passed with the feces. Organisms from lymph node lesions may be shed in urine, vaginal discharges or milk (Winkler and Gale 1970).

Alimentary and respiratory tracts are the usual routes of infection. Cutaneous access through an open wound may occur. Congenital infection is rare (Stamp 1959, Winkler and Gale 1970).

In man and animals the incidence of tuberculosis is directly related to crowding and the degree of association with contaminants in the environment. Severe outbreaks in carnivores fed tuberculous meat have been reported (Winkler and Gale 1970 ).

\section{Signs, Symptoms and \\ Pathogenesis}

Tubercle bacilli that enter the body are engulfed by host phagocytes (disease defense cells that ingest foreign cells). Most bacteria genera are destroyed by phagocytosis, but Mycobacteria multiply, rupture the phagocyte, and are thus distributed in the host to the bloodstream, bronchi, bronchioles, etc. Further phagocytosis occurs, and the phagocytes are eventually arrested at some point, e.g. lung, lymph nodes, intestinal tract, etc. Lesions develop where the phagocyte is arrested. Host reaction leading to the characteristic granuloma lesion of tuberculosis, the tubercle, occurs at the site of localization.

The inner core of a young tubercle consists of phagocytes containing bacteria surrounded by an epithelioid (resembling epithelial) cell layer.
This in turn is surrounded by a layer of giant cells (large phagocytes). As the lesion matures, the inner core goes through a caseous ("cheesy") necrosis (cell death). The resulting acellular core is surrounded by giant cells, epithelioid cells, and an outer layer of monocytes, lymphocytes, plasmacytes and fibroblasts (Hagan and Bruner 1961, Winkler and Gale 1970).

In the typical ungulate response the tubercle undergoes calcification or liquefaction (1iquifying) necrosis followed by cavitation (formation of a cavity). The disease becomes a long term wasting process. Abnormal antler development has been observed in deer. Other signs may include osteomyelitis involving the cervical vertebrae and superficial cervical abscesses (Winkler and Gale 1970).

Carnivores may display a serous nasal discharge, coughing up of bloody sputum, abscessed cervical lymph nodes, hemorrhagic diarrhea, loss of appetite and dullness in pelage (Winkler and Gale 1970).

Pulmonary infections with a chronic cough are characteristic of tuberculosis in wildlife. Alimentary infections with enlarged lymph nodes leading to increased pressure on visceral organs, fever, uterine infections, late abortions and emaciation may also be seen (Winkler and Gale 1970).

\section{Diagnosis}

The classic diagnosis in humans and domestic animals is the intradermal skin test. Tuberculin, a protein derivative from the tubercle bacilli, is injected and, if the host has been exposed previously to the disease, a hypersensitivity reaction manifested 
by a red, firm swelling at the point of injection is observed in 72 hours.

This method appears good for ungulates, fair for carnivores and poor for rodents (Winkler and Gale 1970). The obvious disadvantage of this test for wildlife in the field is the difficulty in confining the animals for 72 hours to read the test. Also, preferential sites of inoculation in wild species have not been established.

An in vitro lymphocyte transformation test has been found to be specific for detecting tuberculosis in cattle (Muscoplat et al. 1975). The test has been tried on a limited number of captive exotics with encouraging results. An advantage of this test over the tuberculin test is that the animal only has to be handled once to obtain a blood sample (Johnson et al. 1978).

The tuberculosis bacteria may be isolated on specific culture media from specimens collected from sputum, gastric contents and other infected tissues, especially lungs. The organism can survive for years at $-20 \mathrm{C}$, and samples for bacteriologic isolation should be frozen if they can not be submitted to a laboratory within a few hours of death. Laboratory growth is slow and may take several weeks (Kubica 1960), but isolation of the organism provides definitive diagnosis (Paterson 1959).

Because the disease does not efficiently stimulate antibody production and because several nonpathogenic (non-disease producing) species of Mycobacteria cross react with the pathogenic ones, serologic techniques are not useful in diagnosing tuberculosis. Diagnosis is usually not difficult in dead animals due to the characteristic appearance of gross and microscopic lesions. Lesions from lungs and lymph nodes are good samples for histologic examination.

\section{Significance and Control}

The major factor contributing to the incidence of tuberculosis is close intra- and interspecific contact and association with infected individuals and the contaminated environment. The organisms are commonly coughed up and may survive up to 6 weeks in the protective sputum. Tuberculosis in domestic animals in most of the United States has been brought under control through eradication of tuberculin positive animals. Because the bacteria can be passed in milk of infected cows, milk pasteurization has also helped in controlling the disease. Public health awareness along with isolation and prolonged drug treatment of infected persons has contributed significantly to lowering the incidence of the disease in people.

However, the incidence of tuberculosis in Alaskan bush communities is not under as good control. The disease is perpetuated by lack of adequate public health control measures. Due to the wide host range of the disease, this human source could serve as a potential source of infection for Alaskan animals and wildlife. In Germany from 1968-72, 197 cattle were found to be infected by the transmission of the tubercle bacille from man (Chaloux 1978). Many people have contact with reindeer that are herded on the Seward Peninsula. These animals could then serve as sources for infection of other species.

Bovine tuberculosis was recently diagnosed in 2 wolves (Canis lupus) 
in Manitoba, and it was felt that this disease was one of the contributing factors in the population decline of the pack (Carbyn 1979). During the period 1973 to 1976, bovine tuberculosis was the cause of death of $39 \%$ of 1206 badgers in Gloucestershire, England (Gallagher 1979).

In animals, eradication of those infected is the only effective means of control. No tuberculosis vaccine is used in this country.

\section{References}

CARBYN, L. N. 1979. Incidence of disease and its potential role in the population dynamics of wolves in Riding Mountain National Park, Manitoba. Portland, Oregon Wolf Symposium.

CHALOUX, P. A. 1978. Worldwide zoonotic aspects of tuberculosis. pp. 219-221. In: Mycobacterial Infections of Zoo Animals. R. J. MONTALI, ed. Smithsonian Institution Press, Washington, DC.

GALLAGHER, J. 1979. Cause of ill health and natural death in badgers in Gloucestershire. Vet. Rec. 105:546-551.

HAGAN, W. A. and D. W. BRUNER. 1961. The $\frac{\text { Infectious }}{\text { Domestic Animals. }} 4$ th ed. Comstock Publishing Assoc., New York, NY. 1033 pp.

JOHNSON, D. W., F. P. SOPER and P. M. RAKICH. 1978. Lymphocyte transformation as a diagnostic tool in tuberculosis of exotic animals. pp. 135-138. In: Mycobacterial Infections of Zoo Animals. R. J. MONTALI, ed. Smithsonian Institution Press, Washington, DC.
KUBICA, G. P. 1960. Tuberculosis: laboratory methods in diagnosis. U.S. Dept. HEW, CDC, At lanta, GA. MUSCOPLAT, C. C., C. O. THOEN, R. M. MCLAUGHLIN, J. R. THOENIG, A. W. CHEN and D. W. JOHNSON. 1975. Comparison of lymphocyte stimulation and tuberculin skin reactivity in Mycobacterium bovis infected Macaca mulatta. Am. J. Vet. Res. 36:699.

PATERSON, A. B. 1959. Tuberculosis: bacteriology. pp. 671-686. In: Diseases Due to Bacteria. Vol. II. A. W. STABLEFORTH and I. A. GALLOWAY, eds. Academic Press Inc., New York, NY.

STAMP, J. T. 1959. Tuberculosis: epidemiology and pathology. pp. 686-713. In: Diseases $\frac{\text { Due }}{\text { B. }} \frac{\text { to }}{\text { W. }}$ STABLEFORTH and I. A. GALLOWAY, eds. Academic Press Inc., New York, NY.

TARSHIS, M. S. 1966. Bacteriology of the mycobacteria. pp. 32-52. In: Clinical Tuberculosis. K. H. PFEUTZE and D. B. RADNER, eds. Springfield, IL.

WINKLER, W. G. and N. B. GALE. 1970. Tuberculosis. pp. 236-248. In: Infectious Diseases of $\frac{\text { Wild }}{\text { Animals }}$ $\begin{array}{lll}\text { Animals. J. W. DAVIS, L. H. } \\ \text { KARSTAD and D. } & \text { 0. TRAINER, eds. }\end{array}$ lowa State Univ. Press, Ames, IA.

WOLINSKY, E. 1973. Mycobacteria. pp. 844-869. In: Microbiology. D. F. DAVIS, R. RULBECCO, H. N. EISEN, H. S. GINSBERG and W. B. WOOD, eds. 2nd ed. Harper and Row, Hagerstown, MD. 


\title{
Tyzzer's (Errington's) Disease
}

\author{
Jamie K. Morton
}

\section{Introduction}

Two diseases with similar manifestations have been reported in the literature. Tyzzer's disease has been associated with laboratory animals, primarily mice and rabbits, while Errington's disease has been associated with free-living animals such as muskrats (Ondatra zibethica). Similarities between Tyzzer's and Errington's disease have been recognized for some time, and recent evidence indicates they are probably a single entity. The causative agent is a pleomorphic, Gram negative, sporeforming, rod-shaped bacteria (Chalmers and MacNeill 1977, Ganaway et al. 1976, Tyzzer 1917, Wobeser et al. 1979).

\section{History}

Tyzzer (1917) described an acute, fatal entero-hepatic disease in Japanese waltzing mice. Epizootics of Tyzzer's disease in laboratory mice have been reported in China, Sweden, England, Japan and the United States. The disease is also recognized in laboratory rabbits (Leporidae), rats (Rattus spp.), gerbils (Gèrbillus spp.), hamsters (Cricetus spp.), cats (Felis catus) and rhesus monkeys (Macaca mulata) (Ganaway et al. 1976).

It was first reported to cause die-offs in a wild species, muskrat, by Karstad (1971). In 1976, Ganaway reported that cottontail rabbits (Sylvilagus floridanus) were extremely susceptible to Tyzzer's disease and suggested that it might be responsible for die-offs observed in Maryland (Ganaway et al. 1976). The causative agent for these cases was identified as Bacillus piliformis.

In 1946 Errington described an epizootic which decimated muskrat populations in lowa marshes. The disease was characterized by hemorrhagic enteritis and focal liver necrosis, but the causative agent was not determined (Karstad 1970).

A similar situation was studied in Wisconsin in the 1950 s by Lord. He concluded that the disease was caused by Clostridium sp., a Gram positive sporeforming bacteria. By injecting the Clostridium into muskrats he induced a disease with symptoms and pathogenicity identical to those described by Errington (Lord et al. 1956).

However, because Clostridium sp. is normally found in the soil, digestive tracts of healthy animals and in the tissues of dead animals, his conclusions were not well accepted (Karstad 1970).

Chlamydia (psittacoses group) was isolated from dead muskrats and snowshoe hares (Lepus americanus) with characteristic Errington's disease lesions following a die-off in Saskatchewan in 1961 (Karstad 1970, Spalatin and Iverson 1970). other organisms implicated as causes of Errington's disease have included Pasteurella tularensis, Pasteurella pseudotuberculosis and Leptospira. Karstad et al. (1970) suggested that Errington's disease be regarded as several diseases with similar gross lesions but different causes (Karstad 1970).

Karstad later demonstrated baccili typical of Bacillus piliformis in 
liver and intestinal lesions in wildcaught muskrats in Ontario (Karstad et al. 1971). Prior to that time, Tyzzer's and Errington's diseases had been regarded as separate entities due to the controversy over the causative agent(s) of Errington's disease. Karstad not only made the first diagnosis of Tyzzer's disease in muskrats, but also suggested that because of gross and histopathologic similarities between Tyzzer's and Errington's, the 2 diseases might be one and the same (Karstad et al. 1971).

Wobeser diagnosed Tyzzer's disease in muskrats in Saskatchewan in 1978. His observations on similarities of lesions and patterns of deaths between Tyzzer's and Errington's disease supported the hypothesis that the 2 diseases might be identical (Wobeser et al. 1978).

Wobeser et al. (1979) published results with the most conclusive evidence of the identity of the 2 diseases. A coincidence provided the opportunity to re-examine some of Errington's original muskrat samples. Paraffin blocks of preserved tissues had been saved and were examined histologically. Organisms typical of Bacillus piliformis, the agent responsible for Tyzzer's disease, were observed in the liver cells. This demonstrated that the organism was present in animals originally diagnosed as having Errington's disease.

\section{Transmission}

Bacillus piliformis is a sporeforming bacteria, and spores are characteristically environmentally stable. Although the exact means of transmission has not been determined, organisms or spores are probably shed in the feces. Thus, once infected animals have been in an area, that area probably remains contaminated for some time. Most likely sources of infection include contaminated housing, bedding (Allen et al. 1965, Chalmers and MacNeill 1977) and feed (Ganaway et al. 1976). Foci of infection tend to be environmentally localized. Wobeser et al. (1978) identified a single muskrat feed house as the only known focus for the disease within 1 marsh. Errington (1963) described finding dead muskrats in specific feed houses and lodges.

Ganaway suggested that places where feces accumulate, aiding in the survival and build-up of spores, would serve as excellent sources of infection for rabbits (Ganaway et al. 1976). He further suggested that any mechanical transfer of feces or spores would aid in spreading the disease.

Exposure in muskrats and rabbits is probably influenced by their gregarious behavior and population densities. Environmental factors such as wind and wave dispersal of contaminated materials also play a role (Errington 1963).

\section{Signs, Symptoms and \\ Pathogenesis}

Tyzzer's disease in laboratory mice is characterized by profuse diarrhea for 2 to 3 days followed by appetite and weight loss and finally death. Mortality is especially high in young mice (Allen et al. 1965).

Reluctance to eat (Karstad et al. 1971) and anal bleeding (Chalmers and MacNeill 1977, Errington 1963) have been observed 12 to 24 hours prior to death in muskrats. Fecal staining of the perineum has been observed in muskrats and cottontail rabbits 
(Chalmers and MacNeill 1977, Errington 1963, Ganaway et al. 1976). The clinical course of the disease is brief (Errington 1963).

Gross lesions of Tyzzer's disease include white or yellowish necrotic foci 1-3 $\mathrm{mm}$ in diameter on the surface and throughout the parenchyma of the liver (Chalmers and MacNeill 1977, Errington 1963, Ganaway et al. 1976, Wobeser et al. 1978). The lumen of the cecum and colon may contain a very fluid, brown fecal material (Chalmers and MacNeill 1977, Karstad et al. 1971, Wobeser et al. 1979). Small hemorrhages may be seen on the small intestine (Errington 1963) and the cecum may appear dark red-black (Wobeser et al. 1979). Congested lungs have also been reported (Errington 1963, Wobeser et al. 1978).

Histologic lesions in the liver consist mainly of areas of coagulation necrosis with no inflammatory response (Chalmers and MacNeill 1977, Wobeser et al. 1978). Necrotic foci are also seen in epithelial cells of the intestine (Chalmers and MacNeill 1977, Karstad et al. 1971).

Infections in laboratory animals usually follow stress or treatment with corticosteroids. The influence of stress on the disease in freeliving animals is not known.

\section{Diagnosis}

Diagnosis of Tyzzer's disease is based on histologically demonstrated B. piliformis in the cytoplasm of cells at the periphery of necrotic foci (Chalmers and MacNeill 1977, Ganaway et al. 1976, Karstad et al. 1971, Wobeser et al. 1978). It is described as long, thin, beaded and filamentous (Chalmers and MacNeill 1977). Giemsa is the preferred stain. The organism has not been grown in cell-free media, but can be grown in the yolk sac of embryonated hen's eggs (Ganaway et al. 1976).

Lesions on the liver and intestines but not on other organs are characteristic of Tyzzer's disease. Infections with Listeria monocytogenes, Francisella tularensis, Pasteurella pseudotuberculosis and Salmonella cause lesions on the spleen as well as the liver (Karstad et al. 1971, Wobeser et al. 1978). Chlamydiosis of muskrats causes 1 iver lesions but not intestinal lesions (Wobeser et al. 1978).

Ganaway used a micro-titer complement fixation (CF) test to detect the disease in cottontail rabbits, but it is not known how long CF titers persist (Ganaway et al. 1976).

\section{Significance and Control}

Tyzzer's disease is a potentially significant factor influencing muskrat and rabbit populations. It is primarily a direct source of mortality. Errington (1963) reported an $80 \%$ depopulation of muskrats in 1 outbreak.

The disease may act secondarily as an influencing factor on behavior. Errington (1963) reported mass emigration of muskrats from established residence areas in which epizootics occurred.

The disease is more significant in young animals than adults. Thus, spring outbreaks tend to involve high numbers of animals due to a spring surplus of young. Fall epizootics, on the other hand, tend to involve a relatively fewer number of animals due to a more stable population at that time (Errington 1963). 
Control of the disease in wild populations is aimed at removing and diluting the source of infection. For muskrats, this would involve a systematic, repeated leveling of lodges and removal of carcasses (Errington 1963).

Although the disease has not yet been diagnosed in Alaska, conditions compatible with the ecology of the disease do exist in the state. This disease should be considered in the differential diagnosis whenever muskrats are necropsied.

\section{References}

ALLEN, A. M., J. R. GANAWAY, T. D. MOORE and R. F. KINARD. 1965. Tyzzer's disease syndrome in laboratory rabbits. Am. J. Pathol. 46:859-882.

CHALMERS, G. A. and A. C. MacNEILL. 1977. Tyzzer's disease in wildtrapped muskrats in British Columbia. J. Wildl. Dis. 13:114-116.

ERRINGTON, P. L. 1963. Muskrat Populations. Iowa State Univ. Press, Ames, IA. 665 pp.

GANAWAY, J. R., R. S. MCREYNOLDS and A. M. ALLEN. 1976. Tyzzer's disease in free-living cottontail rabbits (Sylvilagus floridanus) in Maryland. J. Wildl. Dis. $12: 545-549$.
KARSTAD, L. H. 1970. Errington's disease. pp. 309-310. In: Infectious Diseases of Wild Mammals. J. W. DAVIS, L. H. KAR $\overline{S T A D}$ and D. 0. TRAINER, eds. I owa State Univ. Press, Ames, IA.

KARSTAD, L. H., P. LUSIS and D. WRIGHT. 1971. Tyzzer's disease in muskrats. J. Wildl. Dis. 7:96-99.

LORD, G. N., A. C. TODD and C. KABAT. 1956. Studies on Errington's disease in muskrats. IEII. Am. J. Vet. Res. 17:303-310.

SPALATIN, J. and J. 0. IVERSON. 1970. Epizootic chlamydiosis of muskrats and snowshoe hares. pp. 304-308. In: Infectious Diseases of Wild Mammals. J. W. DAVIS, L. $\bar{H}$. KARSTAD and D. 0. TRAINER, eds. lowa State Univ. Press, Ames, IA. TYZZER, E. E. 1917. A fatal disease of the Japanese waltzing mouse caused by a spore-bearing bacillus (Bacillus piliformis, N. sp.). J. Med. Res. 37:307-338.

WOBESER, G., H. J. BARNES and $K$. PIERCE. 1979. Tyzzer's disease in muskrats: re-examination of specimens of hemorrhagic disease collected by Paul Errington. J. Wildl. Dis. 15:525-527.

WOBESER, G., D. B. HUNTER and P. Y. DROUST. 1978. Tyzzer's disease in muskrats: 0ccurrence in freeliving animals. J. Wildl. Dis. $14: 325-328$. 


\title{
Necrobacillosis
}

\author{
Jamie K. Morton
}

\section{Introduction}

Necrobacillosis is a disease with various manifestations affecting both domestic and wild ruminants. The classification of the causative agent has not been consistent; sometimes more than 1 organism is responsible for the condition (Siegmund 1979, Smith et al. 1972). The term necrobacillosis has been most commonly used to describe the disease or lesion from which the bacteria Fusobacterium necrophorum is isolated. The organism is a Gram negative, non-sporeforming, nonmotile, filamentous, anaerobic (growing only in the absence of oxygen) rod. It is killed in 15 minutes at $55 \mathrm{C}$ and dies after a week at temperatures between $37 \mathrm{C}$ and $4 \mathrm{C}$ (Rosen 1970). Host lesions may be seen in the mouth or throat, vagina, uterus, liver and feet. Fusobacterium necrophorum is part of the normal flora of the mouth and intestinal tracts of many animals, and may gain entry into the body through breaks in the skin or mucous membranes (Rosen 1970, Siegmund 1979).

\section{History}

Necrobacillosis has been recognized in white-tailed deer (Odocoileus virginianus), Columbian black-tailed deer (Odocoileus hemionus columbianus), moose (Alces alces), bighorn sheep (Ovis canadensis), elk (Cervus elaphus), reindeer (Rangifer tarandus) and caribou (Rangifer tarandus) (Hadwen and Palmer 1922, Rausch 1953, Rosen 1970).

Foot rot, one of the more common manifestations of necrobacillosis, was reported by Hadwen in 1922 to be one of the most troublesome of reindeer diseases. Rausch reported that the causative organism had been isolated from typical foot rot lesions in reindeer near Noatak, Alaska. Other researchers reported a high incidence of foot disorders in caribou on the Kurupa, Colville and Canning Rivers in the 1940 s with lesions Rausch (1953) felt were typical of $F$. necrophorum infections. King $(196 \overline{3})$ reported necrophorous infections in caribou collected at Anaktuvuk Pass in 1963. Reindeer with foot disorders suggestive of foot rot have been seen on the Seward Peninsula in Alaska (R. Dieterich, pers. comm.).

\section{Transmission and}

\section{Pathogenesis}

Because $F$. necrophorum is normally found in manure and mud, these bacteria apparently need an injury in the foot or mucous membrane to gain access to healthy tissues. Theories propose that foot injuries may result from rugged terrain, or that cracks occur in the feet as mud on the feet dries and cracks (Rosen 1970, Siegmund 1979, Smith et al. 1972, Zhigunov 1961). The organism also tolerates oxygen better in the presence of Staphylococcus aureus and E. coli, normal bacterial inhabitants of the skin and intestinal tract respectively. Thus, if $\underline{F}$. necrophorum does gain entry, the presence of these other bacteria may help it grow better in tissues. Situations where animals are crowded may enhance the transmission of foot rot among animals due to bacterial seeding of the area with fecal contamination. Organisms on the ground are inadvertently ingested, and the cycle of an animal eating and eliminating the bacteria keeps the organism viable.

Abscesses or ulcers form at the site of entry. If abscesses or 
ulcers drain internally, infections in the feet may spread to the liver, rumen or lining of the abdominal cavity (peritoneum), and organisms from ulcers in the intestine or mouth may seed and infect the liver and lungs respectively (Rosen 1970, Zhigunov 1961).

\section{$\underline{\text { Signs and Symptoms }}$}

Fusobacterium necrophorum abscesses in deer may be seen on the feet, oral cavity, throat, rumen, liver or lungs (Rosen 1970). They contain greenish-yellow pus and have a foul odor. Ulcerated areas may be seen in the oral cavity of deer and reindeer (Rosen 1970, Zhigunov 1961), and similar lesions have been reported on the rumen, uterus, lymph nodes and sometimes the udder in reindeer in Russia (Zhigunov 1961). Foot infections are characterized by swollen joints and necrosis around the top of the hoof or between the toes. As the infection progresses, the joints may stiffen causing lameness (Rosen 1970).

\section{$\underline{\text { Diagnosis }}$}

Diagnosis is based on organism isolation using selective media or by fluorescent antibody techniques to identify the organism in tissues (Rosen 1970).

\section{$\underline{\text { Significance and Control }}$}

Crippling hoof diseases have caused problems in reindeer and caribou in Russia, Scandinavia and Alaska (Hadwen and Palmer 1922, Rausch 1953, Rosen 1970, Zhigunov 1961). Lameness can be detrimental to the animal's ability to dig through the snow for food or to keep up with the herd. No generalization as to the cause should be made. Many of these infections could be caused by $\mathbf{F}$. necrophorum, but brucellosis is also known to cause swollen joints and lameness, and other factors may contribute also.

Moving animals to new grounds or dispersing herds can aid in controlling foot rot when it is likely the animals are congregating in contaminated areas (Hadwen and Palmer 1922, Rausch 1953, Rosen 1970, Smith et al. 1972). Calcium hypochlorite or copper sulfate may be used to disinfect problem areas. Animals in captivity can be chemically or surgically treated (Rosen 1970).

\section{References}

HADWEN, S. and L. J. PALMER. 1922. Reindeer in Alaska. Washington Government Printing Office, Washington, DC. $74 \mathrm{pp}$.

KING, J. A. 1963. Disease survey trips among the caribou herds in Alaska. J. Am. Vet. Med. Assoc. 143:887-888.

RAUSCH, R. L. 1953. On the status of some arctic animals. Arctic $6: 91-148$.

ROSEN, M. N. 1970. Necrobacillosis. pp. 286-292. In: Infectious Diseases of Wild Mammals. J.W.

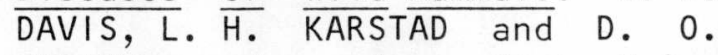
TRAINER, eds. Iowa State Univ. Press, Ames, IA.

SIEGMUND, 0. H., ed. 1979. The Merck Veterinary Manual. Me $\overline{r c k}$ and Co. Inc., Rahway, NJ. 1680 $\mathrm{pp}$.

SMITH, H. A., T. C. JONES and R. D. HUNT. 1972. Veterinary Pathology. Lea and Febiger. Philadelphia, PA. 1521 pp.

ZHIGUNOV, P. S., ed. 1961. Reindeer Husbandry. Izadatel'stvo Sel'skokhozyaistvennoi j Literatury, Zhurnalov i Plakatov, Moskow, 348 pp. 


\title{
Abscesses
}

\author{
Robert A. Dieterich
}

There are a number of bacteria that are capable of causing different types of lesions not commonly classified as specific diseases. For example, abscesses (a localized collection of pus in a cavity formed by the disintegration of tissues) can be caused by many types of bacteria including Staphylococcus, Streptococcus, Corynebacterium, Proteus, etc. Some occur commonly enough to be recognized as specific problems in certain species. An example of this is Corynebacterial infection in domesticated muskox (Ovibos moschatus) (Beckley and Dieterich
1970). One should use care when bacteria are encountered as they can be infectious to man as well. Brucellosis in wildlife does form abscesses at times and is of public health concern.

\section{Reference}

BECKLEY, J. C. and R. A. DIETERICH. 1970. Corynebacterial infection in the domesticated muskox (Ovibos moschatus). Vet. Med./Small Anim. Clin. 65:1063-1067. 
Chapter 3.

PARASITIC DISEASES 


\title{
Introduction
}

\author{
R. Barrett and J. Dau
}

The parasites of all animals have one thing in common; they derive their total existence from a host organism without which they cannot survive. This is a tenuous balance for the parasite to maintain in order to complete its life cycle. Under natural circumstances, this balance is maintained, and the damage done by any class of parasite may be minimal. If the balance shifts toward the parasite, a clinical disease syndrome may become evident. This syndrome may include hemorrhage, disruption of the intestinal mucosa, pneumonia, perforations, abnormal pressures on vital organs or systems, diarrhea or any number of other problems. The parasite syndrome may be complicated by virus or bacterial infections which may further jeopardize the host.

Parasites are generally host specific, but in some instances they cross the host barrier and infect many groups of animals. In any case, expect the unknown while examining an animal for parasites. If you become complacent, you may be reading a headline such as this; "Scabies Blamed For Massive Die-Off of Sheep in the Wrangel Mountains." This headline did occur but not in Alaska; New Mexico had this distinction in 1979. Psoroptes spp. were found infecting the ears and bodies of their bighorn sheep (Ovis canadensis) in the San Andres mountains. This population was estimated at 225 animals in the early 1970s, but by 1978 only 60 to 70 sheep remained. A problem such as this will not occur in Alaska as long as guides, hunters, hikers, etc. use all resources available to promote the early reporting of any suspicious condition whether it is of viral, bacterial, fungal or parasitic etiology. This may enable corrective action to be taken before the problem is beyond control.

While developing the format for this parasitological material, we were forced to decide what parasitic organisms to include, in what detail to cover them and how to arrange the material. A disproportionately large amount of research has been devoted toward the parasitological dimension of wildlife disease mainly because researchers could literally get their hands on the causative agents. This has resulted in a voluminous literature that provides detailed taxonomic descriptions, life histories, pathogenicities, and occasionally control of a multitude of parasitic organisms. Recently, complex mathematical models have been employed in analyzing the interrelationships of variables attributable to the parasite(s), host, environment, time and others, to control and predict the effects of parasites (Gettinby and MacLean 1979). This represents significant progress toward understanding the effects of disease on animal populations.

Since this compendium is aimed primarily at educated laymen, wildlife students and professional field biologists who lack an extensive background in wildlife disease, we have attempted throughout to pare the material presented to the essential elements of the disease conditions. In keeping with this, we decided to include only those parasites we felt 
were most significant to Alaskan wildlife in enough detail to: 1) at least identify these organisms to some gross taxonomic category for finer reticulation with existing keys, 2) provide life cycle information for ecologic considerations, 3) cover pathogenicity and 4) present the significance and control of these conditions where possible. For more thorough coverage of host/parasite relationships and species found in Alaska, refer to Soulsby (1968) and Hopla et al. (1965) respectively.

\section{References}

GETTINBY, G. and S. MACLEAN. 1979. A matrix formulation of the life cycle of liver flukes. Proc. R. Ir. Acad. Sect. B. Biol. Geol. Chem. Sci. 79:155-167.
HOPLA, C. E., W. L. JELLISON and K. A. NEILAND. 1965. Parasites of Alaskan Vertebrates: HostParasite Index. Fed. Aid Wildl. Rest. Proj. $W-6-R$ and $W-15-R$. Univ. Oklahoma Research Inst., Norman, OK. 73 pp.

SOULSBY, E. J. L. 1968. Helminths, Arthropods and Protozoa of Domesticated Animals (Monnig). 6th ed. Will iams and Wilkins Co., Baltimore, MD. 823 pp.

\title{
Trematoda
}

\author{
J. Dau and R. Barrett
}

Endoparasitism has been defined as the relationship in which parasites live inside the bodies of their hosts. This definition can be misleading since many endoparasites begin their parasitic lives as external parasites, and then penetrate their host to become internal parasites. Endoparasitism is a function of infection by any or all of the following types of parasites: tapeworms (Cestoda), flukes (Trematoda), roundworms (Nematoda), spinyheaded worms (Acanthocephala) and Protozoa. The significance of parasite infections ranges from mild to severe depending upon the condition of the host and the type and intensity of infection. All animals have their complement of endo- parasites. Some are host specific (obligate parasites of certain host species) while others infect multiple host species.

\section{Flukes (Trematoda)}

Trematodes comprise 1 of the 2 classes of the phylum Platyhelminthes (flatworms), the other class being the cestodes (tapeworms). Fluke species most significant in Alaska are dorsoventrally flattened and bilaterally symmetric. They possess a gut and 2 suckers; the oral sucker and the ventral hold-fast sucker (acetabulum). Flame cells comprise the excretory system. All are monoecious except the blood flukes 
(Schistosomatidae). Most flukes are associated with the gastrointestinal, respiratory or urogenital tracts; blood flukes occur in the circulatory system.

Flukes utilize molluscan intermediate hosts for development of sporocysts, rediae and cercariae; metacercariae encyst on in aquatic vegetation, invertebrates or vertebrates (i.e. fish). Eggs pass from the definitive host in the feces or urine. They contact water and incubate from 1 week to a month depending on the parasite species, water temperature, light and dissolved oxygen concentration. The eggs hatch to form the first larval generation, the miracidium. Hatching may occur after the eggs are ingested by a suitable molluscan intermediate host or while in the external environment, to produce ciliated, freeswimming miracidia. Once within the mollusc, miracidia lose their cilia and migrate to the hepatopancreas (liver) where they develop into the second larval generation, the sporocysts. Sporocyst germinal cells produce the third larval generation, the rediae, directly or via daughter sporocysts. Rediae in turn give rise to a second generation of rediae or to tail-bearing cercariae. Cerceriae may remain within the mollusc until it is ingested by a suitable host organism, or they may leave the mollusc to become temporarily free-swimming. Cercariae may encyst on vegetation, or the external surface of $f i s h$ or within aquatic invertebrates or fish. The encysted flukes are termed metacercariae; metacercariae are regarded as the infective life stage for their definitive hosts. After the metacercariae are ingested via contaminated water, infected snails or fish, or encysted vegetation, the immature flukes excyst in the intestinal lumen of the definitive host. They then migrate to the site of maturation (lungs, liver, etc.), mature and release eggs to begin the cycle anew (Fig. 1).

For an extensive list of trematodes found in wild mammals, consult Davis and Libke (1968) or Hopla et al. (1965).

\section{References}

DAVIS, J. W. and K. G. LIBKE. 1971. Trematodes. pp. 237-241. In: Parasitic Diseases of Wild Mammals. J. W. DAVIS and R. C. ANDERSON, eds. Iowa State Univ. Press, Ames, IA.

HOPLA, C. E., W. L. JELLISON and $K$. A. NEILAND. 1965 Parasites of Alaskan Vertebrates. Fed. Aid Wildl. Rest. Proj. W-6-R and W-15-R. Univ. Oklahoma Research Inst., Norman, OK. 73 pp. 


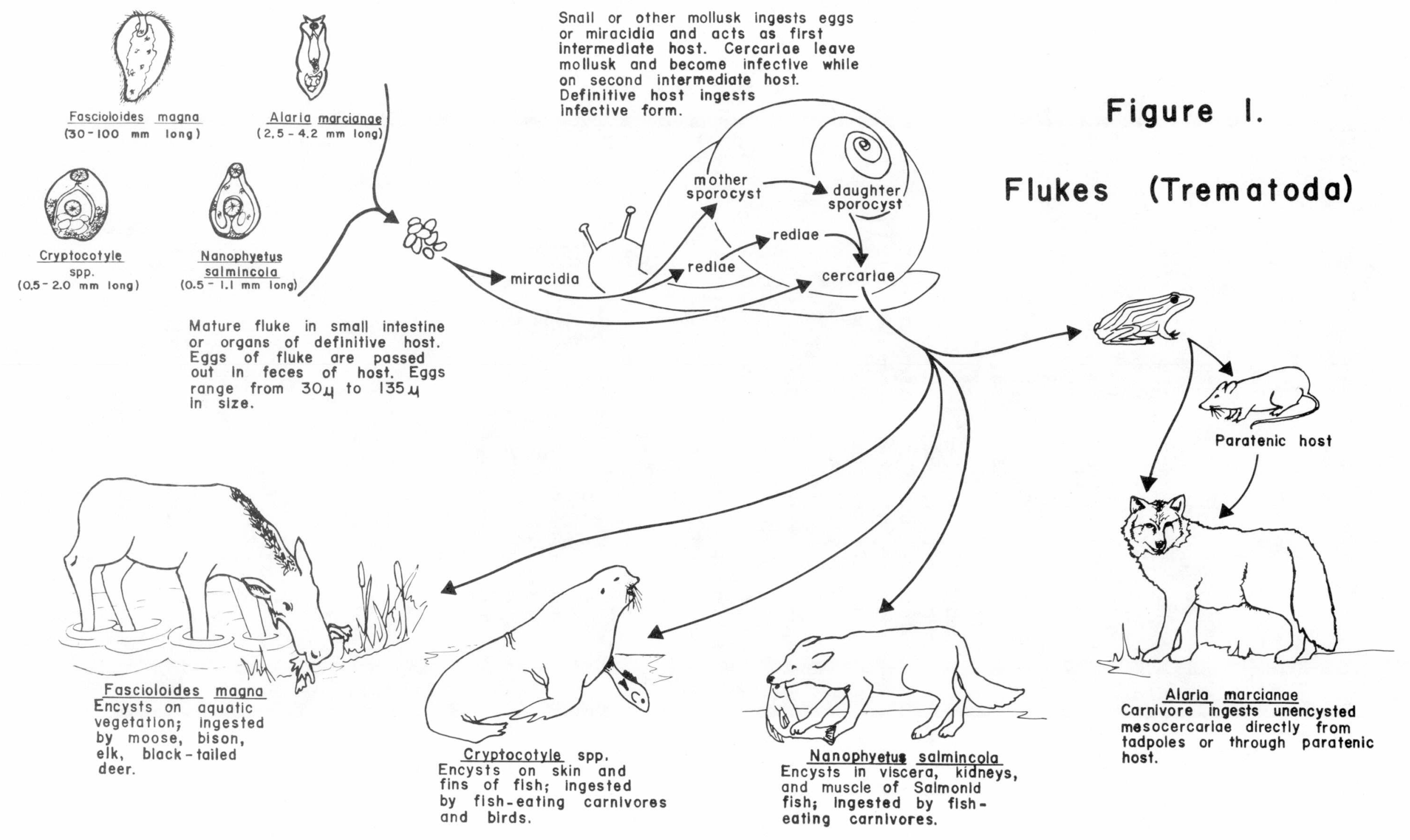




\title{
Fascioloides
}

\author{
R. Barrett and J. Dau
}

Introduction

Fascioloides magna, the "large American fluke," is usually found in the hepatic parenchyma of its definitive hosts but may also occur in the lungs (Davis and Libke 1971). Definitive host species that occur in Alaska include moose (Alces alces), wapiti (Cervus elaphus), bison (Bison bison) and black-tailed deer (Odocoileus hemionus sitkensis) (Davis and Libke 1971). These large, oval flukes are approximately 30-100 $\mathrm{mm}$ by $2-4.5 \mathrm{~mm}$.

\section{Transmission}

Transmission follows that outlined for flukes in general. The eggs hatch in 9 days to 4 weeks depending on environmental conditions; miracidia cannot withstand prolonged exposure to water. Two generations of rediae develop within the snails before cercariae are released into the water to encyst on aquatic vegetation. Metacercariae reach the hepatic parenchyma of definitive hosts 2 to 6 days post ingestion; maturation is complete in 5 months. Adult flukes have been known to live 11 years in experimentally infected animals.

Cervids generally exhibit little tissue response to the presence of these flukes and, as a result, host generated cysts surrounding $\underline{F}$. magna flukes tend to be thin-walle $\bar{d}$. Afferent and efferent bile ducts can penetrate the hepatic cysts, allowing the transfer of eggs. Bison tend to exhibit a strong tissue response to $F$. magna however, and encapsulate the flukes in thick-walled cysts before they completely mature. This reduces the pathogenicity of $F$. magna in bison and precludes the release of eggs. Bison therefore represent a "dead end" in the life cycle of $F$. magna, and the parasite cannot survive in the ecosystem without an alternative definitive host (i.e. moose). Domestic sheep (Ovis aries) suffer severe hepatic hemorrhage and peritonitis when parasitized by as few as 2 to 3 immature flukes; death may ensue before eggs can be produced (Davis and Libke 1971).

Symptoms, Pathogenicity and Diagnosis

Fascioloides magna infections are generally asymptomatic. Diagnosis is based on the presence of mature flukes in affected organs or eggs in fecal and water samples (eggs illustrated in Soulsby 1968). Currently, no means of control are known. The significance of $F$. magna in Alaska has not been establi ished.

\section{References}

DAVIS, J. W. and K. G. LIBKE. 1971. Trematodes: Fascioloides magna. pp. 241-243. In: Parasitic Diseases of Wild Mammals. J. W. DAVIS and R. C. ANDERSON, eds. lowa State Univ. Press, Ames, IA. HOPLA, C. E., W. L. JELLISON and K. A. NEILAND. 1965. Parasites of Alaskan Vertebrates. Fed. Aid Wildl. Rest. Proj. W-6-R and W-15-R. Univ. Oklahoma Research Inst., Norman, OK. 73 pp.

SOULSBY, E. J. L. 1968. Fascioloides. pp. 34-36. In: Helminths, Arthropods and Protozoa of Domesticated Animals (Monnig). 6 th ed. Williams and Wilkins Co., Baltimore, MD. 


\title{
Cryptocotyle
}

\author{
J. Dau and R. Barrett
}

\section{$\underline{\text { Introduction }}$}

Cryptocotyle spp. are intestinal parasites of marine shoreline ecosystems. Definitive hosts that occur in Alaska include herring gulls (Larus argentatus) (Smyth 1962), kittiwakes (Rissa spp.) (Smyth 1962), seals (Phocidae) and mink (Mustela vison) (Dunn 1969). Two Cryptocotyle species have been found in Alaskan mammals; C. lingua in domestic dogs (Canis familiaris) and $C$. jejuna in northern fur seals ( sinus) (Hopla et al. 1965). Cryptotocyle spp. do not mature in waterfowl (Anseriformes). The geographic distribution of Cryptocotyle encompasses marine shorelines of the U.S., Canada and Eurasia; they are common in the Soviet Far East.

Adult Cryptocotyle lingua have been described as having linguaform, spatula-shaped bodies that are approximately $0.5-2.0 \mathrm{~mm}$ by $0.2-0.9$ $\mathrm{mm}$. They possess a spiny cuticle and feeble suckers (Dunn 1969, Soulsby 1968). Cryptocotyle jejuna has the same general body configuration as $\underline{C}$. lingua though is smaller, measuring $\overline{0.5-1.5} \mathrm{~mm}$ by $0.35-0.88 \mathrm{~mm}$ (Soulsby 1968).

\section{Transmission}

Eggs are released into seawater with feces of the definitive host. For $\underline{C}$. lingua, these are ingested by the winkle (Littorina littorea) where the cercariae develop. The cercariae are shed as free-swimming organisms that encyst on the skin and fins of fish (i.e. (Atherina, Gobius, Mullus). Metacercariae appear as black dots visible to the naked eye. Fisheating carnivores incidentally ingest the metacercariae with fish. The parasites excyst (they leave the cyst) in the small intestine of carnivores, mature and produce eggs (Dunn 1969).

\section{Signs Pathogenesis and Diagnosis}

Adult Cryptocotyle spp. inhabit the lumen on villi of the small intestine in their definitive hosts. Large numbers of these flukes cause enteritis with associated degeneration of the epithelium, hemorrhagic erosions and production of viscid mucus (Dunn 1969, Soulsby 1968). Diagnosis in definitive hosts is based on identification of mature flukes in the intestines or eggs in fecal samples. The significance and control of this parasite in its definitive hosts are unknown. Empirical and experimental observations indicate that snails parasitized by Cryptocytyle cercariae move more slowly, and to a lesser extent during seasonal migrations in the intertidal zone than non-parasitized snails. This may indirectly influence snail growth through the effects of altered temperature regimes and immersion patterns on snail feeding activity (Lambert and Farley 1968).

\section{References}

DUNN, A. M. 1969. Cryptocotyle. pp. 101-102. In: Veterinary Helminthology. Lea and Febiger, Philadelphia, PA.

HOPLA, C. E., W. L. JELLISON and K. A. NEILAND. 1965. Parasites of Alaskan Vertebrates. Fed. Aid Wildl. Rest. Proj. W-6-R and W-15-R. Univ. Oklahoma Research Inst., Norman, OK. 73 pp. 
LAMBERT, T. C. and J. FARLEY. 1968. The effect of parasitism by the trematode Cryptocotyle lingua (Creplin) on zonation and winter migration of the common periwinkle, Littorina littorea (L.). Can. J. Zool. 46:1139-1147. SMYTH, J. D. 1962. Cryptocotyle lingua. pp. 177-180. In: Animal Parasitology. Charles C. Thomas, Springfield, IL.
SOULSBY, E. J. L. 1968. Cryptocotyle. pp. 49-50. In: Helminths, Arthropods and Protozoa of Domesticated Animals (Monnig). 6th ed. Williams and Wilkins Co., Balt imore, MD.

\title{
Nanophyetus
}

\author{
J. Dau and R. Barrett
}

Introduction

Nanophyetus salmincola is a small (0. $\overline{5-1.1 \mathrm{~mm}}$ long) white or cream colored fluke that inhabits the small intestine of its final hosts (Soulsby 1968). This fluke shows little host specificity though is limited mainly to fish-eating carnivores. Though this parasite has not been documented in mammals from Alaska, it has been reported elsewhere in a number of species also indigenous to Alaska. Definitive mammalian and avian host species that occur in Alaska are listed in Table 1 with known intermediate hosts. The paucity of information concerning this parasite in Alaska is perhaps more indicative of the lack of pertinent research than the incidence or significance of this fluke. The reported geographic distribution of $N$. salmincola is the Pacific Northwest of the United States (Soulsby 1968) and eastern Siberia (Filimova 1966).

\section{Transmission}

Eggs are passed into fresh water via the feces of the definitive host and hatch in 3 or more months depending on water temperature. Free-swimming miracidia infect snails (Goniobasis plicifera silicula) (Steele 1980) where rediae develop. Cercariae are liberated from the snails in long mucous strands in which they live up to 48 hours. Upon contact with salmonids (i.e. Oncorhynchus spp.) (Soulsby 1968), cercariae penetrate the skin, enter the bloodstream and encyst on kidneys, visceral organs and muscle tissue. The metacercarial cysts, roughly $0.17-0.25 \mathrm{~mm}$ in diameter, can remain viable three and a half months in fish stored above $0 \mathrm{C}$; freezing rapidly destroys the cysts (Soulsby 1968). Metacercariae over 10 days old are infective to carnivores and can remain viable in living fish at least 5 years (Soulsby 1968). It was thought that prepelagic salmonids (fingerlings, fry, smolt, etc.) parasitized by $\underline{N}$. salmincola somehow lost these flukes while at sea. It is now known that this is not true, though the degree of infection may be reduced while salmonids inhabit marine ecosystems. Maturation is rapid with egg production occurring 5 days post infection (Soulsby 1968). 
TABLE 1

Hosts of Nanophyetus salmincola that occur in Alaska

Organism

Reference

First Intermediate Host

Goniobasis plicifera silicula

Second Intermediate Hosts

Chinook Salmon (Oncorhynchus tshawytscha)

Sockeye Salmon (0. nerka)

Pink Salmon (0. gorbuscha)

Coho Salmon (0. kisutch)

Chum Salmon (ㅁ. keta)

$$
\begin{gathered}
1,4,6 \\
4 \\
4 \\
4,6 \\
4
\end{gathered}
$$

\section{Definitive Hosts}

Great Blue Heron (Ardia herodias)

Hooded Merganser (Lophodytes cucullatus)

Belted Kingfisher (Megaceryle alcyon)

Raccoon (Procyon lotor)

Ermine (Mustela erminea)

Mink (Mustela vison)

Beaver (Castor canadensis)

Norway Rat (Rat tus norvegicus)

Wolverine (Gulo gulo)

River Otter (Lutra canadensis)

Sea 0tter (Enhydra lutra)

Domestic Cat (Felis catus)

Lynx (Felis lynx)

Domestic Dog (Canis familiaris)

Coyote (Canis latrans)

Wolf (Canis lupus)

Red Fox (Vulpes vulpes)

Arctic Fox (Alopex lagopus)

Brown Bear (Ursus arctos)

Deer (Odocoileus spp.)

Man (Homo sapiens)

$$
\begin{gathered}
7 \\
3 \\
2,7,8 \\
8 \\
2,5,7 \\
2 \\
5 \\
5 \\
7 \\
5 \\
5,7 \\
8 \\
5,8 \\
2,7 \\
5 \\
5,7 \\
2 \\
5 \\
2 \\
5,7,9
\end{gathered}
$$


Pathogenicity and Diagnosis

Mature N. salmincola infect the mucosa of the duodenum and the small or large intestine. Heavy infections can cause superficial enteritis that may progress into a hemorrhagic stage (Soulsby 1968). Heavy metacercarial infections can be fatal to fish (Bisson and Davis 1976). The direct pathogenic effects of $N$. salmincola on its definitive hosts are less significant than the effects of 2 rickettsial agents transmitted by it.

Neorickettsia helminthoeca, the causative agent of "salmon poisoning disease" (SPD), occurs within N. salmincola. In canids, SPD infections are usually severe and of ten fatal (Davis and Anderson 1971, Soulsby 1968, Steele 1980). Six to 10 days after ingesting fish parasitized by $N$. salmincola that harbor $\mathrm{N}$. helminthoeca, canids experience sudden fever and anorexia. This is followed in roughly 3 days by a purulent discharge from the eyes, vomiting and profuse, often hemorrhagic diarrhea (Soulsby 1968). Mortality of infected animals ranges from 50-90\% (Soulsby 1968). Recovery from SPD produces immunity against further infection by $N$. helminthoeca but not by $\underline{N}$. salmincola.

A closely related but immunologically distinct rickettsia transmitted by $N$. salmincola causes "Elokomin fluke fever." This condition causes an increase in monocytes in the peripheral blood, swelling and infection of lymph nodes, and symptoms resembling mononucleosis in humans (lethargy, anorexia) (Soulsby 1968). Metacercariae of N. salmincola are capable of transmitting both rickettsial agents throughout their 5 year period of existence.

Diagnosis of N. salmincola is based upon identification of mature flukes or eggs in the intestines and feces of definitive hosts respec- tively. Infection of fish by $\mathrm{N}$. salmincola is diagnosed on characteristics of the metacercariae. Diagnosis of salmon poisoning is based on demonstration of $N$. helminthoeca organisms in lymph node biopsies taken during the febrile response of the host. When Giemsastained, N. helminthoeca organisms appear as purple, coccoid bodies approximately $0.3 \mathrm{~mm}$ in size scattered or in compact plaques in macrophages and the cytoplasm of reticular cells (Steele 1980).

\section{Significance and Control}

Practically nothing is known concerning the incidence and significance of $N$. salmincola or either of its rickettsial agents in Alaska. The first intermediate host, Gonobiasis plicifera silicula, is known to occur along coastal British Columbia so may extend into Southeast Alaska. Since N. salmincola is known to occur in Siberia, it probably also occurs in Alaska. Considering the abundance of salmonids in Alaska, the dependence of carnivores and humans on these fish for food, and the fact that many salmon are eaten uncooked (i.e. animal food and cold-smoked or dried fish for human consumption), the potential for fluke and/or rickettsial infection certainly exists. Nanophyetus salmincola is considered more important for its role as a vector for the rickettsiae than for its direct pathogenic effects. Metacercariae of N. salmincola can be serious fish pathogens however. The prevalence of $\mathrm{N}$. salmincola in silver salmon (Oncorhynchus kisutch), may have potential as a biological tag for identification of their populations (01sen 1978).

No mechanisms of control for $\mathrm{N}$. salmonicola are known. Chinook saīmon (0. tshawytscha) are experimentally more resistant to metacercariae 
in warmer water ( $B$ isson and Davis 1976). Rickettsial infections can be successfully treated with tetracycline. Apomorphine will cause vomiting that expells the infective organisms if administered within 3 hours after ingestion of contaminated foods (Soulsby 1968). This is of little practical value, since the presence of causative agents would rarely be known and administration of these drugs to wildlife is unfeasible. Fish should be thoroughly cooked to prevent infection by the fluke or its rickettsiae.

\section{References}

BISSON, P. A. and G. E. DAVIS. 1976. Production of juvenile chinook salmon, Oncorhynchus tshawytscha, in a heated model stream. U.S. Natl. Mar. Fish. Serv. Fish Bull. $74: 763-774$.

DAVIS, J. W. and R. C. ANDERSON. 1971. Trematodes. pp. 237-240. In: Parasitic Diseases of Wild Mammals. lowa State Univ. Press, Ames, IA.

DONHAM, C. R. 1928. Salmon poisoning in dogs. M.S. Thesis, Dept. Vet. Med., Oregon State Univ., Portland, OR.
FARRELL, R. K. 1964. Notes on Nanophyetus salmincola as a reservoir and vector of disease. p. 241. In: Proc. 14th Alaskan Sci. Conf. F. DA AHLGREN, ed. Ak. Div. Amer. Assoc. for the Advancement of Sic., College, AK.

FILIMOVA, L. V. 1966. Distribution of nanophyetosis in the territory of the Soviet Far East. Trudy Gel'mintol. Lab. Akad. Nauk, USSR 17:240-244. (In Russian).

OLSEN, R. E. 1978. Parasites of silver (coho) salmon and king (chinook) salmon from the Pacific Ocean off Oregon. Calif. Fish and Game 64:117-120.

SCHLEGEL, M. W., S. E. KNAPP and R. E. MILLEMAN. 1968. "Salmon poisoning" disease. V. Definitive hosts of the trematode vector Nanophyetus salmincola. J. Parasitol. 54:770-774.

SOULSBY, E. J. L. 1968. Nanophyetus. pp. 61-63. In: Helminths, Arthropods and Protozoa of Domesticated Animals (Monnig). 6th ed. Williams and Wilkins Co., Baltimore, MD.

STEELE, J. H., ed. 1980. Salmon poisoning. p. 355. In: CRC Handbook Series in Zoonoses. Vol. II. Sect. A. H. STOENNER and W. KAPLIN, sect. eds. CRC Press, Inc., Boca Raton, FL. 


\section{Introduction}

Paramphistomum cervi (the "rumen fluke $\mathrm{e}^{\text {II) }}$ is a small (5-12 mm long), pink, conical, ventrally concavedorsally convex parasite of ruminants worldwide. Though it has not been documented from Alaskan cervids, it has been reported to infect reindeer (Rangifer tarandus) (Nikolaevski 1953 from Neiland and Dukeminier 1972) and moose (Alces alces) (Aleksandrov 1962 from Neiland and Dukeminier 1972) in Europe. The limited number of reindeer, less than 25, and single moose from the Seward Peninsula and Fairbanks regions of Alaska respectively, examined by J. Dau during the spring and summer of 1980 , did not harbor mature individuals of this fluke. Mature amphistomes (suborder Paramphistomata), presumably $\underline{P}$. cervi, commonly parasitize moose near Wasilla, Alaska and caribou near Cantwell, Alaska (R. Rausch and R. Dieterich, pers. comm., respectively).

\section{Transmission}

Adult flukes found among villi of the rumen release eggs that are passed in the feces of the ruminant. The unembryonated eggs must reach water to develop and hatch. Ciliated miracidia hatch, and penetrate suitable intermediate snail hosts (species unknown in Alaska). Mother sporocysts develop within the snails followed by development of 2 generations of rediae. Daughter rediae give rise to cercariae which escape from the snail and encyst on aquatic vegetation. The metacercariae are ingested with vegetation by ruminants. Metacercariae leave the metacercarial cysts while in the duodenum and penetrate the duodenal mucosa. The metacercariae develop into juvenile flukes while migrating anteriorly through the mucosa to the abomasum. The young flukes leave the mucosa after reaching the abomasum and travel through the lumen of the abomasum, omasum and reticulum to the rumen. The flukes reach sexual maturity in 2 to 4 months.

\section{Pathogenicity, Significance and Control}

Metacercaria that penetrate the mucosa of the duodenum can cause severe tissue damage while moving through it. This may cause enteritis, diarrhea and even death in severe infections. The significance and control of this parasite in Alaska have not been established.

\section{References}

ALEKSANDROVA, I. V. 1962. Cases of the intensive infection of elk by Paramphistomum cervi in the Kirov Oblast. Zool. Zh. 4:780-782.

NEILAND, $K$. $A$. and C. DUKEMINIER. 1972. A bibliography of the parasites, diseases and disorders of several important wild ruminants of the Northern Hemisphere. Fed. Aid Wildl. Rest. Proj. W-17-R. Alaska Dept. Fish and Game Tech. Bull. No. 3. 151 pp.

NIKOLAEVSKI, L. D. 1953. Paramphistomum cervi in reindeer. Veterinariya 30:29.

OLSEN, 0. W. 1962. Paramphistomum cervi (Schrank, 1790). pp. 128-131. In: Animal Parasites. Burgess Publishing Co., Minneapolis, MN. 


\title{
Alaria
}

\author{
J. Dau and R. Barrett
}

\section{Introduction}

Mature Alaria spp. are known to infect several mammals that occur in Alaska. Red fox (Vulpes vulpes) (Babero and Rausch 1952 as reported by Hopla et al. 1965) and wolves (Canis lupus) (Rausch 1959, Rausch and Williamson 1959) from Alaska have been found naturally infected with $A$. marcianae. Alaskan wolverines (GuTo gulo) have been reported to harbor $A$. mustelae (Dunagan 1957) and àn unidentified Alaria sp. (Rausch 1959). Alaria spp. were reportedly the trematode most frequently encountered in a parasite survey of Ontario mammals; A. marcianae occurred in 10 of $34(29 \%)$ wolves, 1 of $2(50 \%)$ coyotes (Canis latrans), 9 of 10 (90\%) red fox and 1 of 3 (33\%) lynx (Felis lynx) (Pearson 1956). Pearson (1956) lists the final and intermediate hosts of Alaria spp., and their susceptibility to infection based on experimental and empirical observations. Adult $A$. marcianae flukes are 2.5-4.2 $\mathrm{mm}$ long, and usually inhabit the first third of the small intestine.

\section{Transmission}

Trematodes of the genus Alaria utilize 2 intermediate hosts, often a third paratenic host and a final carnivorous host in their life cycle (0lsen 1962). Mature flukes release eggs into the small intestine of the final host. When egg-bearing feces contact fresh water, the eggs hatch into free-swimming, ciliated miracidia. The miricidia penetrate suitable snail hosts (e.g. Heliosoma spp.) and metamorphose into mother sporocysts in the snail renal system. The original mother sporocysts bear daughter sporocysts for approximately 14 months, the average life span of the snails. Daughter sporocysts give rise to free-swimming cercariae that escape from the snail to infect a second intermediate amphibian host; in Alaska, this is the frog Rana sylvatica. Cercariae are more successful penetrating the tegument of tadpoles than mature frogs and will persist throughout tadpole-frog metamorphosis. Larvae of Alaria spp. that infect amphibians are termed mesocercaria. Mesocercariae infect the final carnivorous hosts by 1 of 2 routes: 1) by direct ingestion of the infected frog or tadpole, or 2) by ingestion of a paratenic intermediate host that has acquired mesocercariae from infected tadpoles/frogs. A paratenic host is an animal in which the parasite can survive, but is not adequately suitable for complete development of the parasite. Paratenic hosts tend to accumulate mesocercariae; they may represent an ecological bridge between the aquatic and terrestrial life stages of Alaria spp. Larvae from the tadpole or frog ingested by the paratenic host migrate from the intestine to various tissues where development is arrested. When ingested by a suitable carnivorous final host, the mesocercariae migrate from the gut to the liver or abdominal cavity. The mesocercariae penetrate the diaphragm to reach the lungs where they develop into unencysted metacercariae. The metacercariae move up the trachea, are swallowed, and reach the small intestine where maturation occurs. 


\section{Significance}

Alaria spp. may be less significant in Alaska than other regions that have a greater richness of amphibian species. An ecological bottleneck at the mesocercarial life stage is suggested by the low incidence of mature flukes in wolves from the Brooks Range ( 1 of 162; $0.6 \%$ ) where Rana sylvatica are not abundant as contrasted with the higher incidence of infection in wolves from the Copper River drainage ( 7 of $27 ; 25 \%$ ) where frogs are numerous (Rausch and Williamson 1959).

\section{References}

BABERO, B. B. and R. RAUSCH. 1952. Notes on some trematodes parasitic in Alaskan canidae. Proc. Helminthol. Soc. Washington 19:15-17.

DUNAGAN, T. T. 1957. Studies on the parasites of the edible animals of Alaska. Proj. No. 7955-4. Arctic Aeromed Lab. 18 pp.
HOPLA, C. E., W. L. JELLISON and K. A. NEILAND. 1965. Parasites of Alaskan Vertebrates. Fed. Aid Wildl. Rest. Proj. W-6-R and W-15-R. Univ. Oklahoma Research Institute, Norman, OK. 73 pp.

OLSEN, 0. W. 1962. Alaria. pp. 100-103. In: Animal Parasites. Burgess Publishing Co., Minneapolis, MN.

PEARSON, J. C. 1956. Studies on the life cycles and morphology of the larval stages of Alaria arisaemoides Augustine and Uribe, 1927 and Alaria canis LaRue and Fallis, 1936 (Trematoda: Diplostomatidae). Can. J. Zool. 34:295-387.

RAUSCH, R. 1959. Studies on the helminth fauna of Alaska. XXXVI. Parasites of the wolverine, Gulo gulo L., with observations on the biology of Taenia twitchelli Schwartz, 1924. J. Parasitol. 45:465-484.

RAUSCH, R. and F. S. L. WILLIAMSON. 1959. Studies on the helminth fauna of Alaska. XXXIV. The parasites of wolves, Canis lupus L. J. Parasitol. 45:395-403. 


\title{
Cestoda
}

\author{
J. Dau and R. Barrett
}

Cestodes are flatworms (Platyhelminths) that range in size from several millimeters to many meters in length and parasitize nearly all classes of vertebrates. Mature tapeworms have elongate, usually segmented, flat bodies. Those important in Alaska are polyzoic (having multiple body segments). All require at least 1 intermediate host. They lack a body cavity and alimentary canal. The excretory system consists of flame cells and efferent canals. The tegument, which covers the body, is the major absorptive organ with microvilli somewhat analogous to those lining the vertebrate intestine.

Tapeworms consist of essentially 2 parts: 1) the scolex (head) and 2) the strobila (body). The body of polyzoic cestodes consists of multiple proglottids (body segments). A relatively short, unsegmented neck may occur between the scolex and strobila. Anterior proglottids are youngest; proglottid size and development increases posteriad along the strobila. Mature tapeworms are hermaphroditic; each proglottid possesses male and female reproductive organs. Segments having functional reproductive organs are considered mature. Following cross or self fertilization, reproductive organs degenerate leaving only a dark, eggfilled uterus. Segments in this condition are termed gravid. Gravid segments are passed out of the definitive host singly or in chains; eggs are liberated through disintegration or rupture of the segment. For most cestodes, embryonic development takes place within the uterus so that when freed, the eggs contain a spherical or ovoid embryo termed the oncosphere. The oncosphere develops 3 (rarely 5) pairs of hooks and is termed the hexacanth embryo.

Tapeworm eggs hatch after being swallowed by a suitable intermediate host. The hexacanth embryos penetrate the intestine of this host and migrate to some anatomical site for further development. The hexacanth embryo develops into an infective larva (or metacestode), the type of which depends upon the group of cestodes involved. Cestode larvae, like adults, consist of a scolex and body. Larvae and adult tapeworms differ significantly in body configuration however, the most obvious and general differences being the smaller size and absence of proglottids in cestode larvae ( $\mathrm{Fig} .2$ ).

Larvae of the genus Echinococcus produce protoscolices, a term which refers to the scolex and body of these infective organisms. The terminology that will be used in this section with regard to larvae of cestodes is defined as follows:

1. Cysticercus (= bladderworm)--a larva of the genus Taenia from the wall of which a single scolex arises.

2. Coenurus--a larva in which the bladder wall gives rise to several scolices.

3. Hydatid cyst--a larva of the genus Echinococcus in which the germinal membrane gives rise to brood capsules in which protoscolices are produced.

4. Tetrathyridium--a large, solidbodied larva of the genus Mesocestoides that possesses a single scolex and that is infective for its final host. 

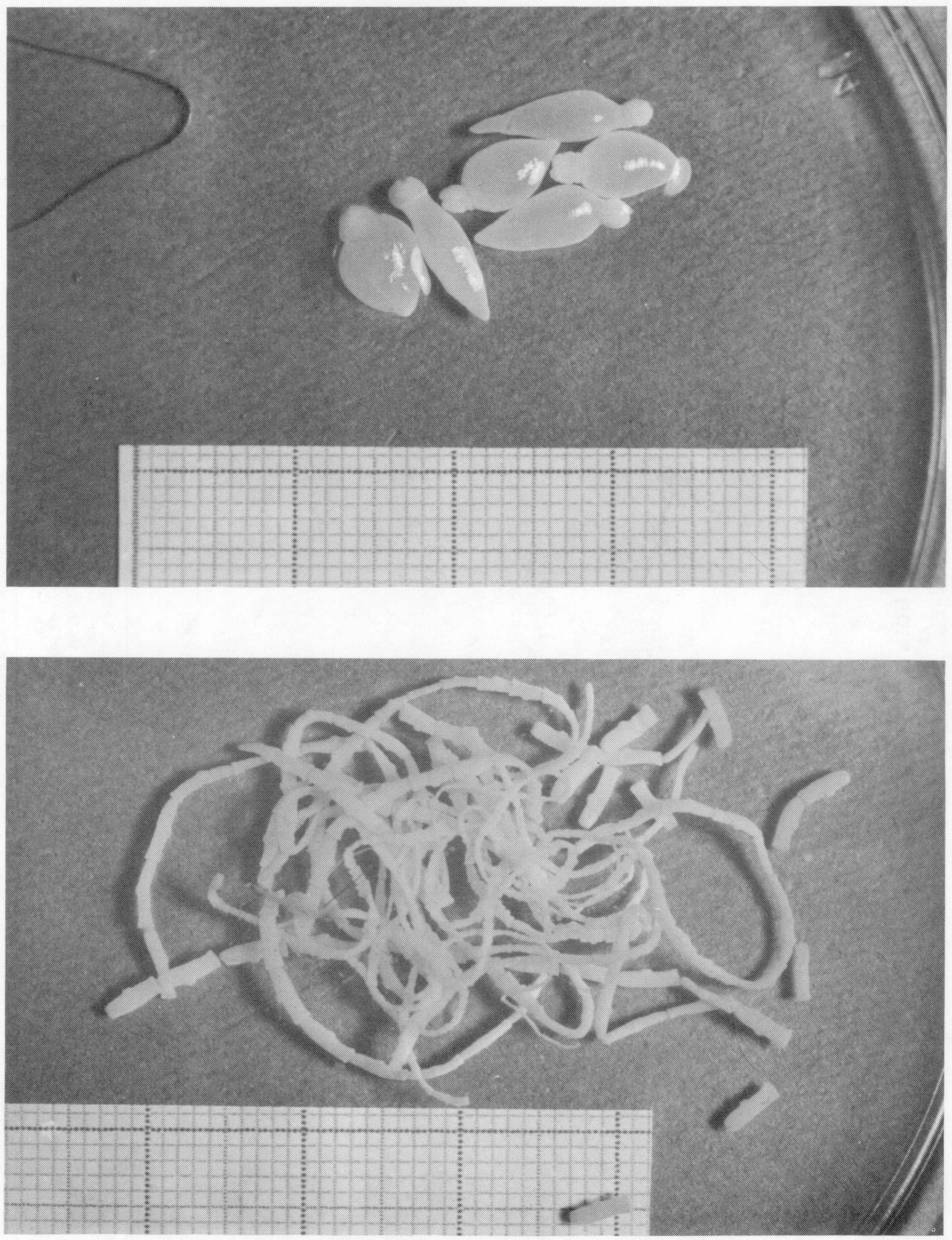

Figure 2. Tapeworm larvae and adults. This illustration shows the disparity in body configuration for adult and larval life stages of these parasites. Scale is in mm (D. Borchert). 


\section{Figure 3. Comparison of two common cestode orders in Alaska.}

\section{ORDER PSEUDOPHYLLIDEA}

Eggs escape through uterine pore. Eggs are operculate, resemble that of trematodes, and need embryonation period in fresh water to hatch into coracidium (free-swimming embryo).

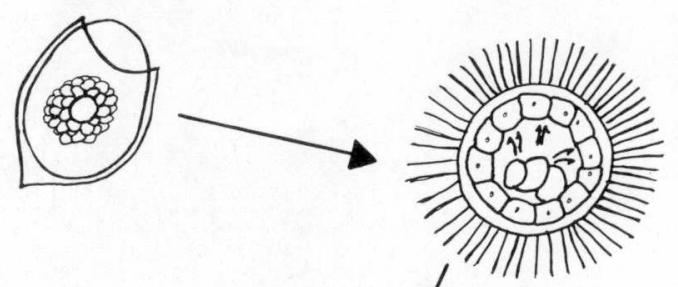

Crustacean (such os Cyclops or Diaptomus) ingests

coracidium and serves as first intermediate host.

Coracidium develops into procercoid larva.

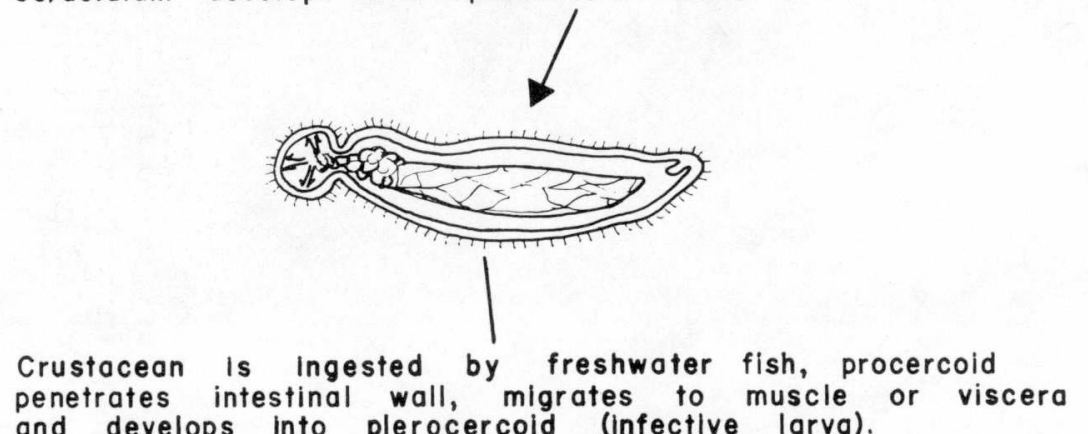

penetrates intestinal wall, migrates to muscle or viscera and develops into plerocercoid (infectlve larva).

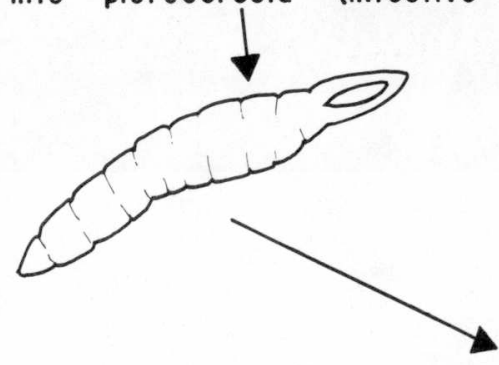

Vertebrate definitive host becomes infected

by ingesting plerocercoid or larval cyst

definitive host tissue, larva develops into

adult cestode.

\section{ORDER CYCLOPHYLLIDEA}

Eggs are released upon rupture or disintegration of gravid proglottids; embryonic development has already occurred in uterus. Upon ingestion by intermediate host the spherical (ovold or spherical embryo with $3-5$ pairs of hooks).

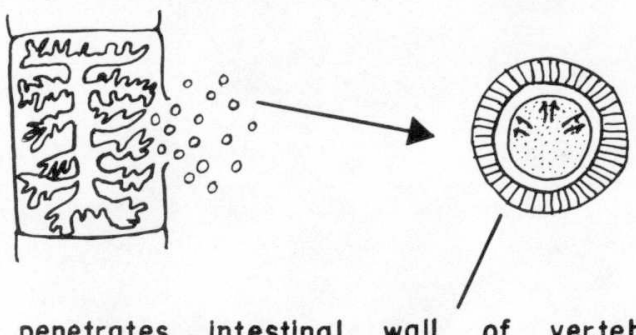

Oncosphere penetrates intestinal wall of vertebrate or Invertebrate intermediate host, migrates to suitable body See common larval cyst morphologies below.
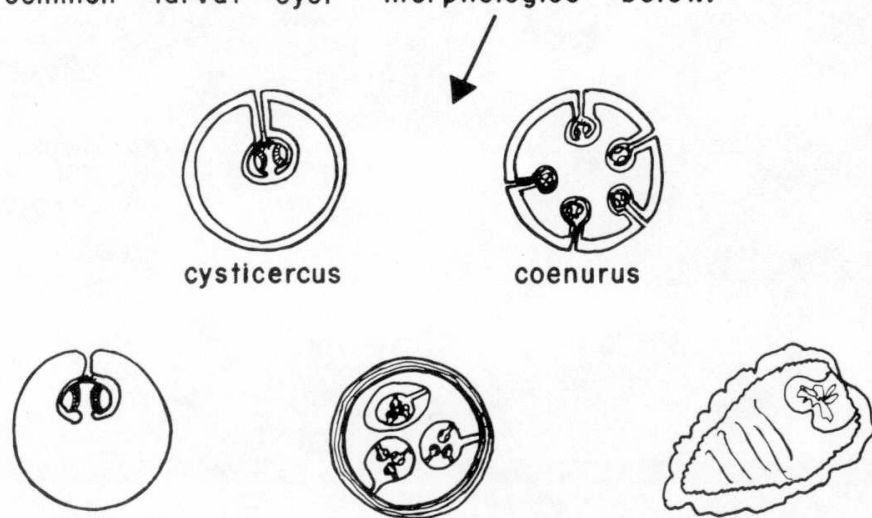

cysticercoid

hydatid cyst

tetrathyridium

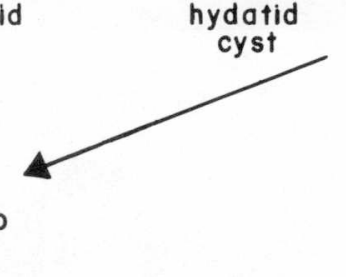


TABLE 2

Anatomical Sites of Localization for Larvae of

Some Cyclophyllidean Cestodes in Mammals of Alaska

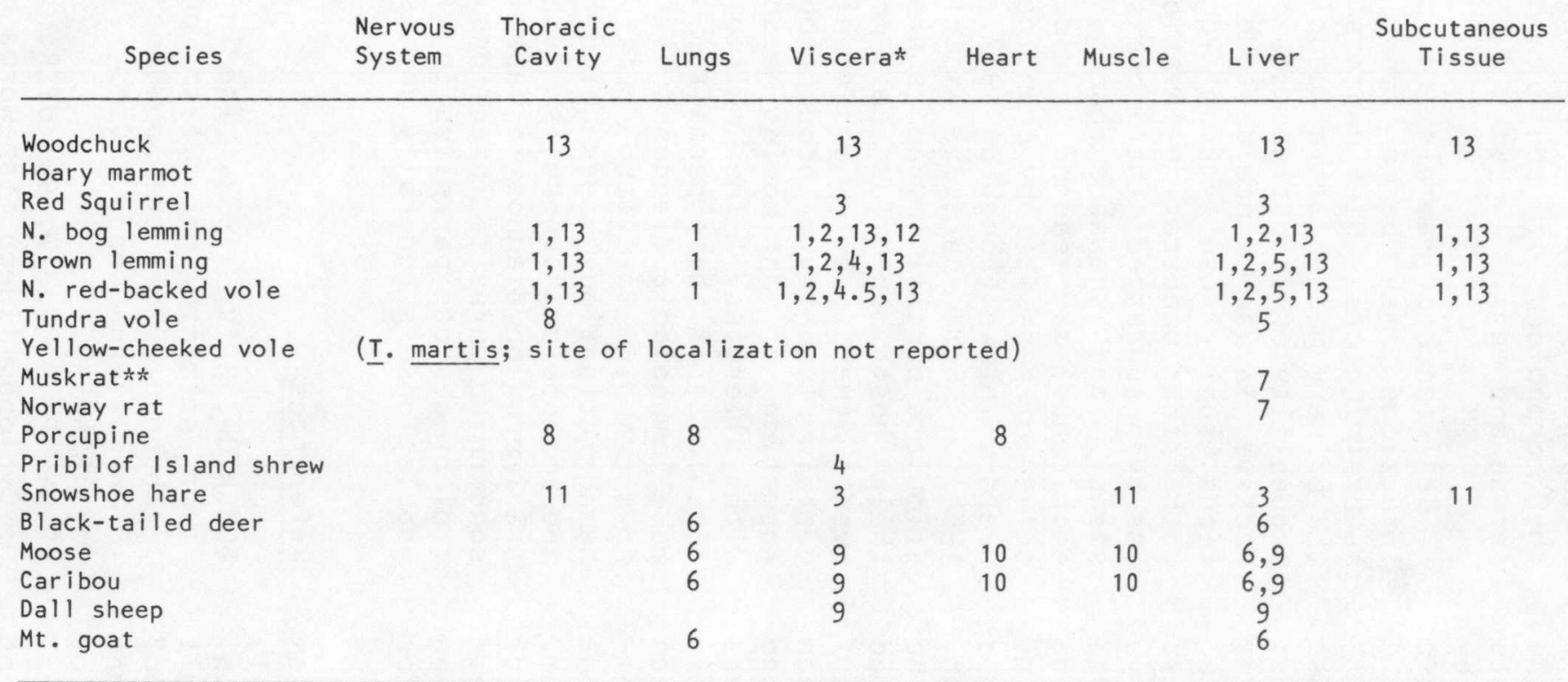

*References to the coelom and those organs and tissues it contains.

**Taenia crassiceps cysticercus was found in muskrat in Ontario (Freeman 1962). Other susceptible intermediate hosts for this parasite include members of Sciuridae and Cricetidae.

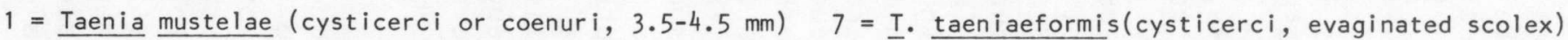

$2=\underline{T} \cdot$ polyacantha (cysticerci)

$3=\underline{T}$. pisiformis (cysticerci, involuted scolex)

4 = Mesocestoides 1 ineatus (tetrathyridia, 1-2 cm)

$4.5=\underline{\text { M. kirbyi }}$ (tetrathyridia)

5 = Echinococcus multilocularis (alveolar cyst)

6 = Echinococcus granulosus (hydatid cyst, $5-10 \mathrm{~cm}$ ) Man can be intermediate host for $\underline{E}$. granulosus and $E$. multilocularis

$8=\underline{T}$. twitchelli (polycephalic, $70 \mathrm{~mm}$ long)

$9=\underline{T}$. hydatigena (cystierci, invaginated scolex, $25 \mathrm{~mm}$ )

$10=\underline{T}$. krabbei (cysticerci, invaginated scolex, $1.8-3 \mathrm{~mm}$ )

$11=\underline{T}$ serialis (coenuri, $5 \mathrm{~cm}$ )

12 = $\mathbf{T}$. rileyi (cysticerci, evaginated scolex)

$13=T$. mustelae (larvae from cysticerci to coenuri)

$14=T$. martis (cysticerci, invaginated scolex, 2-7 mm long) 
5. Cysticercoid--a small larva with one scolex that essentially lacks a body.

Larvae of some tapeworm species are capable of asexual reproduction through endogenous or exogenous budding (budding within or on the external surface). Definitive hosts of cestode parasites are infected by ingesting portions of intermediate hosts that contain infective larvae.

There are 2 orders in the class Cestoda important to Alaskan mammals; Cyclophyllidea which encompasses Mesocestoides, Moniezia, Taenia, Echinococcus, Anoplocephaloides, Paranoplocephala, Andrya, Diandrya and Hymenolepis, and Pseudophyllidea that includes Diphyllobothrium. The scolex of cyclophyllidean tapeworms possesses 2 or 4 suckers and a protrudible rostellum (an anterior, usually tubular portion of the scolex), both of which may be armed with hooks, though suckers rarely so. The scolex of pseudophyllidean cestodes possesses bothria (long grooves) rather than the oval suckers of cyclophyllideans. The neck is less apparent in pseudophyllidean tapeworms, and the life stage comparable to the cyclophyllidean oncosphere is termed the coracidium. Figure 3 compares the life cycles of cyclophyllidean and pseudophyllidean cestodes. Eggs of pseudophyllidean tapeworms are easily mistaken for those of trematodes.

The effects of cestode parasitism varies with regard to the host, parasite and intensity of infection. Usually, pathogenic effects of adult tapeworms are limited to blockage of the intestines from excessive numbers of strobila, generalized toxic or allergic reactions, physical irritation of the intestinal mucosa and nutrient deprivation. Cestodes may provide access to microorganisms that cause secondary infections where scolices of mature tapeworms attach to the intestinal wall of their host. These effects are rarely demonstrable however. Larval infections in intermediate hosts may be more severe than adult worms in definitive hosts since they often destroy tissue. Diagnosis of tapeworm infections is based on the morphology of adult tapeworms, larvae and eggs.

Resistance of mammals to cestode infections may be a function of several factors. Diet appears to be the primary mechanism influencing the probability of ingesting eggs or infective larvae since cestode infections usually result from ingestion rather than penetration invasion. It has been suggested that bears (Ursidae) and snowshoe hares (Lepus americanus) may seasonally rid themselves of parasites by changing their dietary intake (Boughton 1932, Rausch 1954). Some parasite species do stimulate partial immune responses in their hosts such as cell-mediated events (neutrophil, eosinophil and certain lymphocyte production) and formation of antibodies associated with allergies. The multiple antigenicity of these parasite species make immunologic diagnoses difficult. Advances in in vitro cultivation, hemagglutination, agar gel diffusion and immunoelectrophoresis techniques are improving diagnostic capabilities concerning parasite infections. Control of cestode parasites in wildlife populations is generally unfeasible.

\section{References}

BOUGHTON, R. V. 1932. The influence of helminth parasitism on the abundance of the snowshoe rabbit in western Canada. Can. J. Res. 7:524-547.

LEIBY, P. D. and W. G. DYER. 1971. Cyclophyllidean tapeworms of wild carnivora. pp. 174-181. In: Parasitic Diseases of Wild Mammals. J. W. DAVIS and R. C. ANDERSON, eds. lowa State Univ. Press, Ames, IA. 
RAUSCH, R. L. 1954. Studies of the helminth fauna of Alaska. XXI. Taxonomy, morphological variation and ecology of Diphyllobothrium ursi n. sp. provis. on Kodiak Island. J. Parasitol. 40:540-563.
SOULSBY, E. J. L. 1968. Cestodes. pp. 88-93. In: Helminths, Arthropods and Protozoa of Domesticated Animals (Monnig). 6th ed. Williams and Wilkins Co., Baltimore, MD.

\title{
Mesocestoides
}

\author{
J. Dau and R. Barrett
}

\section{Introduction}

Cestodes

$$
\text { of }
$$

the

genus

Mesocestoides are common parasites of Alaskan carnivores (Shults 1970). Their geographic distribution includes Europe, Africa, Asia and North America. Two species have been identified in Alaska; 1) M. kirbyi from coyotes (Canis latrans) (Shults 1970), red fox (Vulpes vulpes) (Dunagan 1957, Shults 1970), arctic fox (Alopex lagopus) (Fay and Williamson 1962, Shults 1970), wolverine (Gulo gulo) (Hopla et al. 1965, Shults 1970) and lynx (Felis lynx) (Shults 1970), and 2) M. lineatus from arctic fox, red fox, wolverine, ermine (Mustela erminea) and lynx (Shults 1970). Additional records of mature $\underline{M}$. lineatus in species indigenous tō Alaska include marten (Martes americana) and mink (Mustela vison) (Shults 1970). Mature M. kirbyi are 360-586 mm long, 2.1-2.9 $\mathrm{mm}$ wide and consist of up to 1362 segments (Shults 1970). Mature M. lineatus are 605-1093 mm long,

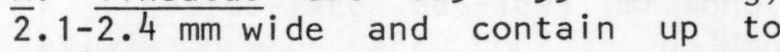
1096 segments.

Tetrathyridia (the larval form of Mesocestoides spp. infective for their definitive hosts) of $\underline{M}$. lineatus have been found in a brown lemming (Lemmus sibericus) and a Pribilof Island shrew (Sorex pribilofensis) both from the Pribilof Islands (Shults 1970). Larvae of $\underline{M}$. kirbyi have been found in red-backe-d voles (Clethrionomys rutilus) (Shults 1970).

\section{Transmission}

Mesocestoides spp. utilize 2 intermediate hosts. The first intermediate host is thought to be a copraphagous orbatid mite (Soulsby 1968). The larval stage that develops within the mite is a small vesicle that essentially lacks a cavity and bears 1 scolex. This larval stage is passively transmitted to the second intermediate host, usually a bird or rodent, but occasionally a carnivore, with ingestion of the mite. Carnivores can thus serve as both second intermediate and final hosts. First stage larvae of Mesocestoides spp. develop into tetrathyridia within the second intermediate hosts.

Tetrathyridia are flat, slender, highly contractile worm-like organisms 1-2 cm long. The larval stage of some species is capable of asexual reproduction in intermediate or definitive hosts through longitudinal division of the scolex 5 days post ingestion (Leiby and Dyer 1971). Tetrathyridia may persist in 
the body cavity, tissues or organs of second intermediate hosts for long intervals. They are passively transmitted to carnivores through carnivory. The tetrathyridia localize and mature in the small intestine of the final hosts. When ingested by an animal other than a preferred definitive host (a paratenic or transport host), tetrathyridia may enter the body cavity, tissues or visceral organs, become dormant and persist in this larval stage.

\section{Symptoms and Pathology}

A dog (Canis familiaris) having a peritoneal infection with a Mesocestoides sp. exhibited anorexia, vomiting and depression. Pathologic changes included reduced blood lymphocytes, decreased serum albumen and midline duodenal displacement (Barasanti et al. 1979). Long term experimental infections with tetrathyridia caused disseminated lesions throughout the liver, lungs, kidneys, epididymis and testicles of mice (Mus sp.) (Todd et al. 1978).

\section{Significance and Control}

The significance of Mesocestoides spp. in Alaska is undetermined. Low, single doses of praziquantel have effectively reduced adult and larval $M$. corti infections in dogs and cats (Felis catus). Mebendazole has reportedly eliminated tetrathyridial infections in mice especially when administered with levamisole (Bennett et al. 1978). In vitro antiparasitic effects have been observed for intestinal extracts taken from mice that had been subcutaneously injected with live tetrathyridia (Niederkorn 1978). Administration of such anthelmintic substances to wildlife is generally unfeasible at this time, however.

\section{References}

BARASANTI, J. A., B. D. JONES, W. S. BAILEY and G. D. KNIPLIN. 1979. Diagnosis and treatment of peritonitis caused by a larval cestode Mesocestoides spp., in a dog. Cornell Vet. 69:45-53.

BENNET, E. M., C. BEHM and C. BRYANT. 1978. Effects of mebendazole and levamisole on tetrathyridia of Mesocestoides corti in the mouse. Int. J. Parasitol. 8:463-466.

DUNAGAN, T. T. 1957. Studies on the parasites of edible animals of Alaska. Proj. Report No. 7955-4. Arctic Aeromed. Lab. 18 pp.

FAY, F. H. and F. S. L. WILLIAMSON. 1962. Studies on the helminth fauna on Alaska. XXXIX. Echinococcus multilocularis Leuckart, 1863, and other helminths of foxes on the Pribilof Islands. Can. J. Zool. $40: 767-772$.

HOPLA, C. E. W. L. JELLISON and K. A. NEILAND. 1965. Parasites of Alaskan Vertebrates. Fed. Aid Wildl. Rest. Proj. W-6-R and W-15-R. Univ. Oklahoma Research Inst., Norman, OK. 73 pp.

LEIBY, P. D. and W. G. DYER. 1971. Mesocestoididae. pp. 220-224. In: Parasitis Diseases of Wild Mammals. J.W. DAVIS and R. C. ANDERSON, eds. lowa State Univ. Press, Ames, IA.

NIEDERKORN, J. Y. 1978. Life cycle of Mesocestoides corti in the dog (Canis familiaris). J. Parsitol. $6 \overline{4: 253}-256$.

SCHMIDT, J. M. and K. S. TODD. 1978. Life cycle of Mesocestoides corti in the dog (Canis familiaris). Am. J. Vet. Res. 39:1490-1493.

SHULTS, L. M. 1970. Mesocestoides kirbyi and $M$. lineatus: 0ccurrence in Alaskan carnivores. Trans. Amer. Micros. Soc. $89: 478-486$.

SOULSBY, E. J. L. 1968. Mesocestoides. pp. 94-96. In: Helminths, Arthropods and Protozoa of Domesticated Animals (Monnig). 6th ed. Williams and Wilkins Co. Baltimore, MD. 
THOMAS, H. and R. GOENNERT. 1978. The efficacy of praziquantel against cestodes in dogs, cats and sheep. Res. Vet. Sci. 24:20-25.
TODD, K. S., JR, J. SIMON and J. A. DIPIETRO. 1978. Pathological changes in mice infected with tetrathyridia of Mesocestoides corti. Lab. Anim. 12:51-54.

\title{
Moniezia
}

\author{
R. Barrett and J. Dau
}

\section{Introduction}

Moniezia spp. are large tapeworms (up to $600 \mathrm{~cm}$ long) that mature in the small intestine of ruminants. Most of the information known about these organisms stems from work with domestic sheep (Ovis aries) and cattle (Bos taurus). These cestodes occur worldwide; in Alaska, they have been identified in reindeer (Rangifer tarandus) (Hopla et al. 1965), moose (Alces alces) (A. Franzmann, pers. comm.), mountain goats (Oreamnos americana), muskox (Ovibos moschatus) and black-tailed deer (0docoileus hemionus sitkensis) (the latter 3 host records observed by R. L. Rausch, pers. comm.). Two species of Moniezia, distinguishable by strobila (body) width and other characteristics, have been identified; $\underline{M}$. expansa (approximately $1.6 \mathrm{~cm}$ wide) and $\underline{M}$. benede $i$ (approximately $2.6 \mathrm{~cm}$ wide).

\section{Transmission}

Eggs are passed into the external environment in gravid segments with feces of the ruminant. The eggs are accidentally ingested by any of several genera of orbatid mites where they mature into cysticercoids (larvae of certain morphology; see glossary) in 2 to 5 months. Infected mites that $\mathrm{cling}$ to vegetation are ingested by the ruminant and the lar- vae are released from the mites by digestive processes. The larvae attach to the intestinal wall of the ruminant and mature in roughly 30 days. The life expectancy of adult worms is approximately 3 months, an unusually brief life span for a cestode. Infections tend to be agespecific, favoring young ruminants less than 5 months of age. Lambs have been reported to pass gravid segments that resemble grains of cooked rice when only 6 weeks old (Soulsby 1968).

\section{Symptoms, Diagnosis} and Control

Moniezia spp. do not appear to be serious wildlife pathogens in Alaska. Heavy infections could cause blockage of the intestines due to the large size of the worms. Diagnosis is based on the morphology of gravid segments and eggs in the feces of host animals or adult worms in the small intestine. A premixed feed of $15 \mathrm{mg}$ oxibendazole per $\mathrm{kg}$ ruminant body weight was reportedly over $71 \%$ effective in eliminating Moniezia infections in domestic cattle with no toxic effects (Williams et al. 1978). The significance of this parasite in Alaskan wildlife has not been established. 


\section{References}

HOPLA, C. E., W. L. JELLISON and K. A. NEILAND. 1965. Parasites of Alaskan Vertebrates. Fed. Aid Wildl. Rest. Proj. W-6-R and W-15-R. Univ. Oklahoma Research Inst., Norman, OK. 73 pp.

SOULSBY, E. J.L. 1968. Moniezia. pp. 96-98. In: Helminths, $\mathrm{Ar}$ thropods and Protozoa of Domesticated Animals (Monnig). 6th ed. Williams and Wilkins Co., Baltimore, MD.
WILLIAMS, J. C., D. SHEEHAN and R. FUSELIER. 1978. Anthelminthic activity of oxibendazole against gastrointestinal parasites of cattle. Proc. Helminthol. Soc. Washington 45:129-131.

\section{Anoplocephaloides, Paranoplocephala, Andrya Hymenolepis}

J. Dau and R. Barrett

Arvicolid rodents (Dicrostonyx, Lemmus, Synaptomys, Clethrionomys and Microtus spp.) are parasitized by a variety of cestode genera. Anoplocephaloides, Paranophlocephala, Andrya and Hymenolepis represent those genera for which adult worms are most frequently found in Alaskan voles (Rausch 1951, 1952). Table 3 summarizes the reported host specificity of these cestode genera in Alaskan voles. The geographic range of distribution for these parasites varies between species. Andrya macrocephala has been recovered from arctic Alaska to Mexico while Paranoplocephala omphalodes is limited to arctic Alaska (Rausch 1951).

Paranoplocephala and Andrya spp. utilize orbatid mites as intermediate hosts for development of cysticercoids (Gleason and Buckner 1979, Rausch 1976). Cysticercoids of Hymenolepis develop in beetles (Rau 1979) and fleas (Rausch and Tiner
1949). Voles become infected by ingesting mites that $\mathrm{cling}$ to vegetation. Though experimental exposure of mites to eggs from individuals of Paranoplocephala spp. indicates infection rates for mites may be low $(1.38 \%)$, peak mite abundance coincides with periods of maximum vole activity resulting in ample opportunity for infection of voles (Rausch 1951).

No correlation reportedly exists between vole density and the frequency or intensity of parasite infections (Rausch 1951, 1976). Quantitative and morphological variations of these vole parasites have been linked to seasonal and geographical differences (Rausch 1976).

These parasites apparently have little pathogenic effect on vole species. 
TABLE 3

Host-parasite Relationships of Some Cestodes

Found in Small Mammals of Alaska

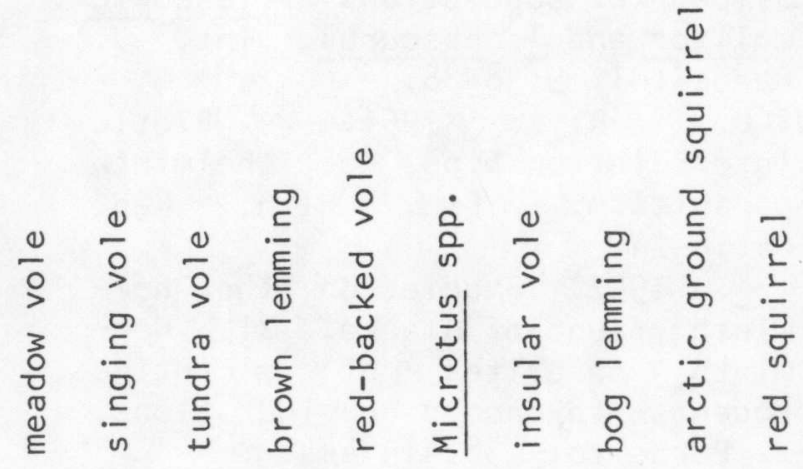

Paranoplocephala omphalodes

Anoplocephaloides infrequens

$\mathrm{X}$ X $\mathrm{X}$

Anoplocephaloides variabilis

$\mathrm{X}$

Anoplocephaloides lemmi

Anoplocephaloides troeschi

$\mathrm{X}$

Anoplocephaloides kontrimavichusi

$\mathrm{x}$

Anoplocephaloides wigginsi

$\mathrm{X}$

Hymenolepis horrida

$\mathrm{X} \quad \mathrm{X} \quad \mathrm{X} \quad \mathrm{X}$

Andrya bialowienzensis

$\mathrm{x}$

Andrya primordialis

(Status in Alaska not clearly established)

Andrya arctica

$\mathrm{x}$ 


\section{References}

GLEASON, L. N. and R. L. BUCKNER. 1979. Orbatid mites as intermediate hosts of certain anoplocephalid cestodes. Trans. Ky. Acad. Sci. 40:27-32.

RAU, M. E. 1979. The frequency distribution of Hymenolepis diminuta cysticercoids in natural, sympatric populations of Tenebrio molitor and T. obscurus. Int. J. Parasitol. 9:58-88.

RAUSCH, R. 1951 Biotic interrelationships of helminth parasitism. Pub. Hlth. Rep. $66: 18-24$.

- 1952. Studies on the helminth fauna of Alaska. XI. Helminth parasites of microtine rodents--taxonomic considerations. J. Parasitol. 38:415-444.
1976. Anoplocephaloides Baer, 1923. Ann. Parasitol. Hum. Comp. $51: 513-562$.

RAUSCH, R. and J. D. TINER. 1949. Studies on the parasitic helminths of the north central states. II. Helminths of voles (Microtus spp.) preliminary report. Amer. Mid. Nat. 41:665-694.

YAMAGUTI, S. 1959. Systema Helminthum. Vol. II. Cestodes. Interscience Publishers, Ltd. London, ENG.

\title{
Diandrya
}

\author{
J. Dau and R. Barrett
}

The genus Diandrya contains 1 species, D. composita, that occurs widely in marmots (Marmota spp.) of western North America. In Alaska, this parasite has been found in hoary marmots (Marmota caligata) and Alaska marmots (M. broweri). Diandrya composita is a relatively large tapeworm having a strobila up to $625 \mathrm{~mm}$ and 817 segments long. This genus is very similar to the genus Andrya from which it apparently evolved.

The lifecycle of $D$. composita is similar to that described for Andrya spp. Both genera utilize an orbatid mite in which cysticercoids develop. The variance in size and development of strobila suggests marmots are continually infected following emergence from hibernation.
The significance of $\underline{D}$. composita in marmots of Alaska has not been established. Rausch (1980) provides a detailed description of this tapeworm. The information presented herein is summarized from that paper.

\section{References}

RAUSCH, R. L. 1980. Redescription of Diandrya composita Darrah, 1930 (Cestoda: Anoplocephal idae) from neararctic marmots (Rodentia: Sciuridae) and the relationships of the genus Diandrya emend. Proc. Helminthol. Soc. Washington $47: 157-164$. 
J. Dau and R. Barrett

Taenia spp. are the most common cestodes of Alaskan mammals. For example, Taenia spp. were found in 182 of $200(91 \%)$ wolves collected from 1949 to 1959 throughout Alaska (Rausch 1959a). Life cycles involve a single intermediate host, usually a rodent or an ungulate and a carnivorous definitive host. Table 3 lists the final and intermediate hosts of Taenia spp. identified in Alaska. The adult tapeworms are generally large, white and inhabit the small intestine of definitive hosts. Bladderworms of most Taenia spp. are cysticerci (each bladder possessing a single scolex) though some larval forms of Taenia spp. are coenuri (each bladder having many scolices), or other morphologic types. Larvae are frequently enclosed within host-generated cysts of fibrous tissue; the extent of encystment is dependent upon the Taenia sp., the area of localization within the intermediate host and hostresponse to the parasite.

Intermediate hosts contract larval infections by ingesting eggs or gravid segments passed in the feces of final hosts. Passive transmission to the definitive host occurs when carnivores ingest larvae within infected prey.

Adult tapeworms, when extremely abundant in host organisms, could theoretically cause blockage of the intestines and compete with the host for nutrients. In addition, mature tapeworms may provide access to viral or bacterial agents where scolices attach to the intestinal mucosa of the host. In his extensive experience with tapeworms of Taenia spp. however, Dr. R. L. Rausch reports he has never seen any evidence that adult cestodes of this genus are pathogenic (pers. comm.). Larvae of Taenia spp. likewise have the potential to be pathogenic, since they of ten destroy tissues of intermediate hosts. Under natural circumstances, intermediate hosts usually do not ingest adequate numbers of eggs to sustain significant damages (R. L. Rausch, pers. comm.).

Diagnosis of infections by tapeworms of Taenia spp. is based on the morphology of the adult tapeworms, gravid segments or eggs in the intestines and feces of the final host. Identification of larvae is based on the morphology of bladderworms. Control of Taenia spp. in wildlife populations is currently impractical.

Though the basis of host-parasite specificity is commonly thought to stem from the physiologic compatability between host and parasite, the ecological agreement between definitive and intermediate hosts is a significant influencing factor. This ecological consideration is exemplified by contrasting the principal final and intermediate hosts of T. pisiformis (coyote, Canis Tatrans--snowshoe hare, Lepus americanus) and I. hydatigena (wolf, c. lupus-moose, Alces alces). All physical and biological components of the environment collectively affect the dynamic relationships between parasites and their hosts. The numerous Taenia spp. that infect a wide variety of Alaskan vertebrates makes this genus attractive for comparative studies of parasite ecology. Table 3 cross-references hostparasite relationships for Taenia spp. in Alaska. 
Host-parasite cross reference for Taenia spp. found naturally in Alaskan mammals

T. crassiceps

$\stackrel{\circ}{\circ}$

T. hydatigena

I. krabbei

T. serialis

T. mustelae

T. pisiformis

I. twitchelli

T. polyacantha

I. taeniaformis

I. rileyi

I. martes

$$
\begin{aligned}
& \text { Definitive Hosts Intermediate Hosts }
\end{aligned}
$$

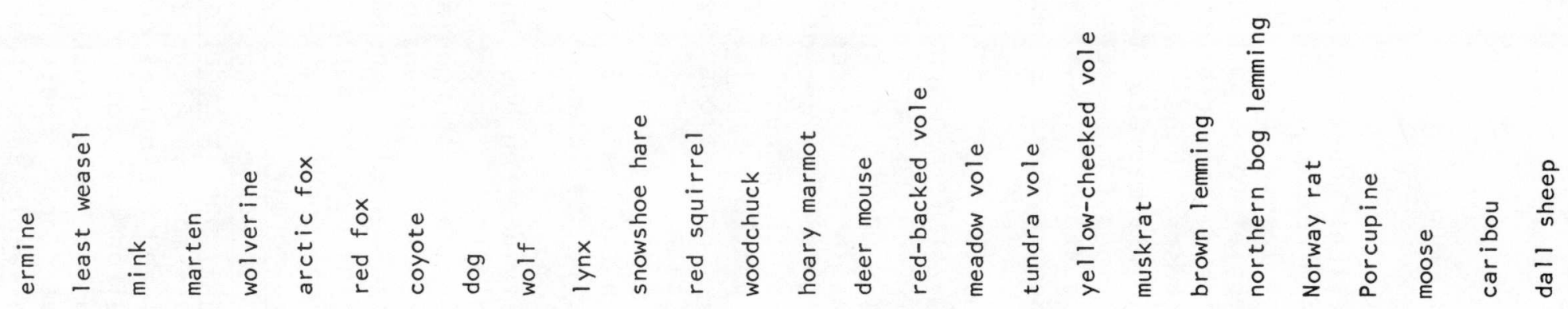




\section{Introduction}

In Alaska, arctic fox (Alopex lagopus) and red fox (Vulpes vulpes) commonly harbor mature T. crassiceps; domestic dogs (Canis familiaris) are parasitized by this tapeworm less frequently. Taenia crassiceps is reportedly the primary parasite of red fox in Ontario (Freeman 1956). Microtines frequently harbor cysticerci. Adult worms are 70-140 $\mathrm{mm}$ long; cysticercus morphology is variable.

\section{Transmission}

Gravid proglottids are passed in the feces of canines. These segments degenerate thus liberating the eggs enclosed within them. Microtine rodents accidentally ingest eggs that cling to vegetation. The oncospheres (embryos) hatch, or are liberated within these rodents and migrate to subcutaneous tissue or to body cavities where cysticerci form. Larvae reproduce asexually through exogenous budding from the parent bladder in spite of host generated encystment. Definitive hosts, i.e. foxes, are infected by eating rodents that harbor cysticerci. Larvae mature within canids 5 to 6 weeks post ingestion.

\section{Symptoms}

Canid infections are asymptomatic. Rodents may exhibit abdominal swelling or enlargement of the ventral portion of the neck. Gross enlargements may hinder the movement of rodents and predispose these intermediate hosts to predation.

\section{Introduction}

Taenia hydatigena resembles $T$. crassiceps in that each utilizes a canid definitive host. Taenia hydatigena is most frequent $\overline{l y}$ found in the wolf (Canis lupus), moose (Alces alces), caribou (Rangifer tarandus) food chain however. Fifty-six of 78 (72\%) wolves collected in Alaska during the period from 1949 to 1959 reportedly harbored mature worms (Rausch 1959b). Coyotes, and to a lesser extent, dogs also serve as definitive hosts in Alaska (Dunagan 1957). Strobila are large, ranging 75-500 cm in length.

Cysticerci are approximately $25 \mathrm{~mm}$ in length. Intermediate hosts identified in Alaska are moose (Dunagan 1957, Rausch 1959b, Rausch and Williamson 1959), caribou (Dunagan 1957, Rausch 1959b) and dall sheep (Ovis dalli) (Dunagan 1957). Twentyseven of 32 (84\%) moose from British Columbia were reported to have cysticerci (Rausch and Williamson 1959); up to 51 cysticerci have been reported from a single, old moose taken in Alaska (Rausch 1959b).

\section{Transmission}

Gravid proglottids pass from canids in the feces. The eggs are eventually ingested by suitable intermediate hosts. Oncospheres are liberated from the eggs in the small intestine of the intermediate hosts and penetrate the intestinal mucosa. They reach the liver via the hepatic portal system. Experimentally infected domestic lambs (Ovis aries) exhibited fibrotic foci in the liver 7 days post infection and macroscopic (1-9 mm) cysticerci in hemorrhagic streaks 10 days post infection 
(Soulsby 1968). Cysticerci may migrate from the liver, often leaving hepatic streaks and/or open pits, into the abdominal cavity 18 to 25 days post infection, or they may become entombed in cysts of host origin (Soulsby 1968). Wolves are infected by ingesting cysticerci with moose/caribou meat.

\section{$\underline{\text { Significance }}$}

Damage to canids is usually minimal. Pathogenic effects of larvae in ungulates are generally localized and of little significance to the health of these intermediate hosts. This parasite is not considered pathogenic for humans.

\section{Taenia krabbei}

\section{Introduction}

Taenia krabbei usually mature in the small intestine of large canids. In Alaska, they have been identified in dogs (Dunagan 1957), coyotes (Dunagan 1957), wolves (Dunagan 1957, Rausch 1959a) and lynx (Dunagan 1957). Mature worms reach $20 \mathrm{~cm}$ in length. Larvae are typical cysticer$\mathrm{ci}$ up to $1.8 \mathrm{~mm}$ and $3.0 \mathrm{~mm}$ long with an invaginated scolex. They have been found in leg, shoulder, tongue and heart muscles of moose and caribou in Alaska (Rausch 1959b). Transmission follows the general wolf--moose food chain. Ova appear in the feces of canines 34 to 37 days post ingestion (Freeman et al. 1961).

\section{Significance}

This Taenia sp. is not considered to be a significant wildlife pathogen in Alaska. Instances of severe infection where host health is impaired probably occur infrequently in both intermediate and definitive hosts. Since cysticerci occur throughout the muscle tissue of intermediate hosts, this parasite may reduce the quality of meat in ungulate game species. Selective harvesting of old individuals, both male and female, may reduce the incidence of $T$. krabbe $i$ infections in wildlife populations.

Taenia serialis

Introduction

Mature T. serialis were identified in 23 of $^{-} 7 \overline{8(29 \%)}$ wolves collected throughout Alaska from 1949 to 1959 (Rausch 1959b). Red fox and coyotes have been found to harbor this tapeworm in the contiguous United States. Though identified frequently in lynx, development is arrested following formation of the scolex and neck. One intermediate host, the snowshoe hare, has been reported from Alaska (Rausch 1959b). Mule deer (Odocoileus hemionus) are also recognized as suitable intermediate hosts outside of Alaska (Hopla et al. 1965).

Adult tapeworms are $40-100 \mathrm{~cm}$ long and possess a small scolex. Larvae are coenuri approximately $5 \mathrm{~cm}$ in diameter when fully developed with a thin, translucent wall.

\section{Transmission}

Mature tapeworms inhabit the small intestine of canids and shed gravid segments or free eggs in the feces of canines. After ingestion by a suitable intermediate host, oncospheres hatch in the small intestine, penetrate the intestinal mucosa and enter the circulatory system. Oncospheres eventually 
localize in subcutaneous connective tissue, muscle tissue or the thoracic cavity. Transmission to final hosts occurs with ingestion of infected intermediate hosts.

\section{Signs, Symptoms and}

Pathogenesis

Heavy infections of T. serialis in hares prevent or retard annual pelage changes, cause lethargy and occasionally abdominal swelling (Hopla et al. 1965). Granulomas (discreet, nodular, granulated lesions of inflammatory tissue) may appear in the liver and lungs during chronic larval infections due to substances released from the parasites that act as toxins. Larval invasion into subcutaneous or muscle tissue has little pathogenic effect.

\section{Significance}

The prevalence of this parasite in Alaska is low. Rausch and Williamson (1959) suggested the incidence of $T$. serialis in wolves may correlate with hare periodicity since the utiliza$t i o n$ of hares by wolves is most intense during periods approximating peak hare densities.

\section{$\underline{\text { Taenia mustelae }}$}

\section{Introduction}

This organism has been identified in several Alaska arvicolids, i.e. the red-backed vole (Clethrionomys rutilus), brown lemming (Lemmus sibericus) and northern bog lemming (Synaptomys borealis) (Rausch 1957 as reported by Hopla et al. 1968), which serve as intermediate hosts. Larvae of $T$. mustelae have also been found in woodchucks (Marmota monax). Final hosts identified in Alaska include the least weasel (Mustela nivalis), ermine ( $\underline{M}$. erminea), mink (․․ vison) and marten (Martes americaná) (Rausch 1977). Mature worms are 25-103 mm long and consist of 25 to 156 proglottids (Leiby and Dyer 1971). Larval forms vary from cysticerci with a single scolex to coenuri. Larvae are usually $3.5-4.5 \mathrm{~mm}$ in length (Schiller 1953), and monocephalic in arvicoline rodents, but range up to $10 \mathrm{~mm}$ in length with polycephalism apparent in muskrats (Ondatra zibethicus) (Rausch 1977).

\section{$\underline{\text { Significance }}$}

Larvae usually localize in hepatic tissue, but have been found subcutaneously and throughout thoracic and visceral organs. Deer mice (Peromyscus maniculatus) experimentally fed 2 gravid proglottids died within 2 weeks due to severe liver damage and hemorrhage (Fay and Williamson 1962).

\section{$\underline{T a e n i a}$ martis}

Taenia martis was infraspecifically divided into 2 taxa (Wahl 1967 as reported by Rausch 1977); T. m. martes of central Eurasia and $\bar{T}$. $\bar{m}$. americana which is found in Alaska. Taenia m. americana superficially resembles $\bar{s}$. mustelae but is somewhat smalle $\bar{r}$ being only 55-71 mm long and 0.832-1.2 mm wide. Adult tapeworms have been infrequently found in marten ( 3 of $43 ; 7 \%$ ) from Alaska. Larvae were identified in yellow-cheeked voles (Microtus xanthognathus) from the Yukon Territory. Larvae very in size and shape, ranging from globular to elongate. They possess a single invaginated scolex and range from 2-7 $\mathrm{mm}$ in length. The low incidence of 
T. martis americana suggests that this parasite is not a significant mortality source in Alaska for either final or intermediate host. Rausch (1977) provides a detailed description of $\underline{T}$. $m$. americana and distinguishes this parasite from $I$. mustelae and I. twitchelli.

\section{Taenia pisiformis}

\section{Introduction}

Freeman et al. (1966) reported T. pisiformis as the cestode most frequently encountered in coyotes of 0 tario; red fox were also susceptible to infection. It has been reported in arctic fox from Alaska (Thomas and Babero 1956). Strobila are 0.6-2.0 m long and $1.0-4.0 \mathrm{~cm}$ wide. Cysticerci commonly infect snowshoe hares and have been reported in red squirrels (Tamiasciurus hudsonicus) (Dunagan 1957). Though reportedly found in lynx (Soulsby 1968), as would be expected considering the dependence of lynx on hares for food, development of these cestodes does not progress beyond the growth of a scolex and neck. Taenia pisiformis has not been recovered from lynx in Alaska (R. L. Rausch, pers. comm.).

\section{Pathogenesis}

Gravid proglottids or rarely, free eggs are passed in the feces of canids. The eggs are ingested by hares and hatch in the small intestine. Oncospheres penetrate the intestinal mucosa, enter the hepatic circulatory system and migrate to the liver. Approximately 30 days later, cysticerci move out of the liver into the body cavity. The liver may or may not indicate the prevalence of cysticerci. In chronic infections, cysticerci may become encysted on the mesenteries.

Extremely heavy larval infections can cause liver damage and reduce the vitality of hares. Increased amounts of pericardial fluid with inflammation and fibrosis of the liver suggest that chronic toxemia occurs (Hopla et al. 1965). Mature tapeworms are not considered serious pathogens except under heavy infestations, or when the vitality of host animals is reduced by other factors (e.g. starvation), thus increasing the relative pathogenicity of these parasites.

\section{Taenia twitchelli}

\section{Introduction}

Wolverines (Gulo gulo) collected in Alaska during 1949 to 1959 exhibited T. twitchelli infections in 50 of $\overline{8} 0$ (75\%) individuals (Rausch 1959a). Strobila range up to $210 \mathrm{~mm}$ and 151 segments long; pregravid segments were up to $3.0 \mathrm{~mm}$ wide (Rausch 1977). Larvae are found free in the pleural cavities of porcupines (Erethizon dorsatum) (Rausch 1959a, 1977), hoary marmots (Marmota caligata) (Rausch 1977) and tundra voles (Microtus oeconomus). Mature larvae are large (at least $70 \mathrm{~mm}$ long), polycephalic organisms with 20 to 24 (average 16 ) roughly tubular vesicles attached to the posterior portion of the bladder from which they arose (Rausch 1977).

\section{Transmission and}

\section{Pathogenesis}

Rausch (1959b) suggested the mechanisms for host specificity that limit T. twitchelli to wolverines may be more ecologic than physiologic; other mustelids do not extensively prey upon porcupines, hoary marmots or tundra voles, and thus aren't exposed to infective $T$. twitchelli larvae. Although 5 of 5 muskrats (Ondatra zibethicus), 1 of 1 red squirrel (Tamiasciurus hudsonicus), 2 of 7 brown lemmings, 2 of 2 meadow voles and 3 of 5 arctic ground squir- 
rels (Spermophilus parryii) were experimentally infected with gravid proglottids (Rausch 1959a); these species are not readily available to wolverines during winter months. It would seem this ecologic immunity must be at least partially reinforced by some physiologic immunity on the part of other mustelids or some natural factor disrupts egg transmission to these other experimentally proven suitable intermediate hosts to prevent infection of weasels, marten, etc. during summer months.

Experimental infections indicate the pathogenicity of $\mathrm{T}$. twitchelli in intermediate host animals is proportional to the number of larvae present (Rausch 1959a). Pathologic tissue changes in these hosts are limited mainly to the lungs. Rupture of pulmonary tissue with hemorrhage makes the larvae of ${ }^{\text {. }}$ twitchelli more pathogenic than most other Taenia spp.

\section{Incidental Taenia Species}

Adult $T$. polyacantha have been reported $i \bar{n}$ arctic fox, dogs and red fox from Alaska (Fay 1960). Cysticerci have been found in redbacked voles (Rausch and Williamson 1959), tundra voles (Rausch and Williamson 1959) and brown lemmings (Rausch 1957 as reported by Hopla et al. 1965). A significant disparity in the frequency of infection between final and intermediate hosts of this tapeworm on St. Lawrence Island was reported by Fay $(1960)$. He reported that about $85 \%$ of the arctic fox population harbored adult worms while only $0.5 \%$ of the microtine rodents on the island harbored larvae. Transmission and pathogenicity of $\mathrm{T}$. polyacantha is similar to T. pisiformis.

The final and intermediate hosts
of $\frac{\text { Taenia }}{\text { taeniaformis are }}$
canids/felids and rodents (prin-
cipally muskrats), respectively. In

Alaska, only the cysticercus 1 ifestage has been reported from Norway rats (Rattus norvegicus) on Adak Island; mice (Mus spp.) are thought to be suitable intermediate hosts ( $R$. L. Rausch, pers. comm.). Mature tapeworms have been found in wolves from Alberta (Freeman 1956). Strobila are 150-600 mm long and 5-6 $\mathrm{mm}$ wide. The high incidence of infections that are comprised of only 1 to 2 worms suggests that definitive hosts acquire partial immunity to $T$. taeniaformis. Cysticerci possess an evaginated scolex. Tumors have been associated with I. taeniaformis infections in rats.

Taenia rileyi has been identified in Alaskan red squirrels, the intermediate host, and lynx, the final host. Sixty-seven of 113 lynx (59\%) from Alberta and the District of Mackenzie harbored mature T. rileyi (van Zyll de Jong 1966). Cýsticerci exhibit an evaginated scolex.

\section{$\underline{\text { References }}$}

DUNAGAN, T. T. 1957. Studies on the parasites of edible animals of Alaska. Proj. Report No. 7955-4. Arctic Aeromed. Lab. 18 pp.

FAY, F. H. 1960. Carnivorous walrus and some arctic zoonoses. Arctic $13: 111-122$.

FAY, F. H. and F. S. L. WILLIAMSON. 1962. Studies on the helminth fauna of Alaska. XXXIX. Echinococcus multilocularis Leuckart, 1863, and other helminths of foxes on the Pribilof Islands. Can. J. Zool. $40: 767-772$.

FREEMAN, R. S. 1956. Life history studies on Taenia mustelae Gmelin, 1790 and the taxonomy of certain Taenoid cestodes from Mustelidae, Can. J. Zool. 34:219-242.

FREEMAN, R. S., A. ADORJAN and D. H. PIMLOTT. 1961. Cestodes of wolves, coyotes and coyote-dog hybrids in Ontario. Can. J. Zool. 39:527-532. 
HOLMES, J. C. and R. PODESTA. 1968. The helminths of wolves and coyotes from forested regions of Alberta. Can. J. Zool. 46:1193-1204.

HOPLA, C. E., W. L. JELLISON and K. A. NEILAND. 1965. Parasites of Alaskan Vertebrates. Fed. Aid Wildl. Rest. Proj. W-6-R and W-15-R. Univ. Oklahoma Research Inst., Norman, OK. 73 pp.

LEIBY, P. D. and W. G. DYER. 1971. Cyclophyllidean tapeworms of wild carnivora. pp. 174-224. In: Parasitic Diseases of Wild Mammals. J. W. DAVIS and R. C. ANDERSON, eds. lowa State Univ. Press, Ames, IA.

RAUSCH, R. L. 1957. Distribution and specificity of helminths in microtine rodents: evolutionary implications. Evol. 11:361-368.

1959a. Studies on the helminth fauna of Alaska. XXXV. On the identity of certain cestodes (Taeniidae) from foxes. Proc. Helminthol. Soc. Washington $26: 125-131$.

1959b. Studies on the helminth fauna of Alaska. XXXVI. Parasites of the wolverine Gulo gulo L., with observations on the biology of Taenia twitchelli Schwartz, 1924. J. Parasitol. $45: 465-484$.

- 1977. The specific distinction of Taenia twitchelli Schwartz, $192 \overline{4 \text { from }}$ T. martis (Zeder, 1803) (Cestoda: Taeniidae). Instituto Biologia Publicaciones Especiales 4:357-366.

RAUSCH, R. L. and F. S. L. WILLIAMSON. 1959. Studies on the helminth fauna of Alaska. XXXIV. The parasites of wolves, Canis lupus. L. J. Parasitol. 45:395-403.
RITCEY, R. W. and R. Y. EDWARDS . 1958. Parasites and diseases of Wells Gray moose herd. J. Mammal. $39: 139-145$.

SCHILLER, E. L. 1952. Studies on the helminth fauna of Alaska. V. Notes on Adak rats (Rattus norvegicus Berkenhout) with special reference to helminth parasites. J. Mammal. 33:38-49.

1953. Studies on the helminth fauna of Alaska. XV. Some notes on the cysticercus of Taenia polyacantha Leukart, 1856, from a vole (Microtus oeconomus operarius $\mathrm{Nelson})$ J. Parasitol. $39: 344-346$.

SOULSBY, E. J. L. 1968. Taeni idae. pp. 113-134. In: Helminths, Arthropods and Protozoa of Domesticated Animals (Monnig). 6th ed. Williams and Wilkins Co., Baltimore, MD.

SWEATMAN, G. K. and P. J. G. PLUMMER. 1957. The biology and pathology of Taenia hydatigena in domestic and wild hosts. Can. J. Zool. 35:93-109.

THOMAS, L. J. and B. B. BABERO. 1956. Some helminths of mammals from St. Lawrence Island, Alaska, with a discussion on the nomenclature of Echinococcus in voles. J. Parasitol. 42:500.

VAN ZYLL DE JONG, C. G. 1966. Parasites of the Canada lynx, Felis (Lynx) canadensis (Kerr). Can. J. Zool. 44:499-509.

WAHL, S. 1967. Etude parasitoecologique des petis mammiferes (Insectivores et Rongeurs) du val de l'Allondon (Geneve). Rev. Suisse Zool. 74:129-188. 


\section{Echinococcus}

R. Barrett and J. Dau

Two species of Echinococcus occur in Alaska: E. granulosus, the causative agent of cystic hydatid disease and E. multilocularis, the causative agent of alveolar hydatid disease. Mature tapeworms of both species are usually less than $6 \mathrm{~mm}$ long with 2 to 6 proglottids. They inhabit the small intestine of canids under natural conditions.

The larvae are hydatid or alveolar cysts (vesicles containing numerous brood capsules each of which gives rise to several protoscolices; a protoscolex is the juvenile scolex budded within the brood capsule). In natural circumstances, larvae of $\underline{E}$. granulosus occur in cervids; those of E. multilocularis are found in rodents. Each cyst of E. granulosus is unilocular (consisting of a single vesicle) and develops from a single oncosphere ("egg"). Conversely, a single oncosphere of E. mul- tilocularis develops into a "parent" hydatid cyst which subsequently gives rise to "daughter" hydatid cysts by exogenous budding. The descriptive specific name multilocularis (multiple locules) refers to the radially arranged, interconnected hydatid cysts characteristic of the fully developed larval form of this species. Larvae of E. multilocularis are designated as "alveolar" hydatid cysts however, since the term "multilocular" hydatid cysts has been reserved for the anomalous form of $\underline{E}$. granulosus seen in ungulates.

Echinococcus granulosus and $\underline{E}$. multilocularis are well defined by biological and development characteristics as well as ecological predator/prey relationships (Rausch 1968). Rausch (1968) compared the following E. granulosus and E. multilocularis as summarized in Table 4.

TABLE 4

A Comparison of Echinococcus granulosus and

E. multilocularis (Rausch 1968)
E. granulosus
E. multilocularis

Adult tapeworm

Hosts

Strobila length

wol ves-dogs

2-6 mm

cervids (man)

uni locular hydatid cysts

slow--adapted to long-lived

intermediate hosts; proto-

scolices develop after 1-2

years foxes-dogs

$1.2-3.7 \mathrm{~mm}$

rodents (man)

alveolar hydatid cysts

rapid--adapted to short-lived

intermediate hosts;

protoscolices develop after

1-2 months 
In Alaska, the 2 species share no intermediate host (i.e. in which the larval development takes place) other than man and no definitive host (i.e. in which the adult tapeworms develop) other than dogs (Canis familiaris). Both species of Echinococcus are found on mainland Alaska; only $\underline{E}$. multilocularis is present on $\mathrm{St}$. Lawrence Island and other islands of the Bering Sea.

A detailed worldwide distributional history and taxonomic review of Echinococcus have been reported (Rausch 1967a, b). Dispersal of infected dogs and domestic ungulates has been instrumental in spreading Echinococcus spp. into new ecosystems (Rausch 1967a).

\section{Echinococcus granulosus}

\section{Introduction}

Echinococcus granulosus is a relatively common tapeworm of wolves (Canis lupus). Sixty of $200 \quad(30 \%)$ wolves collected throughout Alaska from 1949-1959 were found to harbor E. granulosus (Rausch and Williamson 1959). Other intermediate hosts here include caribou (Rangifer tarandus), black-tailed deer (0docoileus hemionus sitkensis) and mountain goats (Oreamnos americanus) (Rausch and Wilitiamson 1959).

In Alaska, strobilar length ranges from 2.0-6.0 $\mathrm{mm}$ with 2 or occasionally 3 segments (Rausch and Nelson 1963). Only the terminal segment is gravid (contains eggs) and it constitutes the major portion of the strobila (body). Larval (or hydatid) cysts usually are $5-10 \mathrm{~cm}$ in diameter in natural intermediate hosts; each is enclosed in a capsule of hostgenerated fibrous tissue. In man, cysts may reach $50 \mathrm{~cm}$ in diameter (Soulsby 1968). Larvae of E. granulosus can develop with varying degrees of success in mammals other than cervids, but in unsuitable hosts they rarely produce protoscolices and cysts of aberrant form are prevalent (Rausch 1968).

Two biologically and ecologically distinct forms of $\underline{E}$. granulosus exist in North America: an indigenous, sylvatic ("wild") form that parasitizes free-living wolves and cervids and a pastoral ("domestic") form of European origin. The domestic form is essentially restricted to domestic cattle and dogs (Rausch 1967a). Though the 2 forms are largely allopatric (occurring separately), the potential for sympatry (occurring together) in Alaska appears to be increasing as agricultural production of protein and dairy products intensifies near large tracts of wilderness. The effects that dogs will have on melding the identities of these recognized forms remains to be seen.

\section{Transmission}

Figure 4 illustrates life cycles of $\underline{E}$. granulosus. The sylvatic life cycle of this parasite currently is most significant in Alaska. It typically follows the wolf-cervid food chain though coyotes reportedly substitute for wolves when the latter are absent (Holmes and Podesta 1968, Rausch and Williamson 1959). Dogs also are definitive hosts, and may constitute the major source of contamination for humans in Alaska. Dogs fed the viscera of moose and caribou are particularly prone to infection.

Eggs shed in the feces of canids are highly resistant to environmental stress and may remain viable for several months (Leiby and Dyer 1971). Eggs clinging to vegetation are ingested by cervids; the oncospheres are liberated in the small intestine. The oncospheres are transported by the circulatory system mainly to the 


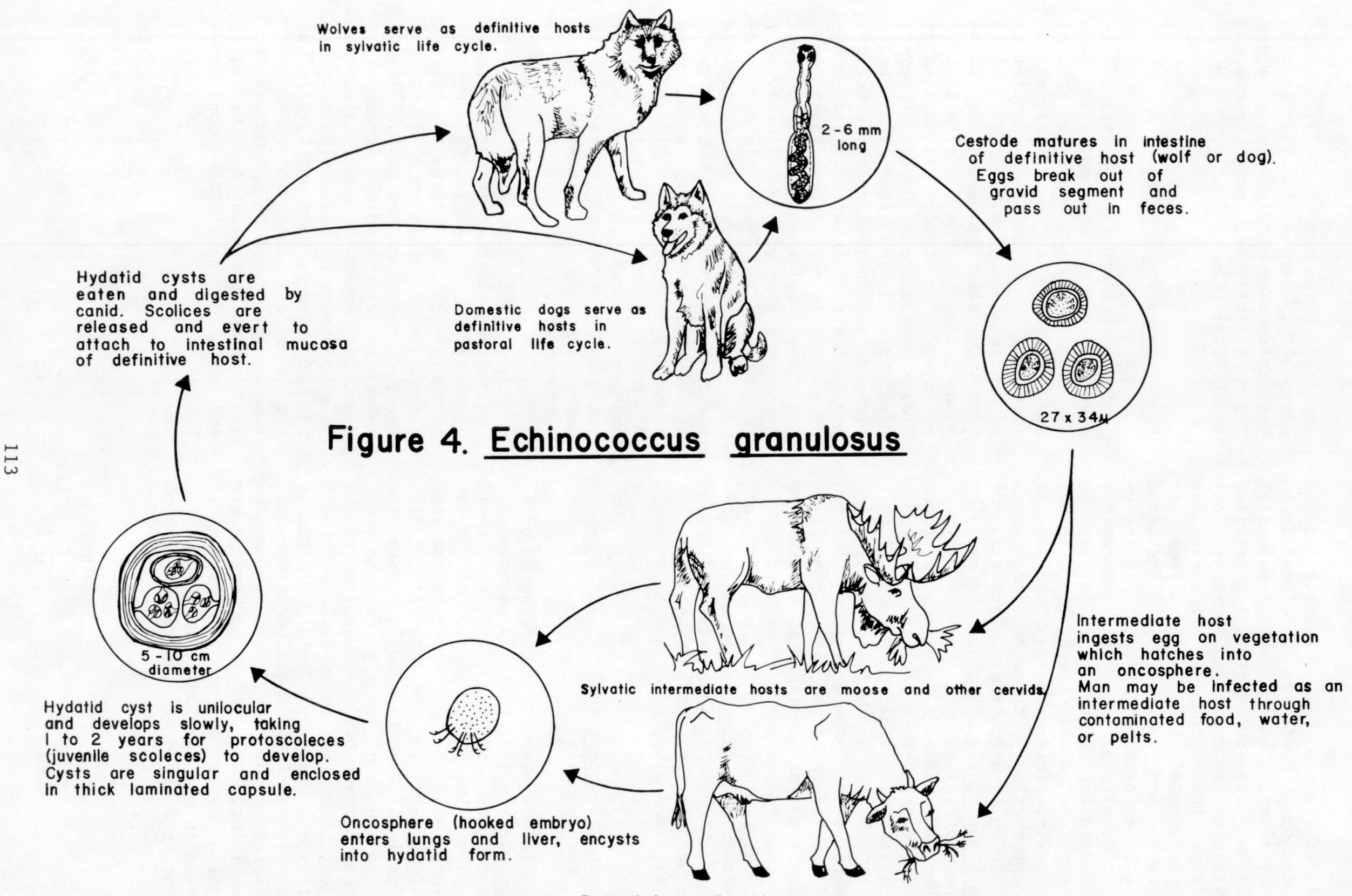

Pastoral intermediate hosts are domestic cattle. 
lungs where the hydatid cysts develop. Cysts may also localize in the liver, though less frequently. Larvae are unilocular, fluid-filled vesicles lined with an inner germinal layer that produces brood capsules approximately 5 months post ingestion (Leiby and Dyer 1971). The brood capsules produce numerous infective protoscolices. Several generations of daughter brood capsules may be contained within the original mother capsule. This capacity for asexual reproduction through endogenous budding enhances the infectivity of Echinococcus spp. larvae and explains how infections comprised of several hundred thousand tapeworms can occur in canids. The prevalence of hydatid cysts in old aged moose (Rausch 1959, Rausch and Williamson 1959) indicates the number of cysts within intermediate hosts is a cumulative function of continuous exposure to eggs. Humans contract hydatid infections by ingesting food or water contaminated by egg-bearing canine feces or through contact with pelts and other fomites (inanimate objects contaminated with infectious organisms).

Signs, Symptoms and Pathogenesis

Infections with mature worms of $E$. granulosus are generally asymptomat $\overline{i c}$ and are not pathogenic to canid hosts. The pathogenicity of larvae in ungulates is dependent on the area of localization and intensity of infection. Most hydatid cysts are found in the lung parenchyma and, less frequently in hepatic tissue just below the capsule. Pathologic tissue changes are mainly limited to displacement of lung/liver tissue and fibrosis of the area surrounding vesicles. Ungulates harboring hydatid cysts in these tissues exhibit no clinical symptoms (if cysts are not very abundant) and remain in good condition (Rausch 1959). Occasionally, larvae localize in kidney, spleen or brain tissue where their effects are more severe and often fatal (Rausch 1952).

\section{Significance and Control}

Though common in both its definitive and intermediate hosts, the low virulence of $\underline{E}$. granulosus in natural hosts reduces its potential as an important wildlife population regulating force. Hydatid disease in humans usually is severe due to the mechanical and toxic effects of vesicles. Infections in humans of Alaska and northern Canada however, reportedly differ from the classic disease form in that most infections are benign (Wilson et al. 1968). Man apparently is a less suitable host for the sylvatic form of $E$. granulosus than of the pastoral form (Wilson et al. 1968). In man, surgical removal of cysts is recommended for pastoral infections whereas cysts of sylvatic origin may allow more conservative treatment. Differential diagnosis between the sylvatic and pastoral forms is based on the patients history of contact with potential wild or domestic animal contaminants.

Rausch (1952) suggested 4 means of controlling E. granulosus in Alaska: 1) education of people having high potential of exposure to hydatid disease (i.e. subsistence villagers) thereby reducing the probability of infection, 2) periodical treatment of dogs to remove the adult tapeworms, 3) development of quarantine programs for domestic animals and 4) elimination of excess dogs in rural villages. The advent of snowmobiles for winter transportation has partially accomplished the latter consideration. 


\section{Echinococcus multilocularis}

\section{Introduction}

Adult tapeworms of $\underline{E}$. multilocularis infect arctic foxes (Alopex lagopus) and to a lesser extent, red foxes (Vulpes vulpes). Dogs and rarely wolves, also harbor adult tapeworms (Rausch and Schiller 1956). In Alaska, E. multilocularis is most frequently found on St. Lawrence and other islands in the Bering Sea and in coastal areas of western and northern Alaska. It is thought to have evolved in Asia and reached Alaska in arctic foxes crossing the pack ice (Rausch and Schiller 1956).

Canids harbor adult tapeworms in the upper third of the small intestine. Strobilar length is $1.2-3.7$ $\mathrm{mm}$ with usually 2 or occasionally 3 to 4 proglottids. Microtine rodents, such as the red-backed vole (Clethrionomys rutilus), brown lemming (Lemmus sibiricus) and tundra vole (Microtus oeconomus) are the principal intermediate hosts (Fay 1973). Alveolar cysts develop from a single embryo and consist of radially arranged subspherical vesicles, usually in the liver. Peripheral vesicles are largest. All vesicles contain brood capsules which generate protoscolices that are infective to foxes. The size of each mass of alveolar cysts varies with the size of the intermediate hosts; severe infections may nearly destroy the liver.

\section{Transmission}

The life cycle of E. multilocularis is diagramed in $\bar{F}$ igure 5 . Transmission from rodents to fox takes place when foxes eat infected rodents. Protoscolices are released within the definitive host by digestive maceration and, to a lesser ex- tent, mastication of the infected rodent. Protoscolices evaginate and attach to the intestinal mucosa of the small intestine where maturation to adult tapeworms is completed in approximately 32 days (Fay 1973). Eggs are passed in gravid segments with feces of the canine. Segments that have been shed expel their eggs through a series of contractions after exposure to the external environment. The eggs are highly resistant to environmental conditions and may be transported by wind, water and insects (Schiller 1954).

Following ingestion of the eggs by a rodent intermediate host, the oncospheres are released in the duodenum by digestive processes. The oncospheres penetrate the intestinal mucosa and reach the liver via the hepatic portal circulatory system. Embryos lodge in the liver parenchyma, usually just beneath the capsule, and develop into primary vesicles in 4 to 12 days depending on the host species. Daughter vesicles subsequently arise through exogenous budding. The number of protoscolices within each alveolar cyst varies but may approach several thousand. Larval development is more rapid for $\underline{E}$. multilocularis than for E. granulosus with infective protoscolices appearing within 2 to 3 months (Rausch 1968). This adaptive feature compensates for the shorter lifespan of rodents than of cervids.

\section{Signs, Symptoms and Pathogenesis}

Mature tapeworms appear to have little pathogenic effect on their final hosts, though infections by 30,000 to 80,000 individuals are common and up to 450,000 tapeworms have been reported from a single fox (Rausch and Schiller 1956). Infections in definitive hosts are self- 


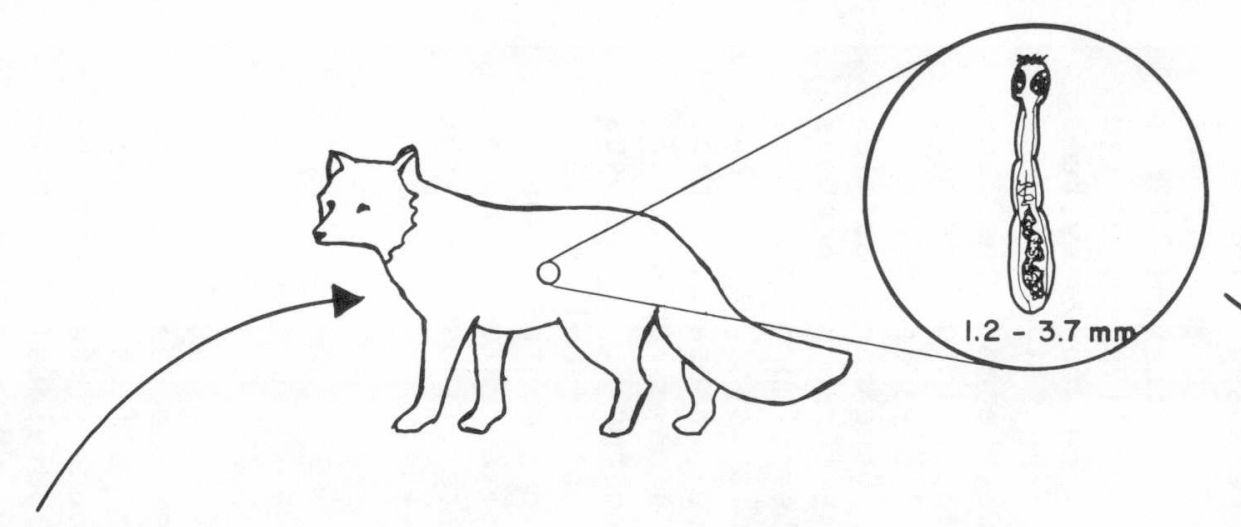

Adult cestode in arctic fox, red fox, and dogs. Eggs from gravid segments are passed in canine feces.

Alveolar cysts in rodent liver

are ingested by canine. Scolices

are released and evaginate to

attach to intestinal mucosa.

Maturation takes about one month.

Figure 5. Echinococcus multilocularis

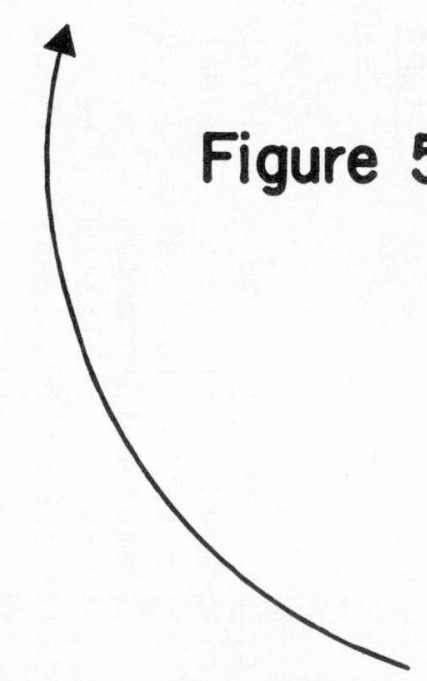

Oncosphere penetrates intestinal mucosa of intermediate host and reaches liver. Embryo develops multiple doughter vesicles by
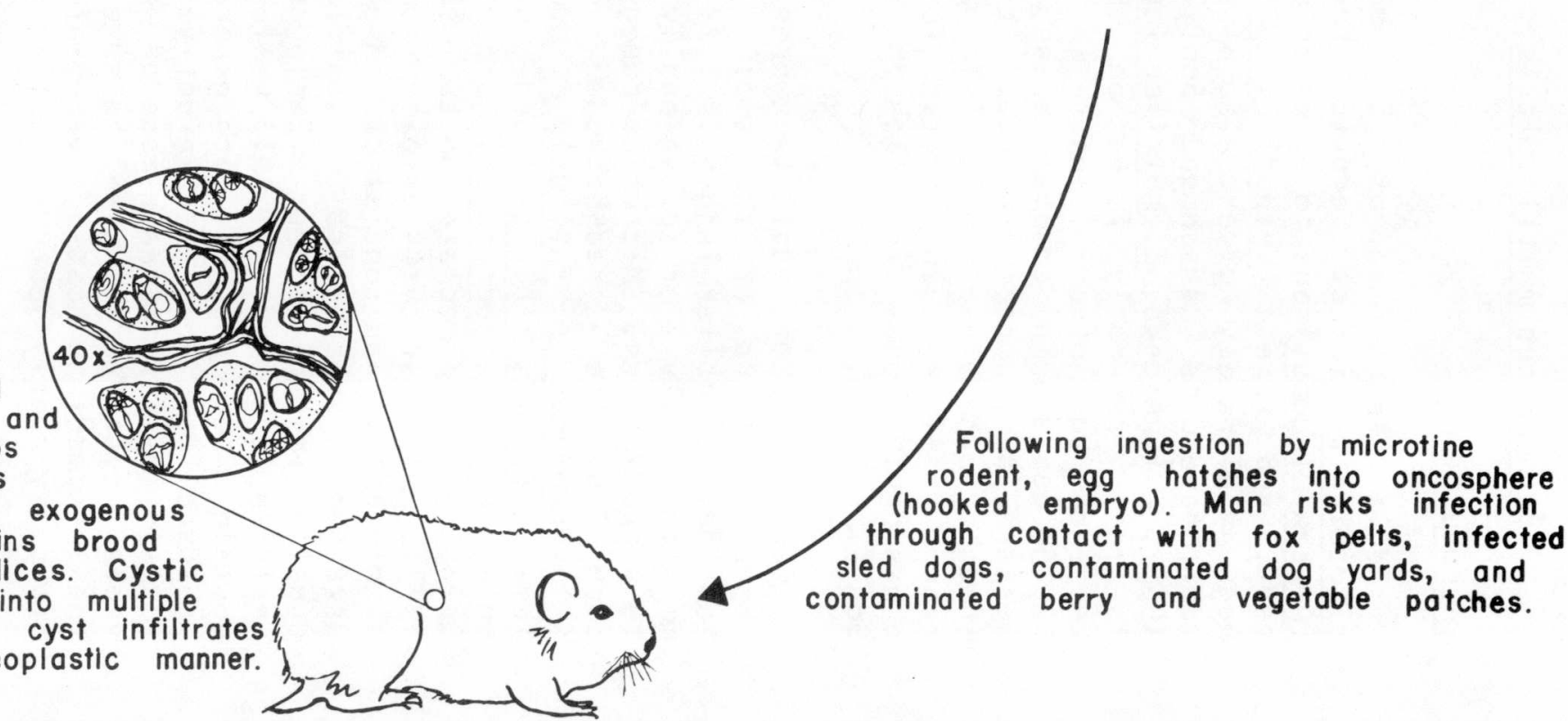

budding. Each vesicle contains brood

chamber becomes subdivided into multiple

vesicles by thin septum, and cyst infiltrates

surrounding host tissue in neoplastlc manner. contaminated berry and vegetable patches. 
limiting as the mature tapeworms live only 3 to 7 months.

Larvae usually are not significant pathogens in their natural intermediate hosts. Alveolar infection in hosts of low suitability, i.e. man, are of ten more severe and may prove fatal when extensive displacement of hepatic tissue impairs the functioning of this organ. In man, larvae often do not complete development, but invade and destroy hepatic tissue continuously. Larval proliferation in man is slow and typically results in central necrosis of the larval mass. Diagnosis is difficult even for experienced clinicians (Rausch 1967a). Surgical removal of the entire larval mass is the only certain means of curative treatment, though is not always possible (Wilson and Rausch 1980). Continuous treatment with mebendazol may be of value in controlling echinococcosis in humans (Wilson and Rausch 1980). Seventy percent of the untreated cases of E. multilocularis in humans were fätal (Wilson and Rausch 1980).

\section{Significance and Control}

The incidence of infection by $E$. multilocularis in foxes on $s \bar{t}$. Lawrence Island during the early to mid-1950s ranged from 40-100\%; for voles, the frequency of larval infection during this same period was 2.0-15.5\% (Rausch and Schiller 1956). The positive correlation between the incidence of E. multilocularis and host abundance suggests the risk of human infection may periodically oscillate with host densities (Fay 1973). The exportation of dogs, rodents and other contaminants (i.e. pelts) from areas where $\underline{E}$. multilocularis is endemic, should be monitored to avoid spreading this parasite into other areas. The 4 control measures cited for $\underline{E}$. granulosis apply equally to E. muTtilocularis.

\section{References}

FAY, F. H. 1973. The ecology of Echinococcus multilocularis Leuckart, 1863, (Cestoda: Taeniidae) on St. Lawrence Island, Alaska. I. Background and rationale. Annal. Parasitol. Hum. Comp. 48:523-542.

HOLMES, J. C. and R. PODESTA. 1968. The helminths of wolves and coyotes from forested regions of Alberta. Can. J. Zool. 46:1193-1204.

LEIBY, P. D. and W. G. DYER. 1971. Echinococcus. pp. 181-188. In: Parasitic Diseases of Wild Mammals. J. W. DAVIS and R. C. ANDERSON, eds. Iowa State Univ. Pres, Ames, IA.

RAUSCH, R. A. 1959. Notes on the prevalence of hydatid disease in Alaskan moose. J. Wildl. Manage. $23: 122-123$.

- 1952. Hydatid disease in boreal regions. Arctic 5:157-174. - 1967a. On the ecology and distribution of Echinococcus spp. (Cestoda: Taeniidae), and characteristics of their development in the intermediate hosts. Annal. Parasitol. Hum. Comp. 42:1-19. - 1967b. A consideration of infraspecific categories in the genus Echinococcus Rudolphi, 1801 (Cestoda: Taeni idae). J. Parasitol. 53:484-491.

1968. Taxonomic characters in the genus Echinococcus (Cestoda: Taeniidae). Bull. Wld. Hlth. Org. 39:1-4.

RAUSCH, R. L. and G. S. NELSON. 1963. A review of the genus Echinococcus Rudolphi, 1801. Ann. Trop. Med. and Parasitol. 57:127-135. 
RAUSCH, R. L. and S. H. RICHARDS. 1971. Observations on parasitehost relationships of Echinococcus multilocularis Leuckart, 1863, in North Dakota. Can. J. Zool. 49:1317-1330.

RAUSCH, R. L. and E. L. SCHILLER. 1956. Studies on the helminth fauna of Alaska. XXV. The ecology and public health significance of Echinococcus sibericensis. Rausch and Schiller, 1954, on St. Lawrence Island. Parasitol. 46:395-419.

RAUSCH, R. L. and F. S. L. WILLIAMSON. 1959. Studies on the helminth fauna of Alaska. XXIV. The parasites of wolves, Canis lupus L. J. Parasitol. 45:395-403.
SCHILLER, E. L. 1954. Studies on the helminth fauna of Alaska. XIX. An experimental study on blowfly (Phormia regina) transmission of hydatid disease. Exper. Parasit. 3:161-166.

SOULSBY, E. J. L. 1968. Taeni idae. pp. 113-134. In: Helminths, Arthropods and Protozoa of Domesticated Animals (Monnig). 6th ed. Williams and Wilkins Co., Baltimore, MD.

WILSON, J. F., A. C. DIDDAMS and R. L. RAUSCH. 1968. Cystic hydatid disease in Alaska. Am. Rev. Resp. Dis. 98:1-15.

WILSON, J. F. and R. L. RAUSCH. 1980. Alveolar hydatid disease. Am. Soc. Trop. Med. Hyg. 29: $1340-1355$.

\title{
Diphyllobothrium
}

\author{
J. Dau and R. Barrett
}

\section{Introduction}

Six species of the pseudophyllidean (see Cestode introduction) cestode Diphyllobothrium reportedly occur in terrestrial Alaskan mammals (Rausch et al. 1967). These are: 1) D. latrum, 2) D. dendriticum, 3) D. Tanceolatum, 4) ㅁ. ursi, 5) ‥ daTliae and 6) D. alascense. other Diphyllobothrium spp. (e.g. D. cordatum and D. pacificium) are known to mainly infect marine mammals. Diphyllobothriid infections are prevalent in areas where vertebrates ingest large quantities of fish on a year round or seasonal basis. In man, the connotation "fish tapeworm" is used to describe infections by Diphyllobothrium.

\footnotetext{
Diphyllobothrium spp. are generally large and yellowish with
}

visible dark uterine areas apparent on proglottids. The neck is usually present, though decreases in length with age for at least some species (Rausch 1954). Scolices are spatula shaped and possess 2 bothria (dorsal or ventral grooves on the scolex of pseudophyllideans roughly analogous to cyclophyllidean suckers).

Diphyl lobothrium

dendriticum reportedly occurs across the greatest geographic range, and in the largest variety of host species in Alaska (Rausch et al. 1967). Diphyllobothrium latum has been identified as the primary diphyllobothriid cestode of humans, its preferred final host (Rausch et al. 1967). Table 5 lists those wildlife species known to harbor larval or adult diphyllobothriid tapeworms in Alaska, and maximum strobilar length and 
Naturally occurring Diphyllobothrium infections reported in Alaska

(From Rausch 1970 except where noted)

1st Intermediate Host 2nd Intermediate Host

Definitive Host

Max. Length $(\mathrm{mm}) \times$ Width $(\mathrm{mm})$

D. latum 1. Diaptomys gracilis

3. Cyclops scutifer

D. dendriticum 1. Cyclops spp.* (Smyth 1962)

2. Diaptomus $\mathrm{spp}$.

D. Ianceolatum 1. Copepod spp.

D. ursi

1. Copepod spp.

D. $\underline{\text { dalliae }}$

1. Copepod spp.

D. alascense 1. Copepod spp. 1. burbot

1. not established

2. burbot (Lota lota)* in Alaska

3. northern pike (Esox lucius)*

1. Salmonidae

2. sticklebacks (Gasterosteus aculeatus $\varepsilon$ Pungitius pungitius)

1. whitefish (Corregonus sardinella)

1. man (Homo sapiens)

2. red fox (Vulpes vulpes)

- arctic fox (Alopex lagopus)

1. black-headed gull

(Larus ridibundus)

2. man

1. bearded seal (Erig-

nathus barbatus)

2. harbor seal (Phoca

vitulina)

3. $\operatorname{dog}($ Canis familiaris)

- man (single

plerocercoid)

1. brown bear (Ursus

2. arctos

2. black bear ( $\underline{u}$.

americanus)

$11,000 \times 23$

coho salmon (o.

kisutch)* (Smyth

1962)

3. man

(Rausch 1954)

perhaps other Oncor-

1. blackfish (Dallia pectoralis)

1. man

3. aretic fox

$522 \times 9$

4. gulls (Larus spp.)

2. (possible) cod

(Eleginus gracilis

3. (possible) sculpin

(Myoxocephalus quad-

1. dogs

2. harbor seal

$730 \times 9$

$1380 \times 9$

$1560-11,3000 \times 8-14$

not reported from Alaska

*Genera or species occurring in, but infection not reported from, Alaska

**Negative results for the presence of pierocoids in fish from Karluk Lake (Kodiak Island)

and the lower Kuskokwim River were reported for dolly varden (Salvelinius malma), coho

salmon, rainbow trout (Salmo gairdineri), sculpins (Cottus spp.), sheefish (Stenodus

leucichthys) and northern pike (Esox lucius). 
width for each Diphyllobothrium spp. Morphological characteristics of Diphyllobothrium spp. found in Alaska have been described in detail for differential identification (Rausch and $\mathrm{Hilli}$ ard 1970).

\section{Transmission}

The life cycle of Diphyllobothrium spp. is illustrated in Figure 6. Mature worms inhabit the upper third of the small intestine in their final hosts. Eggs are usually released from the uterine pore and passed free in the feces of the final host. Single or lengths of gravid segments may be passed in the feces as well. The eggs develop in several weeks after contacting water before they hatch into the first larval stage coracidia (see Cestode introduction). Each coracidium consists of an oncosphere and a ciliated embryophore (the outer cellular covering of the embryo). Coracidia swim slowly in the water column and rapidly die if not ingested by a suitable freeswimming copepod (a small aquatic crustacean). Following ingestion by the copepod, coracidia shed the embryophore and, in 2 to 3 weeks, develop into the second larval procercoid stage. When suitable piscine hosts ingest infected copepods, digestive processes free the procercoids and allow them to penetrate the intestinal wall of the fish. The procercoids then migrate to muscle tissue or visceral organs and develop into plerocercoids (elongate, solid organisms with scolices resembling the mature form). Plerocercoids may persist through the piscine predator/prey trophic system from planktivorous forage fish to carnivorous fish. Plerocercoids of some Diphyllobothrium spp. are found in both freshwater and marine fish species where trophic relationships bridge these ecosystems. Plerocercoids tend to re-encapsulate in the vicinity of the gut in paratenic, predator host fish (Baer 1951).

Definitive mammalian hosts are infected by ingesting raw fish that harbor plerocercoids. By the time digestive processes release the plerocercoids, they have reached the posterior portion of the intestinal tract, so must migrate anteriorly to their preferred area of attachment (Anderson 1978). In ursids, this is approximately $2 \mathrm{~m}$ below the duodenum (Rausch 1954). Plerocercoids of $D$. latum require roughly 2 days to shed their entire larval body prior to formation of proglottids (Anderson 1978). The result is a temporary net biomass loss for this cestode species soon after infection of the definitive host.

Strobilar growth is extremely rapid. A black bear Ursus americanus) experimentally infected with D. ursi plerocercoids exhibited tapeworms up to $960 \mathrm{~mm}$ long and comprised of 620 segments only 10 days post infection (Rausch 1954). In brown bears (Ursus arctos), strobilar growth of $D$. ursi may occur more through increased proglottid size than increased segment numbers. Severe infections are thought to impede the growth of these cestodes.

The intensity of Diphyllobothrium infections varies seasonally for bears and other mammals which undergo hibernation or dormancy (Rausch 1954, Rush 1932). The mechanism causing these variations is thought to be associated with dietary changes that precede denning. Gulls (Laridae) subjected to improper feeding have been observed to spontaneously lose cestode infections (Kuhlow 1953 as reported by Rausch 1954) and marmots have been reported to expel adult cestodes of other genera before hibernation (Dubinin and Leshkovich 1937) as reported by Rausch 1954). Rauschs' (1954) observations and com- 


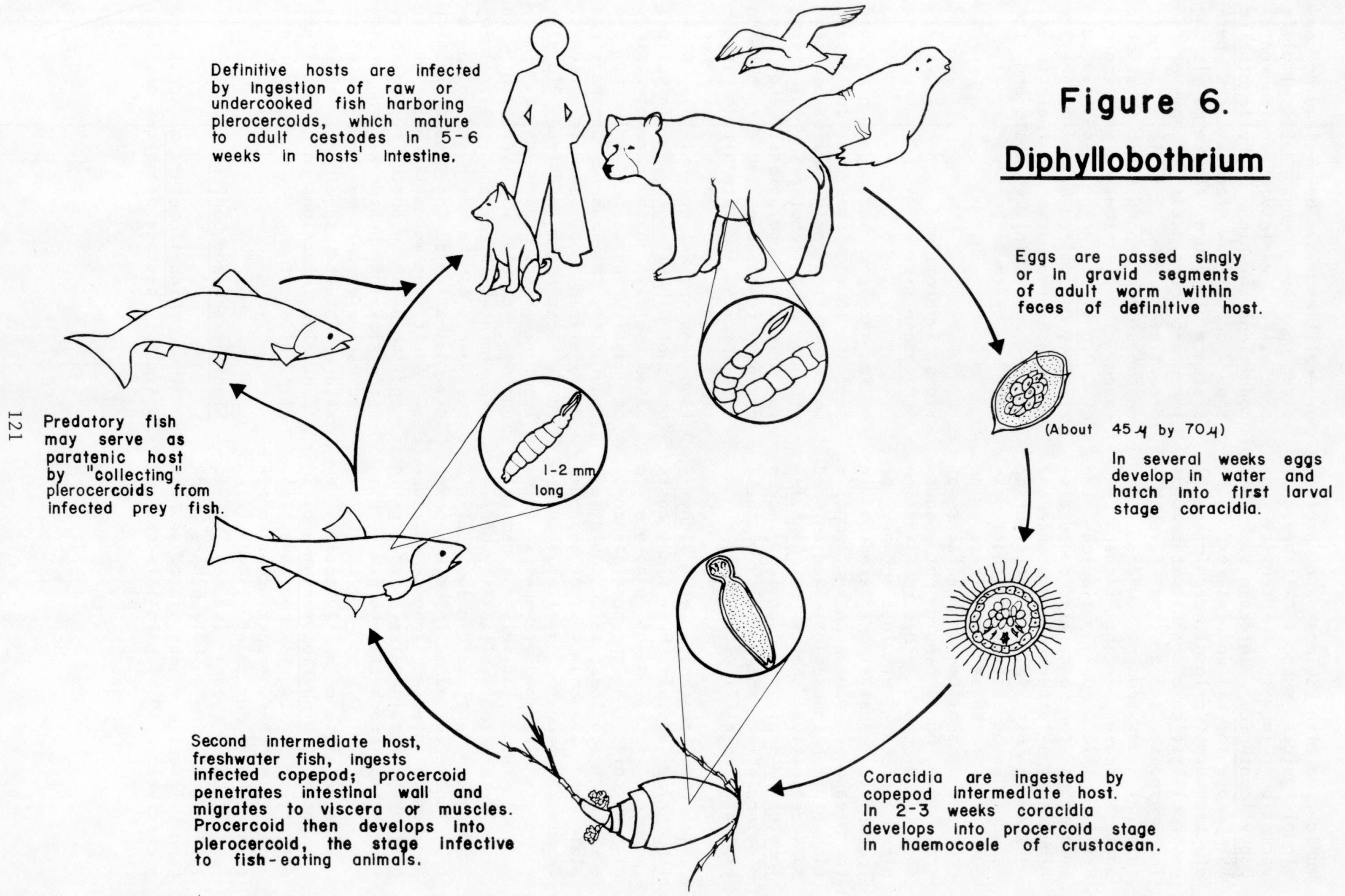


munication with Eskimos and professional guides support this seasonal variation in the intensity of Diphyllobothrium infections. This may be due to: 1) complete expulsion of tapeworms prior to denning or 2) destrobilization of adult tapeworms in response to a less favorable internal environment when host animals cease feeding (Rausch and Hilliard 1970). Only meticulous examinations for parasites could distinguish the latter circumstance and ensure against falsely attributing reduced cestode activity to host expulsion of these parasites. The presence of scolices in the feces of definitive hosts would not necessarily disprove destrobilization since scolex elimination may be incomplete. Spatial differences in the seasonal abundance and composition of fish species utilized as food by definitive hosts of Diphyllobothrium spp., i.e. ursids, makes distinguishing the validity of these explanations difficult.

If the presence of Diphyllobothrium spp. in their final hosts is more a function of exposure to plerocercoids than the persistence of adult worm scolices, this could explain the seasonal loss of cestodes in Alaska where the availability of secondary intermediate hosts (i.e. salmon) is relatively ephemeral. Areas where the availability of fish is more constant could allow definitive hosts to become infected sooner and remain infected longer as suggested by fecal analysis for eggs in such areas (Frechette and Rau 1978).

The persistence of infections by tapeworms of Diphyllobothrium spp. in definitive hosts is controversial. Some researchers report that infections tend to be short lived (i.e. less than 1 year) (Rausch et al. 1967, Wardle 1935) while others contend infections may last several years (Leiper 1936). In this regard, generalities may be misleading considering the number of wildlife species and variety of environmental conditions in which Diphyllobothrium spp. occur in Alaska. The persistence of mature tapeworms is an important consideration however, in determining how rapidly these cestodes can expand their geographic range. The distribution and spread of Diphyllobothrium spp. is ultimately determined by the longevity of these tapeworms in their intermediate and definitive hosts and the mobility of these host species.

\section{Signs, Symptoms and \\ Pathogenes is}

The presence of eggs or gravid segments in the feces of definitive hosts is the only means of identifying diphyllobothriid infections in wildlife populations. Egg morphology reportedly varies between host species, the intensity of infections and with individual worms (Anderson and Halvorsen 1978). Diagnosis of Diphyllobothrium infections based solely on the characteristics of eggs is thus useful only at the population level where many eggs can be analyzed for specific trends. Identification of individual Diphyllobothrium sp. based on egg morphology should be verified with experimental infections. Anthelmintic agents can be administered to individual animals for identification of adult tapeworms if retention and handling of these hosts is feasible. Necropsies of hunter-killed ursids and marine mammals may be employed to secure adult specimens. Diphyllobothriid infections do not manifest clinical symptoms.

\section{Significance and Control}

Though Diphyllobothrium spp. are prevalent cestode parasites of $\mathrm{fish}$, 
marine and terrestrial mammal species including man, their significance as a population regulating force in Alaska has not been established. The relative pathogenicity of this parasite in individual hosts depends on host health and nutrient availability. The ??rocess of inducing cestode expulsion in humans with anthelmintic agents has been described (Rausch and Hilliard 1970). Control in wildlife populations is unfeasible.

\section{References}

ANDERSON, K. 1978. The development of the tapeworm Diphyllobothrium latum (L. 1756) (Cestoda; Pseudophyllidea) in its definitive hosts, with special references to the growth patterns of $\underline{D}$. dendriticum (Nitsch, 1824) and D. ditremum (Creplin, 1827). Parasitol. 77:111-120.

ANDERSON, K. and O. HALVORSEN. 1978. Egg size and form as taxonomic criteria in Diphyllobothrium (Cestoda, Parasitol. 76:229-240.

BAER, J. G. 1951. Cestoda. pp. 139-152. In: Ecology of Animal Parasites. Univ. I11inois Press, Urbana, IL.

DUBININ, V. B. and L. I. LESHKOVICH. 1945. Zhirovye rezervy tarbaganov i ikh zarazhennost' askaridami pred vpadenium $v$ spiachku. Zool Zhur. 24:373-378.

FRECHETTE, J. L. and M. E. RAU. 1978. Seasonal changes in the prevalence of ova of Diphyllobothrium ursi and Baylisascaris transfuga in the feces of the black bear (Ursus americanus). J. Wildl. Dis. $14: 342-344$.
KUHLOW, F. 1953. Ueber die Entwicklung and Anatomie von Diphyllobothrium dendriticum Nitzsch 1824. Zeitschr. Parasitenk. $16: 1-35$.

LEIPER, R. T. 1936. Some experiments and observations on the longevity of Diphyllobothrium infections. J. Helminthol. $14: 127-130$.

RAUSCH, R. 1954. Studies on the helminth fauna of Alaska. XXI. Taxonomy, morphological variation, and ecology of Diphyllobothrium ursi n. sp. provis. on Kodiak Island. J. Parasitol. 40:540-563.

- 1955. Unusual pathogenicity of Diphyllobothrium sp. in a black bear. Proc. Helminthol. Soc. Washington 22:95-97.

RAUSCH, R. and D. K. HILLARD. 1970. Studies on the helminth fauna of

Alaska. XLIX. The occurrence of Diphyllobothrium latum (Linnaeus, 1758) (Cestoda: diphyllobothriidae) in Alaska, with notes on other species. Can. J. Zool. 48:1201-1219.

RAUSCH, R., E. M. SCOTT and V. R. RAUSCH. 1967. Helminths in eskimos in western Alaska, with particular reference to Diphyllobothrium infection and anaemia. Trans. Soc. Trop. Med. Hyg. 61:351-357.

RUSH, W. M. 1932. Diphyllobothrium latum in bear. J. Mammal. 13:274-275.

SMYTH, J. D. 1962. Diphyllobothrium. pp. 239-246. In: Introduction to Animal Parasitology. Charles C. Thomas, Publisher. Springfield, IL.

WARDLE, R. A. 1935. Fish-tapeworm. Biol. Board Can., Ottawa, ONT. 25 pp. 


\title{
Nematoda
}

\author{
R. Barrett and J. Dau
}

The large physical size of mature parasitic roundworms (relative to protozoan parasites), and their tendency to occur in large numbers within their hosts makes these organisms highly visible in most mammals. Thus, in spite of the relatively low species diversity of parasitic nematodes in Alaska, they frequently come to the attention of sportsmen and biologists.

Nematodes exhibit long, cylindrical bodies which taper at both ends. Cuticular annulations (indented rings that circumscribe the body) may give the false impression that nematodes are segmented; they are not. Hooks and thickened areas of the cuticle may be apparent. Though the cuticle may absorb certain nutrients, as well as some anthelmintic agents, ingestion occurs through a primitive alimentary tract. Nematodes are monoecious (separate sexes); females are larger than males.

Unlike cestodes, larval nematodes resemble adults in general body configuration. Larval nematodes usually undergo 4 ecdyses (molts) of the entire cuticle before they reach maturity. The interval between molts consists of 2 phases: 1) an active phase of feeding and growth and 2) a lethargic stage during which the body undergoes structural changes in preparation for the molt.

Nematode life cycles follow a variety of patterns that may or may not employ an intermediate host. Parasitic nematodes usually infect their final hosts while in the third larval stage. The 2 molts that precede the third larval stage may occur within the egg or after hatching. Those species whose larvae temporarily live free in the external environment often retain the cuticle(s) of previous larval stages for protective purposes. These cuticles are shed after the larvae enter the host.

For nematode species that do not utilize an intermediate host, host animals often become infected by ingesting free-living third stage larvae (i.e. Haemonchus contortus). The absence of an intermediate host does not limit these nematodes to this single route of infection however. Infective larvae of Trichinella organisms are ingested with meat in which they are encysted. Third stage Uncinaria lucasi larvae may penetrate the skin of their hosts. Infective Toxascaris leonina larvae remain within their eggs and hatch only after being ingested by their host. These examples show that it is difficult to make generalizations concerning routes of transmission for parasitic nematodes that do not employ an intermediate host.

Parasitic nematodes that utilize an intermediate host follow 1 of 2 life cycle routes. The most common life cycle route entails ingestion of eggs by the intermediate host, usually a rodent. The eggs hatch and eventually, third stage larvae encapsulate in some anatomical region of the rodent. Final hosts become infected by ingesting rodents that contain third stage larvae.

Filarioid (order Filariata) nematodes (i.e. Splendidofilaria spp., see avian disease chapter, or Dirofilaria spp.) complete their life 
cycle without entering the external environment. Larval forms of these species are termed microfilariae; they enter the circulatory system of their final hosts. Blood-sucking arthropods, i.e. mosquitos (Culex spp.) ingest the microfilariae with blood. These larvae develop within the arthropods to a stage that is infective for the final hosts. Infective microfilariae leave the arthropods when the insects land to ingest blood. Microfilariae enter the circulatory system of the final host, migrate to some anatomical site and mature.

Pathologic effects of nematode infections may result from mechanical destruction of host tissue while lar- vae migrate throughout these animals or from the release of toxic substances (i.e. waste products). Thorough descriptions of nematodes are provided by Meyer and 01 sen (1971) and Soulsby (1968).

\section{$\underline{\text { References }}$}

MEYER, M. C. and 0. W. OLSEN. 1971. Essentials of Parasitology. William C. Brown Co., Pub. Dubuque, IA. 305 pp.

SOULSBY, E. J. L. 1968. Nematodes. pp. 142-150. In: Helminths, Artropods and Protozoa of Domesticated Animals (Monnig). 6th ed. Williams and Wilkins Co., Baltimore, MD.

\title{
Baylisascaris
}

\author{
J. Dau and R. Barrett
}

\section{Introduction}

Four species of Baylisascaris occur in Alaska, these are: 1) B. (Ascaris) devosi from wolverines (Gulo gulo) (Rausch 1959, Sprent 1968), mink (Mustela vison) (Sprent 1953) and ermine (M. erminea) (Dunagan 1957, Sprent $1 \overline{9} 68) ; 2$ ) B. (Ascaris) ' columnaris from wolverines (Dunagan 1957); 3) B. (Ascaris) láevis (tarbagan) from woodchucks (Marmota monax), hoary marmots (M. caligata) (Rausch 1951, Sprent 1968), Alaska marmots (M. broweri) (Sprent 1968 ) and arctic ground squirrels (Spermophilus parryii) (Rausch 1951, Sprent 1968); and 4) B. toxascaris transfuga from brown bears (Ursus arctos) (Sprent 1968) and black bears (U. americana) (Rausch 1961). Baylisascaris devosi occurs in marten (Martes americana) (Sprent 1968), but has not been documented in Alaska. Parasites of the genus Baylisascaris occur throughout Alaska.
Fifty-six of 91 free-ranging black bears (U. americanus) were reported to harbor B. transfuga in the small or large intestine (Dies 1979). Black bear cubs in Alaska often become infected with B. transfuga during their first summer and infections are particularly evident in late summer and early fall (Rausch 1961). Baylisascaris devosi was the helminth most frequently encountered in a parasitological survey in the Mackenzie District of the Northwest Territories (29 of 39; 74\%) and is common in wolverines of Alaska (Rausch 1959).

Sprent (1968) provides a table of features for differentiating the species of Baylisascaris and a key for identifying the genera within the family Ascarididae. 
Transmission

The life cycle and body lengths described herein are for $B$. devosi. They were determined experimentally utilizing laboratory mice (Mus sp.), ferrets (Mustela sp.) and marten as reported by Sprent (1953). Life cycles of other Baylisascaris spp. generally follow this description.

Eggs pass freely in the feces of the definitive host. Development to the second larval stage progresses within the eggs; these larvae become infective while within the eggs when about 12 days old. When eggs that contain infective larvae are ingested by a rodent, they hatch and release second stage larvae in the alimentary canal. These larvae pass through the stomach into the intestines. The second stage larvae penetrate the intestinal wall and reach the liver, mesenteries and lungs presumably via the circulatory system. The larvae then lodge in the lung parenchyma and molt to the third larval stage roughly 10 days post ingestion. Third stage larvae are 1.2-1.9 mm long; they tend to migrate anteriorly and localize in and around the head. Third stage larvae are usually encapsulated in host generated connective tissue.

Definitive hosts, i.e. mustelids, are infected by ingesting rodents that harbor infective third stage larvae. The third stage larvae are released from their intermediate hosts by digestive processes in the stomach and small intestine of the final host. Baylisascaris spp. complete their development in the small intestine of the final hosts. Male and female B. devosi roundworms recovered from infections 6 months old were 110-120 mm and 188-238 mm long respectively.

Definitive hosts may develop infections by ingesting eggs and thus bypass the intermediate rodent hosts. Young mustelids are more susceptible to infection by third stage larvae than older mustelids. Fourth stage larvae can infect adult mustelids that have developed a resistance to third stage larvae (Sprent 1953).

Seasonal changes in the incidence of B. transfuga and the empirical observations of black bears passing adult worms in their feces just prior to denning (Rogers 1975) support the theory that bears lose helminth infections at this time (Rausch 1961).

\section{Signs, Symptoms and \\ Pathogenesis}

Light infections are asymptomatic. The mobility of third stage larvae and their prediliction for lung tissue may cause edema (an accumulation of interstitial fluids) and pneumonitis (an inflammation of the lungs) in their intermediate hosts. This is evidenced by dyspnea (labored breathing) and lethargy. Larvae that exit capillaries in the lungs, liver, kidneys and intestines cause petechial (pinhead) hemorrhages. Adult worms may compete with their final hosts for dissolved nutrients and in severe infections, cause intestinal blockage. Anthelmintic agents are effective against adult worms but not larvae. Control of Baylisascaris spp. in wildlife populations is not feasible considering the difficulty in administering anthelmintic drugs, and the persistence of eggs in the external environment.

\section{References}

ADDISON, E. M. and B. BOLES. 1978. Helminth parasites of wolverine, Gulo gulo, from the District of Mackenzie, Northwest Territories. Can. J. Zool. 56:2241-2242. 
ADDISON, E. M., M. J. PYBUS and H. J. RIETVELD. 1978. Helminth and arthropod parasites of black bear, Ursus americanus, in central Ontario. Can. J. Zool. $56: 2122-2126$.

DIES, K. H. 1979. Helminths recovered from black bears in the Peace River region of Northwestern Alberta. J. Wildl. Dis. 15:49-50. DUNAGAN, T. T. 1957. Studies on the parasites of edible animals of Alaska. Proj. Report No. 7955-4. Arctic Aeromed. Lab. 18 pp.

PHILIP, C. B. 1938. A parasitological reconnaissance in Alaska with particular reference to varying hares. 11. Parasitological data. J. Parasitol. 24:483-488.

RAUSCH, R. 1951. Biotic interrelationships of helminth parasitism. Pub. Hlth. Probs. Alaska 66:18-24.
1959. studies on the helminth fauna of Alaska. XXXVI. Parasites of the wolverine, Gulo gulo L., with observations on the biology of Taenia twitchelli Schwartz, 1924. J. Parasitol. $45: 465-484$.

- 1961. Notes on the black bear, Ursus americanus Pallas, in Alaska, with particular reference to dentition and growth. $Z$. Saeugetierkd. 26:77-107.

ROGERS, L. C. 1975. Parasites of black bears of the Lake Superior region. J. Wildl. Dis. $11: 189-192$.

SPRENT, J. F. A. 1953. On the life history of Ascaris devosi and its development in the white mouse and the domestic ferret. Parasitol. 42:244-258.

- 1968. Notes on Ascaris and Toxascaris, with a definition of Baylisascaris gen. nov. Parasitol. 58: 185-198. 


\section{Toxascaris a Toxocara}

J. Dau

Adult worms of these genera exhibit lateral cuticular expansions (cervical alae) located anteriorly that give them an arrow-like body configuration (hence, "arrow worms"). Since worms from species of both genera overlap in size, affect common definitive hosts and manifest similar clinical symptoms, distinguishing between these genera in the field is difficult. Tentative diagnoses may be based in part on the host species from which parasites were identified. Also, Toxascaris spp. tend to flex dorsallym Toxocara spp. ventrally; Toxocari cati has short, wide cervical alae, Toxocari canis possesses long, narrow cervical alae (Schmidt and Roberts 1977). Definitive diagnoses should be based on analysis of egg and life cycle characteristics.

\section{$\underline{\text { Toxascaris leonina }}$}

In Alaska, Toxascaris leonina has been found in black bear (Ursus americanus) (Dubey 1978), lynx ( lynx) (Dubey 1978), arctic fox (Alopex lagopus) (Fay and Williamson 1962, Thomas and Babero 1956) and wolves (Canis lupus) (Rausch and Williamson 1959). It is a common parasite of canids. Four of 5 arctic fox $(80 \%)$ collected in 1961 on St. George Island (Pribilof Islands) and 144 of 200 wolves (72\%) collected throughout Alaska during 1949 to 1959 harbored immature and adult $T$. leonina (Fay and Williamson 196⿸厂 Rausch and Williamson 1959). Infections in wolves averaged 50-60 worms per wolf with up to 175 individuals within a single animal (Rausch and Williamson 1959).

Adult worms are $7-10 \mathrm{~cm}$ long and usually inhabit the small intestine of definitive hosts. Toxascaris leonina may complete its life cycle without an intermediate host. Larvae molt to the infective, second larval stage within the eggs 3 to 6 days post expulsion from the definitive host under optimum conditions. Following ingestion by the definitive host, the second stage larvae hatch and invade the intestinal wall where they remain for 10 to 14 days. The larvae then molt to the third larval stage and return to the intestinal lumen. By 3 to 5 weeks post ingestion, the larvae have molted to the fourth larval stage and grown to approximately $8 \mathrm{~mm}$ long. The worms mature in roughly 6 weeks and begin to produce eggs about 74 days post ingestion (Soulsby 1968). Wolf pups probably contract I. leonina infections at an early age through contact with contaminated feces of infected adults near den sites (Rausch and Williamson 1959).

Small rodents may act as intermediate hosts of T. leonina. In contrast to the sedentary behavior of larvae in their definitive hosts, larvae that infect intermediate rodent hosts migrate throughout the body. Larval growth is arrested in mice after reaching the third larval stage. Definitive hosts are infected by ingesting mice that harbor the third stage larvae.

Toxascaris leonina man;ifests no clinical symptoms. It is not considered a significant wildlife pathogen.

\section{Toxocara}

Two species of Toxocara have been reported from Alaska: 1) I. canis, a 


\section{Figure 7. Common intestinal nematodes in Alaska.}

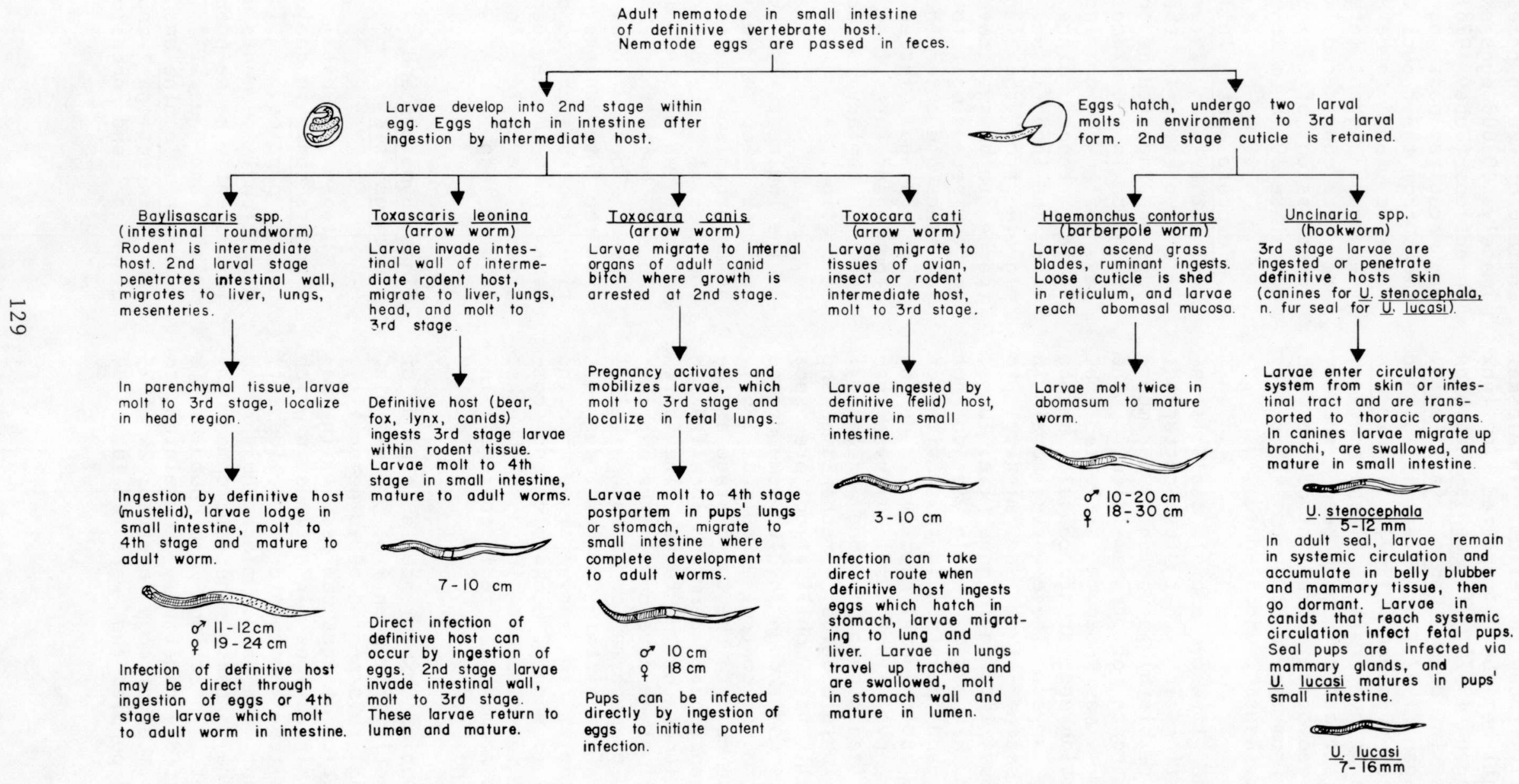


parasite of canids and 2) T. cati, usually found in felids. In Alaska, T. cati has been reported in lynx Tounagan 1957); T. canis has been found in dogs ( Canis familiaris) (Dunagan 1957, Schiller 1952) and wolves (Dunagan 1957). Toxocara canis is very rare in Alaska however, and is thought to have been recently (in geological time) introduced with dogs from the contiguous 48 states (R. L. Rausch, unpub. data).

The life cycle of T. canis is interesting in that pre-parturient (before birth) infection is an important route of transmission. The bitch may become infected by ingesting eggs that contain second stage larvae. These larvae remain in the second stage and migrate to various internal organs (i.e. lungs, liver, kidneys). The larvae do not grow and persist in these sites of localization until reactivated during pregnancy. Not all larvae are mobilized during any one pregnancy; the physiological mechanisms and stimuli for mobilization are not understood though involve some degree of hormonal control (Soulsby 1968). larvae molt to the third stage as the pregnancy nears term and localize in the lungs of the fetus. The fourth larval stage develops in the puppies' lung parenchyma or stomach within 1 week after birth. The nematodes molt to the fifth larval stage when the puppy is about 2 weeks old and complete development in 3 to 4 weeks. Adult worms localize in the small intestine.

Puppies experience a period of susceptibility when 1 or 3 weeks old. During this period of vulnerability, ingestion of eggs can result in patent infections (infections in which parasites complete development) without the larvae migrating through internal organs. The development of patent infections in puppies is reportedly negatively correlated to the number of eggs ingested; 24 of 25 (96\%) puppies that were fed 10 to 100 eggs developed patent infections while 0 of 20 puppies fed approximately 10,000 eggs exhibited patent infections (Dubey 1978). Female dogs may acquire post parturient infections from their pups though such infections tend to be eliminated in a few weeks. Rodent intermediate hosts are not considered important in the transmission of T. canis. Mature worms are $10-18 \mathrm{~cm}^{-}$long.

Toxocara cati follows direct and indirect (via an intermediate host) life cycles. In the direct route, eggs are ingested by the final host and hatch in the stomach. Three to 5 days after being ingested by the felid, second stage larvae reach liver and lung tissue. These larvae migrate from the lungs and up the trachea to be swallowed. They undergo the second molt to the third larval stage and enter the stomach wall. Third stage larvae re-enter the stomach and intestinal lumen, molt to the fourth larval stage and complete maturation.

The indirect life cycle of $T$. cat $\mathrm{i}$ utilizes mice as well as insect and avian organisms as intermediate hosts for larval development. Felids are infected when they ingest intermediate hosts that contain infective larvae. These larvae are released from the intermediate hosts by digestive processes and complete development in the manner described for the direct life cycle. Adult worms are 3-10 cm long.

Signs, Symptoms and Pathogenesis

Intense prenatal infections of Toxocara canis in canids may be fatal for puppies. The extensive migrational movements of larvae may infrequently cause pneumonia, extreme malaise and nausea. Toxocara cati, Toxascaris leonina and light Toxocara canis infections cause recurrent diarrhea and occasionally anemia. 
Such animals exhibit reduced vigor, a dull harsh pelage and a pot-bellied appearance, especially in young animals.

Larvae that localize in atypical sites (i.e. bile ducts) may cause severe pathologic effects. Nervous disorders occur when larvae localize near nervous tissue or when large amounts of toxic by-products released from the parasites are absorbed by the host. Infections are potentially most severe in young animals.

The significance of Toxascaris and Toxocara spp. on wildlife populations of Alaska has not been determined.

\section{References}

DUBEY, J. P. 1978. Patent Toxocara canis infection in ascarid-naive dogs. J. Parasitol. 64:1021-1023.

DUNAGAN, T. T. 1957. Studies on the parasites of edible animals of Alaska. Proj. Report N0. 7955-4. Arctic Aeromed. Lab. 18 pp.

FAY, F. H. and F. S. L. WILLIAMSON. 1962. Studies on the helminth fauna of Alaska. XXXIX. Echinococcus multilocularis Leuckart, 1863, and other helminths of foxes on the Pribilof Islands. Can. J. Zool. $40: 767-772$.
RAUSCH, R. and F. S. L. WILLIAMSON. 1959. Studies on the helminth fauna of Alaska. XXXIV. The parasites of wolves, Canis lupus L. J. Parasitol. 45:3 $\overline{95-403}$.

SCHILLER, E. L. 1952. Studies on the helminth fauna of Alaska. V. Notes on Adak rats (Rattus norvegicus berkenhout) with special reference to helminth parasites. J. Mamma l. 33:38-49.

SCHMIDT, G. D. and L. S. ROBERTS. 1977. Toxocaridae. pp. 460-463. In: Foundations of Parasitology. c. V. Mosby Co., St. Louis, MO.

SOULSBY, E. J. L. 1968. Toxascaris Leiper, 1907; Toxocara Stiles, 1905. pp. In: Helminths, Arthropods and Protozoa of Domesticated Animals (Monnig). 6th ed. Williams \& Wilkins Co., Baltimore, MD.

THOMAS, L. J. and B. B. BABERO. 1956. Some helminths of mammals from St. Lawrence Island, Alaska, with a discussion on the nomenclature of Echinococcus in voles. J. Parasitol. 42:500. 


\section{Uncinaria}

J. Dau

\section{Introduction}

Four species of Uncinaria (hookworms) have been reported from Alaskan mammals: 1) U. stenocephala from arctic foxes (Alopex lagopus) (Dunagan 1957, 01 sen 1958, Thomas and Babero 1956), wolves (Canis lupus) (Rausch and Williamson 1959) and red foxes (Vulpes vulpes) (Dunagan 1957, Schiller and Rausch 1956), 2) U. lucasi from northern fur seaTs (Callorhinus ursinus) ( 01 sen 1958, 1962, Olsen and Lyons 1962), Stellar's sea lions (Eumetopias jubatus) (0lsen 1958) and California sea lions (Zalophus californianus) (01sen 1962), 3) U. rauschi from black bears (Ursus americanus) and brown bears (U. arctos), and 4) U. yukonens is a'so from black and brown bears. Mature U. stenocephla roundworms are 5-12 mm long (Soulsby 1968); adult U. lacasi are 7.4-16 mm long (01sen 1962). Uncinaria stenocephala and $\underline{U}$. lucasi are ecologically very distinct, though are sympatric in the Pribilof Islands. Hookworms do not utilize intermediate hosts (Fig. 7). 01 sen (1968) provides a key to the species of Uncinaria.

\section{Uncinaria stenocephala}

The reported geographic distribution of $\underline{U}$. stenocephala spans Europe and North America. Larvae are not resistant to dessication so limit this parasite to moist areas.

\section{Transmission}

Adult worms localize in the small intestine of their hosts. Eggs pass in the feces of canids and hatch in the external environment. Larvae molt twice while in the external environment to reach the infective, third larval stage. Infective larvae enter the host by being ingested or by penetrating the skin. After invading the host, larvae enter peripheral blood vessels or penetrate the wall of the small intestine and enter the circulatory system in this region. They are then transported to the thoracic organs. Larvae may leave the circulatory system at the pulmonary capillaries by entering alveoli and migrating up respiratory passages. These larvae are eventually swallowed and attach to the wall of the small intestine where they complete development. Larvae that fail to enter alveoli and instead pass through the pulmonary circulatory system reach the systemic portion of the circulatory system and are transported to the visceral organs. These larvae may cause small hemorrhages in the visceral organs before they die. Larvae that reach the systemic circulatory system can infect unborn canids; in prenatal infections, larvae become dormant until after parturition (Soulsby 1968).

\section{Signs, Symptoms and Pathogenesis}

Adult U. stenocephala hookworms are vorácious blood sucking parasites. This is thought to be primarily a respiratory function to acquire dissolved oxygen; only the most easily assimilated food substances are removed from the ingested blood. Mature, individual worms can reportedly remove $0.8 \mathrm{ml}$ to $1.0 \mathrm{ml}$ of blood from their host daily (Soulsby 1968). The cumulative effect of intensive infections can therefore result in a substantial loss of blood for host animals. 
The principal pathologic effect of U. stenocephala roundworms is anemia characterized by an iron deficiency in the host. Anemia results from the effects of the direct loss of blood to these parasites and also from the impairment of absorption by the hosts' intestines. The latter consideration is due to an inflammatory response to the parasites of intestinal tissue that causes fusion of villi and changes in the columnar (absorptive) cells.

Canines develop an immunity to hookworm infections comparable to the "self cure" seen in Haemonchus contortus infections of ruminants. Severe pathogenic effects occur when hookworm infections intensify at a rate faster than the immune system can respond to.

Clinical signs include weakness, emaciation, a dry rough coat, skin irritation where larvae have penetrated and diarrhea that contains bloody mucus. The hosts' blood may appear watery.

\section{Significance and Control}

The significance of $\underline{U}$. stenocephala in Alaska has not been determined. Regional variations in the frequency of infection in this state have been noted. Seven of 27 (25\%) wolves from the taiga community of the Copper River drainage harbored U. stenocephala while only 7 of 162 (4\%) Brooks Range (tundra community) wolves were thus infected (Rausch and Williamson 1959). Man is susceptible to infection (Fullerborn 1927 as reported by 01 sen 1968 ).

\section{Uncinaria lucasi}

Uncinaria lucasi is unique in 2 respects. It is the sole strongylid parasite known to infect fur seal pups. Secondly, its reported geographic distribution is limited to the Pribilof and Kommandorski Islands of the Bering Sea (0lsen 1962).

Transmission

Like U. stenocephala, $\underline{U}$. lucasi follows a direct life cycle. Adult hookworms inhabit the middle to lower portion of the small intestine in pre-pelagic fur seal pups. The adult worms pass eggs throughout the summer via the feces of seal pups. The eggs begin hatching during the first week in September; the proximal explanation for delayed hatching is not understood. It has been suggested that warm weather and the intense contamination of rookeries with urine and feces inhibit hatching until after the seals have departed for the open sea (01sen 1962). Larval development continues throughout the summer, however. Two molts occur within the eggs to produce the third stage larvae that are liberated when hatching occurs in early spring. Third stage larvae overwinter on the rookeries in favorable microhabitats beneath a deep, insulating layer of snow and ice. Neither eggs nor third stage larvae are resistant to cold temperatures $(-20 \mathrm{C})$.

As summer progresses, the number of eggs present in the soil decreases while the number of larvae in the soil increases as the eggs hatch. Seal pups are infected soon after birth by third stage larvae from 2 sources: 1) free-living larvae present in sand on the rookeries that hatched from eggs of the previous year, and 2) infective larvae present in the mother's milk. Free-living larvae invade adults and pups by penetrating their skin, especially on the flippers. These larvae presumably enter the circulatory system and pass through the heart and lungs before being transported throughout the body. These third stage larvae tend to accumulate in 
the belly blubber where they become dormant.

Larvae that are dormant within the seals are said to be in the "tissue phase" of their life cycle. Larvae in the tissue phase are thought to persist in their hosts for long periods of time (duration not specified) (0lsen 1962). In females, some infective larvae localize in the mammary glands, thus facilitating transmission to nursing pups. Larvae that have been ingested are thought to quickly complete 2 molts while in the alimentary canal. These hookworms attach to the mucosa of the small intestine where they complete development approximately 3 weeks post ingestion. Hookworms that localize and mature in the small intestine of fur seal pups are said to be in the "intestinal phase" of their life cycle. Mature worms pass eggs in the pups' feces, thus contaminating the rookery and providing a source of infection for the subsequent cohort of seals.

\section{Signs, Symptoms and \\ Pathogenesis}

The pathogenesis of mature $\underline{U}$. lucasi hookworms is similar to that of $\underline{U}$. stenocephala. Anemia is the principal pathologic effect. Fur seal pups that harbor hookworms in the intestinal phase show a generalized weakness, difficulty breathing and bloody diarrhea that contains large numbers of eggs. The mucous membranes of these hosts appear blanched due to anemia. The small intestine becomes thickened, fragile and exhibits nodules where groups of the hookworms are attached. Free blood is often present in the intestinal lumen. Infections with $\underline{U}$. lucasi hookworms are often acute as evidenced by extensive fat deposits and excellent body condition of dead, heavily parasitized pups (0lsen 1962). Third stage larvae are not considered pathogenic.
Fur seal pups develop an immunity against adult hookworms. These hookworms do not persist in the intestinal phase longer than 5 months in the fur seal pups.

\section{Significance and $\underline{\text { Control }}$}

Uncinaria lucasi represents a significant mortality source for fur seal pups on the Pribilof Islands of Alaska. The fidelity of seals to established rookeries and their gregarious behavior ensure the perpetuation of $\underline{U}$. lucasi. These characteristics aTso facilitate control of this parasite by localizing foci and temporally concentrating periods of contamination. Application of an emulsion of cresylic disinfectant, a nematode larvicide, significantly reduced the incidence of free-living larvae on small, experimental plots. When the larvicide was sprayed over a large area however, no reduction in fur seal pup mortality occurred that year. This should not be interpreted as a failure of the larvicide or as disproving the recognized pathogenicity of $\underline{U}$. lucasi, but rather as an indication of the significance of other mortality sources (i.e. trampling, starvation and exposure) in influencing fur seal pup survivorship.

Neither humans nor arctic foxes are susceptible to $\underline{U}$. lucasi.

\section{Uncinaria rauschi and U. yukonensis}

Hookworms, though not of the genus Uncinaria, were first reported in ursids from polar bears (Ursus maritimus) (Dujardin 1845 as reported by Choquette et al. 1969). Hookworms of this genus commonly inhabit the small intestine of black and brown bears in Alaska (0lsen 1968, Rausch 1961). Since hookworms feed on blood from their host, these parasites are 
not stressed by dietary changes that precede denning, and thus are not seasonally expelled from bears as has been suggested for cestode parasites (Rausch 1961). Two black bears from Alaska, killed in their dens, harbored hookworms; 1 of these bears contained 78 hookworms but only 3 small specimens of Diphyllobothrium ursi (see Cestodes) (Rausch 1961). Infections in individual bears may exceed 400 worms (Choquette et al. 1969).

Uncinaria yukonensis is larger in its overall body size and features than U. stenocephala. Uncinaria yukonensis is roughly $10.5-17.1 \mathrm{~mm}$ long (this range is probably exceeded since the sample from which these dimensions were determined consisted of only 7 individuals from a single black bear) (Wolfgang 1956); U. rauschi is 7.4-10.5 mm long (01sen 1968). The impact of $\underline{U}$. rauschi and $\underline{U}$. yukonensis on bear populations in Alaska has not been determined.

\section{References}

CHOQUETTE, L. P. E., G. G. GIBSON and A. M. PEARSON. 1969. Helminths of the grizzly bear, Ursus arctos L., in northern Canada. Can. J. Zool. 47:167-170.

DUJARDIN, F. $1845 . \quad$ Histoirse naturelle des helminthes ou vers intest inaux. Paris.

DUNAGAN, T. T. 1957. Studies on the parasites of edible animals of Alaska. Proj. Report NO. 7955-4. Arctic Aeromed. lab. 18 pp.

FULLEBORN, F. 1927. Durch Hakenwurmlarven des Hundes (Uncinaria stenocephala) beim Menschen erzeugte "Creeping Eruption". Hamburg Univ. Abhandl. Beg. Auslandsk. Bernhard Nocht Festschr. 26:121-133.
OLSEN, 0. W. 1958. Hookworms, Uncinaria lucasi Stiles, 1901, in fur seals, Callorhinus ursinus (Linn.), on the Pribilof Islands. Trans. 23rd N. Am. Wildl. Conf. 152- 175 .

1962. Uncinaria lucasi Stiles, 1901. pp. 247-249. In: Animal Parasites. Burgess Publishing Co., Minneapolis, MN. 1968. Uncinaria rauschi (Strongyloidea: Nematoda), a new species of hookworms from Alaskan bears. Can. J. Zool. 46:1113-1117.

OLSEN, 0. W. and E. T. LYONS. 1962. Life cycle of the hookworm, Uncinaria lucasi Stiles, of northern fur seals, Callorhinus ursinus, on the Pribilof Islands in the Bering Sea. J. Parasitol. $48: 42-43$.

RAUSCH, R. L. 1961. Notes on the black bear, Ursus americanus Pallas, in Alaska, with particular reference to dentition and growth. Z. Saugetierk. 26:77-107.

RAUSCH, R. $L$. and $F$. S. L. WILLIAMSON. 1959. Studies on the helminth fauna of Alaska. XXXIV. The parasites of wolves, Canis lupus L. J. Parasitol. 45:395-403.

SCHILLER, E. L. and R. RAUSCH. 1956. Mammals of the Katmai National Monument, Alaska. Arctic 9:191-203.

SOULSBY, E. J. L. 968. Uncinaria Frohlich, 1789. pp. 215-219. In: Helminths, Arthropods and Protozoa of Domesticated Animals (Monnig). 6th ed. Williams \& Wilkins Co., Balt imore, MD.

THOMAS, L. J. and B. B. BABERO. 1956. Some helminths of mammals from St. Lawrence Island, Alaska, with a discussion on the nomenclature of Echinococcus in wolves. J. Parasitol. 42:500.

WOLFGANG, R. W. 1956. Dochmoides yukonensis sp. nov. from the brown bear (Ursus americanus) in the Yukon. Can. J. Zool. 34:21-27. 


\title{
Obeliscoides
}

\author{
J. Dau and R. Barrett
}

\section{Introduction}

trichostrongylid $\frac{\text { Obeliscoides }}{\text { cuniculi }}$ Tramily $\begin{array}{r}\text { are } \\ \text { tricos- }\end{array}$ trongylidae) roundworms frequently found in Alaska by hunters while cleaning snowshoe hares (Lepus americanus). Two factors facilitate exposure of sportsmen to these worms: 1) the intense utilization of hares by hunters during periods approximating peak hare density, and 2) the tendency of $\underline{0}$. cuniculi to infect the greatest proportion of hare populations during peaks in hare cycles (Erickson 1944).

As suggested by the provincial name of $\underline{0}$. cuniculi, "stomach worms," these parasites inhabit the lumen of the stomach. They usually localize in the gastric mucosa (the mucus lining between the stomach wall and the bolus of food). The color of $\underline{0}$. cuniculi ranges from white with dark streaking (Graybill 1923) to deep purple-red (J. Dau, pers. obs.). Adult males are 10-14 mm long; adult females are 15-18.5 mm long (Graybill 1923). Female worms do not exceed males in length until the final period of maturation. Obeliscoides cuniculi has been found throughout North America.

\section{Transmission}

Alicata (1932) described the preinfective larval stages of $\underline{0}$. cuniculi. Eggs of these roundworms pass from hares in fecal pellets and hatch in the external environment. First stage larvae exist only briefly (about 65 hours) before they molt to the second larval stage. Most larvae reach the infective, third larval stage 6 days after hatching. Third stage larvae are fairly tolerant to cool ambient temperatures; these organisms remained viable for 30 days at temperatures from 2 to $-4 \mathrm{C}$. Third stage larvae are not highly resistant to dessication. No larvae survived drying for 5 hours at room temperature. Third stage larvae do not grow or develop until they infect a host animal.

Hares are infected as they browse by accidentally ingesting third stage larvae; these larvae do not invade their hosts by penetrating the skin (Alicata 1932). Experimental results indicated that $31-55 \%$ of all larvae ingested become established in hares (Alicata 1932). Establishment of infections in free-living hares occurs during the summer and fall. Within 24 hours post ingestion, third stage larvae penetrate the gastric mucosa and, within 3 days, molt to the fourth larval stage (Sollod et al. 1968). Fourth stage larvae then emerge from the mucosa into the gastric lumen and complete the final molt. Development may be complete in as little as $16-20$ days post ingestion under optimal conditions (Alicata 1932). The intensity of $\underline{0}$. cuniculi infections usually declines in late autumn-early winter. The cause of this decline has not been clearly established. An increased immunity of hares to adult parasites and/or the adverse effects of warm ambient temperatures and dessication on infective larvae during late summer could account for reductions in the number of 0 . cuniculi worms within hares (Gibbs et al. 1977).

Fourth stage larvae that are present in hares at the onset of winter cease developing and overwinter in their hosts at this larval 
stage. The factor(s) that cause this temporary cessation of development are unknown but probably include the effects of crowding by larvae, immunologic responses of the hosts and influences of the external environment. Development of overwintering larvae resumes in March and results in an increase in the ratio of adult to immature worms (Gibbs et al. 1977). This phenomenon is referred to as the "spring rise" in trichostrongylid nematodes. Fourth stage larvae that overwinter in their hosts may be important in maintaining this parasite in ecosystems during years when spring breakup is late.

Signs, Symptoms and Pathogenesis

Light to moderate infections (roughly less than 200 worms per hare) are asymptomatic; they affect neither the quantity nor characteristics of fecal pellets that are excreted by hares or cause blood to appear in hare feces (Worley 1963).

Pathologic tissue changes include petechial (pinpoint) hemorrhages in the stomach, ulcers, gastritis and anemia (Erickson 1944, Gibbs et al. 1977, Sollod et al. 1968). Obeliscoides cuniculi can reportedly cause tissues that line the stomach to slough away and tumors to form on the inner wall of the stomach (Erickson 1944). Heavy infections, i.e. consisting of over 4000 individual worms, could occlude the pylorus and thus prove fatal. Experimental results indicate that hares do not develop an immunity against 0 . cuniculi (Worley 1963).

\section{Significance and Control}

Obeliscoides cuniculi is thought to cause mortalities in wild hare populations (Bookhout 1971, Erickson 1944). Though this parasite has been reported as the sole cause of epidemics in hares (Graybill 1923, Erickson 1944), the effects of these parasites on hares are, as a general rule, probably more insiduous and interrelated with other parasites and conditions in the external environment.

The sedentary nature of hares, their predilection for copraphagy and the tendency for all hares in an area to utilize common runways makes them particularly vulnerable to a number of helminth parasites. of these parasite species that infect hares, 0 . cuniculi frequently infects the greatest proportion of individuals in hare populations (Bookhout 1971, Erickson 1944). Erickson (1944) reported that the prevalence of hares that exhibit concurrent infections with 2 or more nematode species increases during population declines when hare density has peaked. This suggests that factors which influence nematode parasitism in hares act in a density dependent fashion. This could occur if maximum hare density increases the likelihood of ingesting larvae by increasing the absolute amount of feces in an area that could contain larvae, by exposing hares to feces of a greater number of potentially infected hares or by modifying the environment so as to enhance the transmission of infective larvae. The increased frequency of concurrent nematode infections in hares could also be a function of those factors that cause hare numbers to increase and not be directly related to hare density at all. When the proximal reasons for hare periodicity and the host-parasite relationship of these species are better understood, an element of truth from each explanation will probably be found. Ambient temperature, precipitation and the density of hares in a given region influence the relative significance of $\underline{0}$. cuniculi with respect to other parasites of hares (i.e. Passalurus nonanulatus and Trichostrongylus spp.) and dictate how severe the cumulative effects of these helminths will be on individual animals. 
The number of 0 . cuniculi present in individual hares exhibit strong seasonal fluctuations (Gibbs et al. 1977, R. Dieterich, unpub. material). The greatest intensity of infection with adult worms occurs from early spring to late summer. Between late fall and early winter, the total number of these parasites present in hares ranged from 99 (Aldous 1936 as reported by Erickson 1944) to 363 (Bookhout 1971) worms per hare. Up to 4015 worms have been reported from a single hare (Erickson 1944).

Worley and Thompson (1963) provide a review of the efficacy of several anthelmintic agents in reducing $\underline{0}$. cuniculi infections. Experimental infections of laboratory rabbits (Oryctolagus cuniculi) with obeliscoides cuniculi may have value as biological models for studying nematodiasis in large animals, i.e. ruminants, that are difficult to maintain in laboratory facilities. The influence exerted by this roundworm on the density and periodicity of free-living hare populations in Alaska is currently unknown.

\section{References}

ALICATA, J. E. 1932. Life history of the rabbit stomach worm, Obeliscoides cuniculi. J. Ag. Res. 44:401-419.

ALDOUS, C. M. 1936. Food habits of Lepus americanus phaeonotus. J. Mamma 1. 17:175-176.
BOOKHOUT, T. A. 1971. Helminth parasites in snowshoe hares from northern Michigan. J. Wildl. Dis. $7: 246-248$.

ERICKSON, A. B. 1944. Helminth infections in relation to population fluctuations in snowshoe hares. J. Wildl. Manage. 8:134-153.

GIBBS, H. C., W. J. CRENSHAW and $M$. MOWATT. 1977. Seasonal changes in stomach worms (Obeliscoides cuniculi) in snowshoe hares in Maine. J. Wildl. Dis. 13:327-332.

GRAYBILL, H. W. 1923. A new genus of nematodes from the domestic rabbit. Parasitol. 15:340-342.

MAC LULICH, D. A. 1937. Fluctuations in the numbers of the varying hare (Lepus americanus). Univ. of Toronto Studies, Biol. Ser., No. 43. pp. 83-86.

SOLLOD, A. E., T. J. HAYES and E. J. L. SOULSBY. 1968. Parasitic development of Obeliscoides cuniculi in rabbits. J. Parasitol. 54:129-132.

WORLEY, D. E. 1963. Experimental studies on Obeliscoides cuniculi, a trichostrongylid stomach worm of rabbits. Host-parasite relationships and maintenance in laboratory rabbits. J. Parasitol. 49:46-50.

WORLEY, D. E. and P. E. THOMPSON. 1963. Experimental studies on Obeliscoides cuniculi, a trichostrongylid stomach worm of rabbits. 11. Anthelmintic studies in the Dutch rabbit. J. Parasitol. 49:51-54. 


\title{
Haemonchus
}

\author{
J. Dau and R. Barrett
}

\section{$\underline{\text { Introduction }}$}

Haemonchus contortus is often referred to as the "barber pole" stomach worm of ruminants. This translucent nematode takes on a red color when it ingests blood from its host; the white ovaries of female worms wrapped around the red intestine give $H$. contortus its descriptive name. Male $\underline{H}$. contortus nematodes are 10-20 mm long and an even red color; females are 18-30 mm long. This parasite has been reported in Alaska only from caribou (Rangifer tarandus) (Dunagan 1957) though may occur in other ruminants, i.e, moose (Alces alces) or Dall sheep (Ovis dalli). Eggs of a Haemonchus sp. were found in muskox (Ovibos moschatus) fecal samples collected on an Alberta game farm in June 1970 (Samuel and Gray 1974).

\section{Transmission}

As with all trichostrongylid (family Trichostrongylidae) roundworms, $\underline{H}$. contortus does not utilize an intermediate host. Eggs are expelled in the hosts' feces; these hatch and undergo the first 2 larval molts in the external environment. Infective third stage larvae develop in 2 days post expulsion under optimum conditions. Eggs and larvae are vulnerable to adverse environmental conditions so exhibit little overwinter survival. Growth of freeliving larvae is impeded by low ambient temperatures with complete cessation of growth below 9C (Smyth 1962). The severe environmental conditions that characterize much of Alaska probably limit the prevalence of $\underline{H}$. contortus in this state.
The infective, third stage larva loosely retains its molted second stage cuticle to prevent dessication. This retained cuticle prevents feeding and forces larvae to exist on energy and nutrient reserves and further limits the ability of freeliving larvae to survive in the external environment. Third stage larvae are most active during intervals of low light intensity (predawn, dusk and cloudy periods) and will ascend grass blades and other vegetation at these times.

Ruminants accidentally ingest third stage larvae that are clinging to vegetation. The retained second stage cuticle is shed in the forestomachs of the host before larvae attach to the mucosa of the abomasum. Larvae undergo 2 molts while attached to the abomasum before completing maturation ( $\mathrm{Fig} .7$ ).

\section{Signs, Symptoms and}

\section{Pathogenesis}

Due to the similarity of trichostrongylid nematode life cycles, many species of these parasites, including $\underline{H}$. contortus, frequently infect a single ruminant host under natural conditions. The effects of such infections must be considered on a cumulative basis. This masks the pathogenicity of individual parasite species. Experimental infections in domestic sheep (Ovis aries) have shown $\underline{H}$. contortus to be a serious pathogen however, due to its voracious appetite for blood (Soulsby 1968). An infection consisting of 4000 worms may remove up to $60 \mathrm{ml}$ of blood from the host daily (Smyth 1962). Wounds inflicted by the single tooth of 
these worms may provide points of entry for secondary infection by microscopic pathogens.

Acute $\underline{H}$. contortus infections exhibit no clinical symptoms. Chronic infections may show swelling beneath the neck ("bottle jaw"), weakness, diarrhea or constipation and occasionally hair loss. Death results from anemia in the form of reduced erythrocyte and hemoglobin levels and packed cell volume.

Domestic sheep accumulate a localized immunity to $\underline{H}$. contortus parasites that results in the eventual loss of established infections ("self cure") and resistance to subsequent invasions (Soulsby 1968). The significance of $\underline{H}$. contortus in Alaskan ungulates has not yet been determined. Parasite investigations currently being conducted by the University of Alaska on Seward Peninsula reindeer herds may provide a better evaluation of the significance and control of $\underline{H}$. contortus in that area.

\section{References}

DUNAGAN, T. T. 1957. Studies on the parasites of edible animals of Alaska. Proj. Report No. 7955-4. Arctic Aeromed. Lab. 18 pp.

SAMUEL, W. M. and D. R. GRAY. 1974. Parasitic infection in muskox. J. Wildl. Manage. 38:775-782.

SMYTH, J. D. $1962 . \quad$ Tricostrongylidae. pp. $331 . \quad$ In: $\frac{\text { Introduction }}{\text { Parasitology. Charles C. Thomas, }}$ Publisher, Springfield, IL.

SOULSBY, E. J. L. 1968. Haemonchus Cobb, 1898. pp. 235-240. In: Helminths, Arthropods and Protozoa of Domesticated animals (Monnig). 6th ed. Williams \& Wilkins Co., Baltimore, MD. 


\section{Protostrongylus, Muellerius aDictyocaulus}

J. Dau

$\underline{\text { Introduction }}$

Protostrongylus ("brown lungworms"), Muellerius ("hair lungworms") and Dictoyocaulis ("thread lungworms") spp. are metastrongylid (family Metastrongylidae) roundworms collectively known as "lungworms." Protostrongylus and Muellerius spp. are similar in size and life cycle. Dictyocaulus spp. are larger and have a different life cycle than species of either Protostrongylus or Muellerius (Fig. 8). Dictyocaulus, Protostrongylus and Muellerius spp. have similar pathologic effects and clinical symptoms in their definitive hosts (cervids). Lungworms are thought to have reached North America via Eurasian sheep that colonized this continent (Buechner 1960).

Dictyocaulus spp. are perhaps the most widely distributed lungworms in Alaska. These roundworms have been found in free-living Alaskan bison (Bison bison) (Dunagan 1957), muskox (Ovibos moschatus) (Samuel and Gray 1974) and reindeer (Rangifer tarandus) (Hadwen 1922). The only reported Dictyocaulus sp. identified in Alaska was D. viviparus found in a bison (Dunagan 1957). Dictyocaulus filaria has been recovered from captive bison in New York state (Wade et al. 1979). Dictyocaulus viviparus and $\underline{D}$. filaria are white with males 3-8 $\mathrm{cm}$ long and females $5-10 \mathrm{~cm}$ long (01sen 1962).

Protostrongylus spp. have been intensively studied in bighorn sheep (Ovis canadensis) that inhabit the Rocky Mountain states (Buechner 1960, Forrester and Senger 1964). Two Protostrongylus spp. have been identified as serious pathogens in bighorns: $\underline{P}$. stilesi and $\underline{P}$. rushi.
Protostrongylus spp. have been reported in mountain goats (Oreamnos americanus) from Canada and Dall sheep (Ovis dalli) from Alaska. Protostrongylus stilesi occurs mainly in lung parenchyma and is considered more pathogenic than $\underline{P}$. rushi which predominantly inhabit $\bar{s}$ small bronchi. Both $P$. stilesi and $P$. rushi are reddish colored and $16-\overline{3} 5 \mathrm{~mm}$ long ( 01 sen 1962); they cannot be differentiated without microscopic examination. Protostrongylus boughtoni infects the bronchioles and lung parenchyma of snowshoe hares (Lepus americanus)

(Boughton 1932).

Muellerius spp. have been found in Dall sheep from the Brooks Range and reindeer from Nunivak Island (USDA 1969). Larvae from an unidentified Muellerius sp. have been recovered from muskox fecal samples collected on Nunivak Island (R. Barrett, pers. obs.).

\section{Transmission}

Dictyocaulus spp. follow a direct life cycle (they do not employ an intermediate host). Eggs are deposited in the lungs of the host and hatch while being transported via ciliary action up bronchi. The first stage larvae (approximately $0.3 \mathrm{~mm}$ long) migrate up the trachea, are swallowed and eventually pass in the ruminants' feces. During the next 4 days, the larvae molt twice while in the external environment to reach the infective third larval stage. Both the first and second stage cuticles are retained by the third stage larvae. Third stage larvae do not feed, are relatively inactive and rarely venture from the hosts' feces to ascend standing vegetation. It has 


\section{Figure 8. Ruminant Lungworms}

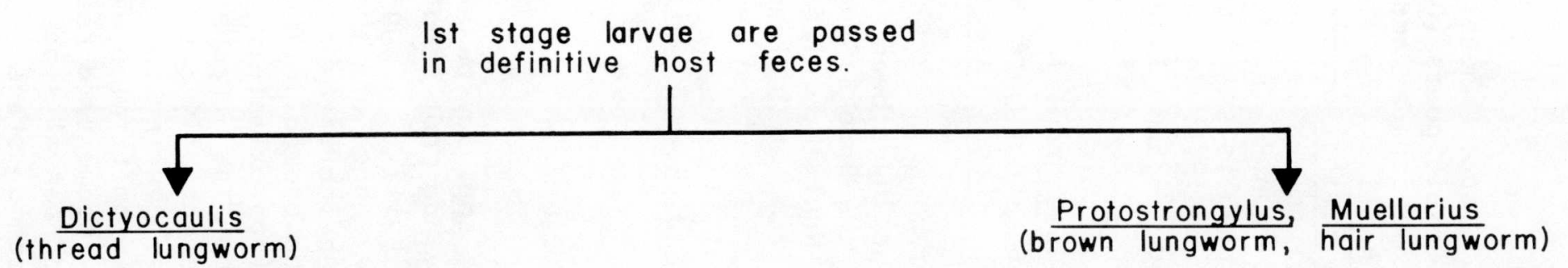

Larvae molt twice in environment to

infective 3 rd stage. Ist and 2 nd cuticle

are retained. Larvae ascend vegetation

and are ingested.

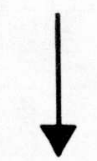

After ingestion by ruminant, larva sheds cuticles, and molts to 4 th stage in intestine.

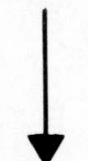

4th larval stage penetrates intestinal wall, enters bloodstream via lymph, passes through heart and lungs, migrates up bronchi. Larvae molt to 5 th stage and overwinter in bronchioles.

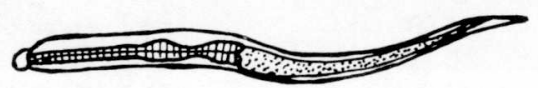

Ist stage larvae

Embryonated eggs are deposited in host's lungs, hatch while being transported up bronchi. Ist stage larvae $(0.3 \mathrm{~mm})$ migrates up trachea, is swallowed, and passes out in feces.

\author{
Protostrongylus, Muellarius
}

Small land snails become Infected by Ist stage larvae; larvae molt to 3 rd stage.
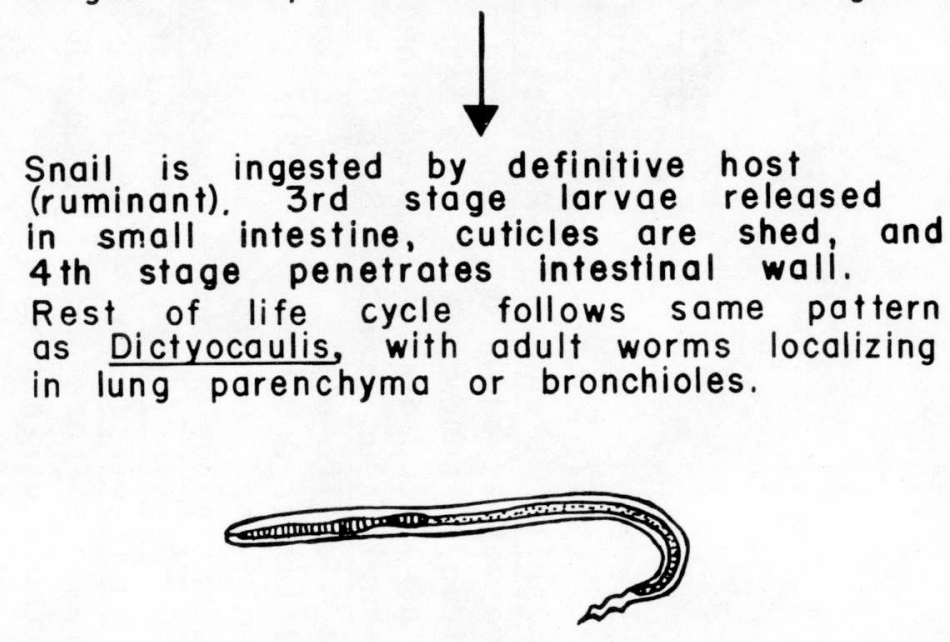

Protostrongylus

Ist stage larvae

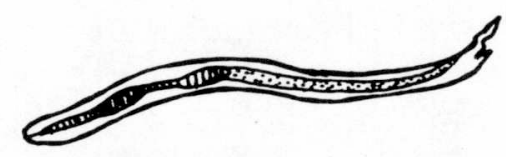

Muellerius

Ist stage larvoe 
been proposed that the extent of feces-herbage contact may be more important in transmitting infective larvae to host animals than the activity of larvae (Michel and Rose 1954). High, lush vegetation and factors that cause the consistency of fecal material to become loose thus "seed" standing vegetation with larvae and increase the potential for infection.

Infective larvae shed the first, second and third stage cuticles after they have been ingested by the host. The fourth larval stage develops in the gastrointestinal tract of the host. These larvae penetrate the intestinal wall, enter the lymph system and are transported to the bloodstream. Approximately 7 days post ingestion, fourth stage larvae reach the lungs, enter alveoli and migrate up the bronchi where they molt to the fifth larval stage. Maturation is complete approximately 22 days post ingestion (Soulsby 1968). Most Dictyocaulus spp. lungworms overwinter as inactive, early fifth stage larvae in the bronchioles of their hosts. Prenatal infection can occur when larvae cross placental membranes.

$\frac{\text { Protostrongylus }}{\text { have nearly identical life }}$ cycles; both utilize intermediate molluscan hosts. Adult worms deposit eggs in the lungs of the definitive host. The eggs hatch in the lungs or are transported to the alimentary canal and swallowed in mucus. The eggs then hatch en route through the hosts' digestive tract. First stage larvae pass from the host in feces or sputum. These larvae are lethargic and fairly resistant to dessication and low ambient temperature (freezing does not kill them). Eventually, the first stage larvae infect small land snails (i.e. Pupilla muscorum) and molt twice while within these hosts to reach the third larval stage. Infection of definitive hosts by third stage larvae occurs when snails that harbor these parasites are swallowed with forage. Third stage larvae are released from the snails in the small intestine of the ruminant by digestive processes. There, the third stage cuticle and retained first and second larval stage cuticles are shed. Fourth stage larvae penetrate the intestinal wall, enter the lymphatic system and eventually reach the bloodstream. The fourth stage larvae are then transported to the lungs where they enter alveoli and localize in lung parenchyma or bronchioles to complete maturation.

A general life cycle for lungworms is diagrammed in Figure 8 .

\section{Signs, Symptoms and \\ Pathogenesis}

The notoriety of lungworm pathogenicity is mainly due to its association with pneumonia. Pneumonia is thought to result from secondary infection of lung tissue that has been affected by lungworms with any of several bacterial (i.e. Corynebacterium pyogenes and Pasteurella spp.) or viral organisms (see Pneumonia in Miscellaneous Disease section of Part 1). Thus, the signs, symptoms and pathogenesis of lungworms usually encompass the lungwormpneumonia complex ("fog fever") and are not limited solely to the parasites themselves. This lungwormpneumonia complex has frequently caused extensive mortality among bighorn sheep populations in the western United States and so, as a result, has received considerable attention from wildlife management agencies. 
During the prepatent (i.e. before maturity) phase of lungworm infections, fourth stage larvae stimulate the infiltration of monocytes and lymphocytes into alveoli causing them to collapse and consolidate. Adult worms in the hosts' bronchi and trachea can cause severe damage to the epithelium and block the passage of air. Fibrosis occurs in areas surrounding lesions during host recovery after the lungworms have died or been expelled.

Clinical symptoms in definitive hosts are evident at both the population and individual animal levels though are vague and offer little more than an indication that some disease is present. Populations of bighorn sheep suffering intense lungworm epizootics show disproportionately high mortality among very young or old aged cohorts. Individual animals exhibit coughing and dyspnea (labored breathing) especially after being disturbed, a reluctance to move, general weakness, inappetance with emaciation and a harsh, dull pelage.

\section{Diagnosis}

The clinical symptoms of lungwormpneumonia infections, ambiguous as they may be, are sufficient to stimulate detailed investigations to determine their cause(s). The presence of lungworm larvae in the feces of definitive hosts indicates the involvement of these parasites. The number of larvae present in the feces is not a good indication of the intensity of lungworm infections however, as this can vary daily for each host animal due to host activity, seasonally due to food, weather and host activity and annually due to fluctuations in climatic regimes and snail numbers
(Forrester and Senger 1964). Estimates of the severity of lungworm infections at the definitive hosts' population level can be based on large samples of fecal material collected over long time intervals and reinforced with necropsies of animals available from that population. The lungs of bighorn sheep parasitized by lungworms often exhibit gray, necrotic nodules up to $2 \mathrm{~cm}$ in diameter (Forrester and Senger 1964). Benign tumors may occur on bronchial epithelium tissue (Soulsby 1968). Frequencies of lungworm infection exceeding $90 \%$ have been reported for some bighorn populations (Forrester and Senger 1964).

\section{Significance and Control}

Though lungworms are present in Alaskan mammals, no severe epizootics have been reported from this state. This could be attributable to any of an array of environmental factors that preclude establishment of these parasites to the extent required to cause extensive mortality or a function of outbreaks being misdiagnosed or not detected. The potential for lungworm-pneumonia epizootics certainly exists in Alaska since the causative organisms and intermediate host animals are present (Dall 1910).

The lungworm-pneumonia complex is undoubtedly a significant population regulating force in some wildlife populations outside of Alaska. This disease complex has been suggested to be the primary natural regulating force of bighorn sheep populations in the Rocky Mountains (Buechner 1960, Forrester and Senger 1964). Boughton (1932) reported that $\underline{P}$. boughton $i$ causes mortality in hare populations even during ebbs in hare density. He suggested that this parasite was capable of causing epidemics in hare 
populations under certain environmental conditions.

The incidence of lungwormpneumonia infections varies seasonally. Moist periods, i.e. from late spring to early summer, increase the activity of both the intermediate host snails and first stage larvae. These periods are thought to present the maximum potential for infection of grazing sheep and goats. Lungworm-pneumonia related mortalities mainly occur during the winter however, when food-stressed animals are least able to physiologically defend themselves against disease (Forrester and Senger 1964). Factors cited as contributing to a lungworm-pneumonia outbreak in bighorn sheep involved a reduction in the quality and quantity of natural forage from: 1) the short term effect of fire in reducing the area available for foraging, 2) competition with livestock for remaining forage, and 3) the employment of supplemental feed (hay) which the sheep were not accustomed to (Buechner 1960).

Control of lungworm-pneumonia outbreaks through the eradication of the parasitic nematodes is infeasible if merely due to the persistence of larvae outside of the definitive hosts. First stage larvae may survive in the hosts' feces up to 2 years; infective larvae are thought to survive within the intermediate snail hosts for 1 to 2 years or possibly longer. Adult lungworms can persist in their definitive hosts for 1 to 2 years. Thus, a 4 to 6 year carry-over of lungworms is possible. Prevention of lungworm-pneumonia outbreaks by manipulating habitat and regulating the density of definitive hosts to maintain healthy cervid populations are probably superior means of minimizing the effects of this wild- life disease. Animals which survive infection by some lungworm species (i.e. Dictyocaulus spp.) acquire a partial immunity in as little as 10 days. Areas where continuous low level exposure to these parasite species occurs probably suffer little detrimental effect from these organisms. The prevalence of lungworms in cervid populations may be reduced by excluding animals from contaminated grazing areas when the availability of alternate feeding areas and control of herd movement prevail. Supplemental feeds that are treated with anthelmintic or immunogenic agents may be of value for reducing the effects of lungwormpneumonia in sheep populations.

\section{References}

BUECHNER, H. K. 1960. The bighorn sheep in the United States, its past, present and future. Wildl. Monog. No. 4. 174 pp.

BOUGHTON, R. V. 1932. The influence of helminth parasitism on the abundance of the snowshoe rabbit in western Canada. Can. J. Res. $7: 524-547$.

DALL, W. H. 1910. Land and Fresh Water Mollusks. Smithsonian Inst., Harriman Alaskan Series. Vol. 13. Doubleday, Page and Co., New York, NY. 171 pp.

DUNAGAN, T. T. 1957. Studies on the parasites of edible animals of Alaska. Proj. Report No. 7955-4. Arctic Aeromed. Lab. 18 pp.

FORRESTER, D. J. and C. M. SENGER. 1964. A survey of lungworm infection in bighorn sheep in Montana. J. Wildl. Manage. 28:481-491.

GOBLE, F. C. and A. MURIE. 1942. A record of lungworms in Ovis dalli (Nelson). J. Mammal. 23:220-221.

HADWEN, S. 1922. Reindeer in Alaska. U.S. Dept. Ag. Bull. No. 1089. Washington, DC. 74 pp. 
KERR, G. R. and J. C. HOLMES. 1966. Parasites of mountain goats in west central Alberta. J. Wildl. Manage. 30:786-790.

MICHEL, J. F. and J. H. ROSE. 1954. Some observations on the freeliving stages of the cattle lungworm in relation to their natural environment. J. Comp. Path. $64: 195-205$.

OLSEN, 0. W. 1962. Superfamily Metastrongyloidea. pp. 268-271. In: Animal Parasites: Their Biology and Life Cycles. Burgess Publishing Co., Minneapolis, MN.
SAMUEL, W. M. and D. R. GRAY. 1974. Parasitic infection in muskoxen. J. Wildl. Manage. 38:775-782.

SOULSBY, E. J. L. 1968. Helminths, $\frac{\text { Arthropods }}{\text { Domesticated }} \frac{\text { and }}{\text { Animals (Monnig). }}$ 6th ed. Williams \& Wilkins Co., Baltimore, MD.

USDA Meat Inspection. Case \#20582, January 14, 1969.

WADE, S. E., W. M. HASCHEK and J. R. GEORGI. 1979. Ostertagiosis in captive bison in New York State: Report of nine cases. Cornell Vet. 69:198-205.

\title{
Dirofilaria
}

\author{
J. Dau and R. Barrett
}

Three species of Dirofilaria occur in Alaska: 1) D. immitis, the "heartworm" of canids and felids, 2) D. scapiceps and 3) D. ursi. Diro$\bar{f}$ ilaria immitis has been found in black bears (Ursus americanus) in southeastern United States (Crum et al. 1978). In canids, mature D. immitis roundworms principally inhabit the right ventricle and pulmonary artery. Microfilariae, the larval form of filarioid (order Filariata) nematodes inhabit the circulatory system of these definitive hosts. Microfilariae are ingested with blood by mosquitos (i.e. Culex spp.) and develop within these arthropods to a larval stage that is infective for the definitive hosts. The infective microfilariae escape from the mosquitos when they land on a canine (or seal) to ingest a blood meal and enter the circulatory system of the definitive host. Adult worms are white and $12-30 \mathrm{~cm}$ long (Soulsby 1968). Heavy infections may interfere with the pulmonary circulatory system. Another filaroid heartworm, Dipetalonema spirocauda, occurs in free-living marine mammals of Alaska and has been documented in: ringed seals (Phoca hispida), spotted seals (P. largha), harbor seals ( . vitulina), ribbon seals (P. fasciata) and bearded seals (Erignathus barbatus) (Eley 1981).

Dirofilaria scapiceps commonly parasitizes snowshoe hares (Lepus americanus) in Alaska. Mature worms localize in the tarsal bursa of these hosts so could impair the mobility of hares if present in large numbers. One hare collected during 0ctober 1980, that harbored 49 individuals of D. scapiceps in the tarsal bursa of both joints (collectively) was extremely reluctant to move (J. Dau, pers. obs.). The cause of morbidity in this animal could not be solely attributed to $D$. scapiceps however, since this particular hare also harbored a light infection (7 cysticerci) of Taenia pisiformis (see Cestodes) and a thorough examination for bacterial organisms was not performed. If D. scapiceps can impede the movement of hares, this would certainly predispose these animals to 


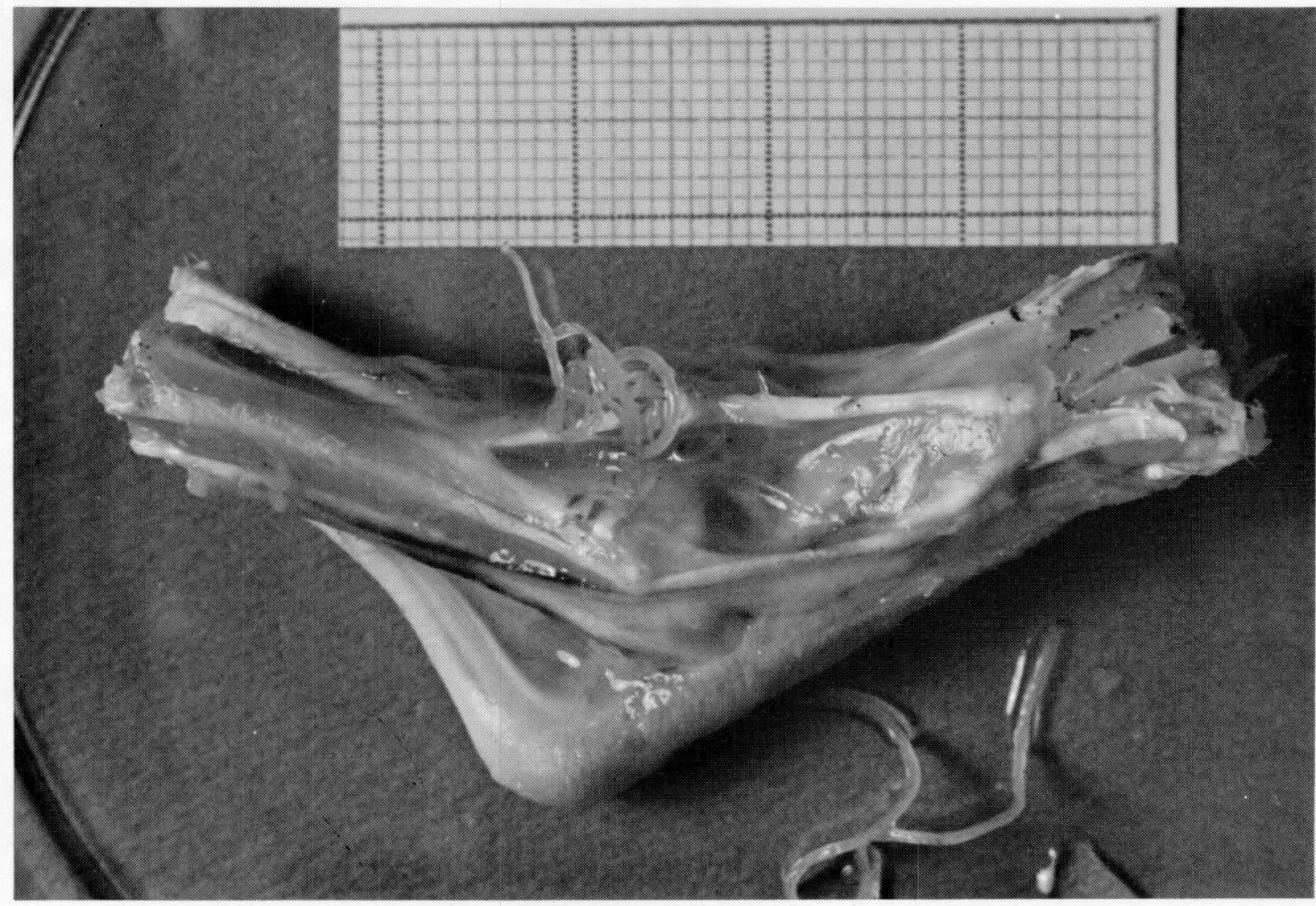

Figure 10. Dirofilaria scapiceps in the tarsal joint of a snowshoe hare (Lepus americanus). The translucent sac of connective tissue that contains these parasites has been broken and several of the worms teased out. Scale is in mm ( $D$. Borchert).

predation. Twenty-two of 40 (55.0\%) hares collected in the Fairbanks area between October 1980 and January 1981 harbored adult $\underline{D}$. scapiceps roundworms in at least 1 tarsal joint.

Hares that support $15+$ worms in a tarsal joint exhibit an enlarged pocket of connective tissue that encloses the worms in an amber colored, serous fluid. This fluid may contain low concentrations of erythrocytes that give it a light red color. The fluid-filled enlargement that characterizes heavy infections is readily apparent when the skin that covers the tarsal joint is removed ( $F i g$. 10). Worms in light infections can be found by disarticulating the tarsal joint. The life cycle of $\underline{D}$. scapiceps is similar to that of $\underline{D}$. immitis.

Dirofilaria ursi was redescribed by Anderson (1952) from worms found in a black bear of Ontario. This nematode occurs in black bears and 
commonly parasitizes brown bears (U. arctos) in Alaska (Rausch 1961). The reported incidence of mature worms in ursids has ranged from $11.1 \%$ ( 3 of 27 brown bear, Yukon and British Columbia; Choquette et al. 1969) to $94.6 \%$ (140 of 148 black bears, Ontario; Addison et al. 1978). Each of 47 black bears from Minnesota examined between April and September, harbored microfilariae in the blood (Rogers 1975). Dirofilaria ursi has been found in subcutaneous tissues (Anderson 1952), around brachial and cervical lymph nodes (Anderson 1952) and in connective tissue of the perirenal and inguinal regions (Addison et al. 1978). The drugs ketamine hydrochloride and xylazine hydrochloride reportedly reduce the intensity of infections by microfilariae in the peripheral blood vessels (Addison et al. 1978).

The significance of $\underline{D}$. immitis, $\underline{D}$. scapiceps and $\underline{D}$. ursi have not been determined for Alaska.

\section{$\underline{\text { References }}$}

ADDISON, E. M., M. J. PYBUS and H. J. RIETVELD. 1978. Helminth and arthropod parasites of black bears, Ursus americanus, in central Ontario. Can. J. Zool. 56:2122-2126.

ANDERSON, R. C. 1952. Description and relationships of Dirofilaria ursi Yamaguti, 1941, and a review of the genus Dirofilaria Railliet and Henry, 1911. Trans. Roy. Can. Inst. 29:35-64.
CHOQUETTE, L. P. E., G. G. GIBSON and A. M. PEARSON. 1960. Helminths of the grizzly bear, Ursus arctos L., in northern Canada. Can. J. Zool. 47:167-170.

CRUM, J. M., V. F. NETTLES and W. R. DAVIDSON. 1978. Studies on endoparasites of the black bear (Ursus americanus) in the southeastern United States. J. Wildl. Dis. 14:178-186.

ELEY, T. J. 1981. Dipetalonema spirocauda in Alaskan marine mammals. J. Wildl. Dis. 17:65-67.

HIGHBY, P. R. 1943. Vectors, transmission, development, and incidence of Dirofilaria scapiceps (Leidy, 1886) (Nematoda) from the snowshoe hare in Minnesota. J. Parasitol. 29:253-259.

RAUSCH, R. L. 1961. Notes on the black bear, Ursus americanus Pallas, in Alaska, with particular reference to dentition and growth. Z. F. Saugetierk. 26:77-107.

ROGERS, L. L. 1975. Parasites of black bears of the Lake Superior region. J. Wildl. Dis. $11: 185-191$.

SOULSBY, E. J. L. 1968. Dirofilaria Railiet \& Henry, 1911. pp. 294-298. In: Helminths, Arthropods and Protozoa Domesticated Animals (Monnig), $6 \overline{\mathrm{th}}$ ed. Williams \&Wilkins Co., Baltimore, MD. 
Introduction

Setarial roundworm species are cosmopolitan parasites of ungulates. One of the 3 species of Setaria that occur in North America, s. yehi, has been reported from Alaskan moose (Alces alces) and reindeer (Rangifer tarandus) (Dieterich and Luick 1971). Setaria yehi has also been found in mule deer (Odocoileus hemionus) from British Columbia and bison (Bison bison) from North Dakota (Becklund and Walker 1969). Adult S. yehi live free in the abdominal cavity of ungulates; microfilariae, the larval form of these parasites, inhabit the circulatory system of ungulates and infect arthropods which serve as intermediate hosts. Some Setaria spp. are known to affect the central nervous system (CNS) of final hosts and cause a paralytic disease known as cerebro-spinal nematodiasis (Innes and Shoho 1953).

\section{Transmission}

Life cycles of Setaria spp. are incompletely understood. Adult worms give rise to microfilariae that enter the circulatory system of the definitive host. The microfilariae then infect suitable blood-sucking arthropods, i.e. mosquitos (Culex spp.), by being ingested with blood. The incubation time for microfilariae of $s$. yehi in the mosquitos is unknown. The larvae leave the mosquito when the arthropod lands on an ungulate to ingest a blood meal. The pathway taken from the site of cutaneous penetration to the site of maturation is unknown.
Signs, Symptoms and

\section{Pathogenesis}

Setaria yehi infections observed in reindeer maintained at the University of Alaska have been asymptomatic; infections were first diagnosed from blood smears collected for routine evaluations. Tentative diagnosis was confirmed through microscopic examination of sediment from blood serum for microfilariae and the presence of adult worms during necropsies. Necropsies revealed from 5 to 20 worms per reindeer. The pathologic effects of these infections included a chronic, mild peritonitis, production of 50-150 $\mathrm{ml}$ of straw colored, serous fluid, formation of fibrous adhesions between mesenteries and intestines and deposition of fibrin on the surface of the liver (Dieterich and Luick 1971).

Setaria spp. have been associated with cerebrospinal nematodiasis. This condition occurs when microfilariae are transmitted by arthropod vectors to ungulate species other than their natural (preferred) definitive hosts (Innes and Shoho 1953). Within these marginal hosts, microfilariae do not enter the circulatory system or the peritoneal cavity but tend to invade the CNS, eyes, liver, heart or lungs (Williams 1955). Complete or partial paralysis occurs in body parts that correspond to the site of localization in the CNS. The presence of immature or mature worms in neural tissue may be evidenced by localized hemorrhage and infiltration of lymphocytes, leucocytes and eosinophils. Paralysis may occur however, without these 
effects. A thorough discussion of the pathology of cerebrospinal nematodiasis has been published (Innes and Shoho 1953).

Reindeer at the University of Alaska that have harbored $\underline{S}$. yehi have never exhibited nervous disorders or reduced reproductive success attributable to these parasites. Examination of cross sections of the spinal chord and brain of infected reindeer revealed no abnormalities. Perhaps this infers that reindeer are preferred hosts of s. yehi.

\section{Significance and Control}

Setaria yehi does not appear to be a significant debilitating force in Alaskan ungulates. Though it occurs in the majority of the reindeer necropsied at the University of Alaska annually (D. Hartbauer, R. Dieterich, pers. comm.), Native reindeer herders on the Seward Peninsula have not found abdominal roundworms in animals slaughtered for meat. Fourteen caribou collected near Cantwell, Alaska, where some of the infected reindeer were held, did not reveal adult worms during gross examination (Dieterich and Luick). Two moose maintained in the immediate vicinity of the University's infected reindeer, 6 domestic cattle (Bos taurus), 2 domestic goats (Capra hir- cus), 4 horses (Equus caballus) and 2 dogs (Canis familiaris), all from the College, Alaska area, did not exhibit microfilariae in their blood in January, 1970 (Dieterich and Luick 1971).

Diethylcarbamazine administered orally to 3 reindeer at a dosage of $50 \mathrm{mg}$ per $\mathrm{kg}$ body weight for 21 days did not reduce concentrations of microfilariae in their blood. One reindeer injected intraperitoneally with an organic phosphate $(13 \mathrm{mg} / \mathrm{kg}$ body weight) did show a reduction in the number of microfilariae present in the blood (Dieterich and Luick 1971).

\section{$\underline{\text { References }}$}

BECKLUND, W. W. and M. L. WALKER. 1969. Taxonomy, hosts, and geographic distribution of the Setaria (Nematoda:Filarioidea) in the United States and Canada. J. Parasitol. 55:359-368.

DIETERICH, R. A. and J. R. LUICK. 1971. The occurrence of Setaria in reindeer. J. Wildl. Dis. $7: 242-245$.

INNES, J. R. M. and C. SHOHO. 1953. Cerebrospinal nematodiasis. Arch. Neurol. \& PHysych. 70:325-349.

WILLIAMS, H. E. 1955. Studies on the bovine filariid Setaria cervi (Rudolphi, 1810). Parasitol. $45: 56-62$. 


\author{
J. Dau and R. Barrett
}

\section{Introduction}

Trichinella spiralis is the causative agent of trichinosis, a notorious disease that infects wild and domestic animals and man over much of the world. Domestic swine (Sus scrofa) are particularly prone to infection by $T$. spiralis. This roundworm has the broadest range of mammalian hosts of all nematodes. The principal route of transmission is via the ingestion of animal flesh that contains infective larvae. Adult worms are small, slender, tapered anteriorly and blunt posteriorly. Males are 1.4-1.6 mm long; females are $3 \cdot 0-4.0 \mathrm{~mm}$ long and ovoviviparous (produce eggs that hatch internally) (01sen 1962). A thorough investigation that involved laboratory examination of tissue samples from more than 2433 Alaskan mammals has been reported (Rausch et al. 1956). During this investigation, larvae were detected in $11.7 \%$ of the total number of animals examined; infected animals represented 23 of 42 species. The results of this study are presented in Table 6 . Carnivores had the highest incidence of infection; examples are: polar bear (Ursus maritimus) $52.9 \%$ and 63.3\% (the latter percentage from $R$. Barrett, unpub. data), brown bear (Ursus arctos) $50.0 \%$; red fox (Vulpes vulpes) $40.8 \%$; and ermine (mustela erminea) $35.5 \%$. Domestic dogs in rural areas exhibited a $61.8 \%$ frequency of infection.

The specific nomenclature of the genus Trichinella is currently under discussion. Historically, spiralis has been considered to be the sole species of Trichinella. As parasitological research progressed in arctic regions however, a strain of this parasite was recognized that was apprently identical to $T$. spiralis of temperate areas in anatomy but was biologically distinct (Rausch 1970). Soviet parasitologists distinguished 2 varieties of $\underline{T}$. spiralis, I. $\underline{\text { s. var. domestica and }}$ T. s. var. nativa, the latter being the arctic form (Britov et al. 1971). Soviet researchers later raised T. $\underline{s}$. var. nativa to specific status based on genetic, ecologic and geographic characteristics (Britov and Boev 1972).

Evidence is accumulating that supports distinguishing the arctic strain of Trichinella from strains indigenous to temperate or tropical regions (Barus et al. 1979, Belosevic and Dick 1979). The question of whether these differences signify the description of $T$. nativa has not been resolved however (Bessunov et al. 1976, Madsen 1976). A comprehensive taxonomic review of the specific nomenclature of Trichenella appears to be warranted. Irrespective of its nomenclatural status, a distinct strain of Trichinella, unique in host specificity and pathogenicity, occurs throughout northern Alaska. The range of this arctic organism should be delimited to determine its southern extent. Trichinella organisms of both arctic and temperate strains probably occur in Alaska (R. Barrett, pers. obs.).

\section{History}

The broad host spectrum of $T$. spiralis could infer that this parasite is phylogenetically very old in relation to other parasitic nematodes (Merkushev 1955). Epidemics described in man resembling trichinosis were reported as early as 
TABLE 6

The Prevalence of Trichinella spiralis in Alaskan Mammals Between 1949 and 1953 (Summarized from Rausch et al. 1956)

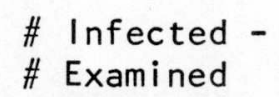

\# Infected -
Av. \# Larvae g Tissue

\section{Soricidae \\ dusky shrew (Sorex monticolis \\ $=$ S. obscuris) \\ masked shrew ( S. cinereus) \\ arctic shrew ( $\bar{s}$. $\overline{\text { arcticus }}$ \\ $=\underline{s}$. tundrens $i \bar{s})$
Ochotonidae
collared pika (Ochotona
collaris)

\section{Leporidae \\ snowshoe hare (Lepus \\ americanus) \\ arctic hare (ㄴ․ arcticus)}

Sciuridae

arctic ground squirrel

(Spermophilus parryii)

$=$ (Citellus undulatus

red squirrel (Tamiasciuris

hudsonicus)

hoary marmot (Marmota

cal igata $=\underline{M}$. $\underline{\text { marmota }}$

caligata)

flying squirrel (Glaucomys sabrinus)

Castoridae

beaver (Castor canadensis)

Cricetidae

brown lemming (Lemmus

sibiricus)

red-backed vole (Clethrionomys

rutilus)

vole (Microtus miuris)

muskrat (Ondatra zibethicus)

collared lemming (Dicrostonyx

torquatus)

meadow (field) vole (Microtus

pennsylvanicus)

tundra vole (… oeconomus)
$0 / 18$

0

$0 / 13$

0

$2 / 53(3.8 \%)$

$0 / 191 * * *$

7.2

0

$1 / 129(0.8 \%)$

0.3

$4 / 94(4.3 \%)$

0.7

$0 / 9$

0

$0 / 1$

0

$1 / 29(3.4 \%)$

3.0

$1 / 18(0.8 \%)$

0.1

$2 / 49(4.0 \%)$

$1 / 57(1.8 \%)$

$1 / 113(0.9 \%)$

0.5 and 0.7

not reported

1.3

\section{$0 / 7$}

0

$0 / 10$

$0 / 234$
0

0 
TABLE 6 (Cont.)
\# Infected -
Av. \# Larvae -
\# Examined
g Tissue

\section{Muridae \\ Norway rat (Rattus \\ norvegicus)}

Erethizontidae

porcupine (Erethizon dorsatum)

Monodont i dae

belukha ( = beluga = white

whale (Delphinapterus leucas)

Balaenidae

bowhead whale (Balaena

mysticetus) (not confirmed)

\section{Canidae}

domestic dog (Canis familiaris)

(Anchorage)

domestic dog (‥ familiaris)

(rural Alaska)

coyote (C. latrans)

wolf ( . . lupus)

red fox (Vulpes vulpes)

arctic fox (Alopex lagopus)

Ursidae

brown bear (Ursus arctos)

black bear (U. americanus)

polar bear ( $\underline{\text { U. }}$ maritimus

= Thalarctos maritimus)

Mustel idae

ermine (Mustela erminea)

least weasel (M. nivalis

$=$ M. rixosa)

woT̄verine (Gulo gulo)

marten (Martes americana)

mink (Mustela vison)

sea otter (Enhydra lutra)

river otter (Lutra canadensis)

Felidae

lynx (Felis lynx)
$28 / 261(10.7 \%)$

not reported

$0 / 3$

0

$1 / 49(2.0 \%)$

0.6

$0 / 15$

0

$0 / 60$

$$
\begin{array}{rr}
102 / 165 & (61.8 \%) \\
1 / 8 & (12.5 \%) \\
51 / 154 & (33.1 \%) \\
31 / 76 & (40.8 \%) \\
16 / 222 & (7.2 \%)
\end{array}
$$

$10 / 20 \quad(50.0 \%)$

12.4

$195 / 308(63.3 \%) * *$

9/17 (52. 9\%)

(7.0 av.)

1.6

$195 / 308(63.3 \%) * *$

$18 / 51(35.3 \%)$

4.0 to 0.7

45.0

3.3

8.4

22.0

$2 / 2(100.0 \%)$

$19 / 38(50.0 \%)$

$0 / 18$

$0 / 43$

$0 / 20$

$0 / 7$

0.1 to 75.0

(7.0 av.) 16.4

0.8 and $1400+$

3.5

0

0

0

$4 / 17(23.5 \%)$
7.2 
TABLE 6 (Cont.)
\# Infected -
\# Examined
Av. \# Larvae -
g Tissue

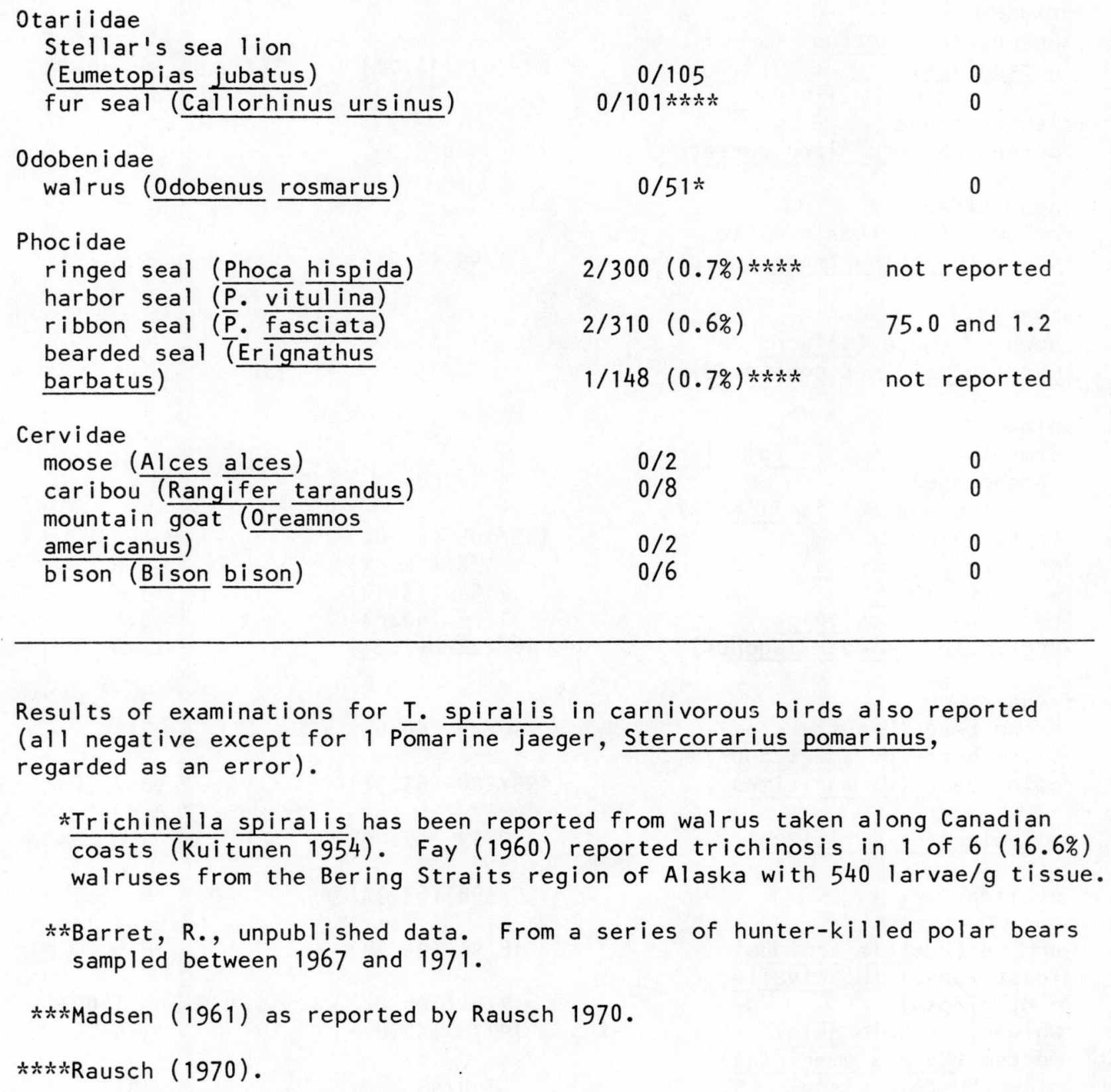

$0 / 2$

$0 / 8$

0

$0 / 2$

$0 / 6$

0

0

Results of examinations for I. spiralis in carnivorous birds also reported (all negative except for 1 Pomarine jaeger, Stercorarius pomarinus, regarded as an error).

*Trichinella spiralis has been reported from walrus taken along Canadian coasts (Kuitunen 1954). Fay (1960) reported trichinosis in 1 of 6 (16.6\%) walruses from the Bering Straits region of Alaska with 540 larvae/g tissue.

$* *$ Barret, R., unpublished data. From a series of hunter-killed polar bears sampled between 1967 and 1971 .

$* * *$ Madsen (1961) as reported by Rausch 1970.

$\star * * *$ Rausch $(1970)$. 
427 B.C. by the Carthaginians. Larvae were first discovered in humans during 1835 by J. Paget. In 1846, animals were recognized as suitable hosts when larvae were recoved from a pig. The first epidemiological breakthrough in trichinosis was made by Zenker in 1860 when he discovered the intestinal and muscle phases of the disease. The presence of T. spiralis in Alaskan Eskimos was suggested in 1914 when several villagers died from a malady resembling trichinosis shortly after eating a belukha whale (Delphinapterus leucas). The presence of I. spiralis in Alaska was confirmed in 1934 when encysted larvae were discovered in polar bear and arctic fox (Parnell 1934 as reported by Fay 1960). Outbreaks of trichinosis in humans that were linked to the ingestion of walrus (Odobenus rosmarus), dogs (Canis familiaris), black bear (U⿳亠口冋rsus americanus) and brown bear in Greenland (Thorborg 1948 as reported by Maynard and Kagan 1963) and Alaska emphasized the importance of wildlife as a source of human infection. It is now realized that the sylvatic (wild) and pastoral (domestic) forms of trichinosis interact in a dynamic, relationship.

\section{$\underline{\text { Transmission }}$}

Trichinella spiralis usually follows a direct 1 ife cycle consisting of 2 phases: 1) an intestinal phase during which third stage larvae copulate and females give birth to first stage larvae, and 2) a muscle phase in which first stage larvae invade muscle tissue.

Terrestrial mammals are infected by ingesting flesh that contains encysted third stage larvae (Fig. 9). It has been suggested that scavenging facilitates the ingestion of $I$. spiralis larvae more than does predation (Merkushev 1955). Since most recognized predators are also facultative scavengers and vice versa, this distinction appears to be moot with respect to The ecology of $I$. spiralis. That scavenging does provide a significant route of infection for carnivores is an important distinction for management and control purposes. Experimental transmission of larvae from feces to domestic swine has been reported though is probably not significant since few viable larvae pass in the feces of host animals (Soulsby 1968). Transmission by scavenger beetles has also been reported; larvae survived 5 to 8 days in these insects (Merkushev 1955 as reported by Zimmerman 1971). Prenatal infection is rare but has been experimentally produced (Soulsby 1968).

Modes of transmission for $I$. spiralis in marine ecosystems are not completely understood. Three possible routes of infection for marine mammals are: 1) direct ingestion of meat that contains infective larvae (carnivory), 2) accidental ingestion of small pieces of detrital meat that contain larvae, and 3 ) ingestion of larvae in vector organisms or their excretia. These routes of transmission are not mutually exclusive and the epidemiology of trichinosis may involve components of each.

Walrus are occasionally carnivorous and therefore could ingest T. spiralis larvae with infected meat. No conclusive evidence has been found to support this hypothes is however (Fay 1960). Belukha whales are predatory but mainly eat fish, organisms in which larvae do not encyst but which may temporarily harbor larvae (Kozlov 1971). Ringed and bearded seals are not known to ingest 


\section{Figure 9.}

\section{Trichinella spiralis}

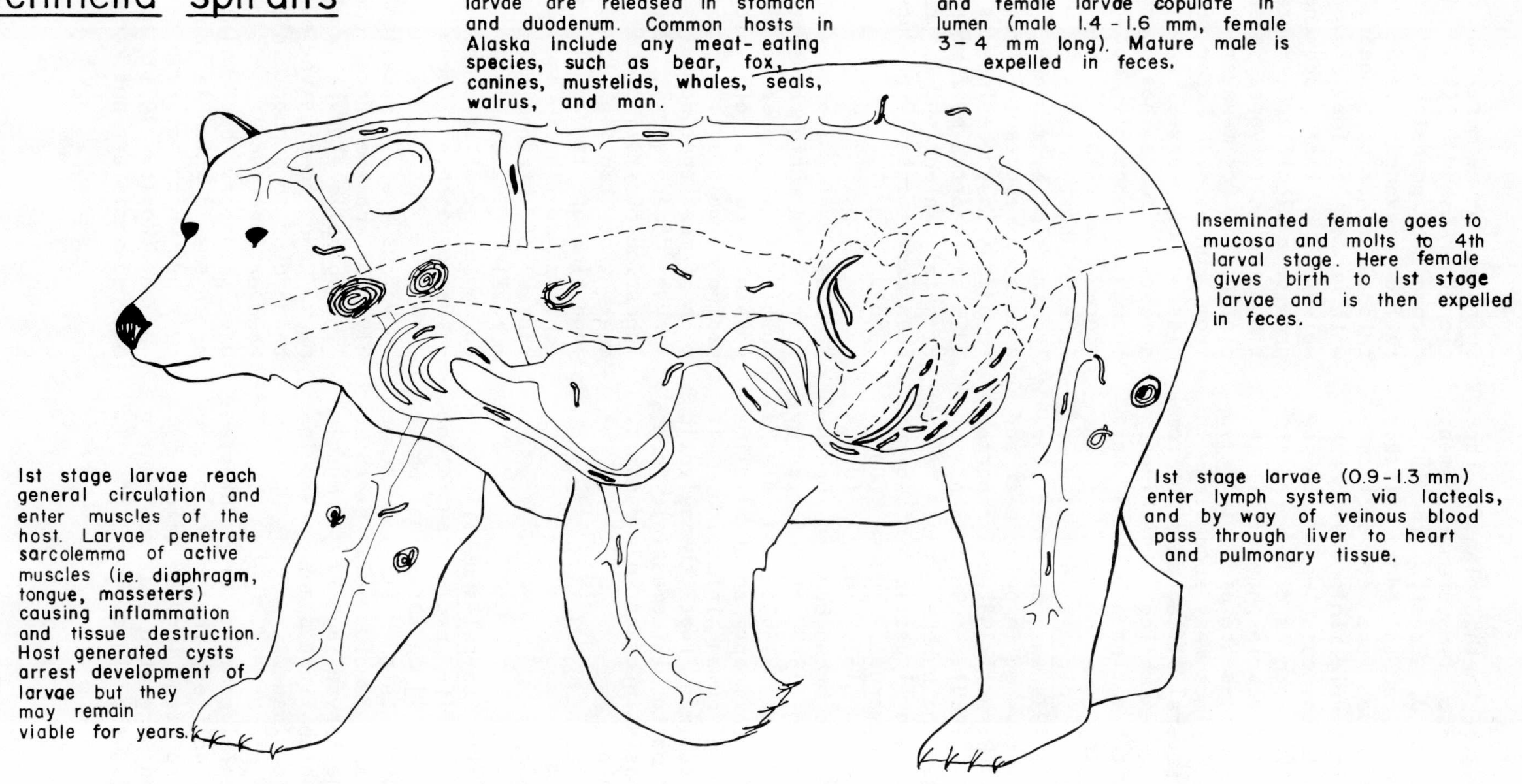

Figure adapted from Olsen, O.W. 1962. Animal Parasites.

Burgess Publishing Co., Minneapolis, MN. p. 331 . 
meat under any circumstances (Fay 1960,1968 ) which precludes carnivory as a mode of transmission.

Amphipods feed extensively on immersed carrion. Non-predatory seals and walruses are attracted to these carcasses by the swarms of feeding amphipods. It is feasible that freefloating bits of meat that contain larvae are accidentally ingested as the amphipods are preyed upon by the seals (Fay 1960, 1968). Carcasses of terrestrial or marine mammals or garbage could provide sources of infection.

The role that vector species may have in the transmission of $T$. spiralis has been examined by severál researchers (Britov 1962, Fay 1968, Hulebak 1980, Kozlov 1971). Birds were experimentally shown to pass infective larvae to fish that, when ingested by a dog and cat (Felis catus), produced encysted larvae (Britov 1962). The validity of this experiment has been questioned however (Fay 1968). Fish and amphipods (i.e. Anonyx spp. and Apherusa glacialis) (Hulebak 1980) can transmit infective larvae to suitable mammalian hosts (Fay 1968, Kozlov 1971). Experimental results indicate that approximately $1 \%$ of the amphipods in a swarm that feeds upon flesh containing about 127 larvae/g tissue can ingest and harbor larvae undigested up to 28 hours (Hulebak 1980). The low frequency of larvae in amphipods could explain the low incidence of infection observed in most marine mammal species (see Table $6)$. It has been suggested that larvae which leave their capsule (excyst) in the digestive tract of an intermediate host are not infective if ingested a second time (Kozlov 1971). Other researchers have reported that excysted larvae are presumably infective (Fay 1968). If excysted larvae are not infective to definitive hosts, the capacity of vector organisms to transmit $T$. spiralis would be greatly diminished.

After the first stage larvae have been ingested with infected meat, they are released from their cysts by digestive processes in the stomach and duodenum. The first stage larvae enter the intestinal mucosa and molt twice to reach the third larval stage. These neotenic larvae (they reproduce before reaching maturity) re-enter the intestinal lumen where copulation occurs. Males are expelled from the host soon after copulation. Inseminated females return to the intestinal mucosa, molt to the fourth larval stage and mature. Females give birth to the first stage larvae while in the intestinal mucosa. They are then expelled from the host; female worms are thought to infect their hosts no longer than 5 to 6 weeks post ingestion (Soulsby 1968).

The first stage larvae enter the lacteals (intestinal lymph nodes), reach the veinous blood supply and are transported through the liver and thoracic organs to muscle tissue. The larvae leave the circulatory system through capillaries that supply muscle tissue; larvae exhibit a prediliction for active muscles, i.e. the diaphragm, tongue and masseters. Larvae penetrate the sarcolemma (a thin sheath of connective tissue that surrounds muscle fibers) as early as 5 days after ingestion of their parents in certain host species (Zimmerman 1971). Individual larvae are usually encapsulated in host generated connective tissue though intense infections may have several larvae within individual cysts. The time required to encyst larvae varies between host species. Larval development is arrested once 
the cysts are completely formed. Larvae are thought to generally live several years in their hosts though infections that have persisted as long as 24 years have been reported (Soulsby 1968). The life cycle is complete when larvae are ingested by a suitable carnivorous mammal.

\section{Signs, Symptoms and Pathogenesis}

Three phases of trichinosis infections are distinguished: 1) an intestinal phase during which ingested third stage larvae sexually mature, copulate and give birth to a new generation of first stage larvae, 2) a muscle phase when newborn larvae leave the intestinal tract of the host to invade muscle fibers and 3 ) a convalescent stage in which host animals recuperate.

The pathogenicity of the intestinal phase of trichinosis is positively correlated with the dosage of larvae ingested by the host. Larvae that enter the intestinal mucosa cause enteritis as evidenced by a thickened, hyperemic (engorged with blood) mucosa and small hemorrhages where larvae have penetrated. The mesenteric lymph nodes become soft and swollen (Zimmerman 1971).

The severity of trichinosis intensifies during the muscle phase of this disease. As larvae invade the sarcolemma, muscles become inflamed. One to 2 days after larvae invade the muscles, microscopic lesions appear due to the degeneration of granulated white blood cells. Cross striation of muscle fibers disappear. Two to 3 weeks post infection the muscle fibers usually exhibit edema (an accumulation of body fluids) and appear swollen. The swelling of parasitized muscle fibers exert pressure on con- tiguous, non-parasitized muscle tissue and stimulates degeneration of these areas as well ( $\mathrm{i}$ immerman 1971). Leukocytes, lymphocytes and macrophages usually appear by the second to third week of muscle invasion. Parasitized muscle tissues permanently degenerate (Merkushev 1955). This consequence of trichinosis is often fatal to host animals.

Convalesence begins with the formation of host-generated cysts that isolate destructive larvae within the host. Cysts consist of an outer capsule of connective tissue and thickened sarcolemma and an inner portion that contains larvae and detritus from destroyed muscle tissue. As larvae are encysted, the edematous swelling recedes and a network of connective tissue replaces muscle tissue that has been destroyed. Calcification of cysts may begin as soon as 3 months post infection; this usually, but not always, results in the death of encapsulated larvae (Zimmerman 1971).

\section{Diagnosis}

Laboratory examinations are particularly important for diagnosing trichinosis. This is due to the complexity of clinical symptoms, the relatively short life span of $T$. spiralis in the intestinal phase, the diminutive size of encysted larvae $(0.9-1.3 \mathrm{~mm}$ long and up to $0.4 \mathrm{~mm}$ wide) (Zimmerman 1971) and the absence of eggs and low proportion of larvae that pass in the feces of host animals.

Post mortem examinations involve the detection of encysted larvae within muscle tissue. Preferred sources of tissue samples are diaphragm, tongue and masseter 
muscles. Three techniques are commonly employed for detecting encysted larvae. These are: 1) the compression method, 2) the artificial digestion method and 3 ) the microscopic tissue section (histologic) method.

The compression method is the simplest and most rapid technique. A $1.0 \mathrm{~g}$ sample of muscle tissue is cut into particles the size of wheat grains, compressed between 2 glass plates and examined microscopically for larvae. The reliability of this method depends heavily on the number of $1 \mathrm{~g}$ samples taken from each animal; low intensity infections may easily be overlooked when sampling effort is low.

The artificial digestion method is used extensively in wildlife investigations (Rausch et al. 1956, Soulsby 1968). Tissue samples, approximately $100 \mathrm{~g}$ each, are removed from animals suspected of harboring T. spiralis. These samples are digested in a solution of pepsinhydrochloric acid. The sediment is concentrated by allowing it to stand undisturbed or through centrifugation. The supernatant is removed and the sediment examined microscopically for larvae. A weakness of this technique is that some larvae may be destroyed during the digestive process and thus, bias estimates of intensity of infection. The larger sample unit of tissue that is examined makes this technique less apt to overlook larvae in mild infections.

Histologic examination for larvae involves sectioning muscle tissue samples into thin slices, staining them and microscopically looking for portions of larvae. Like the compression method, this technique tends to overlook larvae when infections are not intense and sampling effort is low.

Biopsy techniques (removal of tissues or fluids from living animals)
(Hulebak 1980) for detecting T. spiralis include hematology (examination of blood serum). Several publications provide detailed descriptions of these diagnostic techniques (Fay 1968, Gould 1945, Merkushev 1955). Scholtens et al. (1966) provides thorough evaluation of diagnostic techniques used for detecting trichinosis in swine.

\section{Significance and Control}

Trichinella spiralis commonly infects a variety of Alaska mammals (Table 6) though infections are usually subclinical. Trichinosis is a significant disease in Alaska for 2 reasons however: 1) a large proportion of animal species present in the arctic are at least facultatively carnivorous which facilitates transmission of T. spiralis larvae (Rausch et al. 1956) and 2) humans, both $\mathrm{Na}$ tive and White, rely upon wildlife hosts of T. spiralis, i.e. bear and marine mammāls, for important sources of protein. Within Alaska, a strong latitudinal trend in the frequency of infections has been reported (Rausch et al. 1956). Twenty-five of 54 $(80.6 \%)$ red foxes collected in the Brooks Range harbored T. spiralis; only 2 of $13(15.4 \%)$ red foxes taken from the Copper River drainage were thus infected. Ermine (Mustela erminae) showed a similar pattern: 17 of 40 (42.5\%) Brooks Range ermine versus 1 of 11 (9.9\%) Copper River ermine harbored T. spiralis. This trend was not observed in a comparison of wolverines that originated from the 2 areas. Trichinella spiralis occurred more frequently in dogs from rural areas (61.8\%) than urban areas $(0 \%)$ (Rausch et al. 1956).

Most infections in man have occurred in the northern portions of the state (Rausch et al. 1956). Wildlife species attributed as sources of human infection include black, brown and polar bears and 
belukha whales. Several seal species, i.e. the bearded seal (Erignathus barbatus) and harbor seal (Phoca vitulina) and walrus (0dobenus rosmarus), may represent reservoirs of infective T. spiralis larvae for man as well. Transmission between the sylvatic and pastoral forms of trichinosis is not a one way process from wildlife to man. Open pit dumps are considered significant sources of larvae for wildlife species ( $Z$ immerman 1971), i.e. Norway rats (Rattus norvegicus) and black/brown bears. Many coastal villages of Alaska create dumps on near-shore ice that later go to sea and contaminate marine ecosystems. Domestic animal carcasses known to harbor I. spiralis should be destroyed in a way that precludes infection of wildlife (i.e. by incineration).

Thiabendazole, methyridine and several other drugs are available that effectively eliminate larval and adult $T$. spiralis organisms in man and añimals (Soul sby 1968, Zimmerman 1971). Efficient administration of these therapeutic agents to wildlife populations has not yet been developed. Freezing meat for 20 days at $-15 \mathrm{C}$ or 1 day at $-18 \mathrm{C}$ kills the $\mathrm{T}$. spiralis larvae presumably found in domestic animals in temperate regions as does heating meat to $55 \mathrm{C}$ (Maynard 1968). The Trichinella organisms from arctic regions appear to be more resistant to freezing however (R. Barrett, pers. obs.), a significant distinction in the epizootiology of this disease.

\section{References}

BARUS, V., F. TENORA, R. WIGER, T. GENOV and S. KOMANDAROV. 1979. Scanning electron miscroscope studies on males of Trichinella spp. Folix. Parasitol. 26:97-101 (From Bio. Abstr. 1980 69:65336).
BELOSEVIC, M. and T. A. DICK. 1979. Comparison of stages in host intestine with those of an Arctic Trichinella sp. Exp. Parasitol. 48:432-446.

BESSONOV, A. S., R. A. PEN'KOVA. 1976. The strains and species of Trichinella, their role in the epizootiology of trichinelliasis in pigs. Veterinariya 10:47-50.

BRITOV, V. A. 1962. On the role of fishes and crustaceans in the transmission of Trichinella to marine mammals. Zool. Zhur. $41: 776-777$.

BRITOV, V. A. and S. N. BOEV. 1972. Taxonomic rank of various strains of Trichinella and their circulation in nature. Vest. Akad. Nauk Kazak. SSR 28:27-32.

BRITOV, V. A., G. A. ERMOLIN, V. I. TARAKONOV and T. L. NIKITINA. 1971. Genetic isolation of two Trichinella variants. Med. Parazitol. Parazit. Bolezn. 40:515-521. (From Bio. Abstr. 1973 55:31814).

FAY, F. H. 1960. Carnivorous walrus and some arctic zoonoses. Arctic 13: $111-122$.

- 1968. Experimental transmission of Trichinella spiralis via marine amphipods. Can. J. Zool. 46:597-599.

GOULD, S. E. 1945. Trichinosis. Charles C. Thomas, Springfield, IL. $356 \mathrm{pp}$.

HULEBAK, K. L. 1980. Mechanical transmission of larval Trichinella by arctic crustacea. Can. J. Zool. 58:1388-1390.

KOZLOV, D. P. 1971. Sources of Trichinella infection in pinnipeds. Trudy Gel'mint. Lab. Akad. Nauk SSSR 21:36-40. (Fisheries Res. Board Can. Trans. Ser. No. 3010. 8 pp.).

KUITUNEN, E. 1954. Walrus meat as a source of trichinosis in Eskimos. Can. J. Pub. Health 45:30. 
MADSEN, H. 1961. The distribution of Trichinella spiralis in sledge dogs and wild mammals in Greenland under a global aspect. Medd. Grom. 159:1-124.

1976. The life cycle of Trichinella spiralis (Owen, 1835) Railliet, 1856 (Syns. T. nativa Britov et Boev, 1972, T. nelsoni Britov et Boev, $197 \overline{2, \quad T}$. pseudospiralis Garkavi, 1972), with remarks on epidemiology, and a new diagram. Acta. Parasitol. Polonica 24:143-158 (From Helminthol. Abstr. 1977 46:4009).

MAYNARD, J. E. 1968. Trichinosis. pp. 130-131. In: Current Diagnosis 11 . H. F. CONN and R. B.

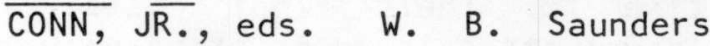
Co., Toronto, ONT.

MAYNARD, J. E. and I. G. KAGAN. 1963. Trichinosis. Practitioner $191: 622-629$.

MERKUSHEV, A. V. 1955. Concerning the rotation of Trichinella invasions in nature and their natural foci. Med. Parazitol. Parazitarn. Bolezni 2:125-130.

OLSEN, 0. W. 1962. Trichinella spiralis. pp. 328-332. In: Animal Parasites. Burgess Publishing Co., Minneapolis, MN.

PARNELL, I. W. 1934. Animal parasites of North-East Canada. Can. Field Nat. 48:111-115.
RAUSCH, R. L. 1970. Trichinosis in the arctic. pp. 348-373. In: Trichinosis in Man and Animals. S. E. GOULD, ed. C. Thomas, Pub. Springfield, IL.

RAUSCH, R., B. B. BABERO, R. V. RAUSCH and E. L. SCHILLER. 1956. Studies on the helminth fauna of Alaska. XXVII. The occurrence of larvae of Trichinella spiralis in Alaskan mammals. J. Parasitol. 42:259-271.

SCHOLTENS, R. G., I. G. KAGAN, K. D. QUIST and L. G. NORMAN. 1966. An evaluation of tests for the diagnosis of trichinosis in swine and associated quant itative epidemiologic observations. Am. J. Epidemiol. 83:489-500.

SOULSBY, E. J. L. 1968. Trichinella Railliet, 1895. pp. 314-318. In: Helminths, Arthropods and Protozoa of Domesticated Animals (Monnig). 6th ed. Williams \& Wilkins Co., Baltimore, MD.

THORBERG, N. V., S. TULINIUS and H. ROTH. 1948. Trichinosis in Greenland. Acta Path. Microbiol. Scand. 35:778-794.

ZIMMERMAN, W. J. 1971. Trichinosis. pp. 127-139. In: Parasitic Diseases of Wild Mammals. J. W. DAVIS and R. C. ANDERSON, eds. lowa State Univ. Press, Ames. IA. 


\section{Acanthocephala}

J. Dau

Parasites of the phylum Acanthocephala are unsegmented, cylindrical and dioecious (separate sexes). The body is conceptually divided into 2 main parts: 1) the presoma which includes the hook-bearing proboscis (hence "spiny-headed") and neck and 2) the trunk which contains the excretory and reproductive systems. Spiny-headed worms lack a digestive tract; nutrients are absorbed through the tegument. The proboscis, the feature that most easily distinguishes this group of parasites, may not be evident in preserved specimens for 2 reasons. First, it may fail to completely evert if not left in fresh water temporarily before being fixed in formalin. Second, it may have been deeply embedded in tissue and was torn from the parasite when collected.

The eggs of acanthocephalans are embryonated when passed in the feces of the definitive host; the embryo is termed an acanthor. The eggs are highly resistant to environmental stress and hatch only after being ingested by an intermediate host (all acanthocephalans utilize at least 1 intermediate insect or arthropod host). Hatching releases the acanthor and allows it to penetrate the gut wall of the intermediate host. The acanthor then enters the body cavity where it progressively develops into a preinfective larval stage termed the acanthella. Further development and encystment of the acanthella produces the cystacanth stage. Cystacanth larvae are infective for their final hosts. This larval stage is adept at re-encysting within organisms (paratenic hosts) that have ingested cystacanths, but are not suitable hosts for the larvae to complete development within. When cystacanths are ingested by a suitable (preferred) host, they are released from their hyaline cysts (clear, structureless cysts) by digestive processes and attach to the intestinal mucosa of the host where development is completed.

The genus Corynosoma is the sole acanthocephalan parasite currently thought to be significant in Alaskan wildlife. 


\section{Corynosoma}

J. Dau

The paucity of information concerning Corynosoma spp. necessitates a relatively brief coverage of this genus. Corynosoma spp. are holarctic (Rausch 1953) and commonly parasitize marine mammals. Table 7 lists the mammalian hosts from which individuals of Corynosoma spp. have been documented in Alaska.

Corynosoma spp. follow the general life cycle outlined in the introduction of Acanthocephala. The first intermediate host of some Corynosoma

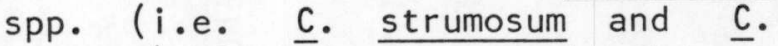
semerme) is thought to be the amphipod Pontoporeia affinis (De Giusti 1971). 0ther amphipods may be suitable intermediate hosts as well. Fish, i.e. the rainbow smelt (0smerus dentex), reportedly serve as paratenic hosts in which cystacanths encyst on the mesenteries (Neiland 1962). Mature individuals of Corynosoma spp. evoke localized tissue response in the small intestine of their definitive host. It has been suggested that considering the well-armed proboscis of these parasites, intense infections could result in severe inflammation and fibrosis of affected tissues (Rausch 1953). The significance of these parasites in Alaskan mammals has not been established. $\underline{\text { References }}$

DE GIUSTI, D. C. 1971. Acanthocephala. pp. 140-157. In: Parasitic Diseases of Wild mammals. J. W. DAVIS and R. C. ANDERSON, eds. Iowa State Univ. Press, Ames, IA.

GOLVAN, Y. J. 1959. Acanthocephales du genre Corynosoma Luehe 1904 parasites de mammiferes d'Alaska et de Midway. Ann. Parasitol. Hum. Comp. 34:288-321.

NEILAND, K. A. 1961. Suspected role of parasites in non-rookery mortality of fur seals (Callorhinus ursinus). J. Parasitol. 47:732.

- 1962. Alaskan species of acanthocephalan genus Corynosoma Luehe, 1904. J. Parasitol. $48: 69-75$.

RAUSCH, R. 1953. Studies on the helminth fauna of Alaska. XIII. Disease in the sea otter, with special reference to helminth parasites. J. Ecol. 34:584-604.

SCHMIDT, G. D. and L. S. ROBERTS. 1977. Acanthocephala in humans. p. 523. In: Foundations in St. Louis, MO.

VAN CLEAVE, H. J. 1953. A preliminary analysis of the acanthocephalan genus Corynosoma in mammals of North America. J. Parasitol. 39:1-13. 


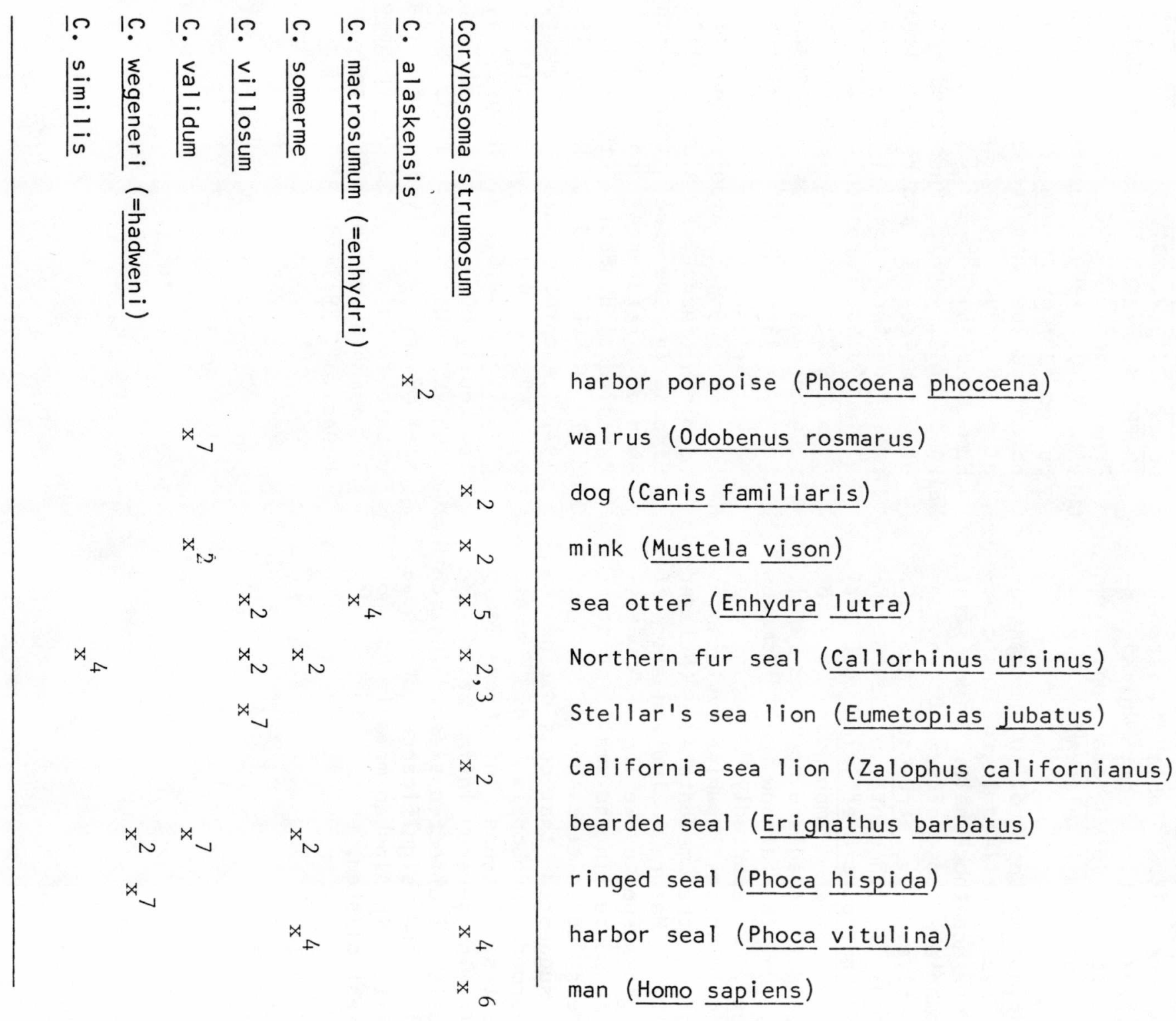




\section{Protozoa}

J. Dau

Protozoans are primitive, single celled, eukaryotic (possessing at least 1 nucleus) organisms contained within a cell membrane. At least 45,000 species in this phylum have been described since Leeuwenhoek employed his primitive magnifying lenses to discover Eimeria stiedae late in the 16th century. Protozoans have evolved to fill a variety of diverse parasitological niches.

One factor partially responsible for the success of protozoan parasites is their simplicity. Organelles function as organs without limiting the ecological distribution of protozoans to the extent that highly adapted functional systems (i.e. digestive or excretory systems) limit vertebrate or even helminth organisms. Protozoan parasites propel themselves with either flagella, cilia, pseudopodia or undulating ridges. Parasites acquire nutrients by ingesting particulate matter or through diffusion. Protozoans are capable of sexual or asexual reproduction. Sexual reproduction may occur by self or cross fertilization. A variety of asexual means of reproduction are known; examples are:
1) schizogony (multiple fission), 2) sporogony (multiple fission following the union of gametes) and 3 ) budding. Many protozoan parasites secrete a highly resistant capsule termed a cyst. This cyst is highly resistant to environmental stress and usually represents the life stage when transmission between hosts occurs.

Protozoan parasites are reportedly the most pathogenic group of organisms in the world (Schmidt and Roberts 1977). Since these organisms are not visible to wildlife field biologists and require a greater dependence on laboratory examination than helminth parasites, protozoans have received only cursorial coverage in this text. The brevity of this section should not be interpreted as representing the significance of protozoan parasites in Alaskan wildlife.

\section{$\underline{\text { References }}$}

SCHMIDT, G. D. and L. S. ROBERTS. sitology. $\frac{\text { Foundations }}{\text { C. V. Mosby }} \frac{\text { of }}{\text { Co. }} \frac{\text { Para- }}{\text { St. }}$ Louis, MO. 


\title{
Trypanosoma
}

\author{
Newton Kingston
}

\section{Introduction}

Trypanosomes are flagellated, protozoan parasites found in the blood and in some cases, in the tissues of all classes of vertebrates (Fig. 11). A single species, Trypanosoma cervi Kingston and Morton, 1975 has been described from members of the deer family, the Cervidae: viz., from elk (Cervus elaphus), mule deer (Odocoileus hemionus), white-tailed deer (ㅁ. virginianus), moose (Alces alcess) and reindeer (Rangifer tarandus) in North America. Another species, Trypanosoma theileri Laveran, 1902 is known from cattle (Bos taurus) in North America (and worldwide) and it is probably this species that affects muskox (Ovibos moschatus) and bison (Bison bison) in the New World. since the parasite of muskox and bison are scanty in the blood of the infected hosts, numbers of these parasites sufficient for identification have not yet been collected.

\section{History}

Trypanosomes were first found in the family Cervidae in 1969 in blood collected and cultured from whitetailed deer in the southeastern United States; about $75 \%$ of deer examined from 7 states were infected. Culturing of blood collected from mule deer in 1972 to 1974 (Clark 1972, Kingston et al. 1975) (with up to $80 \%$ infected) and from elk in 1973 (with up to $90 \%$ infected) (Davies and Clark 1974, Kingston and Crum 1977, Kingston and Morton 1973) from the western U.S. revealed trypanosomes in these species also. After recovery and measurement of bloodstream trypomastigotes, the species of trypanosome in elk was described as Trypanosome cervi by Kingston and Morton in 1975 (Kingston and Morton 1975). Subsequently trypanosomes from mule deer, white-tailed deer and moose were collected and measured by similar methods and were identified as conspecific with $\underline{T}$. cervi (Kingston and Crum 1977, Kiñgston et al. 1975, 1981, Matthews et al. 1977). Since that time, trypanosomes have been collected and cultured from moose, reindeer and muskox (Alaska). The limited numbers of trypanosomes on slides made from blood of these species preclude their identification at present. The collection, description and identification of larger numbers of trypanosomes from reindeer allows their inclusion in this report. The species of trypanosome from reindeer is considered conspecific with I. cervi, thus adding another host species in the family Cervidae to those known to harbor this species of trypanosome. (Descriptions of the trypanosomes from reindeer and muskox will appear el sewhere.)

\section{Transmission and \\ Pathogenesis}

Transmission of trypanosomes from mammalian host to mammalian host is generally by way of an arthropod vector. Keds (Diptera:Hippoboscidae) of sheep and goats have been implicated in transmission of trypanosomes ( $T$. melophagium and $\mathrm{T}$. theodor $\bar{i}$, respectively) of these hosts, and horseflies (Diptera:Tabanidae) are known to transmit the trypanosome of cattle in North America (Hoare 1972). Horseflies have been found infected 

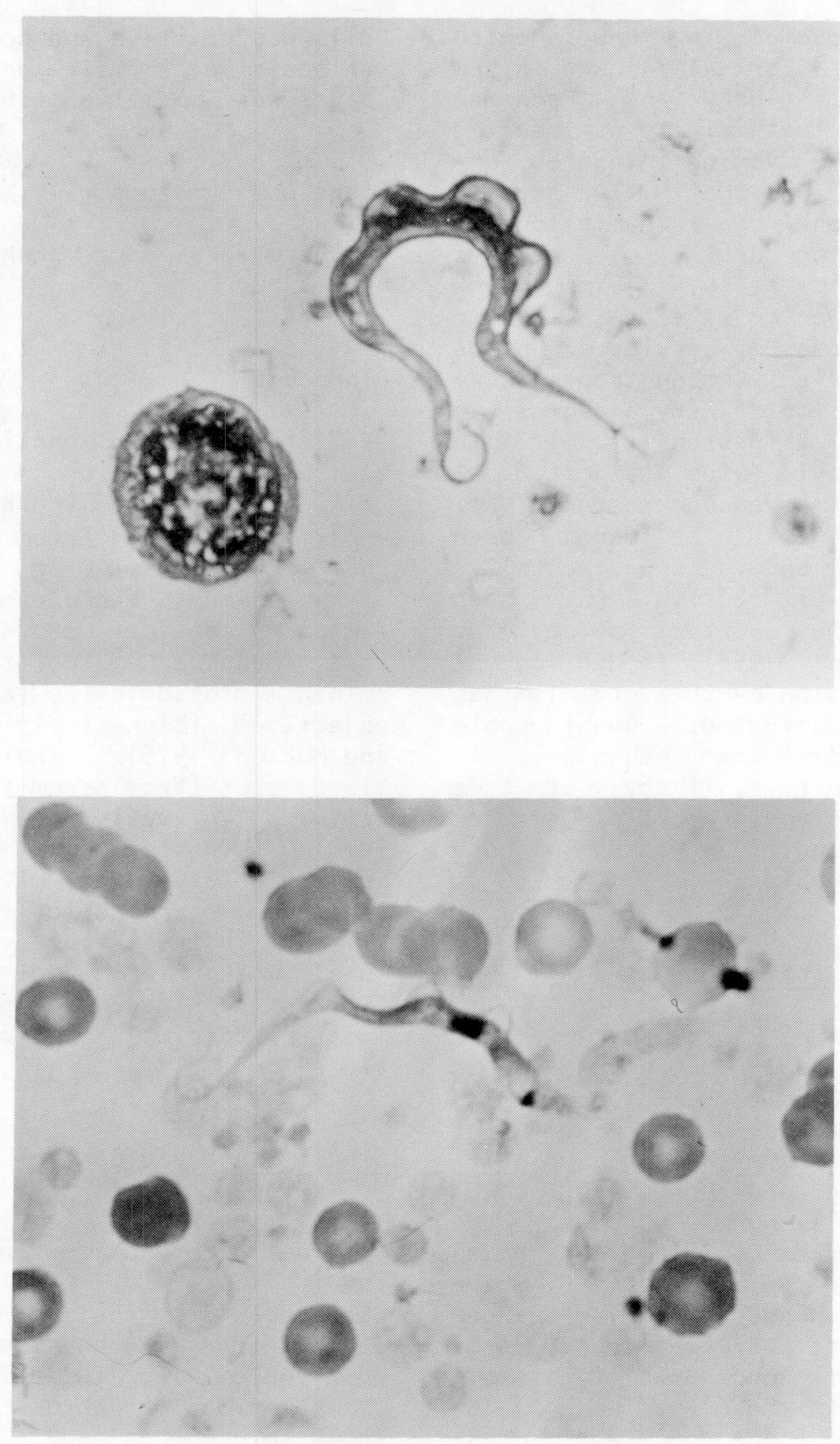

Figure 11. Trypanosomes in blood samples taken from reindeer (Rangifer tarandus (top) and muskox (Ovibos moschatus) (bottom). The anterior flagella is apparent on the left-most end of the organisms in both pictures (N. Kingston). 
with trypanosomes in New Mexico (Davies and Clark 1974) and in New York State (Krinsky and Pechuman 1975) and in the vicinity of infected captive elk in Wyoming (Kingston, unpub. data). No definitive studies have been done on transmission of trypanosomes of cervids.

No pathogenic effects are known for trypanosomes of cervids or bovids in North America, though transplacental transmission of trypanosomes to the cervid (Kingston et al., in press) and bovid fetus (Kingston et al., in press, Van Der Maaten et al. 1973) is known to occur both naturally and experimentally. Trypanosomes ( $T$. theileri) have been found in the aborted bovine fetus (Dikmans et al. 1957) though causation of abortion by this organism has not been demonstrated. Considerable further work is necessary to establish the role, if any, of this organism in cryptic abortion or other pathogenic conditions seen in both cervids and bovids.

\section{Incidence of Infection}

Twenty-one of 24 reindeer examined near Fairbanks in 1979 were detected as infected by direct microscopic examination of the blood (DE vide infra); 11 of 14 adults were positive (11 positive and 2 negative cows, 1 negative bull) and 10 fawns were positive ( 7 male, 3 female). Seven of 13 moose ( 4 captive, 9 freeranging) examined in Wyoming between 1977-1980 were positive (there was 1 positive bull and 1 positive female calf amongst these). Two of 10 yearling and none of 4 adult moose examined from Mt. Mckinley Park and Tanana River Flats, Alaska were positive in 1980 (Shults, pers. comm.).

Five of 13 captive muskox examined near Fairbanks, Alaska were positive for trypanosomes in 1979 ( 1 of 2 bulls was positive and none of 5 cows was positive; 2 male and 2 female calves were positive of 6 examined).

\section{Signs and Symptoms}

There are none seen and none known.

\section{Diagnosis}

Microscopic diagnosis can be accomplished by microhematocrit centrifugation (DE) of freshly collected heparinized blood from the suspect animal. Free-swimming trypanosomes may be seen in the plasma of the infected animal just above the buffy coat (when the hematocrit tube is examined microscopically (at 1.5-10 $x$ objective) (Bennett 1962, Kingston and Morton 1975). Whole blood or blood clot (from serum tubes) may be cultured in veal infusion medium (VIM) (Kingston and Crum 1977). Epimastigotes (young, dividing forms) can be microscopically detected swimming in these cultures 1-3 (or more) weeks following inoculation. Culture techniques are probably the most appropriate for field collections.

\section{Significance and Control}

The importance of trypanosome infection in cervids is not known at the present time. Considering their widespread distribution--by host and geographic area--they appear to cause no problems to their hosts. No transmission of these parasites is known to occur to man. At present, no control procedures are recommended or thought necessary. 


\section{References}

BENNETT, G. F. 1962. The hematocrit centrifuge for laboratory diagnosis of hematozoa. Can. J. Zool. $40: 124-125$.

CLARK, G. G. 1972. Trypanosomes from mule deer in New Mexico and Colorado. J. Wildl. Dis. $8: 325-326$.

DAVIES, R. B. and G. G. CLARK. 1974. Trypanosomes from elk and horseflies in New Mexico. J. Wildl. Dis. $10: 63-65$.

DIKMANS, G., C. A. MAHTHEI, A. H. FRANK. 1957. Demonstration of Trypanosoma theileri in the stomach of an aborted bovine fetus. Cornell Vet. 47:344-353.

HOARE, C. D. 1972. The Trypanosomes of Mammals. Blackwel Scientific Publications, 0xford. 749 pp.

KINGSTON, N. and J. CRUM. 1977. Trypanosoma cervi Kingston and Morton, 1975 in White-tailed deer, Odocoileus virginianus, in the Southeastern United States. Helminthol. Soc. Washington 44:179-184.

KINGSTON, N. and J. K. MORTON. 1973. Trypanosomes from elk (Cervus canadensis) in Wyoming. $\mathrm{J}$. Parasitol. 59:1132-1133.

1975. Trypanosoma cervi sp. n. from elk (Cervus canadensis) in Wyoming. J. Parasitol. 61:17-23.
KINGSTON, N., J. K. MORTON and M. MATTHEWS. $1975 . \quad$ Trypanosomes from mule deer, Odocoileus hemionus, in Wyoming. J. Wildl. Dis. 11:519-521.

KINGSTON, N., E. T. THORNE, G. THOMAS, L. L. MC HOLLAND and M. S. TRUEBLOOD. 1981. Further studies on trypanosomes of game animals in Wyoming II. J. Wildl. Dis. (In press).

KINGSTON, N., B. SWIFT and G. NELMS. Experimental and natural transplacental transmission of Trypanosoma theileri and its possible effects on the bovine fetus. Cornell Vet. (In press).

KISTNER, T. $P$. and $W$. L. HANSON. 1969. Trypanosomiasis in whitetailed deer. Bull. Wildl. Dis. Assoc. 5:398-399.

KINSKY, W. L. and L. L. PECHUMAN. 1975. Trypanosomes in horseflies and deer flies in central New York State. J. Parasitol. 61:12-16.

MATTHEWS, M. J., N. KINGSTON and J. K. MORTON. 1977. Trypanosoma cervi Kingston and Morton, 1975 from mule deer, Odocoileus hemionus, in Wyoming. J. Wildl. Dis. 13:33-39.

VAN DER MAATEN, J. J., W. T. HUBBERT, A. D. BOOTHE, J. H. BRYNER and P. C. ESTES. 1973. I solation of Bovine syncytial virus from maternal and fetal blood. Am. J. Vet. Res. $34: 341-343$. 


\section{Giardia}

J. Dau

Parasites of the genus Giardia are flagellated protozoans of the family Hexamitidae. Protozoans of this family are characterized by 2 readily apparent nuclei that lay side by side. Trophozoites (the active, feeding stage of these organisms comparable to the adult form of helminth parasites) of this genus resemble crude teardrops in form, bear 8 flagella and are less than 25 microns long. Cysts (the encapsulated, highly resistant form of these organisms roughly comparable to the larval stage of helminth parasites) are approximately half the size of trophozoites. Giardia spp. infect canids, felids, rodents and ruminants.

Giardia lamblia inhabits the anterior portion of the small intestine of humans. Trophozoites reproduce through binary fission; the increase of these organisms is thus described by a power function with an exponent of 2. The trophozoites become encysted as they progress down the intestinal tract before being shed in the hosts' feces. Cysts contain either 2 or 4 nuclei. Diarrheic stools may contain up to 14 billion parasites. When swallowed by a human, the parasites excyst in the duodenum and begin the life cycle anew.

Clinical symptoms described for giardiasis in canines include inappetence, listlessness and diarrhea (Barlough 1980). Diarrheic feces usually do not contain blood but often have the texture of oatmeal. These parasites do not lyse host cells but instead ingest the intestinal mucosa. Heavy concentrations of these organisms could impair the absorption of nutrients by host animals and cause dehydration and general weakness.

The diagnosis of giardiasis in humans appears to be increasing in Alaska. Though water is not requisite for maintaining Giardia spp. in ecosystems, contamination of surface water appears to enhance the transmission of these parasites. Giardia spp. can survive in water longer than 2 months at $8 \mathrm{C}$ (versus 4 days at 37C). Also, beaver (Castor canadensis) have been implicated in maintaining these parasites in fresh water systems (Juranek 1979). Considering the abundance of cold, fresh water ecosystems that are inhabited by beaver in Alaska and the dependence of Alaska's populace, especially those people in rural areas on untreated surface water, for drinking water, the potential for epidemics of giardiasis certainly exists in Alaska.

Essentially no research has been conducted concerning the incidence or pathogenicity of Giardia spp. in wildlife. The significance of these organisms in man and wildlife has not been established in Alaska.

\section{References}

BARLOUGH, J. E. 1980. Canine giardiasis. J. Am. Vet. Med. Assoc. $177: 726$.

JURANEK, D. 1979. Waterborne giardiasis. pp. 150-163. In: $\frac{\text { Waterborne }}{\text { Giardiasis. W. TAKUBOWSKI and }}$ J. C. HOFF, eds. EPA Office of Res. \& Dev., Cincinnati, $\mathrm{OH}$. 
LEVINE, N. D. 1973. Giardia Kunstler, 1882. pp. 118-122. In: Protozoan Parasites of Domestic Animals and of Man. 2nd ed. Burgess Pub. Co., Minneapolis, MN.
SCHMIDT, G. D. and L. S. ROBERTS.

1977. Giardia lamblia. pp. $80-85$.
Parasitology. C. Voundations

\section{Eimeria}

J. Dau

\section{Introduction}

Coccidiosis is a disease caused by protozoan organisms of the subclass Coccidia. Eimeria spp. are thought to be the most important genus of this group based on their incidence and pathogenicity so will provide the basis of this brief overview. Most of these ubiquitous organisms are intracellular parasites of the intestinal epithelium though some species may inhabit the visceral organs, i.e. kidneys or liver (Fig. 12). Coccidia occur in a wide range of avian and mammalian species. Though many species of coccidia are recognized, it is thought that the bulk of coccidial species remain to be described. Not all species of coccidia are considered pathogenic.

\section{Transmission}

Eimeria spp. are capable of either sexual (gametogony) or asexual (shizogony) reproduction. Sexual reproduction begins with the meiotic production of gametes that combine to produce an oocyst. 0ocysts are roughly spherical or ovoid and usually less than 35 microns long. They consist of a sporont (a diploid, undifferentiated cell mass) and, for Eimeria spp., are covered by a tough, resistant membrane. 0ocysts are passed in the feces of host animals and are highly resistant to environmental stress.

When the oocysts first enter the external environment they are not infective for host organisms. 0ocysts become infective by the process of sporogony (or sporulation) whereby the sporont gives rise to sporozoites (daughter cells that result from the division of the sporont). When a suitable host ingests infective oocysts, the oocyst wall ruptures and releases the sporozoites. The proximal explanation for sporozoite excystment is unknown. Sporozoites enter the epithelial cells of villi in the small intestine of host animals where they complete development to the trophozoite (active, feeding) stage.

Asexual reproduction begins when the nucleus of the trophozoite mitotically divides to produce a schizont (a trophozoite cell in which only the nucleus has divided, not the cytoplasm). The 2 daughter nuclei are eventually surrounded by an area of protoplasm; these protoplasmsurrounded nuclei are termed merozoites. The number of merozoites that form within a schizont varies between Eimeria spp. The merozoites are released from the schizont to invade other epithelial cells and complete maturation. 


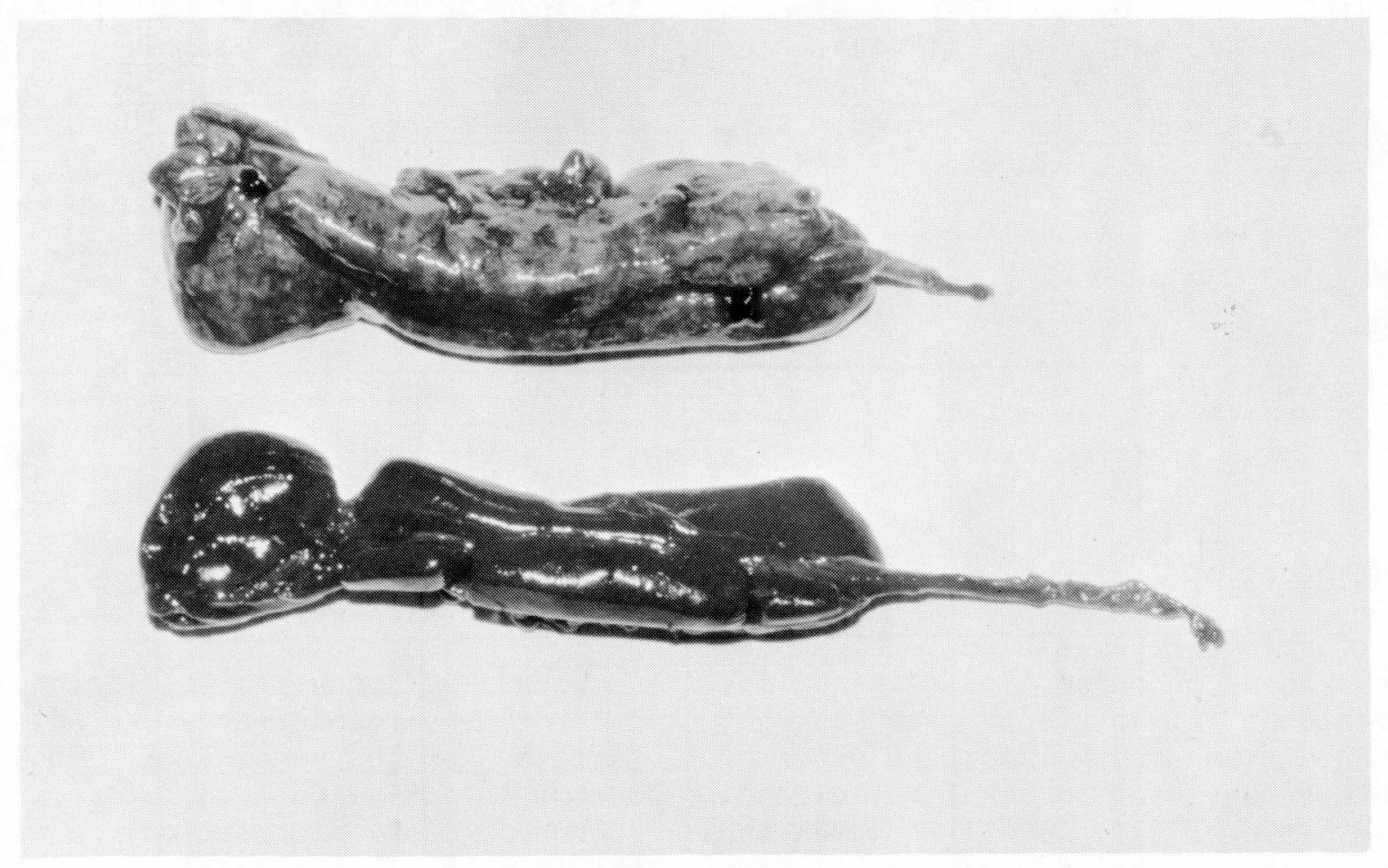

Figure 12. The kidneys of oldsquaw ducks (Clangula hyemalis). The upper kidney was taken from a bird that was suffering from coccidiosis caused by an Eimeria sp. The lower kidney is from a healthy bird for comparison (E. Taylor).

Asexual reproduction of coccidia is a finite process. Therefore, coccidial infections are self-limiting when sources of oocysts do not exist. Docysts are usually present under natural circumstances in fecal droppings however, and thus are continually ingested by host animals.

\section{Significance and Control}

The pathogenicity of coccidian parasites, like helminth parasites and other etiologic agents, is ul- timately influenced by speciesspecific characteristics of hosts and parasites, the attributes of individual animals and the entire array of environmental characterisitcs that comprise the ecosystem inhabited by the host-parasite organisms. The pathogenic effects of coccidia result from the disruption of cells and capillaries by emerging merozoites and concentrations of trophozoites/schizonts. The principal pathogenic effects of this disease are hemorrhage with accompanying anemia and the sloughing of 
intestinal epithelium tissue. Blood clots and cellular debris may form plugs that cause indirect pathogenic effects. Clinical symptoms of coccidiosis include diarrhea which may contain blood, emaciation, general weakness and unthriftiness. Recovery may infer immunity against reinfec$t i o n$ by that species of coccidia; this immunity is not absolute (previously infected animals may harbor light infections with the same organism and stress may destroy the immunity completely) and does not apply to all coccidia species. Coccidiosis is frequently fatal.

Identification of coccidial organisms is usually based on structural characteristics of the oocysts. Other criteria for identifying species are: host specificity, sites of localization within host animals and cross-immunity experiments.

Tentative diagnoses of coccidiosis can be based on clinical symptoms and the presence of oocysts in the feces of host organisms. These parasites commonly occur in most animals however often without detrimental ef- fect. Therefore, the mere presence of oocysts does not necessarily prove that the clinical symptoms observed are due to coccidia.

Many drugs are currently used in domestic animals that effectively control coccidial infections. Treatment of water and artificial feeds may be of value for short-circuiting coccidiosis outbreaks in free-living wildife populations since the disease is self-limiting in individual host animals.

The significance of coccidiosis in Alaskan wildlife has not been established. Levine (1973) provides a thorough coverage of coccidiosis in general and for many species of coccidia as well.

\section{References}

LEVINE, N. D. 1973. The Ap i complexa and the Coccidia proper. pp. 156-254. In: Protozoan Parasites of Domestic Animals and of Man. 2nd ed. Burgess Pub. Co., $\overline{\text { Min- }}$ neapol is, MN. 


\section{Sarcocystis}

J. Dau

Introduction

Sarcocystis spp. are common parasites of Alaskan ungulates. Bison (Bison bison), black-tailed deer (Odocoileus hemionus sitkensis), caribou and reindeer (Rangifer tarandus), moose (Alces alces), mountain goats (Oreamnos americanus) and Dall sheep (Ovis dalli) from Alaska have been found to harbor protozoans of this genus ( $R$. Dieterich, pers. comm.). Marine mammals from Alaska reported to harbor Sarcocystis spp. include northern fur seals (Callorhinus ursinus) (Brown et al. 1974), harbor seals (Phoca vitulina) (Hadwen 1922), bearded seals (Erignathus barbatus) (Bishop 1979) and ringed seals (Phoca hispida) (Migaki 1980). Rausch (1962) reported that collared pikas (Ochotona collaris) often contained these parasites. Black bears (Ursus americanus) (Crum et al. 1978) and coyotes (Canis latrans) (Arther and Post 1977, R. Dieterich, pers. comm) have been reported to harbor Sarcocystis spp.

The specific nomenclature of Sarcocystis is unclear. Though more than 50 species of Sarcocystis have been described (Levine 1973), not all of them are universally accepted as valid. This nomenclatural confusion stems from persistent misconceptions associated with the terminology applied to several life stages of Sarcocystis spp. and from the variability of criteria on which species are currently distinguished. These criteria, namely host specificity, structure of cyst wall and size of trophozoites (the active, feeding stage of these protozoans) vary inter- and intraspecifically, with the age of the intramuscular cysts and with the method employed to preserve the specimens. Until better means of distinguishing recognizable, biologically valid characteristics of Sarcocystis spp. are discovered, the specific nomenclature of this genus will have to be regarded as a convenience rather than as a categorization of biological diversity (Levine 1973).

\section{Transmission}

The specific life cycles for Sarcocystis are largely unknown; only those life cycles for the most common species have been described (Fig. 12). Sarcocystis spp. appear to follow an obligatory predator-prey life cycle. Sexual reproduction occurs in the final carnivorous host and asexual reproduction occurs in the intermediate prey host. Recent investigations indicate that Sarcocystis spp. may be quite host specific (Hudkins and Kistner 1977, Fayer et al. 1976, Fayer and Kradel 1977). The life cycle information presented herein is taken from Levine (1977).

Predators are infected when they ingest meat that contains sarcocysts, also referred to as meronts, cysts or shizonts. The sarcocysts contain merozoites, often erroneously referred to as "spores," that emerge from the sarcocysts in the intestine of the predator. These merozoites enter epithelial cells of the intestine and become either male or female gamonts. Gamonts of each sex produce large numbers of gametes that unite through fertilization to form zygotes. Zygotes surround themselves in resistant walls and are then 


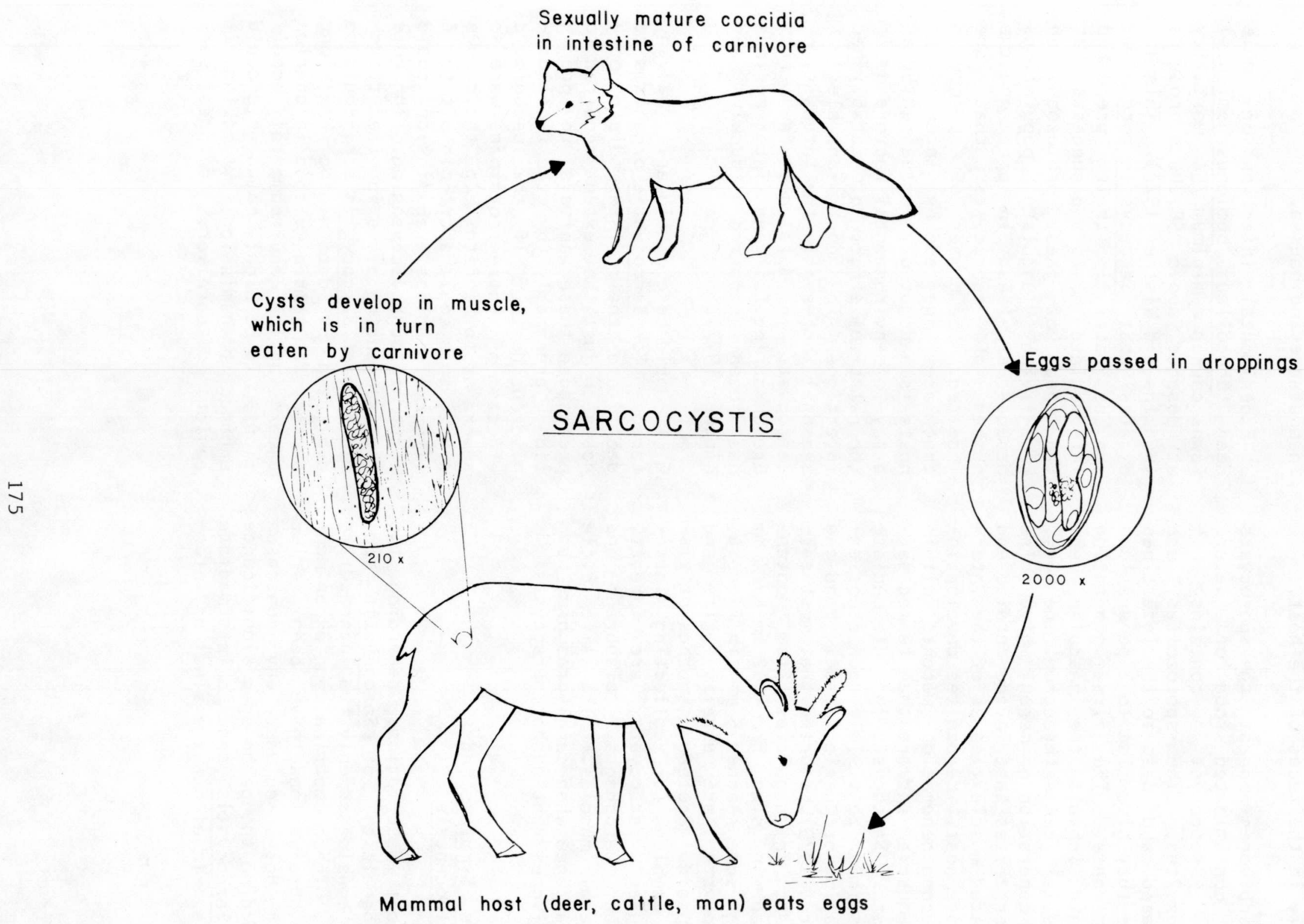

Figure 13 
termed oocysts. Each oocyst gives rise to 2 sporocysts via the process of sporulation; this occurs while in the intestine of the carnivore. The sporocysts leave the oocyst and pass freely in the feces of the host.

Herbivores ingest the sporocysts with contaminated food or water. Each sporocyst contains 4 sporozoites. The sporozoites are released and pass to the intestines where they give rise to 2 generations of meronts. The first generation meronts inhabit the vascular endothelial cells throughout the body. This generation of meronts give rise to merozoites that invade cardiac and striated muscle tissues of the intermediate host. Merozoites develop into second generation meronts within the muscles and are then termed sarcocysts. Sarcocysts in intermediate hosts may be visible upon gross examination of muscle tissue. Pond and Speer (1979) reported that most sarcocysts in ungulates from Montana ranged in size from $0.2 \mathrm{~mm}$ by $1 \mathrm{~mm}$ though some reached $5 \mathrm{~mm}$ in length. Sarcocysts are usually white and ellipsoid. Preferred sources of tissue samples for collecting intramuscular sarcocysts are heart, tongue, esophagus, diaphragm and skeletal muscles. The life cycle begins anew when carnivores prey upon herbivores that harbor sarcocysts.

Signs, Symptoms and

\section{Pathogenesis}

Most descriptions regarding the pathogenicity of Sarcocystis spp. have considered only the intermediate host. These empirical and experimental observations have been varied which has precluded any consensus regarding the pathologic significance of Sarcocystis spp. in intermediate hosts. In either the final or inter- mediate host, the intestinal phase of these parasites may damage the epithelium of this organ. This damage may manifest diarrhea and, in severe infections, cause hemorrhage, inappetance and nausea.

Experimental infection of mule deer (Odocoileus hemionus hemionus) fawns caused a minimum 75\% mortality at dosages above 50,000 sporocysts (Hudkins and Kistner 1977). This indicates that Sarcocystis organisms can be fatal in their preferred intermediate hosts and suggests that the impact of these protozoans on free-living ungulate populations depends at least in part on the number of sporocysts that are ingested. The number of sporocysts that are ingested by intermediate hosts is undoubtedly influenced by an array of environmental factors as is the relative effect of established infections in host animals. A seasonal increase in the intensity of sarcocysts in mule deer fawns from Oregon during fall and winter periods has been reported (Hudkins and Kistner 1977).

It would be evolutionarily advantageous for Sarcocystis organisms to debilitate their intermediate prey hosts to just that extent required to predispose these animals to predation, presumably by the final carnivorous hosts. If the pathologic effects of these protozoans were so severe as to be fatal in their own right, this could short-circuit their life cycle unless transmission could occur through scavenging. The role that scavenging plays in the transmisssion of Sarcocystis spp. could be evaluated by determining how long sarcocysts remain viable in carrion under varying environmental conditions. To be highly pathogenic could enhance transmission by making it easier for carnivores to ingest sar- 
cocysts though this does not appear to be the case under natural circumstances. This would necessitate that sarcocysts be resistant to environmental stress and that environmental conditions (i.e. freezing) prevent the complete degeneration of meat before it can be scavenged. The relative importance of scavenging versus predation is unimportant to the welfare of the intermediate host; it is not of concern how these animals die but whether they die or survive. The distinction between predation and scavenging may be an important consideration however, in controlling these protozoans in wildlife populations. Before measures and means of controlling Sarcocystis can be developed, the ecology and significance of these parasites should be better understood.

\section{References}

ARTHER, R. G. and G. POST. 1977. Coccidia of coyotes in eastern Colorado. J. Wildl. Dis. $13: 97-100$.
BISHOP, L. 1979. Parasite-related lesions in a bearded seal, Erignathus barbatus. J. Wildl. Dis. 15:285-293.

BROWN, R. J., A. W. SMITH and M. C. KEYES. 1974. Sarcocystis in the northern fur seal. J. Wildl. Dis. $10: 53$.

CRUM, J. M., V. F. NETTLES and W. R. DAVIDSON. 1978. Studies on endoparasites of the black bear (Ursus americanus) in the southeastern United States. J. Wildl. Dis. 14:178-186.

FAYER, R., A. J. JOHNSON and P. K. HILDEBRANDT. 1976. Oral infection of mammals with Sarcocystis fusiformis bradyzoites from cattle and sporocysts from dogs and coyotes. J. Parasitol. 62:10-14.

FAYER, R. and D. KRADEL. 1977. Sarcocystis leporum in cottontail rabbits and its transmission to carnivores. J. Wildl. Dis. $13: 170-173$.

HADWEN, S. 1922. Cyst-forming protozoa in reindeer and caribou and a sarcosporidian parasite of the seal (Phoca richardsi). J. Amer. Vet. Med. Assoc. 61:374-382. MIGAKI, G. 1980. Sarcosporidiosis in the ringed seal. J. Am. Vet. Med. Assoc. 177:917-918. 


\section{Besnoitia}

J. Dau

\begin{abstract}
Besnoitia tarandi was first reported in reindeer and caribou (Rangifer tarandus) from Alaska by Hadwen (1922a). In these animals, it causes bones and tendons to become roughened and is thus referred to as "corn meal disease." Hadwen (1922b) stated that old animals are more intensively affected than young animals. Besnoitiosis has also been reported from caribou in northern Canada (Choquette et al. 1967). Besnoitia jellisoni infrequently parasitizes rodents where it produces numerous white cysts throughout the viscera (Jellison 1971). Besnoitia jellisoni has not been reported however from Alaska or northern Canada.
\end{abstract}

The life cycles of Besnoitia spp. that have been described closely resemble the life cycle of Sarcocystis spp. (see this section). Besnoitia spp. utilize 2 hosts: 1) a carnivorous host in which sexual reproduction occurs and 2 ) an intermediate prey host in which aseuxal reproduction occurs (Wallace and Frenkel 1975). Besnoitia spp. differ from Sarcocystis spp. in that unsporulated oocysts are shed in the feces of carnivores; Sarcocystis spp. shed sporulated sporocysts in the feces of carnivores. The cysts of Besnoitia spp. analogus to the sarcocysts of Sarcocystis spp. are termed bradyzoites. Cysts of $B$. tarandi are roughly spherical and are found on the surface of tendons and in the periosteum of bones (Levine 1973). Bradyzoites may be transferred between intermediate hosts; the role that scavenging plays in the transmission from final to intermediate hosts is discussed by Smith and Frenkel (1977). Besnoitiosis can be differentiated from sarcocystis in intermediate hosts on the characteristics and localization of the cysts. The pathogenesis and significance of this protozoan in carnivores and ungulates in Alaska has not been determined.

\section{References}

CHOQUETTE, L. P. E., E. BROUGHTON, F. L. MILLER, H. C. GIBBS and J. G. COUSINEAU. 1967. Besnoitiosis in barren-ground caribou in northern Canada. Can. Vet. J. 8:282-287.

HADWEN, S. 1922a. Notes on the adhesion-tubes of Draconema cephalatum. J. Parasitol. 9:38-39.

1922b. Reindeer in Alaska. U.S. Dept. Ag. Bull. No. 1089. Washington, DC. $74 \mathrm{pp}$.

JELLISON, W. L. 1971. Besnoitiosis. pp. 354-357. In: Parasitic Diseases of Wild Mammals. J. W. DAVIS and R. C. ANDERSON, eds. lowa State Univ. Press, Ames, IA.

LEVINE, N. D. 1973. Besnoitia tarandi (Hadwen, 1922) Levine, 1961) p. 310. In: Protozoan Parasites of Domestic Animals and of Man. 2 nd ed. Burgess Pub. Co., Minneapolis, MN.

SMITH, D. D. and J. K. FRENKEL. 1977. Besnoitia darlingi (Protozoa: Toxoplasmatinae): Cyclic transmission by cats. J. Parasitol. 63:1066-1071.

WALLACE, G. D. and J. K. FRENKEL. 1975. Besnoitia species (Protozoa, Sporozoa, Toxoplasmatidae): Recognition of cyclic transmission by cats. Science $188: 369-371$. 


\section{Ectoparasites}

A review of published information on ectoparasites of game mammals in Alaska revealed many gaps. Much more collecting must be done to answer basic questions such as what species of parasites occur on Alaskan mammals, their geographic distribution and seasonal occurrence, their abundance and effects on their hosts.

\section{Ticks (Acari: Ixodidae)}

Glenn E. Haas

Haemaphysalis leporispalustris (Packard), the rabbit tick, was first recorded from Alaska as a result of Philip's survey of 1937 (Philip 1938, 1939). Specimens were collected from snowshoe hares (Lepus americanus) at Seward and in the vicinity of Fairbanks. The isolation of Francisella tularensis from ticks collected near

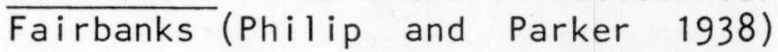
stimulated an expanded survey that showed rabbit ticks ranged from Livengood, Minto and Clear east to Circle Hot Springs and Delta Junction (Geary 1953, Gill 1954, Philip et al. 1954). Rabbit ticks, snowshoe hares and the nearctic strain of tularemia probably spread together into northern North America during postglacial time (Rausch 1972).

Dermacentor albipictus (Packard), the winter tick, abundant on moose (Alces alces), deer (Odocoileus spp.) and neglected range horses (Equus spp.) in regions of Canada (Wilkinson 1979), was intercepted on a horse imported into Alaska (R. E. Barrett, unpub. data). This tick is not known to occur naturally in Alaska, although the record (Klein 1965) of the Dermacentor sp. specimen from a Sitka black-tailed deer (‥ hemionus) on Coronation Island is suggestive.
Chewing Lice (Mallophaga)

Glenn E. Haas

The first record of an ectoparasite from Dall's sheep (Ovis dalli) is of Bovicola jellisoni Emerson from Crescent Mountain, Kenai Peninsula (Kim 1977). Kim extracted about 19,300 specimens from 1 hide, mostly on the sides and belly and least on the head and neck.

\section{Sucking Lice (Anoplura)}

Glenn E. Haas

Several species of sucking lice are known from Alaska. Solenopotes tarandi (Mjoberg) from hides of caribou (Rangifer tarandus) taken at Anaktuvuk Pass and the Utukok River were used for the redescription and illustration of adults by Weisser and Kim (1973) and for a monograph of the genus Solenopotes by $\mathrm{Kim}$ and Weisser (1974).

Tricholipeurus lipeuroides (Megnin) was collected by L. Johnson from Sitka black-tailed deer on Admiralty Island and Baranof Island (N. Wilson, unpub. data)

Sucking lice are also known from the walrus (Odobenus rosmarus) and seals (Phocidae) of Alaska (Kim 1972, 1975, Weber 1950, N. Wilson, unpub. data).

Warbles

Robert A. Dieterich and Glenn E. Haas

The warble fly (0edemagena tarandi L.) is a member of the order Diptera, and is a widespread parasite of 
Rangifer tarandus in Alaska (Washburn et al. 1980, Weber 1950). Adult warble flies are found during the summer months throughout tundra areas inhabited by reindeer and caribou (Rangifer tarandus). These flies somewhat resemble a honeybee in size and body form. Their life cycle is direct, requiring no intermediate hosts (Washburn et al. 1980). The adult female fly lays eggs on the hair of reindeer and caribou on areas of the legs, flanks and lower hips. These eggs hatch in about 6 days to produce tiny larvae that penetrate the skin and migrate to the back of the host. There, the larvae cease migratory movement and are encysted in host generated fibrous tissue. Each encysted larvae pierces the skin of the host to form a small breathing hole. The larvae enlarge during the winter and spring to reach a maximum size of just over 1 inch long by proceeding through a series of molts. These larvae or "grubs" concentrate along the entire dorsal surface of the host, but are found in greatest number in the loin and rump areas. It is common to find 500 to 1000 warbles on the back of 1 animal and as many as 2000 have been documented in severe infections.

In the late spring and early summer, each mature larva exits the back of the host through its breathing hole and falls to the ground to form a pupa. These pupae are a dark brown to black color instead of the light cream color of the mature larva located under the skin. The pupa stage lasts from 3 to 8 weeks before it changes into the adult fly. In turn, the adult fly lives 6 to 8 days before dying. During that period, the female flies deposit their eggs and the cycle starts over again.
Warbles are capable of seriously impairing their host's health. The large numbers of larvae under the skin of the back affects the animal's nutritional balance, causes deleterious allergic responses, frequently causes severe secondary infections involving areas located on the back due to extensive tissue damage, and may also suppress immune responses. Animals weakened by warble infection are more susceptible to other diseases, predation or environmental stress (Zhigunov 1961).

In reindeer herds, harassment by warble flies makes herding difficult due to erratic behavior of the animals in their attempts to avoid this parasite. The value of hides is also decreased due to the scars of breathing-exit holes produced by the larvae.

Yearlings (animals 1 year of age) and cows producing young are usually the most severely affected. It is common for these 2 cohorts (cows with calves) to aggregate during the summer in areas where warble flies can attack. Bulls are more often in scattered groups in windy or barren areas less conducive to warble flies. It is not unusual to find yearlings dead in the late spring due to massive warble fly infection.

Control of warbles has begun in reindeer herds of the Seward Peninsula using an injectable organic phosphate product (Warbex) (Washburn and Dieterich 1969, Washburn et al. 1980). This treatment must be repeated yearly during the period between September and January when the warble larvae are small and located in their host. This product is very effective as a control means if nearly all animals on a range can be treated during a single winter. 
1. The head of a red fox (Vulpes vulpes) submitted to the Northern Regional Laboratory Virology Unit. This animal was rabid; note the porcupine quills in its snout (D. Ritter).

2. A rabid wolf (Canis lupus) from the north slope of Alaska; note the dissheveled appearance of this animal (R. Chapman).

3. Typical scab-like lesions of contagious ecthyma on the muzzle of a muskox (Ovibos moschatus) (R. Dieterich).

4. Open lesions of contagious ecthyma on the lips and nose of a young Dall sheep (Ovis dalli) (R. Dieterich). 


\title{
Diarrhea
}

\author{
Jamie K. Morton
}

Diarrhea is a condition characterized by increased frequency and fluidity of the stools. The feces may also contain white or yellowishwhite mucous or blood. Diarrhea can be caused by a number of factors or organisms including excitement, overeating, malabsorption associated with a tumor (neoplasm), toxins, poison, improper food, pancreatic or liver malfunction, parasites [ $[\mathrm{e} . \mathrm{g}$. tapeworms, roundworms or protozoans), bacteria (e.g. E. coli, Salmonella, Shigella or Mycobacterium paratuberculosis), or viruses e.g. infectious bovine rhinotracheitis (IBR), bovine viral diarrhea (BVD)] . Most of these agents cause an intestinal irritation resulting in malabsorption (impaired absorption of nutrients).

The condition is usually selflimiting. However, whenever diarrhea poses a serious problem, the cause should be determined if possible, as the causative factors have different implications for the rest of the animal population. For example, herds could be dispersed, or the environment cleaned up if toxins or poisons were the cause. Salmonella, Shigella and E. coli are also potential human pathogens which thus pose public health hazards. Johne's disease (caused by Mycobacterium paratuberculosis) is considered a serious disease of wild and domestic animals, and infected animals should not be transplanted. Bovine viruses may cause abortion as well as diarrhea in cattle, thus reproductive potential may be affected.

Captive animals can be treated with certain medications such as Kaopectate, antibiotics for bacterial diarrhea or fluid replacement therapy. Dehydration from severe or prolonged diarrhea can result in death. 


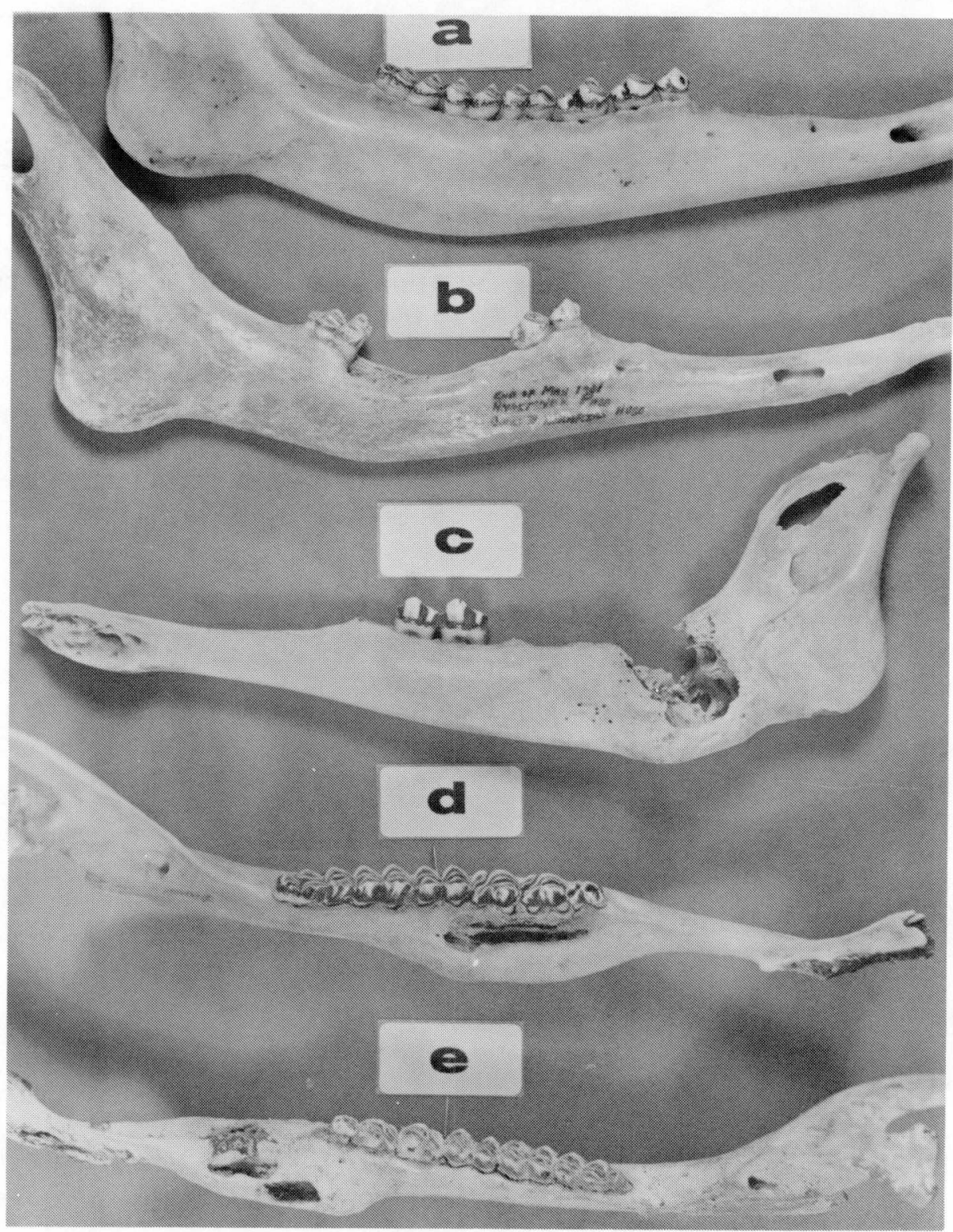


Figure 1. Caribou mandibles from the Western Arctic Herd.

a. Normal mandible.

b. Mandibular lesion probably resulting from trauma. Note missing first and second molars. The alveoli have been filled with cancellus bone with minimal bone destruction. $\mathrm{P} 4$ has been removed for aging.

c. Severe bone lysis probably the result of a dental abscess of the third molar. Note increased ventral ossification. P2, M1, and M2 teeth were lost in handling and storage.

d. Lesion in mid-shaft of mandible, probably the result of dental abscess. Note high degree of osteolysis on the lingual and ventral surface of the mandible. Bone erosion is present at the second root of the third molar (radiograph). Disrupted periodontal membrane is present around roots of all three premolar teeth.

e. Osteolytic lesion in the diastema. There are numerous cavities on dorsal and lingual surfaces of mandible with concurrent bone production on ventral surface.

f. Next page. Radiographs of the mandibles shown in a through e (letters correspond). 


\title{
Mandibular Lesions
}

\author{
Robert A. Dieterich
}

Mandibular lesions are sometimes found on the lower jaws of reindeer and caribou (Rangifer tarandus) (Doerr and Dieterich 1979), Dall sheep (Ovis dalli) (Murie 1930), black-tailed deer (Odocoileus hemionus columbianus) (Cowan 1946) and moose (Alces alces) (Ritcey and Edwards 1958). These lesions consist of swelling and necrosis in the region of dental alveoli or bony exostoses, and cavitation in the diastema and ventral surfaces of the mandible. Anomalies affecting both dental alveoli and the ventral aspect of the mandible are also observed (Fig. 1).

The cause of mandibular lesions has not been firmly established. Trauma, dental abscesses and periodontal disease were cited as the probable causes of most lesions observed in Alaskan caribou (Doerr and Dieterich 1979). This report noted lesions in 7.0 and $4.4 \%$ of mandible pairs collected during 1959-1961 and 1975-1977 respectively. Mandibular lesions occurred most frequently in caribou 7 years of age or older. Thirty-three of 98 (33.7\%) mandibles were missing 1 tooth, while 10 of 98 $(10.2 \%)$ were missing more than 1 tooth. The first molar (M1) was the tooth most commonly lost, although the loss of 2 or more teeth was more common among pre-molars than among molars.

Leader-Williams (1980) reported that the presence of eosinophilic sulfur granules in some of the affected jaws of sub-antarctic reindeer was strongly suggestive of "lumpy jaw." This condition is common in domestic cervids and is caused by the bacteria Actinomyces. However, no bacteria were isolated from the very limited number of mandibles collected. Actinomyces normally inhabit oral cavities as harmless saprophytes, but may invade mandibular tissue via food impaction or trauma. This report suggested that mandibular and dental lesions resulted from environmental peculiarities of the area.

Little is understood about the significance of mandibular lesions. Tooth loss appears to be a direct consequence of this malady. This may predispose to food impaction and provide an avenue of access for secondary infections. Discomfort from these abnormalities may affect feeding patterns and in turn, general body condition.

\section{References}

COWAN, I. M. 1946. Parasites, diseases, injuries and anomalies of the Columbian black-tailed deer, Odocoileus hemionus columbianus (Richardson), in British Columbia. Can. J. Res. D2 $4: 71-103$.

DOERR, J. G. and R. A. DIETERICH. 1979. Mandibular lesions in the western arctic caribou herd of Alaska. J. Wildl. Dis. 15:309-318.

LEADER-WILLIAMS, N. 1980. Dental abnormalities and mandibular swellings in south Georgia reindeer. J. Comp. Pathol. $90: 315-330$.

MURIE, 0. A. 1930. An epizootic disease of elk. J. Mammal. $11: 214-222$.

RITCEY, R. W. and R. Y. EDWARDS. 1958. Parasites and diseases of the Wells Gray moose herd. J. Mammal. 39:139-145. 


\title{
Skin Diseases
}

\author{
Robert A. Dieterich
}

Skin diseases are commonly referred to as dermatoses. The causes of dermatoses are many and range from infectious agents such as viruses, bacteria, and fungi to parasites or hormonal and nutritional etiologies. Wildlife in the warmer areas of the world commonly have skin lesions that are due to external parasites. Alaskan wildlife have infestations of the skin caused by external parasites (see Chapter 3), but in general, these are less significant than in temperate areas of the world. The warble fly of reindeer and caribou does cause severe lesions and is an exception to this general rule.

Hair loss is the most common lesion of skin diseases. When a dermatosis is observed, the presence of parasites should be determined by gross examination. Skinning may facilitate detection of parasites, i.e. warbles, on the skin underside possibly hidden by pelage. The pattern of hair loss should also be noted. Bilaterally symmetric lesions may indicate a physiologic etiology, i.e. a hormonal imbalance or nutritional deficiency, while localized lesions could be induced by bacteria, parasites or other agents. Once the general gross appearance and pattern of the lesion(s) have been noted and hopefully photographed, fresh and formalin fixed tissue samples should be obtained for examination in a diagnostic laboratory.

A listing of causes of skin diseases includes: tumors, cysts, parasites (fleas, ticks, mites, helminths, etc.), hormonal imbalances, nutritional deficiencies or toxicities, infectious agents, and several rather vague conditions of hair loss whose etiology is unknown. often, the condition of the coat may be used as an indicator of the general well-being of wildlife. A dull, rough coat may be a sign of disease not actually located in the skin. Reindeer herders know that animals which do not shed their winter coat by mid-summer are not healthy. Many times, one can pick out a diseased reindeer by looking for a rough, partially shed coat in a herd of otherwise smooth coated animals. Healthy animals typically appear rather ragged while shedding their winter coat. Therefore, diagnoses of dermatoses must consider seasonal cyclic coat changes. 
the eye may be red and swollen indicating conjunctivitis; the clear portion of the eye may be cloudy white in appearance, or there may be actual ulceration indicating erosions of the cornea.

\section{Significance and Control}

Biologists, herders and other wildlife observers often remark that the disease affected only 1 eye. This is true in many cases, but it is likely that in cases where both eyes are affected, the animal suffers from greatly reduced vision, or is blind and is quickly eliminated by predators, starvation or other occurrences.

Individual animals can be treated very successfully with topical or subconjunctivally injected antibiotics if practical. Control of flies, dust and other physical agents is helpful. The incidence of keratitis in reindeer herds is usually low $(1-3 \%)$ but when conditions are optimum, it may reach higher proportions and be a significant problem.

\section{References}

HADWEN, S. and L. J. PALMER. 1922. Eye trouble (keratitis). pp. 56. In: Reindeer in Alaska. USDA Bul1. No. 1089. Government Printing Office, Washington, DC.

REHBINDER, C. 1977a. Clinical and epizootiological studies on keratitis in reindeer. Acta Vet. Scand., Suppl. 66. 26 pp.

$$
\text { - 1977b. Keratitis in rein- }
$$
deer. Relation to the presence of 1st instar larvae of the nostril fly (Cephenomyia trompe L.) in the conjunctival sac and to natural ultraviolet radiation. Acta Vet. Scand. 18:75-85.

- 1977c. Keratitis in reindeer. Relation to bacterial infections. Acta Vet. Scand. $18: 54-64$.

SIEGMUND, O. H. 1979. Infectious keratoconjunctivitis. $\quad$ pp. 225-226. In: The Merk Veterinary Manual. Merck \& Co., Rahway, NJ. 
Robert A. Dieterich

\section{Introduction}

Keratitis is an eye disease of reindeer and possibly caribou (Rangifer tarandus) characterized by blepharospasm (tonic spasms of the eyelids producing more or less complete closure of the eye), lacrimation (tearing), conjunctivitis (inflammation of the white portion of the eye), corneal opacity (clouding of the clear portion of the eye) and ulceration. A similar condition in cattle, sheep and goats is commonly known as "pink eye."

\section{History}

The characteristic symptoms of keratitis have been observed in Alaskan reindeer for many years. It is mentioned in Hadwen's 1922 publication Reindeer in Alaska. He states:

Inflamed eyes are not of uncommon occurrence. The affection seems to be seasonal, starting with the hot weather and disappearing in the autumn. Though the cause of the disease has not yet been ascertained, it would appear to be infectious, and therefore the diseased animals should be slaughtered and not kept as breeding stock [Hadwen and Palmer 1922].

Presently in Alaska, keratitis is observed on an infrequent basis in reindeer herds on the Seward Peninsula. It occurs frequently in the University of Alaska reindeer research herd at Fairbanks. Its occurrence in caribou herds is not well documented.
Transmission and

\section{Pathogenesis}

In domestic animals, keratitis is caused by a variety of physical and infectious agents usually occurring simultaneously in varying combinations. Infectious agents include bacteria, viruses, mycoplasms, rickettsiae and chlamydiae. Physical agents include fly irritation, strong sunlight, dry dusty conditions or coarse, dense vegetation causing eye trauma (Siegmund 1979).

Extensive research has been carried out in Sweden in an attempt to determine the etiology of the disease in reindeer (Rehbinder 1977a, b, c). It was determined that many factors may be involved including UV irradiation, dust, traumatic lesions of the cornea, presence of foreign bodies, the first instar larvae of the nostril fly (Cephenomyia trompe L.) and various bacterial species. In Alaskan reindeer, the disease is reliably and easily cured with a single subconjunctival injection of antibiotics, which indicates that control of bacterial invaders is of primary importance for healing to occur. A bacterial etiology is suspected because viruses do not respond to treatment with antibiotics.

Keratitis is easily diagnosed, as the lesions are visible once the eyelids are separated. One should suspect keratitis any time reindeer or caribou are observed with closed or partially closed eyelids, tears streaking the face, and many times, a white purulent discharge from the corner of the eye. Three main types of lesions are found. The white of 
or yellowish abscesses may be seen on the surface or on cut surfaces of the lungs. In severe cases, fibrinous adhesions may cause the lungs to be stuck to the lining of the thoracic cavity.

\section{Diagnosis}

Pneumonia is most easily diagnosed at necropsy by observing gross lesions as described above. Laboratory identification of the causative agent may be possible if the samples are collected immediately after death. Microscopic examination of tissues preserved in $10 \%$ formalin can give a good picture of the nature of the disease.

\section{$\underline{\text { Significance and } \text { Control }}$}

Pneumonia in wildlife often results following a complexity of factors and may or may not be the cause of epidemics or die-offs. Exceptions to this are the die-offs in wild sheep attributed to lungwormpneumonia complexes. It is possible to treat wild sheep populations for lungworms with medicated feed. Con- trol of physiologic stresses (overcrowding, overgrazing, predator or human harassment, prolonged cold, wet weather, etc.) would probably contribute to the control of pneumonias in wildlife populations. Multivalent vaccines produced for use in cattle may be useful in preventing infections in captive wildlife.

\section{References}

FORRESTER, D. J. 1971. Bighorn sheep lungworm-pneumonia complex. pp. 158-171. In: Parasite Diseases of Wild Mammals. J.W. DAVIS and R. C. $\overline{A N D E R S O N}$, eds. Iowa State Univ. Press, Ames, IA.

MARKHAM, R. J. F. and B. N. WILKIE. 1980. Interaction between Pasteurella hemolytica and bovine alveolar macrophages: cytotoxic effect on macrophages and impaired phagocytosis. Am. J. Vet. Res. 41:18-22.

ROSEN, M. 1970. Pasteurellosis. pp. 214-223. In: Infectious Diseases of Wild Mammals. J. W. DAVIS, L. N. KARSTAD and D. 0. TRAINER, eds. lowa State Univ. Press, Ames, IA. 


\section{History}

One of the most widely recognized significant impacts of pneumonia in wildlife has involved a lungwormpneumonia complex in bighorn sheep (Ovis canadensis). The disease was first diagnosed in bighorn sheep in Montana in the 1920s. Bacteria were soon implicated as secondary invaders to the lungworms, and serologic evidence of respiratory viral infections in bighorn sheep in Montana and Wyoming was reported in the 1960 s. Numerous die-offs of bighorn sheep due to a lungworm-pneumonia complex or bacterial infections have been reported in the United States and Canada since the $1920 \mathrm{~s}$ (Forrester 1971).

Pasteurella multocida has been implicated in pneumonia in various wildlife species since the early part of the century. Species indigenous or closely related to those in Alaska that have been reported specifically with pasteurelloses include caribou (Rangifer tarandus), elk (Cervus elaphus), chipmunks (Eutamias sp.), mink (Mustela vison), muskrats (Ondatra zibethicus), weasels (Mustela sp.), raccoons (Procyon lotor) and California sea lions (Zalophus californianus) (Rosen 1970). Pneumonia has been diagnosed in reindeer (Rangifer tarandus), muskox (Ovibos moschatus), caribou, Dall sheep (Ovis dalli) and moose (Alces alces) in Alaska.

\section{Transmission}

Pasteurella multocida appears to be part of the normal flora in the throats of many animals. It probably acts as an opportunist to provoke infection in a debilitated host. It can be spread from 1 animal to another in droplets of saliva or feces that are ingested (Rosen 1970). Corynebacterium pyogenes also has been isolated from healthy as well as sick animals, and its role in causing infection is not well understood (Forrester 1971). Viruses are probably spread in droplets coughed out by infected animals. Life cycles and transmission of lungworms are covered in another section.

Signs, Symptoms and Pathogenesis

Signs of pneumonia may include oral and nasal mucous discharges, emaciation, coughing, weakness, an uncertain gait, a rough shaggy coat, anemia, diarrhea and conjunctivitis (Forrester 1971). In severe cases the animal may be so weak that it is possible to approach it within a few feet. As with many diseases of wildlife, signs may not be evident until the animal is found dead.

Various stresses may contribute to the pneumonia process. Many pneumonia cases are seen in the spring when the animal's body condition is poorest. The immune system does not function as well in an unthrifty animal. Lungworms may also contribute to a lowered resistance of the respiratory tissues. Certain viruses interfere with normal immune mechanisms and may themselves cause infections. Bacteria appear to be the ultimate cause of severe pneumonia in cattle (Bos spp.), and many are normal inhabitants of the respiratory tract (Markham and Wilkie 1980). Thus, various factors may contribute to lowering the resistance enough to allow the progression of pneumonia.

As a result of the animal's inflammatory response to infection, the lungs may show evidence of lobular congestion (excess blood) and edema (excess intracellular fluid). Bloody froth may be seen in the trachea. The lungs may become firm like liver tissue, a term known as hepatization ("hepar," liver). White 


\title{
Introduction
}

\author{
Robert A. Dieterich
}

There are a number of diseases of general nature that may be the result of 1 or more causes. These diseases can occur in a variety of wildlife species. Conditions such as diarrhea, mandibular lesions, skin diseases, keratitis and pneumonia are covered in this chapter, but there are many more which may be encountered while examining wildlife. The reader should remember that some conditions once considered a specific disease are now known to be actually only symptoms of several diseases. An example of this is anemia. Anemia is a reduction in the number of erythrocytes or hemoglobin in the blood. A large number of nutritional, genetic and infectious processes can cause this symptom.

The reader is referred to the many excellent texts on general pathology and wildlife diseases for details of miscellaneous conditions which are common to humans, domestic animals and wildlife. It is beyond the scope of this compendium to repeat all of this information.

\section{Pneumonia}

\author{
Jamie K. Morton
}

\section{Introduction}

Pneumonia, an inflammation of the lungs, can be caused by a variety of agents including certain bacteria, viruses, lungworms, fungi and foreign bodies. Frequently the condition is the result of a combination of factors. Physiologic stresses such as cold, wet weather, predator harassment or human handling may induce the animal to be susceptible to organisms that would ordinarily be harmless.
Many bacteria have been implicated in pneumonia. Pasteurella multocida, a small Gram negative coccobacillus and Corynebacterium pyogenes, a small Gram positive rod, are among the most frequently encountered. Parainfluenza Type 3 (PI-3), a myxovirus, is probably the most responsible virus, although the viruses of infectious bovine rhinotracheitis (IBR) and bovine viral diarrhea (BVD) may also be involved. Various lungworms contribute to the pneumonia process. 
Chapter 5.

MISCELLANEOUS DISEASES 
TABLE 2 (cont.)

$\begin{array}{ccc} & \text { Tumber } \\ \text { Species } & \text { Type of } & \text { of } \\ \text { Common Name } & \text { Neoplasm } & \text { Cases }\end{array}$

Mus $\frac{\text { musculus }}{\text { (subspecies not }}$
recorded)

Mus musculus morococha

Mus musculus musculus white mouse

Mustela vison

Ovibos moschatus niphoecus

Ovis dalli

$\underline{\text { Rangifer }}$ tarandus

Spermophilus parryi i plesius house mouse

house mouse

(S. Am. E S.U.S.)

mink

muskox

Dall sheep

reindeer

arctic ground squirrel (Alaska range) adenocarcinoma $\quad 1$

lymphosarcoma

myxosarcoma

bronchiogenic

carcinoma

adenocarcinoma

1

fibrosarcoma

1

tumors (uncertain histologic type)

fibropapil loma

adenoma

renal adenocarcinoma

1 squamous cell

carcinoma

(n)

1


TABLE 2

A Partial Listing of Tumors

Found in Alaskan Mammals ${ }^{a}$

Species

Common Name

Type of

Number

Neoplasm

of

Cases

\begin{tabular}{|c|c|c|}
\hline Alces alces gigus & moose (Alaska) & $\begin{array}{l}\text { fibroma } \\
\text { fibropapillomab }\end{array}$ \\
\hline Callorhinus ursinus & northern fur seal & lymphosarcoma \\
\hline Canis lupus & wolf (captive) & tonsillar carcinomac \\
\hline Castor canadensis & American beaver & rhabdomyoma \\
\hline$\underline{\text { Cervus elaphus }}$ & wapiti (American elk) & $\begin{array}{l}\text { embryonal nephromad } \\
\text { myxosarcoma } \\
\text { osteogenic sarcoma }\end{array}$ \\
\hline$\frac{\text { Clethrionomys }}{\text { dawsoni }}$ & $\begin{array}{c}\text { northern red-backed } \\
\text { vole (Fairbanks) }\end{array}$ & $\begin{array}{l}\text { adnexal carcinoma } \\
\text { rhadbomyosarcoma } \\
\text { sarcoma }\end{array}$ \\
\hline Dasycercus cristicauda & $\begin{array}{l}\text { crested-tailed } \\
\text { marsupial "mouse" }\end{array}$ & 1 ipoma \\
\hline Dicrostonyx stevensoni & collared lemming & $\begin{array}{l}\text { adenocarcinoma } \\
\text { adenoma } \\
\text { mixed cell tumor }\end{array}$ \\
\hline Gulo gulo & wolverine & $\begin{array}{l}\text { thymic lymphosarcoma } \\
\text { transitional cell tumor }\end{array}$ \\
\hline$\frac{\text { Microtus }}{\text { fisheri }}$ & insular vole & papilloma \\
\hline$\frac{\text { Microtus }}{\text { macfarlani }}$ & $\begin{array}{l}\text { tundra vole } \\
\text { (Fairbanks) }\end{array}$ & $\begin{array}{l}\text { adenocarcinoma } \\
\text { osteoma } \\
\text { squamous cell } \\
\text { carcinoma }\end{array}$ \\
\hline Microtus xanthognathus & yellow-cheeked vole & $\begin{array}{l}\text { sebaceous gland } \\
\text { adenoma }\end{array}$ \\
\hline
\end{tabular}


be benign or malignant. The actual diagnosis of tissue type and possible malignancy of tumors in wildlife requires the services of a pathologist whose training represents years of advanced study. The wildlife biologist or student should be aware that tumors are found in wildlife and, with proper preservation of small sections of fresh tissues in a fixative such as $10 \%$ formalin, they can be transported to a pathologist for accurate diagnosis.

Skin tumors are the most likely type to be observed in the field because they are somewhat common and easily visible. Reindeer and caribou (Rangifer tarandus) develop skin tumors known as fibropapillomas that appear as raised, roughened, darkly pigmented growths on various locations of the body (Broughton et al. 1942, Fay 1970). Most cause no disability but are unsightly. $0 c-$ casionally, these tumors become secondarily infected, or overgrow vital structures such as the eye and can threaten the general health of the host. In reindeer, those tumors that are predunculated, that is, connected by a thin stalk, are easily surgically removed at the time of handling.

Another type of commonly seen tumor is the oral papilloma of coyotes (Canis latrans) and wolves (Canis lupus) (Greg and Charlton 1973, Samuel et al. 1978). This type of tumor is very similar to the fibropapilloma of reindeer and caribou, but is normally located on the mouth and lips. These growths resemble multiple accumulations of warts, and can be so extensive as to prevent proper eating and can lead to death from malnutrition.

\section{Significance}

Tumors are not usually classified as an infectious or contagious disease, and their significance in wildlife is normally limited to their effect on the single host animal. As in man, malignant tumors or cancers are terminal unless they are removed or arrested. Most skin tumors are harmless and cause only slight discomfort. It is important that tumors be recognized and properly diagnosed so that some unexplained death not be attributed to a cause more significant to herd health.

A partial listing of some types of tumors seen in Alaskan wildlife is presented in Table 2.

\section{$\underline{\text { References }}$}

BROUGHTON, E., T. L. MILLER and L. P. E. CHOQUETTE. 1942. Cutaneous fibropapillomas in migratory barren-ground caribou. J. Wildl. Dis. 8:138-140.

FAY, L. D. 1970. Skin tumors of the Cervidae. pp. 385-392. In: Infectious Diseases of Wild Mammals. J. W. DAVIS, L. H. KAR $\overline{\text { TTAD }}$ and D. 0. TRAINER, eds. Iowa State Univ. Press, Ames, IA.

GREG, A. S. and K. M. CHARLTON. 1973. Electron microscopy of the virus of oral papillomatosis in the coyote. J. Wildl. Dis. 9:359-361.

SAMUEL, W. M., G. A. CHALMERS and J. R. GUNSON. 1978. Oral papillomatosis in coyotes (Canis latrans) and wolves (Canis lupus) of Alberta. J. Wildl. Dis. $14: 165-169$.

SMITH, H. A. 1972. Neoplasia. pp. 190-298. In: Veterinary Pathology. 4th ed. Lea and Febiger, Philadelphia, PA. 
WALLACH, J. 1978. Ursidae. pp. 628-637. In: Zoo and Wild Animal Medicine. M. E. FOWLER, ed. W. B. Saunders Co., Philadelphia, PA. WATSON, G. L. 1968. Spinabifida in a moose. J. Am. Vet. Med. Assoc. $153: 815$.

WOBESER, G. and R. MACLENNAN. 1970. Ventral hernia in white-tailed deer. J. Wildl. Dis. 7:1-2.
WOBESER, G. and W. RUNGE. 1973. Multiple anomalies in a whitetailed deer fetus. J. Wildl. Dis. 9:356-358.

\title{
Tumors
}

\author{
Robert A. Dieterich
}

Introduction and

Description

Tumor is defined as any abnormal mass resulting from the excessive multiplication of cells. Tumors can appear in all areas of the body. Some are obvious to the casual observer while others are hidden deep within the body cavity and are found only at necropsy. The more accurate medical term for these abnormal structures àre neoplasms (neo $=$ new, plasm = cell substance). More precisely, tumors or neoplasms are the new growth of cells which (1) proliferate continuously without control, (2) bear a considerable resemblance to the healthy cells from which they arose, (3) have no orderly structural arrangement, (4) serve no useful function and (5) for the present at least, have a poorly understood origin and cause (Smith 1972). The continuous proliferation of uncontrolled growth of a tumor is what makes it the formidable and destructive process that can end in death of an affected animal.
Tumors are defined as malignant or benign. Malignant tumors or "cancers" are those that grow by infiltration and destroy whatever is in their path. This destruction continues until the life of the host organism is itself destroyed. Tumors may also be benign. Benign tumors are those which grow only expansively and do not reach into new areas. These tumors are less destructive and can be compatible with life. Malignant tumors possess still another method to further their destructive abilities besides direct infiltration. This other method is that of metastasis. When wildly growing malignant tumors reach and invade the walls of blood vessels and lymph channels, small groups of cells can break loose and be carried away to lodge in still other parts of the host. These new growths (metastatic tumors) themselves multiply and destroy still more areas of the body.

Wildlife, like domestic animals and man, develop tumors in various areas of their bodies and these may 
reported sighting an albino caribou in Interior Alaska.

A few diseases with unknown etiology, but considered to be of non-infectious origin have been reported in the literature for wild animals. We have classified them as miscellaneous non-infectious diseases and they include: hepatic fatty necrosis in white-tailed deer in Texas (Blankenship et al. 1976) and spontaneous renal disease in Louisiana beavers (Castor canadensis) (Stuart et al. 1978) and armadillos (Dasypus noremcinctus) (Stuart et al. 1977).

\section{References}

BARRETT, M. W. and G. A. CHALMERS. 1975. Congenital anomalies in a neonatal white-tailed deer in Alberta. J. Wildl. Dis. $11: 497-501$.

BIRD, R. D. 1933. A three-horned wapiti. J. Mammal. 14:164.

BLANKENSHIP, L. H., L. G. ADAMS, R. M. ROBINSON and J. E. ELLISOR. 1976. Hepatic fatty cirrhosis in Texas white-tailed deer. J. Wildl. Dis. 12:396-401.

BROWN, R. J. and J. HARDMAN. 1973. Hydrocephalus in a newborn 1 lama. J. Wildl. Dis. 9:146-147.

CURATOLO, J. A. 1979. A sighting of an albino caribou in Alaska: a review of North American records. Arctic 32:374-375.

DANIEL, M. J. 1967. Polydactyly in red deer in New Zealand. Saugetierkundliche Mitteilungen $15: 149-155$.

DAVIDSON, M. M. 1971. A case of polydactylism in Sika deer in New Zealand. J. Wildl. Dis. $7: 109-110$.

DEMARTINI, J. C. and G. E. CONNOLLY. 1975. Testicular atrophy in Columbian black-tailed deer in California. J. Wildl. Dis. $11: 101-106$.
HOWARD, D. R., J. D. KREHBIEL, L. D. FAY, J. N. STUTH and D. L. WHITENACK. 1976. Visual defects in white-tailed deer from Michigan: Six case reports. J. Wildl. Dis. 12:143-147.

LORENZ, K. 1965. Evolution and Modification of Behavior. Univ. of Chicago Press, Chicago, IL. $121 \mathrm{pp}$.

MILLER, F. L. and A. J. CAWLEY. 1970. Polydactylism in a whitetailed deer from eastern Ontario. J. Wildl. Dis. 6:101-103.

MILLER, F. L. and E. BROUGHTON. 1971. Polydactylism in a barrenground caribou from northwestern Manitoba. J. Wildl. Dis. 7:307-309.

SCANLON, P. F., D. F. URBSON and J. A. SULLIVAN. 1975. A male pseudo-hermaphrodite white-tailed deer resembling an antlered doe. J. Wildl. Dis. 11:237-240.

SAPERSTEIN, G., H. W. LEIPOLD, S. M. KROUCKENBERG and N. A. MUCKENHIRN. 1977. Congenital defects of wild and zoo mammals. Institute of Lab. An. Res. News. National Acad. Sci. 20, No. 4. 23 pp.

SCHLEGEL, M. W., T. A. LEEGE and R. F. LAPEN. 1972. Scrotal hernia in white-tailed deer. J. Wildl. Dis. $8: 320$.

SNYDER, L. H. 1946. The Principles of Heredity. 3rd ed. D. C. Heath and Co., Boston, MA. 450 pp.

STUART, B. P., W. A. CROWELL, W. V. ADAMS and J. C. CARLISLE. 1977. Spontaneous renal disease in Louisiana armadillos. J. Wildl. Dis. 13:240-244.

STUART, B. P., W. A. CROWELL, W. V. ADAMS and D. T. MORROW. 1978. Spontaneous renal disease in beaver in Louisiana. J. Wildl. Dis. 14:250-253.

TROYER, W. A. 1980. Records of white moose in the McKinley Park area, Alaska. Unpubl. Proc. Alaska Interagency Moose Meeting, Mckinley, AK. 


\title{
Congenital and Miscellaneous
}

\section{Non-Infectious Diseases}

\author{
Albert W. Franzmann
}

In wildlife populations, congenital or hereditary diseases and the resulting anomalies are rare. Defects of a crippling nature are generally naturally selected out of the breeding population, thereby lessening their frequency of occurrence. We will occasionally be called upon to examine animals that have survived the rigors of selection and we may see defects and anomalies recognized as hereditable. Perhaps most important in a discussion of heredity diseases is the consideration one should give to predisposition for a behavioral or pathological state that may be transmitted from generation to generation. This is a virtually untouched field in wildlife diseases. Selective forces, particularly those generated by humans, working on a wildlife population may alter succeeding populations through the process of adaptation. Has man already genetically altered wild populations by selecting for those segments that can inherently adjust to agricultural and urban development; or have these populations simply learned accomodation behaviors? Lorenz (1965) reviewed the concept of "learned" and "innate" elements of behavior.

Signs and diagnosis of anomalies are contingent upon each case. Some anomalies in wild mammals have been described: polydactylism in whitetailed deer (Odocoileus virginianus) (Miller and Cawley 1970), red deer (Cervus elaphus) (Daniel 1967), Sika deer (Cervus nippon) (Davidson 1971), and caribou (Rangifer tarandus) (Miller and Broughton 1971); ventral (Wobeser and MacLennan 1970) and scrotal (Schlegel et al. 1972) hernia in white-tailed deer; hydrocephalus in black bear (Ursus americanus) (Wallach 1978) and llama (Llama glama) (Brown and Hardman 1973); testicular atrophy in black-tailed deer (Odocoileus hemionus) (DeMartini and Connolly 1975); 3 antlered elk (Cervus elaphus) (Bird 1933); dwarfism, internal hydrocephalus, porencephaly, inferior brachygnathia, multiple hepatic cysts and renal dysplasia in white-tailed deer (Wobeser and Runge 1973); inferior brachygnathia, atypical incisor tooth alignment, medially folded ears, humero-radial arthrogryposis and bowing of the radius and ulna in a white-tailed deer (Barrett and Chalmers 1975); congenital eye defects in a white-tailed deer (Howard et al. 1976); male pseudohermaphrodism in a white-tailed deer (Scanlon et al. 1975); and spinal bifida in a moose (Alces alces) (Watson 1968). An extensive listing of reported congenital defects of wild and zoo animals has been published (Saperstein et al. 1977).

Albinism occasionally occurs in many mammal species (Snyder 1946). "Albino" moose reports from the Mt. McKinley Park and Healy, Alaska area have appeared in newspapers and magazines almost annually through the mid to late 1970s; however, Troyer (1980) reported that all these sightings were white or part white moose and not true albinos. A spotted moose was seen regularly near Kasilof, Alaska during 1977. An albino bison (Bison bison) made the news in 1975 when efforts were initiated to capture the animal near Chitina, Alaska for the Children's Zoo in Anchorage. Curatolo (1979) 
HOLLEMAN, D. F. and J. R. LUICK. 1976. Radiocesium kinetics in arctic carnivores. Health Physics $30: 241-243$.

- 1978. Using radioecological data to determine prey selection by the Alaskan wolf. pp. 673-681. In: Proc. Environ. Chem. and Cycling Processes. D. C. ADRIANS and J. L. BRISHBIN, eds. DOE Symp. Ser., CONF. 760429, Augusta, GA.

HOLLEMAN, D. F., J. R. LUICK and F. W. WHICKER. 1975. Transfer of radiocesium from 1 ichen to reindeer. Health Physics 21:657-666.

LUICK, J. R., S. J. PERSON, R. D. CAMERON and R. G. WHITE. 1973. Seasonal variations in glucose metabolism of reindeer (Rangifer tarandus L.) estimated with $\mathrm{V}-\mathrm{I}_{\mathrm{C}} \mathrm{Clucose}$ and $3-{ }^{3} \mathrm{H}$ glucose. Br. J. Nutr. 29:245-259.
TURNER, F. B., R. C. ROWLAND and R. A. WOOD. 1966. Nuclear engineering and wildlife: radioactivity in jack rabbits after the Sedan test. J. Wildl. Manage. 30:433-443.

TWARDOCK, A. R. 1970. Radiation and radionuclides (radioisotopes) in animal physiology. pp. 1385-1426. In: Duke's Physiology of Domest ic Animals. $8 \mathrm{th}$ ed. M. J. SWENSON, ed. Cornell Univ. Press, Ithaca, NY. 
Signs and Diagnosis

With the array of potential types and degrees of tissue involvement from radiation, the history of exposure may provide the best clues in making a diagnosis. Low levels of radiation may cause a multitude of pathological possibilities that would be most difficult to attribute to radiation without tissue sampling for the presence of radioactive material. Even with radiation suspected, the problems of degree of radiation and variabilities of resistance make this a most difficult syndrome to assess.

\section{Significance and Control}

Control is obviously based upon prevention of exposure. Unfortunately, this is not under our control as individuals when considering the most damaging of radiation possibilities (nuclear warfare). To this point in time the limitations on atmospheric testing have minimized fallout potential. Wildlife populations, particularly ungulates, are extremely valuable as monitors of radioactive fallout (Alldredge et al. 1974, Eberhardt et al. 1969, Hanson et al. 1975), and the significance of Alaskan mammals in this role is particularly important due to our relative displacement from the human population centers of the world (atmospheric drift).

A benefit of nuclear engineering to wildlife research is through the use of radionuclides (radioisotopes) in physiological research, particularly in tracing metabolic pathways (Twardock 1970). In Alaska, researchers at the Institute of Arctic Biology have utilized radionuclides in reindeer studies of forage ingestion rates (Hanson et al. 1975), transfer of radiocesium from lichens to reindeer (Holleman et al. 1975), glucose metabolism (Luick et al. 1973), water flux (Cameron et al. 1976), total body water and blood volume (Cameron and Luick 1972). Radioecological data were applied to determining prey selection in Alaskan wolves (Canis lupus) (Holleman and Luick 1976, 1978). Radionuclides were recently (1980) used at the Kenai Moose Research Center, Alaska to study rate of rumen passage in moose.

\section{References}

ALLDREDGE, A. W., J. F. LIPSCOMB and F. W. WHICKER. 1974. Forage intake rates of mule deer estimated with fallout cesium-137. J. Wildl. Manage. 38:508-516.

CAMERON, R. D. and J. R. LUICK. 1972. Seasonal changes in total body water, extracellular fluid, and blood volume in grazing reindeer. Can. J. Zool. 50:107-116.

CAMERON, R. D., R. G. WHITE and J. R. LUICK. 1976. Accuracy of the tritium water dilution method for determining water flux in reindeer (Rangifer tarandus). Can. J. Zool. 54:857-862.

EBERHARDT, L. L., W. H. RICHARD, C. E. CUSHING, D. G. WATSON and W. C. HANSON. 1969. A study of fallout cesium-137 in the Pacific Northwest. J. Wildl. Manage. 33:103-112.

FORCUM, D. L. 1968. Reproduction of normal and whole-body irradiated cottontails. J. Wildl. Manage. $32: 180-182$.

HALEY, T. J. and R. S. SNIDER, eds. 1962. Response of the Nervous System to lonizing Radiation. Academic Press, New York, NY. 418 pp.

HANSON, W. C., F. W. WHICKER and J. F. LIPSCOMB. 1975. Lichen forage ingestion rates of free-roaming caribou estimated with fallout cesium-137. pp. 71-79. In: Proc. First Int. Reindeer and Caribou Symp. J. R. LUICK, P. C. LENT, D. R. KLEIN and R. G. WHITE, eds. Biol. Pap. of Univ. of Alaska SRI. Fairbanks, AK. 
SCHWARTZ, C. C., J. G. NAGY and S. M. KERR. 1976. Rearing and training pronghorns for ecological studies. J. Wildl. Manage. 40:464-468.

SIEGMUND, 0. H., ed. 1973. The Merck Veterinary Manual. 4th ed. Merck and Co., Rahway, NJ. 1618 pp.

TUCKER, R. K. and D. G. CRABTREE. 1970. Handbook of Toxicity of Pesticides to wildlife. U.S. Govt. Printing office, Washington, DC. $131 \mathrm{pp}$.
WOOD, S. S. 1977. Hair mineraliza$t$ ion of the snowshoe hare in interior Alaska. M.S. Thesis, Univ. of Alaska, Fairbanks, AK.

\title{
Radiation
}

\author{
Albert W. Franzmann
}

$\underline{\text { Introduction and History }}$

Radiation is the emission and propagation of energy in the form of waves or particles through space or matter (Twardock 1970). Ionizing radiation is radiation having sufficient energy to cause ion pairs to be formed in the medium through which it passes, resulting in excitation and ionization of atoms and molecules (Twardock 1970). In living tissue, the disrupted molecules may be ones essential to metabolism of the organism and the result may be observable biollogic effects (Twardock $1970)$. Sources of radiation are; radioactive decay, $x$-ray machines and accelerators, outer space and nuclear fission.

Mammalian response to ionizing radiation is subject to variables, such as; conditions of radiation exposure, extent of body irradiated, part of body irradiated, species and strain, age, sex, diet, stress and hibernation (Twardock 1970). High levels of radiation result in immediate death (Haley and Snider 1962), lesser levels result in radiation sickness and death within days to weeks, and lower ranges of radiation may be exhibited as delayed effects such as: premature aging and life span shortening; neoplasia (tumors); cataract formation and genetic effects (Twardock 1970).

Wildlife populations were affected by early nuclear testing (Twardock 1970), and captive animals have been used in testing the effects of radiation (Forcum 1968, Twardock 1970). of particular significance in Alaska is the use of ungulates as monitors of radioactive fallout. Tissue from caribou (Rangifer tarandus) and moose (Alces alces) have been collected and used to monitor cesium-137 fallout in the manner described by Eberhardt et al. (1969). Radiation damage to wildlife has not been reported for Alaskan wildlife. 


\section{Significance and Control}

The significance of toxic substance affecting wild mammals lies in the potential of occurrence, not in the present reported paucity of occurrence. Moreover, wildlife populations provide an excellent monitor of the presence of toxic substances and pollutants in the environment. Hair element analysis of toxic elements provided base-line data on relative occurrence of potentially toxic elements in Alaskan moose (Alces alces) (Franzmann et al. 1977) and snowshoe hare (Lepus americanus) (Wood 1977) populations. Heavy metal concentrations in kidneys of raccoons (Hoff et al. 1977) and urban gray squirrels (Sciurus carolinensis) (Mckinnon et al. 1976), and lead in hair of urban and rural small mammals were reported (Raymond and Forbes 1975). Mercury concentrations in hair of coyotes (Canis latrans) (Huckabee et al. 1973) were also reported.

In areas where extensive alteration of the environment may occur, it would be of significant value to obtain base-line values of various toxic pollutants from resident wildlife populations in order to monitor possible subsequent pollution. This may be the most feasible and significant method by which toxic alterations may be quantified and whereby control measures implemented.

\section{References}

CUMBIE, P. M. 1975. Mercury in hair of bobcats and raccoons. J. Wildl. Manage. 39:419-524.

DEAN, R. E. and A. H. WINWARD. 1974. An investigation into the possibility of tansy ragwort poisoning of black-tailed deer. J. Wildl. Dis. 10:166-169.
DITERS, R. W. and S. W. NIELSEN. 1978. Lead poisoning of raccoons in Connecticut. J. Wildl. Dis. $14: 187-192$.

FIMREITE, N. and L. M. REYNOLDS. 1973. Mercury contamination of fish in northwestern Ontario. J. Wildl. Manage. 37:62-68.

FRANZMANN, A. W., A. FLYNN and P. D. ARNESON. 1977. Alaskan moose hair element values and variability. Comp. Biochem. Physiol. 57A:299-306.

HOFF, G. L., W. J. BIGLER and J. G. MCKINNON. 1977. Heavy metal concentrations in kidneys of estuarine raccoons from Florida. J. Wildl. Dis. 13:101-106.

HUCKABEE, J. W., F. CARTAN, G. KENNINGTON and F. CAMINZIND. 1973. Mercury concentration in the hair of coyotes and rodents in Jackson Hole, Wyoming. Bull. Environ. Contam. and Toxicol. 9-38-43.

KARSTAD, L. 1967. Fluorosis in deer. Bull. Wildl. Dis. Assoc. $3: 42-46$.

KIRK, R. W. and S. I. BISTNER. 1975. Handbook of Veterinary Procedures and Emergency Treatment. 2nd ed. W. B. Saunders Co., Philadelphia, PA. 716 pp.

MARSH, H. 1965. Newsom's Sheep Diseases. 3rd ed. Williams and Wilkins Co., Baltimore, MD. 456 pp.

MCKINNON J. G., G. L. HOFF, W. J. BIGLER and E. C. PRATHER. 1976. Heavy metal concentrations in kidneys of urban gray squirrels. J. Wildl. Dis. 12:367-371.

NEWMAN, J. R. and Y. MING-HO. 1976. Fluorosis in black-tailed deer. J. Wildl. Dis. 12:39-41.

RAYMOND, R. B. and R. B. FORBES. 1975. Lead in hair of urban and rural small mammals. Bull. Environ. Contam. and Toxicol. $13: 551-553$. 


\title{
Chemical Toxins
}

\author{
Albert W. Franzmann
}

\section{Introduction}

There are several hundred thousand potentially toxic substances to animals on the market (Kirk and Bistner 1975), and a variety of plants capable of poisoning animals when ingested, including fungi and molds (Siegmund 1973). In freeranging mammals many of these toxins are unavailable, and if available many effects are unknown. Additionally, plants recognized as poisonous to domestic animals may or may not be toxic to wild mammals since sensitivity to toxicants varies greatly from species to species, even within the same genus (Tucker and Crabtree 1970). Conversely, some plants not toxic to domestic animals may affect wild mammals. Animals that have evolved in a particular environment are not likely to utilize plants that are toxic to them; however, if that environment is disturbed, plants not ordinarily present may become a part of that animal's habitat and they may be toxic if consumed. Overgrazing by herbivores, and reduced available habitat may result in consumption of plants not ordinarily part of the animal's diet. With a history of these conditions and sick or debilitated animals reported, plant poisoning must be considered.

Pesticides have been widely used on forests, rangeland and farmland and acute toxicity measured as LD50 (amount lethal to $50 \%$ of a population) has been determined for 108 pesticides on selected wild animals, mostly birds, and a few for mule deer (Odocoileus hemionus) (Tucker and Crabtree 1970).

Poison Control Centers are located throughout the United Atates, and current information may be obtained from these centers regarding a specific poison. Location of the centers may be obtained from the Department of Health, Education and Welfare, Public Health Service, Washington, DC.

The complexity of toxicology in all species and particularly with a paucity of information on wild mammal toxicology negates a discussion on specific toxins. Information on specific poisons and their treatment in domestic animals is available (Kirk and Bistner 1975, Marsh 1965, Siegmund 1973). Reports of toxic substances affecting wild mammals are limited to: lead poisoning of raccoons (Procyon lotor) (Diters and Nielsen 1978); mercury poisoning in mink (Mustela vison) and otter (Lutra canadensis) (Fimreite and Reynolds 1973); fluorosis in white-tailed deer (Odocoileus virginianus) (Karstad 1967) and Columbian black-tailed deer (Odocoileus hemionus columbianus) (Newman and Ming-Ho 1976). The possibility of ragwort poisoning (Senecio spp.) in black-tailed deer was eliminated in an investigation (Dean and Winward 1974), but suspected as causing death in a pronghorn (Antilocapra americana) (Schwartz et al. 1976).

During editing of this compendium, urea fertilizer poisoning of bison (Bison bison) was suspected as the cause of death of several animals near Delta Junction, Alaska (R. Zarnke, pers. comm.). According to Zarnke, the urea fertilizer was stored outside and available to freeranging bison in the area. Tissues were collected and sent to laboratories to confirm the field diagnosis. 


\title{
Dystocia
}

\author{
Albert W. Franzmann
}

Introduction and History

Dystocia (difficult or impossible birth or parturition) may potentially occur in any mammal. In free-ranging mammals the sequel to complicated dystocia is generally death for both mother and young. It is conceivable that following a difficult birth process, the mother may succumb to complications such as a ruptured or prolapsed uterus and/or secondary infection. The young may survive for a short time, but will most likely die of starvation or be killed by predators or scavengers.

At the Kenai Moose Research Center, Alaska, the bodies of a moose (Alces alces) cow and calf were found soon after death and calving complications were assessed as cause of deaths (LeResche 1970). Skeletal remains of a cow moose with the skeletal remains of her calf positioned in the birth canal were found by the author, suggesting death at parturition.

A variety of artiodactylid species were listed as having dystocia cases in zoos, and vaginal and uterine prolapse were a common sequel to dystocia (Boever 1978).

\section{Signs and Diagnosis}

Prolonged labor at birth ( 2 hours or more for artiodactylids) (Boever
1978) with malpresentation and/or malposition of the fetus are the signs of dystocia. The many basic underlying or fundamental causes of dystocia have been described (Benesch and Wright 1952).

\section{Significance and Control}

The impact of dystocia and its negative consequences are negligible in a wildlife population, but the recognition of the possibility of occurrence is a facet of understanding disease in a wildlife population.

\section{References}

BENESCH, F. and J. G. WRIGHT. 1952. Veterinary Obstetrics. Williams and Wilkins Co., Baltimore, MD. $459 \mathrm{pp}$.

BOEVER, W. J. 1978. Artiodactylids. pp. 771-797. In: Zoo and Wild Animal Medicine. M. E. FoWLER, ed. W. B. Saunders Co., Philadelphia, PA.

LERESCHE, R. E. 1970. Moose report. Alaska Dept. Fish and Game, P-R Proj. Segment Rep. 100 pp. 


\title{
Burns
}

\author{
Albert W. Franzmann
}

Introduction and History

Heat, friction, electricity, sun rays and corrosive chemicals persistently contacting wild animals, may cause destruction of epithelium or deeper tissues and may be all classed as burns. Occurrence of burns in Alaskan wildlife is rare and has been reported only for lightning burns (Shaw and $\mathrm{Neiland} \mathrm{1973)} \mathrm{and} \mathrm{rope}$ burns (this section).

\section{Signs and Diagnosis}

The usual lesion of a moderately severe burn in animals is diffuse edema of the skin and subcutaneous tissue, with or without small vesicles and sloughs. Severe burns result in devitalized skin and deep tissue injury. Animals with burns over more than $50 \%$ of the body should be euthanatized. Burns on the body from $15-50 \%$ require systematic treatment, and burns covering less than $15 \%$ of the body can usually be managed without extensive care (Siegmund 1973).

\section{$\underline{\text { Significance and } \text { Control }}$}

The significance of burns in wildlife populations is negligible but should be recognized. Forest fires certainly cause burns to wildlife, and we may be called upon to make a judgement on handling a burn victim. Friction or rope burns do occur when handling and restraining wildlife (Fowler 1978). We have induced rope burn to moose (Alces alces) while slinging and weighing moose (Arneson and Franzmann 1975). Actinic sun rays do not generally negatively affect wildlife; however, in the rare instance of albinism, sunburn may be a limiting factor for these animals.

\section{References}

ARNESON, P. D. and A. W. FRANZMANN. 1975. A winch-tripod device for weighing moose. J. Zoo. Anim. Med. 6:10-12.

FOWLER, M. E. 1978. Restraint and Handling of Wild and Domestic Animals. Iowa State Univ. Press, Ames, IA. 332 pp.

SHAW, G. E. and K. E. NEILAND. 1973. Electrocution of a caribou herd caused by lightning in central Alaska. J. Wildl. Dis. 9:311-313.

SIEGMUND, 0. H., ed. 1973. The Merck Veterinary Manual. 4th ed. Merck and Co., Rahway, NJ. 1618 pp. 


\title{
Electrical Stroke
}

\author{
Albert W. Franzmann
}

Introduction and History

Electrical stroke refers to nerve tissue damage by powerful currents of electricity. Electrocution in animals is usually due to lightning, although accidental contact with high-tension wires occurs (Allen 1979). Electrical shock is rare and has been reported in Alaska only for caribou (Rangifer tarandus) (Shaw and Neil and 1973).

\section{Signs and Pathogenesis}

Death from electrical shock is due to inhibition of the vital nerve centers with resultant respiratory and circulatory arrest (Covault 1963). The force of the shock largely determines the clinical picture. Animals are usually killed instantly, but when the shock is light, the animal may appear dazed for a few hours or up to weeks. These animals generally recover. Singed hair or burns with arboreal designs may be present on the animal (see Burn section, this chapter).

\section{Diagnosis}

A history of electrical storms and the acute death of many animals in a confined area will help confirm a diagnosis. Singed hair may occur in $90 \%$ of animals struck by lightning (Reeks 1927). A Lichtenberg pattern found engraved in the arctic tundra at the electrocution site of caribou was the only sign due to decomposition of the animals (Shaw and Neiland 1973). On post mortem distention of the veins with dark fluid, blood may occur (Covault 1963).

\section{Significance and Control}

Control of lightning strike in wild animals is not possible, and limited for contact with high tension lines, other than by quick repair of downed lines. The significance of these events is negligible. It was estimated that lightning electrocution in an Alaskan caribou herd was expected to occur once every several decades (Shaw and $\mathrm{Ne} \mathrm{i}$ land 1973).

\section{References}

ALLEN, D. L. 1979. Wolves of Minong. Houghton Mifflin Co., Boston, Ma. 498 PP.

COVAULT, C. H. 1963. Diseases due to physical agents. pp. 747-753. In: Diseases of Cattle. W. J. GIBBONS, ed. Am. Vet. Publ., Santa Barbara, CA.

REEKS, R. C. 1927. Lightening and electric shock in animals. Vet. Rec. 7:901.

SHAW. G. E. and K. A. NEILAND. 1973. Electrocution of a caribou herd caused by lightning in central Alaska. J. Wildl. Dis. 9:311-313. 
DOERR, J. G. and R. A. DIETERICH. 1979. Mandibular lesions in the Western Arctic Caribou Herd in Alaska. J. Wildl. Dis. 15:309-318.

FENSTERMACHER, R. 1943. Diseases of white-tailed deer. Cornel Vet. $33: 323-332$.

FOWLER, M. E. 1978. Restraint and Handling of Wild and Domestic Animals. Iowa State Univ. Press, Ames, IA. 332 pp.

FRANZMANN, A. W. and C. C. SCHWARTZ. 1979. Kenai Peninsula moose calf mortality study. Alaska Dept. Fish and Game, P-R Proj. Final Rep. 17 pp.

FRANZMANN, A. W., C. C. SCHWARTZ and R. O. PETERSON. 1980. Moose calf mortality in summer on the Kenai Peninsula, Alaska. J. Wildl. Manage. 44:764-768.

HUDSON, R. J., T. TENNESSEN and $S$. STURKO. 1976. Behavioral and physiological reactions of bison to handling during anthrax vaccination program in Wood Buffalo National Park. pp. G1-G21. In: Wood Buffalo National Park Bison Research. J. STELFOX, ed. Can. Wildl. Serv., Edmonton, ALB.

KIRK, R. W. and S. I. BISTNER. 1975. Handbook of Veterinary Procedures and Emergency Treatment. 2nd ed. W. B. Saunders, Philadelphia, PA. 716 pp.

MILLER, F. L., A. J. CAWLEY, L. P. E. CHOQUETTE and E. BROUGHTON. 1975. Radiographic examination of mandibular lesions in barren-ground caribou. J. Wildl. Dis. $11: 465-470$.

MURIE, 0. J. 1951. The Elk of North America. The Stackpole Co., Harrisburg, PA. 376 pp.
NIELSEN, C. A. 1977. Wolf necropsy report: Preliminary pathological observations. Alaska Dept. Fish and Game, P-R Proj. Special Rep. $129 \mathrm{pp}$.

PENCE, D. B. and $W$. P. MEINZER. 1977. Blindness in a coyote from the Rolling Plains of Texas. J. Wildl. Dis. 13:155-159.

PETERSON, R. L. 1955. North American Moose. Univ. Toronto Press, Toronto, ONT. 280 pp.

RAUSCH, R. A. 1959. Some aspects of population dynamics of the Railbelt moose population, Alaska. M.S. Thesis, Univ. of Alaska, Fairbanks, AK.

SCHLEGEL, M. W., T. A. LEEGE and R. F. LAPEN. 1972. Injurious antler anomaly in a Rocky Mountain elk. J. Wildl. Dis. 8:319.

SCHMIDT, J. L. and D. L. GILBERT, eds. 1978. Big Game of North America: Ecology and Management. Stackpole Books, Harrisburg, PA. $464 \mathrm{pp}$.

SIEGMUND, 0. H., ed. 1973. The Merck Veterinary Manual. 4th ed. Merck and Co., Rahway, NJ. 1618 Pp.

TIMMERMANN, H. R. and M. W. LANKESTER. 1978. Joint disease in ungulates with special reference to moose. Proc. 14th N. Am. Moose Conf. and Workshop. Hal ifax, NS. 14:89-108.

TOMEK, A. 1977. The occurrence of some ecological parameters of the moose in Poland. Acta Theriologica 22:485-508.

WOODFORD, M. H. 1973. Reduction of a rectal prolapse in a wild lioness. J. Wildl. Dis. 9:178-181. 
trauma management in animals is the Handbook of Veterinary Procedures and Emergency Treatment (Kirk and Bistner 1975). Mandatory treatment for accidental wounds include: (1) control hemorrhage, (2) relieve pain and control patient, (3) treat shock, (4) control infection, (5) clean and close wound, (6) provide after care (Siegmund 1973).

Several forms of trauma in wild populations have been described: Malocclusion in rodents and lagomorphs (Fenstermacher 1943); barbed wire, fighting and antler injury in elk (Cervus elaphus) (Murie 1951, Schlegel et al. 1972); eye injury and blindness in a coyote (Canis latrans) (Pence and Meinzer 1977); rectal prolapse in a lioness (Panthero leo) (Woodford 1973); mandibular trauma in caribou (Rangifer tarandus) (Doerr and Dieterich 1979, Miller et al. 1975); fractures in white-tailed deer (Odocoileus virginianus) (Fenstermacher 1943); injuries in black-tailed deer (Odocoileus hemionus) (Cowan 1946); accident, puncture wounds and predation injury to moose (Franzmann and Schwartz 1979, Peterson 1955, Timmermann and Lankester 1978); $50.9 \%$ of 112 wolves (Canis lupus) examined in Alaska had evidence of traumatic injury (skull concussion, damaged teeth, fractures of ribs and long bones, damaged or missing ears or toes and abrasions) (Nielsen 1977). An ecology and management text for North American big game lists injuries for each species covered (Schmidt and Gilbert 1978).

During rut, male moose inflict injuries to each other and particularly to eyes. The author has seen moose on the Kenai Peninsula blinded by eye injuries during the rut. The foregoing references will serve as detailed description for certain forms of trauma. The variability of cause and effect of trauma in wildlife is infinite.

\section{Significance and Control}

In free-ranging animals traumatic injuries are frequent. The impact on the population is generally negligible except in certain instances. High injury rates and death from highway and railroad accidents may have a significant impact on a depressed population. For example, with deep snows in Alaska, moose are forced to cleared highways and railroad beds and the mortality rate may exceed the annual harvest (Rausch 1959) .

Control of most injuries in a wild population is not possible, but certain measures have been taken by various states to minimize highway and road accidents (speed limits, warning signs, fencing and clearing). When possible, highway and railroad construction should not be done through traditional wintering areas for ungulates. Where human development and the livestock industry are in close approximation to freeranging animals, certain types of fencing and construction may cause injury and/or death. Where this is evident corrections should be made.

\section{$\underline{\text { References }}$}

BALL INGER, W. F., R. B. RUTHERFORD, G. D. ZUIDEMA, eds. 1973. The Management of Trauma. 2nd ed. W. B. Saunders, Philadelphia, PA. $785 \mathrm{pp}$.

BASON, P. A. and J. M. HOFMEYR. 1975. Mortalities asociated with wildlife capture operations. pp. 151-160. In: Capture and Care of Wild Animals. Ralph Curtis, Hollywood, FL.

COWAN, I. M. 1946. Parasites, diseases, injuries, and anomalies of Columbian black-tailed deer in British Columbia. Can. J. Res. 24:71-103. 
ents and do not administer corticosteroids (Kirk and Bistner 1975).

\section{References}

DIETERICH, R. A. 1977. Cold injury (hypothermia, frostbite, freezing. pp. 205-206. In: Current Veterinary Therapy VI. R. W. KIRK, ed. W. B. Saunders Co., Philadelphia, PA.
KIRK, R. W. and S. I. BISTNER. 1975. Handbook of Veterinary Procedures and Emergency Treatment. 2nd ed. W. B. Saunders Co., Philadelphia, PA. 716 pp.

SIEGMUND, 0. H., ed. 1973. The Merck Veterinary Manual. 4th ed. Merck and Co., Rahway, NJ. 1618 $\mathrm{PP}$.

TIMMERMANN, H. R. 1979. Morphology and anatomy of the moose bell and its possible function. M.S. Thesis, Lakehead Univ., Thunder Bay, ONT.

\title{
Trauma
}

\author{
Albert W. Franzmann
}

\section{Introduction and $\mathrm{History}$}

In wildife populations trauma (physical wound or injury) is most often observed in the healed state (scars, healed fractures), but occasionally it is observed first hand. Lacerations, abrasions, contusions, fractures, sprains and strains may occur during capture and restraint (Bason and Hofmeyr 1975, Fowler 1978, Franzmann et al 1980). Severe trauma that naturally occurs in a population will result in death if the animal hemorrhages to the point of circulatory collapse, or if the animal is rendered immobile and cannot feed or defend itself. Partially immobilized mammals may fall prey to predators, or may not be able to traverse terrain necessary to feed and in time will die. Injuries and death to animals from automobiles and trains occurs regularly, and in Alaska it is a major mortality factor in certain moose populations (Rausch 1959). Over an 11 year period, 12 of 55 moose (Alces alces) found dead in Poland exhibited mechanical injuries, primarily fractured limbs (Tomek 1977).

\section{$\underline{\text { Signs }}$ and Diagnosis}

External injuries on the animal are easily recognized, but internal injuries and fractures may be difficult to detect in wild animals. Wound treatment is a science in itself, and specific discussion is beyond the scope of this compendium (Ballinger et al. 1973). Basic first aid principles should be applied to animals as well as man. An excellent source of information for basic 


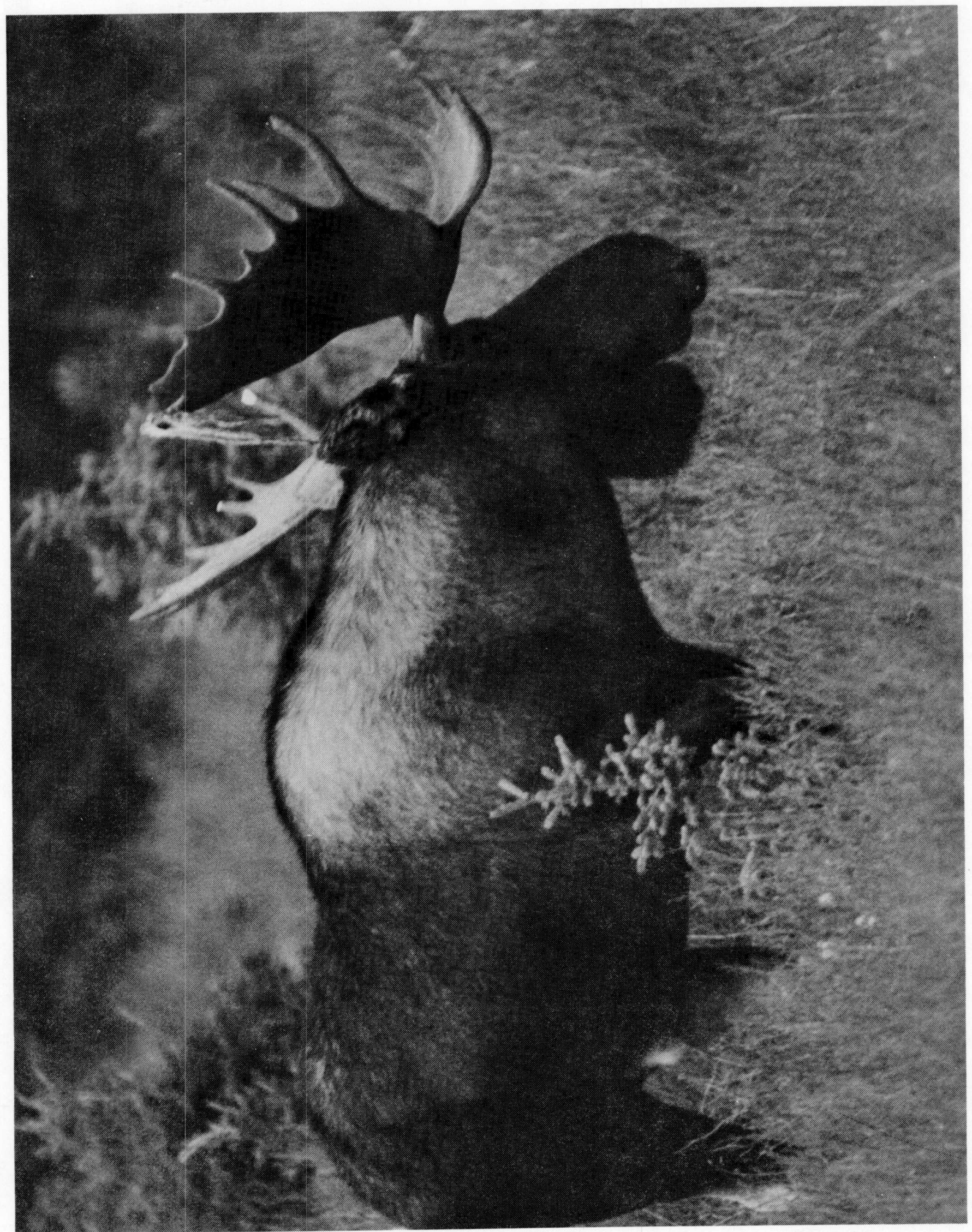

Figure 3. An Alaskan moose with the elongated part of the dewlap or "bell" missing (A. W. Franzmann). 


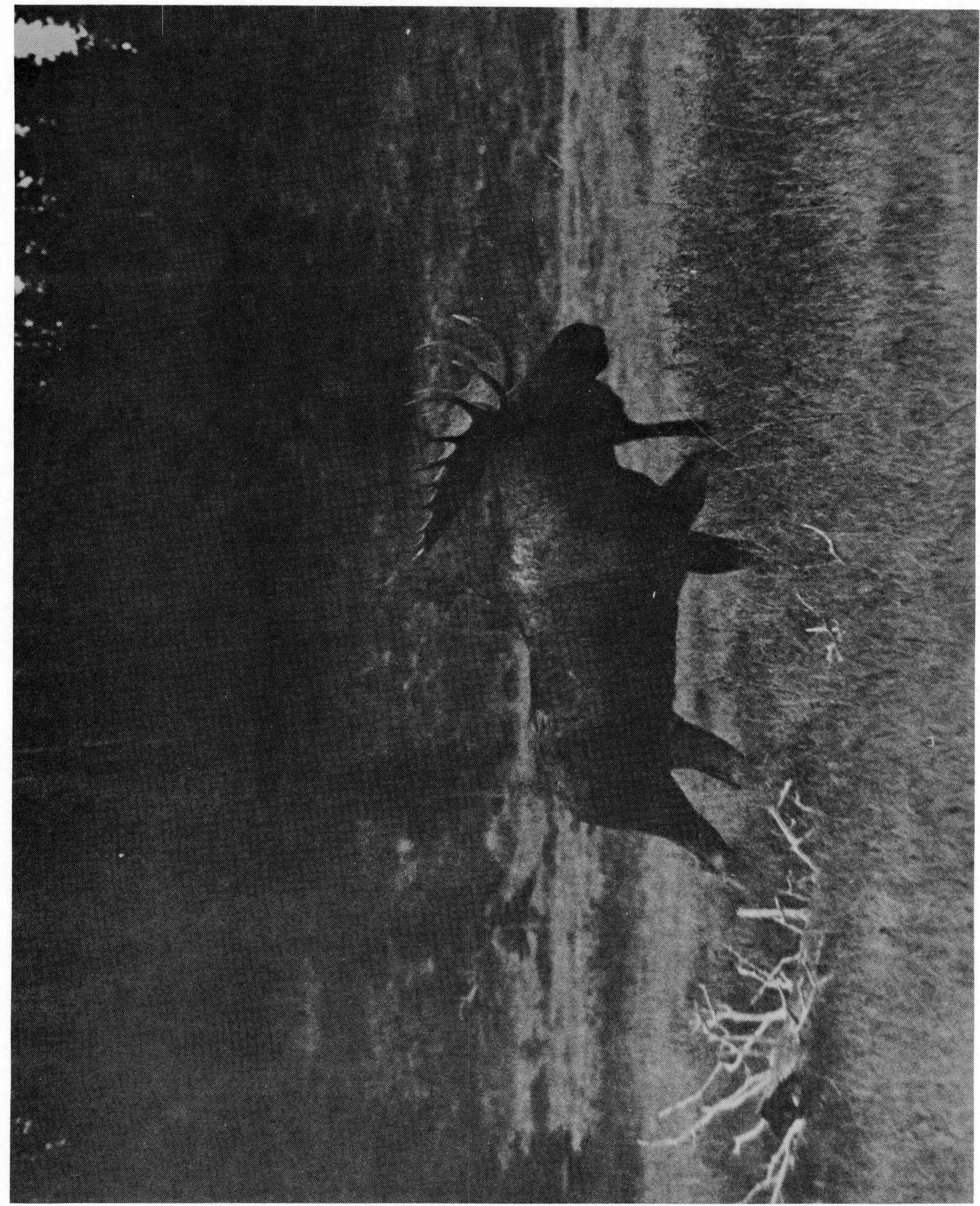

Figure 2. Long dewlap or "bell" on an Alaskan moose (A. W. Franzmann). 


\title{
Frostbite
}

\author{
Albert W. Franzmann
}

Introduction and History

Frostbite or local freezing of tissue results in destruction of superficial tissues with secondary structural and functional disturbances of the smaller surface blood vessels (Siegmund 1973). It may occur in any mammal subjected to prolonged freezing ambient temperatures, generally to extremities such as ears and tail. Nevertheless, reports of frostbite in wild mammals are rare. In Alaskan moose (Alces alces), frostbite of and loss of the bell, particularly in male moose, and loss of the extremities of ears have been seen by the author. Frostbite of the bell is of special interest, since it is the author's belief that most bells on moose in Alaska are shortened by this process. Most moose, and again particularly males, develop an extremely long bell in their early life (Fig. 2). Generally, during winter of the second to fourth year the elongated section of the bell is lost, but the base remains (Fig. 3), perhaps since circulation to the bell extremity is poor (Timmermann 1979). Tick (Dermacentor albapictus) infestation of the bell was suggested as a possible factor in the loss of the bell extremity in Ontario moose ( $H$. Timmermann, pers. comm.). Ticks do not occur on Alaskan moose and thereby are not a factor. Very few aged moose are seen in Alaska with the long pendulous bell.

Dieterich (1977) noted that the
scrotum of male dogs (Canis
familiaris) may be injured by frost-
bite from repeated contact with cold
surfaces or deep snow. This condi-
tion was noted in wolves (Canis
lupus) on the Kenai Peninsula, Alaska

captured during winter for radiocollaring. The affected scrotum was scaly and the skin layers swollen. This condition is not uncommon in Alaskan sled dogs.

Signs

Frostbite first causes the skin to become pale and bloodless. Redness, heat, pain and swelling, loss of hair and peeling of the skin follow. In free-ranging populations this acute phase will seldom be witnessed, but the effects of it will be seen such as: loss of ear extremities, tail, the bell of moose, and irritated and inflamed scrota on wolves and perhaps coyotes (Canis latrans).

\section{Significance and Control}

This disease condition has no measurable impact on a population, but is presented as a condition that should be recognized. If possible, the frostbite victim should receive professional care. Tissue damage and necrosis are greatly increased if thawing and subsequent refreezing occur (Dieterich 1977). The frozen parts should be kept frozen and protected until proper treatment can be initiated (Dieterich 1977).

Frozen tissue should be thawed rapidly in warm water $(38-44 \mathrm{C})$ if refreezing can be prevented. Unnecessary removal of necrotic tissue or amputation should be delayed as healing occurs, as it may take 15 to 20 days to clearly determine the line between healthy and dead tissue (Dieterich 1977). Supportive therapy is indicated to control infection. Do not apply pressure dressing or 
anesthetized (Fowler 1978). Progressive physiologic deterioration occurs with time and lowering body temperature. Dieterich (1977) classified mild hypothermia by body temperatures of 30-32C, moderate hypothermia by temperatures of $22-25 \mathrm{C}$ and profound hypothermia by temperatures of $0-8 \mathrm{C}$. Moderate hypothermia allows survival for approximately 24 hours, while body temperatures of $15 \mathrm{C}$ shorten survival time to 5 or 6 hours. Profound hypothermia narrows survival time to 1 or 2 hours (Dieterich 1977).

\section{Diagnosis}

Lowered body temperature and shivering, along with a good history of the case may be adequate to diagnose an animal with hypothermia. Differentiation from other debilitating diseases must be made.

\section{Significance and Control}

In Alaska, hypothermia may be most often associated with malnutrition (see Malnutrition and Starvation section, this chapter), and as an associate of malnutrition its occur- rence is common during severe winters. Control may not be possible under some circumstances associated with malnutrition (weather extremes, natural disasters), but application of certain wildlife management principles toward improving population nutrition may minimize the occurrence of hypothermia. For control in a single animal the most important procedure is to increase the body temperature by local rubbing and heat application, or by totally warming the animal in a warm water bath at temperatures between $40.5 \mathrm{C}\left(105^{\circ} \mathrm{F}\right)$ and $45.5 \mathrm{C}\left(114^{\circ} \mathrm{F}\right)$ (Fowler 1978). When possible, internal rewarming by peritoneal dialysis is preferred (Dieterich 1977). Supportive therapy is also indicated (Dieterich 1977).

\section{References}

DIETERICH, R. A. 1977. Cold injury (hypothermia, frostbite, and freezing). pp. 205-206. In: Current Veterinary Therapy VI. R. W. KIRK, ed. W. B. Saunders Co., Philadelphia, PA.

FOWLER, M. E. 1978. Restraint and Handling of Wild and Domestic Animals. lowa State Univ. Press, Ames, IA. 332 pp. 


\section{References}

COVAULT, C. H. 1963. Diseases due to physical agents. pp. 747-753. In: Diseases of Cattle. W. J. GIBBoNS, ed. Am. Vet. Publ., Santa Barbara, CA.

FOWLER, M. E. 1978. Restraint and Handling of Wild and Domestic Animals. Towa State Univ. Press, Ames, IA. 332 pp.

FRANZMANN, A. W. and P. D. ARNESON. 1973. Moose Research Center Studies. Alaska Dept. Fish and Game, P-R Proj. Prog. Rep. W-17-5. $116 \mathrm{pp}$.

1974. Immobilization of Alaskan moose. J. Zoo Anim. Med. $5: 26-32$.
FRANZMANN, A. W. and C. C. SCHWARTZ. 1978. Moose Research Center Report. Alaska Dept. Fish and Game, P-R Proj. Prog. Rep. W-17-10. $18 \mathrm{pp}$.

GASAWAY, W. C., A. W. FRANZMANN and J. B. FARO. 1978. Immobilizing moose with a mixture of etorphine and xylazine hydrochloride. J. Wildl. Manage. 42:686-690.

LERESCHE, R. E. and G. C. LYNCH. 1973. A trap for free-ranging moose. J. Wildl. Manage. $37: 87-89$.

\title{
Hypothermia
}

\author{
Albert W. Franzmann
}

Introduction and History

Hypothermia (cold exposure, freezing), is a condition exemplified by low body temperature which results from body heat losses exceeding body heat production necessary to maintain normal body temperature. Contributing factors include: malnutrition, particularly energy intake; exposure and heat loss by conduction and convection; loss of insulating qualities of hair or fur (soiling and moisture); restricted movement (immobilization, physical restraint). For example, a free-ranging Alaskan black bear (Ursus americanus) died from hypothermia in a barrel-trap due to loss of insulating qualities of its hair coat and exposure to a cold and damp environment ( $R$. Modafferi, pers. comm.).
Hypothermia of Alaskan mammals is perhaps more prevalent than we realize. Although most mammals of the north evolved with mechanisms to withstand extreme cold, the predisposing factors to break their resistance are present, particularly malnutrition.

\section{$\underline{\text { Signs }}$}

Low body temperature is the primary sign. At body temperature below $34 \mathrm{C}\left(93.2^{\circ} \mathrm{F}\right)$, thermoregulation in homeotherms (warm-blooded animals) is impaired and at temperatures below $32 \mathrm{C}\left(89.6^{\circ} \mathrm{F}\right)$ the animal is likely to go into a coma and not respond to stimuli (Fowler 1978). Decreased body temperature initiates shivering if the animal is not sedated or 


\title{
Hyperthermia
}

\author{
Albert W. Franzmann
}

Introduction and History

Hyperthermia (heat stroke, heat exhaustion, sun stroke, overheating) is a condition exemplified by high body temperature which results from interference with heat radiation from the body and/or increased heat production through exercise (Covault 1963). Contributing factors include: dehydration; lack of salt; adrenal insufficiency; certain drugs; reduced cardiac efficiency which may result from malnutrition, lack of exercise, infection or intoxication; trauma; infectious disease; and capturing, handling or transporting animals (Fowler 1978). Hyperthermia was induced in moose (Alces alces) (Franzmann and Arneson 1973) during immobilization (Franzmann and Arneson 1974, Gasaway et al. 1978) of trapped animals (LeResche and Lynch 1973) and during helicopter immobilization of black bear (Ursus americanus) and brown bear (Ursus arctos) at the Kenai Moose Research Center. All mammals are susceptible to hyperthermia, but it probably rarely occurs in undisturbed wild animals. Nevertheless, it is not an uncommon syndrome when capturing, handling or transporting wild animals.

\section{$\underline{\text { Signs }}$}

High body temperature, generally $41 \mathrm{C}\left(105.8^{\circ} \mathrm{F}\right)$ and above with increased heart and respiratory rate are initially observed with hyperthermia. With progression of body temperature elevation we observe; dehydration, weakened pulse, restlessness, dullness, incoordination, convulsions and collapse followed by death if the temperature remains very long over 42-43C $\left(107.6-109.4^{\circ} \mathrm{F}\right)$ (Fowler 1978).

\section{Diagnosis}

A history of the case is extremely important, and with most wild animals we may experience hyperthermia to some degree during capture and handling. The high body temperature of the animal with increased heart and respiratory rates are clues. These signs must be differentiated from other syndromes, particularly infectious processes, which may elevate body temperature.

\section{$\underline{\text { Significance and Control }}$}

Treatment is an emergency situation no matter what the cause. An animal with an elevated body temperature should be quickly cooled using water or ice as a spray, bath or enema and adequate ventilation assured. Additional treatment for shock and acidosis may be necessary.

Immobilization, capture, and handling wild animals is a routine for research and management procedure in Alaska, and hyperthermia may be experienced in the process. It is advisable not to capture Alaskan ungulates when ambient temperatures are above $26.6 \mathrm{C}\left(80^{\circ} \mathrm{F}\right)$. Prolonged chase in helicopter immobilization should be avoided. Proper selection and dosage of immobilizing drugs is important. Underdosing etorphine (M-99, D-M Pharmaceutical Inc., Rockville, MD), the immobilizing drug of choice for Alaskan ungulates, may result in hyperexcitability which predisposes hyperthermia. The rate of occurrence of hyperexcitability with etorphine immobilization may be lessened by combining the drug with a tranquilizer (Gasaway et al. 1978). 


\title{
Gastric Ulcers
}

\author{
Robert A. Dieterich
}

\begin{abstract}
Gastric ulcers have been observed in Alaskan snowshoe hares (Lepus americanus) during periods of peak populations (Dieterich and Feist 1980). These lesions appear as brown to black depressed areas in the glandular region of the stomach. At times, the lesions are multiple. Blood discolored to a brown color by gastric juices is found on and near the erosions in the stomach wall. Most lesions are small, measuring a few millimeters in diameter (Iverson et al. 1972). Ulcers of varying sizes are the rule. Ulcers are 1 manifestation of the general adaptive syndrome (GAS) (see General Adaptive Syndrome section, this chapter) and are present in other species of wildlife which are undergoing stressful situations. For example, stomach ulcers have been found in nearly $80 \%$
\end{abstract}

of reindeer (Rangifer tarandus) herded by stressful techniques. The presence of ulcers in the stomach of wildlife can be used as an indication of population stress, but one must be careful to consider all possible causative factors that may be involved.

\section{References}

DIETERICH, R. A. and D. D. FEIST. 1980. Hematology of Alaskan snowshoe hares (Lepus americanus macfarlani) during years of population decline. Comp. Biochem. Physiol. 66A:545-547.

IVERSON, J. O., G. L. HOFF, T. M. YUILL and R. P. HANSON. 1972. Gastric lesions in the snowshoe hare. J. Wildl. Dis. 8:7-9. 


\section{Signs}

In live animals, lameness may be witnessed. The affected joint, generally hip or stifle, reveals degeneration of the articular cartilage and varying degrees of osteophytes (bony out-growth) are present.

\section{Diagnosis}

Diagnosis in wild animals is primarily based upon post mortem examination of joint demonstrating degeneration of cartilage and osteophyte formation.

\section{Significance and Control}

With a limited number of animals in a harvested wild population attaining an aged status, the frequency of occurrence is low; nevertheless, in an unharvested ungulate population such as Isle Royale, Michigan, degenerative joint disease probably plays an important role in increasing the vulnerability of moose to wolf predation by restricting the mobility of the prey (Peterson 1977). This may be true in other populations where aging of a prey species occurs.

A form of control is rendered in ungulate populations where harvesting occurs and where the opportunities for aging are limited.

\section{References}

BOEVER, W. J. 1978. Artiodactylids. pp. 771-815. In: Zoo and Wild Animal Medicine. M. E. FOWLER, ed. W. B. Saunders Co., Philadelphia, PA.

COWAN, I. M. 1946. Parasites, diseases, injuries and anomalies of Columbian black-tailed deer in British Columbia. Can. J. Res. 24:71-103.

CROSS, E. C. 1940. Arthritis among wolves. Can. Field-Nat. 54:2-4.

MAHAM, B. H. and P. S. GIPSON. 1978. Osteoarthritis in a coyote $x$ dog hybrid from Nebraska. J. Wildl. Dis. $14: 395-398$.

PETERSON, R. 0. 1977. Wolf ecology and prey relationships on isle Royale. Nat. Park Ser. Sci. Monograph Series No. II. 210 pp.

SIEGMUND, 0. H., ed. 1973. The Merck Veterinary Manual. 4th ed. Merck and Co., Rahway, NJ. 1618 $\mathrm{pp}$.

STECHER, R. M. 1963. Osteoarthrit is or ankylosing lesions of the spine: Anatomic description of two bears and a bull. Clin. Orthoped. 28:152-162.

TIMMERMANN, H. R. and M. W. LANKESTER. 1978. Joint disease in ungulates with special reference to moose. Proc. $14 \mathrm{th} \mathrm{N}$. Am. Moose Conf. and Workshop, Hal ifax, NS. 14:89-108.

WOBESER, G. and W. RUNGE. 1975. Arthropathy in white-tailed deer and a moose. J. Wildl. Dis. $11: 116-121$. 


\section{References}

BASSON, R. A. and J. M. HOFMEYR. 1975. Mortalities associated with wildlife capture operations. pp. 151-153. In: The Capture and Care of Wild Animals. Ralph Curtis Books, Hollywood, FL.

CHALMERS, G. A. and M. W. BARRETT. 1977. Capture myopathy in pronghorns in Alberta, Canada. J. Am. Vet. Med. Assoc. 171:918-923.

HADLOW, W. J., K. VAN DER WALT and E. YOUNG. 1974. Possible therapy for capture myopathy in captured wild animals. Nature 247:577.

HAIGH, J. C., R. R. STEWART, G. WOBESER and $P$. S. MACWILLIAMS. 1977. Capture myopathy in a moose. J. Am. Vet. Med. Assoc. $171: 924-926$.
FOWLER, M. E. 1978. Medical problems during restraint. pp. 87-88. In: Restraint and Handling of Wild and Domestic Animals. Iowa State Univ. Press, Ames, IA.

FOWLER, M. E. 1978. Capture myopathy. pp. 789-791. In: Zoo and Wild Animal Medicine. W. B. Saunders Co., Philadelphia, PA.

LEWIS, R. J., G. A. CHALMERS, M. W. BARRETT, R. BHATNAGAR. 1977. Capture myopathy in elk in Alberta, Canada: A report of three cases. J. Am. Vet. Med. Assoc. $171: 927-932$.

\title{
Degenerative Arthropathy
}

\author{
Albert W. Franzmann
}

Introduction and History

Degenerative diseases generally refer to tissue deterioration associated with age. These conditions are not prevalent in most freeranging populations due to natural selective forces and most are observed on post mortem examination.

The most commonly observed condition is degenerative arthropathy, also known as degenerative joint or articular disease, osteoarthritis or chronic arthritis. It is generally associated with aging; however, predisposing factors such as metabolic bone disease and copper deficiency may play a part (Siegmund
1973). Degenerative arthropathy of the hip joint from Kenai Peninsula moose (Alces alces) has been seen during examination of moose skeletal remains by the author. It has been reported in white-tailed deer (Odocoileus virginianus) (Wobeser and Runge 1975), moose (Peterson 1977, Timmermann and Lankester 1978, Wobeser and Runge 1975), black-tailed deer (Odocoileus hemionus) (Cowan 1946), coyote (Canis latrans), $x$ dog (Canis familiaris) (Maham and Gipson 1978), wolf (Canis lupus) (Cross 1940) and black bear (Ursus americanus) (Stecher 1963). It has also been described for a variety of zoo artiodactylids (Boever 1978). 
metabolized to lactic acid (Hadlow et al. 1974). The rate at which lactic acid is produced may exceed the rate at which it is removed in the bloodstream and the resultant accumulation leads to acidosis, muscle coagulation and liberation of myoglobin from damaged cells which then appears in the urine.

The common symptoms observed in affected wildlife are mild to severe muscle soreness (lameness), stiffness, weakness, paralysis particularly of the rear legs (Fig. 1) and death (Chalmers and Barrett 1977, Haigh et al. 1977). These symptoms usually appear 1 to 6 weeks after capture. Most commonly, lesions develop within the first week and consist of fairly well demarcated light greyish-brown areas on the muscles. Hemorrhage may be present due to the rupture of muscle groups. In the later stages of the disease, fibrosis and mineralization of necrotic fibers occurs. These changes are often microscopic but significant. The kidneys may be swollen and darker brown than normal from staining due to the excretion of myoglobin. The urinary bladder may contain myoglobin which colors the urine brown. Many times these changes are not evident to the untrained eye and when death occurs suddenly following capture due to myocardial failure, no lesions are apparent.

When muscle cells are damaged, certain cellular enzymes are released into the bloodstream (Lewis et al. 1977). These enzymes can be measured in the laboratory, and those animals having abnormally high levels are at risk. Serum glutamic oxaloacetic transaminase enzymes are found principally in liver, heart and skeletal muscle. Serum creatine phosphokinase is principally found in skeletal muscle, heart and brain. By obtaining serum from animals suspected of having $C M$ and having it analyzed for the presence of these 2 enzymes, one can have confidence in confirming a tentative clinical diagnosis of $\mathrm{CM}$.

Capture myopathy can occur following both physical or chemical methods of restraint and immobilization. Fear and anxiety can contribute to the development of the syndrome as can hyperthermia.

\section{Significance and Prevention}

Capture myopathy can be a significant cause of mortality or disability in wildlife which are being handled by biologists. The fact that this condition can occur several weeks after the initial period of stress must be realized and one can not feel he is not imposing CM losses on captured and released animals simply because they get up and run away (Fowler 1978). Besides outright sudden death due to myocardial failure, there is also death resulting from increased susceptibility to predation or disease in these weakened animals. These types of losses would typically occur several days after capture.

Prevention of overexertion, fear and maintenance of proper thermoregulation are the most important factors to consider. Chases prior to capture are best limited to less than 1 minute in many species when animals are under full flight response. Many professional handlers refuse to pursue an animal after an established safe time has been passed. Frightening noises or activities should be avoided. If severe exertion or stress is inflicted, animals can be treated for shock, and intravenous sodium bicarbonate administered to combat acidosis. When animals are captured and transported, periods of quiet rest should be provided whenever possible. 
SELYE, H. 1946. The general adaptive syndrome and diseases of adaption. J. Clin. Endocrinol. $6: 117-230$.

SELYE, H. 1950. The Physiology and Pathology of Exposure to Stress. Acta, Montreal, PQ. $150 \mathrm{pp}$.

SELYE, H. 1956. The Stress of Life. McGraw-Hill Book Co., New York, NY. 324 .pp.

SELYE, H. 1973. The evolution of the stress concept. Am. Sci. $61: 692-699$.
T-W-FIENNES, R. N. 1965. Atherosclerosis in wild animals. pp. 113-126. In: Comparative Atherosclerosis. J. C. ROBERTS and R. STRASS, eds. Harper and Row, New York, NY.

WIGGERS, K. D., N. L. JACOBSON and R. GETTY. 1971. Atherosclerosis in ruminants. J. Anim. Sci. 32:1037-1041.

\title{
Capture Myopathy
}

\author{
Robert A. Dieterich
}

\section{Introduction}

Capture myopathy (CM) is a syndrome recognized worldwide in many species of mammals, birds and particularly in ungulates (Fowler 1978). Synonyms for this condition include stress myopathy, overstraining disease and white muscle stress syndrome. It is usually associated with the stress of capture, restraint and transportation of wildlife. The usual symptoms are muscular stiffness, weakness, tremors, ataxia, paralysis, myoglobinuria (coffeecolored urine) and death. These symptoms are associated with asymmetrical muscular and myocardial lesions.

\section{History}

Capture myopathy is the result of a physiologic response and therefore the potential of this condition is present in all susceptible species.
The capture of wildlife in Alaska for management and research purposes has increased greatly in the past few years and $C M$ has therefore increased accordingly. Workers in Africa were among the first to recognize the syndrome in the mid.1960s (Basson and Hofmeyr 1975). It has been diagnosed in Alaska in a number of species including moose (Alces alces), reindeer (Rangifer tarandus) and muskox (0vibos moschatus).

\section{Symptoms and Pathogenes is}

The exact mechanisms involved which are responsible for the muscle damage seen in CM are not fully understood but it is believed that severe muscular exertion, straining, prolonged muscular trembling, immobilization and stress all contribute to the development of lesions. Apparently, when there is a sudden stressful increase in muscular activity, muscle glycogen is rapidly 


\section{Significance and Control}

Stress exemplified by the general adaptive syndrome plays a part of every disease process as a predisposing factor and/or a responsive factor. The recognition of this syndrome in medicine is responsible for much of the supportive therapy and nursing care provided a patient. On a population level it is no less important. Good husbandry practices evolved around avoidance of the consequences of this syndrome even prior to it being recognized. In wild populations, again many of the wildlife management principles evolved around the avoidance of the consequences of this syndrome. The capture, handling and transportation of wild animals exemplifies this condition in wild animals (Fowler 1978, Jenkins and Kruger 1975) (also see Capture Myopathy, this chapter). As excitability of Alaskan moose (Alces alces gigas) increased with hand $\overline{i n g}$, blood corticoid levels increased significantly (Franzmann et al. 1975). This was a measured physiological response to the general adaptive syndrome.

Control of the consequences of the general adaptive syndrome in wild populations reverts to an understanding of the ecology of the species, and application of management principles based upon that understanding. In capture and handling techniques we must employ all means possible to minimize stress (Fowler 1978).

\section{References}

BOND, E. 1970. Hepatoma and arteriosclerosis in a wood chuck. J. Wildl. Dis. 6:418-421.
BRODIE, D. A. and H. M. HANSON. 1960. A study of the factors involved in the production of gastric ulcers by the restraint technique. $38: 353-360$.

DIETERICH, R. A. 1969. Medial arterial sclerosis in captive beavers, Castor canadensis. Bull. Wildl. Dis. Assn. 5:115-116.

DIETERICH, R. A. and J. R. LUICK. 1979. An investigation of arterial disease in Alaskan reindeer and caribou. J. Wildl. Dis. $15: 433-435$.

FOWLER, M. E. 1978. Restraint and Handling of Wild and Domestic Animals. Iowa State Univ. Press, Ames, IA. 332 pp.

FRANZMANN, A. W., A. FLYNN and P. D. ARNESON. 1975. Serum corticoid levels relative to handling stress in Alaskan moose. Can. J. Zool. $53: 1424-1426$.

GUTHRIE, D. R., J. C. OSBORNE and $H$. S. MOSBY. 1967. Physiological changes associated with shock in confined gray squirrels. J. Wildl. Manage. 31:102-108.

IVERSEN, J. 0., G. L. HOFF, T. M. YUILL and R. P. HANSON. 1972. Gastric lesions in the snowshoe hare. J. Wildl. Dis. 8:7-9.

JENKINS, W. L. and J. M. KRUGER. 1975. Modern concepts of the animal's physiological response to stress. pp. 172-183. In: The Capture and Care of Wild animals. E. YOUNG, ed. Ralph Curtis Books, Hollywood, FL.

KEITH, L. B., E. C. MESLOW and 0 . J. RONGSTAD. 1968. Techniques for snowshoe hare population studies. J. Wildl. Manage. 32:801-812.

LIBKE, K. G. and H. S. MOSBY. 1968. Stress-induced hemopericardium (Cardiac tamponade) in deer. J. Am. Vet. Med. Assn. 153:813-815.

SARGENT, A. P. 1974. Spontaneous arteriosclerosis in a brown hare. J. Wildl. Dis. 10:130-134. 


\title{
General Adaptive Syndrome
}

\author{
Albert W. Franzmann
}

Introduction and History

In any discussion of diseases in wild mammals, and particularly of non-infectious diseases, stress and the general adaptive syndrome (GAS) must be considered. Stress was defined as the cumulative response of an animal resulting from interaction with its environment via receptors (Selye 1973). The definition alone implies the importance of this syndrome for understanding the mechanism of any disease. A simplified model of the stress mechanism begins with receptors (sensory nerve terminals) which detect change in the animal's environment. The message travels to the central nervous system where a response is transmitted either through the nervous system or through the endocrine system (hormones) to effectors. Effectors are tissues which respond to counteract the challenges to the receptors. The resulting response of the animal depends on the type, duration and intensity of the challenge. Overstimulation.may result in pathologic changes in the body that Selye (1946) termed general adaptive syndrome (GAS). He outlined 3 phases of the syndrome: alarm reaction, stage of resistance and exhaustion phase. This exhaustion phase is of primary concern because it is the period in the GAS when direct effects of overstimulation occur (primarily from excessive corticoid production), and when derangement of the animals adaptive mechanisms play a decisive role in the development of many diseases (Seyle 1946, 1956). The animal during this period is more vulnerable to other challenges. The syndrome occurs in all mammals.
Signs and Diagnosis

Prolonged stimulation and maintenance of the exhaustion phase of the GAS results in adrenocortical hypertrophy (enlarged adrenal gland), lymphoid aplasia (lymph tissue shrinkage), gastrointestinal ulceration and arterial calcification. These lesions are found in man, experimental, domestic and wild animals (Brodie and Hanson 1960, Fowler 1978, Selye 1950). Some examples from wild mammals include: gastric ulcers in snowshoe hare (Lepus americanus) (Iversen et al. 1972); lymphoid aplasia and adrenal hypertrophy (common lesions of wild animals at necropsy) (Fowler 1978); atherosclerosis in captive rodents ( T-W-Fiennes 1965), woodchucks (Marmota monax) (Bond 1970) and beaver (Castor canadensis) (Dieterich 1969); atherosclerosis in free-living brown hare (Lepus europaeus) (Sargent 1974) and in Alaskan caribou (Rangifer tarandus) (Wiggers et al. 1971). In Alaska, an investigation of 34 reindeer and 15 caribou for atherosclerosis and other vascular diseases reported no significant lesions (Dieterich and Luick 1979). Stress induced hemopericardium (an accumulation of blood in the heart sac) was reported in white-tailed deer (Odocoileus virginianus) following its capture ( $L$ ibke and Mosby 1968). Severe hypoglycemia (low blood sugar) was reported in association with acute stress ("shock disease" or "trap sickness") from trapped snowshoe hare (Keith et al. 1968 ) and gray squirrels (Sciurus carolinensis) (Guthrie et al. 1967). 


\title{
Arterial Disease
}

\author{
Robert A. Dieterich
}

Arterial disease occurs in wildlife, but it is not a major cause of death as it is in humans. Actual occlusion of the coronary blood vessels that provide the heart with nutrients, oxygen, etc. is very rare in animals. Two types of arterial disease have been documented in Alaskan wildlife. Atherosclerosis has been found in caribou and reindeer (Rangifer tarandus). Atherosclerosis is a disease of large and medium sized arteries with deposits in the intima of the vessel of yellowish plaques. Arterial changes observed in caribou were quite similar to changes found in man and other animals (Wiggers et al. 1971). It is the opinion of this author that reindeer and caribou living on natural diets do not develop lesions of atherosclerosis significant enough to be a serious threat to their life (Dieterich and Luick 1979).

Medial arterial sclerosis has been diagnosed in captive beaver (Castor canadensis) in Alaska fed cuttings of aspen (Populus tremuloides Michx.) and Purina Lab Chow. Unconfirmed reports indicate that this condition may be present in some wild beavers within the state but only in a mild form. In this type of arterial disease, the arteries are firm and very prominent. When transected, the arteries remain distended and their walls appear to be calcified. If vascular occlusion has not occurred, the animal may be completely healthy or, if partial occlusion has occurred, there may be some signs of limited blood flow to affected areas (Dieterich 1969).

\section{$\underline{\text { References }}$}

DIETERICH, R. A. 1969. Medial arterial sclerosis in captive wild beavers. Bull. Wildl. Dis. Assoc. 5: $115-116$.

DIETERICH, R. A. and J. R. LUICK. 1979. An investigation of arterial disease in Alaskan reindeer and caribou. J. Wildl. Dis. $15: 433-435$.

WIGGERS, K. D., N. L. JACOBSEN and R. GETTY. 1971. Atherosclerosis in ruminants. J. Anim. Sci. 32:1037-1041. 


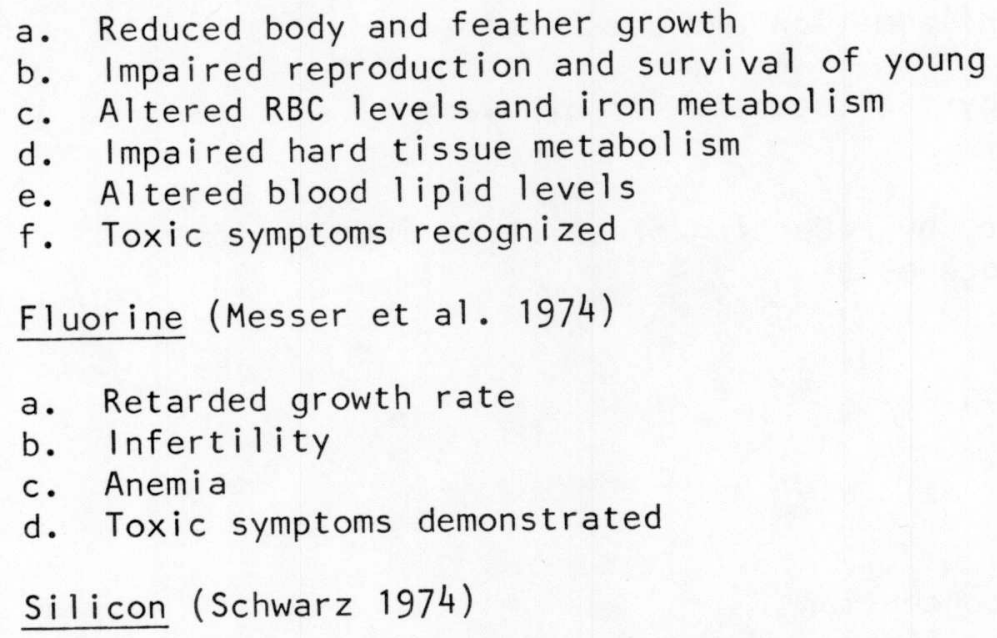

a. Suboptimal reproductive performance

b. Reduced oxidative ability in liver

c. Toxic symptom recognized

Tin (Schwarz 1974)
a. No definite signs recognized, but it has been demonstrated to have a growth stimulant effect
b. Relatively non-toxic 


\section{$\underline{\text { Zinc (Church 1971) }}$}

a. Skin irritability, inflammation and parakeratosis

b. Difficult conception

c. Abnormal estrus and cystic degeneration of ovary

d. Retained placenta

e. Excessive salivation

f. Impaired digestibility by reduction of volatile fatty acids

g. Cessation of spermatogenesis

h. Growth retardation

i. Anorexia

j. Impaired wound healing

k. Relatively non-toxic

\section{Manganese (Church 1971)}

a. Impaired estrus and conception

b. Enlarged joints and stiffness--skeletal abnormalities

c. Weakness and impaired growth

d. Pica

e. Liver degenerative changes

f. Ataxia of newborn

g. Relatively non-toxic

Chromium (Church 1971)

a. Impaired growth and longevity

b. Disturbances in glucose, lipid and protein metabolism

c. Eye disorder--corneal opacity

d. Toxic symptoms recognized

\section{Cobalt (Church 1971)}

a. Listlessness and emaciation

b. Anemia

c. Anorexia

d. Depressed synthesis of B12 in rumen

e. Relatively non-toxic

Molybdenum (Church 1971)

a. Closely related to $\mathrm{Cu}$ and $\mathrm{S}$. High levels depress $\mathrm{Cu}$ and $\mathrm{S}$

b. Renal calculi

c. Relatively non-toxic, but high levels depress other elements

Selenium (Church 1971)
a. Nutritional muscular dystrophy
b. Impaired fertility
c. Persistent diarrhea
d. Depressed growth rate
e. Associated with diseases responding to vitamin $E$ therapy
f. Toxic symptoms recognized 
Phosphorus (Church 1971)

a. Anorexia and weight loss

b. Pica

c. Listless and dull

d. Osteomalacia and ricketts

e. Impaired fertility in females

\section{Sulphur (Church 1971)}

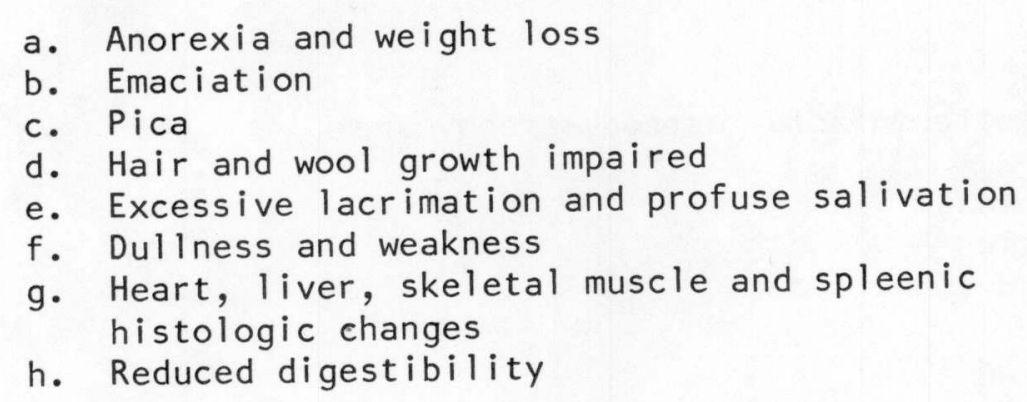

\section{MICRO OR TRACE-ELEMENTS}

\section{lodine (Church 1971)}
a. Thyroid deficiency (enlarged thyroid--goiter)
b. Lowered metabolic rate
c. Birth of hairless, weak or dead young--cretinism
d. Reduced reproductivity (suppressed estrus in females and reduced libido in males)
e. Relatively non-toxic

\section{Iron (Church 1971)}
a. Anemia
b. Anorexia and weight loss
c. Listlessness
d. Gastritis
e. Relatively non-toxic
Copper (Church 1971)
a. Anemia (iron transfer related)
b. Severe diarrhea
c. Depigmentation of hair and defective keratinization of wool and hooves
d. Ataxia and paralysis
e. Fibrosis of cardiac muscles and heart failure
f. Bone deformities (osteoporosis)
g. Reduced fertility and impaired reproductive performance
h. Toxic symptoms recognized 
TABLE 1

An Outline of Some Recognized Mineral

Deficiency Symptoms and Signs

\section{MACRO-ELEMENTS}

Calcium (Church 1971)

a. Skeletal disorders (osteomalacia, osteodystrophy and ricketts)

b. Anorexia and weight loss

c. Lowered milk production

d. Tetany and muscular dysfunction

Chlorine (Hays and Swenson 1970)

a. Principal anion in body fluids

b. Alkalosis

\section{Potassium (Church 1971)}

a. Anorexia and weight loss

b. Listlessness and weakness

c. Pica (depraved appetite)

d. Impaired response to disturbance

e. General stiffness

$f$. Kidney degeneration

g. Histologic change in muscles

\section{Magnesium (Church 1971)}

a. Opisthotonus--retracted head--muscle tremors--convulsions

b. Ataxia

c. Hypersensitivity

d. Increased heat production and fall in energy retention due to tonic muscular activity

e. Reduced appetite

f. Rumen flora changes and reduced digestibility

g. Anemia and jaundice

h. Impaired blood clotting

i. Liver damage and reduced serum albumin and alpha and gamma globulins

j. Serum enzyme changes

Sodium (Church 1971)
a. Pica
b. Decrease body weight
c. Anorexia
d. Listlessness
e. Harsh skin
f. Tetany--collapse and death 
SCHROEDER, H. A., A. P. NASON, I. H. TIPTON and J. J. BALASSA. 1966. Essential trace metals in man: Copper. J. Chron. Dis. 19:1007-1034.

SCHWARZ, K. 1974. New essential trace elements (Sn, V, F, Si): Progress report and outlook. pp. 355-380. In: Trace Element Metabolism in Animals--1l. W. G. HOEKSTRA, J. W. SUTTIE, H. E. GANTHES and W. MERTZ, eds. Univ. Park Press, Baltimore, MD.

SCOTTER, G. W. 1971. Fire, vegetation, soil and barren-ground caribou relations in northern Canada. pp. 209-230. In: Fire in the Northern Environment--A Symposium. C. W. SLAUGHTER, R. J. BARNEY and G. M. HANSEN, eds. Pacific Northwest Forest and Range Exp. Station, Portland, OR.

SMITH, R. L. 1966. Ecology and Field Biology. Harper and Row, New York and London. $686 \mathrm{pp}$.
SPENCER, D. L. and J. B. HAKALA. 1964. Moose and fire on the Kenai. Proc. Third Ann. Tall Timbers Fire Ecology Conf., Tallahassee, FL. 3:11-33.

TOLGYESI, G. and L. BENCZE. 1970. The microelement content of plants consumed by wild mammals, particularly by big game in different game management regions. Pp. 416-418. In: Trace Elements Metabolism in Animals--1. C. F. MILLS, ed. - E. S. Livingstone, Edinburgh, ENG.

UNDERWOOD, E. J. 1977. Trace Elements in Human and Animal Nutrition. $4 \overline{\mathrm{th}}$ ed. Academic $\overline{\text { Press }}$, New York, NY. 545 pp

VIERECK, L. A. 1973. Wild fire in the Taiga of Alaska. J. Quarternary Res. 3:465-495.

WOOD, S. S. 1977. Hair mineralization of the snowshoe hare (Lepus americanus) in interior Alaska. M.S. Thesis, Univ. of Alaska, Fairbanks, AK. 
1977. Alaskan moose hair element values and variability. Comp. Biochem. Physiol. 57A:299-306.

FRANZMANN, A. W., A. FLYNN, P. D. ARNESON and J. L. OLDEMEYER. 1974. Monitoring mineral metabolism via hair element analysis. Proc. 10th N. Am. Moose Conf. and Workshop, Duluth, MN. $10: 1-22$.

FRANZMANN, A. W., R. E. LERESCHE, P. D. ARNESON and J. L. DAVIS. 1976. Moose productivity and physiology. Alaska Dept. Fish and Game, P-R Proj. Final Rep. 87 pp. Multilith.

HAYS, V. W. and M. J. SWENSON. 1970. Minerals. pp. 663-690. In: Dukes Physiology of Domestic Animals. M. J. SWENSON, ed. Cornell Univ. Press, Ithaca, NY.

HOEKSTRA, W. G., J. W. SUTTIE, H. E. GANTHER and W. MERTZ, eds. 1974. Trace $\frac{\text { Element }}{\text { Animals--Il. Univ. Park Press, }}$ Baltimore, MD. 775 pp.

HOPKINS, L. L., JR. 1974. Essentiality and function of vanadium. pp. 397-406. In: Trace Element Metabol ism in Animals--II. W. G. HOEKSTRA, J. W. SUTTIA, H. E. GANTHER and W. MERTZ, eds. Univ. Park Press, Baltimore, MD.

JORDAN, P. A., D. B. BOTKIN, A. S. DOMINSKI, H. S. LOWENDORF and G. E. BELOVSKY. 1973. Sodium as a critical nutrient for the moose of Isle Royale. Proc. 9th N. Am. Moose Conf. and Workshop, Quebec City, PQ 9:13-42.

KIRCHGESSNER, M., ed. 1977. Trace Element Metabolism in Man and Animals--III. Univ. Munchen Press, Freising--Weihenstephan, GER. 684 pp.

KRAMER, P. J. and T. T. KOZLOWSKI. 1960. Physiology of Trees. McGraw-Hi 1 Book Co., New York, NY. 642 pp.
KREFTING, L. W. 1974. The ecology of the Isle Royale moose with special reference to the habitat. Tech. Bull. 297-1974, Forestry Series 15. Agrl. Exp. Sta., Univ. Minnesota, St. Paul, MN. 75 pp.

KUBOTA, J. 1974. Mineral composi$t$ ion of browse plants for moose. Naturaliste Can. 101:291-305.

KUBOTA, J., S. RIEGER and V. A. LAZAR. 1970. Mineral composition of herbage browsed by moose in Alaska. J. Wildl. Manage. 34:565-569.

LUTZ, H. J. 1956. Ecological effects of forest fires in the interior of Alaska. U.S. Forest Service Tech. Bull. 1133. 121 pp.

MARSTON, M. R. 1952. Cobalt, copper and molybdenum in the nutrition of animals and plants. Physiol. Rev. $32: 66-121$.

MESSER, H. H., W. D. ARMSTRONG and L. SINGER. 1974. Essentiality and function of flouride. pp. 425-437. In: Trace Element Metabolism in Animals--II. W. G. HOEKSTRA, J. W. SUTTIE, H. E. GANTHER and W. MERTZ, eds. Univ. Park Press, Baltimore, MD.

MILLS, C. F., ed. 1970. Trace Element Metabolism in Animals--1. E.S. Livingstone, Edinburgh, ENG.

NICHOLAS, D. J. D. and A. R. EGAN, eds. 1975. Trace Elements in Soil-Plant-Animal Systems. Academic Press, New York, NY. 417 pp.

NIELSEN, F. H. 1974. Essentiality and function of nickel. pp. 381-395. In: Trace Element Metabolism in Animals--1I. W. G. HOEKSTRA, J. W. SUTTIE, H. E. GANTHER and W. MERTZ, eds. Univ. Park Press, Baltimore, MD.

OLDEMEYER, J. L., A. W. FRANZMANN, A. L. BRUNDAGE, P. D. ARNESON and A. FLYNN. 1975. Browse quality and the Kenai moose population. J. Wildl. Manage. 41:533-542. 
consideration in selection of areas for vegetative rehabilitation or chemical fertilization of moose browse. These practices are stimulated by new information on moose mineral requirements and mineral status of the area.

It would not be wise to invest great sums of money on mechanical or chemical rehabilitation in areas where vegetative response may favor plants that poorly assimilate certain mineral elements. We could instead, divert our activity to an area that would stimulate plants that have the ability to assimilate desirable elements.

Much research is required to assess the various effects our management plans have on mineral cycling, and even more is required to assess the often subtle effects mineral deficiencies may have on a population. The advent of atomic absorption spectroscopy permits intensive mineral element investigations. Perhaps the lack of definitive information regarding wildlife mineral metabolism, and the general lack of information on the mineral aspect of forage quality for wildlife, stems from our previous inability to study minerals on a practical level. Persons planning research projects should consider adding to the sparse information available relative to minerals and moose by incorporating this into their studies, when feasible. The need for mineral metabolism information is certainly not intrinsic to moose, and these studies on other species should be encouraged.

\section{References}

BALLARD, W. B. and T. SPRAKER. 1979. Unit 13 wolf studies. Alaska Dept. Fish and Game, P-R Proj. Rep. $W-17-9$ and $W-17-10.90$ pp.
CHURCH, D. C. 1971. Digestive Physiology and Nutrition of Ruminants. Vol. II. Oregon State Univ. Book Stores, Corvallis, OR. $801 \mathrm{pp}$.

FLYNN, A. and A. W. FRANZMANN. 1974. Manifestation of copper deficiency in a non-restricted wild animal: The Alaskan moose (Alces alces gigas). pp. 95-99. In: Trace Substances in Environmental Health--VIII. D. D. HEMPHILL, ed. Univ. of Missouri, Columbia, MO.

FLYNN, A., A. W. FRANZMANN and P. D. ARNESON. 1975a. Sequential hair shaft analysis as an indicator of prior mineralization in the Alaskan moose. J. Anim. Sci. 41:906-910.

1975b. Molybdenum-sulphur inter-actions in the utilization of marginal copper in Alaskan moose (Alces alces gigas). pp. 115-124. In: Molybdenum in the Environment. Vol. I. W. R. CHAPPELL and K. K. PETERSON, eds. Marcel Dekker, Inc., New York, NY. FLYNN, A., A. W. FRANZMANN, P. D. ARNESON and O. A HILL, JR. 1974. Determination of past trace element uptake in a wild animal by longitudinal analysis of the hair shaft. Die Naturwissenschaften 61:362-363.

FLYNN, A., A. W. FRANZMANN, P. D. ARNESON and J. L. OLDEMEYER. 1977. Indications of copper deficiency in a subpopulation of Alaskan moose. J. Nutr. 107:1182-1189.

FRANZMANN, A. W. and C. C. SCHWARTZ. 1978a. Moose Research Center Report. Alaska Dept. Fish and Game, P-R Proj. Rep. W-17-10. 18 pp. Multilith.

1978b. Moose calf mortality study, Kenai Peninsula. Alaska Dept. Fish and Game, P-R Proj. Rep. W-17-10. 20 pp. Multilith.

FRANZMANN, A. W., A. FLYNN and P. D. ARNESON. 1975. Levels of some mineral elements in Alaskan moose hair. W. Wildl. Manage. $39: 374-378$. 
burden of this potential problem to the many he already has?

First of all he or she need not be concerned with the specifics of mineral element metabolism, but should be aware of the possibility of deficiencies. More specifically, the manager should be concerned with the possible consequences that land use and management practices may have regarding mineral utilization of the animal in question. Our present state of knowledge will not specifically guide the manager, but awareness of the potential alteration of mineral availability in general, may influence management and land use decisions. Following, are some examples that illustrate this.

1. Land-Use Planning and Management. Moose may assimilate and store a majority of their mineral requirements within the short span of summer. The bulk of their mineral requirements are obtained from primary plant species, but they may require additional supplemental sources such as licks. On the Kenai Peninsula and elsewhere (Krefting 1974), a minor source of total food intake comes from submerged vegetation; however, results from other studies (Kubota et al. 1970, 01demeyer et al. 1975) show these plants to be high in mineral content. On Isle Royale, it was reported that aquatic macrophytes had 500 times more $\mathrm{Na}$ than found in terrestrial vegetation (Jordan et al. 1973). Land use planning and management decisions should consider these potentially important mineral sources ( 1 icks and submerged vegetation) and not exclude access and use by moose.

2. Fire Supression and Prescribed Burning. Whatever the actual cause, there does seem to be a release of nutrients and a fertilizing effect of fire on the organic soils in Alaska (Viereck 1973). Unfortunately, little is known regarding the uptake of micro-elements following fire, but the increase in macro-elements has been noted (Lutz 1956, Scotter 1971). Perhaps more important is the demonstrated population response to fire, such as by moose on the Kenai Peninsula, Alaska (Spencer and Hakala 1964).

Changes in soil and plant composition and quality following fire are influenced by many variables such as parent material, moisture, plant composition prior to fire, intensity of fire and climatic events following fire. Nevertheless, the generalization can be made that browse quality and palatability improve and moose populations respond. Mineral content, including trace minerals, must be considered a positive factor in these events.

3. Forestry Practices. The alteration of mineral cycling in forestry practices is influenced by many factors; however, parent material and subsequent weathering basically determine availability in the forest. The mineral content of hardwood litter usually is higher than that of conifer litter, and bark usually contains 3 to 10 times as high a concentration of minerals as wood (Kramer and Kozlowski 1960). Removal of overstory permits revegetation in an area and browsing animals respond to the increase in available vegetative biomass; however with harvest, appreciable amounts of nutrients are removed (Smith 1966). The long term effects that removal of the forest overstory has on mineral cycling and subsequent availability to animals is difficult to establish, but the ecosystem can remain productive only if the nutrients withdrawn are balanced by an inflow of replacements (Smith 1966).

4. Vegetative Rehabilitation and Fertilization. The variation among plants in their ability to assimilate mineral elements may be cause for 
also support the diagnosis. Moose browse from the Kenai Peninsula had a mean copper value of $5.6 \mathrm{ppm}$ which was 5.3 to 6.9 times lower in copper than for comparable plant species in Minnesota (Franzmann and Schwartz 1978b).

\section{Significance and Control}

Copper deficiency in moose has potential population significance in that basic physiologic processes may be impaired (Hoekstra et al. 1974, Kirchgessner 1977, Mills 1970, Nicholas and Egan 1975, Underwood 1977). Identifying these deficiencies in a population is difficult as the signs may be subtle and effects confused with other conditions. The important consideration is that one must be aware that they can occur, and when a population is showing signs of poor productivity, the possibility of deficiencies existing should not be overlooked. Copper deficiency in moose has been used as an example since it has been identified in Alaska.

Classic symtomatology associated with deficiencies created artificially in a laboratory are not likely experienced in wild populations. It is also unlikely that we will observe "barnyard" type deficiencies in freeranging animals. What are the effects of subtle deficiencies that do not exemplify themselves with known signs, but may affect the well being of the animal or animals and possibly interfere with reproductive success or calf viability?

Essential mineral elements are classified as macro-elements and micro or trace-elements identified. The classification difference corresponds to concentration in tissues, with the trace elements generally expressed in concentrations of parts per million (ppm) and macro-elements in percent. The classic definition of a trace element is any element that exists in concentration equal to or less than $\mathrm{Fe}$ in the body, with iron being the first of the traceelements. The essential macroelements are calcium $(\mathrm{Ca})$, chlorine (Cl), potassium (K), magnesium $(\mathrm{Mg})$, sodium ( $\mathrm{Na}$ ), phosphorus $(P)$ and sulphur ( $S$ ) and they have long been known to be required (Church 1971). The essential micro-elements are iodine (I), iron (Fe), copper ( $\mathrm{Cu}$ ), zinc $(Z n)$, manganese $(M n)$, cobalt (Co), molybdenum (Mo), selenium, (Se), chromium ( $\mathrm{Cr}$ ), tin ( $\mathrm{Sn})$, vanadium (V), fluorine (F), silicon ( $\mathrm{Si}$ ) and nickel ( $\mathrm{Ni}$ ) (Schwarz 1974, Underwood 1977). Twenty additional elements should be considered and investigated as possibly essential (Schwarz 1974).

To elucidate the physiology of these elements and the symptomatology associated with their deficiencies would require a symposium in itself. We will only generalize and state that they all have been established as essential to normal function and their deficiencies are associated with malfunction in basic physiological systems. Some symptoms associated with deficiencies of these elements are listed in Table 1. In addition, all essential elements have recognized toxic levels and associated pathology. The field of mineral metabolism is so complex and interrelated with itself and with other systems, primarily neural and hormonal, that no element can be considered alone. Two classic examples of this are the detrimental effect of high molybdenum intake on copper metabolism (Underwood 1977), and the shift of the Ca: $P$ ratio influenced primarily by phosphorus levels. There are other examples of these types of interractions between minerals which confound our understanding of mineral metabolism.

So what can a wildlife manager do, and why should he or she add the 
a population is a difficult facet of this deficiency to evaluate in a wild population.

This deficiency, as with most deficiencies in a free-ranging population, would not generally be identified by clinical signs. More likely, the identification would come as a result of monitoring mineral metabolism via hair element analysis (Franzmann et al. 1977, 1974). This was the mechanism by which the potential for copper deficiency in moose on the Kenai Peninsula was identified. Three years of moose hair analyses indicated a low copper status in this population. Confirmation of the deficiency was based upon further studies of both the animal and plants consumed by moose.

Hoof overgrowth is a result of copper deficiency and subsequent interference with the establishment of a proper matrix in the formation of "hard" keratin (Marston 1952). The mechanism of copper deficiency interfering with fertility is not thoroughly understood, but low fertility in cattle grazing on copper deficient pastures has been reported (Underwood 1977).

\section{Diagnosis}

Preliminary information from hair mineral element analysis of a population may indicate the potential for copper deficiency. Hair is an ideal recording filament which reflects intake of various elements (Flynn et al. 1975a, 1974). Hair should be plucked from the shoulder hump of Cervidae and a bundle $2 \mathrm{~cm}$ in diameter should be collected, airdried and identified. Samples are analyzed by atomic absorption spectroscopy, and an array of up to 18 elements may be analyzed (Franzmann et al. 1976). Baseline hair element values from wild animals are presently available for only moose (Franzmann et al. 1977) and snowshoe hare (Lepus americanus) (Wood 1977). Researchers in Alaska are presently accumulating hair element values from black bear (Ursus americanus), brown bear (Ursus arctos), wolves (Canis lupus) and coyotes (Canis lat (Ballard and Spraker 1979, Franzmann and Schwartz 1978a, 1978b). Baseline values from species of concern are needed to help assess relative deficiency states.

Clinical signs of copper deficiency are an essential part of the diagnostic procedure. If a hair element profile for a population appears low in copper, clinical manifestation of hoof overgrowth, impaired fertility, and possibly signs recognized in domestics may be identified (Underwood 1977).

Confirmation of copper deficiency requires eliminating the possibility of copper metabolism interference by cadmium, iron, molybdenum and zinc (Flynn and Franzmann 1974, Flynn et al. 1975a, 1977). In addition to low hair copper levels, an important indicator of copper deficiency is blood ceruploplasmin levels. These 2 copper markers have been used to indicate copper problems even when clinical signs were not evident (Flynn et al. 1977). Additional confirmation can be made by analyses of the affected hoof which would have significantly lower copper and sulphur levels than a normal hoof. Sulphur levels are low due to interference in the disulphide bond from the copper deficiency (Flynn et al. 1977). The tensile strength of moose hair with low copper levels is significantly less than hair with normal copper levels (Flynn et al. 1977).

Analysis of plants browsed by moose, and the respective soils may 


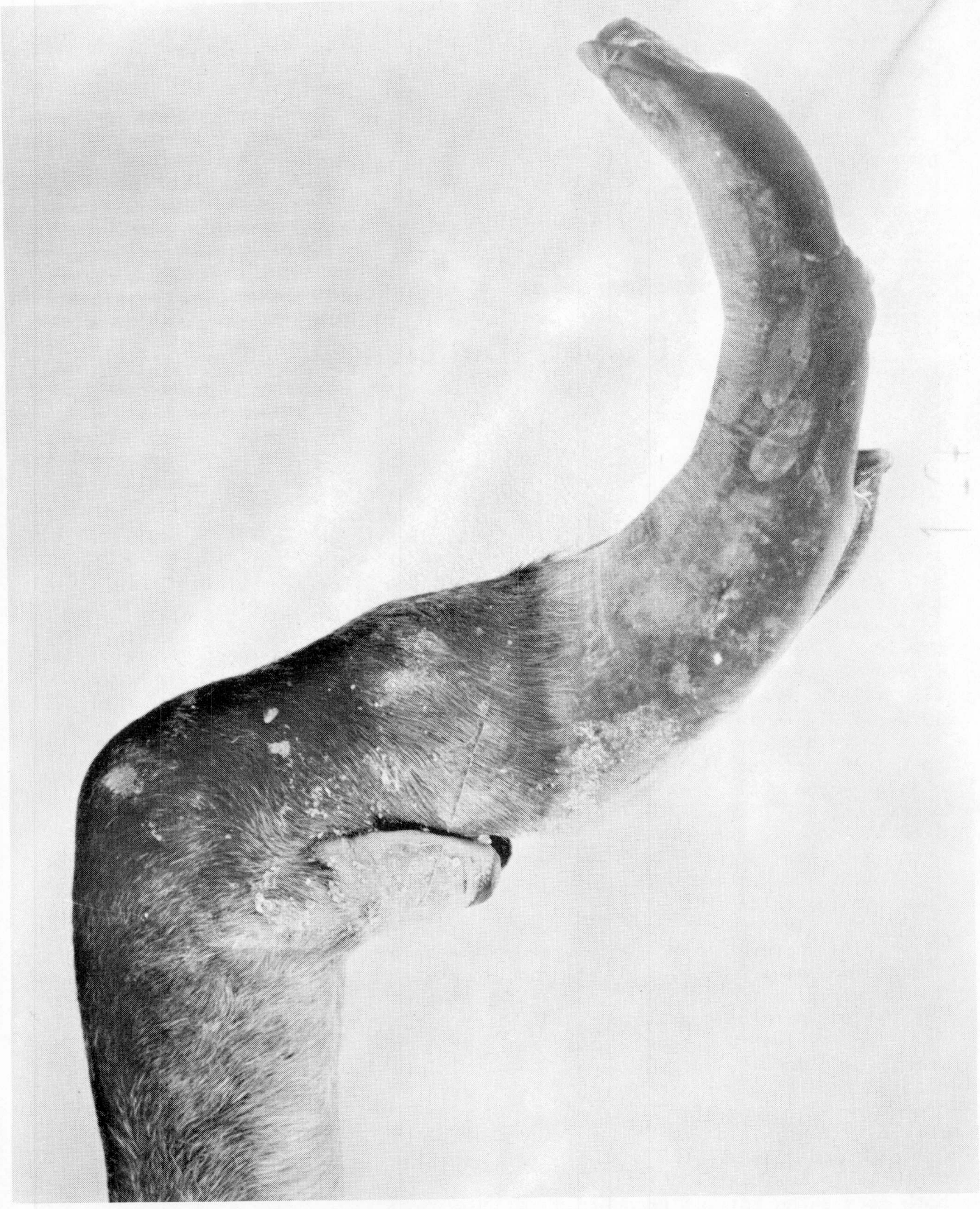

Figure 1. Hoof overgrowth from copper deficiency in an Alaskan moose (A. W. Franzmann). 


\section{References}

CONLOGUE, C. J., W. J. FOREYT, A. L. HANSON and J. A. OGDEN. 1979. Juvenile rickets and hyperparathyroidism in the arctic fox. J. Wildl. Dis. 15:563-567.

DIETERICH, R. A. and R. W. VAN PELT. 1972. Juvenile osteomalacia in a coyote. J. Wildl. Dis. 8:146-148.
FOWLER, M. T. 1978. Metabolic bone disease. pp. 55-76. In: Zoo and Wild Animal Medicine. $\overline{M .} \frac{\text { T. }}{\text { T. }}$ FOWLER, ed. W. B. Saunders Co., Philadelphia, PA.

VAN PELT, R. $W$. and M. T. CALEY. 1974. Nutritional secondary hyperparathyroidism in Alaskan red fox. J. Wildl. Dis. 10:47-52.

\title{
Copper Deficiency
}

\author{
Albert W. Franzmann
}

Introduction and History.

Copper deficiency (hoof overgrowth syndrome) in a subpopulation of Alaskan moose (Alces alces gigas) on the Kenai Peninsula was identified in 1974 based upon hair, blood, hoof and browse mineral element analyses (Flynn and Franzmann 1974, Flynn et al. 1977, Franzmann et al. 1975, 1977, 1974). Clinical manifestation of the disease is exemplified by the hoof overgrowth syndrome (Fig. 1) and possibly by a decline in reproductive rates (Flynn et al. 1977). The Alaskan moose is the only Cervidae in which a copper deficiency has been confirmed. Copper metabolism studies in white-tailed deer (Odocoileus virginianus) and red deer (Cervus elaphus) have been reported (Schroeder et al. 1966, Tolgyes $i$ and Bencze 1970), but clinical signs were not noted nor was a deficiency confirmed. George K. Davis (pers. comm.), University of Florida, indicated that the hoof overgrowth syndrome was frequently observed in cattle in the Florida Everglades, and white-tailed deer in the area around Perry in Taylor County, Florida. In both areas the soil is recognized as being copper deficient.
Copper deficiency in laboratory and domestic animals has been extensively studied and is manifested by anemia, bone disorders, neonatal ataxis, impaired pigmentation and keratinization of hair and wool, infertility, cardiovascular disorders, diarrhea and impaired fatty acid metabolism (Kramer and Kozlowski 1960, Kubota 1974, Schroeder et al. 1966, Scotter 1971).

\section{$\underline{\text { Signs }}$ and Pathogenesis}

The hoof overgrowth syndrome in moose has been sporadically reported on the Kenai Peninsula (Fig. 1). An overgrown hoof is on display at the Damon Museum in Soldotna. Hunters occasionally report a moose with this condition, and several have been trapped and immobilized at the Kenai Moose Research Center. Identification of this condition may be minimized where natural predation occurs, in that these animals would be more susceptible to predation. Nevertheless, the incidence is low, perhaps less than $1 \%$ of the population. Reduced reproductive rates in 
MAUTZ, W. W. 1978. Nutrition and carrying capacity. pp. 321-348. In: Big Game of North America: Ecology and Management. J. L. SCHMIDT and D. L. GILBERT, eds. Stackpole Books, Harrisburg, PA. SIEGMUND, 0. H., ed. 1973. The
Merck Veterinary Manual. 4th ed. Merck and Co., Rahway, NJ. 1618 pp.
WOBESER, G. and W. RUNGE. 1975. Rumen overload and rumenitis in white-tailed deer. J. Wildl. Manage. 39:596-600.

\title{
Metabolic Bone Disease
}

\author{
Albert W. Franzmann
}

\section{Introduction and History}

Fowler (1978) described metabolic bone disease as a prolonged deficiency of calcium, vitamine $D$, or an improper calcium/phosphorus ratio. He has an excellent outline of the disease syndrome and should be referred to for more detailed information.

Conditions associated with the syndrome osteoporosis (porous bone), osteomalacia (soft bone), rickets (disturbed bone formation), fibrous osteodystrophy (defective bone formation) and secondary hyperparáthyroidism (enlarged parathyroid gland) are more of ten seen in caged animals, but may occur in freeroaming species, particularly carnivores. Osteomalacia was reported in a captive coyote (Canis latrans) in Alaska (Dieterich and Van Pelt 1972) and secondary hyperparathyroidism and fibrous osteodystrophy were reported in captive Alaskan red fox (Vulpes vulpes) (Van Pelt and Caley 1974). Rickets and hyperparathyroidism were detected in arctic fox (Alopex lagopus) trapped on St. Paul Island, Alaska (Conlogue et al. 1979).

\section{Signs and Diagnosis}

The metabolic bone disease syndrome exhibited by an individual animal depends on the species, age and duration of deficiency, degree of deficiency and type of deficiency (Fowler 1978). Details of the complexity of signs and diagnosis have been described (Fowler 1978).

\section{Significance and Control}

The incidence of metabolic bone disease is not common in caged or captive wild mammals, but is rarely seen in free-roaming animals. The likelihood of a bone deforming disease requiring a relatively long development time being observed in a wild population is lessened when predators and scavengers are present. 


\title{
Rumen Overload
}

\author{
Albert W. Franzmann
}

Introduction and $\mathrm{History}$

Rumen overload, often called acute overeating, acute indigestion, rumen impaction or ruminal acidosis, results from an abrupt diet change from roughage or browse to large quantities of readily fermentable carbohydrate in ruminant animals (Siegmund 1973). It is characterized by increased acidity in the rumen (Ahrens 1967). Chemical rumenitis may develop in animals that survive the metabolic acidosis of the acute phase (Blood and Henderson 1968).

This syndrome is well recognized in domestic ruminants and was described in white-tailed deer (Odocoileus virginianus) (Wobeser and Runge 1975). The condition has been associated with "rescue" operations of starving wildlife where ruminants are fed readily fermentable carbohydrate crops (corn, hay, wheat, etc.) after they have exhibited signs of malnutrition (Mautz 1978). The result is generally death. In Alaska, the condition was observed by the author in late winter 1972-1973 in a moose (Alces alces) calf in a "rescue" attempt. The syndrome has most likely occurred in other Alaska ruminants and may potentially occur, but winter feeding "rescue" operations and standing crops are not common.

\section{$\underline{\text { Signs }}$ and Diagnosis}

An animal with rumen overload will be anorexic (off feed), dull and exhibit a firm, enlarged rumen (Fincher and Fox 1963). If predisposed by malnutrition, various signs associated with malnutrition may be detected. Diagnosis in wildlife is most often made at necropsy, and is characterized by a rumen containing a large amount of fluid plus grains (Wobeser and Runge 1975). The content is acidic ( $\mathrm{pH} 3.0$ to 6.0) and has a distinct sour odor (Wobeser and Runge 1975). When rumenitis develops, the rumen is inflamed and may have signs of secondary infections (Wobeser and Runge 1975).

\section{Significance and Control}

In Alaska, the occurrence of rumen overload is undoubtedly rare, but as the human population increases we will undoubtedly see an increase in efforts to "rescue" starving freeranging animals resulting in an increased incidence of rumen overload. Secondary control is inherent in understanding the management of starving animals and the consequences of improper feeding of these animals, but primary control lies with application of sound wildlife management principles to minimize starvation in free-ranging ruminants.

\section{References}

AHRENS, F. A. 1967. Histamine, lactic acid and hypertonicity as factors in the development of rumenitis in cattle. Am. J. Vet. Res. 28:1335-1345.

BLOOD, D. C. and J. A. HENDERSON. 1968. Veterinary Medicine. 3rd ed. Balliere, Tindall and Cassell, London, ENG. 927 pp.

FINCHER, M. G. and F. H. FOX. 1963. Acute indigestion. pp. 190-195. In: Diseases of Cattle. W. J. GIBBONS, ed. 2nd ed. Am. Vet. Publ., Santa Barbara, CA. 
HAYS, V. W. and M. J. SWENSON. 1970. Minerals. pp. 663-690. In: $\frac{\text { Duke's }^{\prime} \text { s }}{\text { Animals. }} \frac{\text { Physiology }}{8 \text { th ed. M. J. SWENSON, }}$ ed. Cornell Univ. Press, Ithaca, NY.

KLEIN, D. R. 1964. Range-related differences in growth of deer reflected in skeletal ratios. J. Mammal. 45:226-235.

KLEIN, D. R. 1968. The introduction, increase, and crash of reindeer on St. Matthew Island. J. Wildl. Manage. 32:350-367.

LEOPOLD, A. 1933. Game Management. Charles Scribner's Sons, New York, NY. $481 \mathrm{pp}$.

MAUTZ, W. W. 1978. Nutrition and carrying capacity. pp. 321-348. In: Big Game of North America: Ecology and Management. J. L. SCHMIDT and D. L. GILBERT, eds. Stackpole Books, Harrisburg, PA.

NICHOLAS, D. J. D. and A. R. EGAN, eds. 1975. Trace Elements in Soil-Plant-Animal $\frac{\text { Systems. }}{\text { demic Press, New }}$ York, NY. 417 pp.

ODUM, E. P. 1971. Fundamentals of Ecology. 3rd ed. W. B. Saunders, Philadelphia, PA. 574 pp.

RAUSCH, R. L. 1950. Observations on histopathological changes associated with starvation in Wisconsin deer. J. Wildl. Manage. $14: 156-161$.
RINEY, T. 1955. Evaluating condition of free ranging red deer with special reference to New Zealand. New Zealand J. Sci. and Technol. $36: 429-463$.

ROBINSON, W. L. 1960. Test of shelter requirements of penned white-tailed deer. J. Wildl. Manage. 24:364-371.

RYDER, M. L. 1958. Nutritional factors influencing hair and wool growth. pp. 305-334. In: The $\frac{\text { Biology }}{\text { MONTAGNA }} \frac{\text { of }}{\text { and }} \frac{\text { Hair }}{R .}$ A. Growth. Academic Press, New York, NY.

SMITH, R. L. 1966. Ecology and Field Biology. Harper and Row, New York, NY. 686 pp.

UNDERWOOD, E. J. 1977. Trace Elements in Human and An imal Nutri-

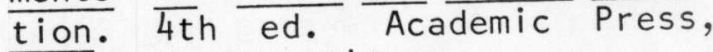
New York, NY. 545 pp.

VERME, L. J. 1962. Mortality of white-tailed deer fawns in relation to nutrition. Proc. Nat. White-tailed Deer Symp. 1:15-38.

WOLFE, M. L. 1978. Habitat changes and management. pp. 349-368. In: $\frac{\text { Big }}{\text { Ecology and }} \frac{\text { Game }}{\text { Management. }} \frac{\text { North }}{\mathrm{J} . \mathrm{L} \text {. }}$ $\frac{\text { Ecology and }}{\text { SCHMIDT and D. Management. J. LILBERT, eds. }}$ Stackpole Books, Harrisburg, PA. 


\section{Significance and Control}

The malnutrition/starvation syndrome is the most prevalent disease affecting Alaskan wildlife. Control of malnutrition and starvation in wildlife populations implies minimizing losses, because some factors influencing this syndrome are not controllable (weather, natural disasters). The application of sound principles of wildlife management to wildlife populations as each situation is presented may improve the welfare of that population and thereby minimize losses from malnutrition and starvation. Various procedures to improve wildlife habitat have been successful and described (Wolfe 1978). Winter feeding of free-ranging ungulates has been done regularly for elk in Wyoming and Washington. The validity of this controversial practice has been reviewed (Mautz 1978).

There is no simple solution to the malnutrition/starvation syndrome and this exemplifies the primary messages of this treatise; (1) an understanding of ecology and wildlife management is basic to wildlife disease investigations, and (2) an understanding of the population from which the animal of concern comes is essential.

\section{References}

ANDERSON, A. E., D. E. MEDIN and D. C. BOWDEN. 1972. Indices of carcass fat in a Colorado mule deer population. J. Wildl. Manage. 36:579-594.

CHEATUM, E. L. 1949. Bone marrow as an index of malnutrition in deer. New York State Conservationist 3:19-22.

COLES, E. H. 1967. Veterinary Clinical Pathology. W. B. Saunders Co., Philadelphia, PA. $455 \mathrm{pp}$.
COOK, R. S., M. WHITE, D. 0. TRAINER and W. C. GLAZENER. 1971. Mortality of young white-tailed deer fawns in south Texas. J. Wildl. Manage. 35:47-56.

COWAN, I. M. 1950. Some vital statistics of big game on overstocked mountain range. Trans. N. Am. Wildl. Conf. 15:581-588.

DASMAN, R. F. 1966. Wildlife Biology. John Wiley and Sons Inc., New York, NY. 231 pp.

DIMOPOULLOS, G. T. 1970. Plasma proteins. pp. 97-129. In: Clinical Biochemistry of Domestic Animals. $\frac{\text { Vol. I. J. J. DANEKA }}{\text { J. }}$ and C. E. CORNELIUS, eds. Academic Press, New York, NY.

FLESH, P. 1954. Hair growth: nutritional factors. pp. 601-661. In: Physiology and Biochemistry of the Skin. S. ROTHMAN, ed. Univ. Chicago Press, Chicago, IL.

FRANZMANN, A. W. and P. D. ARNESON. 1976. Marrow fat in Alaskan moose femurs in relation to mortality factors. J. Wildl. Manage. $40: 336-339$.

FRANZMANN, A. W. and R. E. LERESCHE. 1978. Alaskan moose blood studies with emphasis on condition evaluation. J. Wildl. Manage. 42:334-351.

FRANZMANN, A. W., R. E. LERESCHE, P. D. ARNESON and J. L. DAVIS. 1976. Moose productivity and physiology. Alaska Dept. Fish and Game, P-R Prog., Final Rep. 87 pp. Multilith.

GREER, K. R. 1968. A compression method indicates fat content of elk femur marrows. J. Wildl. Manage. 32:747-751.

GREER, K. R. 1969. Femur marrow reveals the condition of game animals. Montana Fish and Game Dept. Research Bulletin. $10 \mathrm{pp}$.

HARRIS, D. 1945. Symptoms of malnutrition in deer. J. Wildl. Manage. 9:319-322. 
to a point where catabolism occurs. At this time body protein tissue rather than food intake, is used to supply the energy needs of the animal. A decrease in albumin levels may occur with increased catabolism (Dimopoullos 1970).

\section{Signs--Post Mortem}

Fat deposition in most ungulates first disappear over the rump, chronologically followed by the disappearance of subcutaneous fat, kidney and mesenteric fat and pericardial fat (Harris 1945). The last fat deposits to disappear are those of bone marrow (Cheatum 1949), and consequently measuring this fat reserve has been utilized to reveal condition of ungulates (Anderson et al. 1972, Franzmann and Arneson 1976, Greer 1968). Other indices of carcass fat such as bled carcass weight, eviscerated carcass weight, kidney fat index, carcass density, percentage of carcass fat, and depth of back fat have been used to evaluate ungulate body conditions (Anderson et al. 1972). Elk (Cervus elaphus) were considered in excellent condition with femur marrow fat of $80 \%$ or more and in poor condition below $20 \%$ (Greer 1969). Femur marrow fat values below $10 \%$ dry weight may reliably identify moose that have starved (Franzmann and Arneson 1976). Fat deposits can be used as a direct measure of an animal's condition by reflecting its physiological adjustment to the environment (Riney 1955).

Post mortem examination of the quantity and quality of stomach, rumen, or digestive tract contents may assist in assessing potential malnutrition. Skeletal ratios were used to reflect range related growth differences in black-tailed deer (Odocoileus hemionus) (Klein 1964). Histopathological changes in starved Wisconsin white-tailed deer were described as; abundant hemosiderin in the spleen, decreased fat in marrow, a reduction in number and size of follicles in the spleen and fatty degeneration of the heart (Rausch 1950). Signs and causes of neonatal starvation were reported for whitetailed deer (Cook et al. 1971, Verme 1962) and elk (Cowan 1950).

\section{Diagnosis}

Some or all of the above signs may be seen with a variety of diseases with or without a specific etiology. Malnutrition accompanies or may predispose many specific disease syndromes, but it also accompanies a lack of quantity or quality of food intake. It is therefore important that infectious and parasitic diseases be eliminated as causative. Malnutrition confirmation may be strengthened by the history of the population as reflected by reproductive success, behavioral and feeding characteristics and seasonal or cyclic period. Physical examination of the animal and subsequent clinical pathology results will support a malnutrition assessment. Post mortem signs as outlined will provide additional evidence when a dead animal is presented, or when collecting a sample from the population is possible.

Specific forms of malnutrition which begin to attain a specific etiology have been identified and may be the next progression in a diagnosis. Making a specific diagnosis as to a component of nutritional intake entails a detailed diagnostic procedure as outlined for the copper deficiency syndrome in moose (this chapter), and will serve as a guide to diagnosis of other potential mineral deficiencies as outlined for domestic animals (Hays and Swenson 1970, Nicholas and Egan 1975, Underwood 1977). 
less definite regularity. Nevertheless, lack of food supply and subsequent starvation are integral parts of the mechanism, and knowledge of the timing of these events is helpful in wildlife disease assessment.

Early signs of malnutrition may formulate from observing the behavior of a population coupled with a good understanding of the habitat requirements of the population. In ungulates for example, if the animals are concentrating their feeding activity on vegetation not generally considered a select food or known to be of low nutritional quality, we have a clue that malnutrition may occur. Behavioral aberrations such as animals moving and migrating out of traditional use areas may signal food shortages. Increased aggressive behavior as well as timid behavior may add to our case history.

Feeding and behavioral aberrations of a population do not provide a diagnosis, but provide a sound history for the case. It becomes apparent that the observer must have a firm knowledge of the ecology of the species in question to build a diagnosis. Clues from the population history may be subtle, but they may be so obvious that overlooking them would be disastrous.

A physical examination of the live animal may reveal signs that reflect malnutrition, such as; lack of fat cover, poor muscle development, general weakness, poor hair or fur coat, dry and flaky skin and relatively decreased antler and/or skeletal size. A condition scale was developed for white-tailed deer (Odocoileus virginianus) (Robinson 1960) and adopted and used for moose which takes into account the physical characteristics and attitudes of the animal and classifies the animal on a scale from 1 (poorest) to 10 (best) (Franzmann et al. 1976).
Impaired growth or loss of hair is a common and early non-specific response to many nutritional deficiencies in animals (Flesh 1954). Any reduction in food consumption will affect hair growth however, some specific deficiencies have been identified. These deficiencies include; vitamins A, B complex, and $E$, and mineral deficiencies of copper, zinc and iron (Ryder 1958).

Reindeer (Rangifer tarandus) introduced to St. Mat thew Island, Alaska increased from 29 to 6000 over 19 years and subsequently suffered a crash die-off. Body weights following the crash decreased by $38 \%$ for adult females and $43 \%$ for adult males (Klein 1968).

Sampling and analysis of blood components may further assist in identifying malnutrition. Studies of moose blood chemistry and hematology values have provided a tool for comparative evaluation of populations (Franzmann and LeResche 1978). Certain blood values (packed cell volume, hemoglobin, calcium, phosphorus, total protein, albumin, beta globulin and glucose) were used to rank relative condition of moose populations in Alaska. Using these condition influenced blood parameters, the following blood levels in adult moose were concluded to represent moose in average or better condition: packed cell volume--50\%, hemoglobin--18.6 g/dl, calcium 10.4 $\mathrm{mg} / \mathrm{dl}$, phosphorus $5.2 \mathrm{mg} / \mathrm{dl}$, total protein--7.5 g/dl, albumin--4.5 g/dl, beta globulin--0.7 g/dl and glucose--140 mg/dl (Franzmann and LeResche 1978). Levels lower than these from a moose population should alert the observer that there is a physiological aberration. Blood urea nitrogen levels reflect protein intake and cholesterol levels reflect saturated fatty acid intake (Coles 1967). Blood urea nitrogen levels decrease as protein intake decreases 


\title{
Malnutrition and Starvation
}

\author{
Albert W. Franzmann
}

Introduction and History

Malnutrition (poor or inadequate nourishment) and it's negative consequence--starvation, are the most prevalent disease syndromes in freeroaming mammal populations. Both can occur at any time of year. Winter starvation is generally associated with ungulates and other herbivores dependent upon vegetation for food. Some carnivores, omnivores and winter hibernators may experience greater food shortages during summer months. For example, wolves (Canis lupus) on the Kenai Peninsula depend primarily on moose (Alces alces) for food, and are aided in their predation by deep crusted snow and peak pack organization. During summer the pack is less organized (whelping and pup raising) and of course there is no snow. Weight, general condition, and blood parameters in summer are lower than in winter from Kenai Peninsula wolves (Paterson and Franzmann, unpub. data).

The question may arise as to why concern ourselves with a natural phenomenon over which we have little control? Persons concerned with wildlife diseases must be able to recognize malnutrition and starvation in animals. Other diseases may be predisposed by malnutrition, and judgement errors in diagnosis may be made. Conversely, other diseases may predispose malnutrition and starvation and must be recognized.

We will not dwell on the basic causes of malnutrition and starvation which stem from lack of quantity and/or quality of available food because this involves a complex of many factors related to animal habitat requirements. An understanding of basic ecological prin- ciples (Odum 1971, Smith 1966) and wildlife management (Dasman 1966, Leopold 1933) is necessary to background the causes of food shortages in free-roaming populations.

Malnutrition and starvation are as much a part of Alaskan mammal population dynamics as anywhere; no populations are immune. In fact, with many mammal populations at the northern limits of their distribution in Alaska, starvation may have a relatively greater impact than elsewhere.

\section{Signs--Live Animal}

The best measure of the success of a population is that population's productivity as reflected by reproduction, survival and growth of young, and recruitment of breeding animals into the population. Measurement of these qualities may be difficult, but in some poplilations these data are obtained, particularly for game animals by state management agencies. These data may be important in developing a case history, and perhaps critical when extreme situations occur. For example, in the early 1970 s nearly $100 \%$ moose calf mortality was experienced on the Kenai Peninsula due to persistence of snow depths which limited available food.

Fluctuations of animal populations that are more regular than one would expect by chance are called cycles. The 2 most common in Alaska are the 3 to 4 year brown lemming (Lemmus sibiricus) cycle and the 9 to 10 year snowshoe hare (Lepus americanus) and lynx (Felis lynx) cycles (Smith 1966). Many theories have been advanced concerning the cause of these cycles, and other animal cycles of 


\title{
Introduction
}

\author{
Albert W. Franzmann
}

Wildlife disease interest, activity, and reporting have primarily concentrated on parasitology and infectious diseases; disease entities with specific and identifiable etiologies. In the relatively new field of wildlife diseases this is understandable, and is based upon modern trends in human and veterinary medicine. With the discovery of pathogenic agents in the 19th century, a narrowing of the definition of disease from the time of Hippocrates was embraced. Hippocrates defined disease as disharmony within the body, between the body and mind, and between man (or animal) and the environment. The 20 th century redefinition espoused that disease resulted from a collision between a pathogenic agent and a susceptible individual.

The consequence of concentrating on specific etiology in disease has led to emphasis on cure and not prevention. Thereby, the focus was on individuals rather than populations. The pendulum has made its full swing, and many researchers and clinicians today recognize the falacy of a narrow interpretation of disease (Dixon 1978).

One of our goals in this compendium is to make the wildlife disease student cognizant of the broad definition of disease. Wildlife diseases, in particular, are related to populations of animals and their response to environmental forces. Involvement in wildlife diseases generally concerns disharmony syndromes rather than diseases with specific etiologies. Difficulty in identifying causes of disease without a specific etiology and, in the past, a lack of research effort on disharmony syndromes has resulted in a poor understanding of mechanisms involved. For example, the most common disease of free-ranging mammal populations is malnutrition, yet many times this syndrome is not recognized, and the discovery of specific pathogens secondarily associated with the syndrome are labeled as causative. This is not to infer that diseases with a specific etiology do not regularly occur, in fact some non-infectious diseases as well as infectious diseases do have an identifiable single etiology. However, most noninfectious diseases of free-ranging animals are primarily environmentally related, and can be broadly considered as resulting from habitat alteration by natural, mechanical or chemical means resulting in stress, deficiencies or toxicities culminating in impairment and possibly death.

In this treatise we have classified the non-infectious diseases of land mammals as metabolic diseases, stress and degenerative diseases, diseases of physical and chemical origin, and congenital and miscellaneous non-infectious diseases.

\section{Reference}

DIXON, B. 1978. Beyond the Magic Bullet. Harper and Row, New York, NY. 249 pp. 
Chapter 4.

\section{NON-INFECTIOUS DISEASES}


WASHBURN, R. H. and R. A. DIETERICH. 1969. A preliminary report on warble control studies in reindeer. (Abstr.) Proc. 20th Alaska Sci. Conf., AAAS, Alaska Division, 299-300.

WASHBURN, R. H., L. J. KLEBESADEL, J. S. PALMER, J. R. LUICK and D. P. BLEICHER. 1980. The warble-fly problem in Alaska reindeer. Agroboreal is 12:23-28.

WEBER, N. A. 1950. A survey of the insects and related arthropods of Arctic Alaska. Part I. Trans. Amer. Entom. Soc. 76:147-206.
WEISSER, C. F. and K. C. KIM. 1973. Rediscovery of Solenopotes tarandi (Mjoberg, 1915) (Linognathidae: Anoplura), with ectoparasites of the barren ground caribou. Parasitology 66:123-132.

WILKINSON, P. R. 1979. Ixodides. pp. 265-267. In: Canada and
Insect Fauna. H. $\frac{\text { its }}{\text { V. DANKS, }}$ Mem. Entom. Soc. Can. No. 108. $573 \mathrm{pp}$.

ZHIGUNOV, P. S., ed. 1961. Reindeer Husbandry. Izdatel'stvo Sel'skokhozysistvennoi Literatury. 2nd ed. $348 \mathrm{pp}$. 
HAAS, G. E., T. RUMFELT, L. JOHNSON and N. WILSON. 1979. Siphonaptera from mammals in Alaska. Supplement I. Can. J. Zool. 57:1822-1825.

HADWEN, S. and L. J. PALMER. 1922. Reindeer in Alaska. USDA Bulletin No. 1089. Government Printing Office, Washington, DC.

HOLLAND, G. P. 1949. The Siphonaptera of Canada. Can. Dep. Agric. Tech. Bull. 70 .

1963. Faunal affinities of fleas (Siphonaptera) of Alaska with an annotated list of species. pp. 46-63. In: Pacific Basin Biogeography. J. L. GRESSITT, ed. 10 th Pacif. Sci. Congr.

HOPKINS, G. H. E. and M. ROTHSCHILD. 1956. An illustrated catalogue of the Rothschild Collection of fleas (Siphonaptera) in the British Museum (Natural History). Vol. II. Br. Mus. (Nat. Hist.), London, ENG.

HOPLA, C. E. 1965. Alaskan hematophagous insects, their feeding habits and potential as vectors of pathogenic organisms. I. The Siphonaptera of Alaska. Arct. Aeromed. Lab., Ft. Wainwright, AK, Proj. 8241, AAL-TR-64-12. Vol. 1. 267 pp.

JELLISON, W. L. and G. M. KOHLS. 1939. Siphonaptera: a list of Alaska fleas. Pub. Hlth. Rep. 54:2020-2023.

JELLISON, W. L. and C. SENGER. 1973. Fleas of Montana. Mont. Agric. Exp. Sta. Res. Rep. 29.

1976. Fleas of western North America except Montana in the Rocky Mountain Laboratory Collection. pp. 55-136. In: Papers in Honor of Jerry Flora. H. C. $\overline{T A Y} \overline{L O R}$, JR and J. CLARK, eds. W. Wash. St. Coll., Bellingham, WA.

KIM, K. C. 1972. Louse populations of the northern fur seal (Callorhinus ursinus). Amer. J. Vet. Res. 33:2027-2036.
1975. Ecology and morphological adaptation of the sucking lice (Anoplura, Echinophthiriidae) on the northern seal. Repp. P.-v. Reun. Cons. Int. Explor. Mer. 169:504-515.

1977. Notes on populations of Bovicola jellisoni on Dall's sheep (Ovis dalli). J. Wildl. Dis. $13: 4 \overline{27-428}$.

KIM, K. C. and C. F. WEISSER. 1974. Taxonomy of Solenopotes Enderlein, 1904, with redescription of Linognathous panamensis Ewing (Linognathidae: Anoplura). Parasitology 69:107-135.

KLEIN, D. R. 1965. Ecology of deer range in Alaska. Ecol. Monogr. 35:259-284.

PHILIP, C. B. 1938 . A parasitological reconnaissance in Alaska with particular reference to varying hares. II. Parasitological data. J. Parasitol. 24:483-488.

1939. A parasitological reconnaissance in Alaska with particular reference to varying hares. J. Mammal. 20:82-86.

PHILIP, C. B., G. D. GILL and J. M. GEARY. 1954. Notes on the rabbit tick, Haemaphysalis leporispalustris (Packard), and tularemia in central Alaska. J. Parasitol. $40: 484-485$.

PHILIP, C. B. and R. R. PARKER. 1938. Occurrence of tularaemia in the rabbit tick (Haemaphysalis leporis-palustris) in Alaska. Pub. Hlth. Rep. 53:574-575.

RAUSCH, R. L. 1972. Observations on some natural-focal zoonoses in Alaska. Arch. Environ. Hlth. $25: 246-252$.

ROGERS, L. L. and S. M. ROGERS. 1976. Parasites of bears: a review. Part V. Biology of bears, Paper 42. pp. 411-467. In: $\frac{\text { Bears }}{\text { ment. }}-\frac{\text { Their }}{\text { M. R. PELTON, J. Biology }}$, W. $\frac{\text { Mand }}{\text { LENTFER }}$ and G. E. FOLK, eds. 3rd Int. Conf. Bears. IUCN Pubs. n.s. No. 40 . 
dently realizing that it is not likely to be struck twice by the same insect. The animal appears nauseated and walks a few steps with its head elevated, sneezing and showing signs of nasal irritation.

Coughing and nasal discharge occurs, but the effects of nose bot larvae on the host are not as severe as for warbles.

Fleas (Siphonaptera:

Pulicidae and Vermipsyllidae)

Glenn E. Haas

Euhoplopsyllus $\frac{\text { glacialis }}{\text { flea, is a }} \frac{\text { lynx }}{\text { true }}$ (Baker), the hare flea, is a the snowshoe hare in North America and probably of the lynx (Felis lynx) as well (Holland 1949, 1963, Hopla 1965). This flea is recorded from many localities in the Interior and Copper River Basin; on the Killey River, Kenai Peninsula; on Jim Creek near Palmer and near Willow, Susitna Valley (Geary 1953, Gill 1954, Haas et al. 1978, Hopla 1965, Jellison and Kohls 1939, Philip 1938,1939 ).

The life cycle is unusual among fleas in that egg laying and larval development occur on the host, an adaptation for the absence of nesting by hares (R. Elbel, unpub. data).

Chaetopsylla tuberculaticeps (Bezzi), the bear flea, is widely distributed across Europe, Asia and northwest North America on brown bears (Ursus arctos) (Holland 1949, In Alaska, black bears ( americanus) are also sometimes infested (Hopla 1965, Jellison and Kohls 1939, Jellison and Senger 1976), and we have specimens from a coyote (Canis latrans) (Haas et al.
1978). This flea is known from the Kenai Peninsula, Matanuska Valley, Copper Basin, the Interior, Admiralty Island and Chichagof Island (Haas et al. 1978, 1979, Hopla 1965, Jellison and Kohls 1939, Jellison and Senger 1976).

The life cycle of the bear flea has not been described, but in their review paper Rogers and Rogers (1976) suggest it is tied to denning habits which is 1 reason that few black bears are heavily infested, that is, they tend to use a different den each winter and a different bed each night during the summer.

Chaetopsylla setosa Rothschild, a seldom-collected flea of black bears and certain other carnivores in British Columbia and Montana (Holland 1949, Hopkins and Rothschild 1956, Jellison and Senger 1973), was recently collected from a black bear in southeastern Alaska by $L$. Johnson ( $G$. Haas, unpub. data).

\section{References}

BENNETT, G. F. and C. W. SABROSKY. 1962. The Nearctic species of the genus Cephenemyia (Diptera: Oestridae). Can. J. Zool. $40: 431-448$.

GEARY, J. M. 1953. A survey of invertebrate disease vectors in Alaska. Arct. Aeromed. Lab., Ladd Air Force Base, Alaska, Proj. No. 22-1401-0004, Res. Rep. No. 1.

GILL, G. D. 1954. A survey of invertebrate disease vectors in Alaska. Arct. Aeromed. Lab., Ladd Air Force Base, Alaska, Proj. No. 22-1401-0004, Res. Rep. No. 2.

HAAS, G. E., R. E. BARRETT and N. WILSON. 1978. Siphonaptera from mammals in Alaska. Can. J. Zool. $56: 333-338$. 


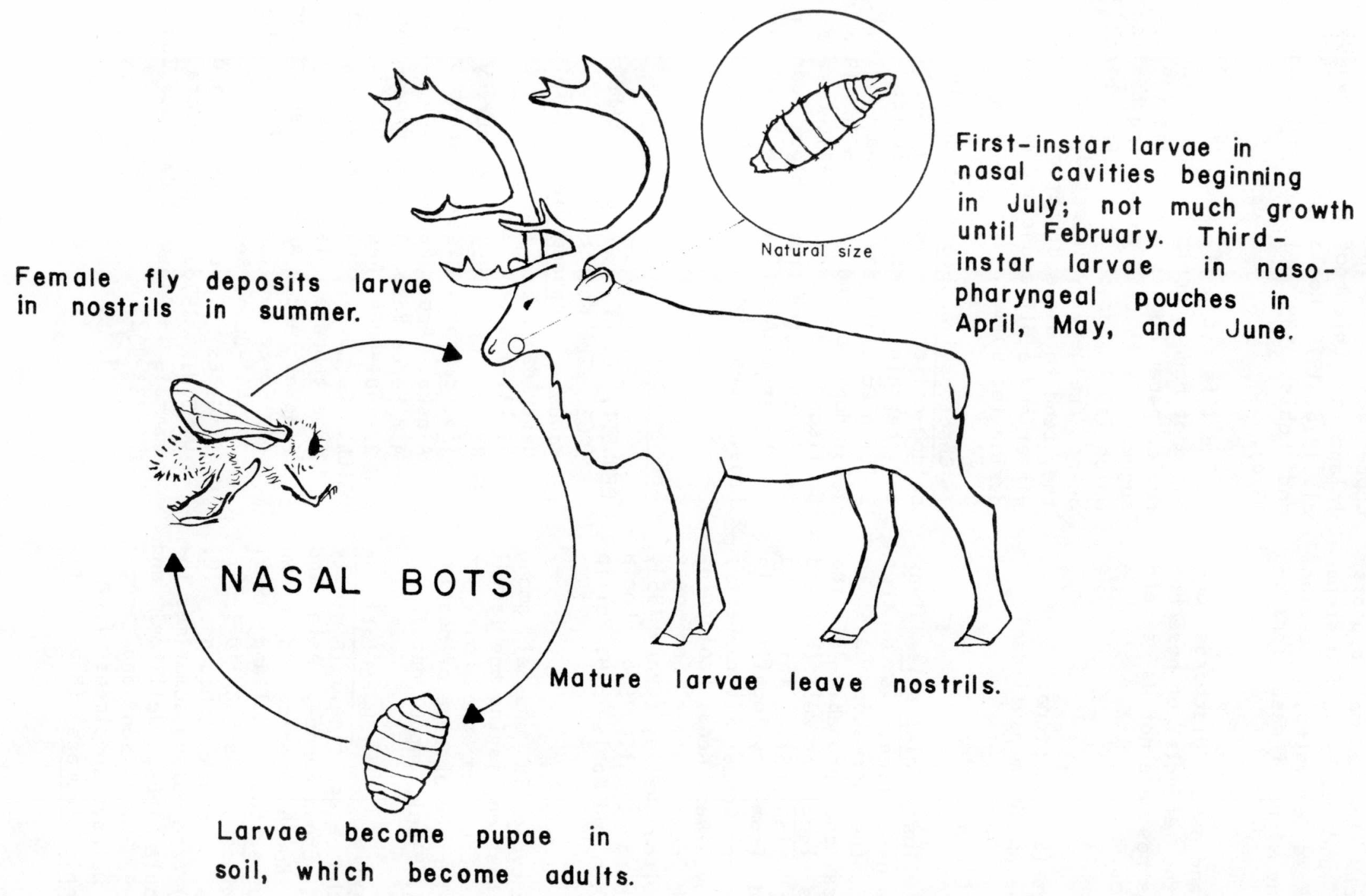

Figure 15 
Normally, $95-99 \%$ of the warbles are killed in each treated animal and, with widespread application, the number of surviving warbles can be reduced to such low numbers that retreatment of the herd will not be needed annually, but only every few years unless untreated animals are introduced into the area. Total eradication of this parasite is difficult; without repeated treatments, warble numbers will eventually increase.

A ballistic implant system is currently being tested at the University of Alaska to treat reindeer for warbles. This system uses a gun powered by compressed air to implant a biodegradable pellet filled with medication. This control method may have application to other species of wildlife in the treatment of their diseases in the future.

\section{Nasal Bots}

Robert A. Dieterich and Glenn E. Haas

Nasal bots (Cephenemyia trompe) are members of the order Diptera (Bennett and Sabrosky 1962). They are commonly found in Alaskan reindeer and caribou (Rangifer tarandus) and white-tailed deer (odocoileus virginianus) in North America (Bennett and Sabrosky 1962). Adult nasal bots closely resemble mature warble flies in appearance. Female bot flies deposit larvae, not eggs, near the openings of the nares. These larvae migrate up the nostrils into the area of the pharyngeal pouches where they remain through the winter. In the late spring and early summer these yellow colored elongate larvae (10-25 mm long) are sneezed out of the nasal area onto the ground and form pupae. The pupae in turn form adults and the life cycle begins again.

A second species of nose bot (Cephenemyia jellisoni) was recorded in Alaska by klein (1965) who found bots in pharyngeal pouches of Sitka black-tailed deer (Odocoileus hemionus sitkensis) in early spring on Coronation and Woronkofski islands. He stated that the bot has been associated with winter deer mortality in Alaska.

It is possible to control nasal bots with the same drug that is used in treating reindeer for warbles (Warbex). The nasal bot's major harm appears to occur when the adult fly is harassing the host during the larva depositing process.

During the summer when mature bots and warbles are prevalent, entire caribou herds frequently panic at the presence of these flies, and race from hill to hill in their attempts to escape. A vivid description of the interaction of the bot fly and reindeer was given by Hadwen (Hadwen and Palmer 1922):

The effect on reindeer...i is most marked. The animals are terrified by the insect's attack, and when it is hovering in front of their noses they assume a terrorstricken look, their eyes staring, their mouths open, and their bodies in a tensely strained attitude. When a reindeer is in this rigid state, the slightest touch on any part of the animal will cause muscular contractions which shake the whole body, just like an electric shock. When the insect deposits its larvae, such a shock follows, it is succeeded by a total relaxation, the deer evi- 
Larvae burrows through skin, migrates to reindeer back, creates a breathing pore, grows larger over winter to become a full-sized warble.

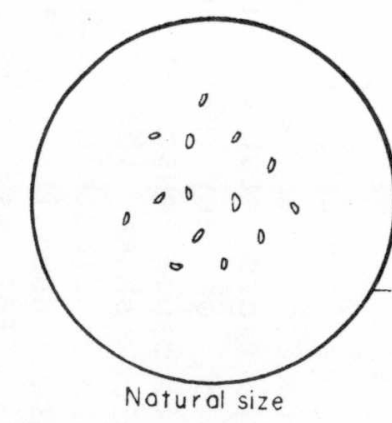

Each egg hatches in

6 days to produce

a tiny larva.

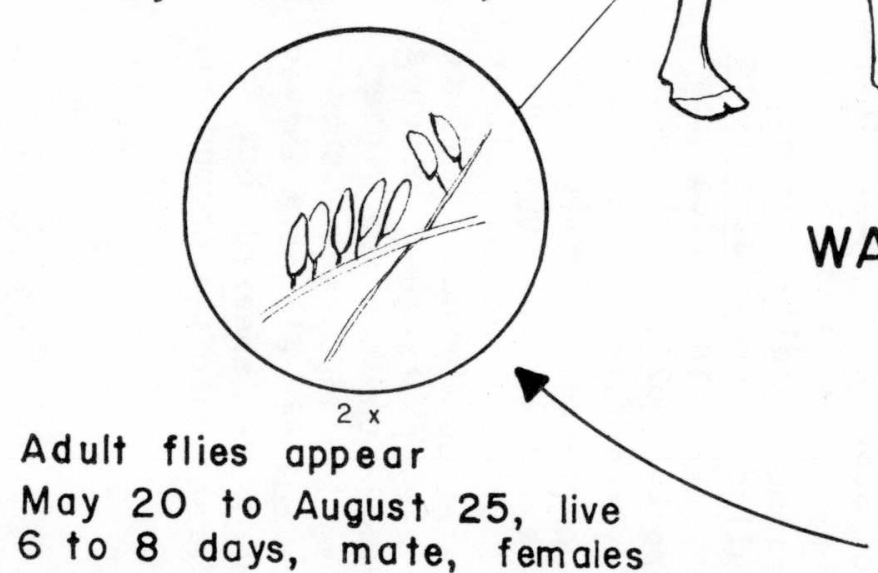

6 to 8 days, mate, females

lay eggs in reindeer hair.
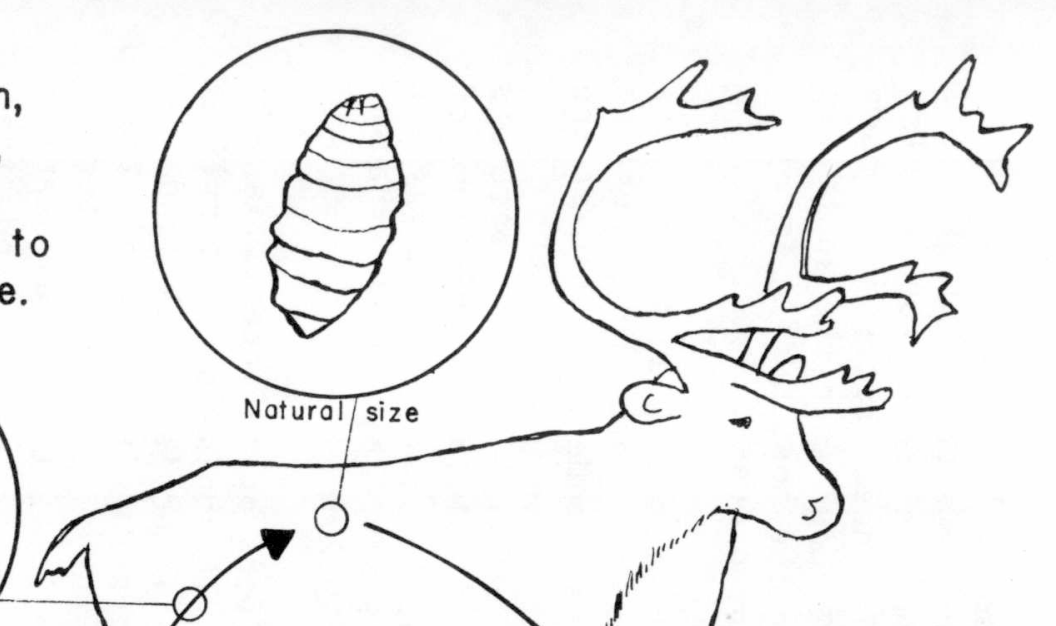

WARBLE FLIES

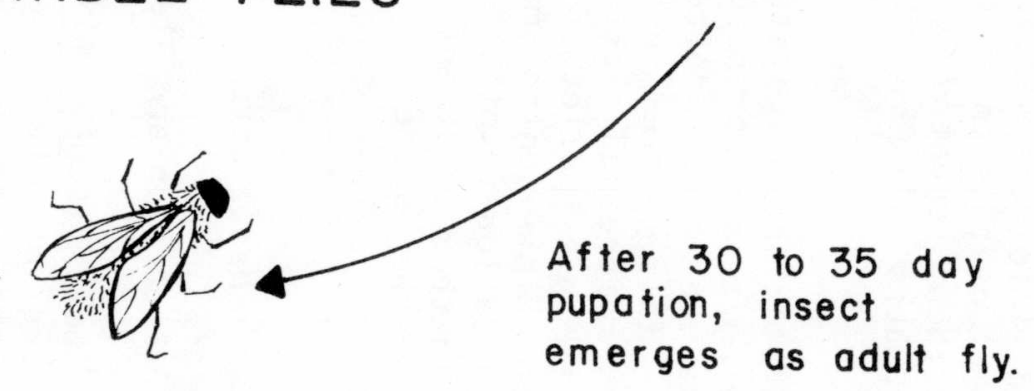

Figure 14 

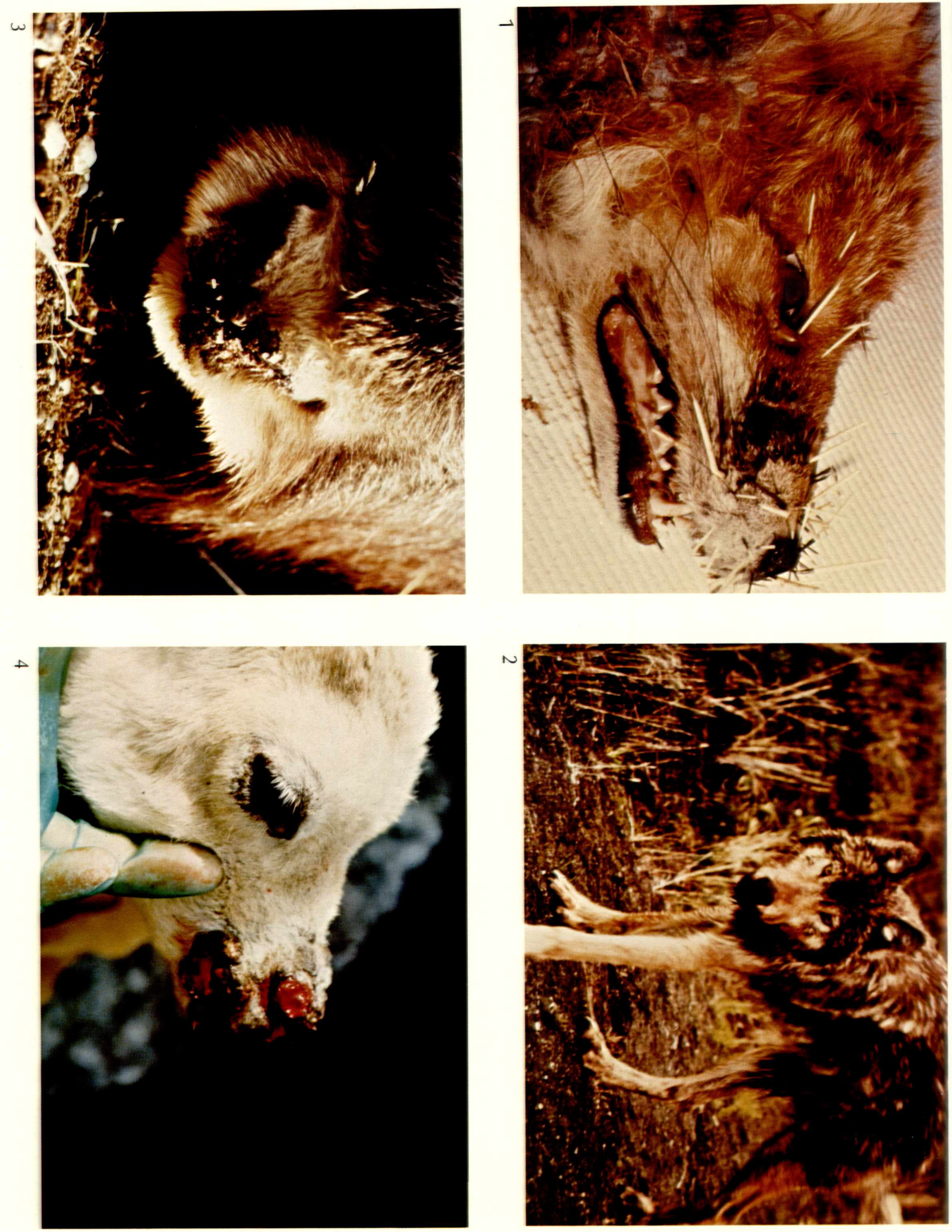
5. Numerous intact and recently ruptured vesicles on the foreflipper of a northern fur seal (Callorhinus ursinus) characteristic of San Miguel Sea Lion Virus (H. Gelberg).

6. Hemorrhagic lesions throughout the lungs of a reindeer (Rangifer tarandus) typical of respiratory virus infection (R. Dieterich).

7. Swollen, fluid-filled carpal joints of a reindeer (R. tarandus) infected with brucellosis. Swelling on the right carpus is extreme; animals infected with brucellosis that exhibit swollen joints usually resemble the smaller, left carpus (J. Dau; R. Dieterich).

8. Enlarged testicles of a caribou (‥ tarandus) infected with brucellosis (D. Hartbauer; R. Dieterich). 

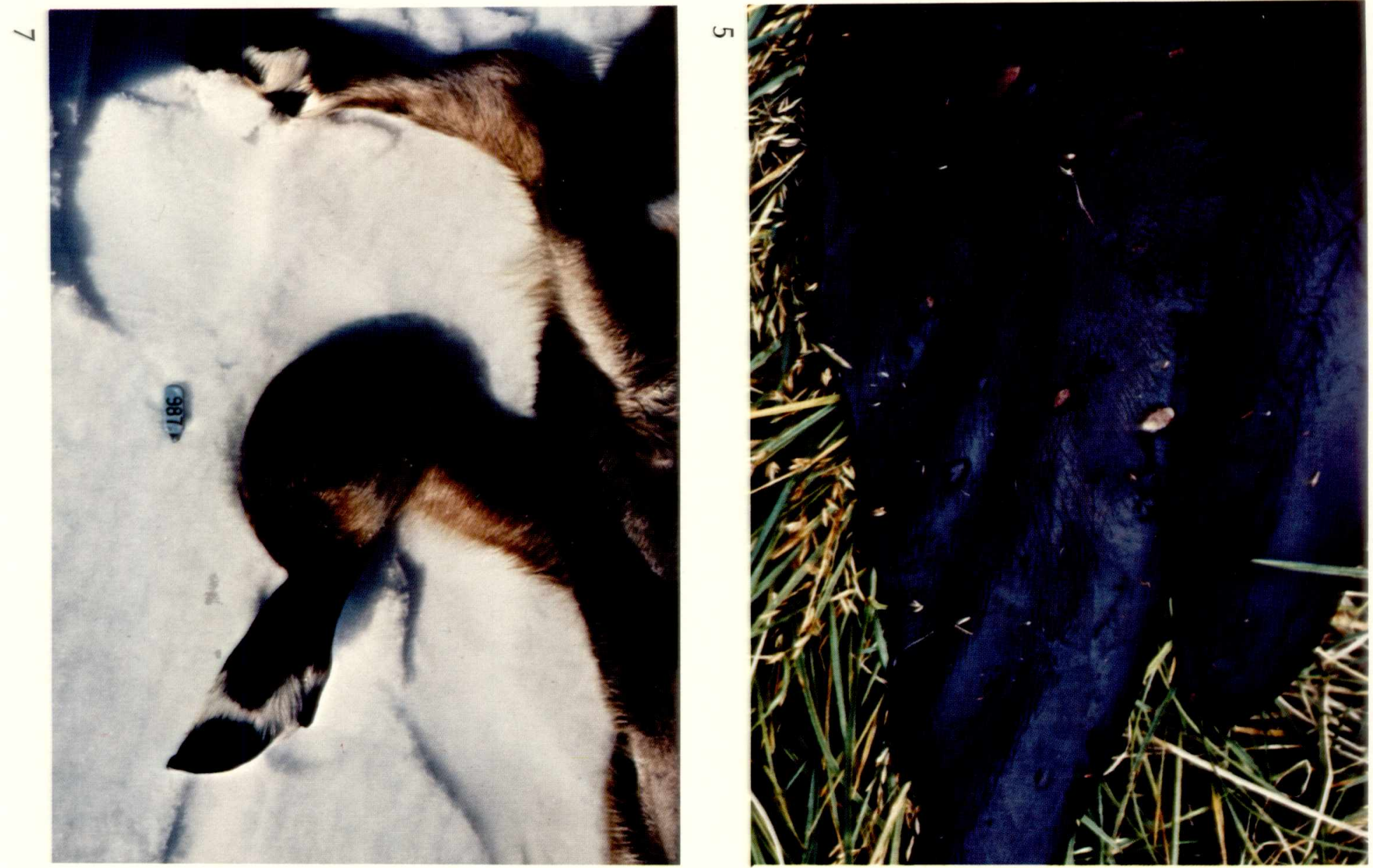

$\infty$

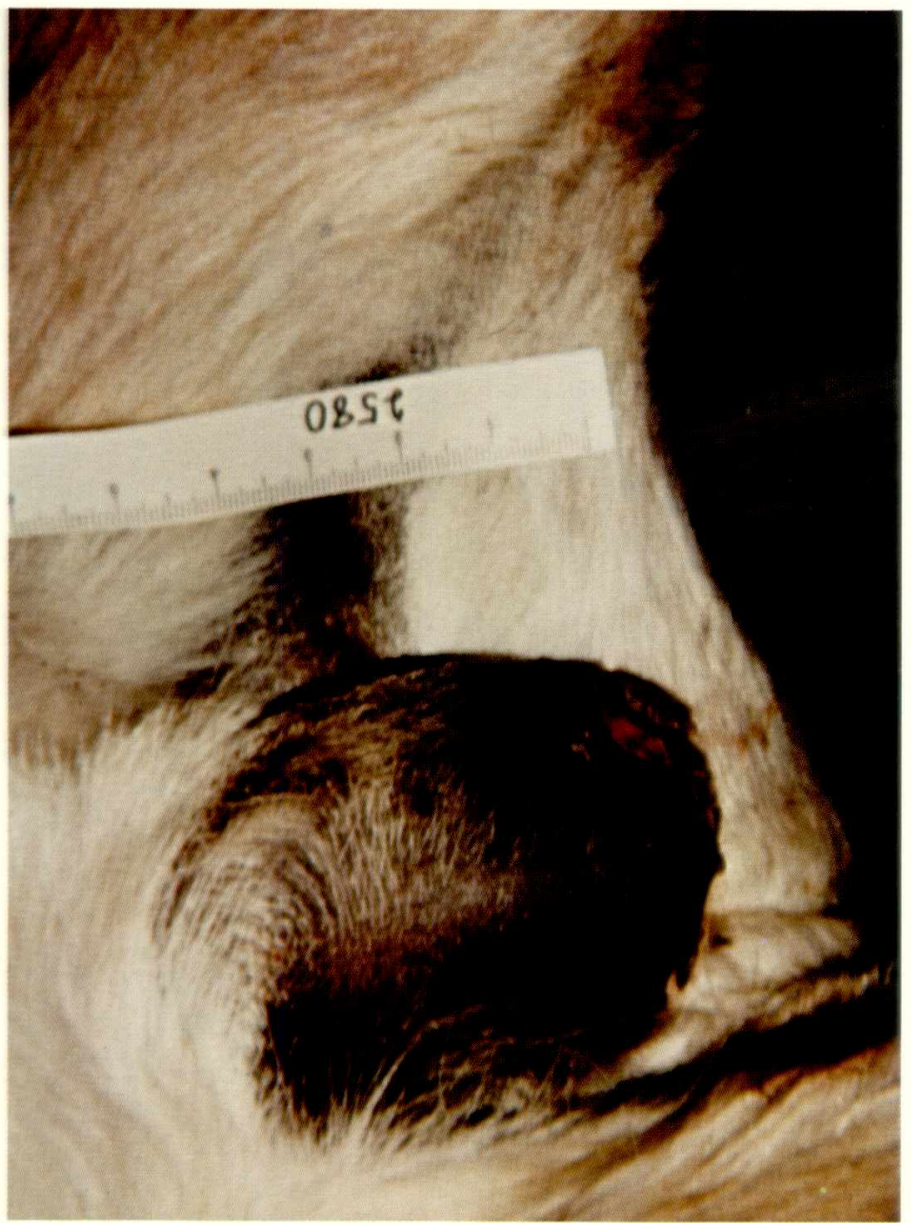

a

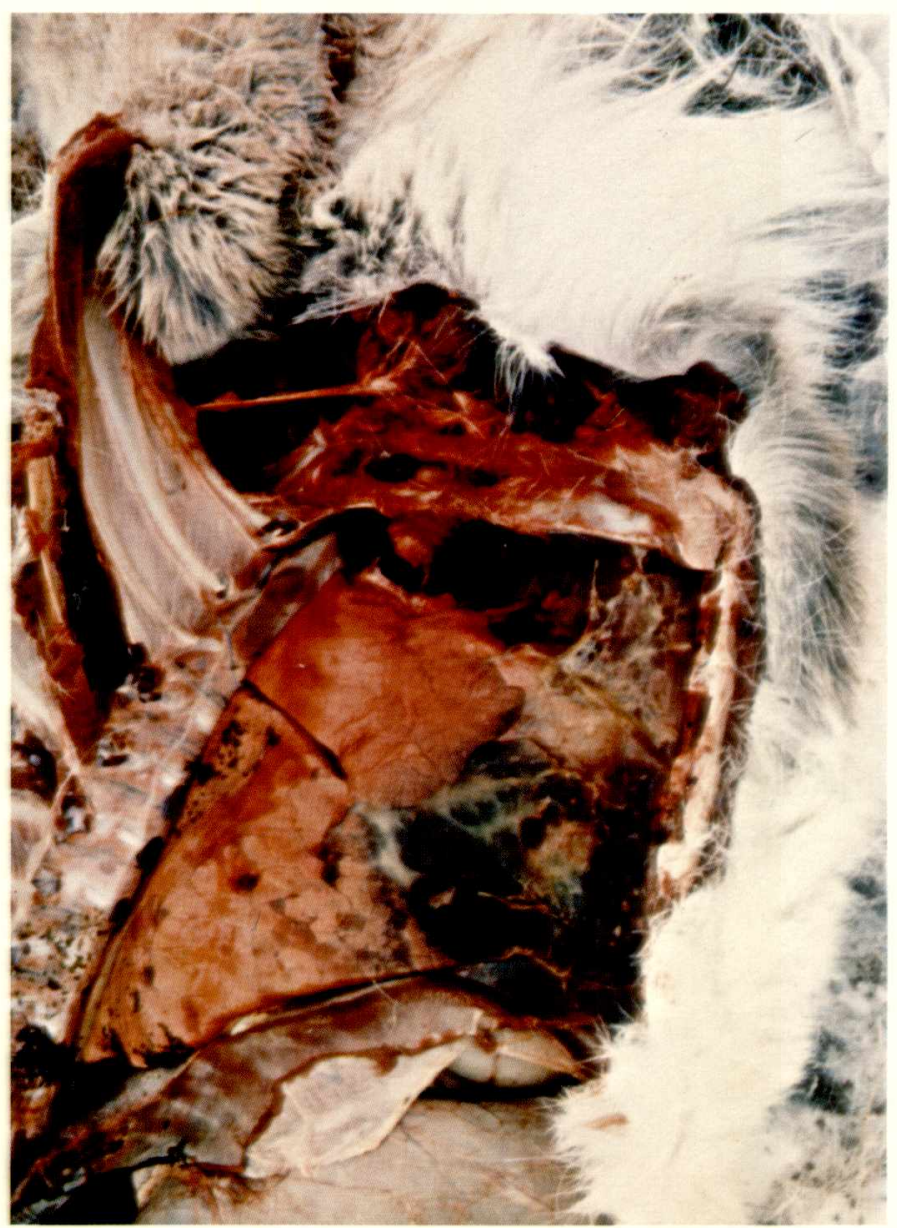


9. Inflammatory tissue response in the liver of a snowshoe hare (Lepus americanus) caused by Francisella tularensis (R. Dieterich).

10. The hoof of a reindeer (R. tarandus) with lesions typical of necrobacillosis. The causative agent of necrobacillosis is Fusobacterium necrophorum (R. Dieterich).

11. The larva of Taenia serialis localized in subcutaneous tissue of a

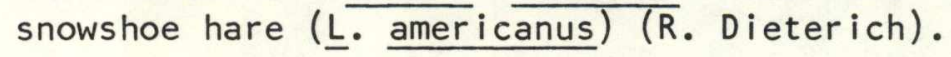

12. Fibrotic scars in the liver of a snowshoe hare (L. americanus) caused by migrating larvae of Taenia pisiformis (R. Dieterich). 

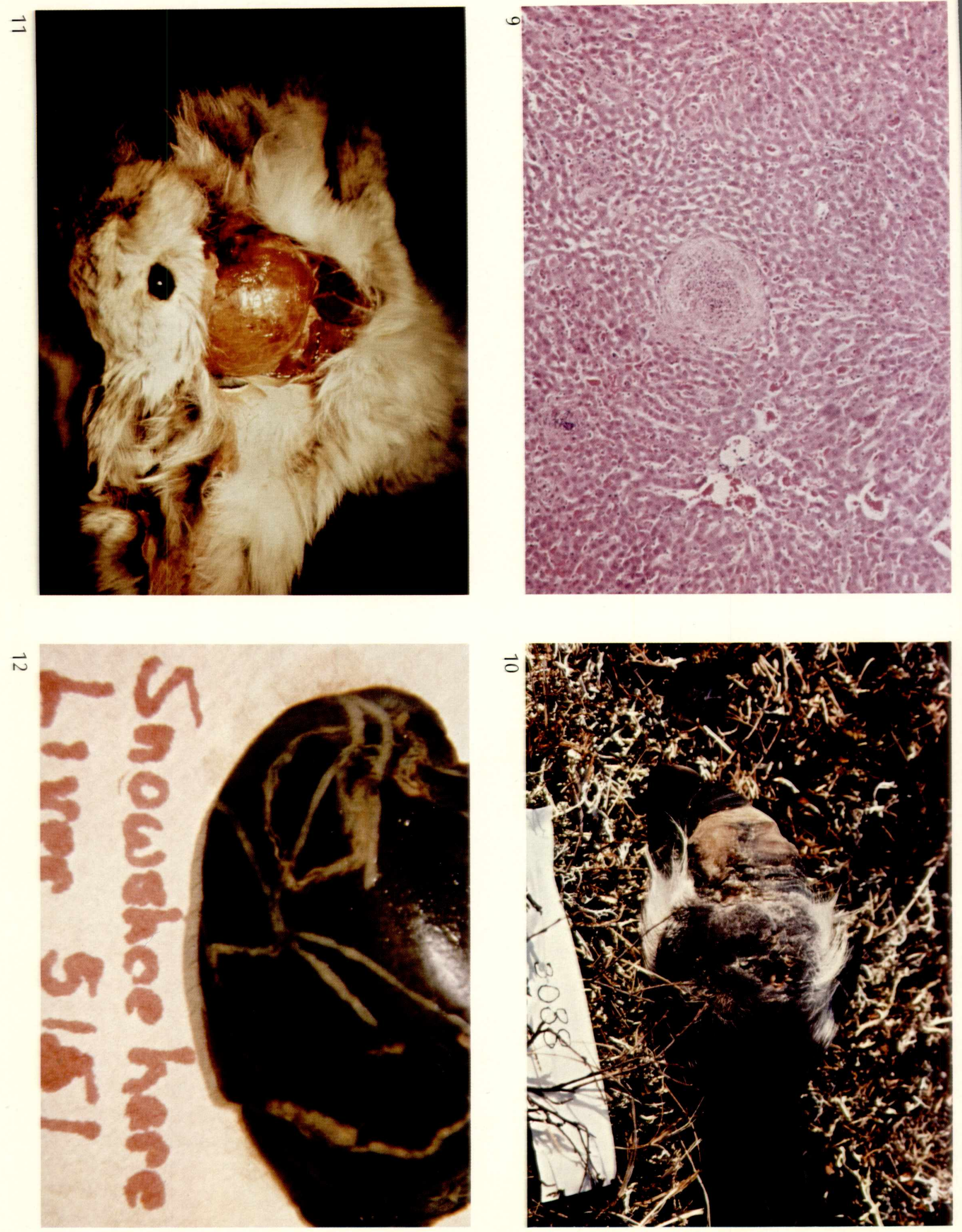
13. Carnivory is an important mode of transmission for many agents of wildlife diseases. This illustration is particularly applicable to the transmission of Diphyllobothrium ursi from its second intermediate host, i.e. chum salmon (Oncorhynchus keta), to its definitve host, i.e. brown bears (Ursus arctos) (A.W. Franzmann).

14. A parasitic roundworm in a spruce grouse (Canachites canadensis) breast (R. Zarnke).

15. Setaria yehi free in the abdominal cavity of a reindeer ( $\underline{R}$. tarandus) (R.

16. Larvae of the arctic form of Trichinella spiralis encysted in muscle tissue. This nematode is the causative agent of trichinosis (R. Dieterich). 

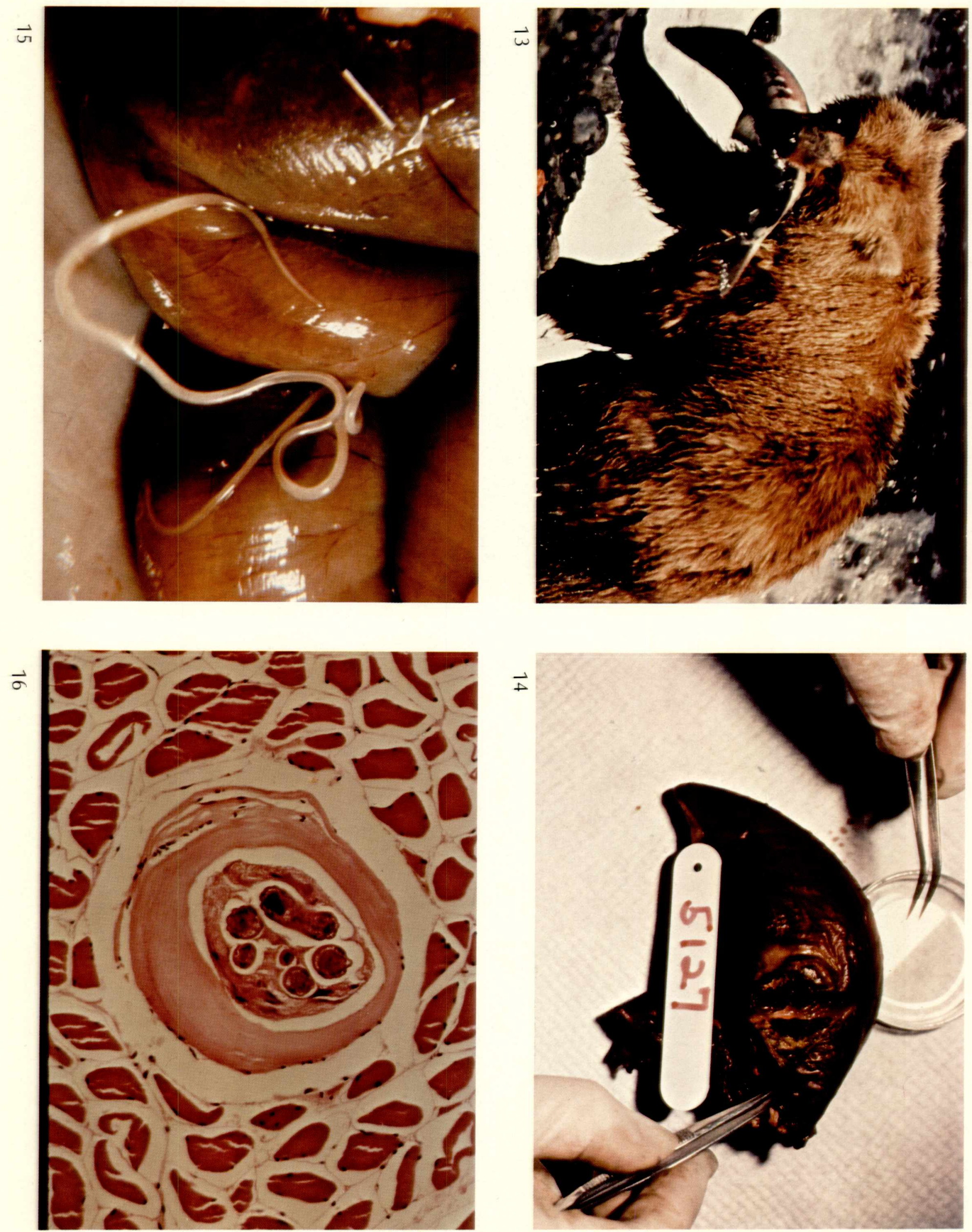
17. The back of a reindeer (R. tarandus) with larvae of the warble fly (Hypoderma (Oedemagena) tarandi). Each lump represents a single larva that is encapsulated in connective tissue under the skin. This photograph was taken in early May just before the larvae begin to emerge from their cysts and enter pupation on the tundra (R. Dieterich).

18. The breathing hole of a warble fly ( $H$. tarandi) larva in the skin of a reindeer (R. tarandus). Larvae emerge from their cysts that are beneath the skin by burrowing out through this hole (R. Dieterich).

19. Warble fly (… tarandi) larvae in cysts of host-generated connective tissue on the underside of a reindeer ( $\underline{R}$. tarandus) hide (R. Dieterich).

20. Larvae of the bot fly (Cephenemyia trompe) in the pharyngeal pouch of a reindeer (R. tarandus). The larvae are the grub-like organisms in the center of the photograph. This picture was taken during early May ( $R$. Dieterich). 

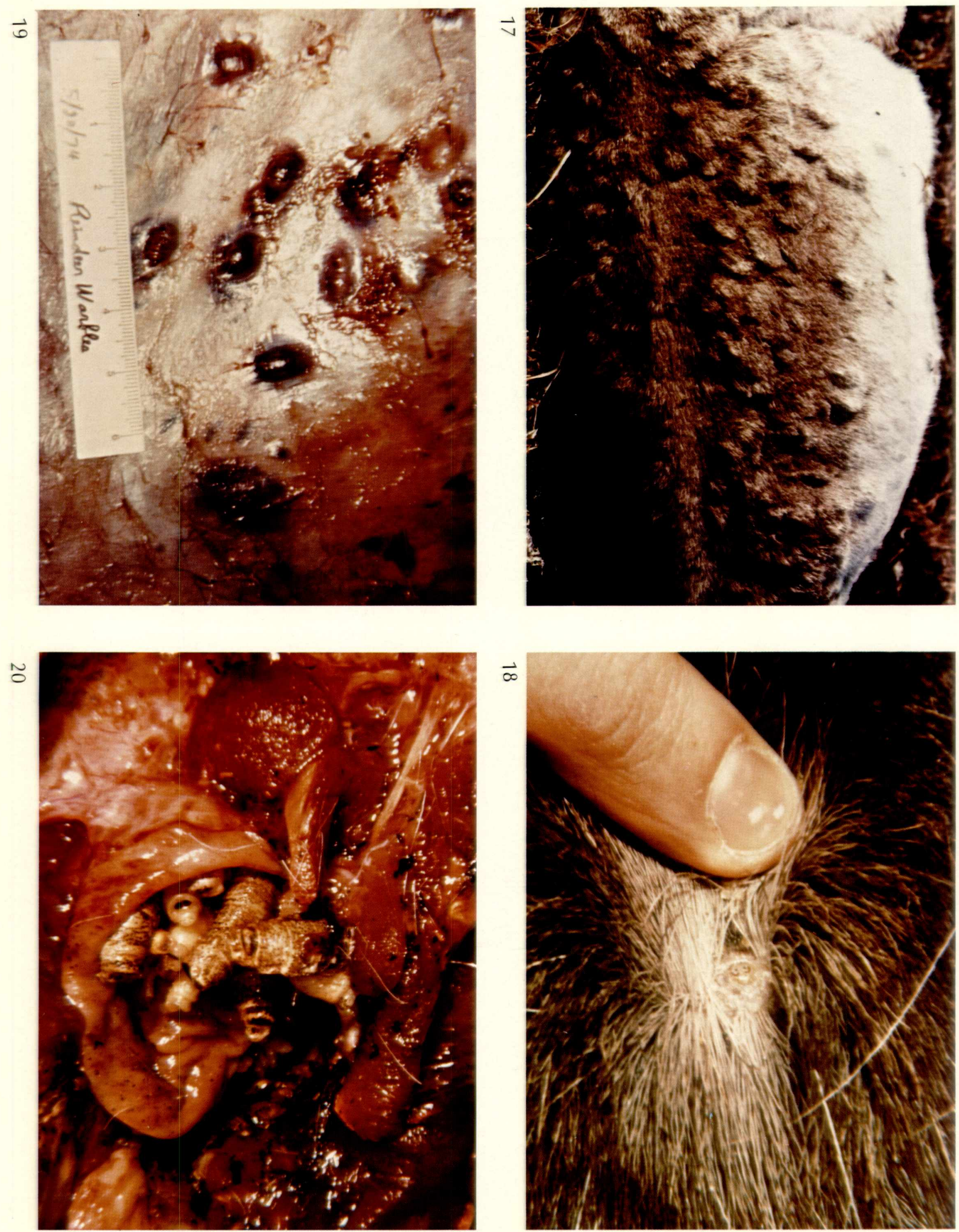
21. Mature warble (ㅂ. tarandi) and bot ( $\underline{\mathrm{C}}$. trompe) flies. The warble fly has a light orange abdomen and is oriented perpendicular to the hair; the bot fly has a darker abdomen and is oriented parallel to the hair, facing the warble fly (photo by J. Wright; identification verified by Dr. D.M. Wood, Biosystematics Research Institute, Ottawa, Ontario).

22. The exudate-filled lungs of a reindeer (R. tarandus) that suffered from pneumonia (R. Dieterich).

23. A tumor (papillomatosis) removed from the skin of a reindeer ( R. tarandus) (R. Dieterich).

24. Keratitis (pink eye) in a reindeer (R. tarandus) (R. Dieterich). 

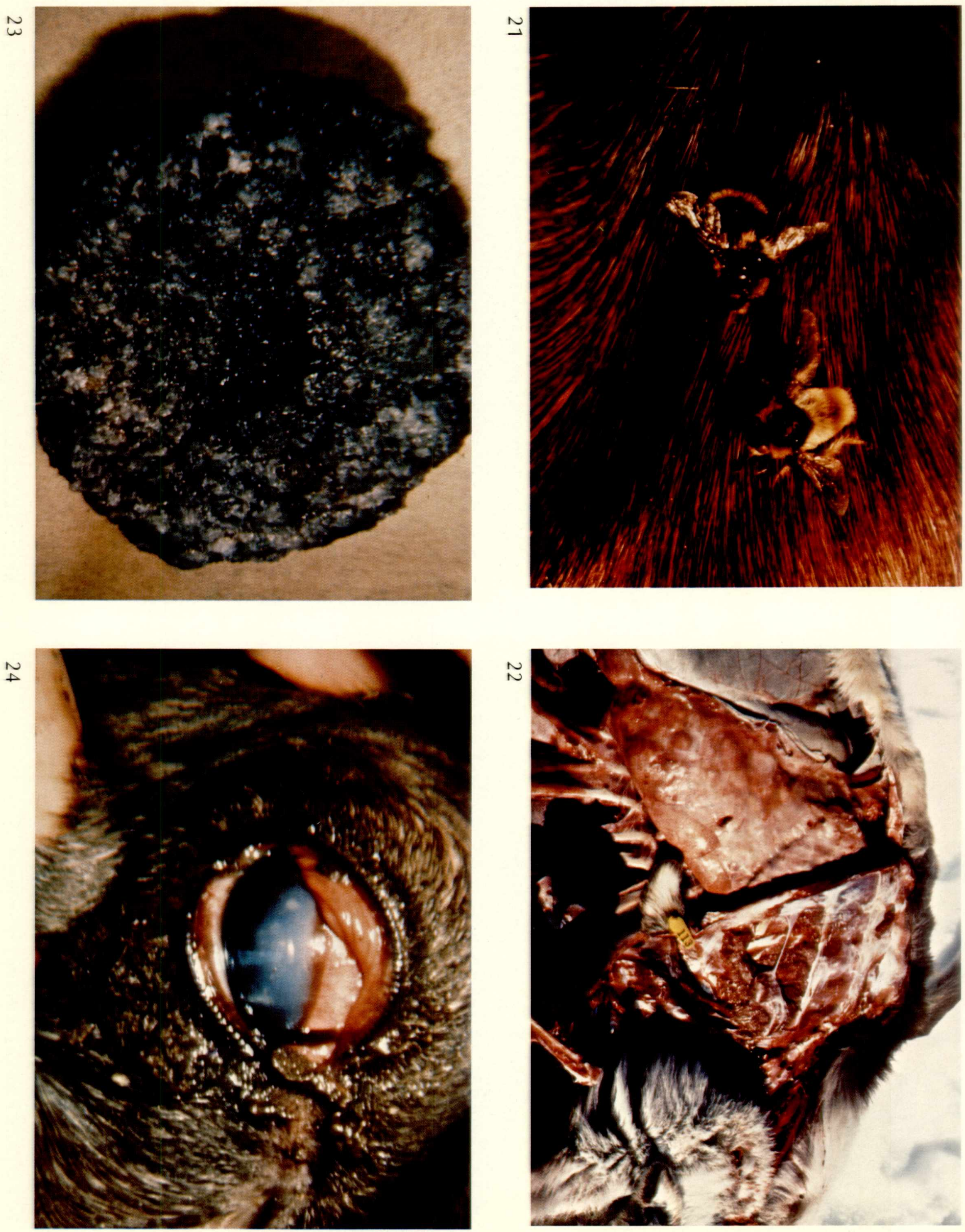
Part II

BIRDS 


\title{
Introduction
}

\author{
L. Michael Philo
}

Documentation of avian diseases in Alaska has been infrequent. This is partially due to lack of reporting rather than to a true scarcity of diseased birds. Considering Alaska's large expanses of uninhabited wilderness, one can easily imagine many, if not most, individual cases and even outbreaks going undetected. Those which are detected may be ignored or perhaps diagnosed but not reported. As a result, present data probably do not begin to reflect the actual prevalence of avian disease in Alaska.

The diseases which we discuss here have been documented rarely, if at all, in this state. However, based on the natural histories of the hosts and the ecology of the infective organisms, it is quite possible that these diseases are actually present.

One can not overemphasize the importance of making every attempt to diagnose and report any obvious or even suspected avian disease. Such information is vital to understanding the epidemiology of these diseases. This, in turn, is important to the management of wild avian species and to the protection of pet birds and commercial flocks which are often victims of disease spread from wild species. 
Chapter I.

VIRAL DISEASES 


\title{
Inclusion Body Disease of Raptors
}

\author{
L. Michael Philo
}

\section{Introduction}

Inclusion body disease of raptors (IBDR) is an acute and uniformly fatal disease of falcons and owls but has not yet been reported in hawks (Halliwell 1979). The herpesviruses which cause the disease have been isolated from falcons, owls and pigeons which have succumbed to the disease. These viruses are closely related to one another (Potgieter et al. 1979) but are distinct from the herpesviruses of duck virus enteritis (Peckham 1975), laryngotracheitis of chickens and Pacheco's parrot disease (Potgieter et al. 1979).

of the species presently known to be susceptible (Burtscher and Sibalin 1975, Graham et al. 1975, Kocan et al. 1977, Peckham 1975, Sileo et al. 1975), those found in Alaska (Robbins et al. 1966, Udvardy 1977) include the American kestrel (Falco sparverius), peregrine falcon (F. peregrinus tundrius), gyrfalcon ( $\bar{F}$. rusticolus), great horned owl (Bubo virginianus) and snowy owl (Nyctea scandiaca).

\section{$\underline{\text { History }}$}

A viral disease syndrome which is apparently identical to IBDR was reported in owls in Austria in 1915 (Burtscher and Sibalin 1975). In 1932 IBDR was reported in the United States in a great horned owl (Sileo et al. 1975). Since the $1960 \mathrm{~s}$ the disease has been repeatedly reported from Germany (Burtscher and Sibalin 1975) and in the 1970 s began to be reported more often in the United States (Graham et al. 1975, Kocan et al. 1977, Potgieter et al. 1979,
Sileo et al. 1975, Ward et al. 1971). None of these reports however, have originated in Alaska.

\section{Transmission}

Experimentally, IBDR can be transmitted by oral, nasal, aerosol or intramuscular inoculation, with an incubation period of 6 to 12 days. The mode of natural transmission however, is unknown (Graham and Halliwell 1978, Halliwell 1979). Similar inclusion body diseases have been reported in a variety of mammalian and other avian species which may serve as prey for raptors (Ward et al. 1971). Because the disease is uniformly acute and fatal in raptors, it appears that the herpesviruses may be poorly adapted to these hosts. These findings have led investigators to speculate that the disease is subclinical in certain prey species which then serve as reservoirs (Graham et al. 1975, Graham and Halliwell 1978).

Signs, Symptoms and

Pathogenesis

Inclusion body disease is invariably fatal in raptors. It attacks primarily the liver and organs involved in blood cell production (i.e. bone marrow, lymphoid tissue, spleen, lung, kidney and remnants of thymus). Most cases develop so rapidly that no signs are seen; the bird is simply found dead, often within a few hours of eating (Graham and Halliwell 1978). In some cases there have been very generalized clinical signs: regurgitation, diarrhea, lethargy, ruffled feathers and 
anorexia, but even these individuals have died within 24 to 72 hours (Graham et al. 1975, Graham and Halliwell 1978, Halliwell 1979). In the field a bird may collapse in midflight or, when approached, fly a short distance and collapse (Sileo et al. 1975).

The most striking finding at necropsy is an enlarged liver heavily studded with tan, yellow or yellowgrey necrotic foci $1-3 \mathrm{~mm}$ in diameter. When the liver is sliced the foci are seen to extend throughout the body of the organ. The spleen too, is often enlarged and studded with necrotic foci. The intestines and bone marrow have tan streaks or nodules of necrosis. When tissue immediately adjacent to these necrotic areas is examined under a light microscope, one can readily see the intranuclear inclusion bodies for which the disease is named (Graham et al. 1975, Kocan et al. 1977, Sileo et al. 1975, Ward et al. 1971).

There is no treatment and no commercially available vaccine (Graham and Halliwell 1978).

\section{Diagnosis}

A positive diagnosis is based on the gross lesions, microscopic lesions, isolation of the virus through growth on cell culture and identification of the virus by virus neutralization or with fluorescent antibody (Burtscher and Sibalin 1975, Peckham 1975).

Good quality close-up photographs of the intact organs with lesions will be helpful in diagnosis and documentation. Small sections of liver, spleen, gastrointestinal tract, bone marrow, kidney, lung, nasal and oral mucosae, thymus (if present) and adrenal gland should be collected and placed in $10 \%$ neutral buffered formalin. As it may not be possible to collect all of these samples, one should collect, as a minimum, sections of all abnormal tissue.

For virus isolation, specimens from liver, spleen and if possible, bone marrow should be collected aseptically and frozen to -20 to $-70 \mathrm{C}$ as quickly as possible. They are stored at these temperatures until further processing.

\section{Significance and Control}

At this time it is difficult to determine the significance of IBDR in Alaska. This is partly because no cases have been reported in the state, but even more important is the fact that so little is known about host range and mode of infection that it is not possible to predict the potential impact upon Alaskan raptors. Some investigators (Ward et al. 1971) feel however, that the potential for disease in wild populations of birds of prey is great.

\section{Acknowledgments}

The assistance of Mr. James R. Dau in assembling the pertinent literature is gratefully acknowledged.

\section{References}

BURTSCHER, H. and M. SIBALIN. 1975. Herpesvirus strigis: Host spectrum and distribution in infected owls. J. Wildl. Dis. 11:164-169.

GRAHAM, D. L., C. J. MARE, F. P. WARD and M. C. PECKHAM. 1975. Inclusion body disease (Herpesvirus infection) of falcons (IBDF). J. Wildl. Dis. 11:83-91. 
GRAHAM, D. L. and W. H. HALLIWELL. 1978. Virus diseases of birds of prey. pp. 260-265. In: Zoo and Wild Animal Medicine. M. E. FOWLER, ed. W. B. Saunders Co., Philadelphia, PA.

HALLIWELL, W. H. 1979. Diseases of birds of prey. Vet. Clin. North Am. Small Anim. Pract. 9:541-568.

KOCAN, A. A., L. N. D. POTGIETER and K. M. KOCAN. 1977. Inclusion body disease of falcons (Herpesvirus infection) in an American kestrel. J. Wildl. Dis. 13:199-201.

PECKHAM, M. C. 1975. Herpesviruses of pigeons, owls and falcons. pp. 256-262. In: Isolation and Identification of Avian Pathogens. S. B. HITCHNER, C. H. DOMERMUTH, H. G. PURCHASE and J.E. WILLIAMS, eds. Arnold Printing Co., Ithaca, NY.
POTGIETER, L. N. D., A. A. KOCAN and K. M. KOCAN. 1979. Isolation of a Herpesvirus from an American kestrel with inclusion body disease. J. Wildl. Dis. $15: 143-149$.

ROBBINS, C. S., B. BRUUN and H. S. ZIM. 1966. Birds of North America. Golden Press, New York, NY. $340 \mathrm{pp}$.

SILEO, L., H. C. CARLSON and S. C. CRUMLEY. 1975. Inclusion body disease in a great horned owl. J. Wildl. Dis. 11:92-96.

UDVARDY, M. D. F. 1977. The Audubon Society Field Guide to North American Birds: Western Region. Alfred A. Knopf, Inc., New York, NY. $854 \mathrm{pp}$.

WARD, F. P., D. G. FAIRCHILD and J. V. VUICICH. 1971. Inclusion body hepatitis in a prairie falcon. J. Wildl. Dis. 7:120-124.

\title{
Duck Viral Enteritis (Duck Plague)
}

\author{
L. Michael Philo
}

\section{Introduction}

Duck viral enteritis is a herpesvirus infection of domestic and wild ducks, geese and swans; no other species are known to be affected. It is acute, contagious, highly fatal and characterized by tissue hemorrhages and free blood in the body cavities (Leibovitz 1971, Sandhu 1978).

\section{History}

From 1923 to the early 1960s duck viral enteritis was reported in the
Netherlands, France, Belgium, India and China (Leibovitz 1971, Sandhu 1978). In 1967 the first reported outbreak in the United States occurred in domestic ducks in New York, and shortly thereafter the disease was found in wild ducks and Canada geese (Branta canadensis) in the area (Leibovitz 1968). Subsequent outbreaks occurred in Maryland, Pennsylvania (Leibovitz 1971), California (Snyder et al. 1973), South Dakota, Wisconsin (Jacobsen et al. 1976) and Alberta (Hanson and Willis 1976). It has not yet been reported in Alaska. 


\section{Transmission}

Duck plague can be transmitted by contact with an infected bird or contaminated environment. Experimental inoculations have shown that the virus is successfully administered via oral, intranasal, intravenous, intraperitoneal, intramuscular and cloacal routes. Water is perhaps the main vehicle for natural transmission since it is a common medium for feeding, drinking and body support. As new susceptible populations of waterfowl arrive at a contaminated site and become infected, the local survival and even increase of the virus are assured. There is also speculation that natural transmission occurs by bloodsucking arthropods (Dardiri and Gailiunas 1969, Leibovitz 1971).

Recovered birds are immune but may remain carriers (Sandhu 1978); virus has been recovered from heal thy wild waterfowl up to 4 years post infection (Burgess et al. 1979).

\section{Signs, Symptoms and}

\section{Pathogenesis}

In natural epornitics as in domestic duck die-offs, most birds are found dead without prior signs of disease. Those in which clinical signs are seen show lethargy, ruffled feathers, diarrhea and increased secretions from the eyes and nose. Dead birds often have soiled or blood-stained feathers around the vent and blood or blood mixed with ingesta flowing from the nostrils (Dardiri and Gailiunas 1969, Hanson and Willis 1976, Jacobsen et al. 1976, Leibovitz 1971, Sandhu 1978, Snyder et al. 1973).

The gross lesions result from vascular damage. There are usually pinpoint and larger hemorrhages of most visceral organs, supporting structures and mesenteries. As the disease progresses, many of these hemorrhagic areas become raised and covered by whitish plaques, particularly on the surface of the liver. The body cavity and lumen of the digestive tract contain free blood (Leibovitz 1971, Sandhu 1978).

\section{Diagnosis}

Preliminary diagnosis can be made on the basis of the gross lesions, but one must also consider avian cholera, erysipelas and duck virus hepatitis in the differential diagnosis. Virus isolation and identification are necessary for confirmation.

Sample collection should begin with the aseptic opening of a carcass and removal of pieces of spleen and liver. These are placed in sterile containers and frozen at -20 to $-70 \mathrm{C}$. Blood samples for virus isolation should be collected from the heart and the serum later removed and frozen at the same temperature; all handling should be aseptic and all containers sterile. Histopathologic sections should be collected from the heart, all abdominal organs and multiple locations along the digestive tract from esophagus to cloaca (Leibovitz 1975). Good quality color photographs, including close-ups of organs with lesions, can be taken after collection of virus isolation samples and will be quite helpful in documentation.

\section{Significance and Control}

As with many avian diseases, the significance in Alaska is difficult to determine. One must keep in mind however, that duck plague has occurred in a number of sporadic outbreaks in North America and that immune carriers exist. Thus, it may be possible for migrating waterfowl to bring the disease into the state. 
This appears to have been the case in Wisconsin (Jacobsen et al. 1976) and Alberta (Hanson and Willis 1976). It is also possible that isolated outbreaks have occurred in remote locations and simply not been discovered.

There is no practical treatment for duck plague, particularly in the wild. There is a commercial vaccine which is effective in domestic ducks (Sandhu 1978), but its effectiveness in wild species is not known. In studies with attenuated virus, mallards (Anas platyrhynchos) responded with antibody formation and resisted challenge, while Canada geese did not produce antibodies and succumbed to the challenge (Butterfield and Dardiri 1969). Therefore the practicality and effectiveness of vaccinating wild populations are highly questionable.

Control measures in domestic waterfowl populations consist of depopulation, sanitation, disinfection and removal of birds from the contaminated environment. This of course is practically impossible to do when an outbreak occurs in a wild population.

Any cases of duck plague should be reported to the Alaska State-Federal Laboratory.

\section{Acknowledgments}

Mr. James R. Dau provided invaluable assistance in collecting and outlining information from the literature.

\section{References}

BUTTERFIELD, W. K. and A. H. DARDIRI. 1969. Serological and immunologic response of wild waterfowl vaccinated with attenuated duck plague virus. Bull. Wildl. Dis. Assoc. 5:99-102.
BURGESS, E. C., J. OSSA and T. M. YUILL. 1979. Duck plague: a carrier state in waterfowl. Avian Dis. 24:940-949.

DARDIRI, A. H. and P. GAILIUNAS. 1969. Response of Pekin and mallard ducks and Canada geese to experimental infection with duck plague virus. Bull. Wildl. Dis. Assoc. 5:235-247.

HANSON, J. A. and N. G. WILLIS. 1976. An outbreak of duck virus enteritis (duck plague) in Alberta. J. Wildl. Dis. 12:258-262.

JACOBSEN, G. S., J. E. PEARSON and T. M. YUILL. 1976. An epornitic of duck plague on a Wisconsin game farm. J. Wildl. Dis. 12:20-26.

LEIBOVITZ, L. $1968 . \quad$ Progress report: duck plague surveillance of American anseriformes. Bull. Wildl. Dis. Assoc. 4:87-91.

1971. Duck plague. pp. 22-23. In: Infectious and Parasitic Diseases of Wild Birds. J. W. DAVIS, R. C. ANDERSON, L. KARSTAD and D. 0. TRAINER, eds. The lowa State Univ. Press, Ames, IA.

- 1975. Duck plague (duck virus enteritis). pp. 251-255. In: Isolation and Identification of Avian Pathogens. S. B. HITCHNER, C. H. DOMERMUTH, H. G. PURCHASE and J. E. WILLIAMS, eds. Arnold Printing Co., I thaca, NY.

SANDHU, T. 1978. Infectious diseases. pp. 193-200. In: Zoo and Wild Animal Medicine. M. E. FOWLER, ed. W. B. Saunders Co., Philadelphia, PA.

SNYDER, S. B., J. G. FOX, L. H. CAMPBELL, K. F. TAM and 0 . A. SOAVE. 1973. An epornitic of duck virus enteritis (duck plague) in California. J. Am. Vet. Med. Assoc. 163:647-652. 


\title{
Avian Pox
}

\author{
L. Michael Philo
}

\section{Introduction}

Avian pox is a viral disease caused by several strains of Poxvirus avium. Most wild and domestic avian species are susceptible to 1 or more of these strains with infections reported in over 60 species $(20$ families) (Kirmse 1967b).

\section{History}

Pox infections have been described in wild and domestic birds since the $1800 \mathrm{~s}$ and distribution appears to be worldwide. Since the turn of the century, the use of histopathology and viral culture techniques has improved the capabilities for diagnosis and documentation of the disease (Karstad 1971), and reports of new hosts and new locations frequently appear in the literature.

Canary pox, a highly lethal strain of poxvirus which infects species of the family Fringillidae (finches) (Fowler 1978), was recently diagnosed in a group of common canaries (Serinus canaria) kept as pets in Fairbanks, Alaska (L. Winterer, pers. comm.). The diagnosis of avian pox in a juvenile green-winged teal (Anas crecca carolinensis) from Minto Flats, Alaska in 1978 represents the first reported case in wild birds in the state as well as the first report of the disease in a migratory duck (Morton and Dieterich 1979).

\section{Transmission}

Avian pox can be transmitted to a susceptible bird by contact with an infected individual or with con- taminated objects such as perches. An open skin lesion, even a tiny abrasion, is sufficient for the virus to enter the body, but the unbroken skin is an effective barrier (Karstad 1971). Skin lesions occur regularly from fighting and pecking, especially at the angles of the mouth (Kirmse 1967a). Raptors commonly have traumatic skin lesions on their feet, facilitating poxvirus infection after contact with contaminated perches or prey (Graham and Halliwell 1978).

Mosquitoes and perhaps other blood-consuming or biting arthropods can act as mechanical vectors, carrying infective virus on contaminated mouthparts. In some cases this appears to have been the only possible source of infection (Halliwell 1972, Karstad 1971, Kirmse 1967a).

Signs, Symptoms and Pathogenesis

The virus strains are classified, according to their host specificity, as mono-, bi-, or tri-pathogenic. For example, the pox strain infective to the yellow-shafted flicker (Colaptes auratus) shows strict host specificity and is thus monopathogenic (Kirmse 1967a); most virus strains however, seem to be tri-pathogenic (Karstad 1971). This flicker is found in Interior Alaska (Robbins et al. 1966), but pox has not yet been reported in the species in the state.

Avian pox is seen in 2 forms, a cutaneous and a diphtheritic form. The former, which is also the more common, appears generally as discrete nodular proliferative lesions which 
eventually develop scabs. These are primarily, though not exclusively, on the unfeathered areas, i.e. legs, feet, eyelids and beak. In the latter form, moist necrotic lesions are found on the mucous membranes of the upper respiratory tract and mouth (Karstad 1971). The wet diphtheritic form has not been reported in raptors, but cutaneous lesions on the face may extend into the mouth and onto the hard palate (Graham and $\mathrm{Hal}-$ liwell 1978). Keratitis, which appears as a clouding of the cornea, has been seen in flickers (Kirmse 1967a). The cutaneous lesions may be so extensive, proliferative and even invasive that the legs and/or face, head and bill are grossly distorted. Distortions of the head appear to be characteristic of Anseriformes (Leibovitz 1969, Pearson and Pass 1975). In some waterfowl, nodular proliferations of the tip of the bill have been seen (Harris et al. 1978, $\mathrm{Hill}$ and Bogue 1978, Morton and Dieterich 1979).

In most cases the birds appear to be in otherwise good health and no internal gross lesions are seen ( $\mathrm{Hal}-$ liwell 1972, Kirmse 1966, Simpson et al. 1975). In wild birds most of the cutaneous lesions are few in number. They regress, presumably due to developed immunity, after a variable amount of time of from several weeks to over 1 year. Duration of infection in wild birds tends to be markedly longer than in domestic species. This, coupled with the generally low mortality in wild birds, probably indicates a hostparasite relationship which has evolved over a long period of time, such that survival of both species is maximized (Karstad 1971, Kirmse 1967a). Some mortality does occur however. In some cases, as with the canary strain, the disease appears to be uniformly fatal, showing generalized signs (Fowler 1978). Within Anseriformes, some species may sustain higher mortality than others (Harris et al. 1978, Hill and Bogue 1978, Leibovitz 1969). Most, if not all cases of mortality are probably only secondarily related to the pox infection. The proliferative lesions can physically interfere with locomotion, acquisition and ingestion of food, respiration and sight (Harris et al. 1978, Hill and Bogue 1978, Leibovitz 1969, Pearson and Pass 1975). Secondary bacterial infection of the lesions is common and can also lead to the demise of the bird or to loss of a toe or foot (Graham and Halliwell 1978, Harris et al. 1978, Karstad 1971, Leibovitz 1969).

\section{Diagnosis}

When sections of affected skin tissue are examined under a light microscope, large intracytoplasmic inclusion bodies called Bollinger bodies are seen and are considered diagnostic (Crawford et al. 1979, Graham and Halliwell 1978, Karstad 1971, Kirmse 1966, Moffatt 1972, Morton and Dieterich 1979). Appearance, under electron microscopy, of immature virus particles in the cytoplasm and mature virions within the inclusion bodies are also considered diagnostic (Pearson and Pass 1975, Simpson et al. 1975). Virus culture is confirmatory, but due to the host specificities of the poxvirus strains and the limited types of cell cultures available, virus isolation is of ten unsuccessful (Karstad 1971).

Samples collected in the field should be multiple sections of cutaneous lesions from face and legs. Sections from dead birds should be deep enough to include the full thickness of the lesion plus adjacent healthy tissue. If samples are col- 
lected from otherwise healthy birds, 1 or more lesions may be sliced from the feet and legs. The specimens are placed in 10\% neutral buffered formalin. If proper preservative is available, duplicate specimens can be collected for electron microscopy. If facilities and equipment are available, sections of lesions can be collected aseptically and placed in sterile containers at -20 to $-70 \mathrm{C}$ to be stored and transported for virus isolation. Good quality color photographs will be helpful in documentation and diagnosis.

\section{Significance and Control}

The significance of avian pox in Alaska is only beginning to be appreciated and is by no means completely understood. Since the greenwinged teal from Minto Flats was a juvenile and had not yet migrated, it had to have acquired the disease within the state (Morton and Dieterich 1979). Thus, the disease did and probably does still occur in Alaska, but it is not known to what extent or in what species. Although the source of the canary infection is unknown, the affected birds had no known contact with wild species either before or after the disease outbreak. The important thing now is to be aware that the disease can and does occur in Alaska and to make every effort to collect all data that become available.

A number of control measures are effective in controlling avian pox in domestic bird populations: mosquito control, dust control, isolation of diseased birds, provision of ample space to minimize cannibalism and in some cases, vaccination (Karstad 1971). Unfortunately these procedures are hardly practical or effective in the field.
Avian pox cases should be reported
to the Alaska State-Federal

Laboratory.

\section{Acknowledgments}

Dr. Lawrence H. Winterer and Mrs. Elizabeth Driscoll were kind enough to discuss in detail, the cases of canary pox in Fairbanks. Their assistance is greatly appreciated.

\section{References}

CRAWFORD, J. A., R. M. OATES and D. H. HELFER. 1979. Avian pox in California quail from Oregon. J. Wildl. Dis. 15:447-449.

FOWLER, M. E. 1978. Infectious and zoonotic diseases. pp. 367-374. In: Zoo and Wild Animal Medicine. M. E. FOWLER, ed. W. B. Saunders Co., Philadelphia, PA.

GRAHAM, D. L. and W. H. HALLIWELL. 1978. Virus diseases of birds of prey. pp. 260-265. In: Zoo and Wild Animal Medicine. M. E. FOWLER, ed. W. B. Saunders Co., Philadelphia, PA.

HALLIWELL, W. H. 1972. Avian pox in an immature red-tailed hawk. J. Wildl. Dis. 8:104-105.

HARRIS, J. M., A. S. WILLIAMS and F. R. DUTRA. 1978. Avian pox in a group of common (California) murres (Uria aalge). Vet. Med./Small Anim. Clin. 73:918-919.

HILL, J. R. and G. BOGUE. 1978. Natural pox infection in a common murre (Uria aalge). J. Wildl. Dis. $14: \overline{337-338 .}$

KARSTAD, L. 1971. Pox. pp. 34-41. In: Infectious and Parasitic Diseases of Wild Birds. J. W. DAVIS, R. C. ANDERSON, L. KARSTAD and D. O. TRAINER, eds. The lowa State Univ. Press, Ames, IA.

KIRMSE, P. 1966. New wild bird hosts for pox viruses. Bull. Wildl. Dis. Assoc. 2:30-33. 
- 1967a. Host specificity and long persistence of pox infection in the flicker (Colaptes auratus). Bull. Wildl. Dis. Assoc. 3:14-20.

- 1967b. Pox in wild birds. An annotated bibliography. Wildl. Dis. 49.

MOFFATT, R. E. 1972. Natural pox infection in a golden eagle. J. Wildl. Dis. 8:161-162.

LEIBOVITZ, L. 1969. Natural occurrence and experimental study of pox and Haemoproteus infections in a mute swan (Cygnus olor). Bull. Wildl. Dis. Assoc. 5: $\overline{130-136 .}$
MORTON, J. K. and R. A. DIETERICH. 1979. Avian pox infection in an American green-winged teal (Anas crecca carolinensis) in Alaska. J. Wildl. Dis. 15:451-453.

PEARSON, G. L. and D. A. PASS. 1975. Fatal pox infection in a roughlegged hawk. J. Wildl. Dis. $11: 224-228$.

ROBBINS, C. S., B. BRUUN and H. S. ZIM. 1966. Birds of North America. Golden Press, New York, NY. $340 \mathrm{pp}$.

SIMPSON, C. F., D. J. FORRESTER and S. A. NESBITT. 1975. Avian pox in Florida sandhill cranes. J. Wildl. Dis. 11:112-115.

\title{
Newcastle Disease
}

\author{
L. Michael Philo
}

\section{Introduction}

Newcastle disease is a highly infectious disease caused by several strains of a myxovirus. It infects domestic waterfowl and poultry, wild waterfowl, perching birds, raptors and many other avian species. Birds may have subclinical to fatal infections with varying involvement of the respiratory tract, gastrointestinal tract and central nervous system (Graham and Halliwell 1978, Palmer and Trainer 1971).

\section{History}

The clinical disease was first described in 1926 in poultry of England and Korea and gradually spread throughout the world. It probably reached the United States by
1935, though it was not identified until 9 years later. It is now endemic in the country (Palmer and Trainer 1971). An outbreak in California in 1971 occurred when a group of infected psittacine birds was imported for sale as pets (Fowler 1978). Several years later the virus was isolated from apparently heal thy Canada geese (Branta canadensis) in the Atlantic flyway (Rosenberger et al. 1975).

\section{Transmission}

The primary avenues of virus infection are probably inhalation and ingestion. The virus is quite viable when free in the environment, so it may be infective in water and dust. It may be eaten in contaminated food or in contaminated or infected prey 
(e.g. earthworms, external parasitic arthropods, other birds). The virus is probably released in exudates, excreta, eggs and carcasses (Palmer and Trainer 1971).

Antibody titers and virus isolation from clinically normal birds plus, in some cases, transmission to susceptible avian test animals have demonstrated the presence of infective carriers among a number of wild and domestic species (BozorgmehriFard and Keyvanfar 1979, Friend and Trainer 1970, Graham and Halliwell 1978, Okoh 1979, Palmer and Trainer 1971, Pearson and McCann 1975). Domestic and wild waterfowl species may have a particularly high resistance to clinical disease (Friend and Trainer 1970, Palmer and Trainer 1971, Rosenberger et al. 1975).

\section{Signs, Symptoms and}

\section{Pathogenesis}

The virus may attack the respiratory system, gastrointestinal tract, central nervous system or any combination with corresponding clinical signs. There may of course, be no signs at all. Mild forms may show combinations of sneezing, head shaking, difficulty in breathing, diarrhea, depression, incoordination, paralysis of legs and wings; the head may be cocked to 1 side and turned or stretched over the back. More serious cases can be fatal (Fowler 1978, Palmer and Trainer 1971). Raptors usually exhibit those signs related to central nervous involvement (Graham and Halliwell 1978). There are usually no gross or microscopic lesions.

\section{Diagnos is}

Clinical signs are not sufficient for diagnosis because they are so variable with host susceptibility and virus strain and because they are so general that they could represent any of a number of other diseases. Definitive diagnosis can only be made by virus isolation and serological identification.

Samples for histopathology should be collected from lung, air sacs, serial sections of gastrointestinal tract, liver, kidney and myocardium. Samples for virus isolation should be collected aseptically from lung, intestine, liver, spleen and brain; they should be frozen, stored and transported in sterile containers at -20 to $-70 \mathrm{C}$.

\section{Significance and Control}

Newcastle disease has not been reported in wild avian species in Alaska, but the presence of subclinical carriers in migratory waterfowl provides the potential for transporting the virus into the state. Perhaps it is even endemic now and has not been identified. Control of outbreaks in wild populations is not practical. There has been at least 1 attempt to vaccinate a wild population with grain soaked in vaccine, but the effectiveness is unknown (Palmer and Trainer 1971).

Care must be taken when handling suspect birds because eye infections and generalized influenza-like infections have occurred in humans (Hanson 1975).

Newcastle disease infection should be reported to the Alaska StateFederal Laboratory.

\section{References}

BOZORGMEHRI-FARD, M. $H$. and $H$. KEYVANFAR. 1979. Isolation of Newcastle disease virus from teals (Anas crecca) in Iran. J. Wildl. Dis. 15:335-337. 
FOWLER, M. E. 1978. Infectious and zoonotic diseases. pp. 367-374. In: Zoo and Wild Animal Medicine. M. E. FOWLER, ed. W. B. Saunders Co., Philadelphia, PA.

FRIEND, M. and D. O. TRAINER. 1970. Serologic evidence of Newcastle disease in captive mallards and swans. J. Wildl. Dis. 6:130-135.

GRAHAM, D. L. and W. H. HALLIWELL. 1978. Virus diseases of birds of prey. pp. 260-265. In: Zoo and Wild Animal Medicine. M. E. FOWLER, ed. W. B. Saunders Co., Philadelphia, PA.

HANSON, R. P. 1975. Paramyxovirus infections. pp. 851-858. In: Diseases Transmitted from Animals to Man. 6th ed. W. T. HUBBERT, W. F. MCCULLOCH and P. R. SCHNURRENBURGER, eds. Charles $C$. Thomas, Springfield, IL.

$\mathrm{OKOH}$, A. E. J. 1979. Newcastle disease in falcons. J. Wildl. Dis. 15:479-480.
PALMER, S. F. and D. O. TRAINER. 1971. Newcastle disease. pp. 3-16. In: Infectious and Parasitic Diseases of Wild Birds. J. W. DAVIS, R. C. ANDERSON, L. KARSTAD and D. O. TRAINER, eds. The lowa State Univ. Press, Ames, IA.

PEARSON, G. L. and M. K. MCCANN. 1975. The role of indigenous wild, semidomestic and exotic birds in the epizootiology of velogenic viscerotropic Newcastle disease in southern California, 1972-1973. J. Am. Vet. Med. Assoc. $167: 610-614$.

ROSENBERGER, J. K., S. KLOPP and $W$. C. KRAUSS. 1975. Characterization of Newcastle disease viruses isolated from migratory waterfowl in the Atlantic flyway. Avian Dis. 19:142-149. 


\title{
Chapter 2.
}

\section{BACTERIAL AND FUNGAL DISEASES}

\section{Avian Cholera}

\author{
L. Michael Philo
}

\section{Introduction}

Avian cholera is an infectious disease of domestic and wild birds and occasionally nonavian species (Korschgen et al. 1978, Rosen 1969). The causative organism is the bacterium Pasteurella multocida, a Gram negative coccobacillus which has bipolar staining characteristics when stained with Wright's, Giemsa's or methylene blue stain.
The disease is most of ten reported as an outbreak in which large numbers of birds, usually waterfowl (Rosen 1971), are found dead. Though deaths have also been reported in small wild passerines (Locke and Banks 1972), crows (Corvus brachyryhnchos) (Zinkl et al. 1977a) and birds of prey ( $\mathrm{Hal}-$ liwell and Graham 1978, Rosen 1972), this is usually in association with, and presumably the result of the outbreak in waterfowl. 


\section{History}

During the 40 years since the first recorded outbreak of avian cholera in this country, most reports are from waterfowl wintering grounds such as California (Oddo et al. 1978, Rosen 1971), Texas (Rosen 1971) and portions of the east coast (Klukas and Locke 1970, Locke et al. 1970); along migratory routes such as Nebraska (Zinkl et al. 1977a) and Saskatchewan (Wobeser et al. 1979); and in summer breeding grounds such as Maine (Korschgen et al. 1978).

Estimated losses for individual species during these die-offs are generally quite variable, from almost none (Rosen 1972) to as high as $20 \%$ ( inkl et al. 1977a) of the individuals in the area. Possibly the largest estimated number of birds of all species to have succumbed during 1 outbreak is 70,000 in California in the winter of 1965-1966 (Rosen 1972). The number of each species lost in these outbreaks does not appear related to number present (Rosen 1971).

\section{Transmission}

The mode of transmission of avian cholera among wild birds is usually undetectable, but there are several possibilities. One is the contamination of water by infective nasal discharges (Rosen 1971, 1972) and feces (Rosen 1969) of diseased waterfowl or by tissues of diseased carcasses (Zinkl et al. 1977a). This is supported by the finding that water from ponds in or around which waterfowl have died of avian cholera contains infective $\underline{P}$. multocida (Korschgen et al. 1978, Rosen 1969, 1971). The bacteria are apparently taken into the bodies of uninfected waterfowl through ingestion of the contaminated water or inhalation of aerosol formed by birds landing and flapping their wings (Rosen 1971). Food supplies can be contaminated by the same infective material.

High density of individuals appears to be another contributing factor in disease transmission and may occur through colonial nesting e.g. common eider ducks, Somateria mollissima dresser i (Korschgen et al. 1978); snow geese, Anser caerulescens (Wobeser et al. 1979); Ross' geese, Anser rossii (Wobeser et al. 1979), roosting e.g. coots, Fulica americana (Klukas and Locke 1970) or forced concentration by storms or unseasonably cold weather (Locke et al. 1970, Zinkl et al. 1977a). These concentrations tend to be in shallow water near shore, which is the area where winds tend to concentrate carcasses and where contaminated feces and nasal discharges are most likely to occur in greatest concentration.

In some outbreaks, diseased domestic fowl, especially chickens and turkeys, may be the source. Contaminated offal may be carried to bodies of water by scavenging gulls and lead to infection of wild waterfowl (Korschgen et al. 1978).

Several investigators hypothesize that migrating waterfowl carry the disease organism north from the wintering sites to their breeding grounds (Rosen 1972, Wobeser et al. 1979). Endemic avian cholera is known to occur in at least 2 wintering sites, California and Texas (Rosen 1971), and would thus serve as the source. Such a hypothesis however, brings up the question of a carrier state. Existence of carriers is controversial, but there does appear to be some support for it. Despite many negative findings (Donahue and 0lson 1969, Rosen 1971), there have been a few isolations of P. multocida from apparently healthy 
waterfowl (Korschgen et al. 1978, Quortrup et al. 1946), and a snow goose which survived a case of avian cholera suddenly died of the disease 3 months later (Rosen 1972).

Another possible mode of transmission is ingestion of contaminated carcasses by scavengers such as gulls (Korschgen et al. 1978, Locke et al. 1970), crows (Zinkl et al. 1977a) and birds of prey (Locke et al. 1970, Rosen 1972). However, many of these species do not show the large losses seen in waterfowl (Klukas and Locke 1970, Locke et al. 1970, Rosen 1972), suggesting a greater resistance.

\section{Signs, Symptoms and \\ Pathogenesis}

Many of the signs of avian cholera are thought due to endotoxins released by the infecting bacteria after they enter the body and are carried to various organs by the bloodstream (Rosen 1971). The disease may be acute, peracute or chronic, though the outbreaks in wild birds seem to be acute or peracute. In these cases the onset is so rapid and mortality so high that hundreds of birds may be found dead and only a few moribund (Rosen 1971).

A bird sick with the acute or peracute form may show anorexia, oral mucous discharge and diarrhea ( $\mathrm{Hal}-$ liwell and Graham 1978). Frothy, bloody nasal discharge has also been noted (Klukas and Locke 1970). Some investigators have observed sick waterfowl swimming in circles (Klukas and Locke 1970) or flying upside down before plunging into the water or onto the ground (Zinkl et al. 1977a). other birds were too weak to swim or fly (Klukas and Locke 1970). The disease progresses rapidly to death, and often a sick bird dies within 5 minutes of the time it is first observed (Zinkl et al. 1977a). A diseased bird becomes incapacitated with the head moved back over the shoulder or forward in a prostrate position and may be seen shivering, convulsing or slowly beating its wings; often the tail, wings and legs are spread (Rosen 1971, Zinkl et al. 1977a).

The chronic form, as seen in captive waterfowl, may occur after the acute form or in older animals. Diarrhea and labored breathing are typical signs (Halliwell and Graham 1978).

Upon examining a fresh carcass, some or all of the following lesions can be seen. Pinpoint hemorrhages may be observed at the surfaces of the heart, mesenteries and gizzard and in the abdominal fat. The liver is often enlarged, copper- or greenish-colored and friable, with numerous small necrotic foci on the surface. The kidneys and spleen are sometimes misshapen and mottled or may appear perfectly normal. The intestines may show hemorrhagic enteritis or no abnormalities at all. The air sacs usually appear normal but may contain yellow fibrinous materal, and the lungs are usually consolidated and hemorrhagic ( $\mathrm{Hal}-$ liwell and Graham 1978, Klukas and Locke 1970, Korschgen et al. 1978, Locke et al. 1970, Rosen 1971, Wobeser et al. 1979. Zinkl et al. 1977a). Despite the lesions, many birds have considerable amounts of subcutaneous fat and ingested food because the disease develops and progresses so rapidly (Locke et al. 1970, Wobeser et al. 1979, Zinkl et al. 1977a).

\section{Diagnosis}

A presumptive diagnosis can be made on the basis of the lesions, particularly the petechial (pinpoint) hemorrhages and the focal necrosis of the liver. This is further supported by finding the typical bipolar bacteria in stained blood smears or liver impressions. Confirmation must 
be made by bacterial isolation and identification (Halliwell and Graham 1978, Rosen 1971).

Samples for culture should be collected and transported to the laboratory using aseptic technique and sterile containers. These should include, if possible, heart blood, liver and lung. If the carcass is not fresh or is decomposed, samples should also be taken from the brain and bone marrow. In the case of waterfowl, a sample of water near shore may be helpful. Blood smears and liver impressions will also be helpful if the carcass is fresh.

If the specimen is fresh, tissue samples may be placed in $10 \%$ neutral buffered formalin for histopathologic examination. These should include liver, lung, kidney, spleen, sections of digestive tract, heart and any other organs which appear abnormal. These results alone will not confirm the diagnosis, but may support a preliminary one and will aid in documentation of the case.

While awaiting confirmation, one should also consider duck plague, erysipelas and Escherichia coli infections in the differential diagnosis, as these diseases have signs in common with avian cholera (Rosen 1971).

\section{Significance and Control}

No outbreaks of avian cholera have been reported in Alaska. Nevertheless, the disease is potentially of great importance in view of some of the previous reports. The outbreaks in Saskatchewan (Wobeser et al. 1979), Nebraska (Zinkl et al. 1977a) and Maine (Korschgen et al. 1978) plus the finding of some apparent carriers (Korschgen et al. 1978, Rosen 1972) demonstrate the potential for the disease to be transported to Alaskan waterfowl breeding grounds from the wintering areas where it is endemic. Furthermore, many of the breeding grounds in Alaska are in remote areas, minimizing the likelihood that outbreaks will be discovered and reported.

If an avian cholera outbreak should be reported, then every attempt must be made to minimize the possibility of its spreading to scavengers and by these scavengers to waterfowl in uncontaminated areas. Scavengers may sometimes be controlled by mass shooting, but this may cause dispersal of infected or contaminated individuals to new areas (Rosen 1971). In the past there has been successful control of some localized outbreaks by collecting and burning all carcasses which can be found.

In addition, if the contaminated bodies of water involved are small and empty directly into a river or ocean, they might be successfully flushed. They are completely drained then allowed to refill (Rosen 1971, Zinkl et al. 1977b). In cases where there are multiple bodies of water as along many Alaskan coastal areas, this method may be not only impractical but unwise as clean ponds may become contaminated if adjacent ones are drained into them. In wetlands, it may be necessary to drain the entire area (Rosen 1971).

In some instances of localized outbreaks, small flocks of waterfowl have been hazed by aircraft so that they move out of the area before becoming infected. This may be a particularly useful method where there is a threat to endangered species, but it requires prior preparation to facilitate obtaining the necessary permits (Zinkl et al. 1977a). In the past, attempts to control large areas of disease outbreak (e.g. thousands of square miles) have been futile (Rosen 1971).

A study is now underway to develop a killed avian cholera bacterin for 
use in captive-reared individuals of an endangered species, the Aleutian Canada goose (J. Price, pers. comm.). It is hoped that the expected 6 month duration of immunity will protect the geese while they are overwintering in California. This should increase the chances of capture-raised birds surviving to breeding ages, when they will hopefully become part of the wild breeding population.

Avian cholera is transmissible to humans, so care must be taken when handling carcasses or live birds which are avian cholera suspects. Humans infected with $\underline{P}$. multocida are often subclinical carriers, but stress can cause them to develop the disease syndrome (Rosen 1975).

All cases of avian cholera should be reported to the Alaska StateFederal Laboratory in Anchorage.

\section{Acknowledgments}

Thanks are extended to Mr. James R. Dau for invaluable assistance in collecting and organizing the pertinent literature.

\section{References}

DONAHUE, J. M. and L. D. OLSON. 1969. Survey of wild ducks and geese for Pasteurella spp. Bull. Wildl. Dis. Assoc. 5:201-205.

HALL IWELL, W. H. and D. L. GRAHAM. 1978. Bacterial diseases of birds of prey. pp. 265-273. In: Zoo and Wild Animal Medicine. M. E. FOWLER, ed. W. B. Saunders Co., Philadelphia, PA.

KLUKAS, R. W. and L. N. LOCKE. 1970. An outbreak of fowl cholera in Everglades National Park. J. Wildl. Dis. 6:77-79.

KORSCHGEN, C. E., H. C. GIBBS and H. L. MENDALL. 1978. Avian cholera in eider ducks in Maine. J. Wildl. Dis. 14:254-258.
LOCKE, L. N., V. STOTTS and G. WOLFHARD. 1970. An outbreak of fowl cholera in waterfowl on the Chesapeake Bay. J. Wildl. Dis. $6: 404-407$.

LOCKE, L. N. and R. C. BANKS. 1972. Avian cholera in cedar waxwings in Ohio. J. Wildl. Dis. 8:106.

ODDO, A. F., R. D. PAGAN, L. WORDEN and R. G. BOTZLER. 1978. The January 1977 avian cholera epornitic in northwest California. J. Wildl. Dis. 14:317-321.

QUORTRUP, E. R., F. B. QUEEN and L. J. MEROVKA. 1946. An outbreak of pasteurellosis in wild ducks. J. Am. Vet. Med. Assoc. 108:94-100.

ROSEN, M. N. 1969. Species susceptibility to avian cholera. Bull. Wildl. Dis. Assoc. 5:195-200.

1971. Avian cholera. pp. 59-74. In: Infectious and Parasitic Diseases of Wild Birds. J. W. DAVIS, R. C. ANDERSON, L. KARSTAD and D. O. TRAINER, eds. The lowa State Univ. Press, Ames, IA.

1972. The 1970-71 avian cholera epornitic's impact on certain species. J. Wildl. Dis. $8: 75-78$.

1975. Pasteurellosis ( $\underline{P}$. multocida). pp. 129-138. In : Diseases Transmitted from Animals to Man. 6 th ed. W. T. HUBBERT, W. F. MCCULLOCH and P. R. SCHNURRENBERGER, eds. Charles C. Thomas, Springfield, IL.

SANDHU, T. 1978. Infectious diseases. pp. 193-200. In: Zoo and Wild Animal Medicine. M. E. FOWLER, ed. W. B. Saunders Co., Philadelphia, PA.

WOBESER, G., D. B. HUNTER, B. WRIGHT, D. J. NIEMAN and R. ISBISTER. 1979. Avian cholera in waterfowl in Saskatchewan, spring 1977. J. Wildl. Dis. 15:19-24.

ZINKL, J. G., N. DEY, J. M. HYLAND, J. J. HURT and K. L. HEDDLESTON. 1977a. An epornitic of avian cholera in waterfowl and common crows in Phelps County, Nebraska, in the spring, 1975. J. Wildl. Dis. 13:194-198. 
ZINKL, J. G., J. J. HURT, J. M.

HYLAND, N. DEY, D. STUDNICKA and D. D. KING. 1977b. Treatment of captive giant Canada geese affected by avian cholera. J. Wildl. Dis. 13:294-296.

\title{
Avian Botulism
}

\author{
Jim Dau
}

\section{Introduction}

Avian botulism ( 1 imberneck, duck disease, western duck sickness) is a paralytic disease usually associated with warm, shallow marsh environments subject to stagnation with high organic detritus levels. The disease affects the peripheral nervous system via toxins produced by 1 or more nonproteolytic, sporeforming, anaerobic types of the bacterium clostridium botulinum. Clostridium botulinum types A, B and $F$ are usually associated with human botulism; types $C$ and $D$ usually affect both birds and mammals; type $E$ affects only humans and certain avian species (Holdeman 1970, Rosen 1971).

Many avian species indigenous to Alaska have been reported susceptible to intoxication. Most of these Alaskan species winter in western North America where the most significant losses recorded have occurred, thus the potential for Alaskan epornitics does exist. Species comprising $95 \%$ of the total mortality of a type $C$ epornitic were pintail (Anas acuta), green-winged teal (Anas crecca) and northern shovelor (Anas clypeata) (McLean 1946). Fay et al. (1965) as reported by Rosen (1971) reported common loons (Gavia immer), herring gulls (Larus argentatus) and ring-billed gulls ( $\mathrm{L}$. delawarensis) to comprise $93 \%$ of the mortality in a type $E$ outbreak.

\section{History}

The first report of a possible botulism outbreak in ducks was made during 1876 (Kalmbach and Gunderson 1934). Subsequent outbreaks in California duck populations prompted intense, diversified research late in the 1920s that encompassed soil chemistry, water analysis, parasitology, bacteriology and pathology. This resulted in identification of $\mathbf{C}$. botulinum type $C$ as the causative agent of western duck sickness and technological developments to control the disease. Recent research has focused on understanding botulism etiology to determine mechanisms that initiate pathogenicity.

Avian botulism occurs worldwide. Though the disease has never been reported in Alaska, current projects under consideration, e.g. the Susitna Hydroelectric Project and the intensifying Delta agricultural effort, may alter these relatively undeveloped areas making them more conducive to botulism outbreaks. 
Transmission

Transmission is the most complex and least understood aspect of avian botulism. The medium of transfer, be it the Clostridium organism per se or its toxin, is still being discussed. Two hypotheses have been proposed explaining avian botulism transmission.

The sludge-bed hypothesis suggests c. botulinum organisms, which normally inhabit the mud or soil of lakes, marshes or fields, proliferate under favorable conditions, e.g. flooding and stagnation. When decaying organic matter accumulates and depletes the oxygen supply, anaerobic conditions stimulate Clostridium growth and production/release of toxin into the environment (Bell et al. 1955). Low concentrations of toxins that are observed in the environment during epornitics do not necessarily falsify this hypothesis if intoxication is a cumulative function of toxin ingestion (Crisley et al. 1968).

The microenvironment hypothesis suggests pathogenic, anaerobic $\underline{c}$. botulinum organisms exist in smal 1 , discrete, particulate environments, e.g. invertebrates or decomposing organic matter that contain requisites for growth independent of the ambient environment (Bell et al. 1955). Toxins are not released from the microenvironment into the macroenvironment; infection results from ingestion of microenvironments containing pathogenic toxins.

High proportions of predaceous and scavenger aquatic invertebrates have been shown to contain $\mathrm{C}$. botulinum type $C$ toxin (Duncan and Jensen 1976). This suggests: 1) invertebrates may concentrate the toxin via feeding in contaminated areas or 2) invertebrates may constitute discrete microenvironments suitable for toxin production. The experimental determination that animal rather than vegetative detritus is the superior medium for toxin production may be due to the presence of the organism in animals at the time of death. If invertebrates constitute the primary mechanism of transmission, a positive feedback cycle approaching exponential growth could occur. Bird carcasses fostering increased invertebrate numbers and causing increased bird mortality would explain the explosive nature of botulism epornitics. Eight blow fly larvae could contain sufficient toxin to kill an adult ring-necked pheasant (Phasianus colchicus). A single contaminated carcass heavily infected with larvae could theoretically be a mortality source for 200 to 300 birds (Lee et al. 1962). Toxins apparently persist through invertebrate larval metamorphoses into pupal life stages (Duncan and Jensen 1976).

Avian botulism exhibits a seasonal periodicity of highest intensity during late summer to early fall. Outbreaks of lower severity occasionally persist throughout the winter. This depressed severity may be due to differential utilization of invertebrates as a food source between seasons or a correlation of toxin production to growth phase of the Clostridium organism (Boroff 1955).

In general, immunity does not result from previous exposure. However, some scavenger species, e.g. gulls (Larus spp.) and vultures (Cathartidae) exhibit immunity for specific C. botulinum types (Rosen 1971).

\section{Signs, Symptoms and \\ Pathogenesis}

Neuromuscular involvement resulting in complete or partial paralysis characterizes avian botulism. The degree of paralysis varies with the amount of toxin 
ingested and with individual host. Clinical symptoms include power ioss expressed as the inability to initiate or sustain flight or loss of leg use; fluid accumulation beneath the nictitating membrane leading to cementation of eyelids; dyspnea; greenish diarrhea; and paralys is of cervical muscles producing a prostrate head and neck posture (1 imberneck) (Rosen 1971).

After ingestion, the toxin is assimilated across the mucous membranes of the intestinal wall into the lymph system, possibly by altering intestinal wall permeability. The toxin is transported to the circulatory system and eventually reaches the myoneural junction of the parasympathetic motor nerves where it interferes with the release of acetyl-choline at the presynaptic sites and causes complete or partial paralysis. Death may result from drowning, respiratory failure, exposure or increased susceptibility to predation (Rosen 1971).

Botulism does not exhibit primary pathologic tissue alterations (Rosen 1971). Secondary pathologic conditions, e.g. eye involvement and plugged vent are occasionally observed. Continued exposure to sublethal toxin levels may invoke chronic infections characterized by emaciation and a distended gall bladder.

\section{Diagnosis}

A tentative diagnosis of avian botulism is suggested by the clinical symptoms previously described. A definitive diagnosis is complicated by the frequency with which $C$. botulinum can be found in healthy birds and by the rapid secondary invasion of the bacteria following death from other causes. Thus, post mortem demonstration of the organism is of little diagnostic value for at- tributing the cause of death to $\boldsymbol{C}$. botulinum.

Definitive diagnoses are usually based on the mouse protection test. Anti-toxin for specific $C$. botulinum types are administered to individual groups of laboratory mice prior to inoculation with serum from a sick bird suspected of harboring a botulism infection. All mice will succumb except those treated with the anti-toxin specific for the $\underline{C}$. botulinum type causing the infection (Rosen 1971).

A fluorescent antibody test using conjugated anti-toxin which binds to erythrocytes of affected ducks has also been used (Rosen 1971).

\section{Significance and Control}

The significance of avian botulism as a mortality source for waterfowl is exemplified by the recorded death of 250,000 ducks during a single epornitic (Rosen 1971). Severe losses in shorebird, marine and upland game bird species have been documented (Rosen 1971).

Environmental conditions predisposing to botulism outbreaks allow epornitics to be predicted. These include high bird densities, flooding, shallow areas of warm stagnant water and large quantities of decaying organic matter.

Methods of control that have been developed lend themselves primarily for use with aquatic or semiaquatic species. These techniques are:

1. Manipulation of water level and flow rate to dilute and remove toxins and maintain aerobic conditions unfavorable for pathogenic Clostridium spp.

2. Removal of organic matter to prevent bacterial growth through 
carcass incineration or wetland burning and disking to decrease vegetative biomass

3. Substrate alteration to minimize shallow water shoreline ecozones

4. Harassment, special emergency hunting seasons or provision of alternate artificial food sources to minimize use of contaminated areas by susceptible species

5. Retrieval and treatment of infected birds (Rosen and Bischoff 1953)

Control is complicated by the presence of the environmentally resistant spores of $\underline{C}$. botulinum and the persistence of toxins through various life stages of invertebrates.

It appears humans are not susceptible to toxins from types C and D. Despite the past practice of market hunters augmenting their waterfowl harvests with botulism mortalities and presumably ample exposure of hunters to the disease, no substantiated cases of human botulism resulting from either toxin type have been recorded (Rosen 1971). Apparently, either the heat labile toxins are destroyed in cooking, the toxin is not assimilated or the biochemical receptors at the presynaptic sites are not compatible with the toxin proteins.

The potential for botulism epornitics in Alaska certainly exists. The high proportion of Alaskan species that winter in areas having histories of botulism epornitics provides a potential source. In light of the ubiquitous nature of Clostridium spp., stress alone may suffice to lower avian resistance to the point where infection results.

Conditions favoring botulism outbreaks often result from environmental alterations that provide inunda- tion of large amounts of organic matter. This has a two-fold effect: (1) it enhances anaerobic conditions and growth of Clostridium organisms and (2) it concentrates avian species by providing areas of high quantity/low quality food. Damming and some agricultural practices that allow high residual amounts of vegetation to go unharvested may fulfill these conditions. This is pertinent to (1) waterfowl management, which often employs both of these techniques to wetlands and (2) Alaska in general, considering proposed hydroelectric projects and developing agricultural areas. Johnson (1970) seemed to have Alaska in mind when he stated:

It is the abrupt change in the environment such as deforestation, reforestation, plowing up of land for agriculture, especially when associated with irrigation and the building or urban communities in otherwise natural environments, that predispose to disease.

\section{References}

BELL, J. G., G. W. SCIPLE and A. A. HUBERT. 1955. A microenvironment concept of the epizoology of avian botulism. J. Wildl. Manage. $19: 352-357$.

BOROFF, D. A. 1955. Study of the toxins of Clostridium botulinum: Relation of autolysis to toxin production. J. Bacteriol. $70: 363-367$.

CRISLEY, F. D., V. R. DOWELL and R. ANGELOTTI. 1968. Avian botulism in a mixed population of resident ducks in an urban river setting. Bull. Wildl. Dis. Assoc. 4:70-77.

DUNCAN, R. M. and $W$. I. JENSEN. 1976. A relationship between avian carcasses and living invertebrates in the epizootiology of avian botulism. J. Wildl. Dis. $12: 116-126$. 
FAY, L. D., 0. W. KAUFMANN and L. A. RYEL. 1965. Mass mortality of waterbirds in Lake Michigan 1963-64. Great Lakes Res. Div., Univ. Michigan Pub. 13. Ann Arbor, MI. $36 \mathrm{pp}$.

HOLDEMAN, L. V. 1970. The ecology and natural history of Clostridium botulinum. J. Wildl. Dis. 6:205-209.

JOHNSON, H. N. 1970. The ecological approach to the study of zoonotic diseases. J. Wildl. Dis. $6: 194-204$.

KALMBACH, E. R. and M. F. GUNDERSON. 1934. Western duck sickness a form of botulism. USDA Tech. Bull. Washington, DC. 411 pp.
LEE, V. H., S. VADLAMUDI and R. P. HANSON. 1962. Blow fly larvae as a source of botulinum toxin for game farm pheasants. J. Wildl. Manage. 26:411-413.

MCLEAN, D. D. 1946. Duck disease at Tulare Lake. Calif. Fish and Game $32: 71$.

ROSEN, M. N. 1971. Botulism. pp. 100-114. In: Infectious and Parasitic Diseases of Wild Birds. J. W. DAVIS, R. C. ANDERSON, L. KARSTAD and D. O. TRAINER, eds. lowa State Univ. Press, Ames, IA.

ROSEN, M. N. and A. I. BISCHOFF. 1953. A new approach toward botulism control. Trans. North Am. Wildl. Conf. 18:191.

\title{
Aspergillosis
}

\author{
L. Michael Philo
}

\section{Introduction}

Aspergillosis is an acute to chronic fungal disease caused by Aspergillus species, primarily $A$. fumigatus (Richard 1975) but occasionally others (e.g. A. glaucas, A. niger) (Altman 1979, A $\bar{r}$ nall 1969). Tt probably occurs in all species of wild and captive birds (Altman 1979), but it has also been reported in man and a number of wild and domestic known as brooder pneumonia, pseudotuberculosis, cytomycosis, bronchomycosis and chick fever (O'Meara and Witter 1971).

\section{History}

The disease was first recognized in wild fowl in the early 1800 s when fungal growth was observed in the lungs and air sacs of a scaup duck
(Aythya sp.) and a jay (0'Meara and Witter 1971, Richard 1975). Since then its occurrence has been documented worldwide (O'Meara and Witter 1971). Although Antarctica is a notable exception, penguins (Sphenisciformes) in captivity commonly become infected (0'Meara and Witter 1971, Redig 1978).

The Alaska State-Federal Laboratory has on record, 3 cases of (species not recorded), a murre (Uria

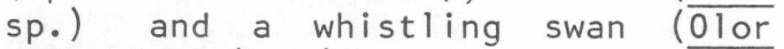
columbianus) (R. Barrett, pers. comm.).

The difficulty in finding published accounts of avian aspergillosis in Alaska should not necessarily be attributed to low incidence of the disease. Most likely it is due to a low likelihood of dead or ill birds being presented to in- 
dividuals or institutions capable of making the diagnosis and perhaps failure to publish individual case reports.

\section{Transmission}

Avian aspergillosis most commonly occurs as a respiratory infection. It usually results from ingestion of contaminated material or from puncture wounds (0'Meara and Witter 1971). The organism is ubiquitous in the soil. It is a saprophyte and has been isolated from decaying corn stalks, cobs and parts of other plants in winter and spring (Herman and Sladen 1958). Generally it constitutes a hazard not in the soil, but after it has become inoculated into the plant or other organic matter which readily supports its growth (Friend and Trainer 1969).

Many investigators hypothesize that the disease occurs by primary exposure, but others feel that most birds have a high level of natural resistance (Altman 1979). A healthy and highly resistant bird which inhales spores may develop and harbor nonproliferating foci of aspergillosis in the lungs or air sacs for up to several years instead of immediately becoming diseased. When the bird is subsequently subjected to stress, its resistance is lowered, the fungus proliferates and the bird quickly succumbs (Redig 1978). This may explain instances where only 1 or a few birds in a flock become infected.

Actually both views are probably correct. In some large outbreaks and die-offs, primary exposure through contaminated food sources has been blamed (Adrian et al. 1978, Pearson 1969). In others, large numbers of birds have died when the stress of winter or competition for limited food supply lowered their natural resistance to previously non- proliferating infections (Herman and Sladen 1958, Redig et al. 1980). The sources of still other outbreaks have remained undetected ( $\mathrm{Zinkl}$ et al. 1977).

One source of natural resistance in wild birds is probably similar to that reported in domestic fowl. There is a mucus sheet lining the respiratory tract. It is continually moved to the outside by cilial action and may remove spores faster than they can be inhaled. However, at humidity less than $10 \%$ or with severe dust this system may be impaired to the point where individual susceptibility is greatly increased (O'Meara and Witter 1971).

Increased susceptibility probably also occurs through use of contaminated nesting materials, high nesting population density, capture and confinement, feeding habits e.g; dump feeding by gulls (Larus spp.) and ravens (Corvus corax), and seedeating by waterfowl (Anseriformes) and young age (especially young of the year) (Adrian et al. 1978, Herman and Sladen 1958). Adverse weather increases susceptibility through stress, release of spores from air strata by raindrops (0'Meara and Witter 1971) or by forcing birds to eat contaminated food (Adrian et al. 1978). Other environmental factors can be of great importance, too. For example, the disease commonly develops in wild waterfowl confined for treatment after being recovered from oil spills. Presumably it is the result of physical and physiological stress (Harris and Smith 1977).

\section{Signs, Symptoms and \\ Pathogenesis}

Aspergillosis in birds is primarily a respiratory disease. The avian respiratory system is quite complex in comparison to the mam- 
malian system, being made up of lungs, air sacs and often air cavities in bones. These provide an extensive surface area upon which the fungus can become established, no doubt a primary contributing factor in the high mortality.

Affected birds may be lethargic and approached to within very close distances, perhaps $10-20 \mathrm{~m}$ or less (Herman and Sladen 1958). When they do flee it will likely be only a short distance and often by walking when too weak to fly. Those more severely affected may be easily caught with a long-handled fish-net or by hand. They are in poor condition, quite listless and showing difficulty in breathing, of ten to the point of gasping. The wings are often drooped and the feathers ruffled. Some individuals may have a cheesy exudate about the tongue, bill and nares (Friend and Trainer 1969). Raptors may exhibit hoarseness if the bronchial tree or syrinx is involved (Redig 1978). Some may have diarrhea and occasionally ataxia or other nervous signs. Death usually occurs within a few days (Herman and Sladen 1958, 0'Meara and Witter 1971). Aspergillus may also occur as a secondary invader, as it did in an outbreak of web necrosis in mute swans (Cygnus olor) (Yates et al. 1969). In some cases birds will show no visible signs of the disease, but necropsy reveals the typical lesions. Massive primary exposure of young birds may cause death before typical lesions have time to develop, but most cases develop slowly enough for them to occur (O'Meara and Witter 1971).

Within the lungs the body responds by forming a fibrous wall around the expanding foci of fungal infection. This results in white nodules which resemble the tubercles of tuberculosis (O'Meara and Witter 1971). The lungs themselves may be firm and dark red, either in whole or in part, such that they resemble the liver in appearance (Adrian et al. 1978). A different appearance occurs where the fungal spores develop on the surfaces of the lungs and digestive organs or on the linings of the chest, abdominal cavity and air sacs. Here typical plaques form. These are masses of fungal fruiting organs and in waterfowl are large and green or bluish to white or yellow, resembling the surface of bread mold (Adrian et al. 1978, O'Meara and Witter 1971). These lesions may be single or multiple, pinhead size to several millimeters in diameter, spongy soft to hard and firmly or loosely attached (O'Meara and Witter 1971). They result in loss of tissue effectiveness plus actual damage by the fungal hyphae. Many necropsies, especially in waterfowl, reveal pinpoint and larger areas of hemorrhage clearly visible beneath the thin epicardium which covers the heart (Adrian et al. 1978). It is also suspected that fungal toxins are produced and have some importance, but this has yet to be confirmed (Herman and Sladen 1958, 0'Meara and Witter 1971). The lungs or air sacs may contain a green dust which is the Aspergillus spores. This seems to be prevalent especially in waterfowl and gulls (Herman and Sladen 1958).

\section{Diagnosis}

Diagnosis is based on the gross appearance of the lesions, microscopic identification of fungal hyphae in the lesions and culture on differential media in the laboratory. In the past, serology has not been a reliable means of diagnosis, but recent work with immunodiffusion (Napolitano et al. 1978) has been shown to be highly reliable and thus shows great promise for the future.

During carcass examination and sample collection, disposable gloves and surgical masks should be worn as 
a precaution. With aseptic technique, swabs may be taken from the trachea, lungs and air sacs and placed in sterile transport medium for transportation to a laboratory. Positive or negative cultures from these locations are not conclusive by themselves, so further samples must be collected to provide sufficient information. Tissue samples containing intact individual lesions should be collected aseptically and placed in sterile containers for culture. Samples of all affected organs should be collected and placed in $10 \%$ neutral buffered formalin for microscopic examination.

\section{Significance and Control}

The status of aspergillosis in Alaska is unknown, but the potential for outbreaks and die-offs is certainly present. It is possible that some have occurred but either have not been discovered or have not been recognized. If and when such an epornitic (ạvian epizootic) is reported in Alaska it is likely that it will be in waterfowl; these species' migratory habits increase the likelihood that they will contact fungal spores, and they are subject to the physical stresses of flying long distances. It will of course, be more difficult to determine the status in less gregarious species because the likelihood of large numbers becoming simultaneously stressed and/or exposed to large concentrations of fungal spores is low, and 1 or a few dead or visibly diseased birds brings much less attention than a large number. Furthermore, occurrence of aspergillosis is more likely to be observed in game species than in non-game species, not due to increased probability of occurrence, but simply because their game status brings them relatively more attention.and importance. Ravens may constitute an exception because they are large, gregarious and often congregated in areas of human habitation.

Practically every avian species is susceptible and the disease is worldwide, so any bird showing signs and/or lesions compatible with aspergillosis should be suspected. Samples should be taken in an attempt to confirm the diagnosis because the occurrence may indicate a concentrated source of Aspergillus spores nearby and therefore a potential epornitic.

It must also be remembered that most mammalian species, including man, are susceptible to the disease, so areas of spore concentration represent a hazard to them, too. Even more important is the possible consumption, by carnivorous mammals, of infected birds which they may easily catch. A danger to humans arises from improper handling of diseased game bird carcasses.

In any wild avian species the diagnosis of aspergillosis is of much greater importance than treatment. Therefore it is advisable to humanely dispatch a diseased bird and collect the appropriate samples rather than to attempt treatment. It is unfortunate that the sanitation and control practices successfully used for game farm and captive birds (0'Meara and Witter 1971) are not practical in free-living populations.

\section{Acknowledgments}

The author gratefully acknowledges $\mathrm{Mr}$. James R. Dau for invaluable assistance in collecting material from the literature. Many thanks are extended to $\mathrm{Mr}$. Richard E. Barrett of the Alaska State-Federal Laboratory for locating records of avian aspergillosis in Alaska. 


\section{References}

ADRIAN, W. J., T. R. SPRAKER and R. B. DAVIES. 1978. Epornitics of aspergillosis in mallards (Anas platyrhynchos) in north central Colorado. J. Wildl. Dis. $14: 212-217$.

ALTMAN, R. B. 1979. Respiratory diseases of caged birds. Vet. Clin. North Am. Small Anim. Pract. 9:527-540.

ARNALL, L. 1969. Diseases of the respiratory system. pp. 263-290. In: Diseases of Cage and Aviary Birds. M. L. PETRAK, ed. Lea and Febiger, Philadelphia, PA.

FRIEND, M. and D. O. TRAINER. 1969. Aspergillosis in captive herring gulls. Bull. Wildl. Dis. Assoc. $5: 271-275$.

HARRIS, J. M. and D. C. SMITH. 1977. Treatment, husbandry and rehabilitation of oil-soaked birds. pp. 694-697. In: Current Veterinary Therapy VI: Small Animal Practices. R. W. KIRK, ed. W. B. Saunders Co., Philadelphia, PA.

HERMAN, C. M. and W. J. L. SLADEN. 1958. Aspergillosis in waterfowl. Trans. North Am. Wildl. Conf. 23:187-191.

NAPOL ITANO, R. L., E. P. DOLENSEK and E. A. RUSSO. 1978. Serodiagnosis of aspergillosis in avian species. pp. 265-268. In: Proceedings of the 1978 Annual Meeting of the American Association of Zoo Veterinarians. Hill's Division Riviana Foods, Topeka, KS.
O'MEARA, D. C. and J. F. WITTER. 1971. Aspergillosis. pp. 153-162. In: Infectious and Parasitic Diseases of Wild Birds. J. W. DAVIS, R. C. ANDERSON, L. KARSTAD and D. O. TRAINER, eds. The lowa State Univ. Press, Ames. IA.

PEARSON, G. L. 1969. Aspergillosis in wintering mallards. Bull. Wildl. Dis. Assoc. 5:404-405.

REDIG, P. 1978. Mycotic infections of birds of prey. pp. 273-276. In: Zoo and Wild Animal Medicine. M. E. FOWLER, ed. W. B. Saunders Co., Philadelphia, PA.

REDIG, P. T., M. R. FULLER and D. L. EVANS. 1980. Prevalence of Aspergillus fumigatus in freeliving goshawks (Accipiter gentilis atricapillus). J. Wildl. Dis. 16:169-174.

RICHARD, J. L. 1975. Aspergillosis. pp. 529-532. In: Diseases Transmitted from Animals to Man. 6th ed. W. T. HUBBERT, W. F. MCCULLOCH and P. R. SCHNURRENBERGER, eds. Charles C. Thomas, Springfield, IL.

YATES, V. J., L. T. MILLER, V. JASTY, C. H. WILLEY and M. HOLTZINGER. 1969. Web necrosis in mute swans--a report of an outbreak. Bull. Wildl. Dis. Assoc. 5:33-34. ZINKL, J. G., J. M. HYLAND and J. J. HURT. 1977. Aspergillosis in common crows in Nebraska, 1974. J. Wildl. Dis. 13:191-193. 


\title{
Avian Tuberculosis
}

\author{
Jamie K. Morton
}

\section{Introduction}

Avian tuberculosis is a contagious, chronic disease caused primarily by Mycobacterium avium, but birds are also susceptible to $\underline{M}$. bovis and more rarely to $M$. tuberculosis. Although it is distributed geographically worldwide, it is most frequent in the North Temperate Zone. The disease has been reported in numerous species indigenous to Alaska including wild geese, ducks, swans and raptors (Buchanan and Gibbons 1974). Twenty serotypes of $\underline{M}$. avium are recognized, types 1 and 2 being the most common (Montali et al. 1976).

\section{History}

Avian tuberculosis was first reported in fowl in London in 1869. Several reports of the disease in captive birds followed. It was first recognized in the U.S. around 1900 but not reported until the early $1920 \mathrm{~s}$. Corvan reported the disease in wild geese and swans in 1941 (Gale 1971). In' 1977 a sandhill crane (Grus canadensis) killed near Palmer, Alaska was reported positive for $\underline{M}$. avium (Thoen et al. 1977).

\section{Transmission}

Avian tuberculosis appears to be a function of feeding habits. Predatory birds acquire alimentary tract infections by ingestion of infected prey. Scavenger species, e.g. gulls (Larus spp.), become infected by ingestion of contaminated sewage effluents. Infections in ring-necked pheasants (Phasianus colchicus tor- quatus) and ruffed grouse (Bonasa umbellus) are thought to stem from association with domestic fowl (Gale 1971). McDiarmid (1956) reported transmission from brooding pigeons to squab.

Lesions in birds may include ulcerating tubercles of the intestinal tract. Thus, large numbers of organisms can be shed with the feces and persist in the soil for months or even years (Karlson 1978).

\section{$\underline{\text { Signs, Symptoms and }}$ \\ Pathogenes is}

Signs of the disease in birds are variable and may be lacking. Emaciation is the most frequent clinical sign. Skin lesions may be observed on the legs, around the eyes or beak or at the wing joints. Liver and spleen are more frequently infected than the lung. Intestinal lesions are also common.

Yellowish-gray nodules are formed as a reaction to the organism. A dry necrosis develops which is in contrast to the liquifying necrosis which is seen in mammalian infections (Gale 1971).

\section{Diagnosis}

The intradermal tuberculin test has been used in domestic fowl. Tuberculin is injected into the wattles or comb and the reaction checked in 48 hours. Feathers are plucked to expose a suitable site on birds without wattles. A whole blood rapid agglutination test has also been found reliable for use in 
chickens. Neither of these methods has proven satisfactory for use in exotics (Karlson 1978, Montali et al. 1976). Applications of the mammalian lymphocyte transformation test are being evaluated (Karlson 1978).

Bacteriologic evidence is based on culturing the spleen, liver, bone marrow or peritoneal tubercles using techniques described for mammals (Karlson 1975). Caseous contents from lesions on the liver or spleen are preferred for histopathologic examination (Karlson 1975).

\section{Significance and Control}

Avian tuberculosis is known to be a significant disease in birds in zoological parks. Mycobacterium avium is also pathogenic for cattle (Bos spp.), deer (0docoileous spp.), mink (Mustela vison), rabbits (Lagomorpha), rats (Rattus spp.), sheep (Ovis spp.) and especially swine (Sus spp.) (Karlson 1978). Although the prevalence of the disease is not known in migratory birds, the disease could become widespread if nesting or resting grounds are contaminated. Humans normally are highly resistant to avian tuberculosis, but have been infected in a few instances.

Control is based on eliminating infected individuals and preventing the use of contaminated areas by noninfected birds. Application of these measures in free-living birds is difficult due to the asymptomatic nature of the disease. The mechanics of at- tempting to limit the use of contaminated areas may themselves contribute to the dispersal of the organ ism.

\section{References}

BUCHANAN, R. E. and N. E. GIBBONS, eds. 1974. Bergey's Manual of Determinative Bacteriology. 8 th ed. Williams and Wilkins Co., Baltimore, MD. $1246 \mathrm{pp}$.

GALE, N. B. 1971. Tuberculosis. pp. 89-92. In: Infectious and Parasitic Diseases of Wild Birds. J. W. DAVIS, R. C. ANDERSON, L. KARSTAD and D. O. TRAINER, eds. lowa State Univ. Press, Ames, IA.

KARLSON, A. B. 1975. Tuberculosis. pp. 99-107. In: Isolation and Identification of Avian Pathogens. S. B. HITCHNER, C. N. DOMERMUTH, N. G. PURCHASE and J. E. WILLIAMS, eds. Am. Assoc. Pathol., Arnold Printing Corp., I thaca, NY.

- 1978. Avian tuberculosis. pp. 21-23. In: Mycobacterial Infections of Zoo Animals. R. J. MONTALI, ed. Smithsonian Institution Press, Washington, $D C$.

MCDIARMID, A. 1956. Some diseases of free-living wild birds in Britain. Bull. Brit. Ornithol. $76: 145$.

MONTALI, R. J., M. BUSH, C. O. THOEN and E. SMITH. 1976. Tuberculosis in exotic birds. J. Am. Vet. Med. Assoc. 169:920-927.

THOEN, C. O., E. M. HIMES and R. E. BARRETT. $1977 . \quad$ Mycobacterium avium serotype 1 infection in Sandhill crane (Grus canadensis). J. Wildl. Dis. $\overline{13: 40}-42$. 
Chapter 3.

\section{PROTOZOAN BLOOD PARASITES}




\title{
Plasmodium
}

\author{
L. Michael Philo
}

\section{Introduction}

Among the avian malarias is a parasitism of the peripheral blood caused by protozoa of the order Haemosporidia, family Plasmodiidae, genus Plasmodium. There are a number of Plasmodium species which infect many species of domestic and wild perching birds, pigeons and doves, waterfowl, gallinaceous birds, raptors and other birds in North America. Still other species cause malarias of man and other mammals (Soulsby 1968).

\section{History}

The first information on avian malaria was acquired about 90 years ago in Algeria, and shortly before the turn of the century an avian Plasmodium species was used for the first demonstration of the transmission of malaria. Throughout the 20 th century more information has been collected on host-parasite relationships and many new avian host species have been discovered (Bennett and Laird 1973).

From 1968 to 1972 a cooperative study between Canada and the United States revealed that waterfowl using the Atlantic flyway had up to $15 \%$ incidence of Plasmodium. This was based on thousands of blood smears from captured and released ducks and geese (Bennett and Laird 1973, Bennett et al. 1974, 1975). The disease has been reported in other eastern locations (Forrester et al. 1980, Kocan and Knisley 1970), the southwest (Loven et al. 1980) and west ( $\mathrm{Clark}$ and Swinehart 1966, Kingston et al. 1976, Stabler et al. 1977). In 1956 a dead gyrfalcon (Falco rusticolis) from the Colville River region in Alaska was diagnosed as having died of avian malaria caused by $P$. relictum (Beebe and Webster 1964).

Avian Plasmodium species are worldwide in distribution, but North American waterfowl may be more heavily parasitized than those of other regions. Waterfowl in the eastern Canadian high arctic may be an exception as most seem to be parasite-free (Bennett and Laird 1973).

\section{Transmission}

The life cycle of avian Plasmodium species is shared by 2 hosts. Asexual reproduction (schizogony) takes place in birds while sexual reproduction (gametogony) requires a bloodsucking arthropod, usually mosquitoes of the genera Culex, Aedes and sometimes Anopheles. In Plasmodium, schizogony occurs in the red blood cells and also in endothelial cells of inner organs.

Sporozoites are introduced into a susceptible avian host by an infected mosquito and eventually reproduce twice, asexually, in skin macrophages and fibroblasts to form merozoites. The second generation merozoites enter either red blood cells or endothelial cells. Those which reproduce in the endothelial cells give rise to merozoites which then enter erythrocytes. Inside the red 
cell the parasite forms a trophozoite, a small cell with a large vacuole which displaces the cytoplasm to the periphery of the parasite cell. The nucleus at 1 pole gives it the classical "signet ring" appearance seen in blood smears. Each of these produces a number of new merozoites by schizogony, digesting hemoglobin in the process. This process is synchronized in all red blood cells and can apparently continue indefinitely.

Eventually some merozoites develop into macrogametes (female) or microgametes (male) within erythrocytes. When these cells are ingested by a mosquito the gametes are released in the midgut and unite to form zygotes called ookinetes. These penetrate the gut wall and form oocysts. Each oocyst nucleus divides to form many sporozoites. After about 10 to 20 days the ooycyst ruptures and the sporozoites migrate to the salivary glands. (For more detail see Soulsby 1968).

In many avian species, circulating parasite levels drop in winter and increase again in spring, presumably in response to changes in host hormone levels (Applegate 1971, Applegate and Beaudoin 1970, Loven et al. 1980, Stabler et al. 1977). The spring increase corresponds to increased incidence of infection within avian populations because it coincides with emergence of the arthropod vectors. Under favorable conditions the rate of transmission equals or exceeds the rate needed to replace birds which succumb directly or indirectly to the infection (Applegate et al. 1971, Beaudoin et al. 1971).

Signs, Symptoms and Pathogenes is

The vast majority of birds diagnosed as infected with Plasmodium were not noted as having clinical signs (Altman 1977, Bennett et al. 1974, 1975, Clark and Swinehart 1966,
Halliwell 1979, Kocan 1968, Kocan and Knisley 1970, Stabler et al. 1977). However, the disease can certainly not be considered nonpathogenic. Affected birds may be listless, weakened and anemic (Ward 1978); young birds may have a depressed growth rate (Forrester et al. 1980) in addition to the other signs. In some cases, up to $100 \%$ of the red cells can be infected, with no mortality (Kocan 1968). Enlarged spleens are often seen on necropsy (Forrester et al. 1980), probably in response to the requirement for removal of large numbers of damaged erythrocytes. The signs can be sufficiently mild to be unnoticed when large numbers of birds are handled during screening studies. They can be severe enough to cause death, but the level of mortality among wild bird populations appears to be very low.

\section{Diagnosis}

Avian malaria caused by Plasmodium species can be diagnosed by identification of the intraerythrocytic schizonts and gametocytes in blood smears (Altman 1977). Morphology of the gametocytes, along with avian species affected, is then helpful in differentiating among species of the parasite (Keymer 1969, Soulsby 1968). Latent infections or those with only gametocytes in the red blood cells are very difficult to diagnose, particularly since the gametocytes closely resemble those of Haemoproteus (Keymer 1969, Kocan 1968). Samples for histopathology should be collected from reticuloendothelial tissues (e.g. lung, thymus, spleen, lymphoid tissue, bone marrow) and liver, but these may still not lead to definitive diagnoses in those difficult cases.

Birds are thought to remain infected for life, but after initial infection and parasitemia, the parasite may not be seen on peripheral blood smears until relapse 
of the disease. Even histopathology may not be helpful. The technique of subinoculation or isodiagnosis is needed to detect these cases. Blood from a suspect donor is inoculated into a recipient whose blood is then periodically examined with smears. Latent infections in donors will produce detectable parasitemia in the recipients. This method has revealed a $60 \%$ infection rate in a population of Canada geese (Branta canadensis) while peripheral smears of the donors showed no Plasmodium at all (Herman et al. 1966).

Differential diagnosis includes pox, ornithosis and pantothenic acid deficiency (Keymer 1969).

\section{$\underline{\text { Significance and }}$ Control}

The significance of Plasmodium infections in Alaskan birds is difficult to determine because the incidence and severity in the state are unknown. However, based on what is known about the disease in other locations, one might expect to find at least a low level of parasitism among the migratory and perhaps resident bird populations.

The disease appears to predispose certain avian populations to increased mortality from weather, predators and other diseases by decreasing overall resistance and body condition (Forrester et al. 1980). The extent to which this occurs in Alaska is unknown but may be found to be very important.

Another consideration pointed out by avian malaria investigators (Bennett et al. 1975) is that the avian malarias must be considered and their significance understood in planning for "improved wetlands." The increased crowding of migratory waterfowl in these wetlands may increase mortality by these vector-borne parasitisms.
In isolated infections of domestic fowl or captive populations of wild birds, control of mosquitoes and treatment of affected birds with quinacrine hydrochloride or chloroquine phosphate have been successful (Altman 1977, Kingston et al. 1976). Unfortunately these measures are not practical in natural populations.

\section{References}

ALTMAN, R. B. 1977. Parasitic diseases of caged birds. pp. 682-687. In: Current Veterinary Therapy VI: Small An imal Practice. R. W. KIRK, ed. W. B. Saunders Co., Philadelphia, PA.

APPLEGATE, J. E. 1971. Spring relapse of Plasmodium relictum infections in an experimental field population of English sparrows (Passer domesticus). J. Wildl. Dis. 7:37-42.

APPLEGATE, J. E. and R. L. BEAUDOIN. 1970. Mechanisms of spring release in avian malaria: effect of gonadotropin and corticosterone. J. Wildl. Dis. $6: 443-447$.

APPLEGATE, J. E., R. L. BEAUDOIN and D. C. SEELEY, JR. 1971. The effect of spring relapse in English sparrows on infectivity of malaria to mosquitoes. J. Wildl. Dis. $7: 91-92$.

BEAUDOIN, R. L., J. E. APPLEGATE, D. E. DAVIS and R. G. MCLEAN. 1971. A model for the ecology of avian malaria. J. Wildl. Dis. 7:5-13.

BEEBE, $F$. L. and $H$. M. WEBSTER. 1964. North American Falconry and Hunting Hawks. North American Falconry and Hunting Hawks Press, Denver, C0. $315 \mathrm{pp}$.

BENNETT, G. F. and M. LAIRD. 1973. Collaborative investigations into avian malarias: an international research programme. J. Wildl. Dis. 9:26-28. 
BENNETT, G. F., W. BLANDIN, H. W. HEUSMANN and A. G. CAMPBELL. 1974. Hematozoa of the Anatidae of the Atlantic flyway. I. Massachusetts. J. Wildl. Dis. $10: 442-451$.

BENNETT, G. F., A. D. SMITH, W. WHITMAN and M. CAMERON. 1975. Hematozoa of the Anatidae of the Atlantic flyway. II. The maritime provinces of Canada. J. Wildl. Dis. $11: 280-289$.

CLARK, G. W. and B. SWINEHART. 1966. Blood protozoa of passerine birds of the Sacramento (Calif.) region. Bull. Wildl. Dis. Assoc. 2:53-54. FORRESTER, D. J., P. P. HUMPHREY, S. R. TELFORD, JR. and L. E. WILLIAMS, JR. 1980. Effects of blood-induced infections of Plasmodium hermani on domestic and wild turkey poults. J. Wildl. Dis. 16:237-244.

HALLIWELL, W. H. 1979. Diseases of birds of prey. Vet. Clin. North Am. Small Anim. Pract. 9:541-568.

HERMAN, C. M., J. 0. KNISLEY JR. and E. L. SNYDER. 1966. Subinoculation as a technique in the diagnosis of avian Plasmodium. Avian Dis. 10:541-547.

KEYMER, I. F. 1969. Parasitic diseases. pp. 393-452. In: Diseases of Cage and Aviary Birds. M. L. PETRAK, ed. Lea and Febiger, Philadelphia, PA.
KINGSTON, N., J. D. REMPLE, W. BURNHAM, R. M. STABLER and R. B. MCGHEE. 1976. Malaria in a captively-produced F1 gyrfalcon and in two F1 peregrine falcons. J. Wildl. Dis. 12:562-565.

KOCAN, R. M. 1968. The canvasback duck (Aythya valisineria): a new host record for Plasmodium. Bull. Wildl. Dis. Assoc. 4:86-87.

KOCAN, R. M. and J. O. KNISLEY. 1970. Incidence of malaria in a wintering population of canvasbacks (Aythya valisineria) on Chesapeake Bay. J. Wildl. Dis. $6: 441-442$.

LOVEN, J. S., E. G. BOLEN and B. W. CAIN. 1980. Blood parasitemia in a south Texas wintering waterfowl population. J. Wildl. Dis. $16: 25-28$.

SOULSBY, E. J. L., ed. 1968. Helminths, Arthropods and Protozoa of Domesticated Animals (Monnig). 6th ed. Williams and Wilkins Co., Baltimore, MD. 824 pp.

STABLER, R. M., C. E. BRAUN and T. D. I. BECK. 1977. Hematozoa in sage grouse from Colorado. J. Wildl. Dis. 13:414-417.

WARD, F. P. 1978. Parasites and their treatment in birds of prey. pp. 276-281. In: Zoo and Wild Animal Medicine. M. E. FOWLER, ed. W. B. Saunders Co., Philadelphia, PA. 


\title{
Leucocytozoon
}

\author{
L. Michael Philo
}

\section{Introduction}

Another of the avian malarias is a protozoan blood parasite of the order Haemosporidia, family Haemoproteidae, genus Leucocytozoon. The scope of avian species infected is as extensive as that of Plasmodium: passerines, pigeons and doves, waterfowl, gallinaceous birds, raptors and others, but these parasites are found only in birds (Cook 1971, Soulsby 1968, Ward 1978).

\section{History}

Leucocytozoon infection was first reported in 1915 when it was found in a domestic duck in Ontario, Canada (Herman et al. 1975). Nearly 20 years later it was reported in wild ducks and a domestic goose. It was found in Canada geese (Branta canadensis) in the late 1930s. Since then it has been reported throughout North America but its incidence is worldwide (Bennett and Laird 1973, Herman et al. 1975).

Screening studies in the northwestern section of the Atlantic flyway have revealed a prevalence of up to $14 \%$ among wild waterfowl (Bennett et al. 1974, 1975, Kocan and Knisley 1970), while somewhat higher percentages were found for wild ducks from northwestern Canada (Williams et al. 1977). In the Seney National Wildlife Refuge in northern Michigan, up to $80 \%$ of adult Canada geese may be infected and goslings sustain significant losses from the parasite (Herman et al. 1975).

In 1965, 162. rock ptarmigan (Lagopus mutus), age 6 weeks to over
2 years, were captured near Eagle Summit, Alaska. Nearly $90 \%$ had Leucocytozoon infection. Three willow ptarmigan (ㄴ. lagopus) from Eagle Summit all had Leucocytozoon parasites. No clinical signs were reported in any of these birds (Stabler et al. 1967).

\section{Transmission}

Leucocytozoon has a life cycle similar to that of Plasmodium, but these parasites, in contrast to Plasmodium, undergo only exoerythrocytic schizogony. This occurs in the endothelial and parenchymatous cells of the lungs, lymphoid tissue, liver, heart, kidney, brain, spleen and other organs. Some merozoites form megaloschizonts in these tissues beginning 4 to 6 days after infection; these are very large and are useful in making a diagnosis. Gametocytes appear in the circulating leukocytes or erythrocytes 6 to 8 days post infection, causing the host blood cells to become grossly distorted and spindle shaped (Keymer 1969, Soulsby 1968). The insect vectors for this parasite are black flies (family Simuliidae) of the genera Simulium and rarely Prosimulium (Cook 1971, Soulsby 1968).

\section{Signs, Symptoms and Pathogenes is}

Although most birds found to be infected are not reported as having clinical signs (Bennett et al. 1974, 1975, Loven et al. 1980, Stabler et al. 1967, 1977, Williams et al. 1977), Leucocytozoon simondi appears to be the most lethal protozoan blood 
parasite of wild waterfowl (Bennett et al. 1974). Infection in avian adults appears to be chronic (Williams et al. 1977) and mortality probably occurs only rarely, if at all (Kocan and Knisley 1970, Ward 1978). The disease has its most severe effects on young birds of perhaps all affected wild and domestic species, and mortality can be quite high (Cook 1971, Desser et al. 1978, Herman et al. 1975, Karstad 1965, Keymer 1969, Soulsby 1968). Intensity of clinical signs varies from mild to severe to death. Diseased birds may show varying degrees of weakness, neurological signs, anemia, poor growth, anorexia (poor appetite) and difficulty in breathing, often with rapid onset (Cook 1971, Desser et al. 1978, Herman et al. 1975, Karstad 1965, Soulsby 1968). Those which succumb do so either as a direct result of the disease or to some other cause to which their weakened condition leaves them susceptible. Leucocytozoonosis can be the major cause of losses of young of the year in waterfowl populations and take up to $90 \%$ of the young during epizootics. Those which survive retain the infection and become carriers (Desser et al. 1978, Herman et al. 1975).

Necropsy may show no gross abnormalities or may reveal an enlarged spleen (Desser et al. 1978).

\section{Diagnosis}

Diagnosis can be made by clinical signs plus identification of the gametocytes in blood smears. The absence of pigment granules is an important point in distinguishing Leucocytozoon from other avian protozoan blood parasites. The gametocytes are not always present, so it is wise to take specimens for histopathologic examination from lung, heart, brain, lymphoid tissues (including thymus, lymph nodes and intestinal sections), liver, spleen and muscle. These are examined for presence of schizonts or megaloschizonts.

\section{$\underline{\text { Significance and } \text { Control }}$}

As with Plasmodium, one must be aware of the potential epizootics which might occur from Leucocytozoonosis in waterfowl breeding grounds. However, the results are apt to be more devastating in a Leucocytozoon outbreak than with Plasmodium as many or most of the young of the year may be lost. It is thought that this protozoan may be an important population-limiting factor in waterfowl in North America (Bennett et al. 1974), but the significance for Alaska is unknown. The parasite may be important to other Alaskan avian populations, particularly the various species of ptarmigan. Unfortunately, it is extremely difficult to determine just how important it is in these species because there are so few data.

Control of the disease in wild avian populations is not practical.

\section{Acknowledgments}

The author greatfully acknowledges Mr. Jerry McGowan of the Alaska Department of Fish and Game for access to his personal collection of literature on Alaskan avian species.

\section{References}

BENNETT, G. F. and M. LAIRD. 1973. Collaborative investigations into avian malarias: an international research programme. J. Wildl. Dis. 9:26-28. 
BENNETT, G. F., W. BLANDIN, H. W. HEUSMANN and A. G. CAMPBELL. 1974. Hematozoa of the Anatidae of the Atlantic flyway. I. Massachusetts. J. Wildl. Dis. $10: 442-451$.

BENNETT, G. F., A. D. SMITH, W. WHITMAN and M. CAMERON. 1975. Hematozoa of the Anatidae of the Atlantic flyway. II. The maritime provinces of Canada. J. Wildl. Dis. $11: 280-289$.

COOK, R. S. 1971. Leucocytozoon Danilewsky 1890. pp. 291-299. In: Infectious and Parasitic Diseases of Wild Birds. J.W. DAVIS, R. C. ANDERSON, L. KARSTAD and D. 0. TRAINER, eds. The lowa State Univ. Press, Ames, IA.

DESSER, S. S., J. STUHT and A. M. FALLIS. 1978. Leucocytozoonos is in Canada geese in upper Michigan. 1. Strain differences among geese from different localities. J. Wildl. Dis. 14:124-131.

HERMAN, C. M., J. H. BARROW, JR. and I. B. TARSHIS. 1975. Leucocytozoonosis in Canada geese at the Seney National Wildlife Refuge. J. Wildl. Dis. 11:404-411.

KARSTAD, L. 1965. A case of leucocytozoonosis in a wild mallard. Bull. Wildl. Dis. Assoc. $1: 33-34$.

KEYMER, I. F. 1969. Parasitic diseases. pp. 393-452. In: Diseases of Cage and Aviary Birds. M. L. PETRAK, ed. Lea and Febiger, Philadelphia, PA.
KOCAN, R. M. and J. O. KNISLEY. 1970. Incidence of malaria in a wintering population of canvasbacks (Aythya valisineria) on Chesapeake Bay. J. Wildl. Dis. $6: 441-442$.

LOVEN, J. S., E. G. BOLEN and B. W. CAIN. 1980. Blood parasitemia in a south Texas wintering waterfowl population. J. Wildl. Dis. $16: 25-28$.

SOULSBY, E. J. L., ed. 1968. Helminths, Arthropods and Protozoa of Domesticated Animals (Monnig). 6th ed. Williams and Wilkins Co., Baltimore, MD. $824 \mathrm{pp}$.

STABLER, R. M., N. J. KITZMILLER and R. B. WEEDEN. 1967. Blood parasites in rock ptarmigan from Eagle Summit, Alaska. J. Parasitol. 53:1297.

STABLER, R. M., C. E. BRAUN and T. D. I. BECK. 1977. Hematozoa in sage grouse from Colorado. J. Wildl. Dis. 13:414-417.

WARD, F. P. 1978. Parasites and their treatment in birds of prey. pp. 276-281. In: Zoo and Wild Animal Medicine. M. E. FoWLER, ed. W. B. Saunders Co., Philadelphia, PA.

WILLIAMS, N. A., B. K. CALVERLEY and J. L. MAHRT. 1977. Blood parasites of mallard and pintail ducks from central Alberta and the Mackenzie Delta, Northwest Territories. J. Wildl. Dis. $13: 226-229$. 


\title{
Haemoproteus
}

\author{
L. Michael Philo
}

Haemoproteus is a protozoan blood parasite of the order Haemosporidia, family Haemoproteidae. It is worldwide in many avian and some reptilian species (Soulsby 1968).

This protozoan has been found in most surveys for avian hematozoa in North America, occurring in up to $50 \%$ of the birds examined. In some cases it was the most common blood parasite found (Bennett and Laird 1973, Bennett et al. 1974, 1975, 1980, Clark and Swinehart 1966, Kocan and Knisley 1970, Loven et al. 1980, Marx 1966, Stabler et al. 1977). Results of 1 of the surveys showed that the parasite occurred in up to $10 \%$ of the mallard (Anas platyrhynchos) and pintail (A. acuta) ducks collected in the Northwest Territories of Canada (Williams et al. 1977).

The life cycle is similar to those of Plasmodium and Leucocytozoon. Gametogony and sporogony occur in blood-sucking louse flies (order Diptera, family Hippoboscidae) and midges (family Ceratopogonidae, genus Culicoides). In contrast to Plasmodium, only developing and mature gametocytes occur in host erythrocytes, while schizogony occurs only in endothelial cells of blood vessels in the lungs, liver, spleen, kidneys and other internal organs. Histological sections of these tissues along with the blood smears are diagnostic (Cook 1971, Soulsby 1968).

This species is considered nonpathogenic or mildly pathogenic for most or all wild avian species (Cook 1971, Keymer 1969, Soulsby 1968), though there are rare reports of clinical signs and mortality, especially in young birds (Cook 1971,
Julian and Galt 1980, Keymer 1969, Soulsby 1968). Control measures for wildfowl infections are not practical, but this is of little importance because of the low pathogenicity.

The main significance of the infection is in distinguishing it from the other protozoan blood parasites. If gametes were to appear in a Plasmodium infection before erythrocytic schizogony occurred this could be mistaken for Haemoproteus infection (Cook 1971). In such a case, distinguishing between the 2 can be difficult, but the typical halter shape of the Haemoproteus gametocytes along with histopathologic findings may be sufficient to make the proper determination.

\section{References}

BENNETT, G. F. and M. LAIRD. 1973. Collaborative investigations into avian malarias: an international research programme. J. Wildl. Dis. 9:26-28.

BENNETT, G. F., W. BLANDIN, H. W. HEUSMANN and A. G. CAMPBELL. 1974. Hematozoa of the Anatidae of the Atlantic flyway. I. Massachusetts. J. Wildl. Dis. $10: 442-451$.

BENNETT, G. F., A. D. SMITH, W. WHITMAN and M. CAMERON. 1975. Hematozoa of the Anatidae of the Atlantic flyway. Il. The maritime provinces of Canada. J. Wildl. Dis. $11: 280-289$.

BENNETT, G. F., H. WITT and E. M. WHITE. 1980. Blood parasites of some Jamaican birds. J. Wildl. Dis. $16: 29-38$. 
CLARK, G. W. and B. SWINEHART. 1966. Blood protozoa of passerine birds of the Sacramento (Calif.) region. Bull. Wildl. Dis. Assoc. 2:53-54.

COOK, R. S. 1971. Haemoproteus Kruse 1890. pp. 300-308. In: Infectious and Parasitic Diseases of Wild Birds. J. W. DAVIS, R. C. $\overline{A N D} \overline{E R S O N}$, L. KARSTAD and D. 0. TRAINER, eds. The lowa State Univ. Press, Ames, IA.

JULIAN, R. J. and D. E. GALT. 1980. Mortality in muscovy ducks (Cairina moschata) caused by Haemoproteus infections. J. Wildl. Dis. 16:39-44.

KEYMER, I. F. 1969. Parasitic diseases. pp. 393-452. In: Diseases of Cage and Aviary Birds. M. L. PETRAK, ed. Lea and Febiger, Philadelphia, PA.

KOCAN, R. M. and J. O. KNISLEY. 1970. Incidence of malaria in a wintering population of canvasbacks (Aythya valisineria) on Chesapeake Bay. J. Wildl. Dis. $6: 441-442$.
LOVEN, J. S., E. G. BOLEN and B. W. CAIN. 1980. Blood parasitemia in a south Texas wintering waterfowl population. J. Wildl. Dis. $16: 25-28$.

MARX, D. J. 1966. Some blood parasites from Minnesota and Wisconsin birds. Bull. Wildl. Dis. Assoc. 2:6-8.

SOULSBY, E. J. L., ed. 1968. Helminths, Arthropods and Protozoa of Domesticated Animals. 6 th ed. Williams and Wilkins Co., Baltimore, MD. 824 pp.

STABLER, R. M., C. E. BRAUN and T. D. I. BECK. 1977. Hematozoa in sage grouse from Colorado. J. Wildl. Dis. 13:414-417.

WILLIAMS, N. A., B. K. CALVERLEY and J. L. MAHRT. $1977 . \quad$ Blood parasites of mallard and pintail ducks from central Alberta and the Mackenzie Delta, Northwest Territories. J. Wildl. Dis. $13: 226-229$.

\title{
Trypanosoma
}

\author{
L. Michael Philo
}

Several species of trypanosomes have been found in birds, but they are considered nonpathogenic (Keymer 1969, Soulsby 1968). They are worldwide in distribution (Bennett and Laird 1973) and have been observed in a variety of wild fowl in Canada (Bennett 1961, Williams et al. 1977) and in rock ptarmigan (Lagopus mutus) and willow ptarmigan (L. lagopus) at Eagle Summit, Alaska (Stabler et al. 1967).
The vectors may be louse flies (family Hippoboscidae), black flies (family Simuliidae), or mosquitoes (Aedes aegypti) (Bennett 1961, Keymer 1969). Trypanosoma is probably of little significance in Alaska other than its occurrence as a nonpathogenic parasite. 


\section{Acknowledgments}

Mr. Jerry McGowan of the Alaska Department of $\mathrm{Fish}$ and Game kindly allowed the use of his personal collection of avian literature; his cooperation is greatly appreciated.

\section{References}

BENNETT, G. F. 1961. On the specificity and transmission of some avian trypanosomes. Can. J. Zool. 39:17-33.

BENNETT, G. F. and M. LAIRD. 1973. Collaborative investigations into avian malarias: an international research programme. J. Wildl. Dis. 9:26-28.
KEYMER, I. F. 1969. Parasitic diseases. pp. 393-452. In: Diseases of Cage and Aviary Birds. M. L. PETRAK, ed. Lea and Febiger, Philadelphia, PA.

SOULSBY, E. J. L., ed. 1968. Helminths, Arthropods and Protozoa of Domesticated Animals (Monnig). 6 th ed. Williams and Wilkins Co., Baltimore, MD. $824 \mathrm{pp}$.

STABLER, R. M., N. J. KITZMILLER and R. B. WEEDEN. 1967. Blood parasites in rock ptarmigan from Eagle Summit, Alaska. J. Parasitol. 53:1297.

WILLIAMS, N. A., B. K. CALVERLEY and J. L. MAHRT. $1977 . \quad$ Blood parasites of mallard and pintail ducks from central Alberta and the Mackenzie Delta, Northwest Territories. J. Wildl. Dis. $13: 226-229$. 


\section{Chapter 4.}

\section{MISCELLANEOUS CONDITIONS}

\section{Lead Poisoning}

Jim Dau

Introduction and History

Lead poisoning has been recognized as a significant mortality source in waterfowl since the late $1800 \mathrm{~s}$. It is a non-infectious condition caused by ingestion of spent shotshell pellets introduced into the environment by hunters. Research has embraced essentially 3 aspects of the disease: (1) determination of waterfowl losses attributable directly or indirectly to lead poisoning, (2) evaluation of its physiologic effects and (3) investigation for lead shot substitutes.
Potentially all invertebrate and vertebrate species worldwide may be susceptible to the disease; however, feeding habits and food preferences of certain waterfowl species, the methods employed to hunt them and public awareness concerning game species have focused attention on the effects of lead poisoning in this avian group. Species which tend to probe shallow water substrates for tubers and seeds, e.g. mallards (Anas platyrhyncos), pintails (A. acuta) and Canada geese (Branta cañadensis), appear most likely to ingest lead 
shot (Bellrose 1959, Cook and Trainer 1966, March et al. 1976). Waterfowl species that prefer small seeds and animal matter are not as likely to ingest lead shot as are species which exploit coarser foodstuffs, e.g. corn. Large species, e.g. whitefronted geese (Anser albifrons), tend to ingest more pellets than small species, e.g. mallards.

\section{Signs, Symptoms and Pathogenes is}

Ingestion of shot appears to be strictly accidental; lead shot is not preferentially ingested as grit (Bellrose 1959). The number of pellets ingested is related to the availability of shot in feeding areas and by the size of the bird species. Areas most conducive to lead poisoning are those subject to heavy hunting pressure, especially where annual blinds are situated near preferred feeding locales, and having solid rather than mucky substrates in calm, shallow, ice free water. Detritus accumulation and settling of the high specific gravity pellets into aquatic substrates makes the annual carry over of shot negligible (Bellrose 1959).

Lead poisoning usually peaks soon after the closure of waterfowl hunting seasons (late fall to early winter) when accumulation of spent shot has reached its maximum. Waterfowl previously denied access to these areas by gunning pressure resume feeding there and ingest the shot with food.

Ingested shot is retained by birds if not passed in the feces within 3 days. Approximately $65 \%$ of the lead volume is absorbed within this initial period regardless of dose. Assimilation of the remaining $35 \%$ requires roughly 45 days (Cook and Trainer 1966). Lead shot in the gastrointestinal tract does not necessarily prove fatal. Birds may recover from low doses or after passing the shot, without lasting effects or reduced fertility (Cook and Trainer 1966). The effects of assimilated lead are cumulative, however.

Tolerance to ingested lead varies among species. For example, laughing gulls (Larus atricilla) are reported more resistant than waterfowl. Prefledging laughing gulls had lead concentrations equal to concentrations found in adults, indicating an equilibrium of dissolved lead in soft tissues before fledging (Munoz et al. 1976).

Lead appears to affect birds in mainly 2 ways: (1) via food deprivation and (2) through mitochondrial damage (March et al. 1976). Lead interacts physiologically with calcium and may replace it in certain blood proteins. Since high calcium levels depress food intake, it is suspected that lead can do the same. Weight loss and starvation result. Impairment of the mitochondria depresses the capability for aerobic lipid metabolism which is necessary for sustained activity. This causes the affected birds to become inactive and eventually moribund.

Ingested lead is metabolically degraded by gastric juices into lead salts (molecular compounds produced by biochemical reactions whereby lead displaces the hydrogen of an acid). Lead salts are absorbed from the small intestine into the circulatory system where they associate with erythrocytes. These lead constituents have an affinity for amino and sulfhydryl ligands. Lead chemically bound with these groups may cause inhibition of enzymatic activity and active transport of potassium in the erythrocytes resulting in anemia. Which lead-macromolecule (i.e. proteins) complexes form determines the fate and toxicity of assimilated lead. 
Lead accumulation occurs primarily in bone $(60 \%)$, liver $(25 \%)$ and kidney (4\%) tissue. Lead not assimilated is passed in bile excretions, urine and feces. Lead may be absorbed intramuscularly; thus, the effects of lead shot wounds in waterfowl are not limited solely to traumatic (mechanical) tissue destruction, but include the biochemical effects of lead toxicity (Oehme 1978). Dogs (Canis familiaris) have been observed through radiography to carry large numbers ( 50 or more) of shotshell pellets intramuscularly without apparent effect, however (R. Dieterich, pers. comm.). Absorption and mobilization of lead stores are influenced by diet, vitamin D levels, hormonal activity, acidosis (relative reduction of alkali reserves in the blood and other body fluids) and chelation (Oehme 1978). More detailed discussions of lead poisoning toxicology are provided by Hammond (1978) and Osweiler and Van Gelder (1978).

Gross symptoms of lead poisoning include anorexia with emaciation (Bellrose 1959, Cook and Trainer 1966, March et al. 1976), esophageal impaction distended gall bladder and bile stained liver (Cook and Trainer 1966, Munoz et al. 1976), lethargy (Bellrose 1959, Cook and Trainer 1966, March et al. 1976), swollen head and greenish diarrhea (Cook and Trainer 1966). Histologic tissue changes include lung hyperemia and hemorrhage, patchy necrosis of kidneys, heart and gizzard and insolubility of interstitial heart fat. Acute manifestations of the disease from large doses may preclude macroor microscopic tissue changes and kill birds prior to emaciation.

\section{Diagnosis}

\footnotetext{
Diagnosis is based upon clinical signs, histopathologic tissue changes, increased lead levels in
}

plasma and tissues and presence of shot as detected through radiographic techniques and direct examination. Preferred samples are intact gizzards and liver and brain tissue. Soft tissues show only recent exposure to lead; life-time lead accumulation is revealed by bone and feathers (Munoz et al. 1976).

\section{Significance and Control}

Surprisingly few pellets will significantly reduce a bird's likelihood of survival beyond 2 months post ingestion. Two number 6 pellets decrease a drake mallard's probability of survival by $25 \% ; 6$ number 6 pellets by 50\%. In 1959 when hunting pressure was much lower than today, it was reported that approximately $25 \%$ of wild mallards in North America ingested 1 or more pellets annually (Bellrose 1959). If this seems unfeasible, consider hunters average 1 duck for every 5 shots fired (Bellrose 1959). This translates into 1400 pellets introduced into waterfowl habitat per duck taken if hunters use a 12 gauge shotgun with number 6 shot. A study that involved 45 whistling swans (0lor columbianus) reported an average pellet count of 50 per bird with a maximum of 201 pellets recovered (Cook and Trainer 1966).

Consequences of lead poisoning, besides direct mortality, are increased susceptibility to hunting, predation and disease and decreased ability to migrate in terms of rate and distance (Bellrose 1959). The significance of this condition is a function of the inherent tolerance and feeding habits/food preferences of the species involved (Bellrose 1959, Munoz et al. 1976), shot availability and stress (Bellrose 1959). Sportsmen may underestimate the significance of this condition by assuming all dead and dying birds observed are merely unrecovered 
cripples. Also, since mortalities peak after waterfowl hunting seasons close, most intoxications probably go unnoticed. The ramifications of reduced weight gain and lowered ability to migrate are not fully known.

Alaska is no more exempt from this condition than any state where duck hunting occurs. Though the legal duration of waterfowl hunting seasons in northern Alaska are generous, the actual period of hunting before autumn waterfowl out-migrations is rather short. Thus, long term accumulation of shot is reduced in this area.

It has been suggested that birds may be more susceptible to lead intoxication during breeding seasons (Cook and Trainer 1966). This may be significant considering this state's unspoken condonation of spring subsistence hunting for waterfowl and proposed legislation that would legalize this practice. As Alaska becomes more populated and big game hunting becomes more restrictive, duck hunting may intensify and increase the potential for lead poisoning.

Waterfowl management decisions outlawing lead shot in certain flyways have forced technological advances in the development of steel substitutes for lead shot to minimize the incidence of lead poisoning.
References

BELLROSE, F. C. 1959. Lead poisoning as a mortality factor in waterfowl populations. 111. Nat. Hist. Surv. Bull. 27:235-288.

COOK, R. S. and D. O. TRAINER. 1966. Experimental lead poisoning of Canada geese. J.Wildl. Manage. $30: 1-8$.

HAMMOND, P. B. 1978. Metabol ism and metabolic action of lead and other heavy metals. pp. 87-99. In: Toxicity of Heavy Metals in the Environment. Part 1. F. W. OEHME, ed. Marcel Dekkär, Inc., New York, NY.

MARCH, G. L., T. M. HOHN, B. A. MCKEOWN, L. SILOE and J. C. GEORGE. 1976. The effects of lead poisoning on various plasma constituants in the Canada goose. J. Wildl. Dis. 12:14-19.

MUNOZ, R. V., JR., C. S. HACKER and T. F. GESELL. 1976. Environmentally acquired lead in the laughing gull, Larus atricilla. J. Wildl. Dis. 12:139-142.

OEHME, F. W. 1978. Mechanisms of heavy metal inorganic toxicities. pp. 69-85. In: Toxicity of Heavy Metals in the Environment. Part I. F. W. OEHME, ed. Marcel Dekkar, Inc., New York, NY.

OSWEILER, G. D. and G. A. VAN GELDER. 1978. Epidemiology of lead poisoning in animals. pp. 143-171. In: Toxicity of Heavy Metals in the Environment. Part 1. F. W. OE HME, ed. Marcel $\overline{\text { Dek- }}$ kar, Inc., New York, NY. 


\section{Splendidofilaria}

Jim Dau

Two species of this parasitic roundworm occur in Alaska: $\underline{S}$. pectoralis and $S$. papillocerca. Avian hosts of both species are apparently restricted to the family Tetraonidae. Two parasitological surveys of nongallinaceous birds from British Columbia and portions of Alaska failed to discover Splendidofilaria spp. The reported geographic distribution of these parasites encompasses the area from southcentral British Columbia to the Cariboo region of Alaska. They are frequently found in the LivengoodFairbanks-Delta region of this state and may appear in large proportions of grouse in some populations ( $J$. Dau, pers. obs.)

Splendidofilaria pectoralis has been found in blue grouse (Dendragapus obscuris), spruce grouse (Canachites canadensis), ruffed grouse (Bonasa umbellus) and sharptailed grouse (Pedioecetes phasianellus) from British Columbia and Alaska. Splendidofilaria papillocerca has been documented in whitetailed ptarmigan (Lagopus lagopus) from Russia (Sonin $1 \overline{966 \text { as }}$ reported by Gibson 1968). Filarioid nematodes, probably $\underline{S}$. pectoralis, in tetraonids of Alaska were first reported by Babero (1953).

Both $\underline{s}$. pectoralis and $\underline{S}$. papillocerca are white and inhabit subcutaneous tissue in the pectoral region. Mature male $S$. pectoralis roundworms average $12 . \overline{8} \mathrm{~mm}$ long; females are considerably larger, averaging $40.4 \mathrm{~mm}$ in length. The posterior portion of the males and females of both species is curved in the shape of a buttonhook. Splendidofilaria papillocerca was described from a very limited sample of worms ( 1 male and 2 females); the male was $10.6 \mathrm{~mm}$ long and the females were 35.7 and $31+\mathrm{mm}$ long. Gibson (1968) provides a list of characteristics for differentiating the 2 species.

The life cycle of Splendidofilaria spp. is incompletely understood. The first larval stage of this parasite is termed a microfilaria. Microfilariae inhabit the circulatory system of the definitive host. Blood-sucking arthropods are thought to ingest the microfilariae when they feed on an infected bird. Within the arthropods, the microfilariae develop to the stage that is infective for the final hosts. These larvae escape from the intermediate hosts when the arthropods land to ingest another blood meal. The stimulus that prompts the microfilariae to escape from the arthropods and the means by which microfilariae determine the suitability of prospective hosts, if such a means exists, are unknown.

Gibson (1967) reported finding fourth stage larvae of $\underline{s}$. pectoralis in tetraonids as early as July 24. By August all worms collected were considered mature. Microfilariae were present by early September. The rapid host response of tetraonids quickly encapsulates and destroys these parasites. This suggests that the realized lifespan of Splendidofilaria spp. in their definitive hosts may be short. The higher incidence of infection in grouse chicks (16 of $32 ; 50.0 \%$ ) than in adults ( 5 of $19 ; 26.3 \%$ ) suggests that intermediate hosts may tend to feed on young birds or that recovery leads to immunity. 
While skinning grouse and ptarmigan, sportsmen frequently find the adult roundworms coiled in masses of connective tissue. These parasites can easily be removed from the carcass, do not impair the taste or appearance of the meat and do not represent a threat to human health. The significance of Splendidofilaria spp. in Alaskan tetraonids has not been established.

\section{$\underline{\text { References }}$}

BABERO, B. B. 1953. Studies on the helminth fauna of Alaska. XVI. A survey of the helminth parasites of ptarmigan (Lagopus spp.). J. Parasitol. 39:538-545.
GIBSON, G. G. 1967. Splendidofilariae pectoralis n. sp. (Filarioidea:Onchocercidae) from tetraonid birds in northwestern North America. Can. J. Zool. 45:1135-1147.

1968. Splendidofilaria papillocerca (Lubimov, 1946) and Microfilaria lagopodis (Haaland, 1928) (Filarioidea) from ptarmigan in northwestern North America. Can. J. Zool. 46:21-27.

SONIN, M. D. 1966. Filariata of animals and man and the diseases they cause. Part I. Aproctoidea. (In Russian). Osnovy Nematodologi i, Vol. 17. 
25. Necrotic foci on the liver of a gyrfalcon (Falco rusticolus) with inclusion body disease of raptors (IBDR) caused by a herpesvirus (L.G. Swartz).

26. Necrotic foci on the spleen of a gyrfalcon (F. rusticolus) with IBDR (L.G. Swartz).

27. Avian pox in a green-winged teal (Anas crecca carolinensis) ( $R$. Dieterich).

28. Plaques characteristic of aspergillosis on the air sacs of a gull (Larus sp.) (R. Dieterich). 

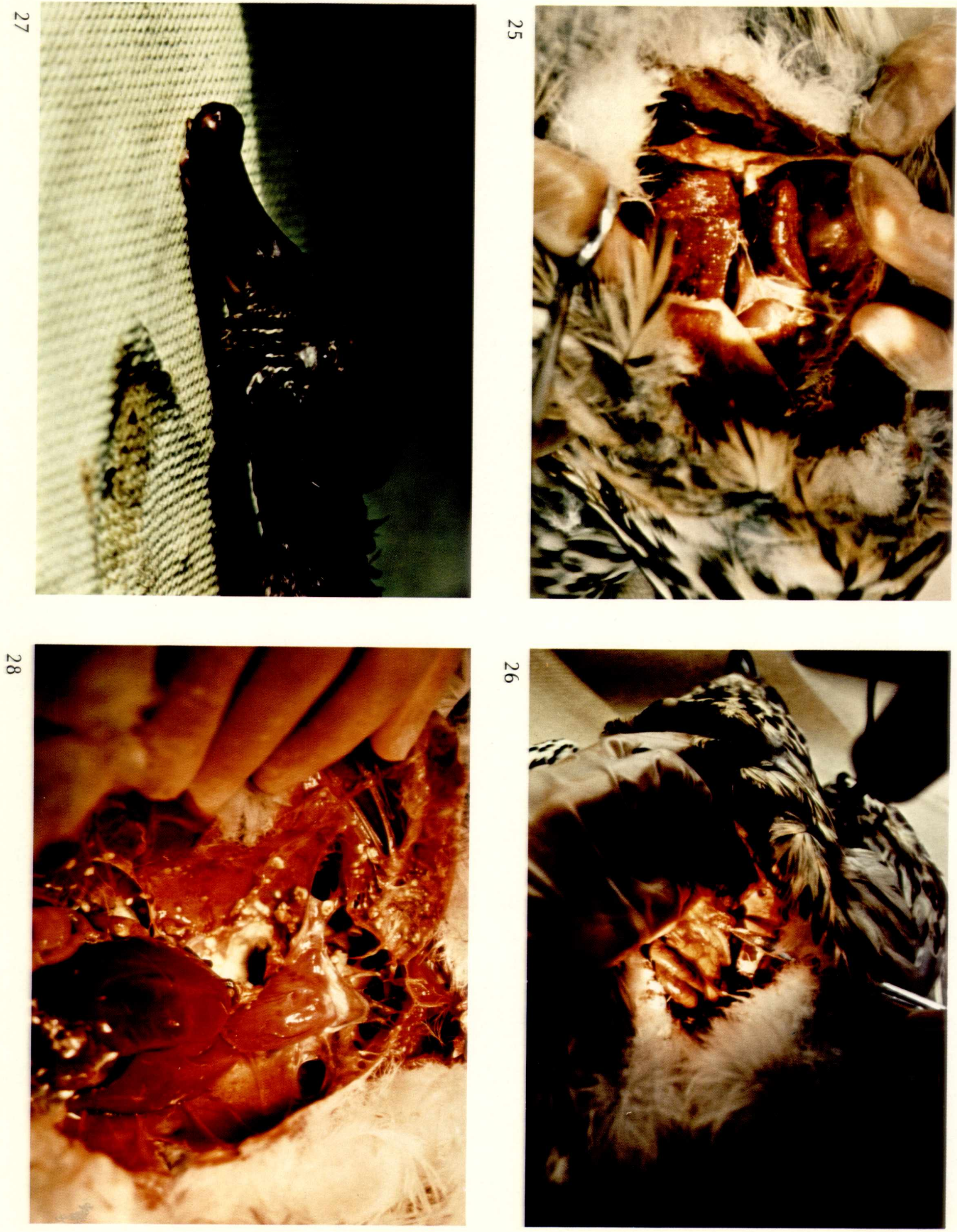
Part III

FISH 


\title{
Introduction
}

\author{
Roger S. Grischkowsky
}

Disease in fish populations is not limited to hatchery operations. Natural incidences of specific diseases vary from absent to present in minor percentages to omnipresent. The significance of disease in wild fish populations is little known. Known natural disease involvements have rarely been associated with major mortalities. Sick fish behave abnormally and are quickly preyed upon. In hatcheries the presence of an infectious agent under the right set of environmental conditions, ones which favor the pathogen and not the host, frequently results in mortalities of epizootic proportions.

Stress events can mediate the susceptibility of a host to an agent. Unsatisfactory water quality parameters are stressors as are other external factors such as the confinement, handling and density of fish, predation, competition and numerous others. Most diseases of fish are stress mediated. Often stress causes the difference in expression between the asymptomatic carrier state and the diseased state.

Mortality investigations and disease or carrier surveys are ways in which diseases can be studied at the population level. Three aspects which should be immediately considered during mortality investigations are: 1) environmental assessment, 2) nutritional consideration and 3) disease. Even when a disease is detected, a cause and effect relationship is often difficult to establish. Dead fish must be fresh to be of any value in determining whether a disease was involved in their demise. Live fish are best for having signs of the disease and reflecting abnormal behavior patterns.

Surveys which enumerate diseases and pathogen incidences can effectively indicate the potential for disease related mortality, and point out populations which may be more susceptible to the affects of stressors. Populations showing substantial incidences are probably ones from which individual fish should best be kept out of hatchery operations, or ones in which preventative measures are appropriate for disease control.

The chapters that follow are discussions of diseases either found in wild or hatchery related populations of $f i s h$, and have the potential to occur in Alaskan populations.

Note: The use of a trade name doesn't indicate endorsement by the authors or Alaska Department of Fish and Game. 
Chapter I.

VIRAL DISEASES 


\title{
Infectious Hematopoietic Necrosis Virus
}

\author{
Roger S. Grischkowsky
}

Introduction

Infectious hematopoietic necrosis (IHN) virus is an RNA rhabdovirus which causes disease in hatchery reared, incubated or susceptible juvenile fish or eggs. It is restricted to North America (Amend et al. 1973) and Japan (Sano 1976, Sano et al. 1977). Susceptible species include sockeye salmon (Oncorhynchus nerka), chinook salmon (Oncorhynchus tshawytscha), chum salmon (Oncorhynchus keta) (Sano 1976, Sano et al. 1977) and rainbow and steelhead trout (Salmo gairdneri). The virion is present in gonadal fluid of spawning adult fish. Vertical transmission between adult and eggs establishes infection in progeny. Waterborne transmission is effective for experimental challenge as water flows past incubating eggs in redds or incubators and as particles are shed from overt infected alevins, fingerlings or smolts to other juvenile susceptible species in the immediate vicinity. Mortality occurs during the freshwater incubation or rearing period for salmon and steelhead or the first 2 years for rainbow trout. Epizootics are not uncommon in artificially propagated stocks and have been documented in nature (Wil$1 \mathrm{i}$ ams and Amend 1976). Mortalities in Alaska due to IHN virus have taken place only in sockeye hatcheries, although it has been detected in a few chinook fish lots.

The anterior kidney (called the head kidney) is the hematopoietic (erythrocyte producing) organ in $\mathrm{fish}$. This is the target organ of IHN virus disease. Death is from a severe electrolyte and fluid imbalance due to renal failure (Amend and Smith 1975).

\section{History}

Infectious hematopoietic necrosis virus has caused loss of sockeye salmon artificially propagated in Alaska. Substantial mortalities of juveniles have occurred at Kitoi Bay Hatchery on Afognak Island near Kodiak (1973-1975), Sitka estuarine rearing pens and Auke Creek Hatchery in Southeast Alaska (1975) and at Big Lake Hatchery (1979). Mortalities during these epizootics have ranged from $10-99 \%$ with the higher portion of the range most frequently encountered.

\section{Alaska}

The virus is distributed throughout all sockeye salmon populations in Alaska (Grischkowsky and Amend 1976). It is detected in asymptomatic carrier adult gonadal fluids of fish at or near spawning and moribund juveniles dying from the disease. In this state the virus has been detected in only a few chinook salmon populations and not in trout. In Alaska it continues to prevent sustained artificial propagation of the sockeye salmon.

\section{Washington}

The virus history on the west coast other than Alaska commenced with the investigation into a contagious disease outbreak of sockeye salmon thought to be of virus origin at the U.S. Fish and Wildlife Service station, Leavenworth, Washington in 1951 (Rucker et al. 1953). Fish involved were sockeye and kokanee, a landlocked variety of the same species. A filterable agent was im- 
plicated in this outbreak by its production of the infection in healthy salmon with injections of bacteria-proof, filter-processed suspension from moribund fish showing characteristic signs. Previous mortalities as early as the 1948 sockeye salmon brood were probably caused by the same infectious agent (Rucker et al. 1953). The source of the infectious agent was indicated as raw sockeye viscera from Alaska, British Columbia and Washington used without pasteurization for fish feed in that hatchery (Rucker et al. 1953). It was from this early work that the basis for transmission, fish signs, necropsy gross abnormalities, deformation of survivors, kidney necrosis and isolation and identification of the agent later to be termed IHN virus was established.

\section{British Columbia and Washington}

A British Columbia epizootic from a virus later called IHN virus occurred at 2 different hatcheries near Chilliwack. The mortalities involved sockeye salmon and rainbow trout in 1967 (Amend et al. 1969). Amend and Wood (1972) conducted an IHN virus survey from the Fraser River of British Columbia to the Columbia River between Washington and Oregon. Its presence was detected in 2 Washington sockeye salmon stocks but not in chinook, coho (Oncorhynchus kisutch) or chum salmon tested. Outbreaks of the disease in rainbow trout occurred in a private hatchery by 1973 in Washington (Amend 1975).

\section{Oregon}

Oregon records of IHN virus epizootics commenced in 1958 at the Willamette River Salmon Hatchery in sockeye salmon juveniles. Its characteristics and the pattern of the mortality closely resembled that reported in 1953 in Washington (Wingfield et al. 1969). The virus from that disease outbreak has been used as the type source for what was then termed the Oregon Sockeye Virus (OSV). The virus is present in some Pacific salmon and rainbow trout in that state (Amend et al. 1969).

\section{California}

California's IHN virus problems have centered around juvenile chinook salmon reared at the Coleman National Fish Hatchery and Nimbus Hatchery near Northern Californian tributaries of the Sacramento River (Wingfield and Chan 1970). The name initially given that agent was the Sacramento River Chinook Disease (SRCD).

\section{Other States}

Rainbow trout IHN virus epizootics have also occurred in Minnesota (Plumb 1972), Idaho, Montana, South Dakota (Amend et al. 1973), Colorado and West Virginia (Wolf et al. 1973). These rainbow trout outbreaks appeared to have originated in egg shipments from 1 hatchery (Amend 1975).

\section{Transmission}

Infectious hematopoietic necrosis virus is transmitted vertically from asymptomatic carrier spawning adults to progeny via gonadal fluids which surround the egg at deposition. It is not known whether IHN virus gains entrance to the egg interior either during oogenesis or prior to micropore closure after extrusion. Mass mortalities in Alaska due to the virus, at Kitoi Bay and Big Lake hatcheries, indicate embryonic infection prior to hatching or immediately 
after the chorion is broken during the hatching process. Infection has occurred in disinfected eggs initially on pathogen-free well water. Surface water from Meadow Creek at Big Lake Hatchery was used to accelerate development at or near hatching. That water intake is adjacent to a sockeye salmon spawning ground of typically high virus incidence levels. The virus is generally not detectable in water supplies, but the author has isolated it in the effluent from moribund sockeye salmon in troughs. Other investigators (Wingfield and Chan 1970) have implicated fish to fish waterborne transmission. They placed chinook juveniles native to that area below a hatchery with chinook experiencing an epizootic of IHN virus. Within 7 to 10 days the test fish showed signs. Mortality reached $40 \%$. The purpose of this experiment was to determine how eggs from virusnegative adults could result in virus-diseased fish. Waterborne transmission can explain that cccurrence.

The latent period following exposure preceding the advent of signs and mortality was reported as 6 to 12 days for sockeye alevins (Amend 1975) and 7 to 10 days for chinook juveniles (Wingfield and Chan 1970) by waterborne challenge. Waterborne transmission was confirmed and was approximately as effective as dietary transmission using rainbow trout (Amend 1975).

Adult asymptomatic carriers are reservoirs of infection. Females have consistently higher virus incidences than males. Attempts to find other reservoirs of infection have failed. Fish examined in Alaska for IHN virus with negative findings include rainbow trout, coho salmon, chum salmon, pink salmon, arctic grayling (Thymallus arcticus), threespine stickleback (Gasterosteus aculeatus), dolly varden (Salvelinus malma), long-nose sucker (Catostomus catostomus) and an unidentified sculpin (Cottus sp.). These above tested fish were taken from IHN virus present systems.

Infected sockeye alevins, not succumbing immediately after hatching, migrate from the emergence site to the nursery lake. After a lacustrine residence of 1 to 3 years, the smolts migrate out to sea. After 1 to 3 years they return to the spawning ground, infect the progeny by the transovarian mode and complete the transmission cycle. One natural epizootic of sockeye from IHN virus has been studied in depth by Williams and Amend (1976). The egg to fry survival was the lowest ever recorded in the Chilko River of British Columbia from the 1972 brood. Fry to smolt survival for that year group approached normal level. This indicates the occurrence of IHN virus mortality primarily during the migration to the nursery lake. The progeny from the 1976 adult return of the 1972 brood resulted in only onethird of that expected from the egg deposition. The virus was again isolated (Williams and Amend 1976). Amend (1975) similarly indicated signs usually occur 21 to 35 days post hatching after a natural challenge.

Signs and Pathogenes is

\section{Behavior}

The disease first appears in juvenile salmonids as behavior abnormalities. At that point, initial differences are not diagnostic for IHN virus but generalized to possibly include other health problems. One of the first behavioral signs of this disease is anorexia (failure to feed, 
loss of appetite). In the case of alevins, feeding will not have commenced. The gastrointestinal tract upon necropsy is empty and juveniles appear pinheaded or emaciated with the head protruding laterally. Fish will swim in the tail down position primarily at the effluent end of troughs. Juveniles begin accumulating at the container screens and rolling over or floating at the surface. Mortality is typically rapid. Any stress such as dip netting, will cause immediate death as exhibited by this rolling over phenomenon.

Listlessness is common during the initial behavior abnormality stage. Rapid irrigation is present during this period while fish are swimming, rolled over ventral up at the surface or held against the outlet screen is present. Fish when prodded may swim frantically in the tail down mode. Carrier adults are asymptomatic.

\section{Gross External Pathology}

As mentioned previously, pinheadedness results from not having commenced feeding. Trailing fecal casts are present in many fish. Petechiae or small hemorrhagic spots, grouped in a strawberry pattern, appear early and mid-ventrally and at the base of fins and the anal vent. Deformation of the vertebral column, both laterally (scoliosis) and dorsoventrally (lordosis), is common in the later stages of the epizootic and in survivors (see Figs. 1 and 2 ).

\section{Gross Internal Pathology}

Internal characteristics pathognomonic for IHN virus include petechiae on pyloric caecae and musculature and pale kidney. Advanced cases show hemorrhagic areas internally which developed from the petechial sites. Hemorrhagic areas and petechia can be distinguished from blood released during dissection by attempting to wipe it off. Loose blood can be removed easily while that resulting from a diseased condition is protected from removal by a mucous layer. Hematocrits are so low as to make blood collection difficult or not possible. Hematocrits gathered would be reduced from the normal $35-40 \%$ cells to low twenties or high teens.

\section{Microscopic Pathology}

The target organ for this disease is the anterior kidney. This hematopoietic (blood cell producing) organ shows massive necrosis during the progression of IHN virus disease. The normal sockeye juvenile kidney is shown in color Figure 38. Major voids after necrosis can occur with edema and destruction of parenchyma surrounding tubules. Other pathognomonic microscopic characteristics are below included from various studies by Yasutake, Amend, Chambers and Mead (Amend et al. 1969, Amend and Chambers 1970, Yasutake 1970, 1975, Yasutake and Amend 1972). In the anterior kidney there is an increase in macrophage-like cells, a decrease in undifferentiated blast cells, chromatin margination of macrophage nuclei, pyknotic and necrotic lymphoid cells, extensive pyknosis and karyorrhexis and leucopenia (reduction in number of leucocytes) (Saunders 1974). Kidney pathology is detectable in 3 days post exposure. Less pronounced necrosis occurs in spleen, liver, pancreatic and granular cells. Splenic tissue shows the same cellular changes as kidney but less conspicuously. Minor hepatic parenchyma degeneration appears as localized necrotic foci and karyorrhexis. Pan- 


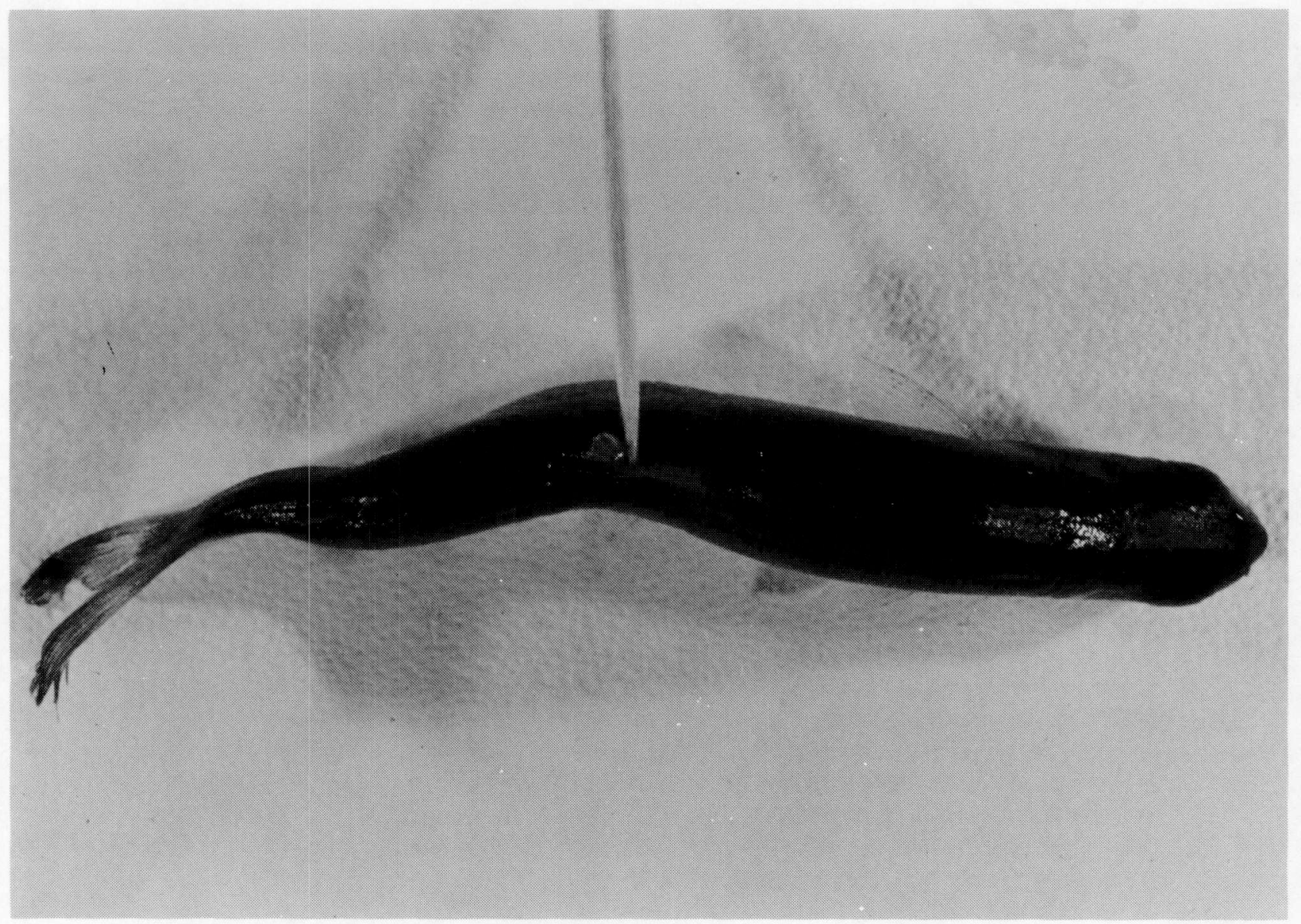

Figure 1. Lordosis in a sockeye smolt typical of that caused by IHN virus. The fish was caught at Frazer Lake where the virus is common in sockeye salmon adults (R. Grischkowsky). 


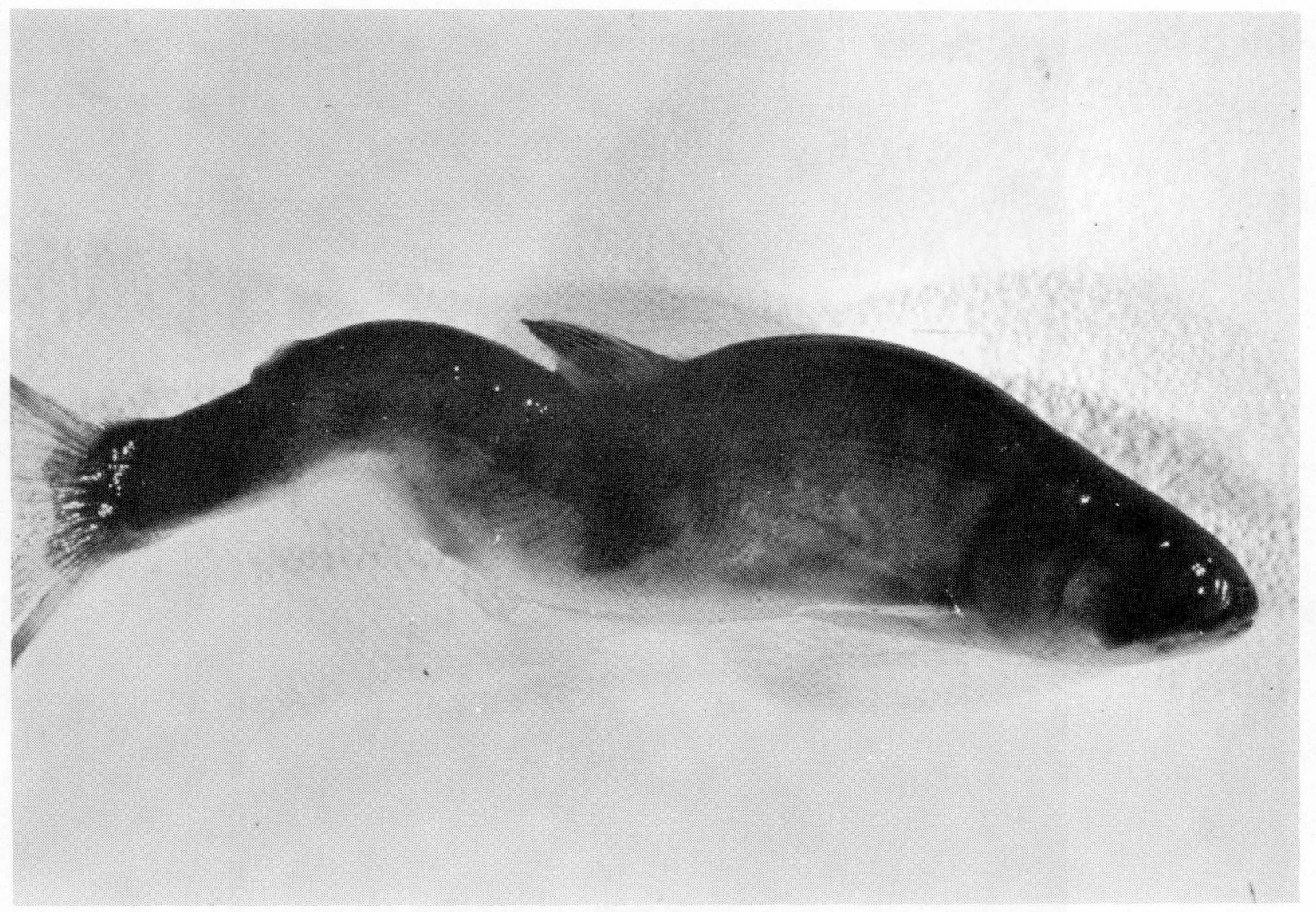

Figure 2. Scoliosis in a sockeye smolt thought to be caused by IHN virus from Frazer Lake, Alaska (R. Grischkowsky). 
creatic changes induced by IHN virus are dominated by large intracytoplasmic inclusions in islet and acinar cells. Extensive granulation of the stomach and intestine walls occurs. These granules are concentrated in the subcompactum and stratum granulosum of the submucosas. Death of infected fish probably occurs from a severe electrolyte and fluid imbalance from renal failure (Amend and Smith 1975).

\section{Cytopathic Effect}

Necrotic changes of fathead minnow epithelial (FHM) cells used for diagnosis of IHN virus is not generally detectable until 3 or more days post inoculation. Plaques first form with localized changes followed by a retreating edge of affected cells which degenerate. The result is change of a confluent monolayer (see Fig. 3) to lumps of grape cluster like pyknotic cells. Considerable thickening of the nuclear margin is first detectable. Thickening of the nuclear membrane and enlargement of the nucleus are visible with careful discernment. Rounding of the cells as pyknosis occurs, results in floating cells sloughed off from the growing surface (see Fig. 4 ).

\section{Virus Morphology and Characteristics}

According to Amend and Chambers (1970) IHN virus is bullet shaped with an RNA core and a 158-181 x 90-91 $\mathrm{nm}$ size and is sensitive to $\mathrm{pH}$, ether and heat. It has been classified as a rhabdovirus and was successfully serially cultured in a human diploid cell strain and a hamster cell line both at 18C (Clark and Soriano 1974).
Diagnosis

The presence of the agent is diagnosed by virology and histopathology means.

\section{Virus Culture}

Culture of the virus in FHM cells is possible when present in gonadal fluids of susceptible species at or near spawning. It is more readily detectable in ovarian fluid than seminal fluid. Organ or whole fish cultures of juveniles dying from the disease are also effective. The anterior kidney is the currently preferred tissue. Samples are preliminarily processed by centrifugation and addition of antibiotics to prevent contamination. Inoculated cells held at $12 \mathrm{C}$ show CPE in 3 to 14 days. Cells exhibiting CPE are cultured twice to a final dilution of $10^{-6}$. Negative samples are blind pass cultured twice to a final dilution of $10^{-4}$. Diluting while subculturing eliminates toxicity induced by contaminants. Blind passing removes any protein or enzyme masking effect. Samples showing typical CPE in serial cultures can be serum neutralized. Cultures exhibiting atypical CPE should be serum neutralized for confirmation. Control cultures should always be included. Heat lability of IHN virus requires maintenance of sample temperatures at less than $21 \mathrm{C}$ for sustained periods ( $P$ ietsch et al. 1977). Gonadal fluids can be frozen prior to preliminary or final processing, but should not be kept fresh for more than 5 days (at 5C). Whole fish or organ samples should not be frozen due to selective virus reductions. 


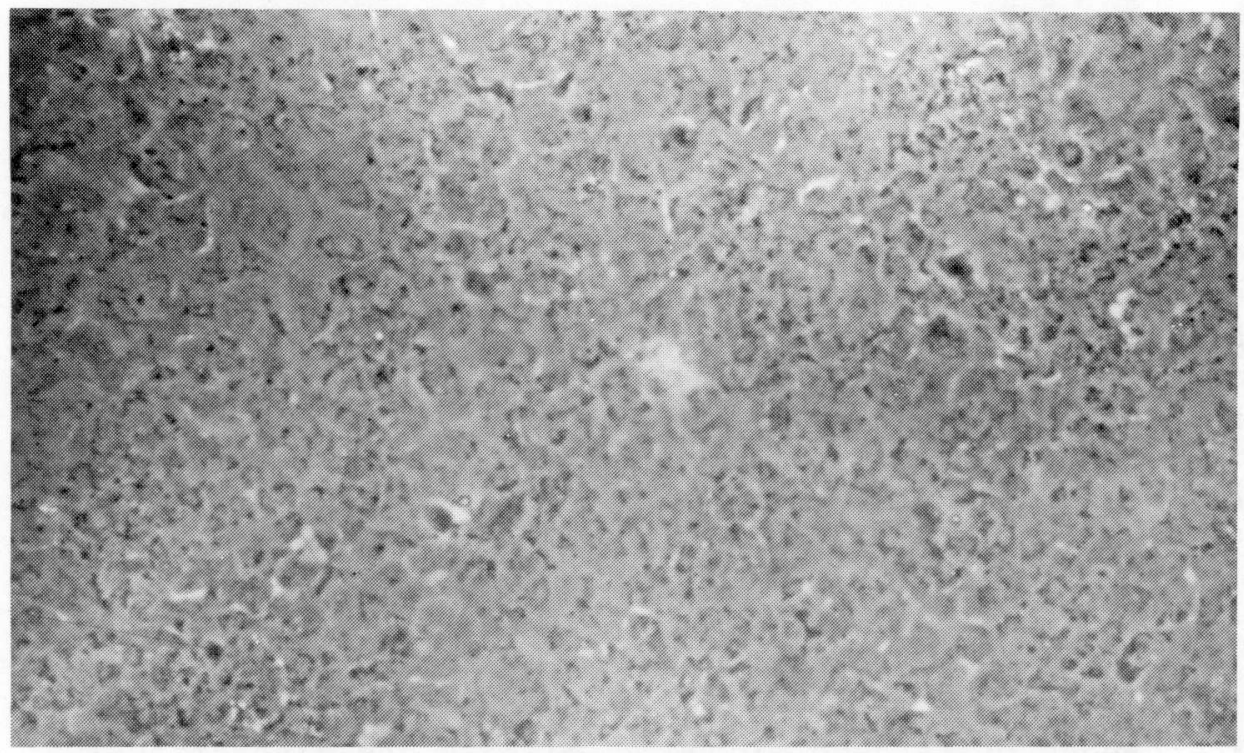

Figure 3. Confluent fathead minnow epithelial cells not infected with virus $(100 x)(R$. Grischkowsky).

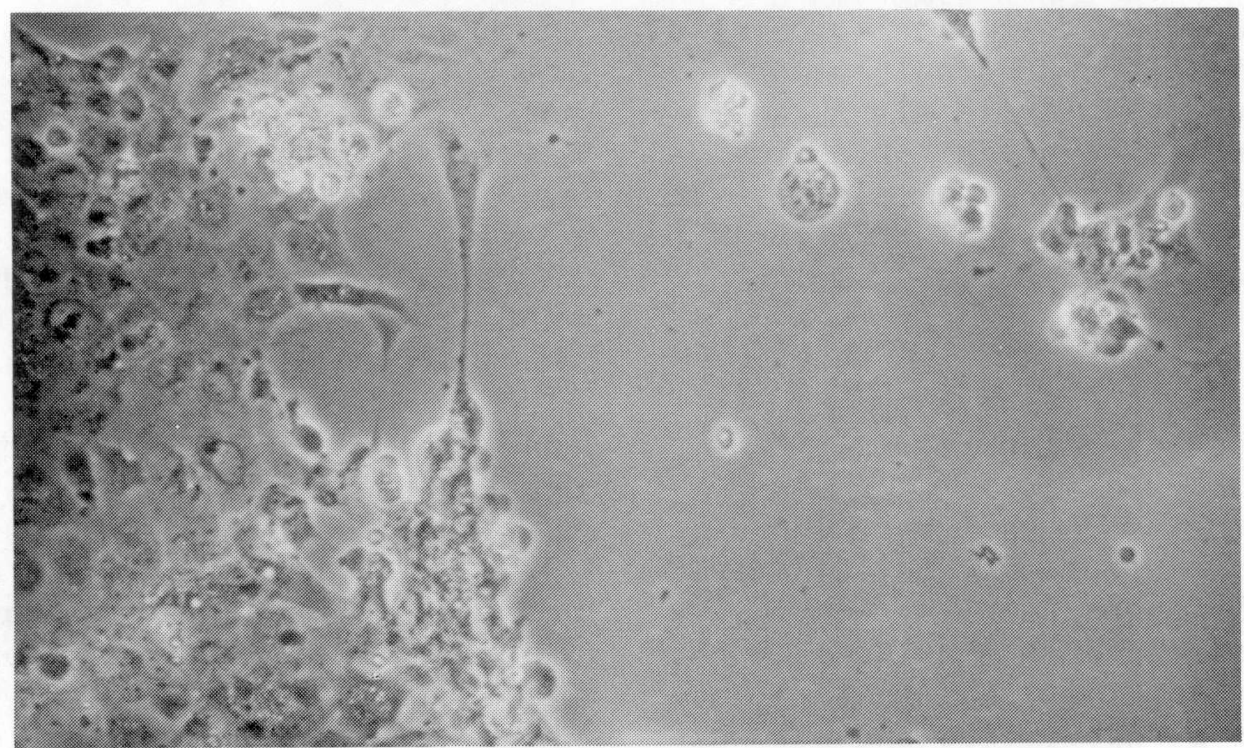

Figure 4. Fathead minnow epitheal cells infected with infectious hematopoietic necrosis virus. Cytopathic effects including plaque formation, rounding up of cells, lifting off substrate surface and floating "grape clusters" are present (100X) (R. Grischkowsky). 


\section{Histopathology}

Paraffin histotechnique provides the most rapid means of identification. The presence of several of the previously described pathognomonic traits in susceptible species is diagnostic for IHN virus. Fish of the same species and similar stage of development should always be used as comparisons. Slides exhibiting deliberate post mortem necrosis and environmental deterioration should be used as additional controls.

\section{Significance and Control}

\section{Significance}

Infectious hematopoietic necrosis virus has caused the death of tens of millions of sockeye salmon juveniles in Alaska since 1973 (see color Fig. 39). Chinook salmon stocks are now showing a minor incidence and as such are threatened. Combined, these are the most valuable commercial salmon species. It has necessitated the destruction of sockeye and chinook salmon fish lots and the elimination of programs designed to increase numbers of some stocks. This disease is the limiting factor for sockeye salmon artificial propagation in Alaska.

\section{Control}

Control of IHN virus disease is at best very difficult. In general, the best combination is to use virus-free stocks in a disinfected hatchery with depurated surface waters or well water.

Water depuration. Clear water is the first priority. If infected or carrier fish are upstream from the hatchery the water is contaminated. Well water if possible, should be utilized. It is generally pathogenfree. If well water is not desired due to temperature incompatibility, a heat exchanger should be considered to change the water temperature appropriately. If surface water must be used, it should be depurated using ultraviolet (UV) irradiation, ozone $\left(\mathrm{O}_{3}\right)$ generation or chlorinationdechlorination. Risks to fish and hatchery workers are present with $0_{3}$ and chlorination water and air residual. Irradiation by UV presents a visual hazard and may allow raw water to pass during an electrical failure if gravity flow is used.

Surface disinfection. Surface disinfection of eggs may allow the use of carrier stocks while reducing disease loss and occurrence. An organic iodine complex called iodophor is routinely used for surface disinfection of salmonid eggs. Its efficacy against IHN virus is substantiated (Amend and Pietsch 1972), but some disagreement exists relative to its effectiveness against naturally exposed eggs. lodophor was effective against all known salmonid viruses at $25 \mathrm{ppm}$ iodine for 5 minutes. Chlorine as sodium hypochlorite at 10 ppm Chlorine for 30 minutes was also effective against IHN virus (Amend and Pietsch 1972). Amend et al. (1973) performed laboratory and field tests of an iodophor with rainbow trout. After contamination of eggs with ovarian fluid containing IHN virus, half the eggs were disinfected for 5 minutes at $50 \mathrm{ppm}$ iodine. After 6 weeks, $3 \%$ of control fish died as opposed to $80 \%$ of the treatment group. The disease was not detected in the control fish. A rainbow trout hatchery with annual IHN virus disease in juveniles has received $75 \mathrm{ppm}$ iodine for a 10 minute egg disinfection since 1970 with no reoccurrence of the disease (Amend et al. 1973).

Elevated water temperature. Elevated water temperature has been used as a control measure for this disease. Amend (1970) determined 
that the disease could be controlled if water temperature was raised to $18 \mathrm{C}$ for 4 to 6 days commencing within 24 hours post infection. Reinfection must be prevented as fish are susceptible afterwards (Amend 1970). Carrier fish survivors may result from this method (Amend 1970). Water temperatures above $15 \mathrm{C}$ tend to increase fish resistance to the virus. The effect is apparently not due to the inability of the virus to grow at 15-18C (Clarke and Soriano 1974) but perhaps from interferon protection of the host (Amend 1970). Virus inactivation in vitro increased directly with temperature but survival of the virus was still substantial at $27 C$ (Pietsch et al. 1977). Inactivation was complete at $32 \mathrm{C}$ within 8 hours (Pietsch et al. 1977).

Rearing juvenile chinook salmon in the Sacramento River at $15 \mathrm{C}$ is thought adequate for control of the disease (Wingfield and Chan 1970). Elevated water temperatures may have limited applicability to sockeye salmon in Alaska as high temperature lethality of the host must be considered.

Selective breeding. The possibility of increasing the number of fish that can tolerate the disease by selective breeding was suggested by McIntyre and Amend (1978). In that paper, heritability (contribution from 1 generation to the next) for IHN virus in sockeye salmon was about $30 \%$ as determined by survival by fry of IHN virus challenges.

Vaccination. Use of a killed or attenuated live vaccine to protect salmonids against IHN virus disease has some potential and considerable difficulties. Government approval must be achieved prior to use and interstate transport. Research, development and licensing costs are extensive and difficult to bear for 1 agency or company. One field test of 1 IHN virus vaccine was performed in
Alaska at Crooked Creek and Kitoi Bay hatcheries of the Kenai Peninsula and Afognak Island respectively. Only low levels of protection were gained. Immunocompetence and time of infection commencement pose difficult technical problems.

In Alaska, infection has occurred at or near hatching. Protection of fish at that time would be necessary for a vaccine to contribute meaningfully. At the present an IHN virus vaccine is a hope for the future.

\section{$\underline{\text { References }}$}

AMEND, D. F. 1970. Control of infectious hematopoietic necrosis virus disease by elevating the water temperature. J. Fish Res. Board Can. 27:265-270.

mission of infectious hematopoietic necrosis virus in rainbow trout. J. Wildl. Dis. $11: 471-478$.

- 1976. Prevention and control of viral diseases of salmonids. J. Fish. Res. Board Can. 3:1059-1066.

AMEND, D. F., W. T. YASUTAKE and R. W. MEAD. 1969. A hematopoietic virus disease of rainbow trout and sockeye salmon. Trans. Amer. Fish. Soc. 98:796-804.

AMEND, D. F. and V. C. CHAMBERS. 1970. Morphology of certain viruses of salmonid fishes. II. In vivo studies of infectious hematopoietic necrosis virus. J. Fish. Res. Board Can. $27: 1385-1388$.

AMEND, D. F. and J. P. PIETSCH. 1972. Virucidal activity of two iodophors to salmonid viruses. J. Fish. Res. Board Can. 29:61-65.

AMEND, D. F. and L. SMITH. 1975. Pathophysiology of infectious hematopoietic necrosis virus disease in rainbow trout: hematological and blood chemical changes in moribund fish. Infect. and Immun. 11:171-179. 
AMEND, D. F. and J. W. WOOD. 1972. Survey for infectious hematopoietic necrosis (IHN) virus in Washington salmon. Prog. FishCult. 34:143-147.

AMEND, D. F., W. T. YASUTAKE, J. L. FRYER, K. S. PILCHER and W. H. WINGFIELD. 1973. Infectious hematopoietic necrosis. In: Symposium on the Major Communicable Fish Diseases in Europe and Their Control. EIFAC, FAO. Tech. Paper 17, Suppl. 2:80-98.

AMEND, D. F. and J. R. NELSON. 1977. Variation in the susceptibility of sockeye salmon Oncorhynchus nerka to infectious haemopoietic [hematopoietic] necrosis virus. J. Fish Biol. 11:567-573.

CLARK, H. F. and E. Z. SORIANO. 1974. Fish rhabdovirus replication in non-piscine cell culture: a new system for the study of rhabdovirus-cell interaction in which the virus and cell have different temperature optima. Infect. and Immun. 10:180-188.

GRISCHKOWSKY, R. S. and D. F. AMEND. 1976. Infectious hematopoietic necrosis virus: prevalence in certain Alaskan sockeye salmon, Oncorhynchus nerka. J. Fish. Res. Board Can. 33:186-188.

MCINTYRE, J. D. and D. F. AMEND. 1978. Heritability of tolerance for infectious hematopoietic necrosis in sockeye salmon (Oncorhynchus nerka). Trans. Amer. Fish. Soc. 107:305-308.

PIETSCH, J. P., D. F. AMEND and C. M. MILLER. 1977. Survival of infectious hematopoietic necrosis virus held under various environmental conditions. J. Fish. Res. Board Can. 34:1360-1364.

PLUMB, J. A. 1972. A virus caused epizootic of rainbow trout (Salmo gairdneri) in Minnesota. Trans. Amer. Fish. Soc. 101:121-123.

RUCKER, R. R., W. J. WHIPPLE, J. R. PARVIN and C. A. EVANS. 1953. A contagious disease of salmon possibly of virus origin. Fishery Bull. 76 from Fishery Bull. of the Fish and Wildl. Serv. 54:35-46.
SANO, T. 1976. Viral diseases in Japan. Fish Pathology 10:221-226.

SANO, T., T. NISHIMURA, N. OKAMOTO, T. YAMAZAKI, $H$. HANADA and $Y$. WATANABE. 1977. Studies on viral diseases of Japanese fishes VI. Infectious hematopoietic necrosis (IHN) of salmonids in the mainland of Japan. J. Tokyo Univ. Fish. $63: 81-85$.

SAUNDERS, W. B. CO. 1974. Dorland's lllustrated Medical Dictionary. 25th ed. W. B. Saunders Co., Philadelphia, PA. 1748 pp.

WILLIAMS, I. V. and D. F. AMEND. 1976. A natural epizootic of infectious hematopoietic necrosis in fry of sockeye salmon (Oncorhynchus nerka) at Chilko Lake, British Columbia. J. Fish. Res. Board Can. 33:1564-1567.

WINGFIELD, W. H., F. L. FRYER and K. S. PILCHER. 1969. Properties of the sockeye salmon virus (Oregon strain). Proc. Soc. Exper. Biol. Med. 130:1055-1059.

WINGF IELD, W. H. and L. D. CHAN. 1970. Studies on the Sacramento River Chinook Disease and its causative agent. pp. 307-318. In: A Symposium on Diseases of Fishes and Shell fishes. S. F. SNIESZKO, ed. Amer. Fish. Soc. Spec. Publ. No. 5, Washington DC.

WOLF, K., M. C. QUIMBY, L. L. PETTIJOHN and M. L. LANDOLT. 1973. Fish viruses: isolation and identification of infectious hematopoietic necrosis in Eastern North America. J. Fish. Res. Board Can. 30:1625-1627.

YASUTAKE, W. T. 1970. Comparative histopathology of epizootic salmonid virus diseases. pp. 341-350. In: A Symposium on Diseases of Fishes and Shellfishes. S. F. SNIESZKO, ed. Amer. Fish. Soc. Spec. Publ. No. 5, Washington $D C$.

1975. Fish viral diseases: clinical, histopathological and comparative aspects. pp. 247-271. In: The Pathology of Fishes. W. E. RTBELIN, ed. Univ. Wisconsin Press, Madison, WI. 
YASUTAKE, $W . T$. and D. F. AMEND. 1972. Some aspects of pathogenesis of infectious hematopoietic necrosis (IHN). J. Fish. Biol. 4:261-264.

\title{
Viral Erythrocytic Necrosis
}

\author{
John Burke
}

\section{Introduction}

Viral erythrocytic necrosis (VEN) (Evelyn and Traxler 1978) has not been observed in Alaskan fish, but it is probably present in the marine environment and at least 2 anadromous fish, pink (Oncorhynchus gorbuscha) and chum (Oncorhynchus keta) salmon. Because there have never been extensive surveys for VEN in Alaska; the signs of the disease are not grossly obvious; and the disease is present in adjacent waters of British Columbia, it would follow that VEN will eventually be found in Alaska.

Knowledge of VEN is only recent and very sparse. The viral agents of the disease have not been isolated, grown in cell culture, or satisfactorily taxonomically placed. The conditions predisposing the disease are not known, and the marine reservoir of the virus has not been identified (Evelyn and Traxler 1978).

\section{History}

What were thought to be intraerythrocytic protozoans have been noted in lizards for more than 65 years (Johnston and Davies 1973).
More recently, some of these presumed protozoans have proven to be viral inclusion bodies. Similar misinterpreted inclusions have been noted in the erythrocytes of other reptiles and amphibians (Johnston and Davies 1973).

Laird and Bullock (1969) encountered this abnormal condition which they called piscine erythrocytic necrosis (PEN) in several marine species. The condition was characterized by an inclusion in the cytoplasm of affected erythrocytes and was clearly pathological to Atlantic cod (Gadus morhua). The etiology of the disease was not determined, but the authors were aware that it could have been viral. Walker (1971), while looking at cod from the same area, found cytoplasmic inclusions associated with large icosahedral virions which he compared to lymphocystis virus. The range of known marine hosts was listed for Atlantic fishes by Walker and Sherburne (1977).

Appy (Appy et al. 1976) used electron microscopy to examine infected cod erythrocytes and found virus associated with the inclusions. Walker and Sherburne (1977) also examined 
infected cod erythrocytes with electron microscopy and noted a small cytoplasmic viroplasm with an adjacent coherent group of large icosahedral virions. Both Appy (Appy et al. 1976) and Walker and Sherburne (1977) described infected cells and the associated virions. Infected cells had basophilic cytoplasmic inclusions, with or without an attached group of granules (virions), nuclear destruction (pyknosis and karyorrhexis), and were generally distorted. The inclusion was round, compact and Fuelgen positive (contained DNA) and of ten had attached granules. Electron micrographs showed that the round inclusion was viroplasm and that the granules were virions (Walker and Sherburne 1977). The virion had a sharply defined envelope with a side-to-side diameter of $300 \mathrm{~nm}$. The nucleoid was electron dense and spheroidal, approximately 230 in diameter. Each virion had a surcoat of unknown origin, virus or erythrocyte. The intensity of infection in Atlantic cod ranged from less than $.01 \%$ to greater than $99 \%$ of the mature erythrocytes (Walker and Sherburne 1977).

Reno et al. (1978) used electron microscopy to examine Atlantic herring (Clupea harengus harengus) showing this condition. These $145 \mathrm{~nm}$ in diameter, than previously described in cod. They presumptively classified the virus within the Iridoviridae.

More recently, Evelyn and Traxler (1978) called attention to a disease of pink and chum salmon which was characterized by a single cytoplasmic inclusion in affected erythrocytes. This condition had been seen as early as 1964 in chum salmon and was recognized in cultured pink salmon in 1972. Sockeye (Oncorhynchus nerka), chinook (Oncorhynchus tshawytscha), and coho (Oncorhynchus kisutch) salmon reared in pens adjacent to those holding infected pink and chum salmon did not have the disease. An attempt to experimentally transmit erythrocytic necrosis virus (ENV) to the sockeye, chinook and coho salmon was unsuccessful. Evelyn and Traxler (1978) proposed changing the name of the disease from PEN to viral erythrocytic necrosis (VEN), feeling VEN was more descriptive and would more widely designate the disease in other fish and animals.

Most recently, MacMillan and Mulcahy (1979) found the presence of VEN ranged from $0-17 \%$ in geographically different chum salmon stocks in Puget Sound, Washington. In addition, they found the disease incidence varied from $4-59 \%$ in different Pacific herring (Clupea harengus pallasi) stocks from Puget Sound.

\section{Transmission}

MacMillan and Mulcahy (1979) were able to experimentally transmit the disease to chum, pink, coho, sockeye, chinook, and Atlantic (Salmo salar) salmon and rainbow (Salmo gairdneri), brown (Salmo trutta) and brook (Salvelinus fontinalis) trout with an intraperitoneal injection of virus taken from naturally infected chum salmon. Chum salmon and brook trout were infected by waterborne virus from chum salmon, and chum salmon were infected with virus taken from herring. The differences in species susceptibility between the results of Evelyn and Traxler (1978) and those of MacMillan and Mulcahy (1979) were related to the smaller, presumed more susceptible fish used by the later authors.

Transmission experiments have clearly shown that VEN is infectious and that its agent is a virus (Evelyn and Traxler 1978). The disease has not been seen in fish held only in freshwater, though it was experimentally transmitted through freshwater to a susceptible host (MacMillan and Mulcahy 1979). Natural infection of 
salmonids has only been seen in chum and pink salmon which share the behavioral pattern of migration to saltwater shortly after hatching. The above information suggests that the causal agent is of marine origin, an agent these 2 species contact while still of susceptible size.

\section{Signs and \\ Pathogenesis}

The signs of VEN are neither obvious nor pathognomonic. Infected fish are anemic, which can be seen in pale gills, heart, liver, kidney and alimentary tract. Fish infected by ENV had low hematocrits $(2-10 \%$ common) and their blood clotted slowly or not at all (Evelyn and Traxler 1978). Histopathological changes occurred in the kidney which exhibits active hematopoiesis (Evelyn and Traxler 1978). MacMillan et al. (1980) suggested that the progression of the infection might relate to the developmental stage of infected erythrocytes seen in the blood. Two or 3 weeks after injection with the virus, experimental fish showed a significant erythroblastosis with erythroblasts (immature erythrocytes) of the periferal blood containing VEN inclusions. This condition designated later stages of infection (MacMillan et al. 1980).

\section{Diagnosis}

Diagnosis is based on giemsastained blood smears. The fish were considered ENV positive if any inclusion-bearing erythrocytes were detected in the examination of 50 microscopic fields (100x objective lens). If inclusion-bearing erythrocytes were not found in this examination, the fish were designated ENV free (Evelyn and Traxler 1978).

\section{Significance and Control}

Little can be said about the significance of VEN or its control. Evelyn and Traxler (1978) noted that the disease was diagnosed over a wide temperature range $(6.5-19 \mathrm{C})$, but that it seemed most severe during the summer. This knowledge may eventually have some bearing on control of the disease. The course of VEN appeared to be chronic with low mortality rates (up to $0.3 \%$ per day) among pen-reared pink and chum salmon. Natural outbreaks of VEN in pink and chum salmon were usually complicated by vibriosis or bacterial kidney disease (Evelyn and Traxler 1978).

MacMillan et al. (1980) examined some of the secondary consequences of ENV infections. Erythrocytic necrosis virus-infected $f i s h$ had a three-fold greater mortality rate from vibriosis, a significantly decreased tolerance to oxygen depletion, and a decreased ability to regulate serum sodium and potassium in saltwater. This combination of secondary effects of ENV infection might prove a problem during saltwater pen rearing of susceptible salmonids. There is some speculation (J. MacMillan, pers. comm.) that the disease may debilitate infected adult salmon so that they do not have the strength to spawn when their required spawning migrations are greater than several miles upstream from saltwater.

Viral erythrocytic necrosis is probably widespread in the Pacific and Atlantic marine environments. It is possible that salmonids are not a principle host in the dynamics of the disease, and that they represent an offshoot from the main course of infection. Until the vectors and dynamics of ENV transmission are known, little can be done to control the disease. 

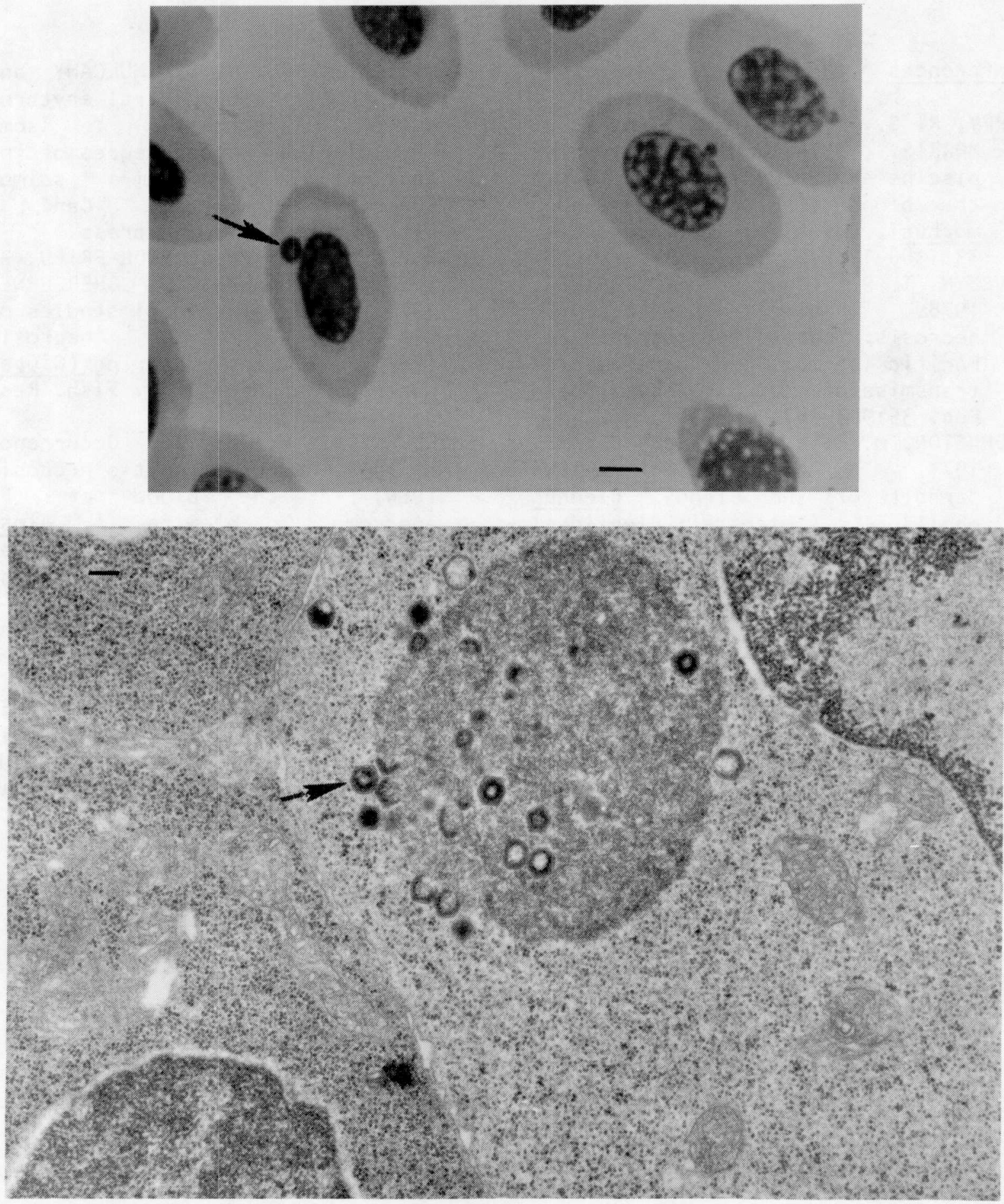

Figure 5. (Top) Viral erythrocytic inclusion (arrow) in the cytoplasm of an erythrocyte from the peripheral blood of a chum salmon ( $\underline{0}$. keta). Bar $=2.5$ micrometers (J.P. MacMillan).

(Bottom) Erythrocytic necrosis virion (arrow) associated with a cytoplasmic inclusion in an erythrocyte from the peripheral blood of a chum ( $\underline{0}$. keta) salmon. Bar $=2$ micrometers (J. P. MacMillan). 


\section{References}

APPY, R. G., M. D. B. BURT, and T. J. MORRIS. 1976. Viral nature of piscine erythrocytic necrosis in the blood of Atlantic cod (Gadus morhua). J. Fish. Res. Board Can. 33:1380-1385.

EVELYN, T. P. T. and G. S. TRAXLER. 1978. Viral erythrocytic necrosis: natural occurrence in Pacific salmon and experimental transmission. J. Fish. Res. Board Can. 35:903-907.

JOHNSTON, M. R. L. and A. J. DAVIES. 1973. A Pirhimocyton-like parasite of the blenny, Blennus pholis L. (Teleostei; Bleniidae) and its relationship to Immanoplasm Neumann, 1909. Int. J. Parasitol. 3:235-241.

LAIRD, M. and W. L. BULLOCK. 1969. Marine fish hematozoa from New Brunswick and New England. J. Fish. Res. Board Can. $26: 1075-1102$.

MACMILLAN, J. R. and D. MULCAHY. 1979. Viral erythrocytic necrosis (VEN) disease: incidence in Puget Sound, artificial transmission and species susceptibility. J. Fish. Res. Board Can. 39:196-200.
MACMILLAN, J. R., M. D. MULCAHY and M. LANDOLT. 1980. Viral erythrocyt ic necrosis: some physiological consequences of infection in chum salmon (Oncorhynchus keta). Can. J. Fish. Aquat. Sci. In press.

RENO, P. W., M. PHILLIPPON-FRIED, B. L. NICHOLSON and S. W. SHERBURNE. 1978. Ultrastructural studies of piscine erythrocytic necrosis (PEN) in Atlantic herring (Clupea harengus harengus). J. Fish. Res. Board Can. 35:148-154.

SHERBURNE, S. W. 1977. Occurrence of piscine erythrocytic necrosis (PEN) in the blood of the anadromous alewife, Alosa pseudoharengus, from Maine coastal streams. J. Fish. Res. Board Can. $34: 281-286$.

WALKER, R. 1971. PEN, a viral lesion of fish erythrocytes. Am. Zool. 11:707.

WALKER, R. and S. W. SHERBURNE. 1977. Piscine erythrocytic necrosis virus in Atlantic cod, Gadus morhua, and other fish: ultrastructure and distribution. J. Fish. Res. Board Can. 34:1188-1195. 


\title{
Infectious Pancreatic Necrosis Virus
}

\author{
John Burke
}

\section{Introduction}

\begin{abstract}
Infectious pancreatic necrosis (IPN) disease results from a viral infection of salmonids. Infectious pancreatic necrosis virus (IPNV), found in North American salmonids, was the first piscine virus isolated. The virus is made up of double stranded, segmented RNA and is considered somewhat ambiguously a reolike virus.
\end{abstract}

In recent years IPNV has been spread to almost every major trout growing area in the world. The acute form of IPN primarily affects juvenile fish and occasionally larger fish. The disease also commonly occurs inapparently as a benign carrier state in yearling and adult fish.

\section{History}

Acute catarral enteritis was described by M'Gonigle (1940). At the time, this condition was considered a physiological disease of brook trout (Salvelinus fontinalis) and Atlantic salmon (Salmo salar) from hatcheries in Canadian Maritime Provinces. The original description of IPN was published in 1955 (Wolf 1976, Wood et al. 1955) and in 1960 the viral agent was isolated (Wolf et al. 1960). Since these early publications more than 100 reports have been published on the virus or the disease it causes. Some of the more interesting work is summarized and listed by Wolf (1976).

Probably the most significant historic aspect of IPN is its spread by fish culturists to nearly every major trout growing area of the world. It is thought the disease was moved from North America throughout both Europe and Japan by transporting infected fish or eggs. The disease also has likely been taken to Australia and Tasmania in the same manner.

Virtually all salmonids can be infected by IPNV. This virus also occurs, or at least exhibits long term survival, in the guts of fish-eating birds and some invertebrates (Sonstegard 1970). This disease does not occur in Alaska at present, and this is one of the foremost reasons for fish and egg transfer regulations in the state.

\section{Transmission}

The 3 primary routes of IPNV transmission have been by water, feed, and eggs from carrier adults who have survived an epizootic. During an epizootic the virus is shed into the water with the feces of infected fish. Uninfected fish downstream from the epizootic can become infected with this waterborne virus. All of the fish infected with IPNV do not die, some recover and develop to maturity. These fish may appear healthy and yet are often carriers of IPNV which is found in feces, peritoneal washings and in spermatic and ovarian fluids. The virus is then passed to the next generation with the eggs of these carrier females (Seeley et al. 1977).

\section{Signs and Pathogenesis}

The first sign of an IPN epizootic is a sudden increase in mortality. The most healthy appearing fry are often the first affected. Whirling 
is common among terminally infected fish with death following in several hours. External signs often include darkening, exopthalmia, ascites and hemorrhage at the base of fins. Internally, petechia (pin point hemorrhages) in the area of pyloric caeca and a pale liver and spleen can denote the disease. The digestive tract is usually empty with a clear to milky mucous filling in the stomach and anterior intestine (sometimes called a mucous plug). These signs are very similar among fish suffering from several different viral diseases, so that virus isolation and the history of the affected stock is necessary for diagnosis and direction (Wolf 1966).

Histopathological examination shows that pancreatic tissue is a target organ. This tissue contains cytoplasmic inclusions and shows pyknosis (nuclear shrinkage) and karorrhexis (a pyknotic nucleus undergoing fragmentation) of infected cells. The liver and spleen also demonstrate pathological changes. Nonspecific hyalin degeneration (characterized by a monogeneous pink appearance under the light microscope) is another unexplained major histopathological change seen in IPNV infected fish (Snieszko et al. 1957).

\section{Diagnosis}

Clinical signs of the IPN may be useful in diagnosis, but their absence cannot be relied upon to indicate the absence of the virus. Diagnosis of IPN must involve virological (isolation) and identification (neutralization) tests. The virus can be isolated on a number of fish cell lines, rainbow trout gonad (RTG-2), fathead minnow (FHM), and bluegill (BG-2) are used most commonly in this procedure. Incuba$\mathrm{tion}$ at $20 \mathrm{C}$ has been recommended
(Amend and Wedemeyer 1970) but $18 \mathrm{C}$ is more commonly used. Characteristic CPE (cells feather, elongate, are spiderlike as compared with IHN where cells round up) can usually be noted in from 24 to 48 hours after inoculation. Positive confirmation requires isolation of the virus as described above and serologic neutralization.

The most sensitive test for the detection of IPN involves the use of internal organ homogenates (whole fish with fry) or the sex products from spawning adults. Unfortunately, sampling the internal organs requires sacrifice of the fish and sampling sex products can only be done during spawning. To circumvent this problem, feces are sampled and have proven fairly reliable, though not as sensitive as the first 2 samples mentioned in the detection of carrier fish.

\section{Significance and Control}

The significance of the initial mortality among fry is related to several factors. The first variable is the age of the fish, which become more resistant to the disease as they get older. The second major is the particular strain of IPNV involved. The virulence of different virus strains varies as do resultant mortalities, from 10-90\% (Wolf 1976). With this range of prognoses for IPNV epizootics and the knowledge that survivors become vertical transmitters of the disease, it is obvious that the best treatment for the disease is avoidance. Since IPNV is not in Alaska and there are strict regulations designed to prevent its importation, it is possible the disease can be avoided. If the virus is present, fish culturists must be concerned with water purification, carrier detection, egg and brood stock movement, and even 
sea gulls and invertebrates which can sustain the virus for long periods of time after a meal of infected fish or detritus. There is no successful treatment for IPNV infected fish nor is there a totally successful management scheme to control the disease once it has spread throughout a system. Wolf (1966) advises that the sole means of handling the problem in an infected watershed is to anticipate a loss of one half or more and to provide extra eggs.

\section{$\underline{\text { References }}$}

AMEND, D. F. and G. WEDEMEYER. 1970. Approved procedure for determining the absence of infectious pancreatic necrosis (IPN) virus in certain fish and fish products. U.S. Fish Wildl. Serv., Fish Dis. Leaflet No. $27.4 \mathrm{pp}$.

M'GONIGLE, R. H. 1940. Acute catarrhal enteritis of salmonid fingerlings. Trans. Amer. Fish. Soc. $70: 297-303$.

SEELEY, R. J., A. PERLMUTTER and V. A. SEELEY. 1977. Inheritance and longevity of infectious pancreatic necrosis virus in the zebra fish Brachydanio rerio (HamiltonBuchanan). Appl. Env. Microbiol. $34: 50-55$.
SNIESZKO, S. F., E. M. WOOD and W. T. YASUTAKE. 1957. Infectious pancreatic necrosis in trout. AMA Archives of Pathol. 63:229-233.

SONSTEGARD, R. A. 1970. Descriptive and Epizootiological Studies of Infectious Pancreatic Necrosis of Salmonids and Lymphosarcoma of Esox Masqumongy. Ph.D. Thesis, Univ. of Guelph, ONT.

WOLF, K., S. F. SNIESZKO, C. E. DUNBAR and E. PYLE. 1960. Virus nature of infectious pancreatic necrosis in trout. Proc. Soc. Exper. Biol. Med. 104:105-108.

WOLF, K. 1966. Infectious pancreatic necrosis (IPN) of salmonid fishes. U.S. Fish Wildl. Serv., Fish Dis. Leaflet No. 1. 4 pp. - 1976. Fish viral diseases in North American 1971-1975, and recent research of the Eastern Fish Disease Laboratory, USA. Fish Path. 10:135-154.

WOOD, E. M., S. F. SNIESZKO and W. T. YASUTAKE. 1955. Infectious pancreatic necrosis in brook trout. AMA Archives of Pathol. 60:26-28. 
Chapter 2.

BACTERIAL DISEASES 


\author{
A. J. Didier, Jr.
}

\section{Introduction}

Bacterial kidney disease (BKD) is a systemic infection caused by a small (0.3-0.5 um $\times 0.5-1.0$ um), nonmotile, non-acid fast, Gram positive, diplobacillus. The proposed name for this organism is Corynebacterium salmonis (Sanders and Fryer 1978). A11 salmonid fish (subfamily Salmoninae) are considered susceptible to infection. There are no known cases of BKD in non-salmonids.

An additional name change was recently proposed by Sanders and Fryer (1980) which would result in the BKD causative agent being called Renibacterium salmoninarum gen. nov., sp. nov.

\section{History}

An infection which became known as "Dee disease" was first reported in Scotland in 1933 (Bullock et al. 1971, Mackie et al. 1933). The etiologic agent was a small Gram positive bacillus which produced lesions in the spleen and other organs of Atlantic salmon (Salmo salar). In 1935, a similar condition was reported in trout in Massachusetts (Belding and Merrill 1935, Bullock et al. 1971). Since that time, BKD has been reported frequently in cultured salmonids in the United States, and its geographic range has been extended to include Canada, Japan and France (American Fisheries Society 1979). Bacterial kidney disease may now be one of the most prevalent bacterial diseases of salmonid fishes.
In Alaska, BKD was first detected at Petersburg in 1975. Substantial losses of coho salmon (Oncorhynchus kisutch) smolts at the state salmon hatchery there were directly attributable to this disease. Infected fish had been shipped to state facilities in Juneau and Sitka; BKD was later detected at both sites. In 1978 , this author conducted a survey of salmon stocks in 26 watersheds of Southeast Alaska and found BKD to be much more widespread than previously realized (unpub. data). Coho, chum (Oncorhynchus keta) or pink salmon (Oncorhynchus gorbuscha) carriers were detected in half of the systems sampled, although the incidence levels in these systems varied widely. Carrier rates were generally less than $10 \%$ but several were greater than 25\%. Carriers were found in virgin stream systems as well as in those which had received transplants of hatchery reared fish. Recently, others have detected BKD in Dolly Varden char (Salvelinus malma) from the Juneau area and in coho salmon from Seward (R. Grischkowsky, pers. comm.) As further sampling programs are conducted in other areas of Alaska, it is likely that the geographic range of the disease will be extended.

\section{Transmission}

The bulk of current information indicates that a primary path of BKD transmission is vertically from adults to juveniles through the egg (Bullock et al. 1978, Klontz 1978). Oral transmission is possible but infection is not always certain (Wolf 
and Dunbar 1959, Wood and Wallis 1955). Transmission through the water has been possible only when the skin has been broken or abraded prior to exposure (Wolf and Dunbar 1959). Success of infection is dependent on a variety of factors, including species specific susceptibility to the disease. of the major trout species, brook trout (Salvelinus fontinalis) are the most susceptible, brown trout (Salmo trutta) are intermediate and rainbow trout (Salmo gairdneri) are most resistant (Bullock et al. 1971). Among the Pacific salmons, BKD is most frequently observed in sockeye (Oncorhynchus nerka), chinook (Oncorhynchus tshawytscha) and coho, since all these species typically require long hatchery rearing periods. Sanders et al. (1978) showed that sockeye were more sensitive to BKD than either coho or steelhead trout ( $\underline{S}$. gairdneri). Susceptibility differences also appear within a given species. For example, Suzumoto et al. (1977) found different levels of resistance to BKD in coho salmon which had different genotypes for transferrin, an iron binding plasma protein.

Water quality and ecological parameters also play a role. Warren (1963) surveyed federal salmon hatcheries in the United States and found that the severity of BKD incidents increased as water hardness decreased. It was noted that these observations could be due to several indirect factors, such as the effect of water chemistry on the salmonid immune response, or its influence on possible intermediate BKD vectors. Mitchum et al. (1979) attributed increased BKD incidence in the headwaters of their test stream to lower volumes and velocities of water, frequent pools, softer water, and stress resulting from severe annual water, level fluctuations. After noting that fish with BKD were also frequently infected with parasites, Wood and Yasutake (1956) suggested that parasites may contribute to BKD incidence by either lowering a fish's general resistance to infection or by providing a portal of entry for the BKD organism.

In hatchery environments, diet is considered a major factor influencing BKD incidence. Wood (1974) credited the use of diets containing cottonseed meal rather than corn gluten with significantly reducing BKD in Washington state hatcheries. He also indicated that coho salmon fed dry diets suffered higher BKD losses than those fed moist formulas. Wedemeyer and Ross (1973) found that the incidences of BKD in lots which were fed corn gluten and cottonseed meal formula diets were similar, but demonstrated that the non-specific stress of infection was greater in groups fed corn gluten.

Bacterial kidney disease infections can occur over a wide range of temperatures, but the progression rate of the disease is highly temperature related. Sanders et al. (1978) tested coho and sockeye salmon and steelhead trout, and found that BKD infection and mortality could occur in all species at temperatures from 3.5-20.5C. However, mean time of death following infection at 6.7 was 60 to 71 days while at 15-20.5C, it was only 21 to 34 days. Sockeye suffered severe mortalities over the entire temperature range while coho and steelhead suffered peak mortalities at $6 \cdot 7-12.2 \mathrm{C}$. In the latter 2 species, the disease appeared to be somewhat depressed at temperatures above $12.2 \mathrm{C}$. 
Signs and Pathogenesis

Typically, the course of BKD is slow and the epizootic appears as a continual, chronic loss. Acute losses have been reported, generally at the moderate temperatures $(13-18 \mathrm{C})$. In either case, external signs may include exopthalmos, abdominal swelling, and blisters or blebs in the skin. These blisters are filled with a clear amber to cream colored purulent fluid. If broken, the lesions appear as large open sores. Abscesses have also been reported in the musculature and in the orbit of the eye.

Internally, the kidneys are often pale and swollen. Macroscopic lesions containing bacteria, leucocytes and cell debris may appear in the kidney, liver or spleen (color Fig. 37). Ascitic fluid may be present in the abdomen. The intestine is usually distended and filled with a white to yellow viscous fluid. At water temperatures below $8.3 \mathrm{C}$, a white, false membrane may envelope the heart, liver, spleen, gonads or air bladder.

It should be noted that in the early stages of an infection or in the carrier state, affected fish may show none of these gross pathological signs. In these cases, presence of the organism may be demonstrated through microscopic examination of tissues or tissue smears.

Several descriptions of the histopathology of BKD are available (Smith 1964, Wood and Yasutake 1956, Young and Chapman 1978). To comparative pathologists, it is said to resemble mammalian renal diseases such as glomerulonephritis. After becoming systemic and penetrating the internal organs, focal necroses occur in the kidney, liver and spleen. Bacteria may be found in large numbers at all these sites. In the kidney, the condition is also characterized by fat deposits, and edema in the glomerulus and renal tubules. The false membrane mentioned earlier differs from the true diphtheritic membrane caused by Corynebacterium diphtheriae in that it is superficial and does not penetrate the organs it covers.

\section{Diagnosis}

Bacterial kidney disease may be difficult to diagnose in that it may occur simultaneously with other fish diseases such as furunculosis (Aeromonas salmonicida), bacterial septicemia (Aeromonas hydrophila) and infectious pancreatic necrosis virus. In these cases, BKD may not be the primary cause of death. Presumptive diagnosis of BKD is usually possible through observation of the gross pathology and examination of gram stained tissue smears from target organs. There are few other Gram positive fish pathogens, and the size and shape of Corynebacterium salmonis is usually distinctive. Fresh or frozen sample materials are preferred, but formalin preserved tissues are also usable.

Various strains of $\underline{c}$. salmonis are serologically homologous and distinct from non-BKD organisms (Bullock et al. 1974), so positive diagnosis can be based on the use of the fluorescent antibody technique (FAT). Direct and indirect staining techniques are available (American Fisheries Society 1979, Bullock and Stuckey 1975). Both use specific anti-BKD serum and in both cases, the primary dye is fluorescein isothiocyanate (FITC) with a 


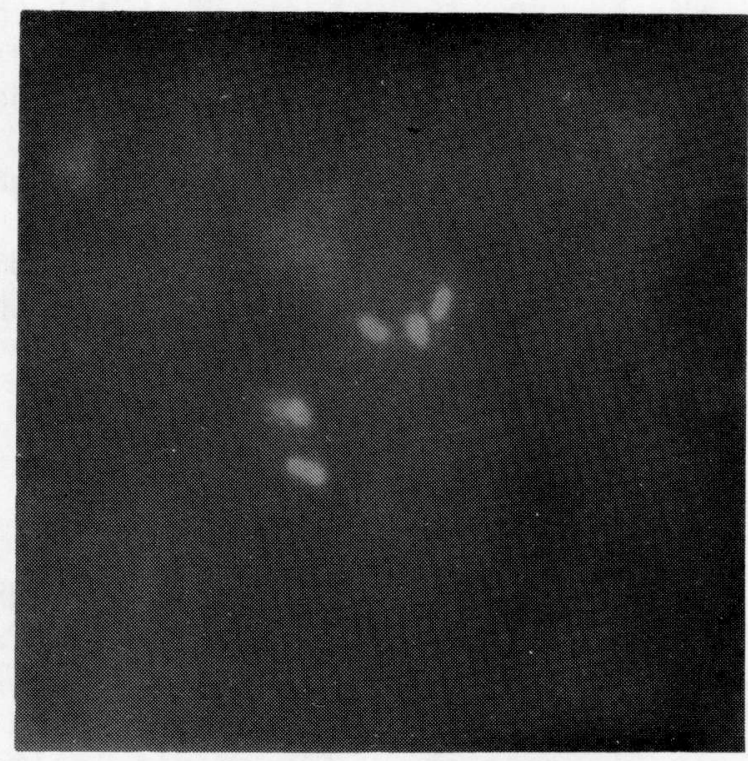

Figure 6. Cells of Corynebacterium salmonis stained with the direct fluorescent antibody technique (1000X) (A. Didier).

rhodamine counter stain to reduce background fluorescence. When stained with this preparation and examined with an incident light fluorescence microscope, cells of $\underline{C}$. salmonis fluoresce with a brilliant green color against a dark background (Fig. 6). Since the FAT is relatively fast, simple and definitive, it is usually the method of choice in most diagnostic laboratories. Potential test tissues include kidney, liver, spleen, lower intestine, fecal material and gonadal fluids. Fresh or frozen materials may be used.

Serologic diagnosis is also possible using immunodiffusion in agar gels (Chen et al. 1974). The technique requires little elaborate equipment, and is useful in providing positive confirmation of overt cases. However, practice has shown it to be relatively insensitive in detecting asymptomatic carriers (Evelyn 1978). Fresh materials are preferred for this procedure.

Culture of the organism is a third method for positive diagnosis commonly used with other bacterial pathogens, but with $\underline{C}$. salmonis there are a number of distinct problems. The organism has an absolute requirement for L-cysteine hydrochloride, and the best medium currently available is a pH 6.5 nutrient agar fortified with cysteine and blood serum (Evelyn 1977). Even under optimum conditions however, incubation periods of 10 days to 3 weeks may be required for development of visible colonies from the initial inoculation. If other bacteria are present, overgrowth of $\underline{C}$. salmonis with other faster growing forms is a constant problem. Sample dilution with ordinary saline may inactivate the 
organism, and there even appears to be anti-BKD activity in tissue fluids. In spite of these difficulties, culture may be one of the most sensitive detection techniques currently available (Evelyn 1978). Fresh materials are required for this procedure.

\section{Significance and Control}

The significance of BKD in hatchery operations has been well documented. At some Alaskan facilities, losses of coho salmon finger 1 ings have ranged from 2-5\% per month during the final months of freshwater or estuarine rearing. Mortalities can also occur in salmon smolts during their transition to seawater, and in prespawning adult salmon after they return from the ocean. Annual BKD epizootics in feral trout populations were reported by Mitchum et al. (1979). Clearly, BKD can have a detrimental impact at any point in the life history of salmonid fishes. However, Suzumoto et al. (1977) concluded that BKD resistance played only a small role in the overall viability of coho salmon at the Big Creek Hatchery (Oregon) when they found that the genotype which was most resistant to BKD comprised only a smal! portion of the total population. Either there were other factors which were more important, or there were negative traits associated with BKD resistance.

Since the incidence of BKD in individual stream systems varies so widely, it is likely that the impact of BKD must be evaluated on a caseby-case basis. In systems where the carrier rate is less than $10 \%$, BKD is detrimental to individual fish, but its impact on the population as a whole is probably small. At higher carrier rates, the negative influence may be more noticeable.
Some degree of prevention in hatcheries is possible using standard iodophor disinfections of fish eggs. Corynebacterium salmonis is sensitive to these compounds (Smith 1964) and any bacteria clinging to the external surfaces of the eggs should be killed. Bullock et al. (1978) reported that iodophor disinfection did not eliminate the disease but reduce its severity. Apparently, some bacteria are transmitted within the egg and cannot be reached by surface disinfection.

Treatment of BKD epizootics has always been difficult, probably because the bacteria may occur intracellularly. In the past, sulfonamides were used to control BKD epizootics but the disease usually reappeared once the treatments were stopped. Wolf and Dunbar (1959) tested the efficacy of 34 agents for controlling BKD and found that erythromycin was the drug of choice. Recently, Klontz (1978) has reported considerable success in treating BKD through use of the water soluble form erythromycin phosphate. Prespawning adult salmon losses were prevented with subcutaneous injections of erythromycin phosphate at $11.0 \mathrm{mg} / \mathrm{kg}$ body weight. Juvenile losses were prevented by water hardening freshly spawned eggs for 30 minutes in a 1-2 $\mathrm{mg} / \mathrm{L}$ solution of erythromycin phosphate. However, the use of this chemical has not been approved by the Food and Drug Administration in fish to be used for human consumption. Several groups are actively seeking an exemption from this restriction for newly fertilized fish eggs.

Control through either acquired or genetic immunity is not an available option. Evelyn (1971) showed that salmonids do form antibodies against c. salmonis, but the level of response was highly variable. Considerable work is still necessary to determine the proper methods of an- 
tigen preparation and presentation before a BKD vaccine becomes a reality. The work of Suzumoto et al. (1977) indicated that there may be some genetic basis to BKD resistance, but much more work is needed to determine the specific factors involved, and whether or not they are linked to other undesirable traits. For the present, control of BKD will depend on rigid sanitation at hatchery facilities, disinfection of all cultured lots of fish eggs, and restriction of the movement of infected fish.

\section{$\underline{\text { References }}$}

AMERICAN FISHERIES SOCIETY. 1979. Fish Health Blue Book--Procedures for the Detection and Identification of Certain Fish Pathogens. D. MCDANIEL, ed. Rev. ed. Amer. Fish. Soc. Fish. Health Section. $118 \mathrm{pp}$.

BELDING, D. L. and B. MERRILL. 1935. A preliminary report upon a hatchery disease of the Salmonidae. Trans. Am. Fish. Soc. 65:76-84.

BULLOCK, G. L., D. A. CONROY and $S$. F. SNIESZKO. 1971. Book 2A: Bacterial diseases of fishes. pp. 1-51. In: Diseases of Fishes. S. F. SNIESZKO and H. R. AXELROD, eds. TFH Publications, Inc., Neptune, NJ.

BULLOCK, G. L. and H. M. STUCKEY. 1975. Fluorescent antibody identification and detection of the Corynebacterium causing kidney disease of salmonids. J. Fish. Res. Board Can. 32:2224-2227.

BULLOCK, G. L., H. M. STUCKEY and P. K. CHEN. 1974. Corynebacterial kidney disease of salmonids: Growth and serological studies on the causative bacterium. Appl. Microbiol. 28:811-814.

BULLOCK, G. L., H. M. STUCKEY and D. MULCAHY. 1978. B.K.D. vertical transmission. AFS Fish Health Section Newsletter 6:1-2.
CHEN, P. K., G. L. BULLOCK, H. M. STUCKEY and A. C. BULLOCK. 1974. Serological diagnosis of corynebacterial kidney disease of salmonids. J. Fish. Res. Board Can. 31:1939-1940.

EVELYN, T. P. T. 1971. The agglutinin response in sockeye salmon vaccinated intraperitoneally with heat-killed preparation of the bacterium responsible for salmonid kidney disease. J. Wildl. Dis. $7: 328-385$.

- 1977. An improved growth medium for the kidney disease bacterium and some notes on using the medium. Bull. Off. Int. Epiz. $87: 511-513$.

1978. Sensitivities of bacterial kidney disease detection methods with special remarks on the culture method. pp. 15-16. In: Proc. Joint 3rd Biennial Fish Health Section and 9th Annual Midwest Fish Disease Workshops, Kansas City, Mo.

KLONTZ, G. W. 1978. Prevention of bacterial kidney disease in adult and juvenile salmonids. pp. 11-14. In: Proc. Joint 3rd Biennial Fish Health Section and 9 th Annual Midwest Fish Disease Workshops, Kansas City, MO.

MACKIE, T. J., J. A. ARKWRIGHT, T. E. PRYCE-TANNATT, J. C. MOTTRAM, W. D. JOHNSTON and W. J. M. MENZIES. 1933. Second interim report of the Furunculosis Committee. HM Stationery Office, Edinburgh. 65 $\mathrm{pp}$.

MITCHUM, D. L., L. E. SHERMAN and G. T. BACTER. 1979. Bacterial kidney disease in feral populations of brook trout (Salvelinus fontinalis), brown trout (Salmo trut$\underline{\mathrm{ta}}$ ), and rainbow trout (Salmo gairdneri). J. Fish. Res. Board Can. 36:1370-1376.

ROSS, A. J. and C. A. SMITH. 1972. Effect of two iodophors on bacterial and fungal fish pathogens. J. Fish. Res. Board Can. 29:1359-1361. 
SANDERS, J. E. and J. L. FRYER. 1978. Corynebacterium salmonis sp. nov. The causative agent of bacterial kidney disease; selected biochemical properties and pathogenesis in salmonid fishes. pp. 28-33. In: Proc. Joint 3rd Biennial Fish Health Section and 9th Annual Midwest Fish Disease Workshops, Kansas City, MO.

- 1980. The taxonomic position of the causative agent of bacterial kidney disease. Fish. Health News. U.S. Fish Wildl. Serv. 9:vii.

SANDERS, J. E., S. PILCHER and J. L. FRYER. 1978. Relation of water temperature to bacterial kidney disease in coho salmon (Oncorhynchus kisutch), sockeye salmon (0. nerka), and steelhead trout (Salmo gairdneri). J. Fish. Res. Board Can. 35:8-11.

SMITH, I. W. 1964. The occurrence and pathology of Dee disease. Freshwater and Salmon Fish. Res. $34: 1-12$.

SUZUMOTO, B. K., C. B. SCHRECK and J. D. MCINTYRE. 1977. Relative resistances of three transferrin genotypes of coho salmon (Oncorhynchus kisutch) and their hematological responses to bacterial kidney disease. J. Fish. Res. Board Can. 34:1-8.

WARREN, J. W. 1963. Kidney disease of salmonid fishes and the analysis of hatchery waters. Prog. Fish Cult. 25:121-131.
WEDEMEYER, G. A. and A. J. ROSS. 1973. Nutritional factors in the biochemical pathology of corynebacterial kidney disease in the coho salmon (Oncorhynchus kisutch). J. Fish. Res. Board Can. 30:296-298.

WOLF, K. and C. E. DUNBAR. 1959. Test of 34 therapeutic agents for control of kidney disease in trout. Trans. Am. Fish. Soc. $88: 117-124$.

1959. Methods of infecting trout with kidney disease and some of the effects of temperature on experimental infections. U.S. Fish Wildl. Spec. Sci. Rep. Fish. 286. $8 \mathrm{pp}$.

WOOD, E. M. and W. T. YASUTAKE. 1956. Histopathology of kidney disease in fish. Am. J. Pathol. 32:845-857.

WOOD, J. W. 1974. Diseases of Pacific Salmon: Their Prevention and Treatment. 2nd ed. and treatment. Dept. Fish., Hatchery Div. State of Washington, Olympia, WA. $82 \mathrm{pp}$.

WOOD, J. W. and J. WALLIS. 1955. Kidney disease in adult chinook salmon and its transmission by feeding to young chinook salmon. Research Briefs, Fish. Comm., OR. $6: 32-40$.

YOUNG, C. L. and G. B. CHAPMAN. 1978. Ultrastructural aspects of the causative agent and renal histopathology of bacterial kidney disease in brook trout (Salvelinus fontinalis). J. Fish. Res. Board Can. 35:1234-1248. 


\title{
Bacterial Septicemias Caused by Aeromonas and Pseudomonas
}

\author{
Jill E. Follett
}

\section{Introduction}

Septicemias in freshwater caused by bacteria of the fishes Aeromonas and Pseudomonas are common worldwide. These Gram negative, oxidase positive bacteria are generally saprophytic, but may invade fish as opportunistic pathogens. Infections tend to be low grade with a low level of mortalities. Usually, infections are precipitated by some environmental stress, or a previous infection with a pathogenic bacterium or a protozoan parasite.

\section{History}

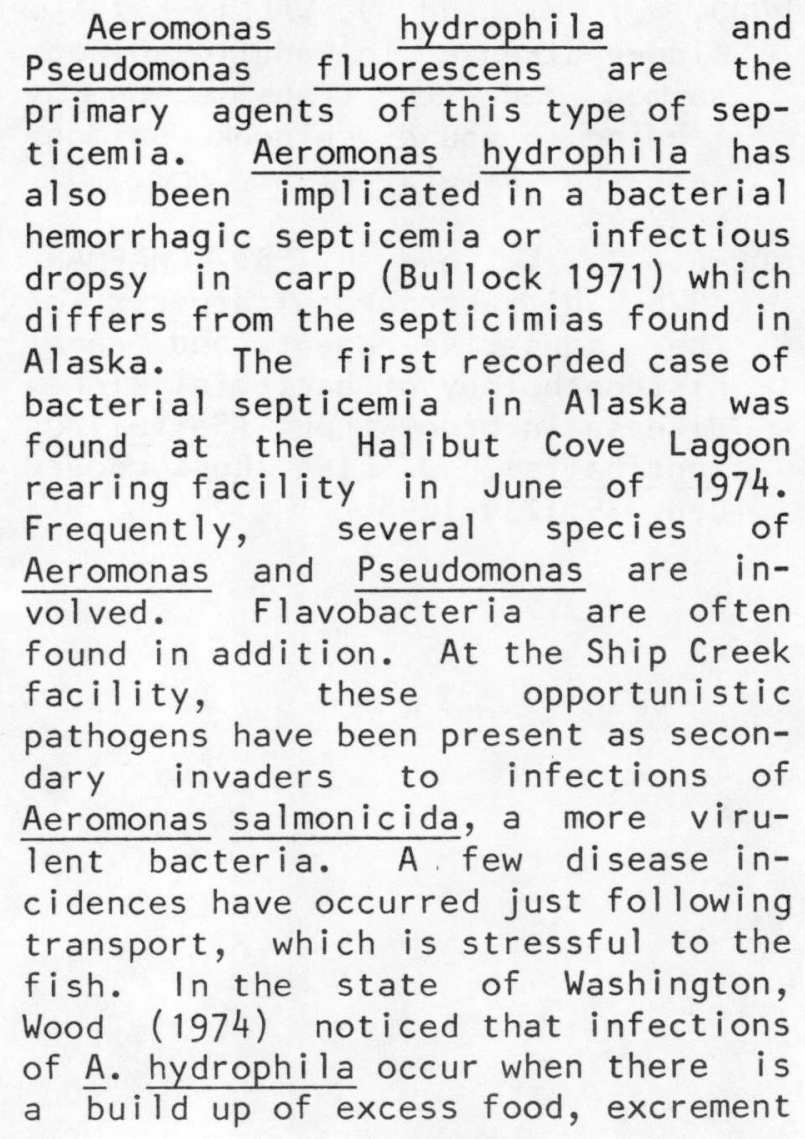

and dead fish followed by cleaning of the ponds. The cleaning exposes fish to high levels of bacteria which have been allowed to multiply in the organic debris.

\section{Transmission}

Aeromonads and pseudomonads occur naturally in the water especially when it is high in organic matter. Accumulation of excess food, excrement and dead fish allow ideal breeding places for the bacteria. When the opportunity arises, they will invade the fish through the intestinal tract or external lesions. Bullock (1965) was able to experimentally infect goldfish (Carassius auratus) with a Pseudomonas only after a few scales were removed, allowing access to the interior of the fish. Once an infection is established, the infected fish probably transmit organisms to the non-infected ones.

\section{Signs and Pathogenesis}

The establishment of an aeromonad or pseudomonad septicemia is almost always precipitated by some other stress factor such as poor environment, handling, or pre-existing infection of some other type. Once established, the pathogenesis is similar to that of other Gram negative bacteria. Endotoxins released from cell walls upon autolysis of dead cells cause the disease process. The signs will not differentiate whether the septicemia is caused by an aeromonad or pseudomonad. 
Frequently, in these low grade infections there are no signs, just a low level of mortalities. Infected fish may be lethargic 2 to 3 days prior to death and gather in quiet areas of the ponds or raceways. Internally, capillaries break down in the body cavity and organs.

Bloody or ascitic fluid may be present in the body cavity. As the disease progresses, hemorrhages in the body wall can be seen internally and occasionally externally. Bloody fluid may be extruded from the vent if pressure is applied. The kidney may be soft, the spleen and other organs pale. Other signs that may be present include hemorrhaging at the base of fins, pale gills and exopthalmia (protuding eyes) (Snieszko and Bullock 1976). Signs and pathology resemble those of other septicemias caused by Gram negative bacteria.

\section{Diagnosis}

This disease occurs primarily in freshwater. Definitive diagnosis can be made on the basis of signs, and isolation and identification of the etiologic agents. Since signs may be few and resemble those of other diseases, usually the causative agent must be isolated.

Isolation of the agents can be done on blood or tryptic soy agar (TSA). If the isolate is a short to medium, Gram negative, oxidase positive, motile rod, it can be presumptively identified as a pseudomonad or aeromonad. Occasionally 1 of these will be non-motile (Bullock 1965). Further differentiation is based on biochemical tests. Biochemical characteristics of $\underline{A}$. hydrophila include glucose fermentation, production of 2,3-butanediol and starch hy- drolysis. It is the only known 2,3-butanediol positive organism in the aquatic environment. Until recently, it was named $\underline{A}$. liquefaciens and will be frequently seen as such in the literature. Biochemical characteristics of $P$. fluorescens include oxidative or alkaline reaction in glucose, production of fluorescent pigment and no starch hydrolysis. The greenish-yellow fluorescent pigment is usually visible in daylight in cultures on TSA within a week. It is elicited by media containing $0.15 \%$ each of dipotassium phosphate and magnesium sulfate. Examination with ultraviolet light should reveal the presence of fluorescing pigments. 0 ther aeromonads and pseudomonads may be isolated from septicemias. Species of the former produce a fermentative reaction, and the latter an oxidative or alkaline reaction, in Hugh and Leifson's O/F medium (see section on Furunculosis). Additional methods for identification are described by Bullock (1961, 1965).

\section{Significance and Control}

\section{Significance}

Presently, this disease is responsible for losses at several hatcheries at various times. Since the organisms are naturally occurring, it is not feasible to completely eliminate them. Losses are not usually large and can be reduced by proper fish culture. Wild fish are not likely to become naturally infected unless conditions became unfavorable. The organisms can also cause human infections in a weakened host, but is is unlikely to be transmitted from fish. 


\section{Control}

Prevention. Prevention is a matter of good fish culture practices. Ponds should be cleaned regularly and dead fish picked daily. Handling stress should be minimized. Eliminate reservoirs of infection which allow bacteria to multiply. Prophylactic treatment prior to tagging or transporting may diminish losses. To reduce hauling stress, calcium chloride can be added to the transport water.

Treatment. Treatment is done with oxytetracycline (OTC) at a dosage of $1 \mathrm{~g} / \mathrm{kg} \mathrm{fish/day/15}$ days. Occasionally, organisms resistant to OTC will be isolated. In these cases, treatment can be done with sulfamethazine at $1.1 \mathrm{~g} / \mathrm{kg}$ fish/day/15 days (R. Grischkowsky, pers. comm.).

\section{References}

BULLOCK, G. L. 1961. The identification and separation of Aeromonas liquefaciens from Pseudomonas fluorescens and related organisms occurring in diseased fish. Appl. Microbiol. 9:587-590.
- 1965. Characteristics and pathogenicity of a capsulated Pseudomonas isolated from goldfish. Appl. Microbiol. 13:90-92.

1971. Identification of fish pathogenic bacteria, Book 2B. pp. 1-41. In: Diseases of Fishes. S. F. SNIESZKO and H. R. AXELROD, eds. TFH Publications, Neptune, NJ.

BULLOCK, G. L., D. A. CONROY and S. F. SNIESZKO. 1971. Bacterial diseases of fishes, Book 2A. pp. 1-51. In: Diseases of Fishes. S. F. SNIESZKO and H. R. AXELROD, eds. TFH Publications, Neptune, $\mathrm{NJ}$.

SNIESZKO, S. F. and G. L. BULLOCK. 1976. Diseases of freshwater fishes caused by bacteria of the genera Aeromonas, Pseudomonas, and Vibrio. U.S. Fish and Wildl. Serv. Fish. Dis. Leaflet No. 40. $10 \mathrm{pp}$.

WOOD, J. 1974. Diseases of Pacific Salmon: Their Prevention and Treatment. State of Washington, Department of Fisheries, Hatchery Division, WA. $82 \mathrm{pp}$. 


\title{
Bacterial Gill Disease
}

\author{
Jill E. Follett and Roger S. Grischkowsky
}

\section{Introduction}

Bacterial gill disease is characterized by proliferation of gill epithelium and presence of myxobacteria on the gills. It is common in cultured salmonids and found occasionally in warmwater fish. Several types of myxobacteria may be isolated from the gills but a causative organism is not always found. Although other bacteria such as Pseudomonas spp. and columnaris organisms can cause gill disease, the term bacterial gill disease usually refers to that caused by miscellaneous myxobacteria. Apparently, it is precipitated by poor environmental conditions and stress. Death is caused by fish being unable to get adequate oxygen due to gill clubbing, or to the massive amounts of bacteria on the gills.

\section{History}

Bacterial gill disease was first described by Davis (1926, 1929) as a disease of trout (Salvelinus fontinalis and Salmo gairdneri) in Vermont. It has since been found to affect all species of salmonids throughout the world. Warmwater fishes such as bass (Micropterus sp.) and crappie (Pomoxis sp.) have also been affected by a similar disease (Davis 1953).

Historically, gill disease in which myxobacteria were found was called the eastern or bacterial type. By definition, no bacteria were found in the western type (Rucker et al.
1952). It is now known to be caused by a deficiency of pantothenic acid and is referred to as nutritional gill disease. By 1935, a western type of bacterial gill disease was found, thus eliminating the need for geographical identification. The use of vitamin fortified diets has virtually eliminated the occurrence of nutritional gill disease. Several other agents, both infectious and non-infectious, can have detrimental effects on gills.

\section{Alaska}

The first report of bacterial gill disease in Alaska occurred in coho (Oncorhynchus kisutch) at Fire Lake Hatchery in May 1975. The rising water temperature was suspected as being partially responsible. During 2 weeks in May of 1976, mortalities due to bacterial gill disease reached $25 \%$ in a lot of coho being held at Starrigavan rearing facility. Conditions which led to the outbreak included the feeding of starter mash and overcrowding of the fish in plastic wading pools. Treatment with Diquat $^{\mathrm{a}}$ and spreading out the fish reduced mortalities.

Another outbreak occurred at Big Lake Hatchery in chinook salmon (Oncorhynchus tshawytscha) during the fall of 1976. Several additional incidences have occurred at the Fire Lake facility, most probably due to the eutrophic water supply.

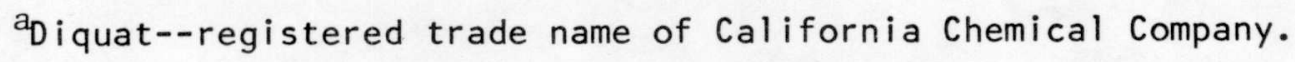




\section{Transmission}

Since myxobacteria are commonly found in soil and water, transmission initially occurs from water to fish. After an outbreak has begun, fish to fish horizontal transmission occurs as the disease spreads.

Bacterial gill disease has been successfully induced by exposing fingerling trout ( $\underline{S}$. fontinalis or $\underline{S}$. gairdneri) to stress factors (Bullock 1972). These factors were a combination of crowding, high levels of ammonia, low levels of DO (dissolved oxygen) and presence of particulate matter. The stressed fish were probably exposed to additional unfavorable factors which were unknown. Use of only 1 factor did not induce the disease.

Attempts to transmit bacterial gill disease with cultures of myxobacteria or mixed eubacteria, or by using dead, diseased trout have not been successful. However, the disease has been transmitted when live diseased trout ( $\underline{s}$. fontinalis or s. gairdneri) were added to a tank of stressed fingerlings (Bullock 1972). Transmission occurred more rapidly than induction; in 3 to 5 days as opposed to 11 days. Bullock suggested that bacterial cultures failed to cause gill disease because they may have lost their virulence upon subculture. The failure of dead, diseased trout to transmit disease could possibly be attributed to an overgrowth of saprophytic bacteria on the gills of dead trout which masked the effect of any pathogenic form.

Bacterial gill disease is primarily an environmental disease and secondarily a bacterial disease. When $f i s h$ are stressed by unfavorable conditions such as low DO, crowding or eutrophication and the bacteria are favored, an outbreak may be precipitated. Most outbreaks occur in the springtime when fish are ac- tively growing and these conditions are likely to exist. The use of chemicals is an additional factor. A scenario proposed for a disease outbreak is that formalin treatment of eggs to control fungus will coagulate protein (Klontz 1979) and mucus on the gills of the alevins causing epithelial proliferation and gill clubbing. Additionally, fry are treated with chemicals such as quarternary ammonium germicides and Diquat which increase chemical clubbing. If fish are overfed with mash, this will increase suspended solids leading to eutrophication, increased biological oxygen demand, decreased oxygen and increased ammonia. These conditions favor the pathogen, not the host, and an outbreak will be precipitated.

The incubation period ranges from 3 to 7 days. Yearlings and older fish are less susceptible than fry. Hatchery fish develop a low antibody titer against myxobacteria which protects them from the disease (Snieszko 1970).

\section{Signs and Pathogenesis}

\section{Behavior}

The first indication of bacterial gill disease is that the fish cease feeding. They tend to be lethargic, pale and to ride high in the water. Frequently, affected fish will line up against the current near the water inlet in an attempt to obtain additional oxygen. Irrigation of the gills usually increases. The gill cover or operculum may be abnormally extended. Epizootics can be explosive with losses exceeding $50 \%$ in 24 hours (McDaniel 1979).

\section{Gross Pathology}

Upon gross examination of the gill, excessive mucus secretion is 
usually seen. Gills may appear swollen, congested and deeper red than normal. In severe cases, the gill may become infected with fungus (Davis 1953).

\section{Microscopic Pathology}

A normal salmonid gill is shown in Figure 7. When gills are infected, they appear clubbed and the filaments fused, especially at the distal ends (Fig. 8). Long, slender myxobacteria can be seen in wet mounts or stained preparations (Fig. 9). Hyperplasia of the gill lamellae begins at the distal end and may extend inward as opposed to nutritional gill disease where hyperplasia begins at the base of the lamellae and extends outward (Amlacher 1970). Some authors (Rucker et al. 1952, Wood and Yasutake 1959) have also described a "plaque" which appears to be a developmental feature in bacterial gill disease. It consists of single, globuse cells projecting from the lamellae. Frequently, these cells are filled with intensely basophilic granules. As the hyperplasia develops, the cells either disappear or are obscured by other cell types. An increase in the typical filamentous bacteria occurs at this point.

\section{$\underline{\text { Diagnosis }}$}

Bacterial gill disease is diagnosed by the microscopic examination of gills revealing hyperplasia, swelling, clubbing, fusing of filaments and lamellae, and the presence of large numbers of myxobacteria. Since several different morphological types of myxobacteria have been isolated from gill disease, more than 1 species may be involved in causing the disease (Bullock 1972, Wood 1974). Myxobacteria associated with gill disease differ immunologically from those associated with columnaris disease, and probably also from those causing cold water disease (Snieszko 1970), although Flexibacter columnaris has been implicated ingill disease (Klontz 1979). General characteristics of the myxobacteria include long, thin Gram negative rods (about 0.5 um $\times 5-10$ um) which may exhibit a swinging motion when attached to gill surfaces, or lie flat on the gill surface in parallel whorls. They may also appear in clumps or haystacks, or in a continuous layer lying side by side in some cases (Davis 1953). Infected tissue can be crushed and stained to reveal the masses of bacteria.

Myxobacteria are difficult to grow in culture, but can be isolated on semi-solid media (Anacker and Ordal 1959). Aerobic incubation at $25 \mathrm{C}$ is preferred. Yellow or white colonies with spreading edges are formed. No microcysts or fruiting bodies are produced. Myxobacteria may exhibit creeping motility on surfaces.

\section{$\underline{\text { Significance and } \text { Control }}$}

\section{Significance}

Occurrence of bacterial gill disease can cause a large percentage of losses in a short time. It is endemic in some hatcheries. Poor environmental conditions weaken the fish, making them unable to resist infection by the pathogen. When the disease occurs, it indicates that good fish culture practices are not being followed. If this is a common occurrence at a facility, the culture techniques and water quality should be analyzed.

\section{Control}

Prevention. Since bacterial gill disease is primarily environmental and secondarily bacterial, it can be prevented by proper environmental conditions. One precipitating factor 


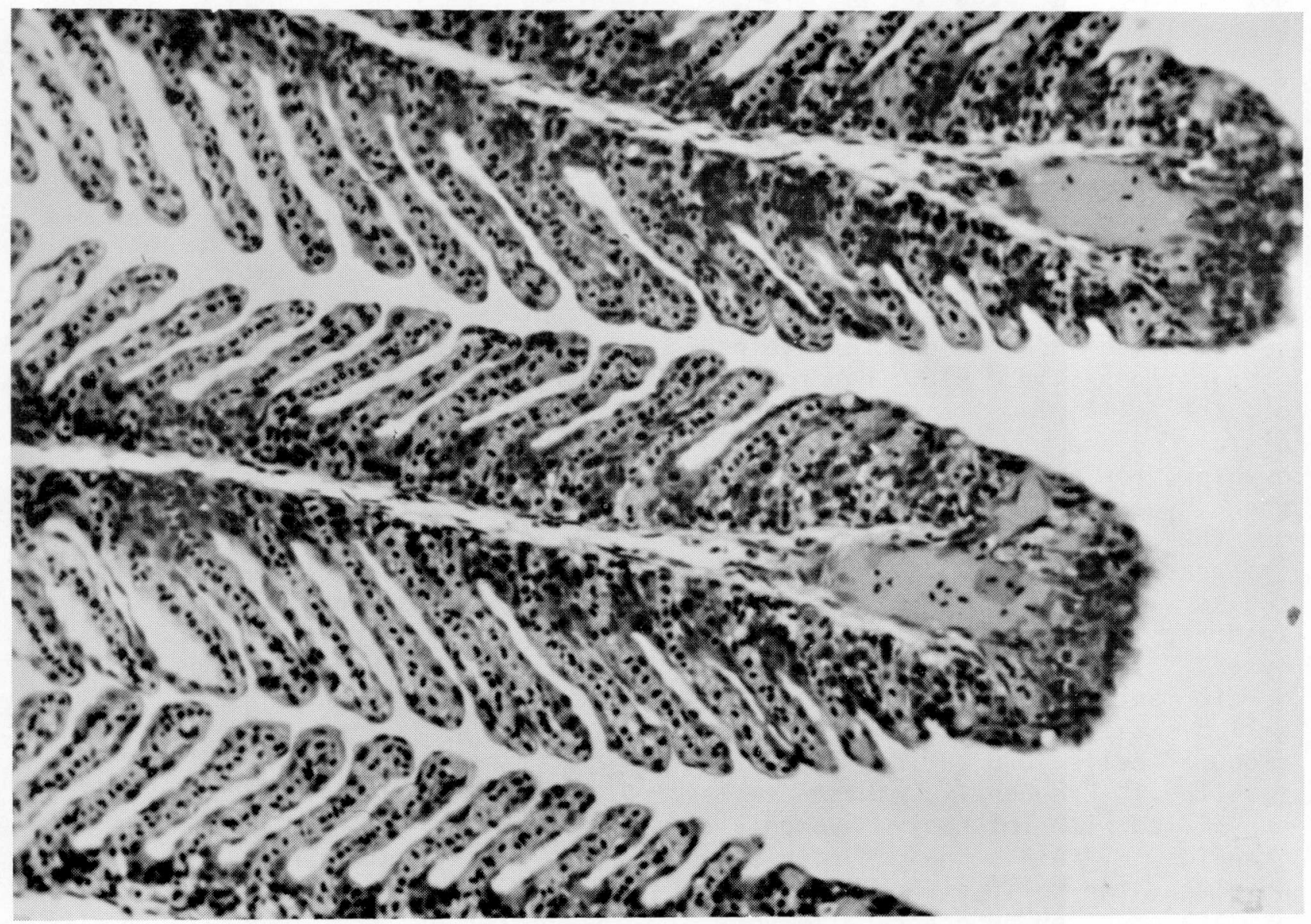

Figure 7. A normal salmonid gill. Hematoxylin-eosin (H E E) stain (100X) (R. Grischkowsky). 


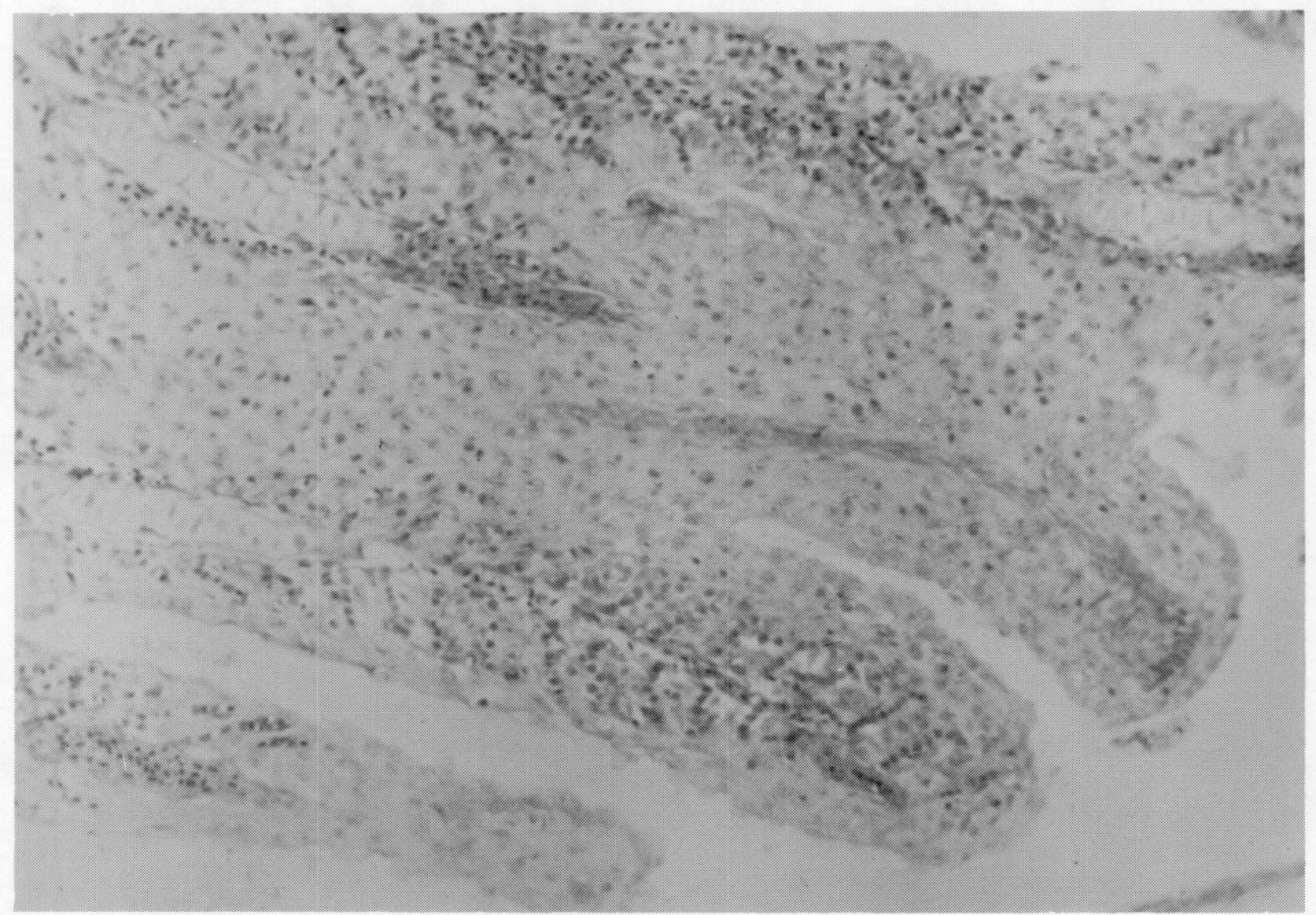

Figure 8. A salmonid gill showing fusing of the gill filaments. HE E stain (100X) (W. Yasutake). 


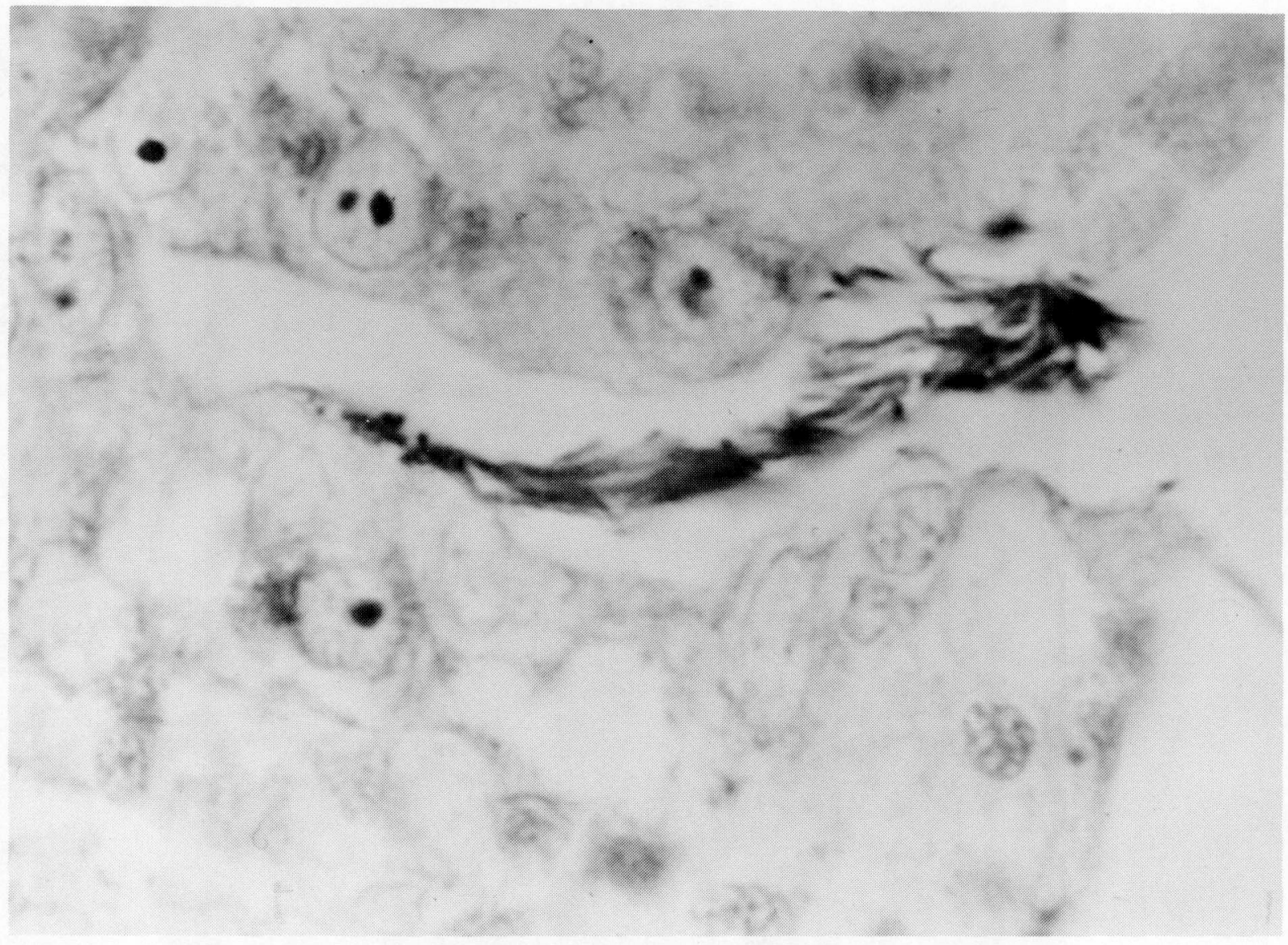

Figure 9. Bacterial gill disease. Long, dark organisms are myxobacteria in an infected gill (450X) (W. Yasutake). 
is crowding. Wood (1974) found that nearly all outbreaks in chinook salmon were associated with pond feeding levels approaching .02L kg of feed/day/L/minute inflow. Coho appeared to be more resistant in a similar rearing environment. He recommended keeping pond feeding levels below .014-.018 kg feed/day/L/minute for chinook. Spreading out feeding times will also help to prevent high accumulation of metabolites.

Another way to prevent gill disease is by using a water supply free of silt, mud and organic debris. If refuse water is used, it should be filtered, the ammonia oxidized, and water current sufficient to remove excess food and feces.

Excessive use of chemicals such as formalin, Diquat and quaternary ammonium compounds should be avoided as they can cause gill clubbing and allow the bacteria to become established in the gills. Overfeeding with mash can also be detrimental to the gills.

Treatment. Treatment will depend on what precipitated the outbreak. In every case, steps should be taken to increase the $D O$ and reduce suspended solids within the water. If fish are crowded, they should be spread out and water flows increased. If $\mathrm{fish}$ have been on mash, feeding should cease for a few days and tanks cleaned to remove excess food and debris. The source of gill irritation must be removed before the gills will heal. The use of salt dips can be helpful, and may be better than toxicity caused by some chemicals.

Chemicals have been frequently utilized for treatment. Borg (1960) reported a considerable degree of control from a quaternary ammonium compound. Unfortunately, this only treats the signs and unless the causes are addressed, the disease will reoccur. Also, excessive use of chemicals will cause further gill clubbing. Chemical treatments which appear to be effective are Diquat baths (2-4 ppm Diquat cation) for 1 hour on 3 to 4 consecutive days; benzalkonium or benzethonium chlorides at $2 \mathrm{ppm}$ active ingredient for 1 hour on 3 to 4 (Wood 1974), and a quaternary ammonium compound at $4 \mathrm{ppm}$ for 1 hour daily not to exceed 3 days during any 14 days (Klontz 1979). Diquat treatments destroy ATPase required for smoltification and should not be used within 30 days of saltwater release. They appear to be toxic to coho salmon. Potassium permanganate baths at $2 \mathrm{ppm}$ for 1 hour on 1 to 3 days may be more effective than either of the above, but some problems may be associated with its use. Its toxicity and efficacy will vary with water quality and temperature. Anytime a new treatment is tried, it should be tested with a few fish before application to the entire lot. One other method of control is copper sulfate dips. A 1 minute dip at $500 \mathrm{ppm}$ is utilized. This disease is best controlled by prevention rather than resorting to chemicals.

\section{References}

AMLACHER, E. 1970. Textbook of Fish Diseases. T.F.H. Publications, Neptune, NJ. 302 pp.

ANACKER, R. L. and E. J. ORDAL. 1959. Studies on the myxobacterium Chondrococcus columnaris. I. Serological typing. J. Bacteriol. 78:25-32.

BORG, A. F. 1960. Studies on myxobacteria associated with diseases in Salmonid fishes. Wildl. Dis. 8:1-85, 2 microcards.

BULLOCK, G. L. 1972. Studies on selected myxobacteria pathogenic for fishes and on bacterial gill disease in hatchery-reared salmonids. U.S. Fish and Wildl. Serv. Tech. Paper No. 60. 29 pp. 
DAVIS, H. S. 1926. A new gill disease of trout. Trans. Amer. Fish. Soc. 56:156-160.

- 1927. Further observations on the gill disease of trout. Trans. Amer. Fish. Soc. $57: 210-212$.

- 1953. Culture and Diseases of Game Fishes. Univ. Calif. Press, Berkeley, CA. 332 pp.

KLONTZ, B. 1979. Fish Health Management Vol. IT. Fish. Resources and off. of Continuing Ed. Univ. Idaho, ID. 142 pp. - 1979. New insights into respiratory diseases of salmonids. Northwest Fish Culture Conf., Portland, OR.

MCDANIEL, D. 1979. Fish Health Blue Book. Procedures for the Detection and Identification of Certain Fish Pathogens. Amer. Fish. Soc. $118 \mathrm{pp}$.
RUCKER, R. R., H. E. JOHNSON and G. M. KAYDAS. 1952. An interim report on gill disease. Prog. Fish-Cult. 14:10-14.

SNIESZKO, S. F. 1970. Bacterial gill disease of freshwater fishes. U.S. Dept. Interior, Fish and Wildl. Serv., Fish Dis. Leaflet No. 19. $4 \mathrm{pp}$.

WOOD, E. M. and W. Y. YASUTAKE. 1959. Histopathology of Fish: V. Gill Disease. Prog. Fish-Cult. $19: 7-13$.

WOOD, J. W. 1974. Diseases of Pacific Salmon: Their Prevention and Treatment. 2 2nd ed. State of Washington Department of Fisheries. Hatchery Division Manual. $82 \mathrm{pp}$.

\title{
Cold-Water Disease
}

\author{
Roger S. Grischkowsky
}

\section{Introduction}

Cold-water disease, also known as peduncle disease and low temperature disease (Pacha and Ordal 1970) is a disease of coho salmon (Oncorhynchus kisutch), chinook salmon (Oncorhynchus tshawytscha), sockeye salmon (Oncorhynchus nerka) and rainbow trout (Salmo gairdneri) (Rucker et al. 1953). Primarily affected are coho salmon juveniles. It is typically caused by myxobacteria of the Cytophaga psychrophila type. Initial infections of the dorsal and peduncle area worsen until large lesions occur or even the peduncle falls off or the vertebral column becomes visible. Low water tempera- ture is involved in the onset and continuation of this fatal condition. The agent causes an acute septicemia in gill respiratory cells, kidney lesions and renal tubule necrosis (Wood and Yasutake 1956). Cytophaga psychrophila is a relatively new designation and has an uncertain generic designation. It is probably a Flexibacter but characterization of more isolates is necessary (Buchanan and Gibbons 1974). The known disease distribution in the continental U.S.A. is predominantly in the Northwest (American Fisheries Society 1979) and in Alaska. 


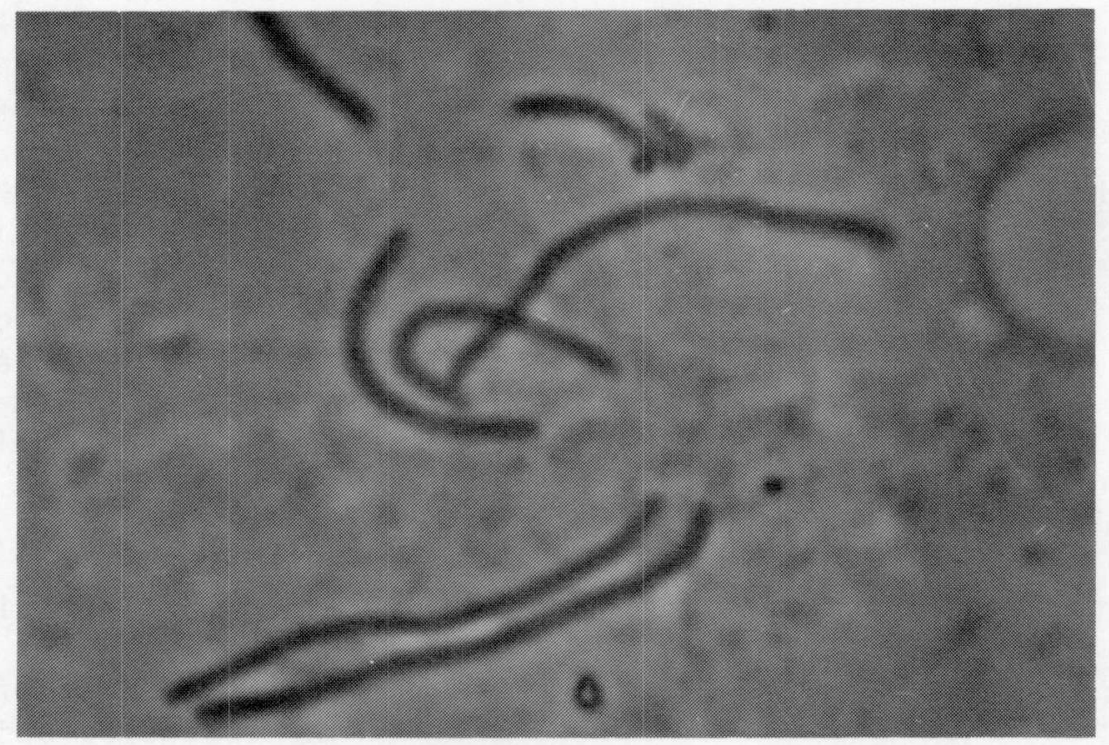

Figure 10. Myxobacteria from coho (Oncorhynchus nerka) lesions (phasecontrast 2000X) (R. Grischkowsky).

\section{$\underline{\text { History }}$}

Cold-water disease was first reported in 1946 (Davis 1970) in juvenile rainbow trout within a West Virginia hatchery. The name c. psychrophila was assigned in $1 \overline{9} 60$ as a new species because of its low temperature growth capability, and the ability to kill fish under cold conditions (Borg 1960). That finding resulted from an outbreak in coho salmon in Washington state.

In Alaska, cold-water disease was found by the author during the winter of 1973-1974 affecting coho salmon at Halibut Cove Lagoon. These were juveniles in estuarine rearing pens at super cooled water temperatures approximately $0.5 \mathrm{C}$. Severe peduncle erosion resulted in mortality of approximately half of the fish during the coldest portion of the winter. Myxobacteria were present in lesions as were other bacilli and cocci. Figure 10 displays myxobacteria isolated from such an outbreak in Alaska. 
Transmission

Cold-water disease is transmitted waterborne or from fish to fish (Bullock and Snieszko 1970). Transmission occurs both in freshwater and seawater.

$\underline{\text { Signs }}$ and Pathogenesis

\section{Signs}

Tail darkening, loss of dermis or lesions on the dorsal, or posterior surfaces for fish under low temperature conditions are characteristic of cold-water disease. Fish affected will not be feeding as is also typical with most other fish diseases.

\section{Pathogenesis General}

The condition begins with a whitening of the adipose fins, discoloration proceeds to the base and to the posterior end of the caudal peduncle. A color change to dark brown of the peduncle and disappearance, erosion, of the adipose fins occur until the dermis on the entire peduncle is broken down exposing musculature. Eventually the vertebral column is exposed, and the caudal fin falls off if the fish does not die first. Within muscle tissue, hemorrhage occurs breaking down blood vessels and releasing bacteria promoting waterborne transmission (Davis 1970). Inflammation and swelling contribute to the musculature destruction. Yolk sacs of alevins may be ruptured by direct bacterial action (Bullock and Snieszko 1970).

\section{Temperature Considerations}

The optimum temperature for lab growth of $\mathrm{C}$. psychrophila is $15-20 \mathrm{C}$ whereas the optimum temperature for the disease is 4-10C (Wood 1974). The suggested reason for this difference is that fish are less resistant at $10 \mathrm{C}$ or lower, and young fish are less resistant than older fish. By the time the water temperature is higher than $10 \mathrm{C}$ the $\mathrm{fish}$ are larger and more resistant. Temperature increases, once infection is established, to more than $20 \mathrm{C}$ do not reduce mortalities (Wood 1974). The disease typically develops at 7-10C with an incubation period of less than 10 days (Bullock and Snieszko 1970). In this state the infection is generally a $4 \mathrm{C}$ or lower occurrence.

\section{Stages of Infection}

A chronic form of infection results in lordosis and scoliosis while the acute form, (more typical) includes lesions, darkening and mortality (American Fisheries Society 1979). Losses are inversely proportional to the stage of development of the fish. The most susceptible time for fish infection is 2 to 3 weeks within the time of yolk absorption (Wood 1974).

\section{Diagnosis}

Cold-water disease is best presumptively diagnosed by the presence of lesions in susceptible species under low temperature conditions, and by the presence of myxobacteria in these lesions as quickly determined by phase-contrast examination of a wet mount or by Gram stain of lesion scrapings. The agent's cellular morphology is characteristic: long thin rods of uniform width usually curved with blunt ends (see Fig. 1). The size of these rods is about $.75 \times 1.5-5$ um (Davis 1970, Pacha and Ordal 1970). They appear essentially non-motile in wet mount and Gram negative. 
Culture attempts from lesions are sometimes unsuccessful. Reliance upon cultures should not be made due to recovery difficulties. The lesion, a complex entity, is impossible to duplicate in the lab. Dilute semi-solid media can be used to grow most myxobacteria. The presence of some seawater will enhance the growth of many of these bacteria, and should routinely be added when fish held under estuarine conditions are being investigated. Colonial morphology of c. psychrophila includes yellow, translucent colonies that are spreaders on a cytophaga agar (American Fisheries Society 1979). These should exhibit a twitching type motion on semi-solid agar or moist plates. This motility is historically termed "gliding." The organism is aerobic and grows readily at $4-23 \mathrm{C}$ in $.8 \% \quad \mathrm{NaCl}$ but not $2 \%$ (Pacha and Ordal 1970).

Confirmatory diagnosis can be made using a rapid slide agglutination test using a prepared antiserum, or by performing biochemical testing to determine a pattern essentially identical with $\underline{C}$. psychrophila (American Fisheries Society 1979). These results include sensitivity to chlortetracycline, bacitracin, dihydrostreptomycin, erythromycin, neomycin, penicillin and oxytetracycline (OTC). Isolates are proteolytic and degrade gelatin, casein albumen and also tyrosine (American Fisheries Society 1979). Generally the presumptive testing is all that's necessary. Techniques sensitive enough to detect asymptomatic carriers may be unavailable (American Fisheries Society 1979).

\section{Significance and Control}

\section{Significance}

The disease can be quite serious affecting as much as $50 \%$ of juvenile coho during a winter season (Pacha and Ordal 1970). In Southcentral Alaska, the disease limits overwintering of coho in estuarine rearing pens. Mortalities are low level but consistent, amounting to major portions of cultured fish. Afflicted fish do not recover from the acute stage.

\section{Control}

Temperature. Temperature increases to $12.8 \mathrm{C}$ have resulted in a sharp decline in mortality, and an inability to isolate the organism after 1 week according to 1 publication (Rucker et al. 1953). Placing fish unaffected under warmer conditions should increase their resistance.

Removal of affected fish. Culling of obviousiy affected fish as they will not recover, will reduce the reservoir of infection and should reduce both waterborne and $\mathrm{fish}$ to fish transmission.

Chemotherapy. Effective chemical treatment of this condition is difficult. Its presence internally and externally makes the task rigorous. To treat the septicemia, a therapeutic blood level must be reached. Lesions can be treated primarily by topical application of concentrated solution. The use of an oral route for chemical administering is complicated by the generalized anorexia (loss of appetite), a part of that disease. The affect is summarized: "The fish that eat it, don't need it; and, the fish that need it, don't eat it" (Wood 1974).

A nitrofuran has been used for rapid control of this disease (Wood 1974). It is rapidly taken up directly through the integument and gills. The recommended dose is a 1 hour bath at 10-15 ppm (Wood 1974). 
Sulfamethazine is effective when blood levels reach or exceed 5-6 $\mathrm{mg} / 100 \mathrm{ml}$ (Wood 1974). Due to anorexia, therapy must be initiated before serious outbreak occurs. Chemical baths are ineffective at the yolk sac stage (Amend 1970). Sulfamethazine and sulfisoxazole at 200 $\mathrm{mg} / \mathrm{kg} /$ day have been recommended as oral sulfonamides as has OTC at 50 $\mathrm{mg} / \mathrm{kg} /$ day $/ 10-14$ days (Bullock and Snieszko 1970). Chemotherapy by that route should be combined with benzalkonium chloride application for external disinfection (Bullock and Snieszko 1970).

Control of this disease under cold-water conditions is difficult at best.

\section{$\underline{\text { References }}$}

AMEND, D. F. 1970. Myxobacterial infections of salmonids: prevention and treatment. pp. 258-265. In: A Symposium on Disease of Fishes and Shellfishes. S. F. SNIESZKO, ed. Amer. Fish. Soc. Spec. Publ. No. 5.

AMERICAN FISHERIES SOCIETY. 1979. Fish Health Blue Book--Procedures for the Detection and Identification of Certain Fish Pathogens. D. MCDANIEL, ed. Rev. ed. Amer. Fish. Soc. Fish. Health Section. $118 \mathrm{pp}$.

BORG, A. F. 1960. Studies on myxobacteria associated with diseases in salmonid fishes. Wildl. Diseases 8:1-85, 2 microcards.
BUCHANAN, R. E. and N. E. GIBBONS, eds. 1974. Bergey's Manual of Determinative Bacteriology. 8 th ed. Williams \&Wilkins Co., Baltimore, MD. $1268 \mathrm{pp}$.

BULLOCK, G. L. and S. F. SNIESZKO. 1970. Fin rot, coldwater disease and peduncle disease of salmonid fishes. Fish Disease Leaflet No. 25. U.S. Dept. Interior Bur. Sport Fish and Wildl., Eastern Fish Disease Laboratory, Leetown, WV. 3 pp.

DAVIS, H. S. 1970. Culture and Diseases of Game Fishes. Univ. Calif. Press, Berkeley, CA. 332 $\mathrm{PP}$.

PACHA, R. E. and E. J. ORDAL. 1970. Myxobacterial infections of salmonids: prevention and treatment. pp. 243-257. In: A Symposium on Disease of Fishes and Shellfishes. S. F. SNIESZKO, ed. Amer. Fish. Soc. Spec. Publ. No. 5, Washington, $D C$.

RUCKER, R. R., B. J. EARP and E. J. ORDAL. 1953. Infectious diseases of Pacific salmon. Trans. Amer. Fish. Soc. 83:297-312.

WOOD, E. M. and W. T. YASUTAKE. 1956. Histopathology of fish. III. Peduncle ("cold-water") disease. Prog. Fish-Cult. $18: 58-61$.

WOOD, J. W. 1974. Disease of Pacific Salmon: Their Prevention and Treatment. 2nd ed. State of Washington, Dept. of Fish. Hatchery Div. $82 \mathrm{pp}$. 


\section{Furunculosis}

Roger S. Grischkowsky

Introduction

Furunculosis is a disease of $\mathrm{fish}$ caused by a short bacillus Aeromonas salmonicida (see Fig. 11). It is typically restricted to freshwater, but saltwater occurrence exists. The disease results in boil-like furuncles on $\mathrm{fish}$ and ultimate death in most cases (see Fig. 11). Susceptible genera include Pacific salmon (Oncorhynchus) and trout (Salmo). All salmonids are susceptible although differences occur, and trouts are the most susceptible (Snieszko 1969). Of the trouts, brook trout (Salvelinus fontinalis) is the most susceptible and rainbow trout (Salmo gairdneri) the least (Snieszko 1969).

\section{History}

\section{U.S. and Worldwide}

Furunculosis is believed to have originated in western U.S. rainbow trout stocks and was thought disseminated by general distribution of Shasta rainbow trout in $1880 \mathrm{fish}$ cultural operations (Fish 1937). It was first described as Bacterium salmonicida, the causative agent of trout epizootics in Germany (Emmerich and Weikel 1894). Simultaneously it was in Michigan trout as Bacterium truttae (March 1903). Primarily a salmonid disease, it was reported in non-salmonids by several authors in 1911 and 1912: grayling (Thymallus thymallus), tench and pike, chub (Squalinus cephalus) and dace (Leuciscus leuciscus) (Arkwright 1912, Plekn 1911, Surbeck 1911). In the $1930 \mathrm{~s}$ an intensive research ef- fort resulted in the finding of furunculosis in Rocky Mountain whitefish (Prosopium williamsoni) (Duff and Stewart 1933), carp (Cyprinus carpio), catfish (Silurus glanis) and sculpins (Cottus spp.) (Mackie et al. 1930). A hatchery plagued with furunculosis in Berlin, New Hampshire was disinfected after destruction of all fish in 1938 without reoccurrence (Thompson et al. 1973). The causative agent was given the generic term of Aeromonas in 1953 (Anderson 1972). Other names have included Bacillus salmonicida and Proteus salmonicida (Buchanan and Gibbons 1974).

Furunculosis was first found in Swedish trout farms (Salmo trutta, Salmo salar, s. gairdner $\bar{i}$ and Salvel inus namaycush) in 1951 (Johanson 1973). By 1959 the disease spread to 15 fish farms, but only 2 are still registered as having that agent. It is found in wild Swedish fish populations. A mass mortality in minnows (Phoximus phoximus) occurred in the Norwegian Lake due to an abberrant achromogenic strain (Hastein et al. 1978). The agent was found in chinook (Oncorhynchus tshawytscha), steelhead trout ( . gairdneri) and coho salmon Oncorhynchus kisutch) in Oregon hatcheries (Fryer and Conrad 1965), the chestnut lamprey (Ichtyomyzon castaneus) in Michigan (Hall 1963) and goldfish (Carassius auratus) (Mawdesley-Thomas 1969, Shotts et al. 1978). Epizootic magnitude infections have been reported in carp (Cyprinus carpio), catfish (Silurus glanis) and sculpins (Cottus spp.) (Mackie et al. 1930), brassy minnow (Hybognathus hankinsoni), northern 
red belly dace (Chromosus eos), brook sticklebacks (Culaea inconstans), yellow perch (Perca flavescens) flathead minnow ( $\overline{\text { Pimephales promelas }}$ ) and the creek chub (Semotilus atromaculatus) (McFadden 1970). Furunculosis is now present almost everywhere salmonids are raised with the exceptions of Australia, New Zealand and Tasmania (Snieszko 1973).

\section{Alaska}

Aeromonas
discovered in Alaska by the author in
January 1975 in coho salmon juveniles
in estuarine rearing pens off Douglas
Island. A year later it was found in
rainbow trout adults and chinook sal-
mon juveniles in 2 Anchorage hatch-
eries (see Fig. 12). Sheefish (Stenodus leucichthys) from the Yukon River died from the agent during ripening at 1 of the Anchorage hatcheries in 0ctober 1976. The following year marked the finding of $A$. salmonicida in arctic grayling (Thymallus arcticus) in Tolsona Lake. The disease has become the major limiting factor to salmonid production at an Anchorage hatchery. Fifty percent of coho juveniles and prespawning rainbow adults are routinely lost each season due to that disease. Chinook there are affected but to a lesser extent. These joint infections with Saprolegnia parasitica, an aquatic phycomycete, are common and a difficult combination to control. This joint involvement has recently been reported with minnows. Mortalities are early fall to late summer. Prespawning and postspawning rainbow trout usually die from it November through March while coho juveniles die during late spring and early summer. Similar temporal variations have been reported in coho of Washington state where late spring to early summer is the classical outbreak with aberrant occurrences late autumn to early winter and late winter to early spring. The use of heated cooling water from the fossil fuel electric generation plant there provides ideal temperature water for furunculosis development even during the coldest winter months. 0 il spills at the base uphill from the hatchery since the 50's has resulted in hydrocarbon exposure for those fish. It is possible this exposure has reduced immune response of the fish and increased susceptibility to furunculosis.

\section{Transmission}

Transmission of furunculosis is primarily fish to fish. In hatcheries this occurs by generalized use of nets, spawning gloves and other utensils. Desquamation, mucus removal and inoculation with a net are accomplished simultaneously. Waterborne and transovarian transmission have been implicated (Sinderman 1974, Snieszko 1973, Wood 1974). It is an obligate pathogen found in water, only when contaminated by diseased or carrier fish. In Alaska it has not been found in water even at locations of acute mortality. Investigation into $\underline{A}$. salmonicida's capability to survive in water showed it had $\leq 20 \%$ survival after 2 days at 26.7-37.8C (Ross and Smith 1974). It has a sensitivity to elevated temperature and was less able to survive in water than Aeromonas liquifaciens and Pseudomonas fluorescens.

\section{$\underline{\text { Signs }}$ and Pathogenesis}

\section{External Signs}

External effects of furunculosis include anorexia (failure to feed), 

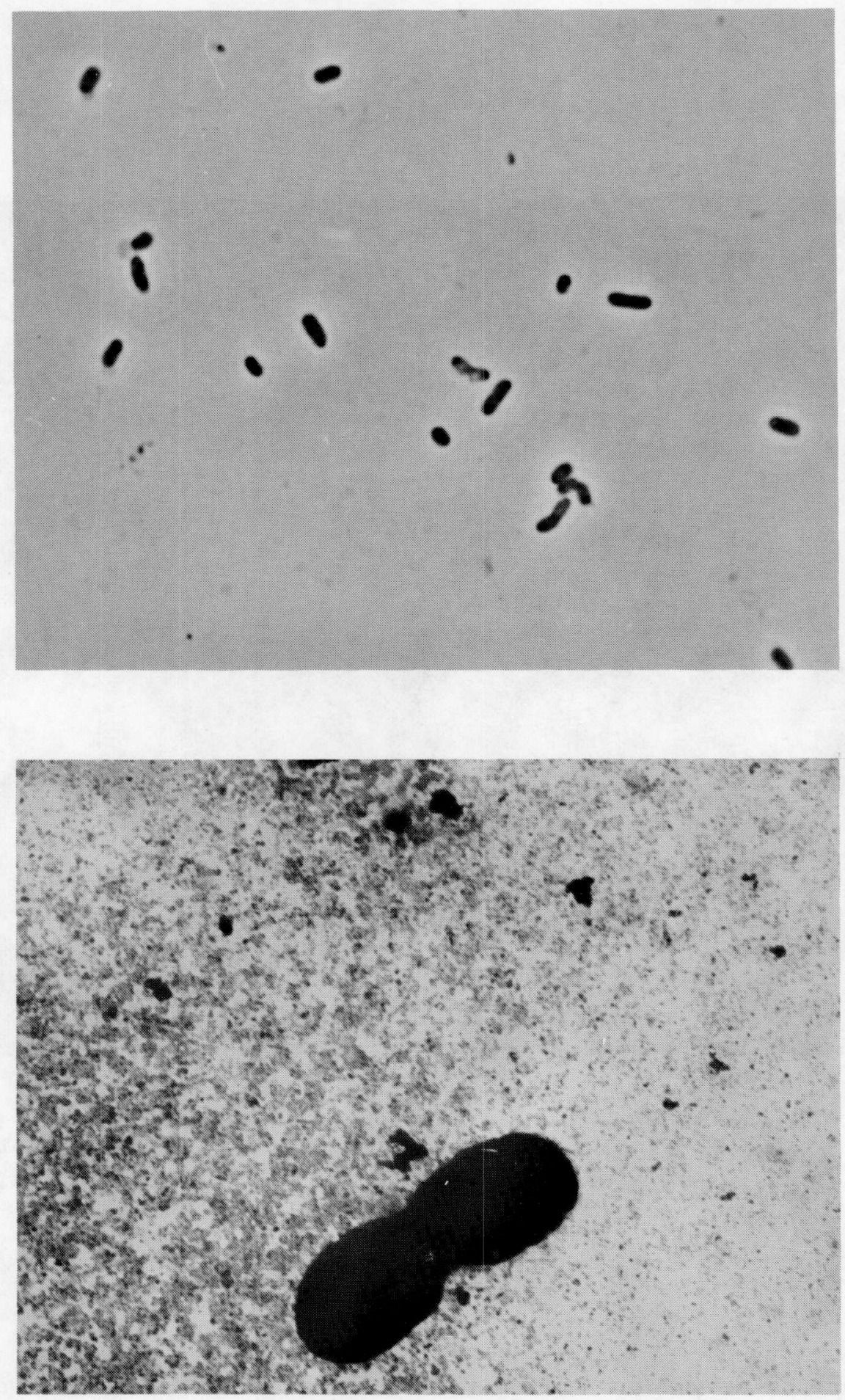

Figure 11. Aeromonas salmonicida rods about $0.6 \times 0.8$ micrometers (upper picture phase-contrast 2000X; lower picture electron micrograph $25,088 \mathrm{X}$ ) (R. Grischkowsky). 


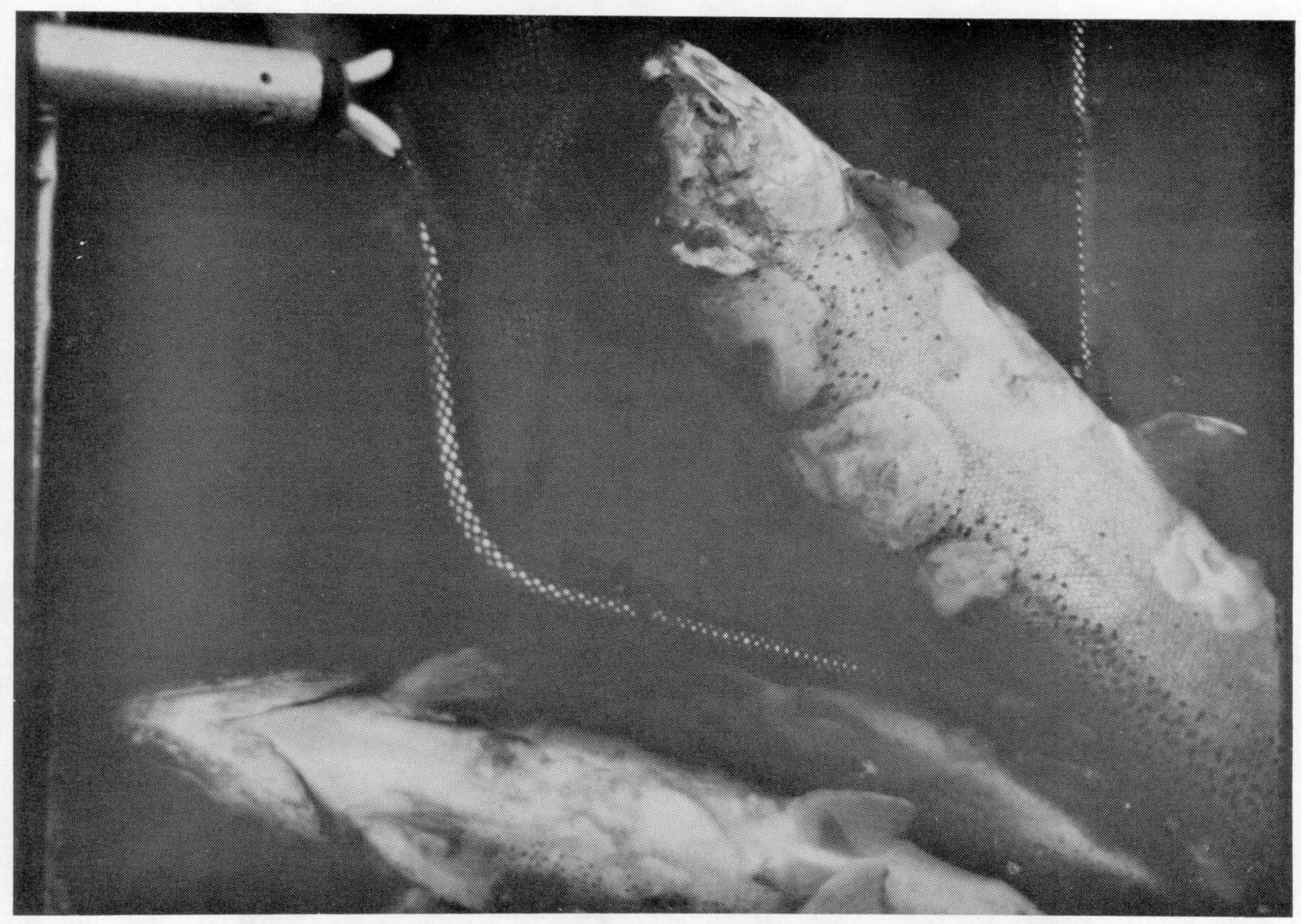

Figure 12. Rainbow trout with joint infection. Head and ventral lesions are caused by Aeromonas salmonicida while white blotches represent Saprolegnia peripherally and furunculosis centrally (R. Grischkowsky).

lesions (particularly on anterior ventral or dorsal areas), hemorrhagic areas (progressing from petechia to open body cavity penetration) and erythremia of eyes, base of fins and anal vent (see color Fig. 30 and Fig. 12). Dark pigmentation around outer edges of lesions and around eye and mouth as well as destruction of upper and lower jaw bones have also been reported (Hastein et al. 1978).

Lesion involvement includes brain, eye and muscle tissue internally (Hastein et al. 1978). 
Internal Signs

Furunculosis is a systemic infection. Kidney necrosis is present during the most pronounced infection stages (Snieszko 1969). Petechiae on mesentaries in pancreatic tissue are pronounced signs. Gross necrosis at the site of furuncle formation is prevalent (see color Figs. 31 and 32). This proceeds from petechial to full penetration of the body cavity. Internal pathology additionally includes localized gross hemorrhages in intestine and liver and darkened hypertrophied spleens (McFadden 1970) (see color Fig. 32).

\section{Pathogenesis}

Incubation period for A. salmonicida is 1 week optimalTy and up to 10 days for susceptible strains (several hours for experiment infections) (Snieszko 1969, 1973). At < $7 \mathrm{C}$ the infection becomes latent (Snieszko 1969). Although the optimum is $13-21 \mathrm{C}$, outbreaks have occurred at less than 2C, (Wood 1974) and mortality is directly proportioned to the temperature (Groberg et al. 1978). The investigators found $2-26 \%$ mortality at 3.9 and $6.7 \mathrm{C}$ and $93-100 \%$ at $20.5 \mathrm{C}$ for coho and chinook salmon and steelhead trout after i.m. (intramuscular) or i.p. (intraperitoneal) injection.

Stages of infection. The 4 forms of the disease designated are acute, subacute, chronic and latent with mortalities and signs most numerous in the acute stage and completely lacking in latent form. It has been hypothesized that summer temperatures favor the host (op. cit.). Low temperature takes the disease from chronic to latent, and high temperature from chronic to acute (Herman 1973).

$\frac{\text { Details }}{\text { occurs }} \frac{\text { of }}{\text { early }} \frac{\text { infection. }}{\text { in the Bacteremia }}$ process. In capillaries, bacteria break down the walls of the vessels allowing blood and serum to leak out into surrounding tissue causing a furuncle (Wood 1974). Large numbers of cells are needed to initiate infection (Wood 1974). Lesions are formed from either inside or outside. These are characterized by pathological changes in epidermis and stratum spongiosum with edema, distortion of scales, hyperemia, minor hemorrhages, leucocytic infiltration, myopathy of underlying musculature and myotomal (myomere) muscle fiber damage (Hastein et al. 1978). Acetate was also found incorporated more rapidly into lipids by livers from $A$. salmonicida infected brook trout in vitro and in vivo (Shieh 1976).

Blood parameters. Major blood parameter changes from furunculosis in carp included decrease in blood sugars, increase in creatine and nonprotein nitrogen and a reduction in fibrin clot capabilities.

Virulence factors. Smooth (S) and rough (R) forms of A. salmonicida have been discovered (Añderson 1972, 1973). The $S$ form was found to be the virulent one. Lipopolysaccharide (LPS) was present as an additional external layer in the $S$ form. The LPS was endotoxin. A glycoprotein has been isolated from broth culture supernatant fluids which is more prevalent in virulent strains (Fuller et al. 1977).

\section{Diagnosis}

\section{Gross Detection}

Fish first will be off feed and appear listless early during a furunculosis outbreak. Petechiae form ventrally first, easily seen in contrast to white ventral salmonid surfaces. Erythremia becomes apparent at anal vent, eyes and fin bases. 
Internal petechiae in pancreatic tissue and mesentaries are also visible early. These signs are not specific for that disease and are similar to those seen with infectious hematopoietic necrosis virus and vibriosis.

\section{Bacteriology}

Basic procedures for fish furunculosis diagnosis is provided in the Fish Health Blue Book (American Fisheries Society 1979). Media containing tyrosine or phenylalanine promote the production by $\underline{A}$. salmonicida of water soluble brown pigment (Wolke 1975) (see color Fig. 29). It is also produced by some Aeromonas hydrophila (Paterson 1974). Three subspecies of A. salmonicida have been suggested: salmonicidaclassical, achromogenes-atypical achromogenic strains for salmonids, and nova atypical strains emerged from nonsalmonids (McCarthy 1978). Only minimal DNA homology divergence was detected from these subgroups (McCarthy 1978). An alternate division includes salmonicida, achromogenes and masouicida, a term for the masou (Oncorhynchus masou) infections (Buchanan and Gibbons 1974).

Colonies typically are clear, glossy, smooth, round and entire. They are Gram negative, non-motile, cytochrome or Kovacs oxidase positive which ferment glucose (see Fig. 13). The vibrio-stat $0 / 129 \quad(2,4$ diamino-6, 7-di isopropyl pteridine) does not inhibit $A$. salmonicida nor does 2,3-butanediol, typically the tests used to differentiate it from vibrios and $\underline{A}$. hydrophila.

A green medium for presumptive identification of aeromonads causes them to appear bright yellow (McCoy and $\mathrm{Pil}$ cher 1974).
Triple sugar iron agar reaction is alkaline slant and hydrogen sulfide or acid butt. Variability exists in novobiocin sensitivity and hemolysis on $5 \%$ human blood agar. Other typical characteristics include penicillin resistance, oxytetracycline (OTC), polymyxin $B$ and erythromycin sensitivity (see Fig. 14).

\section{Fluorescent Microscopy}

Fluorescent antibody technique (FAT) is used successfully to detect and identify A. salmonicida in cultures or fish tissues. Methods established involve direct or indirect fluorescence technique and incident light (American Fisheries Society 1979, Bullock and Stuckey 1975). Fluorescein isothiocyanate is conjugated with anti-A. salmonicida identification (Rabb et al. 1969).

\section{Significance and Control}

\section{Significance}

In Alaska, furunculosis is a limiting factor for fish production at 1 hatchery and affects programs involving wild grayling and sheefish as it is frequently present in those species. When present it restricts rainbow trout culture.

\section{Control}

One of the best examples of furunculosis control is in Sweden (Johanson 1973). After the first outbreak of furunculosis in 1951, it spread by 1959 to 15 fish farms. Strict sanitation prevented further spread. These were effective, as wild fish populations there do not have the agent. In 1959 a health control program was established. This included monitoring, destruction of 


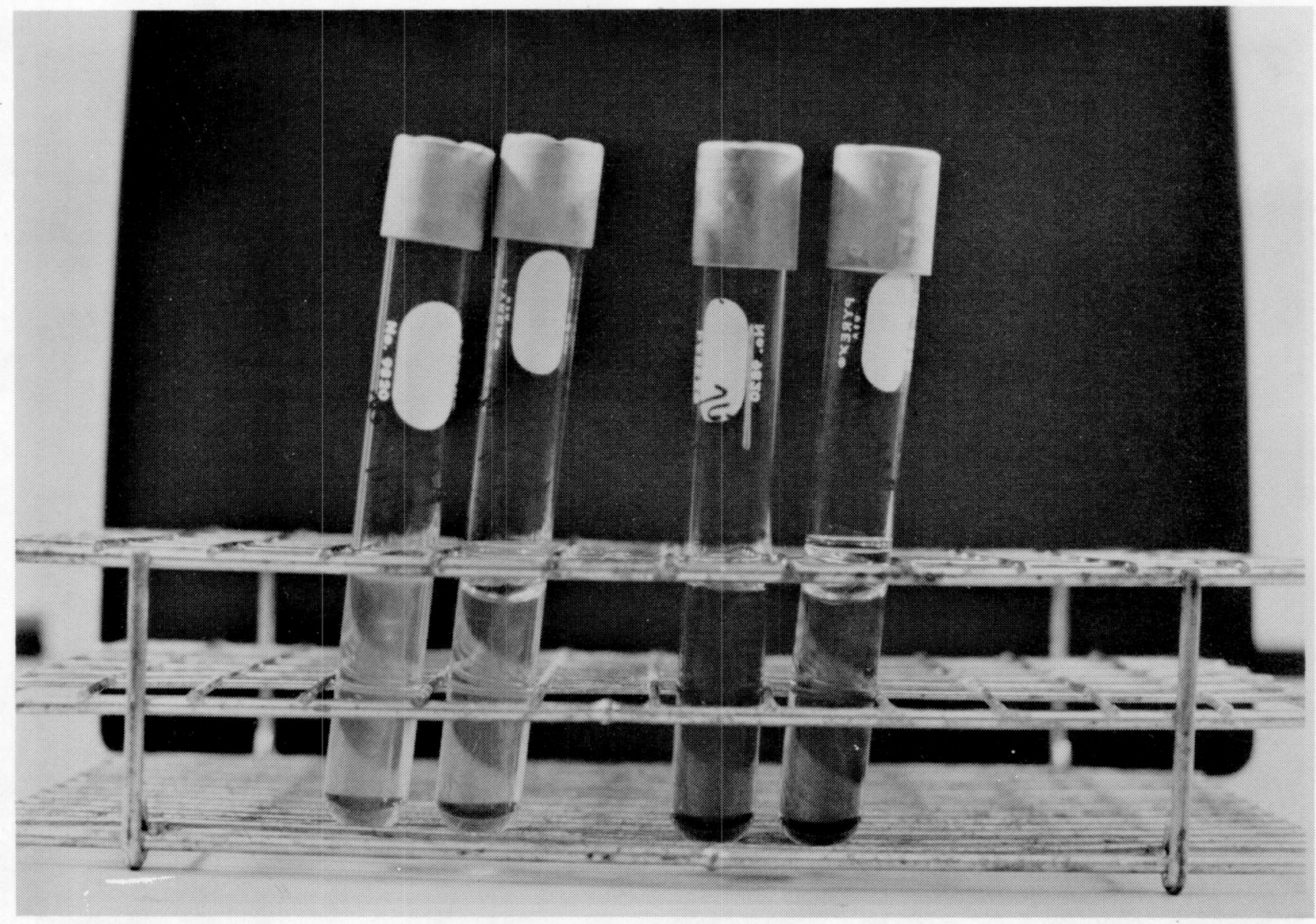

Figure 13. Oxidation and fermentation of glucose testing of Gram negative fish bacilli. The two left most tubes (yellow) indicate the aeromonad or vibrio reaction which is oxidative and fermentive. The first and third tubes were provided essentially anaerobic conditions using heating to expell gasses and sterile mineral oil over lay. These appear yellow with utilization of glucose. The right most two show different reactions. Tubes are green showing no reaction ( $R$. Ward). 


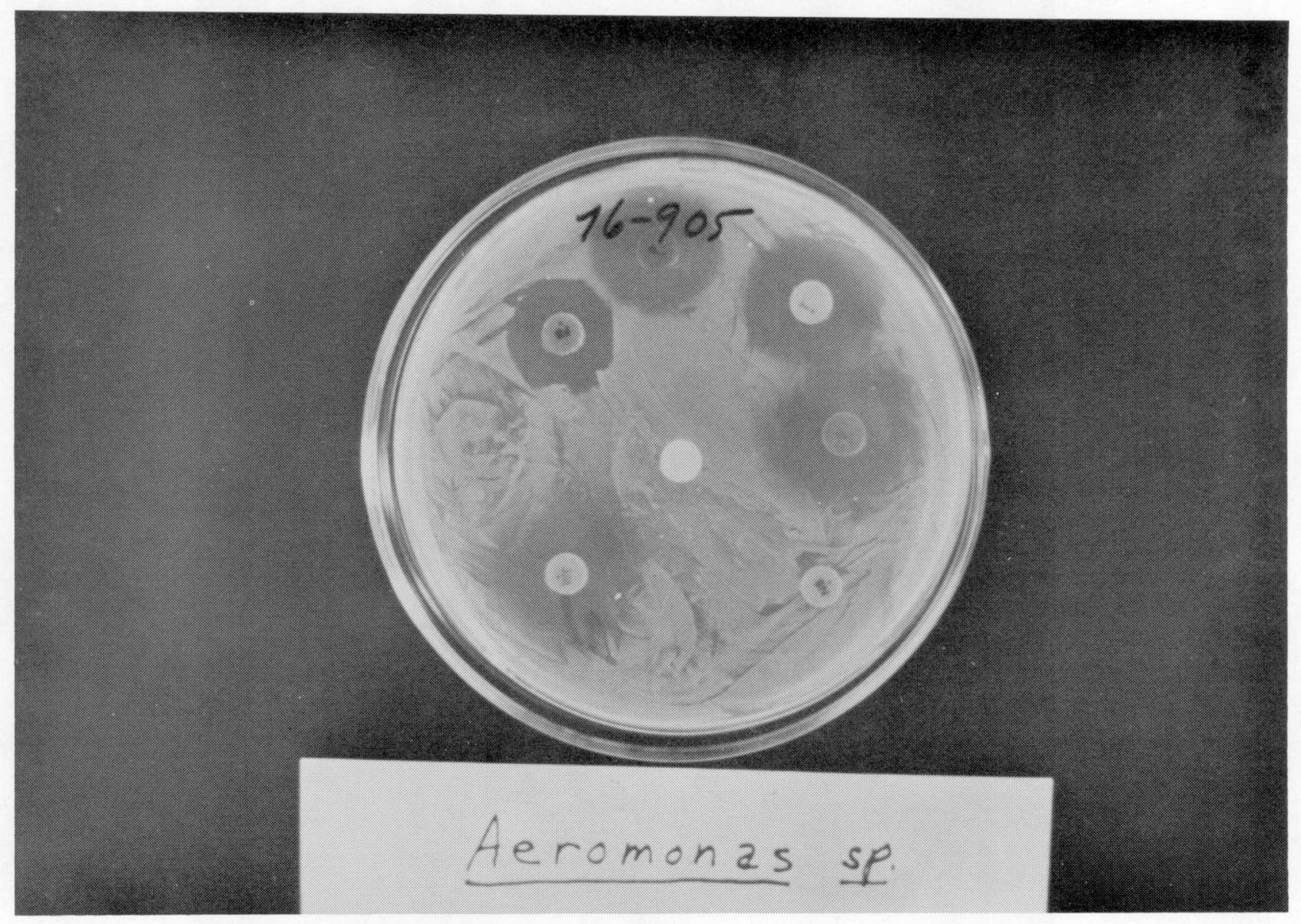

Figure 14. Aeromonad culture spread plated on Mueller-Hinton agar to test antibiotic disc sensitivity. This isolate shows the novobiocin (NB) resistance, oxytetracycline $(T)$ sensitivity, erythromycin sensitivity, ("E" disc not clear but located between "T" and "NB"), sulfamerazine (SM) sensitivity, $0 / 129$ vibrio static substance (center unlabeled disc) resistance, polymicin B sensitivity and penicillin sensitivity (R. Grischkowsky). 
diseased fish and disinfection with nonanadromous fish and by chemotherapy when anadromous $\mathrm{fish}$ are involved. Utensils were routinely disinfected or separate equipment used for each rearing unit, disinfection of hauling trucks, change from fresh diet to pelleted, replacement of earthen ponds with concrete and fiberglass, high water flow used $\left(10-151 / \mathrm{minute}^{2} / \mathrm{m}^{2}\right)$ and low density established $\left(<5 \mathrm{~kg} / \mathrm{m}^{2}\right)$. Fish disease control legislation was adopted to include an import license for live and egg transport allowed only for fish certified free of that agent, all fish movement was diagnosed to allow time for investigation, egg transport was allowed only from involved hatcheries only after egg surface disinfection, and stocking of fish allowed only if free from infectious diseases. An international fish disease control program later addressed transportation, forms and certification procedures, fish farm health status indicators, listing of pathogen hatchery codes and designation of certifiable diseases (Food and Agriculture Organization 1977). By 1973 only 2 hatcheries were still registered as having that agent.

Environmental changes. Reported environmental changes to control furunculosis include lower water temperature (Sinderman 1974, Snieszko 1973), higher dissolved oxygen (Kingsberry 1961, Snieszko 1973), stringent environmental sanitation (Herman 1968, Snieszko 1973), reduced density (Snieszko 1973) and water depuration or use of well water (Herman 1968). Densities of less than $2.5 \mathrm{fish} / \mathrm{m}^{3}$ are reported to prevent furunculosis spread (Snieszko 1973).

Fish destruction. Destruction of diseased hatchery stock is effective in preventing introduction to new areas. Removal of infected fish within the hatchery, carrier fish from the water source, hatchery disinfection, and fish replenishment with clean fish are effective combinations in eliminating furunculosis. This type of control has real potential due to the apparent difficulty of A. salmonicida to propagate outside a host (Snieszko 1973). He found its survival to be in seawater 1 day, freshwater 1 week, sewage or organic matter water 1 month.

Chemotherapy. Control recommendations for this agent are OTC at 2.5-3.5 g/45.4 kg/day (Snieszko 1969), potentiated sulfonamide at 50 $\mathrm{mg} / \mathrm{kg} / \mathrm{d}$ (Bullock et al. 1974), sulphamerazine at $120-220 \mathrm{mg} / \mathrm{kg}$ body weight and nitrofurans at $25-50 \mathrm{mg} / \mathrm{kg}$ body weight/day (Ghittino 1972). Use of OTC (orally, i.m. and i.p.), sodium sulfamethazine, and a nitrofuran derivative have not been effective in Alaska. Resistance from excessive drug use is a reason for lack of control. Aeromonas salmonicida was found to have plasmid exchange with Pseudomonas and Enterobacteriaceae which allows for the exchange of drug resistance factors between plasmids and subsequent redistribution to other bacteria (0lsen and Wright 1976). The agent could donate or receive $\mathrm{R}$-factors. It is doubtful that chemotherapy will be an effective means of control in the near future.

Selective breeding. Selective breeding of trout for resistance to furunculosis has a potential for controlling the disease (Sinderman 1974, Snieszko and Hoffman 1963). The susceptibility of trout was found, in 1933 , to increase with age. Great variation in susceptibility was noted (Davis 1970, Furunculosis Committee of Great Britain 1933). This difference in susceptibility was thought chiefly a matter of heredity. 0lder trout rarely die from the agent except immediately prior to and during spawning when mortality was heavy 
(Davis 1970). Selective breeding to control furunculosis in brook and brown trout was proposed in 1954 (Wolf 1954). Considerable variation with brook trout to furunculosis was found, and slower growing fish were more resistant (Snieszko et al. 1959). The first hatchery trials from this effort resulted in control mortalities of $8.6-14.5 \%$ versus 0.6-1.9\% for selected as resistant fish over 1-6 months (Ehlinger 1964).

Outbreaks of furunculosis in brook trout, thought to be resistant to this disease, have occurred (Snieszko 1964, 1973). These fish were asymptomatic carriers requiring only an adverse environment for disease onset.

Oral preparations have been treated and found both ineffective in protecting against $\underline{A}$. salmonicida (Spence and Fryer 1965, Udey and Fryer 1978) in coho salmon and effective against salmon and trout (Klontz and Anderson 1970). Active immunity has been conferred using coho salmon via i.p. and parental injections of whole cell bacterins with adjuvants and endotoxins (Paterson and Fryer 1974, Udey and Fryer 1978).

Passive immune serum from rainbow trout has been shown to provide temporary protection in coho salmon juveniles (Spence and Fryer 1965).

The nature of immune responses to A. salmonicida have been investigated. These findings apply to this type of furunculosis control. A survey for naturally acquired immunities to $A$. salmonicida in brook trout in Pennsylvania hatcheries showed a range of reactive individuals of $0.8-87 \%$ in 24 populations (Krantz and Heist 1970). The percent reactive individual increased with age of the populations.

A comparison between the development of agglutin titer in rabbits, and trout (brook and brown, $\underline{s}$. trutta) indicated the response of $\overline{\text { trout }}$ to bacterial antigenic stimulation was similar to that of rabbits but with a delay of (2 to 3 months) antibody formation (Krantz et al. 1970).

Precipitations were demonstrated by gel diffusion after a single parental exposure to the soluble antigen of $A$. salmonicida (Anderson and Klontz $1 \overline { 9 } 7 0 \longdiv { \text { . No component similar } }$ to rabbit gamma globulin was detected. Four IgM-like subunits were found in purified anti-A. salmonicida antibodies. These were 175 macroglobulins ( $\mathrm{C}$ isar and Fryer 1974).

Vaccination. Vaccination has some potential for furunculosis control. Research in Alaska has not suggested any protection of trout by vaccination. Both active immune and passive immune experiments have failed to increase survivals from lab challenges.

\section{References}

AMERICAN FISHERIES SOCIETY. 1979. Fish Health Blue Book--Procedures for the Detection and Identification of Certain Fish Pathogens. D. MCD $\overline{A N} I E L$, ed. Rev. ed. 1979. Amer. Fish. Soc. Fish. Health Section. $118 \mathrm{pp}$.

ANDERSON, D. P. 1972. Virulence and persistence of rough and smooth forms of Aeromonas salmonicida inoculated into coho salmon (Oncorhynchus kisutch). J. Fish. Res. Board Can. 29:204-206.

- 1973. Investigations of the lipopolysacchoride fractions from Aeromonas salmonicida smooth and rough forms. pp. 175-179. In: Symposium on the Major Communicable Fish Diseases in Europe and Their Control EIFAC Tech. Pop. $\overline{17}$ (Suppl. 2). 
ANDERSON, D. P. and G. W. KLONTZ. 1970. Precipitating antibody against Aeromonas salmonicida in serums of inbred albino rainbow trout (Salmo gairdneri). J. Fish. Res. Board Can. 27:1389-1393.

ARKWRIGHT, J. A. 1912. An epidemic disease affecting salmon and trout in England during the summer of 1911. J. Hyg. 12:391-412.

BUCHANAN, R. E. and N. E. GIBBONS, eds. 1974. Bergey's Manual of Determinative Bacteriology. $8 \mathrm{th}$ ed. Williams \& Wilkins Co. Baltimore, MD. 1268 pp.

BULLOCK, G. L., H. M. STUCKEY, D. COLLINS, R. L. HERMAN and G. MAESTRONE. 1974. In vitro and in vivo efficacy of a potentiated sulfomamide in control of furunculosis in salmonids. J. Fish. Res. Board Can. 31:75-82.

BULLOCK, G. L. and H. M. STUCKEY. 1975. Fluorescent antibody identification and detection of the Corynebacterium causing kidney disease of salmonids. J. Fish. Res. Board Can. 32:2224-2227.

CISAR, J. 0. and J. L. FRYER. 1974. Characterization of anti-Aeromonas salmonicida antibodies from coho salmon. Infect. and Immun. 9:236-243.

DAVIS, H. S. 1970. Culture and Diseases of Game Fishes. Univ. Calif. Press, Berkeley, CA. 332 $\mathrm{pp}$.

DUFF, D. C. B. and B. J. STEWART. 1933. Studies on furunculosis of fish in British Columbia. Contrib. Can. Biol. Fish. $8: 103-122$.

EHLINGER, N. F. 1964. Selective breeding of trout for resistance to furunculosis. N.Y. Fish Game J. $11: 78-90$.

EMMERICH, R. and E. WEIKEL. 1894. Uber eine durch Bakterien erzengte Seuche unter den Forellen. Archirif. Hyg. 21:1-21.
FOOD AND AGRICULTURE ORGANIZATION OF THE UNITED NATIONS. Government consultation of an international convention for the control of the spread of major communicable fish diseases, 1977. Control of the spread of major communicable fish diseases. FAO Fish. Rep. 192. $44 \mathrm{pp}$.

FIELD, J. B., L. L. GEE, C. A. ELVEHJEM and C. JUDAY. 1943. The blood picture in furunculosis induced by Bacterium salmonicida in fish. Archives of Biochem. pp. 277-284.

FISH, F. 1937. Furunculosis in wild trout. Copeia. 1:37-40.

FRYER, J. L. and J. F. CONRAD. 1965. Some observations on furunculosis in adult Pacific salmon and steelhead trout. Prog. Fish-Cult. 27:99-100.

FULLER, D. W., K. S. PILCHER and J. L. FRYER. 1977. A leukocytolytic factor isolated from cultures of Aeromonas salmonicida. J. Fish. Res. Board Can. 34:1118-1125.

FURUNCULOSIS COMMITTEE OF GREAT BRITAIN. 1933. Second interim report. H.M. Stationery Office, Edinburgh, Scotland. 81 pp.

GHITTINO, P. 1972. The principal aspects of bacterial fish diseases in Italy. pp. 25-38. In: Diseases of Fish. L. E. MAWDESLEY-THOMAS, ed. Symp. Zool. Soc. Lond. Vol. 30.

GRIFFIN, P. J., S. F. SNIESZKO and S. B. FRIDDLE. 1953. A more comprehensive description of Bacterium salmonicida. Trans. Amer. Fish. Soc. 82:129-138.

GROBERG, W. J. JR., R. H. MCCOY, K. S. PILCHER and J. L. FRYER. 1978. Relation of water temperature to infection of coho salmon (Oncorhynchus kisutch), chinook salmon ( 0 . tshawytscha), and steelhead trout (Salmo gairdneri) with Aeromonas salmonicida and $A$. hydrophila. J. Fish. Res. Board Can. 35:1-7. 
HALL, J. D. 1963. An Ecological Study of the Chestnut Lamprey, Ichthyomyzon castaneus Girard in the Manistec River, Michigan. Ph.D. Thesis, Univ. Michigan, Ann Arbor, MI. 106 pp.

HASTEIN, T., S. J. SALTVEIT and R. J. ROBERTS. 1978. Mass mortality among minnows, Phoximus phoximus (L.) in Lake Twe itevatn, Norway, due to an aberrant strain of Aeromonas salmonicida. J. Fish Dis. 1:214-249.

HERMAN, R. L. 1968. Fish furunculosis. Trans. Amer. Fish. Soc. 97:221-230.

1973. A review of the prevention and treatment of furunculosis. pp. 171-173. In: Symposium on the Major Communicable Fish Diseases in Europe and Their Control. EIFAC Techn. Paper 17 (Suppl. 2).

JOHANSON, N. 1973. Furunculosis in Swedish fish farms 1951-1971. pp. 189-192. In: Symposium on the Major Communicable Fish Diseases in Europe and Their Control. EIFAC Techn. Paper 17 (Suppl. 2).

KINGSBERRY, 0. R. 1961. A possible control of furunculosis. Prog. Fish-Cult. 23:136-137.

KLONTZ, G. W. and D. P. ANDERSON. 1970. Oral immunization of salmonids: a review. pp. 16-20. In: A Symposium on Diseases of Fishes and Shellfishes. S. F. SNIESZKO, ed. Spec. Publ. 5. Amer. Fish. Soc. Washington, DC.

KLONTZ, G. W. and J. W. WOOD. 1973. Observations on the epidemiology of furunculosis disease in juvenile coho salmon (Oncorhynchus kisutch). pp. 181-186. In: Symposium on the Major Communicable Fish Diseases in Europe and Their Control. EIFAC Tech. Paper 17 (Suppl.2).

KRANTZ, G. E. and C. E. HEIST. 1970. Prevalence of naturally acquired agglutination antibodies against Aeromonas salmonicida in hatchery trout in Central Pennsylvania. J. Fish. Res. Board Can. 27:969-973.
KRANTZ, G. E., J. M. REDDECLIFF and C. E. HEIST. 1963. Development of antibodies against Aeromonas salmonicida in trout. J. Immunol. 91:757-760.

MCCARTHY, D. H. 1978. Taxonomy of the genus Aeromonas. pp. 25-28. In: Proc. of the Joint $3 r d$ Biennial Fish Health Section and 9th Annual Midwest Disease Workshops. August 15-18, 1978.

MCCOY, R. H. and K. S. PILCHER. 1974. Peptone beef extract glycogen agar, a selective and differential Aeromonas medium. J. Fish. Res. Board Can. $27: 2365-2370$.

MCFADDEN, T. W. 1970. Furunculosis in nonsalmonids. J. Fish. Res. Board Can. 27:2365-2370.

MACKIE, T. J., J. A. ARKWRIGHT, T. E. PRYCE-TENNANT, J. C. MOTTROM, W. D. JOHNSTON and W. J. M. MENZIES. 1930. Interim report of the furunculosis committee. H.S. Stationery Office, Edinburgh, Scotland. $65 \mathrm{pp}$.

MARCH, M. C. 1903. A more complete description of Bacterium truttae. Bull. U.S. Fish Comm. for 1902 . $22: 411-415$.

MAWDESLEY-THOMAS, L. E. 1969. Furunculosis in the goldfish (Carassius auratus). J. Fish. Biol. 1:19-23.

MONTEM, E. 1968. Smolt production of the Swedish State Power Board and the economical importance of diseases. Bull. Off. Int. Epizoot. 69:1687-1690.

OLSEN, R. H. and C. D. WRIGHT. 1976. Interaction of Pseudomonas and Enterobacteriaceae plasmids in Aeromonas salmonicida. J. Bacteriol. 128:228-234.

PATERSON, W. D. 1974. Biochemical and serological differentiation of several pigment producing aeromonads. J. Fish. Res. Board Can. 31:1259-1261. 
PATERSON, W. D. and J. L. FRYER. 1974. Immune response of juvenile coho salmon (Oncorhynchus kisutch) Aeromonas salmonicida cells administered intraperitoneally in Freund's complete adjuvant. J. Fish. Res. Board Can. 31:1751-1755.

1974. Effect of temperature and antigen dose on the antibody response of juvenile coho salmon (Oncorhynchus kisutch) to Aeromonas salmonicida endotoxin. J. Fish. Res. Board Can. 31:1743-1749.

PLEKN, M. 1911. Die furunkulose der salmoniden. Centralbl. Bakteriol. Abt. 1 Orig. 60:609-624.

RABB, L., J. W. CORNICK and L. A. MCDERMOTT. 1969. A macroscopic slide agglutination test for the presumptive diagnosis of furunculosis in fish. Prog. Fish-Cult. $26: 118-120$.

ROSS, A. J. and C. A. SMITH. 1974. Effect of temperature on survival of Aeromonas liquefaciens, Aeromonas salmonicida, Chondrococcus columaris and Pseudomonas fluorescens. Prog. Fish-Cult. 36:51-52.

SHIEH, H. S. 1976. Effect of Aeromonas salmonicida (Furunculosis) infection on the incorporation of 1-14C acetate into lypids of trout liver. Infect. and Immun. 14:836-838.

SHOTTS, E. B., D. G. ELLIOTT, D. G. MCCARTHY and J. D. TALKINGTON. 1978. Characterization of the causative agent of ulcerative furunculosis in goldfish. pp. 21-24. In: Proc. of the 6th Joint Biennial Fish Health Section and 9 th Annual. Midwest Fish Disease Workshops. Aug. 15-18, 1978.

SINDERMAN, C. J. 1974. Diagnos is and control of mariculture disease in the United States. Nat. Mar. Fish. Serv. NOAA-76121001 Highlands, NJ. 306 pp.

SNIESZKO, S. F. 1964. Remarks on some facets of epizootiology of bacterial fish diseases. Div. Indust. Microbiol. 5:97-100.
1969. Fish furunculosis fish disease leaflet--17. U.S. Dept. Interior, Bur. Sport Fish. and Wildl. Eastern Fish Disease Lab., Leetown, WV. 2 pp.

- 1973. Furunculosis of salmonidae. pp. 157-162. In: Symposium on the Major Communicable Fish Diseases in Europe and Their Control. European Inland Fish. Advisory Comm. Tech. Paper 17 (Suppl. 2) Food and Agriculture Organization of the United Nations.

SNIESZKO, S. F., C. E. DUNBAR and G. J. BULLOCK. 1959. Resistance to ulcer disease and furunculosis in Eastern brook trout Salvelinus fontinalis. Prog. Fish-Cult. 21:111-116.

SNIESZKO, S. F. and G. L. HOFFMAN. 1963. Control of fish diseases. Lab. Animal Care 13:197-206.

SPENCE, K. D. and J. L. FRYER. 1965. Active and passive immunization of certain salmonid fishes against Aeromonas salmonicida. Can. J. Microbiol. 43:397-405.

SURBECK, G. 1911. La furunculose des poisson dans les eaux libres. Bull. Suisse Peche Piscicult. $10: 162-165 ; 11: 173-175$.

THOMPSON, P. E., W. A. DILL and G. MOORE. 1973. The major communicable fish disease of Europe and North America. EIFAC Tech. Paper 17 (Suppl. 1). 48 pp.

UDEY, L. R. and J. L. FRYER. 1978. Immunization of fish with bacterins of Aeromonas salmonicida. Mar. Fish. Rev. 40:12-17.

WOLF, L. E. 1954. Development of disease resistant strains of fish. Trans. Amer. Fish. Soc. $83: 342-349$.

WOLKE, R. E. 1975. Pathology of bacterial and fungal diseases affecting fish. pp. 58-62. In: The Pathology of Fishes. W. E. $\overline{R I B E L} \overline{I N}$ and G. MIGAKI, eds. Univ. Wisconsin Press, Madison, WI.

WOOD, J. W. 1974. Diseases of Pacific Salmon: The ir Prevention and Treatment. 2nd ed. Washington Department of $\mathrm{Fisheries,01ym-}$ pia, WA. 82 pp. 


\section{Vibriosis}

Roger S. Grischkowsky

Introduction

Vibriosis is a bacterial disease which affects a wide range of vertebrates and invertebrates. All salmonids and many other teleost fishes are susceptible. The causative agent is generally Vibrio anguillarum but sometimes other vibrios are involved. The cause, a marine bacillus, has wide ranging growth capabilities making it an excellent pathogen. Primarily the infection occurs in estuarine conditions, but freshwater juvenile mortalities have taken place, and anadromous adults can bring vibrios from the neritic waters to the freshwater environment where prespawning vibriosis mortalities have also occurred.

Vibrio disease in fishes is a systemic infection, septicemia, which results most frequently in death of the fish. Necrosis of the abdominal muscles results in penetration of the gut in highly infected fish. Migration across gut tissue layers is 1 mode of action in vibrios. Access to humoral fluids results in rapid septicemia.

\section{History}

Vibrios have been known to be responsible for epizootics of vertebrate fishes for many years. Anderson and Conroy (1979) compiled a review article that shows vibrioassociated (… anguillarum, Vibrio ichthyodermis and Vibrio piscium) diseases in 42 species of teleost fishes in 16 countries. Fishes in the U.S. list are smelt (Atherinops afinis), herring (Clupea pallasi), killifish (Fundulus parvipinnis), Pacific cod (Gadus macrocephalus), goby (Gillichtys mirabilis), blenny (Hypsoblennius gi lberti), 3 Pacific salmon pink (Oncorhynchus gorbuscha), chum (Oncorhynchus keta), chinook (Oncorhynchus tshawytscha), rainbow trout (Salmo gairdneri) and queenfish (Seriphus politus). The clinical signs shown by fish infected with vibrios include discoloration of the skin, hemorrhages of body surface including the eye, septicemia and a decrease in hematocrit. Bergman (1909) first described $\underline{v}$. anguillarum which he implicated as the causative agent of "Rotseuche des Aales, pestris rubra anguillarum," red pest of eels. The disease had had epizootic reports in 1718, 1790, $1825,1850,1864,1867,1892$ (Canestrini 1893), 1901, 1903 in Denmark, Germany and Holland and in 1884, 1885, 1889, 1898 and 1899 in the Adriatic Sea (Bergman 1909). July was isolated as the worst month for occurrence of this disease in either fresh or saltwater (Canestrini 1893). These early reportings gave a syndrome of red flecks, lesions and hemorrhagic areas of the surface of the head (Bergman 1909). Vibrio anguillarum was there described as having the following characteristics: 1.5-2.7 um long $x 1$ um wide, Gram negative, liquifies gelatin, grows best at $16 \mathrm{C}$ but grows to $35 \mathrm{C}$, not harmful to warm-blooded animals but pathogenic for eels, salamanders, goldfish, frogs and sticklebacks.

Portions of this work are from the author's copyrighted dissertation. 
Nybel in anguillarum

(1935) as described $\underline{v}$. monotrichous, Gram negative, wi thout spores or capsules, gelatin liquified, gelatin stab cholera-like, without gas but fermentative in dextrose and maltrose, H2S not produced, without reducing capability, halophilic, upper temperature range 37-40C and pathogenic for fish. Buckman (1952) isolated $\underline{V}$. anguillarum from the underside of gills, in body fluid, brain tissue, heart blood, kidney, liver, intestines and in internal lesions of the musculature of flat fish (Pleuronectes platessa). The syndrome was a general reddening, lesions and ulcerations of the opercula, nasal orbitals and bony exterior of the head. Rucker et al. (1953) found vibrio disease present in Pacific salmon: pink, chum, chinook and coho (Oncorhynchus kisutch). A similar pathology was found also in Pacific herring, Clupea pallasi, seined in Puget Sound. The syndrome was extensive hemorrhages throughout the musculature with massive concentrations of vibrios in these lesions followed by heavy mortalities from April through the end of summer whenever the water temperature approached 10C. Schaperclaus (1953) determined the temperature range of V. anguillarum to be 6 to $37-40 \mathrm{C}$ while the salt range was $0.07-8.5 \%$. The same disease of eels in Europe and the Baltic Sea resulted typically in 15-25\% mortality after 1 to 10 days with a syndrome of red patches on the head, hemorrhagic areas and red lesions of the musculature. Vibriosis has been reported of the pike, called "Hechtpest" (Pike pest), which killed $5-62 \%$ of the fish per year during the period 1926-1931 with a syndrome of lesions, paleness, listlessness and hemorrhagic areas on the dorsal head surface (Schauperclaus 1953).

The vibrio disease known in Europe for more than 2 centuries was not found extensively in America until 1951 when it was found in chum salmon fingerlings in Deception Pass (Puget Sound) saltwater (Rucker 1959). The reported syndrome was redness at the base of the fins and on the sides of the fish, inflammation of intestinal tract and a general septicemia. In 1952 Rucker and Ordal found the same disease in rainbow trout and steelhead trout ( $\underline{S}$. gairdneri) in a Vancouver, Washington hatchery (Rucker 1959).

Isabel Smith (1961) isolated $\underline{V}$. anguillarum from finnock (immature Salmo trutta) in Scotland, showing a syndrome of hemorrhagic vent and firis. The same organism was found pathogenic for finnock, eels, brown trout ( $\underline{S}$. trutta), perch, plaice ( $\underline{P}$. platessa) and saithe (Pollachius virens) (Smith 1961). Muroga and Egusa (1967) found $\underline{V}$. anguillarum responsible for an endemic disease of ayu (Plecoglossus altivelis) in Lake Hamana in Japan. Haastein and Holt (1972) found $V$. anguillarum in coalfish (Gadus virens), Atlantic salmon (Salmo salar) and cod (Gadus morhua), flounder (Pleuronectes flesus), plaice and dab (Limanda 1 imanda). The isolation was apparently associated with a mortality in Norwegian waters which had the highest rate during summer and late fall.

The first proven outbreaks of piscine vibriosis in Canada were recorded by Evelyn (1971) in 4 species of Pacific salmon (chum, sockeye, pink and chinook salmon) cultured in seawater at facilities in Nanaimo and West Vancouver, B.C. The mortalities occurred during July 1958 and August and September 1969 with the following syndromes: redness at the base of paired fins, vent and gill isthmus, diffuse hemorrhages on the body surface and musculature. Cardwell and Smith (1971) cited a small outbreak of vibrio sp. in chinook salmon in seawater on a floating laboratory on Elliot Bay in 
Puget Sound. The bacteria apparently resulted in damage to erythropoietic system or accelerated destruction of red blood cells. An almost complete mortality of a half million chinook salmon occurred from vibriosis at Lint Slough near Waldport, Oregon in 1968 (Cisar and Fryer 1969).

In Alaska, $V$. anguillarum and Vibrio sp. are responsible for major losses of coho, sockeye (Oncorhynchus nerka), chinook and pink salmon. Vibrios were responsible for $90-98 \%$ mortality of all coho, sockeye and chinook salmon during July through September at the Little Port Walter, Baranof Island estuarine rearing pens (ERP) in 1972. Heavy vibriosis losses prevented seawater acclimation of fingerlings at this facility (Heard and Martin 1973). Previous years of rearing, 1969 and 1971, had lower mean water temperatures and no apparent mortality. Survivors of this 1973 epizootic exhibited reduced growth rates.

Pink salmon juveniles in an ERP at Kitoi Bay, Afognak Island, near Kodiak, experienced substantial mortalities during October and November of 1975 from $V$. anguillarum. More recently, mortalities have been substantial at Starrigavan ERP near Sitka in October 1976 in chinook salmon, at Halibut Cover Lagoon ERP in sockeye salmon and Beaver Falls ERP near Ketchikan in chum salmon, both during the summer of 1977 and at Halibut Cover Lagoon ERP in pink salmon. The chum salmon mortality was in the vicinity of $50 \%$ while the pink salmon mortality totaled $96.7 \%$.

\section{Transmission}

Initial infection is thought to be waterborne, but $f i s h$ to $f i s h$ once it is established in a population (Bullock 1977). The incubation period after natural or waterborne exposure typically is 3 to 21 days (Bullock
1977). In i.p. (intraperitoneal) injections with rainbow trout and chinook salmon, death occurred in 5 days when susceptible species were used with virulent strains.

Removal of slime layer portions and desquamation contribute to transmission facility. Virulent strains are such good antigens, exposure by almost any method is adequate to commence an infection. Exposure to seawater containing vibrios is adequate for commencement of the disease at $12 \mathrm{C}$ or greater and has been successfully used as a challenge procedure (Fryer et al. 1976).

\section{$\underline{\text { Signs }}$ and Pathogenesis}

\section{External Signs}

The characteristic syndrome observed in moribund or dead fish injected with $\underline{\mathrm{V}}$. anguillarum or other vibrios or fish naturally infected with vibriosis is as follows: red eye, hemorrhagic areas present including cranial caps, opercula cover and anal opening, darkening of the dorsal surface, bloating and open penetration of abdominal cavity and pale pinḱ gills (see Fig. 15). Bleeding throughout the body surface was so pronounced that white filter paper underneath the specimens soon became red stained. The syndrome as noted agrees with those early reports for vibriosis (Bergman 1909, Buckmann 1952, Rucker 1959, 1972, Schaperclaus 1954).

\section{Internal Signs}

Vibriosis internally will most promiminently show erythremia, petechiae and hemorrhagic areas in internal organs and mesenteries. Destruction of kidney tissue has been found using histopathology of moribund coho salmon. Other changes have included muscle necrosis, hemor- 


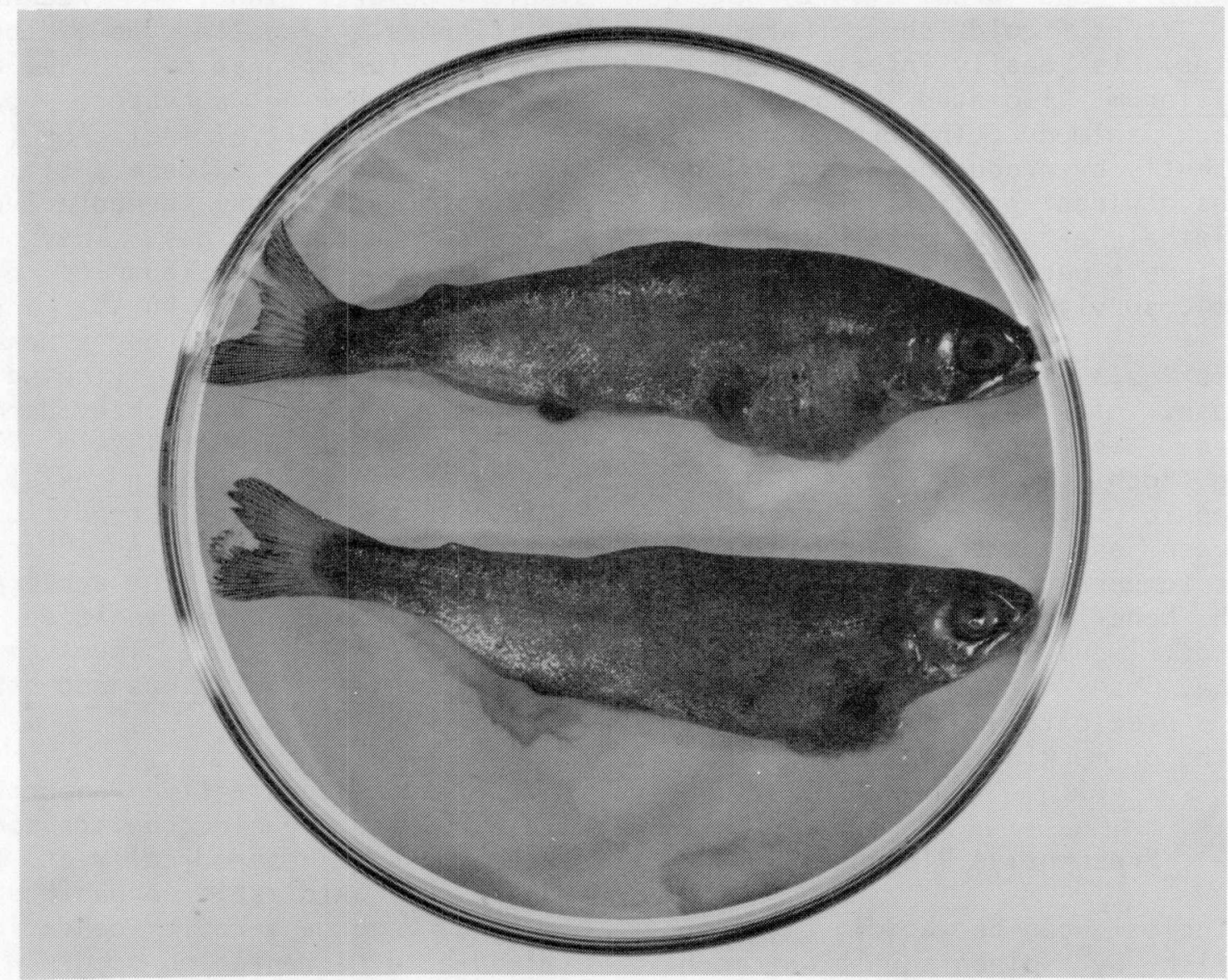

Figure 15. Chinook salmon (Oncorhynchus tshawytscha) showing signs of vibriosis after being injected with a vibrio Vibrio anguillarum pathogenic to fish. Penetration of the gut and hemorrhage at the anal vent and around eyes are present. Erythremia is general. The filter paper under the fish shows this condition (1.1X) ( $R$. Grischkowsky).

rhages between muscle fibrils and inflammation of the intestinal tract (Fryer et al. 1972, McCarthy et al. 1974).

\section{Pathogenesis}

Exposure to the agent by a susceptible species is followed by a septicemia and organ involvement.
Petechiae develop at mesenteries, pancreatic tissue sites and ventral areas. Lesions follow with gut penetrations and death at any time during disease development.

Specific isolates of the bacterium are effective against specific hosts while other vibrios are pathogenic for other even unrelated species. 
Umbreit and Ordal (1972) determined that goldfish (Carassius auratus) is easily infected with $\mathrm{V}$. anguillarum isolated from young chinook salmon. The organism acted apparently by producing a toxin and it was at least partially responsible for lethal effects obtained. When dose levels were relatively high $\left(10^{5}\right.$ cells), survivors were immune.

Vibriosis is a stress mediated disease. Its presence in eutrophic waters is greater than in obligotrophic waters. These eutrophic environments favor the pathogen and not the host. Elevated water temperature is another factor which benefits the pathogenesis to the detriment of the host. In Alaska, many of the epizootics have been precipitated by handling, grading or marking.

\section{Presumptive Diagnosis}

Vibriosis can be rapidly diagnosed in fish by spleen squash wet mount examination using a light or phasecontrast microscope. The presence of very motile rods, usually slightly curved in the spleen indicates a septicemia. Fish raised in estuarine salinity conditions, summer water temperatures with hemorrhagic areas internally or externally are presumptively positive for the disease.

\section{Isolation}

Kidney spleen' or lesion culture, using almost any total bacterial count agar or broth plus $1.5-2 \% \mathrm{NaCl}$ or $50 \%$ seawater are effective for isolating from symptomatic fish.

\section{Identification}

Vibrio is a genus of bacteria containing Gram negative rods characteristically motile by means of a single polar flagellum (recently peritrichous flagella have been reported) (Baumann et al. 1973), and frequently showing curvature particularly on initial isolation (see Fig. 16). They are oxidase positive, but dissimulate sugars fermentatively without production of gas. They possess molar percent guanine plus cytosine values in the 40-48 range. The eighth edition of Bergey's Manual recognized $\underline{\mathrm{V}}$. anguillarum (including V. piscium and $V$. ichthyodermis (Buchanan and Gibbons 1974). Two main types of $V$. anguillarum exist in Pacific Northwest fish: traditional fast growing strain and an abberant slow growing isolate. The aberrant strain has not been isolated in Alaska although disease involvement with Vibrio alginolyticus and other vibrios exists.

Vibrios are facultative anaerobes with a respiratory electron transport system for growth aerobically at the expense of oxidizable organic substrates. Under anaerobic conditions a mixed-acid fermentation occurs with the production of lactic, succinic and formic acids and ethanol without gas (Stanier et al. 1970).

A method for differentiation of Vibrio and Pseudomonas was presented by Shewan et al. (1954). According to the authors, rapid differentiation of asporogenous Gram negative bacilli could be accomplished by testing sensitivity towards penicillin, oxytetracycline (OTC) and a vibriostatic compound called 0/129. Neither Vibrio nor Pseudomonas were sensitive to penicillin. Pseudomonas was found insensitive to $0 / 129$ and variable in sensitivity towards OTC. Vibrios were sensitive to both of the mentioned inhibiting agents. A determinative scheme for identification of Gram negative asporogenus rods classified as Pseudomonas, Aeromonas and vibrios was prescribed by Bain and Shewan (1968). Gram negative, motile, polar flagellated, 


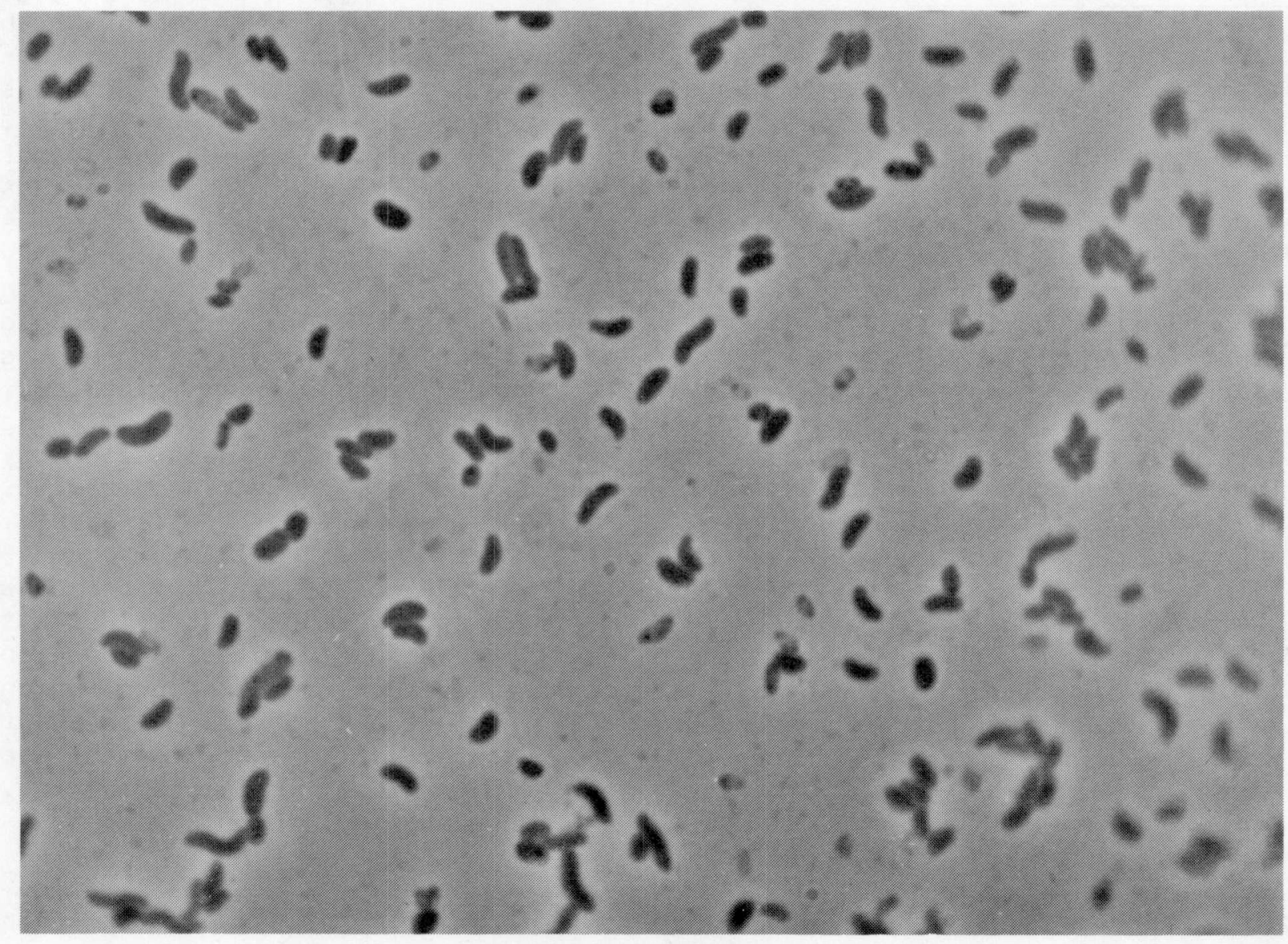

Figure 16. Vibrio anguillarum culture of a strain found pathogenic for chinook salmon (‥ tshawytscha). Typical cellular morphology is shown: curved bacilli, rounded ends, singly and in pairs generally $0.5 \times 1.5-3.0 \mathrm{micrometers} \mathrm{(R.} \mathrm{Grischkowsky).}$

oxidase positive rods are tested in Hugh and Leifson's medium with $\mathrm{NaCl}$ added, against $0 / 129$ and arginine dihydrolase. Pseudomonas species are not fermentative in Hugh and Leifson's medium while Aeromonas species do ferment glucose usually with gas production but are insensitive to $0 / 129$. Vibrios are sensitive to $0 / 129$ and fermentative in glucose but without gas. Vibrio spp. pathogenic for poikilothermic animals are arginine dihydrolase + and lysine decarboxylase -.
In summation, among the motile Gram negative rods, Pseudomonas is easily identified by its oxidative only characteristic in Hugh and Leifson's medium. Aeromonas and vibrios are fermentative, but the former is insensitive to $0 / 129$ while the vibrios are quite sensitive to this vibriostatic agent. One exception is Aeromonas shigelloides which is sensitive to this agent. 


\section{Significance and Control}

Historic losses to vibriosis in Alaskan ERP's from 1974-1976 have totaled $18 \%$ of 1.9 million fish. Many investigators agree vibriosis is 1 of the principal deterrents to estuarine rearing and further development of mariculture at many places in the world (Fryer et al. 1976, Sinderman 1974). It doesn't result in mortalities every year and is affected by meteorological and hydrographic conditions. When mortalities occur in Alaska due to vibrios, they do so simultaneously at several places throughout the state. other locations traditionally have no incidence of the disease. Vibrio abundance in seawater is generally May to October in Southeast waters and July to October in Southcentral Alaskan waters. These are peak levels and traditional vibriosis season timings. Release of fish prior to abundant vibrio levels will reduce the possibility of that disease reducing survival.

Significance in Alaska of this agent requires a site by site analysis and determination of historic involvement.

\section{Control}

Control methods for vibriosis include environmental changes, chemotherapy, selective breeding and vaccination. Efficacy of control measures in Alaska has varied considerably according to species, rearing site, water temperature, fish density, handling and general level of health.

\section{Environmental changes. Environ-} mental requirements of fish are highly specific for maintenance of optimum fish health. Specific environmental conditions provide the balance between host and pathogen. Salinity decreases, density reduc- tions and temperature lowerings during an epizootic will improve fish survival. Although vibriosis can occur at all times of the year, it is most prevalent at 14-20C. In Alaska, vibrio epizootics occur generally at $8 \mathrm{C}$ or higher. Temperatures in excess of $12-14 \mathrm{C}$ are infrequent. Densities of $8-16 \mathrm{~kg} / \mathrm{m}^{3}$, common to ERP's may stimulate the disease condition (Novotny and Harrell 1975). Reduced densities, as long as feeding threshold response is maintained, should reduce mortalities. Placing affected fish into freshwater may reduce mortality during an epizootic. Although freshwater vibriosis occurs, it is rare. Once septicemia occurs, freshwater exposure will not decrease mortalities. Osmoregulatory stress may increase death rates. Reduction of stress before an exposure which could produce an outbreak may prevent the disease. Stressors harmful to fish in saltwater include marking, grading, handling and crowding.

Chemotherapy. Sulfamethazine, sulfamerazine, OTC and nitrofuran compounds have been suggested for the control of vibriosis with salmonids (Fryer et al. 1972). Rucker cited OTC as the antibiotic of choice for decreasing mortalities of Pacific salmon from latent pathogens including vibrios (Rucker et al. 1953). $\mathrm{He}$ recommended $250 \mathrm{mg} / \mathrm{kg}$ of $\mathrm{fish}$. Chemotherapy has only been marginally effective in Alaska. Once an epizootic is started, treatments will be of little value. As entrance into seawater, warm water temperature and vibrio incidence are generally predictable and prophylactic treatments with OTC are recommended. Such treatments, $4.5 \mathrm{~g} / 45 \mathrm{~kg} / 15$ days, are thought beneficial administered 2 weeks prior to a major stressor. Also prerelease OTC treatments may reduce neritic migrations vibrio affects. Treatments at subclinical levels may promote drug resistance factors. 
Selective breeding. Selective breeding has been suggested as a means of vibriosis control (Bullock 1977). Selection experiments have been conducted with Atlantic salmon parr (Gjedrem and Aulstad 1974). A difference in resistance between river strains was found. Heritability was estimated as 0.12 and 0.07 for respective sire and dam components. The results indicated selection against vibrio disease would yield genetic change.

Immunization. Immunization of fish against pathogenic vibrios has received considerable attention. Fish form antibodies when antigenic substances are injected. Large numbers of fish in hatchery situa$t$ ions make oral immunization the most practical. In an early experiment, a lyophilized sonicate was prepared from $V$. anguillarum cells. At Lint Slough near Waldport, Oregon, mortality was decreased from $98 \%$ in controls to $45 \%$ in the vaccinated group at 12.4C (Fryer et al. 1972). Snieszko (1970) showed fish gain some immunity by the intestinal route by an apparent exchange between bacterial antigens within the intestine into tissues via leucocytes. He found oral immunization conferred a low degree of protection under favorable conditions, but was less effective under stress conditions.

Vibrio bacterins, killed broth culture preparations, have been administered to steelhead trout and Pacific salmon using oral, vacuum infiltration, hyperosmotic, dip and spray methods. Evaluation of immunizations have included ERP survival, lab challenge and adult returns. Results from these efforts relate to size, species, site, weather, release timing and stress.

Oral vaccination affords low level protection adequate for protection of host under favorable conditions. Under stress, low level protection may not be adequate (Snieszko 1970). Chinook salmon were afforded protection from natural vibriois challenge at Lint Slough by oral administering of $\underline{V}$. anguillarum lyophilized sonicate of cells (Evelyn 1971). Protection of steelhead trout against vibriosis was afforded by orally fed V. anguillarum live low-virulence cultures (Braaten and Hodgins 1976). Oral vaccination is not as effective as spray technique in protecting coho salmon (Garrison and Gould 1976). Results using oral administering are variable. No tests have been made in Alaska. Immune response build up requires 2 weeks or more.

To afford protection, the build up must occur prior to exposure to the antigen. For vibrio disease protection, fish after vaccination must be kept in freshwater or seawater with no vibrio abundance. Anamnestic secondary increase in immunogenic response has not been demonstrated in $\mathrm{fish}$. Accordingly, boosters are not generally administered. Vacuum infiltration utilizes a vacuum chamber for pressure reduction and bacterin uptake. Hyperosmotic techniques utilized $6-8 \% \quad \mathrm{NaCl}$ dip to remove body fluids followed by isotonic bacterin dips to result in uptake via the lateral line system, gills and directly through integument (Amend and Fender 1976). These techniques were determined to be unnecessary with this agent as it is such an effective antigen easily taken up by immersion at 1 atmosphere.

Vibrio bacterin administering is effective in reducing ERP mortalities from vibriosis. In Alaska, vibrio disease oocurs sporadically. Protection is needed where historically vibrio disease has been present.

A prophylactic program of bacterin administering is beneficial during the rearing stage of anadromous salmonids. Adult return increases have not routinely occurred after vibrio bacterin application. 
Coho salmon vaccinated during field trials with coded wire tags in Alaska have not had significantly different returns. Pink salmon survivals were significantly higher than non-vaccinated controls. Vibriosis protection has not been an all or none phenomenon. Vaccinated $\mathrm{fish}$ can sustain vibriosis mortalities.

\section{References}

AMEND, D. F. and D. C. FENDER. 1976. Uptake of bovine serum albumin by rainbow trout from hyperosmotic solutions: a model for vaccinating fish. Sci. 192:793-794.

ANDERSON, J. I. W. and D. A. CONROY. 1979. Vibrio disease in marine fishes. pp. 266-272. In: A Symposium of Disease of Fishes and Shellfishes. S. F. SNIESZKO, ed. Am. Fish. Soc. Special Publ. No. 5, Washington, $D C$.

BAIN, N. and J. M. SHEWAN. 1968. Identification of Aeromonas, Vibrio and related organisms. pp. 79-84. In: Identification Methods for Microbiologists, Part B. B. M. GIBBS and D. A. SHAPTON, eds. Academic Press, New York, NY.

BAUMANN, P., L. BAUMANN and J. L. REICHELT. 1973. Taxonomy of marine bacteria: Beneckea parahaemolyticus and Beneckea alginolyticus. J. Bacteriol. $113: 1144-1155$.

BERGMAN, A. M. 1909. Die rote Beulenkrankheit des Aals. Berichte a.d. Kgl. Bayer. Biolog. Versuch. Munchen. 2:10-54.

BRAATEN, B. A. and H. O. HODGINS. 1976. Protection of steelhead trout (Salmo gairdneri) against vibriosis with a living lowvirulence strain of Vibrio aguillarum. J. Fish. Res. Board Can. 33:845-847.

BUCHANAN, R. E. and N. E. GIBBONS, eds. 1974. Bergey's Manual of Determinative Bacteriology. $8 \mathrm{th}$ ed. Williams and Wilkins Co., Baltimore, MD. 1246 pp.
BUCKMANN, A. 1952. Infectionen mit Glugea stephani und mit Vibrio anguillarum bei Schollen (Pleuronectes platessa L.). Kurze Mitt. aus d. Fischereibiol. Abt. Max-Planck-Inst. Meeresbiol. Wilhelmshaven 1:1-7.

BULLOCK, G. L. 1977. Vibriosis in fish. Fish Disease Leaflet 50. U.S. Dept. Inter.. U.S. Fish and Wildl. Serv. Eastern Fish Disease Lab, Leetown, WV.

CANESTRINI, G. 1893. La malattia dominante delle anguille. Atti Ist. Veneto Ser. 7:809-814.

CARDWELL, R. D. and L. S. SMITH. 1971. Hematological manifestations of vibriosis upon juvenile chinook salmon. Prog. Fish-Cult. $33: 232-234$.

CISAR, J. 0. and J. L. FRYER. 1969. An epizootic of vibrios in chinook salmon. Bull. Wildl. Dis. Assoc. $5: 73-76$.

EVELYN, T. P. T. 1971. First records of vibriosis in Pacific salmon culture in Canada and taxonomic status of the responsible bacterium, Vibrio anguillarum. J. Fish. Res. Board Can. 28:517-525.

FRYER, J. L., J. S. NELSON and R. L. GARRISON. 1972. Vibriosis in fish. Progress in Fishery and Food Science. Univ. of Wash. Publ. in Fish. New Series $5: 129-133$.

FRYER, J. L., J. S. ROHOVEC, G. L. TEBBIT, J. S. MCMICHAEL and K. S. PILCHER. 1976. Vaccination for control of infectious diseases in Pacific salmon. Fish. Pathol. $10: 155-164$.

GARRISON, R. L. and R. W. GOULD. 1976. Development of a bacterin and oral immunization procedure to control vibriosis of juvenile salmonids reared in saltwater. Annual Progress Report, Anadromous Fish Project. July 11975 to June 30 1976. Oregon Dept. Fish and Wildl., Portland, Oregon and Dept. Microbiol., Oregon State Univ., Corvallis, OR. (Unpublished). 
GJEDREM, T. and D. AULSTAD. 1974. Selection experiments with salmon I. Differences in resistance to vibrio disease of salmon parr (Salmo salar). Aquaculture 3:51-59.

GRISCHKOWSKY, R. S. 1973. Studies of the Nature of Pacific Oyster (Crassostrea gigas, Thunberg) Mortality. I. Implications of Bacterial Pathogenicity and II. Pathogenicity Testing of Vibrios on Chinook Salmon (Oncorhynchus tshawytscha, Walbaum) and Pacific Oysters. Ph.D. Thesis, Univ. of Washington, Seattle, WA. 152 pp.

HAASTEIN, T. and G. HOLT. 1972. The occurrence of vibrio disease in wild Norwegian fish. J. Fish Biol. 4:33-37.

HEARD, W. R. and R. M. MARTIN. 1973. Progress report on feasibility of salmon smolt production in estuarine husbandry pens at Little Port Walter. Progress Report, July 1 to December 31 1972. U.S. Dept. of Commerce. Nat. Marine Fish. Serv. Auke Bay Lab., Auke Bay, Alaska. 18 pp. (Unpublished).

MCCARTHY, D. H., J. P. STEVENSON and J. S. ROBERTS. 1974. Vibriosis in rainbow trout. J. Wildl. Dis. $10: 2-7$.

MUROGA, K. and S. EGUSA. 1967. Vibrio anguillarum from an endemic disease of ayu in Lake Hamana. Bull. Jap. Soc. Sci. Fish. $33: 636-640$.

NOVOTNY, A. J. and L. W. HARRELL. 1975. Vibriosis a common disease of Pacific salmon cultured in marine waters of Washington. Washington State Univ., Extension Bull. 663. 8 pp.
NYBEL IN, 0. 1935. Untersuchungen uber den bei Fischen krankheitserregenden Spaltpilz Vibrio anguillarum. Mitt der Anstalt F. Binnenfishcherei bei Drottningholm, Stockholm 8:1-62.

RUCKER, R. R. 1959. Vibrio infections among marine and freshwater fish. Prog. Fish-Cult. 21:22-25. 1972. Fish disease therapy: past, present, and future. Progress in Fishery and Food Science, Univ. of Wash. Publ. in Fish. New Series 5:135-140.

RUCKER, R. R., B. J. EARP and E. J. ORDAL. 1953. Infectious diseases of Pacific salmon. Trans. Amer. Fish. Soc. 83:297-312.

SCHAPERCLAUS, $\quad 1954$. Fischkrankheiten. 3rd ed. Akademie-Verlag, Berlin. 708 pp.

SHEWAN, J. M., W. HODGKISS and J. LISTON. 1954. A method for the rapid differentiation of certain non-pathogenic, asporgenous bacilli. Nat. 173:208-211.

SINDERMAN, C. J. 1974. Diagnos is and Control of Mariculture Diseases in the United States. National Marine Fisheries Service. NOAA-76121001 Highlands. NJ. 306 $\mathrm{pp}$.

SMITH, I. W. 1961. A disease of finnock due to Vibrio aguillarum. J. Gen. Microbiol. 24:247-252.

SNIESZKO, S. F. 1970. Immunization of fishes: a review. J. Wildl. Dis. $6: 24-30$.

STANIER, R. Y., M. DOUDOROFF and E. A. ADELBERT. 1970. The Microbial World. $3 r d$ ed. Prentice-Hall, Inc., Englewood Cliffs, NJ. 873 pp.

UMBREIT, W. W. and E. J. ORDAL. 1972. Infection of goldfish with Vibrio anguillarum. Am. Soc. Microbiol. News 338:93-96. 


\section{Enteric Redmouth}

Joseph Sullivan

\section{Introduction}

Enteric redmouth is a systemic bacterial disease associated with Yersinia ruckeri. It has been called "Hagerman redmouth" and simply "redmouth," although the latter term has also been applied to diseases with the same clinical signs involving other bacteria. Yersinia ruckeri is a member of the Enterobacteriaceae, the only one commonly found to be pathogenic to salmonids. Normally an intestinal dweller, it causes disease when it is given the opportunity to cross the gut wall barrier.

The salmonids it has been reported to infect are rainbow trout (Salmo gairdneri), cutthroat trout (Salmo clarki), Atlantic salmon (Salmo salar), brown trout (Salmo trutta), brook trout (Salvelinus fontinalis), chinook salmon (Oncorhynchus tshawytscha), coho salmon (Onchorynchus kisutch) and sockeye salmon (Oncorhynchus nerka) (American Fisheries Society 1979). It can probably infect any salmonid. A reservoir host in the Hagerman Valley of Idaho has been reported to be crayfish (McDaniel 1971), but both there and at 1 location in Alaska (Woody Springs near Kotzebue), $\underline{Y}$. ruckeri was isolated from spring water where no known reservoir host exists.

\section{History}

Enteric redmouth was first recognized as a problem by Rucker (1966) in the 1950's in rainbow trout at the Hagerman National Fish Hatchery. Near perfect conditions existed for the disease to spread and it did. Vast quantities of good quality spring water at the optimum temperature of $15 \mathrm{C}$ soon made the Hagerman Valley the most important locus for the commercial production of rainbow trout in the United States. The aquifer itself contained $\underline{Y}$. rucker $i$ so that exposure to the bacteria was nearly inevitable. Huge quantities of fish were shipped out to many locations and $\underline{Y}$. rucker $i$ with them.

Originally called "redmouth," McDaniel (1971) proposed the name "Hagerman redmouth" to distinguish it from similar diseases involving nonenteric bacteria. However, as the disease became better known, researchers found that some old stock cultures of unidentified Gram negative, cytochrome oxidase negative rods were, in fact, $\underline{Y}$. rucker $i$ and some could not be traced back to the Hagerman Valley. Bullock et al. (1977) reported 2 cultures taken in 1952 from rainbow and brook trout at the Leetown National Fish Hatchery, West Virginia and several others from a 1960 Australian epizootic in rainbow trout. The Leetown cultures were at least as old as Rucker's original isolate and the Australian fish had not been exposed to American diseases for many years. "Enteric redmouth" was more descriptive, removed the questionably valid stigma from the Hagerman Valley and has been in use since 1975 (Busch and Ewing 1975).

The ERM (enteric redmouth) bacterium received its formal appelation, $\underline{Y}$. ruckeri, and was biochemically described in 1978 by Ewing et al. (1978). Considering the properties that other authors 
(Analytab Products 1978, Busch 1978) and we in the Fish Pathology Section of the Alaska Department of Fish and Game have observed, it appears that either the species is very plastic or that a species complex is involved. Some of the differences may be due to the temperatures at which the cultures were incubated or the sensitivity of the tests used ( 0 'Leary et al. 1979).

The first record of ERM in Alaska was made by McDaniel (1971). Subsequent communications with him revealed the case to be a prespawning sockeye mortality at Karluk Lake in 1969.

Enteric redmouth has only been identified a few other times in Alaska, primarily with the recent acquisition of sensitive identification techniques. It is not known whether ERM was endemic to the state or was imported here. On the one hand, rainbow trout from Hagerman, Idaho were imported into Southeast Alaska in 1964, 2 years before ERM was first reported in the literature. Southeast hatcheries and hatcheries that have exchanged stocks with Southeast hatcheries, have yielded Gram negative cytochrome oxidase negative rods from their fish since then. Positive identifications of these cultures were never made, but 2 stocks of fish later diagnosed as having ERM were among those previously shown to be carrying the unidentified bacteria. Conversely, Y. ruckeri was reported from water at Woody Springs which, untreated, may be incapable of supporting fish. Though seemingly parallel to the situation in Idaho, subterranian host animals could exist in the Hagerman aquifer whereas they probably cannot survive in Woody Springs. However, if not endemic, it is hard to see where this may have originated. Perhaps both have some veracity.

Even if $\underline{Y}$. ruckeri was not endemic, and Gräm negative, cytochrome oxidase negative rods were ERM, the shuffling of stocks among hatcheries may have distributed the disease to many of the planted areas of the state. Its occurrence hereafter should come as no great surprise.

\section{Transmission}

Simplistically, Y. ruckeri is horizontally spread through the water multiplying in the gut, passing out with the feces and being ingested by other fish. Disease occurs when the bacteria cross the gut wall and initiate a septicemia, but this is not necessary for the organism to be transmitted. In the Hagerman Valley along the Snake River, the ultimate source appears to be the aquifer itself. George W. Klontz (pers. comm.) isolated $Y$. ruckeri from water taken several feèt into the aquifer. But Klontz and Huddleston (1979) found that the ERM bacteria in spring water samples survived only 2 to 3 weeks leading them to surmise that a host is necessary. No potential subterranean hosts are known there, but conditions are good for their existence.

Klontz and Huddleston (1979) also noted that spring dwelling crayfish had a $30 \%$ Y. ruckeri carrier rate at 500 or greater organisms per gram of visceral homogenate. As with many diseases, removal of carriers ( $f$ ish, crayfish etc.) from the water source is important. In 1967, escaped fish from the incoming spring water above the raceways at the Hagerman National Fish Hatchery (the "type locality" for ERM) were removed; this prevented further access to the area, and effectively subdued the disease as a problem (Klontz and Huddleston 1979). There has been only 1 outbreak of the disease since then.

Recirculating systems without depurating devices are analogous to systems with infected fish in the water source. They may likewise keep 
the numbers of bacteria artificially high in the water, paving the way for a disease outbreak.

\section{Signs and Pathogenesis}

The clinical signs for ERM are roughly the same as those for other systemic Gram negative bacterial diseases. The hemorrhaging and erosion around the mouth or "redmouth" is, as previously stated, common to many diseases. Acute and subacute infections may display exophthalmia, swollen abdomen (from ascites) and reddened opercula and $f$ in bases and inflamed, hemorrhagic vent (color Fig. 33). Unless the environmental conditions have also been poor, the fins are usually not frayed. Longer term "screen hangers" may be very thin and hollow bellied, may have lost pigment control and be darker fish. Bloody diarrhea can often be expressed from the anus, unlike other septicemias. This sign is also common to returning adult salmon infected with Ceratomyxa shasta, but this protozoan disease is not known to occur in Alaska.

Internally there may be inflammation and hemorrhaging in most of the visceral organs (color Fig. 34) including the visceral fat, and some hemorrhaging may be seen in the muscles along the abdominal wall. The hematopoietic tissues may undergo necrosis. There may be edema in the spleen, liver and kidney. The liver may become a pale, cream color (color Fig. 35). Fluids may accumulate in the abdominal cavity, stomach and intestine. The inflamed, hemorrhagic lower intestine (color Fig. 36) with bloody diarrhea is particularly notable with ERM. Other septicemias may result in the inflammation of the intestine, but enteric redmouth begins here and therefore this sign is more common with ERM.
Yersinia ruckeri may reside indefinitely in the lower intestine of fish without causing disease. Normally, the bacteria, although multiplying, are being shed with the feces and a balanced condition exists. However, when some stress takes the fish off their feed, the fish stops defecating and the numbers of bacteria increase. The overabundance initiates necrosis of the mucosa. When the stress is removed and feeding resumes, this dead tissue is sloughed. This exposes the underlying layers, access to the blood vascular system therefore occurs and the septicemia begins. Acute outbreaks occur about 3 to 5 days after the stress is removed. The disease may then become chronic with an eventual loss of $30-70 \%$.

\section{Diagnosis}

Although the bloody diarrhea is very indicative, diagnosis cannot be made only on the basis of clinical signs. Diagnosis is made by the isolation and identification of the causative organism, the slide agglutination test or the indirect fluorescent antibody test. The Fish Health Section of the American Fisheries Society (1979) allows a presumptive identification of $\underline{Y}$. ruckeri if the organism is a Gram negative, cytochrome oxidase negative rod, does not produce indole in tryptone broth, gives an alkaline/acid reaction on triple sugar iron agar (a change from "acid/acid" in the 1975 edition), ferments glucose and is motile. Motility is variable (our observations and O'Leary et al. 1979), but the rest agrees with previous descriptions. If the organism does not fit within the more restrictive boundaries of previously described strains, confirmatory diagnosis may rest on the slide agglutination or fluorescent antibody test. 
These are at least 3 serotypes of ERM and without testing against all of them, false negative fluorescent antibody and slide agglutination tests may result. The procedures may be found in the Fish Health Section of the American Fisheries Society (1979).

\section{Significance and Control}

Occurrence of ERM in the wild fishes of Alaska is probably insignificant. However, since diseases at hatcheries affect the numbers of $\mathrm{fish}$ released, there is an effect.

Hatchery epizootics of enteric redmouth are primarily a sign of poor culture techniques. Without stress, the fish can usually coexist with $\underline{Y}$. rucker $\mathrm{i}$ and not develop the disease. Any sort of stress that would cause a disturbance of normal digestive processes could precipitate an outbreak.

Rucker (1966) recommended a therapeutic treatment of sulfamerazine at $20 \mathrm{~g} / 100 \mathrm{~kg}$ of $\mathrm{fish} /$ day/5 days followed by 3 days of oxytetracycline at $5.0 \mathrm{~g} / 100 \mathrm{~kg} /$ day. Klontz (1979) recommended that $6.6 \mathrm{~kg}$ of each $/ 100 \mathrm{~kg}$ be given simultaneously. This may cause less renal damage due to the sulfamerazine and create less likelihood of the bacteria becoming resistant to both drugs. Use of these drugs requires a 3 week withdrawal period for marketing or stocking from the last date the drug was fed to the fish.

For systemic bacterial infections (which would include ERM), Poupard (1978) recommends a 5:1 mixture of a conventional sulfonamide, e.g. sulfadiazine and a potentiated sulfoamide, e.g. trimethoprin at 5.5 $\mathrm{g} / 100 \mathrm{~kg} \mathrm{fish} / 5-10$ days. This should give enhanced antimicrobial effects without the toxicity and palatability problems of higher doses.
Problems with resistance to these drugs have arisen in Alaska, although poor environment may also have hindered the fish's ability to respond to the disease.

Although poor fish culture techniques will eventually be expressed in some sort of mortalities or failure to meet goals, avoiding exposure of $\mathrm{fish}$ to $\underline{\mathrm{Y}}$. ruckeri is the wisest approach.

If exposure has occurred and is henceforth unavoidable, precautions can still be taken. Eliminate any continuous source of infection, practice good sanitation, calculate the carrying capacities of the facilities, stay within them and accomplish what needs to be done with a minimum of stress.

Because of the required withdrawal period, this should not be done if the fish are being sorted for stocking or processing.

There are at least 2 commercially available vaccines against ERM. If the disease is a known problem it might be wise to consider immunizing the fish against it. An early trial with 1 of these was so stressful that fish carrying infectious pancreatic necrosis virus became clinically ill and suffered $47 \%$ mortalities (Busch et al. 1978). The recent versions are more safe and are applied in a dip treatment or under a shower. Vaccination is not cheap, not without stress, and should not be used as an alternative to good fish culture.

\section{References}

AMERICAN FISHERIES SOCIETY. 1979. Fish Health Blue Book. Procedures for the Detection and Identification of Certain Fish Pathogens. Amer. Fish. Soc. FIsh Health Section. $118 \mathrm{pp}$. 
ANALYTAB PRODUCTS. 1978. Analytical Profile Index. Enterobacteriacea and 0ther Gram Negative Bacteria. Ayerst Laboratories Inc.

BULLOCK, G. L., H. M. STUCKEY and E. B. SHOTTS, JR. 1977. Early records of North American and Australian outbreaks of enteric redmouth disease. Fish Health News 6:96-97.

BUSCH, R. A. 1978. Enteric redmouth disease (Hagerman strain). Mar. Fish. Rev. 40:42-51.

BUSCH, R. A., N. E. BURMEISTER and A. L. SCOTT. 1978. Field and laboratory evaluation of a commercial enteric redmouth vaccine for rainbow trout. Proc. Joint Third Biennial Fish Health Sect. and Ninth Ann. Midwest Fish Dis. Workshops. $67 \mathrm{pp}$.

BUSCH, R. A. and A. J. EWING. 1975. Establishment of an asymptomatic carrier state infection of enteric redmouth disease in rainbow trout. J. Fish. Res. Board. Can. 32:2429-2432.

EWING, W. H., A. J. ROSS, D. I. BRENNER and G. R. FANNING. 1978. Yersinia ruckeri sp. nov., the redmouth (RM) bacterium. Int. J. Syst. Bacteriol. 28:37-44.
KLONTZ, G. W. 1979. Fish Health management: II. Concepts and Methods of Fish Health Epidemiology. Fish. Resources and Off. Continuing Educat., Univ. Idaho, ID. 142 pp.

KLONTZ, G. W. and T. R. HUDDLESTON. 1979. Enteric redmouth of rainbow trout in Idaho: 1976 status report. $13 \mathrm{pp}$. (Unpublished).

MCDANIEL, D. W. 1971. Hagerman redmouth...a new look at an old fish problem. Am. Fish and U.S. Trout News 15:14-28.

O'LEARY, P. J., J. A. ROHOVEC and J. L. FRYER. 1979. A further characterization of Yersinia ruckeri (enteric redmouth bacterium). Fish Pathol. 14:71-78.

POUPARD, C. J. 1978. Therapy of fish diseases. pp. 268-275. In: The Pathology of Fishes. R. J. $\overline{R O B} E \overline{R T S}$, ed. Bailliere Tindall, London, ENG.

RUCKER, R. R. 1966. Redmouth disease of rainbow trout (Salmo gairdneri). Bull. Off. Int. Epizoot. 65:825-830. 
Chapter 3.

PARASITIC DISEASES 


\title{
Costiasis
}

\author{
A. K. Hauck
}

\section{Introduction}

Costiasis, the fish disease caused by the cosmopolitan, flagellated, protozoan ectoparasite Ichtyobodo sp., is of considerable importance in the propagation of both warm and cold-water fish (Ellis and Wootten 1978, Fish 1940). Although Ichtyobodo has been recorded as infesting salmonids in saltwater (Wedemeyer et al. 1976, Wood 1974), it is normally considered a serious pathogen only in freshwater. This obligate parasite is capable of seriously infesting all species of pond fishes, salmon and trout (Fish 1940).

Ichtyobodo spp. belong to the class Zoomastigophorea, order Kinetoplastida and family Bodonidae (Margolis and Arthur 1979). There are 2 described species of Ichtyobodo: 1. necator (= necatrix) and 1. pyriformis, both capable of parasitizing salmonids and producing costiasis (Davis 1953). Ichtyobodo necator (Pinto 1928) was originally described as Bodo necator (Fish 1940). Later it was relegated to the genus Costia from which the term costiasis was derived.

Conditions which will precipitate costiasis include state of susceptibility and immunocompetence of the host and environmental conditions such as crowding, low dissolved oxygen and excessive size variation among the fish (Robertson 1979, Wedemeyer et al. 1976).

\section{History}

Costiasis is widely distributed in Europe and North America (Amlacher
1970, Davis 1953, Margol is and Arthur 1979). It most likely occurs universally, since the parasite has cosmopolitan distribution.

Recorded Canadian hosts for 1. necator are the threespine stickleback (Gasterosteus aculeatus), sockeye salmon (Oncorhynchus nerka) and brook trout (Salvelinus fontinalis) (Margolis and Arthur 1979).

In Alaska, several Ichtyobodo infestations occurred in 1975; costiasis in East Creek Hatchery (Lake Nunavaugaluk) sockeye salmon and in Halibut Cove Lagoon (Kachemak Bay) coho salmon (Oncorhynchus kisutch), and infestations of Auke Bay (Juneau) sockeye salmon, of Kitoi Bay Hatchery (Kodiak Island) sockeye salmon and of Crystal Lake Hatchery (Petersburg) coho salmon. The Auke Bay and Kodiak records both occurred concurrent with an epizootic caused by infectious hematopoietic necrosis virus in the same groups of fish. In addition, Ichtyobodo was isolated in 1977 from ship Creek Hatchery (Anchorage) rainbow trout (Salmo gairdneri) and Little Port Walter (Baranof Island) chinook salmon (Oncorhynchus tshawytscha). Since 1977, the parasite has not developed infestations of significance or caused serious fish mortalities in Alaska.

\section{Transmission}

Reproduction of Ichtyobodo is accomplished asexually by longitudinal fission. As the attached organism approaches division, 4 additional flagella are produced making a total of $8 \mathrm{flagella}$. Accompanying the newly divided organism are 4 of these flagella, which by motile means will direct it to either the same 
host or to a new host. Being an obligate parasite, Ichtyobodo must find a suitable host within approximately 1 hour after division to survive (Amlacher 1970). Attachment of the daughter organism to a host was thought to be accomplished by means of the flagella (Fish 1940), possibly because the flagella are not visible in attached forms. However, more recent electron microscope studies have shown lchtyobodo to attach by means of a flat disc (Rogers and Gaines 1975).

\section{$\underline{\text { Signs }}$ and Pathogenesis}

Early Ichtyobodo investigators have suggested that the organism parasitizes debilitated fish in poor condition (Amlacher 1970, Robertson 1979). More recent reports, while indicating this may be true of older fish and brood stock with residual lchtyobodo infestations, have found no correlation between fish condition and intensity of infestation. These same reports postulate that of primary importance in establishing a state of costiasis are the host's state of susceptibility and immunocompetence. Environmental variables and state of health are considered secondary (Robertson 1979).

Environmental aspects which will facilitate costiasis are crowded conditions, low dissolved oxygen, excessive size differences of $\mathrm{fish}$ and $\mathrm{pH}$ (Amlacher 1970, Wedemeyer et al. 1976). Water temperatures do not appear to be important since infestations have been known to occur between 3.5 and 16C (Robertson 1979).

The host's age is also an important factor in establishing disease. Ichtyobodo apparently has a preference for young fry or fingerlings, and costiasis is seldom of consequence in older fish. In fact, the most severe epizootics have occurred in fry within a few weeks after the first feeding (Fish 1940, Wood 1974).

The parasite has been found to show a definite periodicity in its infestations. After being discovered on first feeding fry, lchtyobodo quickly increases in numbers, and the peak of infestation is at about 4 weeks. Following this is a progressive decline in intensity of infestation after 11 and 13 weeks on gills and skin, respectively. After this period the parasite is virtually absent (Reichenbach-Klinke 1973). A progressive series of 3 phases seems to occur in the Ichtyobodo infestation of salmonid fry: gills only, gills and skin and skin only. When a heavy infestation is found on the skin, few parasites are seen on the gills (Robertson 1979).

Signs generally are similar to those common with infestations of external parasites, e.g. loss of appetite and flashing. An increase in daily mortalities will ensue. In advanced cases, a blue-gray film appears on the surface of the fish. This coloration is caused by the marked mucous production and general hyperplasia of the body epithelium in response to the irritant (Fish 1940, Rogers and Gaines 1975).

Gill alterations include acute hyperplasia and cell necrosis. Fusion of secondary lamellae, apparently to enclose the parasite, has been observed. The host also responds by producing large numbers of both goblet cells on secondary lamellae and melanin granules adjacent to primary lamellar blood vessels. Prominent lymphocyte infiltration has also been observed (Ellis and Wootten 1978, Fish 1940). Skin lesions include hyperemia (increased blood resulting in distended blood 
vessels) and necrosis (Amlacher 1970, Robertson 1979). Also evident is a marked reduction in epidermal cytoplasmic content (Robertson 1979). This reduction in cytoplasm may be caused by the fact that the parasite extends small microtubules into the host cell through which vacuolated food is returned to the host (Rogers and Gaines 1975).

The tissue alterations in Alaskan fish from East Creek Hatchery and Halibut Cove Lagoon showed severe hyperplasia and fusion of secondary lamellae (color Fig. 41). Increased number of goblet cells, cytoplasm reduction and lymphocyte infiltration (Ellis and Wootten 1978, Fish 1940, Rogers and Gaines 1975) were not evident in the above Alaskan samples.

Death is caused by osmoregulatory failure followed by hemodilation and circulatory failure (Robertson 1979). Death has been reported to be caused by the presence of the parasite only on the gills (Savage 1935); however, infestation of the skin is also considered important in causing death of the host (Robertson 1979).

\section{Diagnosis}

Costiasis is seldom accurately diagnosed possibly because of the small size and sedentary nature of the causative organism ( Fish 1940).

The dorsal fin epithelium and tips of gill filaments are 2 areas of the fish providing the most likely sites for infestation of Ichtyobodo (Fish 1940). The velvet-like, bluish cast on the skin should not be considered diagnostic for Ichtyobodo, since this same sign is caused by Chilodonella, Trichodina, Oodinium, Gyrodactylus and water hyperalkalinity (Reichenbach-Klinke 1973).
Temporary wet mounts, particularly in $0.7 \%$ physiological saline, should be examined immediately after preparation, since the organism will not normally live longer than 5 minutes on a temporary mount. If more time is required, filaments from live specimens should be fixed in neutral formalin for later examination.

Two forms of 1. necator may be found in epithelial smears: parasites attached to epithelium and a smaller number of free-swimming forms (Fish 1940). The attached forms are pear-shaped (color Fig. 42); the free-swimming forms are ellipsoid in outline, measure approximately $8 \times 4 \mathrm{u}$ and are dorso ventrally compressed (EII is and Wootten 1978, Fish 1940).

Few structural details may be discerned in the living parasite. When properly fixed and stained, 1 or more large vacuoles, smaller, darker staining inclusion bodies, and a dark, spherical nucleus surrounded by a halo are visible. The freeswimming forms have 4 flagella, 2 long and 2 short, which arise from the anterior end, lie along a funnel shaped groove on the organism's ventral side. The long flagella frequently extend beyond the body of the organism (Fish 1940, ReichenbachKlinke 1973). The flagella are not readily visible on the attached form (Fish 1940).

\section{Significance and Control}

Ichtyobodo is considered by many as the most important flagellate protozoan parasite of salmonid culture (Robertson 1979). Heavy infestations of up to 15 parasites/host epithelial cell have been observed (Fish 1940). 
Although normally considered a problem of fry, costiasis has also been known to occur in older fish. Heavy mortalities to Atlantic salmon (Salmo salar) smolts have occurred when the fish were infested by Ichtyobodo prior to transport (Ellis and Wootten 1978). Also, unsuccessful adaptation of chinook salmon smolts to seawater has been known to occur as a result of residual Ichtyobodo infestations (Wood 1974). This sheds additional light on the ability of the parasite to affect its host's osmoregulatory mechanism.

Wounds produced by Icthyobodo will also facilitate infection by secondary invaders (Fish 1940, Rogers and Gaines 1975) and result in additional mortalities.

Controls for this parasite are both mechanical and chemical. The elimination of crowded conditions, increase of dissolved oxygen, and effective grading will help prevent or relieve the problem. There are many chemical controls (Amlacher 1970, Fish 1940, Hoffman and Meyer 1974). Those effectively used on salmonids include acetic acid, formalin and pyridyl mercuric acetate (PMA) (Hoffman and Meyer 1974). Before treatment, the chemical should have current FDA or EPA registration as appropriate for food fish.

To prevent the possibility of costiasis from occurring in seawatercultured salmonids, it is important to ensure that smolts are uninfested before transport. If fish are infested, transport in medicated freshwater might properly control the pathogen (Ellis and Wootten 1978).

Although the occurrences of costiasis in Alaska presently are few, it will most likely become a more significant Alaskan disease entity in the future, as new fish hatchery and incubation facilities become operational.

\section{References}

AMLACHER, E. 1970. Textbook of Fish Diseases (transl. by D.A. Conroy and R. L. Herman). TFH Publications Inc., Neptune City, NJ. 302 pp.

DAVIS, H. S. 1953. Culture and Disease of Game Fishes. Univ. Calif. Press, Berkeley, CA. 332 $\mathrm{pp}$.

ELLIS, A. E. and R. WOOTTEN. 1978. Costiasis of Atlantic salmon, Salmo salar L. smolts in seawater. J. Fish. Dis. 1:389-393.

FISH, F. F. 1940. Notes on Costia necatrix. Trans. Amer. Fish. Soc. $70: 441-445$.

HOFFMAN, G. L. and F. P. MEYER. 1974. Parasites of Freshwater Fishes: a Review of Their Control and Treatment. TFH Publications Inc., Neptune City, NJ. 224 pp.

MARGOLIS, L. and J. R. ARTHUR. 1979. Synopsis of the parasites of fishes of Canada. Bull. Fish. Res. Board Can. 199:269.

REICHENBACH-KLINKE, H. -H. 1973. Fish Pathology. TFH Publications Inc. , Neptune City, NJ. 512 pp.

ROBERTSON, D. A. 1979. Hostparasite interactions between lchtyobodo necator (Henneguy, 1883) and farmed salmonids. J. Fish Dis. 2:481-491.

ROGERS, W. A. and J. L. GAINES, JR. 1975. Lesions of protozoan diseases in fish. pp. 117-141. In: The Pathology of Fishes. W. E. RIBELIN and G. MIGAKI, eds. Univ. Wisconsin Press, Madison, WI.

SAVAGE, A. 1935. Notes on costiasis. Trans. Amer. Fish. Soc. 65:332-333.

WEDEMEYER, G. A., F. P. MEYER and L. SMITH. 1976. Environmental Stress and Fish Diseases. TFH Publications Inc., Neptune City, NJ. $192 \mathrm{pp}$. 
W00D, J. W. 1974. Diseases of Pacific Salmon: Their Prevention and Treatment. 2nd ed. Dept. Fisheries, Hatcheries Div., State of Washington, WA. $82 \mathrm{pp}$.
WOOTTEN, R. and E. A. NEEDHAM. 1978. Parasitology of teleost fishes. pp. 144-182. In: Fish Pathology. R. J. ROBERTS, ed. Balliere, Tindall, London, ENG.

\title{
Ichthyophthiriasis
}

\author{
A. K. Hauck
}

\section{Introduction}

Ichthyophthiriasis, commonly known as white-spot disease or "ich," is caused by Ichthyophthirius multifilis Fouquet, 1876. This protozoan is an obligate parasite belonging to the class Ciliatea, order Hymenostomatida and family Ophryoglenidae (Margolis and Arthur 1979). It is a holotrichous ciliate, i.e. it has a uniform covering of cilia over its entire body.

Ichthyophthirius multifilis enjoys cosmopolitan distribution, being known to parasitize feral, hatchery and aquarium freshwater fish (Cheng 1973, Meyer 1972). Its marine counterpart, which closely parallels 1. multifilis in life cycle and produces a similar pathology in fish, is Cryptocaryon irritans (Rogers and Gaines 1975).

This parasite knows no host boundaries, although catfish (Silurus glanis), salmonids, yellow perch (Perca flavecens) and eels are considered to be the most susceptible hosts (American Fisheries Society 1979).
History

Ich was first described from France in 1876. Since that time, it has been reported from all European countries, North America, China, India, Indonesia, Australia and the USSR. Ich is most likely found in every country which has engaged in foreign fish exchange, particularly those which have imported goldfish and other aquarium fishes (Hoffman 1970).

In Canada its recorded hosts include the freshwater drum (Aplodinotus grunniens), white sucker (Catostomus commersoni), threespine stickleback (Gasterosteus aculeatus), brown bullhead (Ictalurus nebulosus), smallmouth bass (Micropterus dolomieui), largemouth bass (Micropterus salmoides), whitebass (Morone chrysops), yellow perch, (Perca flavescens), Atlantic salmon (Salmo salar), brook trout (Salvelinus fontinalis), coho salmon (Oncorhynchus kisutch), chinook salmon (Oncorhynchus tshawytscha), cutthroat trout (Salmo clarki), rainbow trout (Salmo gairdner i) and brown trout (Salmo trutta) (Margol is and Arthur 1979). 
In Alaska the known history of ich is limited to 3 recordings: in 1975 ich produced heavy prespawning mortalities in sockeye salmon (Oncorhynchus nerka) at Meadow Creek (near Big Lake), in 1978 ich caused heavy mortalities in threespine sticklebacks of Six Mile Lake (near Anchorage), and in 1979 ich trophozoites were observed on skin, nares, and gills of Big Lake Hatchery sockeye salmon which were dying from Saprolegnia infection of nare and brain tissues. It is noteworthy that the threespine sticklebacks (mortality of 1978) also showed concurrent infections of the larval cestode Schistocephalus solidus, the external protozoan Trichodina sp., the eye fluke Diplostomulum sp., the intestinal fluke Crepidostomum sp. and an unidentified nematode in the lamina propria (connective tissue of the stomach). In spite of this, the ich infestation was considered the primary cause of mortality.

At this writing the parasite has not been found on Alaskan fish north of $62^{\circ}$ latitude. This northern range corresponds nicely with the report that 1. multifilis has not been found north of $60-65^{\circ}$ northern latitude in Russia (Hoffman 1970).

\section{Transmission}

The life cycle of 1 . multifilis is both direct and complex. The infective form or theront, is a freeswimming form which seeks contact with a new piscine host. Death occurs if the theront fails to locate a host within 48 hours; however, at optimum temperatures (approximately 22C), infective stages have been known to survive for 96 hours. The theront possesses an incompletely developed cytostome (mouth), which becomes completely developed only after the ciliate has established itself under the host's epidermis. When finding a host, the theront penetrates the epithelium, with aid from strong ciliary movement, to the hypodermis, where the parasite becomes enclosed in host epithelial tissues, matures and grows (Cheng 1973, Meyer 1972, Reichenbach-Klinke 1973).

The mature parasite is thought to gain nourishment by imbibing host fluids and consuming host tissues, possibly detached by both the growth and the rotatory movement of the organism (Meyer 1972, ReichenbachKlinke 1973). In preparation for its benthic, encystment stage apart from the host, the organism is known to deposit glycogen and amylopectin food reserves in its cytoplasm (Cheng 1973). Maturation normally occurs within 10 to 14 days in water temperatures $>15 \mathrm{C}$ and in 35 days at temperatures $<10 \mathrm{C}$. When the organism has attained a certain maturation stage, it penetrates the host's epithelium and leaves the skin or gills (Meyer 1972). The organism may swim for 2 to 6 hours before attaching to a substratum (Meyer 1972). Little preference is shown in the choice of substratum, since benthic forms have been found on surfaces of aquariums or ponds, on plants, debris, or some even remain free floating (Davis 1953, Meyer 1972). After attachment, the organism secretes a thin gelatinous membrane over its surface. This membrane forms a cyst and aids in holding the organism in place (Meyer 1972). During the benthic stage, asexual fission occurs inside the cyst, producing between 250 to 2000 tomites. The number of tomites formed depends upon the size of the matured organism at encystment. The tomites are pyriform, entirely ciliated, and measure between 18-45 um. After 7 to 24 hours, the tomites are released from the cyst, become free-swimming theronts and select a new host (Cheng 1973, Meyer 1972, Reichenbach-Klinke 1973). It has been noted that the food reserves 
stored by the mature organism are completely depleted prior to encystment of the tomites (Cheng 1973).

The completion of the life cycle requires between 4 to 40 days, depending on water temperatures and conditions (Hoffman 1970). Ichthyophthirius multifilis is considered sensitive to lack of oxygen and low $\mathrm{pH}$, although its ability to reproduce in such great numbers enhances the completion of its life cycle and survival (Amlacher 1970, Johnson 1974).

\section{Signs and Pathogenesis}

In contrast to many fish diseases which occur after the host has become weakened, 1. multifilis reportedly will infest any fish host, regardless of its state of health, to complete its life cycle (Johnson 1974). There is some confusion in this precept however, since 1 investigator found that 2 to 3 days after disturbances in mucous metabolism had occcurred, 1. multifilis infestations occurred at the site of the disturbance (Gaines and Rogers 1975).

The action and movement of the parasite on the host and the host's response to the parasite are important mechanisms in the pathogenesis of Ichthyophthirius. Trophozoites will involve in lateral movement below the epidermis producing corridors and collecting in common chambers ( $\mathrm{Am}$ lacher 1970, Bauer 1958). Some investigators have indicated I. multifilis has penetrated into the $\overline{\text { der- }}$ mis, blood vessels, cephalic muscles, conjunctiva and corneal epithelium and lamina propria (Amlacher 1970, Dukes 1975).

Others have found 1 . multifilis beneath the intestinal mucosa of small aquarium $f i s h$ and in the visceral cavity of sticklebacks (Hoffman 1970). Possibly the maturity of the organism plays a role in these extreme cases, since the destructive action of young theronts on fish is considered much greater than that of mature parasites (Amlacher 1970).

In response to the actions of 1 . multifilis, the host undergoes ce $\bar{l}-$ lular epithelialization at the sites of infection and engulfs the young parasites by host cell hyperplasia. This occurs within 6 days of initial infection. Stasis (diminution or stoppage of blood flow) is said to be produced in the dermis. Pathological signs and lesions produced by Ichthyophthirius include: increased mucous production, desquamation, hyperplasia, reduction of goblet cells in the areas of epithelialization, appearance of small, white pustules on gills and entire body surface, and skin erythema (redness). The gills become swollen, anemic, lose epithelium, turn yellow-grey in color and become necrotic. Infected fish will lose their appetite, congregate near the source of incoming water or at the edges of the pond and become sluggish, emaciated and suffer respiratory inhibition (Bauer 1958, Davis 1953, Dukes 1975, Johnson 1974, Meyer 1972, Reichenbach-Klinke 1973, Rogers and Gaines 1975). If the infestation is heavy or respiration is severely inhibited, death will ensue.

The Alaskan threespine sticklebacks which underwent an Ichthyophthirius epizootic in August of 1978, showed the following tissue alterations: young parasites were completely surrounded by host hyperplastic epidermis, more mature parasites had penetrated to the dermis but not beyond (color Fig. 43). A marked reduction of goblet cells was particularly evident in the epidermis directly surrounding the parasite. Gills only showed hyperplasia at the site of parasite encystment. A moderate hyperemia of secondary lamellae was evident, although no aneurysms were present. 
Fish which have survived ich are said to develop temporary immunity, which over a period of several months, enables them to resist the attachment of the infective forms of the organism (Hoffman 1970, Johnson 1974, Reichenbach-Kl inke 1973). This however, is not a complete immunity because the host can still act as an asymptomatic carrier (Johnson 1974). Rainbow trout, in response to 1. multifilis, have been reported capable of producing a serum element which purportedly kills the parasite on contact (Amlacher 1970). Much more work is needed to come to a complete understanding of the immune responses of fish to ich. It is known however, that stressors such as oxygen shortages, rapid environmental changes, etc., will lessen the ability of a fish to produce effective immunity. Also, environmental stressors will decrease the immunity of symptomatic carriers and as a result, disease may become active once again (Johnson 1974).

\section{Diagnosis}

In selecting a fish for examination, living fish are preferred, although the parasite may be found on dead fish if little time has elapsed since death (Amlacher 1970). Location of the white spots on the skin, making the fish appear that it has been sprinkled with salt, is presumptive diagnosis (Reichenbach-Klinke 1973).

Preparation of skin scrapings and examination of the gills will help confirm the diagnosis of Ichthyophthirius, and should always be done regardless of the appearance of the white spots on the skin (Johnson 1974). Confirmation can be made from microscopically examining the parasite for the following: large size (0.5-1.0 mm diameter), uniform cilia giving the organism a rolling motion, the anterior end being slightly protracted and containing a cytostome (mouth) and a large, horseshoe-shaped macronucleus (color Fig. 44). The cytoplasm of the organism frequently contains vacuoles and pigment deposits, and adhering to the macronucleus is a smaller, spherical micronucleus (American Fisheries Society 1979, Meyer 1972, Reichenbach-Klinke 1973).

\section{Significance and Control}

\section{Control}

The best control of Ichthyophthirius disease, as in all fish culture operations, is prevention. The prevention of Icthyophthirius may be accomplished by prohibiting the dissemination of fish stocks which have known records of the parasite to watersheds where the parasite has no record of incidence. Frequently however, fish transports are necessary. The purchase of treated and/or certified Ichthyophthirius-free fish does not insure those fish are free of the organism, since the subepidermal 1. multifilis trophozoites are sheltered from treatments and may not appear on asymptomatic carriers. Therefore, potential carrier fish should be quarantined for several days prior to introducing them into uninfected waters to assist in removal of adult epidermal forms (Johnson 1974).

Ichthyophthirius multifilis has notoriously been disseminated worldwide by both tropical and bait fish transports. Therefore, the release of aquarium fish into natural waters, and the use of bait fishes should be prohibited.

Because of the long periods involved with cold water temperatures to ascertain infection, quarantine of suspected carriers is most likely an impractical control in Alaska. 
Ichthyophthirius multifilis ceases development at high water temperatures (approximately 30C) (Johnson 1974). Although this is an effective control of Ichthyophthirius for warm water and aquarium fish (Meyer 1972), it is obviously impractical for coldwater hatchery situations in Alaska. In Alaska where hatchery water temperatures are suboptimal for Ichthyophthirius, handling stress will still lower the resistance of the fish to the parasite.

In hatchery situations, the elimination of wild fish populations from the hatchery water is important, since wild fish will act as carriers of 1. multifilis (Johnson 1974). If the parasite is found in hatchery water, then the best prevention is to change to ground water (Johnson 1974). If the parasite is known to infect hatchery fish, then a practical method of controlling the problem is to hold the infected fish in swiftly running water for several days or weeks until all the mature parasites have dropped off. The swift current will carry the parasites away before they have opportunity to multiply and reinfect the hatchery fish (Davis 1953).

Other effective methods of controlling 1. multifilis in the hatchery are: to treat brood fish with formalin, to use artificial spawning techniques followed by iodophore disinfection of eggs, to separate adults from eggs before the eggs hatch (Wedemeyer et al. 1976), and to permit equipment to disinfect for long periods between use in concentrated potassium permanganate or acriflavine hydrochloride (Amlacher 1970, Hoffman and Meyer 1974, Johnson 1974).

The chemical controls (Hoffman and Meyer 1974, Meyer 1972, ReichenbachKlinke 1973) for ich infestations are too numerous to mention. Generally, the most effective chemicals are for- malin and malachite green (Hoffman 1970). However, if fish are to be used for human or animal food, they can be treated with drugs and chemicals only in accordance with current federal (FDA and EPA) laws and regulations (Meyer 1972). Neither formalin nor malachite green are currently registered for use on food fishes.

Chemical baths will effectively control and eliminate theronts, tomites and emerged adults. However, no drugs, chemicals, or treatments are known which will effectively control the trophozoite and encysted, benthic stages (Amlacher 1970, Meyer 1972, Reichenbach-Klinke 1973). In low water temperatures, encysted, benthic stages will hatch normally after 20 days. Therefore, baths must necessarily be extended to effectively eliminate emerging adults, preferably to periods longer than 20 days (Johnson 1974, ReichenbachKl inke 1973).

The stressful effect of extended bath treatments necessary in cold waters may be more harmful to the fish than the parasite. Therefore, the best control of ich in Alaska as elsewhere is prevention.

\section{Significance}

The holotrichous ciliate, 1 . multifilis, although providing no $\overline{i l l}$ effects to humans (Johnson 1974), probably causes more damage to $\mathrm{fish}$ populations over the entire world than any other single parasite (Rogers and Gaines 1975). The parasite's direct life cycle and the ability of a mature organism to produce many infective elements aid the successful completion of its life cycle (Johnson 1974). I ch is also dangerous because of its lack of host specificity, destructiveness to host tissues and difficulty to eradicate. Also, the parasite reportedly is 
capable of producing medicationresistant strains, causing host immunity to be short-lived (Reichenbach-Klinke 1973).

The hatchery environment provides the ideal home for this ciliate. Investigators have shown that 1 . multifilis has a natural propensity for infesting free-swimming fishes over benthic fish. This, combined with the often crowded situation in the hatchery environment, causes infestation to exceed the natural rate and enhances the contagious potential of the parasite (Johnson 1974).

Inapparent (asymptomatic) infestations with 1. multifilis are considered common. The consequences of release of such carriers into another fish population may result in a serious health problem for the established stock (Johnson 1974, American Fisheries Society 1979).

Since ich is not normally of significance in waters where temperatures rarely approach the parasite's opt imum (approximately 21-24C) (Roberts 1975), one might theorize that this disease will only occur in Alaskan waters when summer temperatures produce warm water conditions. This possibility is supported by the fact that all 3 known occurrences of the disease in Alaska were during summer months.

\section{References}

AMERICAN FISHERIES SOCIETY. 1979. Fish Health Blue Book. Procedures for the Detection and Identification of Certain Fish Pathogens. D. MCDANIEL, ed. Rev. ed. Amer. Fish. Soc. Fish. Health Section. $118 \mathrm{pp}$.

AMLACHER, E. 1970. Textbook of Fish Diseases. (transl. from German). TFH Publications Inc., Neptune City, NJ. 302 pp.
BAUER, 0. N. 1958. Parasitic diseases of cultured fishes and methods of their prevention and treatment. pp. 265-298. In: Parasitology of Fishes. (transl. from Russian). V. A. DOGIEL, et al., eds. TFH Publications Inc., Neptune City, NJ.

CHENG, T. C. 1973. General Parasitology. Academic Press Inc., New York, NY. 965 pp.

DAVIS, H. S. 1953. Culture and Diseases of Game Fishes. Univ. California Press, Berkeley, CA. $332 \mathrm{pp}$.

DUKES, T. W. 1975. Ophthalmic pathology of fishes. pp. 383-398. In: The Pathology of Fishes. W. E. RIBELIN and G. MIGAKI, eds. Univ. of Wisconsin Press, Madison, WI.

GAINES, J. L. and W. A. ROGERS. 1975. Some skin lesions of fishes. pp. 429-441. In: The Pathology of Fishes. W. E. RIBELIN and G. MIGAKI, eds. Univ. of Wisconsin Press, Madison, WI.

HOFFMAN, G. L. 1970. Parasites of North American Freshwater Fishes. Univ. California Press, Berkeley, CA. 486 pp.

HOFFMAN, G. L. and F. P. MEYER. 1974. Parasites of Freshwater Fishes: A Review of Their Control and Treatment. TFH Publications Inc., Neptune City, NJ. 224 pp.

JOHNSON, S. K. 1974. Ich disease of fishes. Texas Agr. Exp. Sta. Bull. L-1210. 5 pp.

MARGOLIS, L. and J. R. ARTHUR. 1979. Synopsis of the parasites of fishes of Canada. Bull. Fish. Res. Board Can. 199:269.

MEYER, F. P. 1972. Parasites of Freshwater Fishes. 11. Protozoa. Ichthyophthirius multifilis. USFWS, Fish Dis. Leaflet No. 2.4 pp.

REICHENBACH-KLINKE, H. - H. 1973. Fish Pathology. TFH Publications Inc., Neptune City, NJ. 512 pp. 
ROBERTS, R. J. 1975. The effects of temperature on diseases and their histopathological manifestations in fish. pp. 477-496. In: The Pathology of Fishes. W. E. RIBELIN and G. MIGAKI, eds. Univ. of Wisconsin Press, Madison, WI.

ROGERS, W. A. and J. L. GAINES, JR. 1975. Lesions of protozoan diseases in fish. pp. 117-141. In: The Pathology of Fishes. W. E. RIBELIN and G. MIGAKI, eds. Univ. of Wisconsin Press, Madison, WI.
WEDEMEYER, G. A., F. P. MEYER and L. SMITH. 1976. Environmental Stress and Fish Diseases. TFH Publications Inc., Neptune City, NJ. 192 pp.

\title{
Hexamitiasis
}

\author{
A. K. Hauck
}

\section{Introduction}

Hexamita (Octomitus) (Dujardin $1841)$ is the generic name designating a group of polymastigine, flagellate protozoans which are cosmopolitan or worldwide in distribution. Hexamita is represented by many free-living, commensal and parasitic species. Recorded symbiotic hosts include leeches, trematodes, univalve and bivalve mollusks, arthropods, frogs, salamanders, snakes, turtles, birds, monkeys, rodents and fish (Uzmann and Hayduk 1963).

Some confusion exists regarding the taxonomy of this parasite. Octomitus intestinalis (Schmidt) and Octomitus intestinalis truttae (Schmidt) were both described in 1920. Octomitus salmonis (Moore) was described in 1922 from trout in hatcheries throughout the State of New York. The generic name (Octomitus) was later changed to Hexamita. Octomitus intestinalis truttae and $\underline{0}$. salmonis are probably the same organism. Also, H. intestinalis and $H$. salmonis a re most likely the same organism. If so, they should all be called $H$. intestinalis (G. Hoffman, pers. comm.). Since the organism has been widely known in recent times as $H$. salmonis and has been reported predominantly from salmonid hosts, it will be referred to here as $\underline{H}$. salmonis.

Hexamita salmonis is a common resident in the gastrointestinal tract of juvenile salmonids where it may result in the condition known as hexamitiasis. Although the pathogenicity of $\underline{H}$. salmonis has been challenged (Uzmann et al. 1965), it has been reported to cause catarrhal enteritis with desquamation of the intestinal mucous membrane by other investigators (Sano 1970). Hexamita, on the other hand, has been documented as extremely pathogenic to fish (Reichenbach-Klinke 1973). Hexamita sp. endoparasites are also considered to play an important role in the "hole-in-the-head" disease or "Lochkrankheit" of cichlids (Reichenbach-Klinke 1973). 
Those species of $f$ ishes known to be susceptible to Hexamita spp. infections include: brown trout (Salmo trutta), Atlantic salmon ( $\overline{\text { Salmo }}$ salar), rainbow trout (Salmo gaird$\overline{\text { neri) }}$, cutthroat trout (Salmo clarki), lake trout (Salvelinus namaycush), brook trout (Salvelinus fontinalis), Dolly Varden trout (Salvelinus malma), Arctic grayling (Thymallus arcticus), round whitefish (Prosopium cylindraceum), sockeye salmon (Oncorhynchus nerka), chinook salmon (Oncorhynchus tshawytscha), pink salmon (Oncorhynchus gorbuscha), chum salmon (Oncorhynchus keta), coho salmon (Oncorhynchus kisutch), burbot (Lota lota), threespine stickleback (Gasterosteus aculeatus), longnose sucker (Catostomus catostomus) and tropical discus fish (Symphysodon discus) (Bykhovskaya-Pavlovskaya et al. 1962, Hoffman 1970, Margol is and Arthur 1979).

\section{History}

The $\underline{H}$. salmonis endoparasite enjoys worldwide distribution. The occurrence of fish hexamitiasis accompanying infections with Hexamita appears to occur less frequently (Uzmann et al. 1965). The parasite is reported to be endemically present at trout and salmon hatcheries throughout the United States and Canada (Davis 1953, Uzmann et al. 1965). Early investigators reported that it was responsible for heavy mortalities among fingerling trout (Davis 1953), but evidence that Hexamita actually causes mortalities and reduces growth is still being debated. A causal relationship is reportedly not apparent (Uzmann et al. 1965).

In Alaska, mortalities of juvenile salmonids presumptively caused by Hexamita have been reported on 2 occasions from a Southcentral hatchery. Both cases occurred at Fort Richardson Hatchery (near Anchorage) toward the latter part of December in 1978 and 1979. Mortalities first appeared in the 1978 brood Crooked Creek chinook fingerlings. Heavy infections of the parasite were observed in wetmounts of gut scrapings from both moribund and dead fish from this lot. Healthy fingerlings showed light to moderately intense infections. Within days of the initial report, Hexamita was isolated from Ship Creek chinook, Halibut Cove Lagoon chinook and Ennis Alaska rainbow trout. Parasites were not isolated from intestinal contents of the Swanson River rainbow trout also being reared at the hatchery. Total mortalities attributed to $\underline{H}$. salmonis at Fort Richardson Hatchery were $20.6 \%$ and $1.2 \%$ for 1978 and 1979 respectively. Hexamita salmonis was later isolated from a group of normal-appearing adult fish located in the hatchery's water source. These included feral Dolly varden which had entered the hatchery's water system through Ship Creek (a feeder stream to the hatchery) and a collection of unidentified salmonids which had earlier escaped from the hatchery's rearing ponds.

\section{Transmission}

Hexamita salmonis is transmitted horizontally from both asymptomatic and moribund carriers. Successful transmission is assisted in the parasite's ability to rapidly reproduce in vast numbers of organisms through 2 forms: trophic and intracellular (Davis 1953).

The trophic form reproduces by binary fission. During this reproductive process, the organisms become rounded and their internal, cellular structures, except the flagella, divide into equal parts. New flagella quickly develop on the daughter flagellate cells. The entire process occurs rapidly under favorable conditions. 
The intracellular form has been reported as occurring in the epithelium of the intestine and pyloric caeca (Davis 1926, 1953). This form first appears as a small round cell which divides into many smaller cells, each identical to the original. These daughter cells spread throughout the host's intestinal epithelium and repeat the cycle. In time these intracellular forms of Hexamita purportedly develop into the flagellate form located in the intestinal lumen.

When environmental conditions are less favorable, the trophic form will develop into a cyst which can live for a considerable period outside the host. The parasite is transmitted by a larger, noninfected fish preying on a smaller, infected fish or by passive ingestion of cysts which have been excreted with alimentary wastes (Bykhovskaya-Pavlovskaya et al. 1962). 0lder fish normally will not develop clinical signs of the disease, but they may become reservoir hosts which transmit the parasite horizontally to new hosts via al imentary wastes.

Although the intracellular form of hexamitiasis does manifest itself as a pathological condition of some importance, the identity of $\mathrm{H}$. salmonis as its causal agent has not been proven (Uzmann et al. 1965).

\section{Signs and Pathogenesis}

One of the initial signs of hexamitiasis is behavioral in nature. Fish lose their appetites and decrease or completely terminate food intake (anorexia). Emaciation then results in a "pinhead" appearance, where the fish show a large head, attenuated body and pinched-in abdomen. The fish then become weak and listless and are frequently seen swimming alone near the water surface and at the tail of the raceway. In some cases, fish have been observed swimming on their sides or in a corkscrew motion. Spasmodic twitching along the flanks has also been reported (Davis 1953, Wood 1974).

Internal clinical signs include catarrhal enteritis (inflammation of intestine with mucous exudate) with desquamation (sloughing) of the intestinal mucous membrane (Sano 1970). The exudate is described as a yellowish, thick fluid (Wood 1974). Inflamed spots on the gut and stomach wall have also been reported. Some confusion exists with gross findings however, since other investigators report finding swollen bellies, abdominal cavities containing clear, ascitic fluid and lack of intestinal inflammation (Ferguson 1979). Gross findings and signs seen with the occurrences of hexamitiasis in Alaska have included emaciation, some side swimming, spasmodic side twitching and gut void of fecal materials, but containing both thick and serous yellowish fluids. The corkscrew swimming, inflamed spots on the gut and stomach wall, swollen bellies, and ascitic fluid in abdominal cavity are signs which have been inapparent in the 2 Alaskan cases of the disease.

Although the parasite is most commonly located in the fish gastrointestinal tract, I have isolated these flagellates from the liver of heavily infected fish. Therefore, in heavy infections, Hexamita also has access to the bloodstream.

In spite of excellent distributional, morphological and life-history information on Hexamita, the pathogenicity of the organism is a matter of debate. Some authors maintain that the organism is not a primary pathogen and that hatchery mortalities associated with Hexamita appear following inadequate feeding (Amlacher 1961) or reflect the effect of other stressful conditions (Uz- 
mann et al. 1965). Others consider the organism the cause of both chronic and acute mortalities (Davis 1953, Roberts and Shepherd 1974). Another author, after associating Hexamita with an increase in fish mortalities, did not find a simple cause and effect relationship applicable to $\underline{H}$. salmonis and the disease entity hexamitiasis (Ferguson 1979). Since no other pathogen was detected in association with the mortalities, it was suggested that Hexamita is a more important pathogen than the lack of pathological findings would indicate and should not be underestimated under certain environmental conditions (Ferguson 1979). During the Alaskan enzootics, the healthy-appearing fry and fingerlings were frequently found harboring vast numbers of $\underline{H}$. salmonis without exhibiting either $\bar{c}$ inical or behavioral signs of hexamitiasis. During the course of the Alaskan outbreaks, once those healthy, infected fish ceased feeding (anorexia), they soon showed the listless behavior and emaciated condition, and the prognosis for those individuals became either unfavorable or grave. In spite of efforts to deliver a medicated feed, those anorexic fish would not resume feeding and subsequently died of starvation. During both Alaskan enzootics, the pathogen was isolated in heavy intensities of infection from fry, fingerlings, smolts and adult fish. However, the majority of mortalities in both instances occurred in fry which were approximately $400 \mathrm{fish}$ per pound $(1.14 \mathrm{gm} / \mathrm{fish})$. Mortalities of both smaller and larger fish were appreciably lower than those of the fish of the above size. A possible hypothesis explaining the recurring mortalities at the same time each year, therefore, is that certain environmental qualities occur (low water flow, maximum water recirculation, etc.) which result in favorable growth conditions for the parasite. At this time the $1 \mathrm{~g}$ chinook are just beginning to feed adequately and are adversely affected by the parasite. Concurrently both larger and smaller fish, many with equally heavy infections, are relatively free of mortalities.

\section{Diagnosis}

The most reliable method of diagnosing the condition hexamitiasis is by microscopical examination of contents from the anterior end of the intestine. Fecal contents from any part of the gastrointestinal tract are appropriate when intensities of infection are heavy. A temporary wet mount is prepared by immersing suspect fecal materials in a drop of saline or water. An examination under low power using a compound microscope is usually sufficient, at which magnification the parasites appear as colorless, piriform organisms rapidly darting in all directions. Identification may be confirmed by the pear-shaped body (color Fig. 45) with 2 anterior nuclei, 6 anterior flagella, 2 posterior flagella and 2 parallel axostyles (rigid rods entending the length of the body).

\section{Significance and Control}

Hexamita's significance as a problematic fish pathogen greatly diminished with the advent of moist pellet diets. It is unknown whether there is a cause and effect relationship here (Wood 1974).

Conditions which seem to predispose hexamitiasis are inadequate diet, overcrowding, considerable lot size variation, unsuitable water supply, low dissolved oxygen, suboptimal water quality and poor sanitation (Davis 1953, Uzmann et al. 1965, Wood 1974).

The present incidence of $\underline{H}$. salmonis in only a single Alaskañ hatch- 
ery can hardly be considered important as far as overall impact on Alaskan fish. Further, the continuing debate regarding the importance of the parasite as a primary pathogen might further diminish its current importance in Alaska. The fact remains however, that $H$. salmonis either directly or indirectly resulted in the deaths of approximately $20 \%$ of Fort Richardson Hatchery's chinook salmon over a period of 2 months in late 1978 to early 1979. The importance of this parasite as an Alaskan fish pathogen may be judged by its future impact on Alaskan fishes.

The most effective method of combating these organisms is prophylactic. The incidence of infection may be held in check when fish are held under as natural conditions as possible and overcrowding is avoided. When the incidence of infection increases, the intensity of infection will likewise increase and chemotherapy should be considered.

Since 1940, many experimental chemotherapeutic controls of Hexamita have been conducted (Hoffman 1970). Common treatments historically recommended included mercurous chloride, $\mathrm{N}$-carbamoylarsanilic acid, or $\mathrm{MgSO}_{4}$. The first 2 cures have of ten been considered harder on the fish than the disease. In fact, experimentation has indicated that unmedicated control coho salmon show growth and food conversion superior to that of medicated fish, despite high levels of Hexamita infection (Uzmann and Jesse 1963).

Recent recommendations have been for the continued use of $\mathrm{MgSO}_{4}$ salts ( $3 \%$ in the feed for 2 to 3 days) (Wood 1974) and furazolidone (75 $\mathrm{mg} / \mathrm{kg}$ of $\mathrm{fish} / 14$ days) (Ferguson 1979). Chemotherapy used during the Alaskan outbreaks came after experi- mentation with mercurous chloride 11-2-hydroxyethyl)-2-methyl-5-nitroimidazole and $\mathrm{MgSO}_{4}$ salts. There was little difference between the effectiveness of $\mathrm{MgSO}_{4}$ salts and mercurous chloride; 1-(2-hydroxyethy 1$)$ -2-1-(2-hydroxyethyl)-2-methyl-5-nitroimidazale was less effective. Although $\mathrm{MgSO}_{4}$ salts adequately purged gastrointestinal tracts and resulted in the reduction of mortalities, its effect was only temporary. After a few days, mortalities due to Hexamita were again higher than normal. A combination of treatments using $\mathrm{MgSO}_{4}$ salts (3\% in the feed for 3 days) followed with 2 consecutive days of Diquat $^{\mathrm{a}}$ bath (2 ppm, for 1 hour) was found to very effectively control Hexamita. Although not tried, it is believed that a second treatment of $\mathrm{MgSO}_{4}$ salts and Diquat administered 7 to 14 days after the first would be even more effective. The salts of $\mathrm{MgSO}_{4}$ are not considered harmful to either the organism or fish, whereas Diquat apparently impacts the survival of the parasite's encysted stage.

\section{References}

AMLACHER, E. 1961. Taschenbuch der Fisch Krankheiten. Fischer Verlag, Jena DDR. (transl. by D. A. Conroy and R. L. Herman). TFH Publications, 1970. 310 pp.

BYKHOVSKAYA-PAVLOVSKAYA, I. E., A. V. GUSEV, M. N. DUBININA, N. A. IZYUMOVA, T. S. SMIRNOVA, I. L. SOKOLOVSKAYA, G. A. SHTEIN, S. S. SHUL 'MAN, V. M. EPSHTEIN. 1962. Key to Parasites of Freshwater Fish of the USSR. Zool. Inst., Acad. Sci. USSR (English transl.) TT 64-11040, OTS, Dept. Commerce, Washington, DC. $919 \mathrm{pp}$.

DAVIS, H. S. 1926. Octomitus salmonis, a parasitic flagellate of trout. Bull. of the Bureau of Fish., WA 42:9-26.

aDiquat - registered tradename of the California chemical Company. 
1953. Culture and Diseases of Game Fishes. Univ. of California Press, Berkeley, CA. 332 pp. ELKAN, E. and $H$. - H. REICHENBACHKLINKE. 1974. Color Atlas of the Disease of Fishes, Amphibians and Reptiles. TFH Publications Inc., Neptune, NJ. 256 pp.

FERGUSON, H. W. 1979. Scanning and transmission electron microscopical observation on Hexamita salmonis (Moore, 1922) related to mortalities in rainbow trout fry Salmo gairdneri Richardson. J. Fish Dis. 2:57-67.

HOFFMAN, G. L. 1970. Parasites of North American Freshwater Fishes. Univ. of California Press, Berkeley, CA. 486 pp.

MARGOLIS, L. and J. R. ARTHUR. 1979. Synopsis of the Parasites of Fishes of Canada. Bull. Fish. Res. Board Can. 199:269 pp.

REICHENBACH-KLINKE, H. -H. 1973. Fish Pathology. TFH Publications Inc., Neptune, NJ. 512 pp.
ROBERTS, R. J. and C. J. SHEPHERD. 1974. Handbook of Trout and Salmon Diseases. Fishing News (Books) Ltd, Surrey.

SANO, T. 1970. Etiology and histopathology of hexamitiasis and an IPN-like disease of rainbow trout. J. Tokyo Univ. Fish. $56: 23-30$.

UZMANN, J. R. and $S$. H. HAYDUK. 1963. In vitro culture of the flagellate protozoan Hexamita salmonis. Sci. 140:290-292.

UZMANN, J. R. and J. W. JESSE. 1963. The Hexamita (=0ctomitus) problem: a preliminary report. Prog. FishCult. 25:141-143.

UZMANN, J.R., G. J. PAULIK and S. H. HAYDUK. 1965. Experimental hexamitiasis in juvenile coho salmon (Oncorhynchus kisutch) and steelhead trout (Salmo gairdneri). Trans. Amer. Fish. Soc. 94:53-61.

WOOD, J. W. 1974. Diseases of Pacific Salmon: Their Prevention and Treatment. 2nd ed. Washington Dept. of Fish., Olympia, WA. $82 \mathrm{pp}$. 


\title{
Trichodiniasis
}

\author{
A. K. Hauck
}

\section{Introduction}

Trichodiniasis is the infestation of $\mathrm{fish}$ by ciliated protozoans of the genus Trichodina. Trichodina spp. are of class ciliatea, order Peritrichida and family Urceolariidae (Margolis and Arthur 1979).

Although probably the most common external obligate parasites of freshwater fishes worldwide, trichodinids also parasitize marine fishes. Almost all fish are vulnerable to infestation by Trichodina, although some are specific for certain fishes or groups of fishes (American Fisheries Society 1979).

There are more than 100 species of Trichodina recorded from freshwater and marine fishes; approximately 60 of these are from marine fishes (Goldstein 1971, Lom 1970). There are about 20 freshwater species of Trichodina known in the U.S., which are more commonly found parasitizing warm-water fishes than trout and salmon (Davis 1953, Goldstein 1971). Freshwater $f$ ish also show a seasonal variability in incidence, with greater incidences seen in warmer than colder waters (Lom 1970).

Sites of infestation of Trichodina vary with habitat. Freshwater fish are most commonly infected by Trichodina on the body and fins with less frequent infestations of gills (Davis 1953) and urinary bladder. In contrast to freshwater fishes, the marine parasites attack primarily the gills of their hosts (Lom 1970).

In addition to parasitizing fish, Trichodina spp. are known to parasitize the alimentary tracts of the soft-shell clam (Mya arenaria), the skin, gills, and urinary bladders of juvenile and adult frogs and toads, the gills of other amphibians (Necturus and Triturus) and to exist as commensals on hydras (Cheng 1973). It is not known if Trichodina infests other marine invertebrates (Lom 1970).

\section{$\underline{\text { History }}$}

Trichodina was first described by Ehrenberg in 1831. This old genus has since been divided into 5 genera: Trichodina, Tripartiella, Foliella, Trichodinella and Dipartiella, all of which parasitize fish (BykhovskayaPavlovskaya et al. 1962). In this treatise, only parasites of the genus Trichodina and their effects on fish will be considered.

Trichodiniasis is widely distributed in Europe, Russia and North America (BykhovskayaPavlovskaya et al. 1962, Davis 1953, Hoffman 1970, Margolis and Arthur 1979). It most likely occurs universally, since the parasite is known to have cosmopolitan distribution in both fresh and marine waters.

In Canada, 12 species of Trichodina have been described from both marine and freshwater fishes. of these, 10 were from marine and 2 were from freshwater fish. Marine species include: Trichodina cottidarum, T. decipiens, T. domerguei saint johns $\bar{i}$, T. elizabethae, T. galyae, T. jarmilae, T. lairdi, $\bar{T}$. oviduct $i, T$. rectuncinatā and I. vañcouverense; freshwater species include: T. domergue $i$ and $T$. tenuidens (Margol is and Arthur 1979). 
Freshwater hosts recorded from Canada (Margol is and Arthur 1979) are the threespine stickleback (Gasterosteus aculeatus), quillback (Carpiodes cyprinus), longnose sucker (Catostomus commersoni), mottled sculpin (Cottus bairdi), slimy sculpin (Cottus cognatus), carp (Cyprinus carpio), northern pike (Esox lucius), brassy minnow (Hyboganthus hankinsoni), brown bullhead (Ictalurus nebụlosus), pumpkinseed (Lepomis gibbosus), burbot (Lota lota), Newfoundland eelpout (Lycodes lavalaei), largemouth bass (Micropterus salmoides), white bass (Morone chrysops), hornyhead chub (Nocomis biguttatus), common shiner (Notropis cornutus), blacknose shiner (Notropis heterolepis), yellow perch (Perca flavescens), logperch (Percina caprodes), trout perch (Percopsis omiscomaycus), fathead minnow (Pinephales promelas), black crappie (Pomoxis nigromaculatus), ninespine stickleback (Pungitius pungitius), Atlantic salmon (Salmo salar), brook trout (Salvelinus fontinalis) and creek chub (Semotilus atromaculatus). Sal twater hosts recorded from Canada (Margolis and Arthur 1979) include longhorn sculpin (Myoxocephalus octodecemspinosus), shorthorn sculpin (Myoxocephalus scorpius), padded sculpin (Artedius fenestralis), lumpfish (Cyclopterus lumpus), moustache sculpin (Triglops murrayi), radiated shanny (Ulvaria subbifurcata), sea raven (Hemitripterus americanus), winter skate (Raja ocellata) and thorny skate (Raja radiata).

From salmonids of North America, Trichodina californica has been reported from the gills of chinook salmon (Oncorhynchus tshawytscha), T. fultoni from gills of rainbow trout (Salmo gairdneri), T. truttae on gills of cutthroat trout (Salmo clar$\mathrm{ki})$ and Trichodina spp. from brook trout (Hoffman 1970).
In Alaska, Trichodina spp. have been isolated in 1975 from Kitoi Bay Hatchery (Kodiak) sockeye salmon (Oncorhynchus nerka); in 1976 from Little Port Walter (National Marine Fisheries Service, NOAA, Baranof Island) coho salmon (Oncorhynchus kisutch); in 1977 from Ship Creek Hatchery (Anchorage) rainbow trout, from Sheldon Jackson College Hatchery (Sitka) coho salmon, and from Sand Point School Hatchery pink salmon (Oncorhynchus gorbuscha); in 1978 from Sand Point School Hatchery pink salmon and from Six Mile Lake (Anchorage) threespine stickleback. In May 1979, trichodiniasis occurred in rainbow trout and coho salmon at Ship Creek Hatchery (Anchorage), and 1 ight infestations were reported in pink salmon at Tutka Bay Hatchery (Homer). Trichodiniasis also occurred in June 1979 in sockeye salmon at Kasilof Hatchery (Kenai Peninsula).

\section{Transmission}

Fish are infested by Trichodina through direct transmission. There are no intermediate hosts necessary for successful completion of the life cycle. Reproduction is normally accomplished by binary fission, although these parasites are capable of sexual reproduction by conjugation. The daughter organisms are smaller than the adults and contain only half the number of skeletal parts (Davis 1953).

After its procreation, the daughter organism will either attach immediately to the parent host or seek a new host. The length of time the parasite is capable of surviving apart from the host depends upon water temperatures. Also, the length of time the parasite will remain on the dead host depends upon water temperatures. The parasite reportedly will depart from the dead fish within 12 hours, 72 hours, and 140 hours at 
respective temperatures of $25 \mathrm{C}, 11 \mathrm{C}$ and 5C (Davis 1953). Heavy infestations of Trichodina have been reported to occur within 12 hours (Davis 1953); therefore, at optimum water temperatures, the parasite can reproduce and spread quickly.

\section{$\underline{\text { Signs }}$ and Pathogenesis}

Trichodiniasis is characterized by the appearance of white, irregular blotches on the head and dorsal surface of the fish. Fins may become frayed in heavy infestations. Fish react to the parasite by flashing, becoming weak, losing their appetite and becoming sluggish, particularly when infestations are heavy. Viewed under a bright light, the infected fish will exhibit a white, translucent film covering the body. This film is produced by hyperplasia, excess mucous production and necrosis of the epidermis. Scales are loosened and skin erythema appears. The hyperplasia of the epidermis benefits the parasite since it will feed on the hyperplastic cells (Davis 1953, Reichenbach-Klinke 1973).

Trichodina spp. are known to infest the skin and gills and infect the urinary bladder and ureters of fish (Amlacher 1970, Margol is and Arthur 1979, Reichenbach-Klinke 1973). Gill epithelium is frequently eroded exposing the gill filaments. Trichodinids have been reported ingesting erythrocytes and producing secondary edema of secondary lamellae (Kingsford 1975, Rogers and Gaines 1975). Most likely some haemodilution as well as introduction of secondary invaders into the gill tissues occurs. The skin is reportedly destroyed to the subcutis tissues (below the epidermis and dermis) (Reichenbach-Klinke 1965) in heavy, untreated cases. This would also facilitate the introduction of secondary invaders through the skin. The effects of Trichodina to urinary bladder and ureters are not recorded. Most likely some desquamation and host inflammatory response occur in these tissues. Heavy infestation will lead to death if the condition remains untreated.

The 1979 incidence of trichodiniasis at Kasilof Hatchery (Kenai Peninsula) produced minor hyperplasia of the epidermis. In this instance, a trichodinid was found in subcutis tissues, but only hyperplasticity was involved (color Fig. 47).

\section{Diagnosis}

Trichodinids are among the most highly specialized and structurally complicated protozoans. Isolation of Trichodina can be done through temporary wet mounts from skin/gill scrapings. Diagnosis of trichodiniasis is simplified because of the complicated structure of the animal.

The trichodinid body is saucershaped with a concave aboral (ventral) side and a convex adoral (dorsal) side. On the aboral side is an adhesive disk which serves as a holdfast organ. The disk is composed of a complicated arrangement of 3 concentric rings. The inner ring is called the denticulate ring (Davis 1953). The great variation in the number and shape of the denticles of this ring is used in species differentiation (Davis 1953, Reichenbach-Klinke 1965). The convex dorsal side is smooth and bears 3 girdles of cilia known as the adoral ciliature. This ciliature makes a complete circuit of the adoral surface and leads into the lateral mouth. At the ventral margin of the body is a band of long cilia known as the ciliary girdle, by which the organism moves over the host's surface 
(Davis 1953, Hoffman 1970). Within the body are a large, horseshoeshaped macronucleus, a small, round micronucleus, a large contractile vacuole, and other smaller vacuoles (color Fig. 48).

Diagnosis of trichodiniasis should not be done by visualization of signs alone, because those signs may all be caused by the effect of other parasites, i.e. Ichtyobodo, Chilodonella, Gyrodactylus, Oodinium, etc. (Reichenbach-Klinke 1973).

\section{Significance and Control}

\section{Control}

Trichodiniasis is considered a stress-mediated disease of salmonids. It may be precipitated by overcrowded conditions (especially in fry and fingerlings), low dissolved oxygen or excessive size differences among fish (Wedemeyer et al. 1976). Prevention, reduction, or elimination of stressful conditions will help prevent the occurrence of mortalities from a trichodinid infestation. When the situation warrants the use of chemicals to control an epizootic, Trichodina is easily controlled in salmonids with the use of many treatments, too numerous in extent to mention here (Davis 1953, Hoffman and Meyer 1974, Reichenbach-Klinke 1973).

\section{Significance}

It may be difficult to determine the relative importance of Trichodina as a pathogen, since they are frequently accompanied by other parasites and possibly secondary invaders (Rogers and Gaines 1975). This ciliate under normal conditions is said to rarely give rise to pathological manifestations of disease, being found sporadically on healthy fish. On a weakened host, its reproduction can reach massive proportions (Amlacher 1970, Lom 1970). Light infestations may facilitate the entry of secondary invaders, but heavy infestations have resulted in heavy mortalities (Kingsford 1975). In fact, severe epizootics of freshwater fish caused by this ciliate have been recorded. The only recorded marine epizootic involved checkered puffers (Schoeroides testudineus) (Lom 1970).

In Alaska, this parasite is not now, nor will it likely be in the future, a serious pathogen. The cold water temperatures experienced here undoubtedly influence its ability to rapidly multiply. It is normally seen only during the few warm months of the year, and then it usually appears under stressed hatchery conditions. When an epizootic has occured, the parasite was easily treated and controlled by using chemicals such as formalin and salt.

Trichodinids are known to be hyperparasitized by small amoeba (Cheng 1973). In this host-parasite relationship, trichodinids may serve as transfer hosts, because the amoeba may use them as a temporary refuge and vehicle for reaching a definitive fish host. Due to the unknown pathological significance of this relationship, this may give Trichodina additional future importance as a pathogen or pathogen carrier.

\section{References}

AMERICAN FISHERIES SOCIETY. 1979. Fish Health Blue Book. Procedures for the Detection and Identification of Certain Fish Pathogens. D. MCD $\overline{A N} I E L$, ed. Rev. ed. Amer. Fish. Soc. Fish. Health Section. $118 \mathrm{pp}$. 
AMLACHER, E. 1970. Textbook of Fish Diseases (transl. from German). TFH Publications Inc., Neptune City, NJ. 302 pp.

BYKHOVSKAYA-PAVLOVSKAYA, I. E., A. V. GUSEV, M. N. DUBININA, N. IZYUMOVA, T. S. SMIRNOVA, I. L. SOKOLOVSKAYA, G. A. SHTEIN, S. S. SHUL'MAN, V. M. EPSHTEIN. 1962. Key to Parasites of Freshwater Fish of the USSR (transl. from Russian). NTIS TT-64-11040. 919 pp.

CHENG, T. C. 1973. General Parasitology. Academic Press Inc., New York, NY. 965 pp.

DAVIS, H. S. 1953. Culture and Disease of Game Fishes. Univ. California Press, Berkeley, CA. $332 \mathrm{pp}$.

GOLDSTEIN, R. 1971. Diseases of Aquarium Fishes. TFH Publications Inc., Neptune City, NJ. 126 pp.

HOFFMAN, G. L. 1970. Parasites of North American Freshwater Fishes. Univ. California Press, Berkeley, CA. 486 pp.

HOFFMAN, G. L. and F. P. MEYER. 1974. Parasites of Freshwater Fishes: A Review of Their Control and Treatment. TFH Publications Inc., Neptune City, NJ. 224 pp.
KINGSFORD, E. 1975. Treatment of Exotic Marine Fish Diseases. ARCO Publ. Co. Inc., New York, NY. 92 pp.

LOM, J. 1970. Protozoa causing diseases in marine fishes. pp. 101-123. In: A Symposium on Diseases of Fishes and Shellfishes. S. F. SNIESZKO, ed. Am. Fish. Soc. Spec. Publ. 5.

MARGOLIS, L. and J. R. ARTHUR. 1979. Synopsis of the parasites of fishes of Canada. Bull. Fish. Res. Board Can. 199:269.

REICHENBACH-KLINKE, H. -H. 1965. The Principal Diseases of Lower Vertebrates. Book 1: Diseases of Fishes. TFH Publications Inc., Neptune City, NJ. 205 pp. - 1973. Fish Pathology. TFH Publications Inc., Neptune City, NJ. 512 pp.

ROGERS, W. A. and J. L. GAINES, JR. 1975. Lesions of protozoan diseases in fish. pp. 117-141. In: The Pathology of Fishes. W. E. RT $\overline{I B E L} \overline{I N}$ and G. $\overline{M I G A K I}$, eds. Univ. of Wisconsin Press, Madison, WI.

WEDEMEYER, G. A., F. P. MEYER and L. SMITH. $1976 . \quad$ Environmental Stress and Fish Diseases. TFH Publications Inc., Neptune City, NJ. $192 \mathrm{pp}$. 


\title{
Saprolegniasis
}

\author{
A. K. Hauck
}

\section{Introduction}

Saprolegnia spp. are a universal component of natural freshwaters (Hoffman 1969, 1970). Most species are considered saprophytes, except Saprolegnia parasitica which has shown evidence of being a primary pathogen (Wolke 1975). As saprophytes commonly called "water molds," Saprolegnia are capable of growth and reproduction throughout the year on a wide variety of substrates including fish carcasses, eggs and other dead and decaying organic matter found in freshwater. There is evidence that Saprolegnia may also affect fish which inhabit low salinities (Wolke 1975). Saprolegnia has been labeled as an opportunistic facultative parasite, e.g. an organism which does not absolutely depend on parasitism, but will adapt to it if the opportunity presents itself (Neish 1977).

Confirmed fungal disease agents of fish, which infect fish under both natural and experimental conditions, make up approximately 3 orders, 10 genera and 22 species. The order Saprolegniales includes approximately 90 species of fungi (Wolke 1975). In the strict definition, the term Saprolegnia is a genus of the class Phycomycetes. Unfortunately, many fungal organisms found associated with piscine mycosess have been improperly identified as Saprolegnia. As a result, the name Saprolegnia has erroneously developed into a term commonly applied to several fish fungal disease agents (Wolke 1975). In order to adequately discuss Saprolegnia spp. as a fish pathogen, it is necessary to consider both the age of the infected fish host, and the pathogen's ability to function as a primary disease agent. Therefore, Saprolegnia will be discussed as it affects eggs, juvenile, and adult fish and as a primary or secondary pathogen.

\section{History}

Susceptibility of dead eggs and fish to Saprolegnia is generally universal in freshwater habitat. Because of its sedentary nature and ability to function as both a facultative parasite and saprophyte, it historically represents the most important fish egg pathogen. In Alaska, Saprolegnia is omnipresent in feral adult salmon populations which are or have completed spawning.

Saprolegnia is found at all Alaska State and private hatcheries where fish egg incubation occurs. In these cases it functions as a saprophyte on dead eggs and as an opportunist in spreading infection to adjacent, healthy eggs. It has also been found in most hatcheries which conduct fish rearing programs. In these cases it has been associated as a saprophyte on dead fish, as a secondary, opportunistic invader to fin and tail rot conditions, and with other dermal lesions caused by bacterial pathogens and abrasions from handling trauma. For example, it has been associated as a secondary invader of dermal vesicles resulting from the bacterial causative agent of furunculosis, Aeromonas salmonicida, in presmolt chinook (Oncorhynchus tshawytscha), presmolt coho (Oncorhynchus kisutch), and adult rainbow trout (Salmo gaird- 


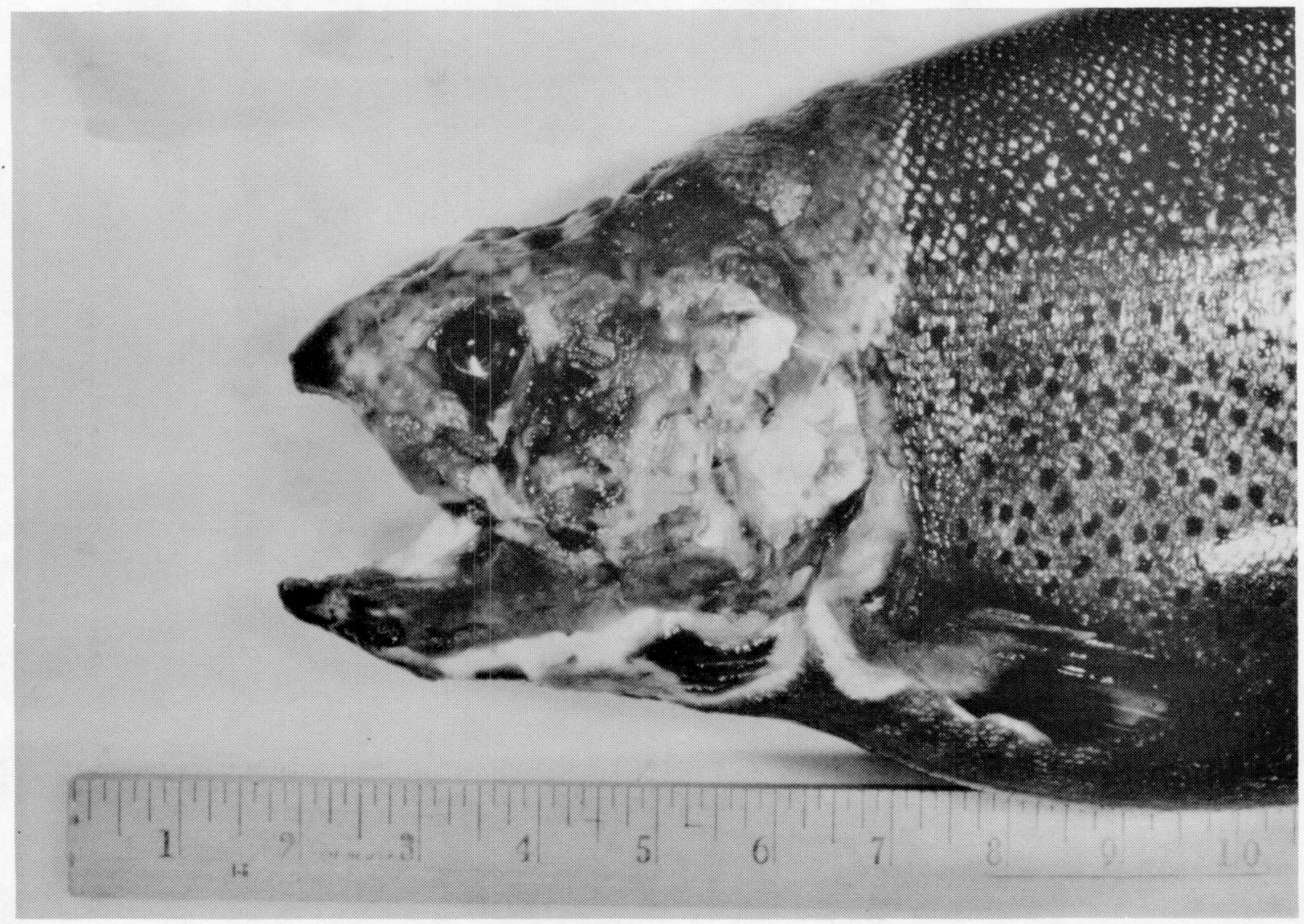

Figure 17. Saprolegnia infestation and erosion of head epidermal tissues of Alaskan adult rainbow trout (Salmo gairdneri) (A.K. Hauck).

neri) at Ship Creek Hatchery in Anchorage. In many of the adult rainbow trout at the same facility, Saprolegnia has been found associated with chronic head and body lesions characterized by severe erosion of the epidermis ( $\mathrm{Fig}$. 17). The head lesions are similar in appearance to those caused by ulcerative dermal necrosis (Reichenbach-Klinke 1973). Many of these rainbow trout were likewise infected with A. salmonicida.
As a primary pathogen, Saprolegnia has been found in Alaska at 2 hatchery facilities. In 1977 it was implicated as the cause of death in sockeye (Oncorhynchus nerka) fry at Kasilof Hatchery; and in 1980 it occurred in Big Lake Hatchery sockeye fry. In both instances infections were associated with the nares. 


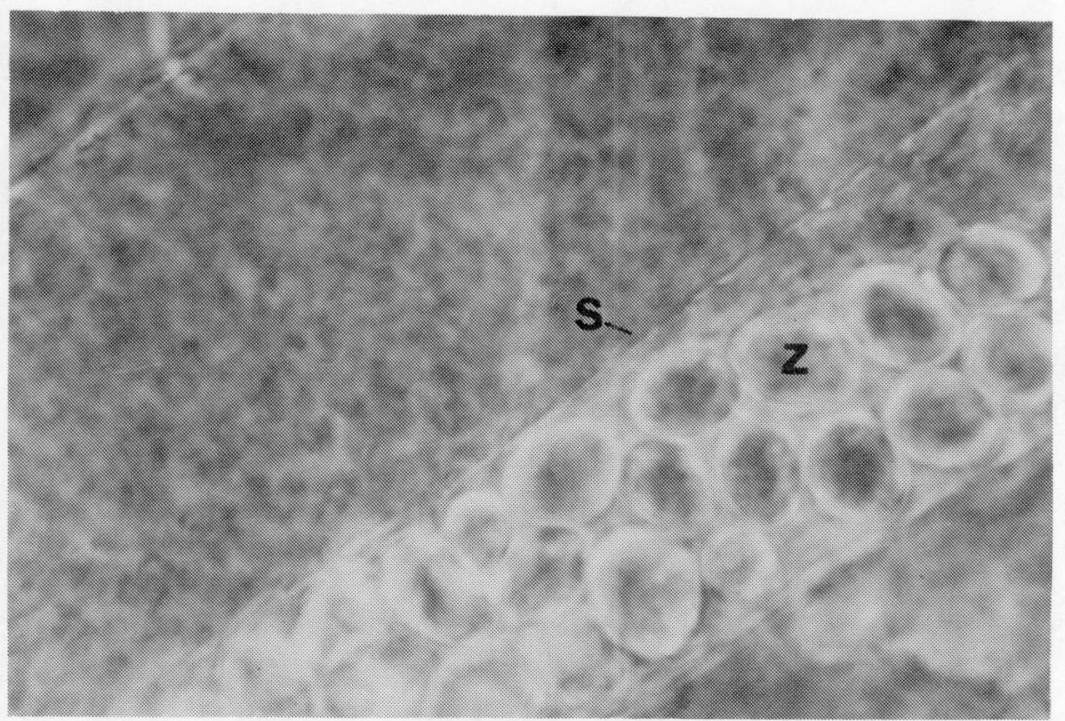

Figure 18. Asexual zoospores ( $\mathrm{Z}$ ) within sporangium (s) (1000X, phasecontrast) (R. Grischkowsky).

\section{Transmission}

Reproduction of Saprolegnia spp. is accomplished through both as exual and sexual means. Asexual reproduction occurs through the release of flagellated zoospores, (Fig. 18) from a sporangium (Fig. 19) which is located at the tip of a fungal filament. Each of the zoospores may be transmitted via water to other fish and eggs where it develops into new hyphal filaments. Between 24 and 48 hours are required to complete a minimum life cycle from reproductive spore to mycelium (mass of filaments) to reproductive spore (Hoffman 1969, 1970).

Sexual reproduction is accomplished through male antheridia and female oogonia sex organs (Fig. 20), the products from which develop into independent filaments which are capable of both asexual and sexual reproduction (Wolke 1975).

It has been reported that both healthy and infertile fish eggs will resist fungus invasion for a month or more, whereas dead eggs succumb to invasion several days after turning white (Hoffman 1969). Invasion of normal eggs by Saprolegnia may be attributed to the presence of organic matter on the eggs, although there is no evidence supporting this theory (Davis 1953). Host specificity also may be a factor influencing transmission of this pathogen (Wolke 1975). 


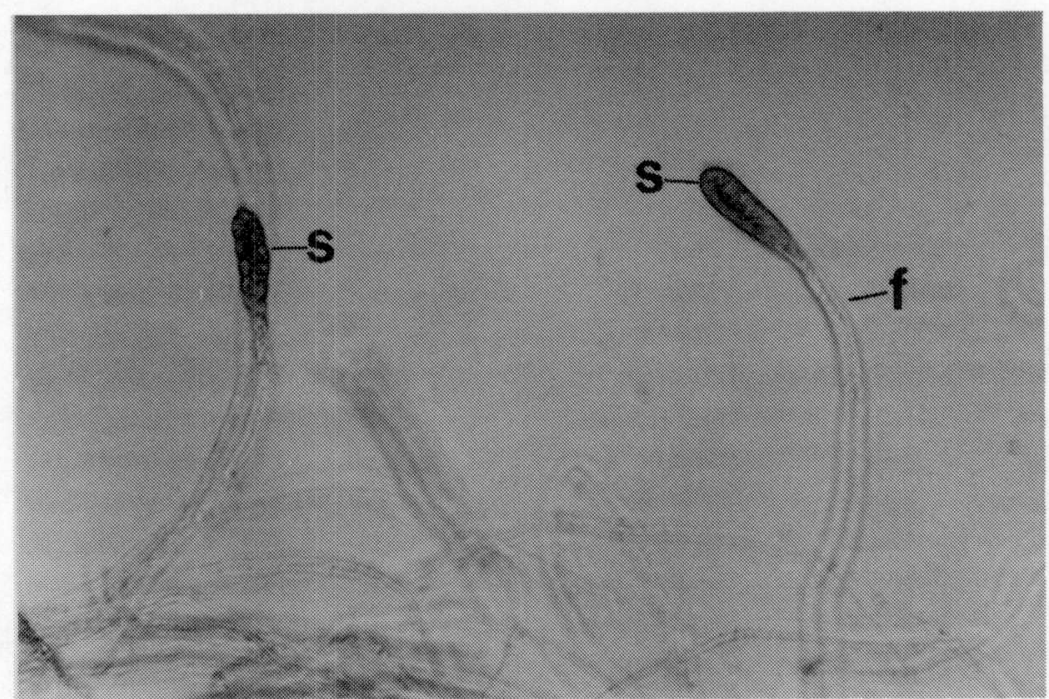

Figure 19. Asexual sporangia (s) at tips of fungal filaments ( $f$ ) (100X, phase-contrast) (R. Grischkowsky).

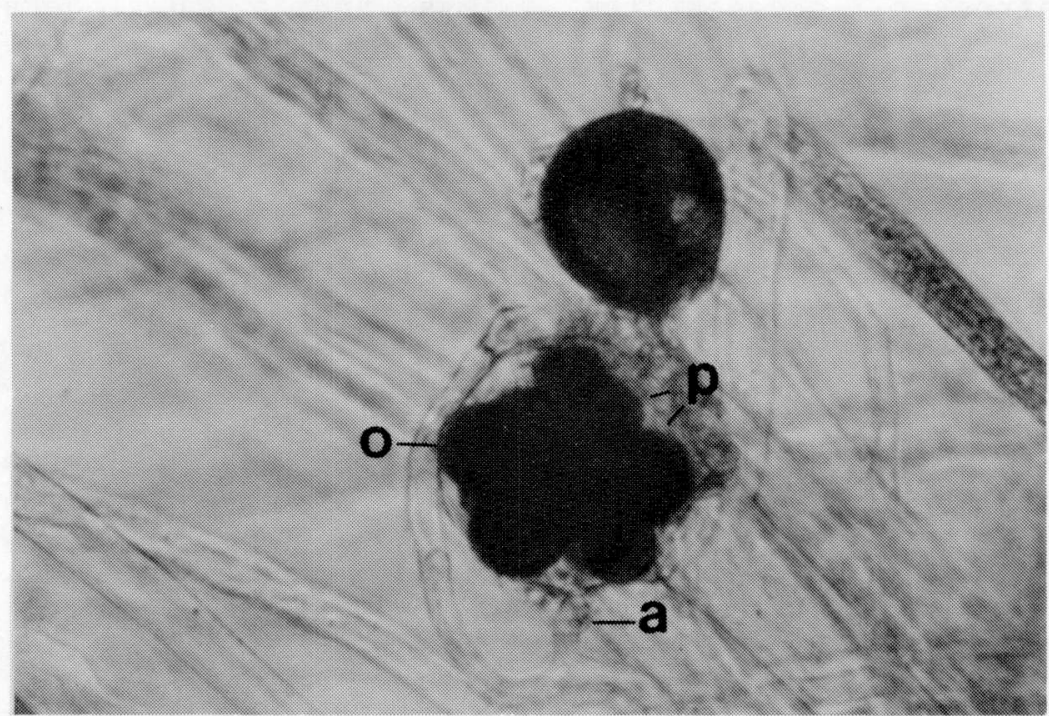

Figure 20. Sexual reproduction involving female oogonium (o) containing black, spherical oospores ( $p$ ) and male antheridium (a) (400X) (R. Grischkowsky). 
Signs and Pathogenesis

Due to the confusion which has reigned in the identification of fungal pathogens, the pathogenicity of the agents has been complicated (Wolke 1975). In many cases it is difficult to determine if the agent acts as a saprophyte, an opportunist, a primary pathogen or as a combina$t i o n$ of these.

Saprolegniasis frequently appears to be associated with some adverse environmental condition (Wolke 1975). Fungal lesions often appear on $\mathrm{fish}$ and eggs after handling, under conditions of crowding, trauma from spawning, high water temperatures, in the presence of pollutants, and in conjunction with other diseases and conditions. For example, treatment of fish with sulfa-medicated diet will weaken gill epithelium and increase gill infection by Saprolegnia (Wood 1974). The initial effect of the environmental condition is the impairment of normal defenses and reparative capacities. Also, the environmental stressor increases the plasma corticosteroid production, which alone will lower the resistance of the fish to infection (Neish 1977, Wedemeyer 1970). This increase in corticosteroid production has been positively correlated with saprolegniasis incidence (Roth 1972). In addition, the depletion of ascorbic acid reserves of a fish due to anorexia (loss of appetite) further impairs that fish's ability to repair tissue damage (Ashley et al. 1975). This impairment is intensified by increases in plasma corticosteroids (Wedemeyer 1970). The effect of decreased immunocompetency from increased corticosteroid levels, combined with the decreased ability of the fish to repair tissue damage, might provide the mechanism whereby normally nonpathogenic Saprolegnia organisms become pathogens (Neish 1977).
Abrasions and wounds on $\mathrm{fish}$ constitute the major route of infection of Saprolegnia (Wolke 1975). The wounding of a fish, although facilitating the infection by Saprolegnia, does not insure infection (Neish 1977). Lesions infected by Saprolegnia can occur anywhere on the body of the fish, but are most commonly found on the dorsal surface of the head, anterior to the dorsal fin, near the adipose fin and on other fins. Lesions are often associated with ulcers, vesicles and wounds resulting from mechanical trauma. In some cases, fish with fungal lesions on some parts of the body also have had obvious wounds which have appeared to be uninfected by Saprolegnia (Neish 1977). Eyes and gills may also be infected. Fish suffering with concurrent bacterial infections frequently have mycosis of the gills.

Skin lesions vary in condition depending on the age of the lesion (Wolke 1975). Early lesions appear pale with a central zone of scale loss and a peripheral zone of erythema (redness). Advanced lesions may be ulcerated and expose underlying muscle. Histologically, Saprolegnia sp. first destroys the epidermis, penetrates the basement membrane and dermis, and in some cases is found in the hypodermis and musculature. Microscopic lesions show mycelial masses over zones of necrotic and ulcerated epithelium. Individual hyphae extend into dermis and between muscle fibers. Inflammatory response is normally absent or very slight, with lymphocyte and macrophage infiltration into infected areas (Wolke 1975). A leucocytic infiltration has also been reported in cases when the Saprolegnia infection was seen with dermal ulcers (Neish 1977). Necrosis and hyaline degeneration of striated muscle is also seen in the lesion (Wolke 1975). 
As the infection progresses, fish become increasingly lethargic and congregate in quieter areas of the stream. The fish frequently engage in frenzied bouts of activity between rest periods. There appears to be no correlation between Saprolegnia infected surface area and time of death. Time of death is a function of the growth rate of the fungus, the initial site of infection, the type of tissue infected, and the ability of the fish to withstand the invader. There is no evidence that either toxins or systemic infections are produced by Saprolegnia (Neish 1977). Death of adult salmonids injected with Saprolegnia has been postulated to be caused by osmoregulatory breakdown, followed by hemodilation and circulatory failure (Richards and Pickering 1979).

The disease-causing mechanisms of Saprolegnia infections, when the organism was implicated as a primary pathogen in sockeye salmon fry at Big Lake and Kasilof Hatcheries, needs further discussion. Although obviously moribund, the fish initially did not exhibit the presence of external infectious agents. A small mycelial tuft was later noticed in some fish at the nare opening. Histopathologic examination of head tissues showed mycelial masses in the nares and surrounding connective tissues. In some sections, hyphal filaments were located in the forebrain tissues (telecephalon) (color Fig. 46) and around the eyes. Host leucocytic inflammatory response was absent, but the lesion was hemorrhagic. Growth of the mycelium appeared to originate in the nare epithelium and extend both to the exterior of the fish through the nare aperture as well as internally into connective, brain, and some optic tissues (conjunctiva, cornea and iris). Death was presumed to have been caused by Saprolegnia invasion of nervous tissues. There was no evidence of systemic invasion, as death may have occurred before the infection can became systemic. Toxins should not be ruled out as producers of lethal side effects. More work is required in this area.

Pathogenesis associated with fish eggs remains largely undocumented. It is assumed that the mechanisms causing saprolegniasis of eggs are similar to those seen with fish.

\section{Diagnosis}

The presence of Saprolegnia spp. on fish or fish eggs is indicated by a white-tan, cottony growth on the surface of the animal or enveloping the infected egg(s) (Hoffman 1969). The mycelium is more easily seen when infected materials are inspected under water.

Presumptive identification of Saprolegnia may be accomplished by observing, under temporary wet mount, typical nonseptate, irregularly branching filaments (hyphae), each of which is approximately 20 um in diameter. Saprolegnia may be further characterized by the termination of older filaments into both club-shaped sporangia containing zoospores and round oogonia (female sexual reproductive structures). Also characteristic of the genus Saprolegnia, is the ability of a new sporangium to proliferate into the space occupied by an older sporangium (Hoffman 1969, 1970).

Saprolegnia invaderis has been diagnosed differentially from Saprolegnia parasitica in its ability to initiate internal infection, where it proliferates and causes the death of the fish ordinarily before the mycelium appears on the fish exterior. Saprolegnia parasitica, on the otherhand, invades from an external wound or lesion, producing the mycelial mass on the surface of the fish prior to death (Davis 1953). The identifi- 
cation of the earlier mentioned fungal agent, which infected the nare and brain tissues of the Alaskan sockeye salmon fry before being visually observed at the fish surface, presumptively may have been $S$. invaderis due to the unusual mechanisms of infection.

\section{Confirmatory}

Saprolegnia identification may be accomplished by observing zoospore release via lateral exit papillae. The use of oogonial structures for identification is unreliable because the natural production of oogonia is an alleged rare event (Neish 1977). Oogonia are reportedly obtainable by culturing the organism on special media (Scott et al. 1963).

Because of the multitude of species involved and the complexity of the freshwater fungal organisms, the need for a trained mycologist to make a final diagnosis is obvious.

\section{Significance and Control}

Fish are known to differ in susceptibilities to infection by Saprolegnia, salmonids being highly susceptible (Wolke 1975). On the basis of current evidence, many of the fungi causing saprolegniasis are primary pathogens. For example, $\mathrm{S}$. parasitica is known to be pathogenic to both injured and uninjured fish after exposure to zoospores (Wolke 1975). Also, experimentation with $\mathrm{S}$. declina shows it capable of being a primary pathogen in young coho and chinook salmon (Neish 1977). Differences among species of Saprolegnia in ability to perform as primary pathogens have been attributed to differences in virulence, with $S$. parasitica and $S$. ferax considered highly virulent. Epizootics caused by fungal pathogens have been reported to occur, mainly with chronic dermal or branchial infections (Wolke 1975).
In Alaska, Saprolegnia has been implicated as an agent producing death in fish either directly or indirectly in conjunction with many conditions. At Ship Creek Hatchery (Anchorage), Saprolegnia has been found infecting salmonids reared in the environmentally polluted (petroleum) and pathogen contaminated (A. salmonicida) waters. Saprolegnia infections have been associated with fin and tail rot conditions at salmonid hatcheries, where juvenile fish have experienced gas supersaturation or other stress related conditions. Fungal infections have also been seen on prespawning salmon mortalities in both Alaska and elsewhere (Neish 1977), and on normal spawning Alaskan salmon in both hatchery and feral situations. Saprolegniasis has been associated with cold-water disease, with fish suffering from infections caused by other disease agents, with fish stressed from reuse water systems, with decaying organic matter in freshwater and with all Alaskan salmon hatcheries. Saprolegnia also constitutes the most important fish egg diseases in Alaska in its ability to notoriously attack dead eggs and later encompass and kill adjacent live eggs (Wolke 1975).

The significance of Saprolegnia as an Alaskan fish pathogen is further compounded since asexual zoospores are more numerous at cold water temperatures. In addition, many strains of Saprolegnia have sexual stages that occur at low temperatures. Also, because skin heals more slowly in cold water temperatures, fish with dermal abrasions and lesions tend to be more vulnerable to infection with Saprolegnia (Wolke 1975).

Control of Saprolegnia is accomplished through mechanical and chemical methods for both eggs and fish. Mechanical control is effected by removing and disposing dead and infected eggs and fish as frequently as the condition appears or is 
suspected and not less than 2 to 3 times a week. When frequent removal is impractical, chemical controls should be applied.

There are many chemical controls for Saprolegnia (Hoffman and Meyer 1974, Knittel 1966, Wood 1974); however, the majority of documented control methods have not been approved by FDA or EPA for use on food fish. In Alaska both malachite green and formalin drips and flushes have been used. These chemicals will most likely never be registered for use with food fish, since registration research terminated in 1977 (Warren 1978). There is promise in the use of Diquat ${ }^{a}$, in controlling Saprolegnia in eggs and juveniles (Wood 1974). Hatchery facilities located near saltwater have the great advantage of using periodic saltwater flushes over eggs to effectively inhibit the growth of Saprolegnia. Those facilities, which do not have geographical proximity to natural saltwater, will find that the addition of sodium chloride to freshwater to treat for Saprolegnia is counterproductive. If large quantities of salt are required in producing a specific salinity, both the cost and materials required become impractical. It is hoped that chemicals, which are proven fungicides or fungistats and properly registered by the FDA or EPA, will soon be forthcoming for hatchery use.

\section{References}

ASHLEY, L. M., J. E. HALVER and R. R. SMITH. 1975. Ascorbic acid deficiency in rainbow trout and coho salmon and effects on wound healing. pp.769-786. In: The Pathology of Fishes. W. E. RIBELIN and G. MIGAKI, eds. Univ. of Wisconsin Press, Madison, WI.

DAVIS, H. S. 1953. Culture and Diseases of Game Fishes. Univ. of California Press, Berkeley, CA. $332 \mathrm{pp}$.

HOFFMAN, G. L. 1969. Parasites of freshwater fish. I. Fungi. (Saprolegnia and relatives) of fish and fish eggs. U.S. Fish and Wildl. Serv., Fish Dis. Leaflet No. 21. 6 pp.

- 1970. Parasites of North American Freshwater Fishes. Univ. of California Press, Berkeley, CA. $486 \mathrm{pp}$.

HOFFMAN, G. L. and F. P. MEYER. 1974. Parasites of Freshwater Fishes. A Review of Their Control and Treatment. TFH Publications Inc., Neptune City, NJ. 224 pp.

KNITTEL, M. D. 1966. Topical application of malachite green for control of common fungus infections in adult spring chinook salmon. Prog. Fish-Cult. 28:51-53.

NEISH, G. A. 1977. Observations on saprolegniasis of adult sockeye salmon, Oncorhynchus nerka (Walbaum). $\frac{\text { J. Fish Biol. }}{\text { J. }}$ $10: 513-522$.

REICHENBACH-KLINKE, H. -H. 1973. Fish Pathology. TFH Publications Inc., Neptune City, NJ. 512 pp.

RICHARDS, R. H. and A. D. PICKERING. 1979. Changes in serum parameters of Saprolegnia-infected brown trout Salmo trutta. L. J. Fish. Dis. 2: $\overline{197-206}$.

$a_{D i q u a t}$ - registered trade name of the California Chemical Company. 
ROTH, R. R. 1972. Some factors contributing to the development of fungus infections in freshwater fish. J. Wildl. Dis. 8:24-28.

SCOTT, W. W., J. R. POWELL and R. L. SEYMOUR. 1963. Pure culture techniques applied to the growth of Saprolegnia species on a chemically defined medium. Virginia J. of Sci. 14:42-48.

WARREN, J. W. 1978. The role of the U.S. Fish and Wildlife Service in drug registration. PP. 59-60. In: Proceedings of the Joint Third Biennial Fish Health Section and Ninth Annual Midwest Fish Disease Workshops; 1978 August 15-18; Kansas City, Mo.
WEDEMEYER, G. 1970. The role of stress in disease resistance of fishes. pp. 30-35. In: A Symposium on Diseases of Fishes and Shellfishes. S. F. SNIESZKO, ed. Am. Fish. Soc., Spec. Publ. 5.

WOLKE, R. E. 1975. Pathology of bacterial and fungal diseases affecting fish. pp. 33-116. In: The Pathology of Fishes. W. E. $\overline{R I B E} \overline{L I N}$ and G. MIGAKI, eds. Univ. of Wisconsin Press, Madison, WI.

WOOD, J. W. 1974. Diseases of Pacific Salmon: Their Prevention and Treatment. 2nd ed. Dept. Fisheries, Hatcheries Div., State of Washington. $82 \mathrm{pp}$.

\title{
Whirling Disease
}

\author{
A. K. Hauck
}

\section{Introduction and History}

A major North American wildlife disease that may be significant sometime in Alaska's future is whirling disease. Whirling disease is a serious hatchery disease in Central Europe, from where it has spread to the United States (California, Connecticut, Massachusetts, Michigan, Nevada, New Jersey, Ohio, Pennsylvania, Virginia, West Virginia), throughout Europe, Russia, New Zealand and South Africa (Hoffman 1970, American Fisheries Society 1979) because of both natural and man-induced transfers. Susceptible hosts include all species of salmon, trout and grayling (Thymallus arcticus) (American Fisheries Society 1979). Caused by the myxosporidian, protozoan parasite Myxosoma cerebralis, whirling disease is the most dreaded myxosporidian disease of young rainbow trout.

\section{Transmission}

Transmission is accomplished by passage of spores from infected to uninfected fish. After spores are ingested, trophozoites are released in the intestine. These are carried in the bloodstream primarily to the head cartilage where they reproduce. After a few months, infection spreads down the vertebrae. At the 26 th vertebra, damage to sympathetic nerves, which regulate pigment production in the tail, occurs and the blackened tail sign appears. Both dark tails and whirling will continue for a considerable period after the initial infection. If the fish survives, the bone abnormalities will remain through its lifetime, and it will become a natural reservoir for the parasite (Reichenbach-Klinke 1973). 


\section{Signs and Pathogenesis}

Signs and lesions produced by this disease include lying near the bottom of the pond in an exhausted state, circular tail chasing, black discoloration of the caudal region, loss of balance, mandibular deformities, scoliosis (horizontal spinal curvature), lordosis (vertical spinal curvature) and deformities of the head and spine (Reichenbach-Klinke 1973).

\section{Significance and Control}

Control may be accomplished by destruction of infected fish, filling ponds with calcium oxide or calcium cyanamide (Hoffman and Meyer 1974), and never importing rainbow trout (Salmo gairdneri) or other susceptible species from areas of known incidence into areas where the disease does not exist. At the present there is no successfully known medication for whirling disease (Hoffman and Meyer 1974). Whirling disease is the most dreaded myxosporidian-caused disease of salmonids in the world.

\section{References}

AMERICAN FISHERIES SOCIETY. 1979. Fish Health Blue Book. Procedures for the Detection and Identification of Certain Fish Pathogens. D. MCDANIEL, ed. Rev. ed. Amer. Fish. Soc. Fish Health Section. $118 \mathrm{pp}$.

HOFFMAN, G. L. 1970. Intercont inental and transcontinental dissemination and transfaunation of fish parasites with emphasis on whirling disease (Myxosoma cerebralis). pp. 69-81. In: A Symposium of Diseases of Fish and

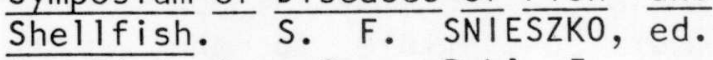
Am. Fish. Soc. Spec. Publ. 5.

HOFFMAN, G. L. and F. P. MEYER. 1974. Parasites of Freshwater Fishes: A Review of Their Control and Treatment. TFH Publ ications Inc., Neptune City, NJ. 224 pp.

REICHENBACH-KLINKE, H. -H. 1973. Fish Pathology. TFH Publications Inc., Neptune City, NJ. 512 pp. 


\title{
Ceratomyxosis
}

\author{
A. K. Hauck
}

Introduction and History

Ceratomyxa shasta is a myxosporidian protozoan parasite which produces the disease ceratomyxosis. Ceratomyxosis was originally reported as the cause of high mortalities in hatchery rainbow trout (Salmo gairdneri) in northern California (Wales and Wolf 1955). Since that incidence, its range has extended north to Oregon, Washington, Idaho and British Columbia. Juvenile salmonids, especially rainbow trout, are most susceptible to the disease. Hosts include coho salmon (Oncorhynchus kisutch), chinook salmon (Oncorhynchus tshawytscha), sockeye salmon (Oncorhynchus nerka), chum salmon (Oncorhynchus keta), rainbow and steelhead trout (Salmo gairdneri), cutthroat trout (Salmo clarki), brown trout (Salmo trutta), Atlantic salmon (Salmo salar) and brook trout (Salvelinus fontinalis) (American Fisheries Society 1979). In Canada, the parasite has been found within the last 5 years in adult and juvenile chum salmon ( $\mathrm{Fisheries}$ and Environment of Canada 1977, Margolis and Evelyn 1975) from hatcheries and open seas, respectively. Those hatcheries include the Capilano River Hatchery (Vancouver) and Quinsam River Hatchery (Vancouver Island) (Fisheries and Environment of Canada 1977). This parasite, although not affecting Alaskan fish at this writing, is considered a significant pathogen because of its apparent northwardly-expanding distribution.

Transmission

Salmon are known to become infected when exposed to infected water
(Sanders et al. 1970, Wood 1974). It is known that water temperatures above 10C will initiate infection. All freshwater locations where $C$. shasta occurs are associated with a lake or reservoir, which may be necessary for successful completion of its life cycle (Wood 1974). At the present time the life cycle of this parasite is largely a mystery.

\section{$\underline{\text { Signs }}$ and Pathogenesis}

Signs include sluggish behavior, fish seeking quieter waters, swollen abdomen, exophthalmia. Lesions include pyloric caecal and intestinal swelling, nodule formation in gut lumen, lesions of kidney, liver and spleen and muscle abcesses. Death is reportedly caused by perforated gut lesions and peritonitis (American Fisheries Society 1979, Wood 1974).

\section{Diagnosis}

Diagnosis may be accomplished by locating spores in wet mounts of contents from the lower intestinal wall, gall bladder or lesions under light microscopy (American Fisheries Society 1979). The kidney beanshaped spores contain 2 polar capsules and are approximately $8 \times 17$ um in dimension (Margolis and Evelyn 1975).

\section{Significance and Control}

Control may be accomplished by lowering hatchery water temperatures, killing infected hosts, and pretreatment (chlorination and ultraviolet irradiation) of infected waters (American Fisheries Society 1979). 
The transfer of young or adult $\mathrm{fish}$ from areas of known infection to noninfected areas should be avoided at all costs.

\section{References}

AMERICAN FISHERIES SOCIETY. 1979. Fish Health Blue Book. Procedures for the Detection and Identification of Certain Fish Pathogens. D. MCDANIEL, ed. Rev. ed. Amer. Fish. Soc. Fish Health Section. $118 \mathrm{pp}$.

FISHERIES AND ENVIRONMENT OF CANADA, ENHANCEMENT SERVICES BRANCH. 1977. Salmonid enhancement program, annual report for 1977. J. E. MCDONALD, ed. Hazeldine Press Ltd., Vancouver, BC. 84 pp. MARGOLIS, L. and T. P. T. EVELYN. 1975. Ceratomyxa shasta (Myxosporida) disease in chum salmon (Oncorhynchus keta) in British
Columbia. Fish. Res. Board Can. 32: $1640-1643$.

MARGOLIS, L. and J. R. ARTHUR. 1979. Synopsis of the parasites of fishes of Canda. Bull. Fish. Res. Board Can. 199:269.

SANDERS, J. E., J. L. FRYER and R. W. GOULD. 1970. Occurrence of the myxosporidian parasite Ceratomyxa shasta in salmonid fish from the Columbia River Basin and Oregon coastal streams. pp. 133-141. In: A Symposium on Diseases of Fishes and Shellfishes. S. F. SNIESZKO, ed. Am. Fish. Soc. Spec. Publ. 5.

WALES, J. H. and H. WOLF. 1955. Three protozoan diseases of trout in California. Calif. Fish \& Game $41: 183-187$.

WOOD, J. W. 1974. Diseases of Pacific Salmon: Their Prevention and Treatment. 2nd ed. Dept. Fisheries, Hatcheries Div., State of Washington. $82 \mathrm{pp}$. 
Chapter 4.

ENVIRONMENTAL DISEASES 


\title{
Gas Bubble Disease
}

\author{
A. K. Hauck
}

\section{Introduction}

Gas bubble disease (GBD) is a condition in fish similar to the bends in humans, caused by supersaturation of water with air. Supersaturation occurs when water contains more air than it can normally hold under normal conditions of temperature and pressure. Under high pressure or at low temperatures water can contain more air (Garton 1976).

Gas bubble disease will affect all species of fish which inhabit supersaturated waters. Threshold levels, those gas levels where significant mortalities begin to occur, will vary for both species and age of fish (Davis 1975, Nebeker et al. 1980). Invertebrates are likewise affected by gas supersaturated water (Nebeker et al. 1976).

Gas supersaturated water has both artificial and natural causes. Supersaturation is a common problem in water below dams (Ebel et al. 1975) and also may be caused by thermoelectric power plants. At dams, the impact of water cascading over the spillway carries air into the deep basin where, under pressure from overlying waters, the air goes into solution in the water. As the water returns to a lesser depth, the pressure is relieved and the water becomes supersaturated. At thermal power plants, saturated water is heated. The warmer water holds less air at equilibrium, so it becomes supersaturated (Garton 1976). Heated water from thermonuclear and steamelectric generating stations has been documented as having produced supersaturated water lethal to fish
(Demont and Miller 1971, Rucker and Kangas 1974).

Plunge pools at the base of waterfalls or at the base of dam spillways are another source of supersaturated water (Rucker and Kangas 1974, Wood 1974). A common artificially caused source of excess gas in water is air entering the intake of a water pump. Also, excess gas may appear in a gravity flow water system if air is permitted to enter into the intake pipe to a hatchery (Stroud et al. 1975).

Water from natural springs and wells is frequently unsatisfactory for rearing fish because of excess nitrogen. If under pressure, this subterranean water will absorb excessive nitrogen and other gases from the pressurized air. It will also react with organic matter and lose oxygen. For these reasons, spring and well waters frequently need aeration before use in fish culture (Rucker 1972, Rucker and Kangas $1974)$.

Melting snow water has reportedly caused GBD in hatcheries. Snow water is saturated with air at low temperatures and may become supersaturated with air as it warms (Rucker 1972). Natural lake and bay waters have been known to cause fish kills through algal photosynthetic activity and warming of surface waters (Stroud et al. 1975).

What was initially known as "the nitrogen problem" is today known as air supersaturation (Garton 1976). Primarily because of newer, more precise analytical methods have 
researchers been able to study total dissolved gas (TDG) pressures in water. Results of this experimentation have produced the knowledge that total gas supersaturation levels are much more important than either individual gases or varying combined gas ratios, since fish mortalities will not occur unless TDG levels exceed $100 \%$ (Nebeker et al. 1976).

\section{History}

Air supersaturation was first noted in hatchery and aquarium facilities in the early 1900s. At that time GBD was ascribed as the cause of the condition (Garton and Nebeker 1977). Dissolved nitrogen gas was postulated to be the cause of GBD and received primary research emphasis. Supersaturation caused by entrainment of air in spillwater first became a problem on the Columbia River when Bonneville Dam was constructed in 1938. As more dams were constructed, the problem intensified with the result that the entire river could be supersaturated during some periods of the year (Ebel 1969). Because of the potential economic and natural loss to salmonid stocks exposed to supersaturated water in the Columbia River system, a Nitrogen Task Force was formed by the U.S. Secretary of the Interior and the Tri-State Governors Conference (Idaho, Washington and Oregon) in the early 1970s. This task force, which consisted of 23 public and private agencies, was organized to investigate the problem at both field and laboratory levels (Garton and Nebeker 1977). The results of the task force accounts for the voluminous literature on gas supersaturation problems relating to fish.

Knowledge of the occurrence of GBD in Alaska is limited to artificial situations. The scope of the problem is not extensive in Alaska because of the lack of dams, reactors and other artificial sources of gas supersaturated water. Known gas supersaturation problems have occurred at Fort Richardson Hatchery (Anchorage), at Crystal Lake Hatchery (Petersburg) and at Klawock Hatchery (Prince of Wales Island). Mortalities due to GBD were low at the above facilities.

\section{Signs, Symptoms and Pathogenesis}

Before GBD will occur, a specific supersaturated gas level or threshold must be reached. The threshold will vary with age of the fish, species and level of exposure to supersaturated water. Eggs, embryos, and newly hatched fry have been found to be unaffected by high levels of gas supersaturated water ( $127 \%$ TDG). At about day 16 posthatch when the fish begin swimming up, death occurs rapidly at this level (Nebeker et al. 1978). Newly hatched fry, when held in supersaturated was as low as $103 \%$ TDG for long periods, may develop GBD. This has been confirmed with chinook salmon fry Oncorhynchus tshawytscha) at Fort Richardson Hatchery (Anchorage) in January 1979.

There are differences in the sensitivity of juvenile and adult salmonids to air-supersaturated water. The threshold level, where most salmon and trout begin to exhibit adverse effects of exposure to supersaturated water, is about 114\% (Garton 1976, Garton and Nebeker 1977) (Table 1).

Considerable research effort has been directed to identify the effect of individual gases on fish survival. Carbon dioxide gas at levels from $1.7-22.0 \mathrm{mg} / 1$ has been found to not 
TABLE 1

Comparative sensitivities of ages and species of salmonids to air-supersaturated water

Species* Threshold

Order of Decreasing Age Sensitivity

1. sockeye salmon

$113.9 \%$

smolt - juvenile - adult

(Oncorhynchus nerka)

2. steelhead trout

$114.2 \%$

juvenile - smolt - adult

(Salmo gairdneri)

3. chinook salmon

$114.7 \%$

(only adults tested)

(‥ tshawytscha)

4. coho salmon

$115.7 \%$

adult - smolt - juvenile

(‥ kisutch)

*Species are 1 isted in order of decreasing sensitivity; sensitivities are based on time to 20\% mortality (Garton and Nebeker 1977, Nebeker and Brett 1976). 
affect survival of salmonids held in constant gas supersaturated water levels (Nebeker et al. 1976, Rucker 1972). Experimentation on oxygen/nitrogen ratios has shown that mortalities decreased significantly when the ratio of $\mathrm{O}_{2} / \mathrm{N}_{2}$ is increased (increase in $\mathrm{O}_{2}$, decrease in $\mathrm{N}_{2}$ ), while maintaining a constant total percent saturation. However, much more extensive and severe signs and lesions of GBD appear at high $\mathrm{O}_{2} / \mathrm{N}_{2}$ ratios. Although oxygen appears to play a significant role in the formation of external emphysema, death occurs sooner with low $\mathrm{O}_{2} / \mathrm{N}_{2}$, indicating $\mathrm{N}_{2}$ to be the more lethal of the gases. Total gas saturation is a more important consideration than individual gas levels, since fish mortalities from GBD will not occur unless the total dissolved gas saturation exceeds $100 \%$ regardless of $\mathrm{O}_{2} / \mathrm{N}_{2}$ ratios (Nebeker et al. 1976, 1979, Rucker 1976).

Signs of GBD in juvenile and adult salmonids are frequently similar. At acutely lethal levels of supersaturation ( $\geq 120 \%$ TDG), signs exhibited by fry are loss of equilibrium, abnormal buoyancy, floating at the surface, violent head shaking, terminal convulsions, flared opercula, blindness and death with the mouth open. Adult salmonids become blind, will release excess gas from the buccal cavity, rub their sides against the tank, tend to be inactive, and remain as close to the bottom of the tank as possible.

The severity, and rate of development of peripheral lesions seem to correlate directly to time of exposure, age of $f i s h$ and level of supersaturation (Stroud and Nebeker 1976). Lesions are attributable to the accumulation of gas in blood vasculature (emboli $i$ ), in tissues (emphysema), or by excessive pressure in hollow organs (tympanites).
Fry show hemorrhage of vitelline vessels, rupture of yolk-sac membranes and denaturation of yolk materials. Lesions seen in fry and adults are generally identical, e.g. subcutaneous bubble accumulations on the head, in mouth, in fin rays, in lateral line, and on gill arches. other tissue alterations are gill hemorrhaging, hyperemia (increased blood in tissues from distension of vessel) at the base of fins, opercular and branchiostegal edema, petechial hemorrhage in fins, skin, muscle, gonads, nares and brain. Also, hyperemia of intestinal epithelium, exophthalmia (popeye) and hyphema (blood in the anterior chamber of the eye) are common lesions. (Nebeker et al. 1976, 1978, 1979, Stroud et al. 1975).

Some lesions are reversible without permanent damage, provided the supersaturated water conditions are removed (Stroud et al. 1975) or lowered to $<103 \%$ TDG. Fish held at acute levels of saturated water frequently die without exhibiting external lesions (Stroud et al. 1975). In sublethal levels of supersaturation, external lesions and gas bubbles are more extensive because the $f i s h$ have longer exposures to the gases before death (Stroud et al. 1975, Stroud and Nebeker 1976).

It is hypothesized that increased muscular activity will result in higher blood temperatures causing soluble gases to come out of solution and move into general circulation. Fish held in supersaturated waters frequently will die shortly after disturbances caused by human activity, e.g. netting, cleaning, etc. (Stroud and Nebeker 1976). This may be attributed to the increase in muscular activity precipitated by the disturbance. A combination of the environmental and physiological conditions release a "cascading emboli effect," during which small bubbles 
coalesce into large bubbles (Stroud and Nebeker 1976). Death is caused by hemostasis (interruption of the flow of blood by blockage of circulation) by gas bubbles (Stroud et al. 1975, Stroud and Nebeker 1976).

\section{Diagnosis}

Gas bubble disease may be diagnosed as the cause of mortalities simply after observing the signs and lesions. A measurement of the gas saturation may be necessary when no signs or lesions appear or confirmation of diagnosis is desired.

Analytical methods for determining dissolved gas levels in water are many. Modern, acceptable methods include gas chromatography, mass spectrometry, Van Slyke manometry and saturometry (Rucker 1972). The first 3 methods are impractical and expensive for routine needs. The Weiss saturometer is inexpensive, simple in operation and will provide quick, accurate results under routine hatchery, field and experimental conditions (Fickeisen et al. 1975).

Both the increase in water temperature and the agitation of the water sample will result in inaccurate gas readings. Therefore, it is important to examine the water on site if gas supersaturation is thought to be a problem.

\section{Significance and Control}

In an experiment conducted by the U.S. EPA, the gonadal products of adult salmon held in supersaturated (110\% TDG) conditions were found to be normal in appearance and consistency, and approximately $90 \%$ of the eggs hatched. Therefore, there is no evidence of any adverse effect of gas supersaturation to sex products or the ability of resulting embryos to hatch (Bouck et al. 1976). The embolic lesions will appear at 106\% TDG, and fish will survive until hemostasis occurs. If gas levels are acute $(\geq 120 \%)$, the fry will succumb before signs or lesions indicate a problem exists. If supersaturated conditions are $\geq 103 \%$ TDG, salmonid fry are stréssed and may later develop conditions which lead to death, e.g. coagulated yolk, fin rot, tail rot, etc. (Wood 1974).

Gas supersaturation problems have probably been more widespread in Alaska than current information would indicate. If water is supersaturated at acute levels, the signs and lesions will not have sufficient time to appear before death occurs. When water contains low levels $(<105 \%)$, frequently the condition is neither suspected nor do the fish show typical lesions or signs. Because of the delayed effects of levels < $105 \%$ on fish, the theory (Garton 1976) that limiting supersaturation levels to $110 \%$ will protect salmonids and other sensitive species from GBD is not considered valid in this instance.

Measures controlling supersaturated water are both natural and artificial in scope. Fish and other aquatic organisms are able to reduce their exposure to supersaturation by seeking greater depth and greater water pressures (an increase of 1 meter in depth will lower the supersaturation by $10 \%$ ) (Garton 1976).

At greater depths, the colder water temperatures will also influence salmonid survivals. When juvenile steelhead trout were tested from $9-18 \mathrm{C}$ at a constant $116 \%$ saturation, each degree (C) increase resulted in decreased time to $50 \%$ death (TM50) by about 30 hours. At $9 \mathrm{C}$ the TM50 was 330 hours, whereas at $18 \mathrm{C}$, the TM50 was about 50 hours. Increased temperatures will sig- 
nificantly decrease survival time of steelhead and chinook salmon, but no significant effect was apparent for either sockeye or coho salmon (Nebeker et al. 1979).

Any method which will forcefully expose large water surface area to air will help eliminate excess gas. Methods include spraying, dripping, aerating, baffling, etc. Elimination of the plunge pool effect or of air entrainment into a water pump intake or line will prevent excess gas from accumulating. Maintaining a sufficiently voluminous head of water above a gravity flow intake will also eliminate gas entrainment.

At power plants, capabilities to reduce and break up spillway flows (Ebel et al. 1975), increasing flows through turbines, surfacing the cooler subsurface waters, and eliminating thermal plumes are a few methods of controlling gas supersaturation. It is expedient that effectively designed supersaturation controls be provided at the inception of any project which will impact water temperatures and pressures and possibly produce supersaturated water.

\section{$\underline{\text { References }}$}

BOUCK, G. R., A. V. NEBEKER and D. G. STEVENS. 1976. Mortality, saltwater adaptation, and reproduction of fish exposed to gas supersaturation. U.S. EPA Ecological Res. Ser. EPA-600/3-76-050. 55 $\mathrm{pp}$.

DAVIS, J. C. 1975. Minimal dissolved oxygen requirements of aquatic life with emphasis on Canadian species: a review. J. Fish. Res. Board Can. 32:2295-2332.

DEMONT, J. D. and R. W. MILLER. 1971. First reported incidence of gas bubble disease in the heated effluent of a steam electric generating station. Proc. 25th Ann. Meeting, S. E. Assoc. Game and $\mathrm{Fish}$ Comm.
EBEL, W. J. 1969. Supersaturation of nitrogen in the Columbia River and its effect on salmon and steelhead trout. Fishery Bull. $68: 1-11$.

EBEL, W. J., H. L. RAYMOND, G. E. MONAN, W. E. FARR and G. K. TANONAKA. 1975. Effect of atmospheric gas supersaturation caused by dams on salmon and steelhead trout of the Snake and Columbia Rivers. NOAA Proc. Report, N.W. Fish. Center. 111 Pp.

FICKEISEN, D. H., M. J. SCHNEIDER and J. C. MONTGOMERY. 1975. A comparative evaluation of the Weiss saturometer. Trans. Amer. Fish. Soc. $104: 816-820$.

GARTON, R. R. 1976. Four-year study on gas bubble disease provides data to protect aquatic life. Research Highlights, Corvallis Environ. Res. Lab, U.S. EPA (May): 01, 1-6.

GARTON, R. R. and A. V. NEBEKER. 1977. A multiple approach to solving the gas supersaturation problem. pp. 4-19. In: Recent Advances in Fish ioxicology Symposium. R. A. TUBB, ed. Oregon State University, Corvallis, OR. U.S. EPA Ecological Res. Ser., July 1977. EPA-600/3-77-085.

NEBEKER, A. $V$. and J. R. BRETT. 1976. Effects of airsupersaturation on survival of Pacific salmon and steelhead smolts. Trans. Amer. Fish. Soc. $105: 338-342$.

NEBEKER, A. V., G. R. BOUCK and D. G. STEVENS. 1976. Carbon dioxide and oxygen/nitrogen ratios as factors affecting salmon survival in air-supersaturated water. Trans. Amer. Fish. Soc. 105:425-429.

NEBEKER, A. V., D. G. STEVENS and J. R. BRETT. 1976. Effects of gas supersaturated water on freshwater aquatic invertebrates. pp. 51-65. In: Gas Bubble Disease. D. H. FICKEISEN and M. J. SCHNEIDER, eds. Proc. of a workshop held at Richland, WA, Oct. 8-9, 1974. CONF-741003, ERDA Tech. Inf. Center, Oak Ridge, TN. 
NEBEKER, A. V., D. G. STEVENS and R. K. STROUD. 1976. Effects of airsupersaturated water on adult sockeye salmon (Oncorhynchus nerka). J. Fish. Res. Board Can. 33:2629-2633.

NEBEKER, A. V., J. A. ANDROS, J. K. MCCRADY and D. G. STEVENS. 1978. Survival of steelhead trout (Salmo gairdneri) eggs, embryos, and fry in air-supersaturated water. J. Fish. Res. Board Can. 35:261-264.

NEBEKER, A. V., A. K. HAUCK and F. D. BAKER. 1979. Temperature and oxygen/nitrogen gas ratios affect fish survival in airsupersaturated water. pp. 299-303. In: Water Reseach, Vol. 13. Pergamon Press Ltd., Great Britain.

NEBEKER, A. V., D. G. STEVENS and R. J. BAKER. 1979. Survival of salmon smolts in seawater after exposure to air-supersaturated water. Prog. Fish-Cult. 41:30-32. NEBEKER, A. V., A. K. HAUCK, F. D. BAKER and S. L. WEITZ. 1980. Comparative responses of speckled dace and cutthroat trout to airsupersaturated water. Trans. Amer. Fish. Soc. 109:760-764.

RUCKER, R. R. 1972. Gas bubble disease of salmonids: A critical review. Tech. Papers of the Bureau of Sport Fish. and Wildl. No. 58. $11 \mathrm{pp}$.

RUCKER, R. R. and P. M. KANGAS. 1974. Effect of nitrogen supersaturated water on coho and chinook salmon. Prog. Fish-Cult. $36: 152-156$.
RUCKER, R. R. 1976. Gas bubble disease of salmonids: variation in oxygen-nitrogen ratio with constant total gas pressure. pp. 85-88. In: Gas Bubble Disease. D. H. FIC KEISEN and M. J. SCHNEIDER, eds. Proc. of a workshop held at Richland, WA, Oct. 8-9 1974. CONF-741003, ERDA Tech. Info. Center, Oak Ridge, TN.

STROUD, R. K., G. R. BOUCK and A. V. NEBEKER. 1975. Pathology of acute and chronic exposure of salmonid fishes to supersaturated water. pp. 435-449. In: Chemistry and Physics of Aqueous Gas Solutions. W. A. ADAMS, ed. The Electrochemical Society, Princeton, NJ.

STROUD, $R$. $K$. and $A$. $V$. NEBEKER. 1976. A study of the pathogenesis of gas bubble disease in steelhead trout (Salmo gairdneri). pp. 66-71. In: Gas Bubble Disease. D. H. FICKEISEN $\frac{\text { and }}{M \text {. J. J. }}$ SCHNEIDER, eds. Proc. of a workshop held in Richland, WA, Oct 8-9, 1974. CONF-741033, ERDA Tech. Info. Center, Oak Ridge, TN. WOOD, J. W. 1974. Disease of Pacific Salmon: Their Prevention and Treatment. 2nd ed. Dept. Fisheries, Hatcheries Div., State of Washington. $82 \mathrm{pp}$. 


\section{Saltwater Gill Disease}

Roger S. Grischkowsky

\section{Introduction}

Saltwater gill disease is a harmful condition of dense Chaetoceros, phytoplankter growth typically Chaetoceros convolutus, which affects some salmonids during marine periods of captivity in estuarine rearing pens. The Chaetoceros cells (see Fig. 21) have very sharp and long setae which penetrate gill lamellae reducing oxygen exchange and resulting in blood loss. These phytoplankters can reach concentrations thick enough to stop the flow of water through a 4 inch line when gravity fed into a number 10 phytoplankton net in 2 hours. Such concentrations provide adequate impalement opportunity to affect the population. The detection of direct setae affect on lamellae is difficult.

\section{History}

Saltwater gill disease as it has become known was first recorded in 1961 when lingcod (Ophiodon elongatus) were held temporarily in surface holding pens (Bell 1961). Then the actual penetration of gill lamellae by the spines was observed. First observations in salmonids, primarily sockeye (Oncorhynchus nerka) but including other Pacific salmon species, were in 1976 (Kennedy et al. 1977). Both the 1961 and 1976 findings were in the Nanaimo, B.C. area.

Another case was reported in salmon in estuarine rearing pens at Camano Island, San Juan Island group (L. Harrel, pers. comm.). The disease in Alaska has occurred at 2 locations, Little Port Walter National Marine Fisheries Service Research Station on Baranof |sland and Kitoi Bay of Afognak Island. The former problem was in 1976 while the latter was 1977 and 1978. The 3 mortalities were in estuarine rearing pens during spring or summer in pink salmon (Oncorhynchus gorbuscha). In each incident Chaetoceros was the dominant phytoplankter and visible blooms were occurring.

\section{Transmission}

The agent is not really transmitted, but will grow when introduced into different locations as the nutrients, water and light conditions allow.

\section{Signs and Pathogenesis}

\section{Signs}

The first sign noticed during the initial portions of a mortality will probably be respiratory distress exemplified by rapid irrigation. The fish will also be off feed. The commencement of mortalities should bring enough focus on the estuarine container to notice a phytoplankton bloom. Microscopic examination will confirm the diatom presence.

\section{Pathogenesis}

Typical pathogenesis has been reported from investigation into the mortality at Camano Island (L. Harrel, pers. comm.). During this dieoff from concentrated $C$. convolutus, detailed involvement with this diatom within gill lamellae was demonstrated using histopathology. Spines had penetrated lamellae and caused direct mortality through blood loss and reduced oxygen exchange capacity. This penetration of spines from 


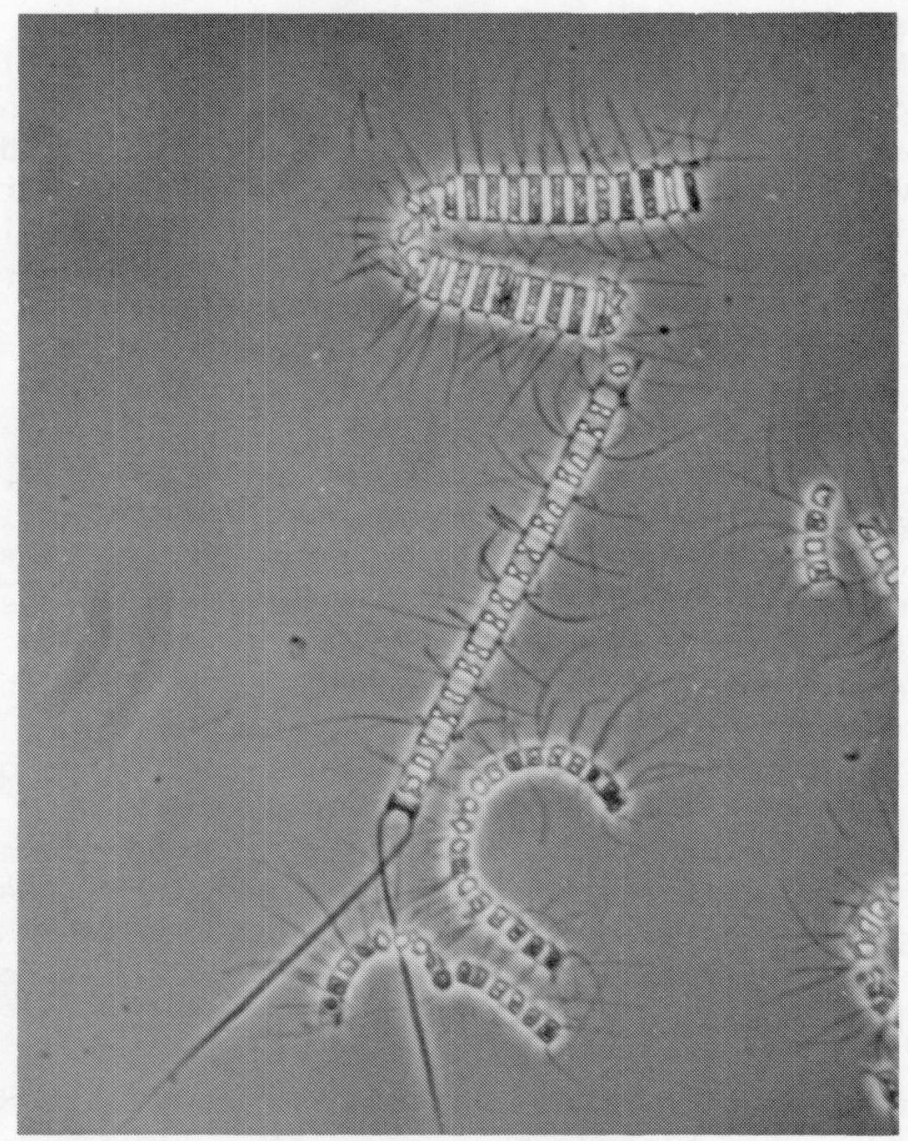

Figure 21. Chaetoceros taken from a conical shaped vertical raceway holding pink salmon (0. gorbuscha) at Little Port Walter, National Marine Fisheries Service Research Station, on Baranof Isl. (1000X, phasecontrast) (R. Grischkowsky).

diatoms into the gill tissue of $1 \mathrm{ing}-$ cod and Pacific salmon had been previously reported (Bell 1961, Brett et al. 1978). Densities of 32,000 cells/l have been detected in all stocks and species of Pacific salmon. During this mortality deaths ranged from $0-8 \%$ in chinook salmon (Oncorhynchus t.shawytscha) to $50 \%$ in sockeye salmon.

\section{Diagnosis}

Chaetoceros includes the greatest number of species of planktonic diatoms, and is the most important one in abundance of species and number of cells in the eastern Pacific (Cupp 1943). This includes about 150 species with most being neritic. Chaetoceros convolutus has a width of 15-23 um (Cupp 1943). The genus is easily recognized by the essentially straight or slightly bent chains and distinct setae projecting from the chain cells (see Fig. 21). In Alaska several variations of the reported $\underline{C}$. convolutus morphology have been noticed. The involvement of Chaetoceros in these mortalities is apparent but speciation has not been attempted. 
The diatom's presence in concentrations dense enough to cause mortality is associated with several meteorologic and hydrographic conditions. Two weeks prior to a bloom is typically clear skies, bright sunshine, rising water temperature (15-21C), initially clear water and light onshore winds (Brett et al. 1978).

\section{Significance and Control}

Chaetoceros is a limiting factor of fish farming. Early intense blooms have the potential for complete decimation of Pacific salmon in estuarine environment (Brett et al. 1978). Sockeye salmon are the most vulnerable typically, while major mortalities have occurred in coho (Oncorhynchus kisutch), chinook, pink and chum (Oncorhynchus keta) salmon (Brett et al. 1978). During the 1973-1976 period at Nanaimo, losses in sockeye salmon have been light to heavy and negligible in the other listed Pacific salmon species (Kennedy et al. 1977). No bloom was reported in 1976 and those noted were between mid-September and midNovember.

No control is really possible. of potential benefit are filtering of water coming into estuarine rearing pens to exclude the agent and early release of fish. The author has tried both. The filtering approach is labor intensive when phytoplankton nets are used, and is ineffective once a bloom has started or affected a pen. Release may increase survival but no evaluation has been made.

\section{References}

BELL, G. R. 1961. Penetration of spines from a marine diatom into the gill tissue of 1 ingcod 0 phidon elongatus. Nature 92:279-280.

BRETT, J. R., W. GRIFFIOEN and A. SOLMIE. 1978. The 1977 crop of salmon reared on the Pacific Biological Station Experimental Fishfarm. Fish. and Environment Canada. Fish. and Mar. Serv. Tech. Rpt. No. 845. 17 pp.

CUPP, E. E. 1943. Marine Diatoms of the West Coast of North America. Univ. California Press, Berkeley, CA. 237 pp.

KENNEDY, W. A., W. GRIFFIOEN and A. SOLMIE. 1977. The 1976 crop of salmon reared on the Pacific Biological Station Experimental Fishfarm. Fish. and Environment Canada. Fish. Mar. Serv. Tech. Rpt. No. 726. 21 pp. 


\title{
Coagulated Yolk Disease
}

\author{
Jill E. Follett
}

Introduction

Coagulated yolk disease, also referred to as white-spot, is a noninfectious malady of incubating eggs or the yolk sacs of alevins. It is manifested by the appearance of white flecks of coagulated yolk in the eggs and later in the fry. These white flecks are actually the indication of an abnormality. The disease occurs sporadically and the cause or causes are poorly understood. Afflicted fish do not recover. A "white-spot" infection that is caused by the protozoan Ichthyophthirius should not be confused with coagulated yolk disease.

\section{History}

This disease occurs where fish eggs are incubated. The earliest reported case was found in brook trout (Salvelinus fontinalis) by Agersborg in 1933 (Mazuranich and Nielson 1959). He attributed it to insufficient water flow or too little oxygen because of overcrowding. In 1939, Leach recorded white-spot disease in Loch Leven (Salmo trutta) fry and rainbow trout (Salmo gairdneri) eggs (Mazuranich and Nielson 1959). He speculated that it was due to physical injury. Various incidences of the disease have been recorded but no common cause has been implicated. Recent experiments at Dworshak National Fish Hatchery and National Fisheries Research Center have shown that neither malachite green nor severe shocking alone cause coagulated yolk (Nelson 1979). Bacteria are often associated with the disease, but appear to be secondary invaders, not the causative agents (Wood 1974).
In Alaska, the first reported cases of coagulated yolk disease occurred in 1975 at Crystal Lake Hatchery in chinook salmon (Oncorhynchus tshawytscha) and Deer Mountain Hatchery in chum salmon (Oncorhynchus keta).

Transmission

The disease is not infectious and cannot be transmitted from fish to fish.

$\underline{\text { Signs }}$ and Pathogenesis

\section{Gross Pathology}

The first sign is the presence of white opaque flecks of coagulated yolk in the egg or in the alevin (color Fig. 40). The spots are normally at the surface of the yolk and randomly distributed. In fry, the coagulated yolk will be seen a few days after hatching. The spots may enlarge and coalesce with time. Pinheading, anemic gills, white or frayed fins may also be noticed. Fry will begin to die about midway through the fry stage. In lighter infections, the mortality does not start until near the end of the fry stage.

It has been reported that "tail rot" in Alaska may be caused by coagulated yolk disease. The unabsorbed yolk somehow interferes with the fish's ability to maintain epithelium and this can lead to "tail rot" or "fin rot." The affected fins or tails develop a whitish appearance. The "tail rot" is usually a self limiting condition but coagulated yolk is fatal. Wood (1974) refers to instances where 
white flecks of coagulated yolk may appear and persist after hatching but disappear later and cause no problem. This is not commonly found.

\section{Histopathology}

The yolk sac of an infected fry may appear not to decrease as the fry grows as it does in normal fry. In actuality, histological studies show that development in an infected larvae proceeds much slower than in a normal one. The yolk may be resorbed but at a considerably slower rate. Shishanina (1959) explains this as the effect of pathological changes connected with metabolic disturbances in different organs. He believes that these abnormal physiological processes are caused by some disturbance in the physiochemical regime necessary for proper development of the salmonid larvae. These abnormal processes lead to the appearance of white inclusions. The inclusions are most likely denatured proteins. Resultant defects in organs and alterations in their functions produce a toxic dystrophy which causes the death of the larvae.

\section{Pathogenes is}

Historically, the pathogenesis of coagulated yolk disease has been attributed to a number of factors. Rough shocking of eggs or early shocking, excessive use of malachite green on eggs, rough handling, high temperatures and the presence of heavy metals in the water have all been incriminated at various times. A combination of factors probably contribute to its occurrence. Mazuranich and $\mathrm{Nielson}$ (1959) found that the disease in Pacific salmon (0. tshawytscha and Oncorhynchus kisutch) fry was not related to temperature or oxygen deficiency, but was caused by frequent use of malachite green. Environmental factors such as gas supersaturation above 102-103\%, unfavorable temperatures, toxic ions such as copper and zinc in the water supply, and mineral deficient egg incubation water have also been implicated (Wedemeyer et al. 1976). Rucker (1975) indicated that excess nitrogen gas did not cause coagulated yolk in his study. Overcrowding has been associated with white-spot, but recent data indicated that it occurred regardless of the seeding density (Nelson 1979). Water flow seems to be important. Flow that is too low will cause low dissolved oxygen, while flow that is too high will cause physical injury. Egg lots that have been split between facilities, but treated the same otherwise, may have an outbreak in 1 facility but not others. Several hatcheries have mentioned that use of an artificial substrate reduced the incidence of white-spot (Nelson 1979). A study on yolk sac malformation salmon indicated that chum, pink (Oncorhynchus gorbuscha) and sockeye (Oncorhynchus nerka) salmon alevins were susceptible to coagulated yolk when provided with smooth substrate but chinook and coho (‥ kisutch) were not (Edami 1973). In sockeye salmon, malformation increased with temperature increase from $8 \mathrm{C}$ to $12-14 \mathrm{C}$ and when water velocity was increased at $14 \mathrm{C}$. Presently, coagulated yolk cannot be pinned down to any 1 cause but seems to be induced by many.

Soft water facilities reported twice as many white-spot problems as hard water stations in Nelson's (1979) study. Spring chinook and coho salmon were susceptible primarily in soft water, while other species were affected in either hard or soft water. 


\section{Diagnosis}

The appearance of white flecks or masses of coagulated yolk in eggs or alevins is diagnostic. The fish commonly develop rigid, frayed, or whitish pectoral fins and suffer high mortality.

\section{Significance and Control}

This disease can cause significant losses in eggs and fry of reared fish. The disease has not been reported in wild fish, but probably occurs before fry emerge from the gravel. No treatment is known. Control is currently achieved only by prevention. The following good fish culture practices may reduce the incidence: keep nitrogen gas supersaturation levels below or at $100 \%$, maintain appropriate water temperatures, avoid excessive use of malachite green, minimize rough handling, avoid early or over shocking and observe proper loading and waterflow. Soft water stations may want to increase salts to prevent deficiencies. When the causes are more clearly understood, disease control may be more defined.

\section{References}

EDAMI, H. 1973. Yolk sac malformation in Pacific salmon in relation to substrate, temperature and water velocity. J. Fish. Res. Board Can. 30:1249.
MAZURANICH, J. J. and W. E. NIELSON. 1959. White-spot disease of salmon fry. Prog. Fish-Cult. $21: 172-176$.

NELSON, R. C. 1979. White-spot and coagulated yolk disease survey and report. Dworshak/Kooskia NFH Complex, Ahsahka, ID. (Unpublished).

RUCKER, R. R. 1975. Excess nitrogen gas in water not a cause of coagulated yolk disease in chinook salmon. Prog. Fish-Cult. 37:101-102.

SHISHANINA, A. D. 1959. Data on the etiology and pathogenesis of white-spot disease of salmon larvae. Academy of Sciences, USSR, Conference Proc. No. 9; Proc. of Conference on Fish Diseases. Israel Program for Scientific Translations (for NSF, Washington), Jerusalem, 1963. TT 61-31058 U.S. Dept. of Commerce, Washington, DC. 1959:97-101.

WEDEMEYER, G. A., F. P. MEYER and L. SMITH. 1976. Diseases of Fishes Book 5: Environmental Stress and Fish Diseases. TFH Publications, Neptune City, NJ. 192 pp.

WOLF, K. 1970. White-spot disease of fish eggs and fry. Fish Disease Leaflet No. 22. National Fisheries Research Laboratory, U.S. Fish and Wildl. Serv., Leetown, WV. $3 \mathrm{pp}$.

WOOD, J. W. 1974. Diseases of Pacific Salmon, Their Prevention and Treatment. 2 2nd ed. State of Washington, Dept. of Fisheries, Hatchery Div., Olympia, WA. 82 pp. 
29. Reaction of Aeromonas salmonicida on tyrosine containing agar. Brown diffusible pigment is produced after 48 hours (R.A. Ward).

30. Aeromonas salmonicida dorsal head lesions in rainbow trout. Hemorrhagic areas are prevalent throughout cephalic outer tissue (R.A. Ward).

31. Petechiae in a salmonid on ventral surface showing the beginning of hemorrhagic area in submucous (W.T. Yasutake).

32. Furunculosis lesion in coho salmon (Oncorhynchus kisutch). Internal petechiae present in a major erythrymic involvement of the body cavity (W.T. Yasutake). 
$\stackrel{\omega}{\omega}$

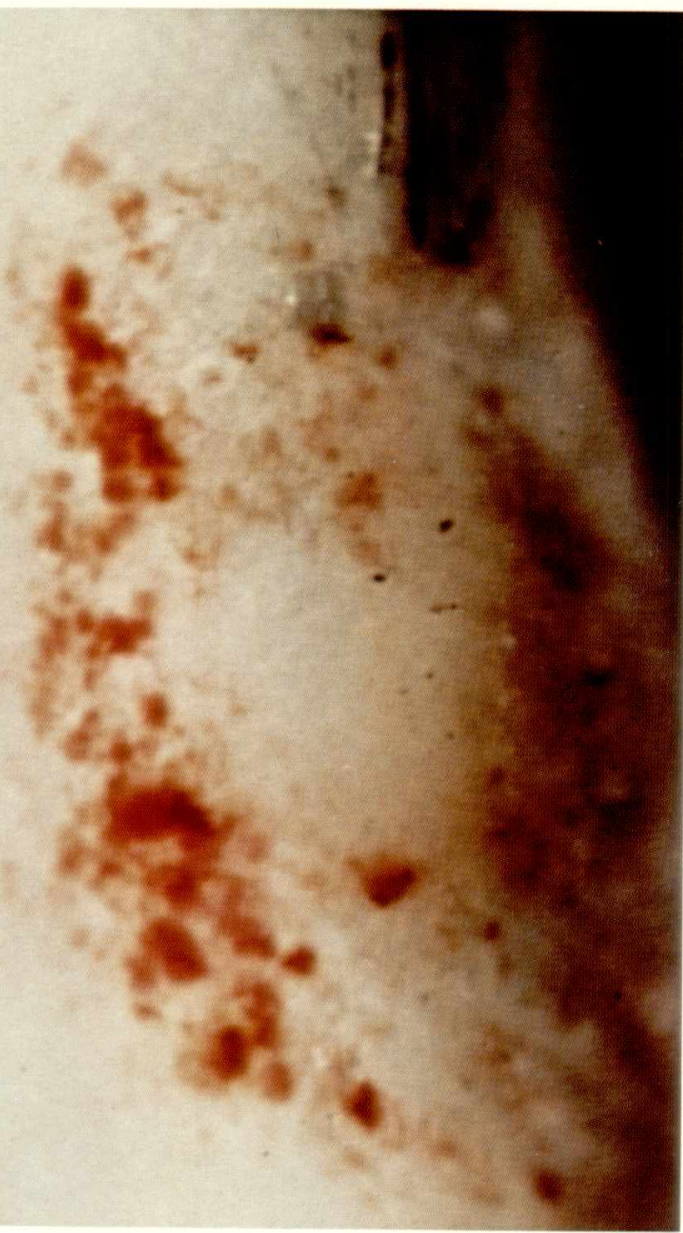

N

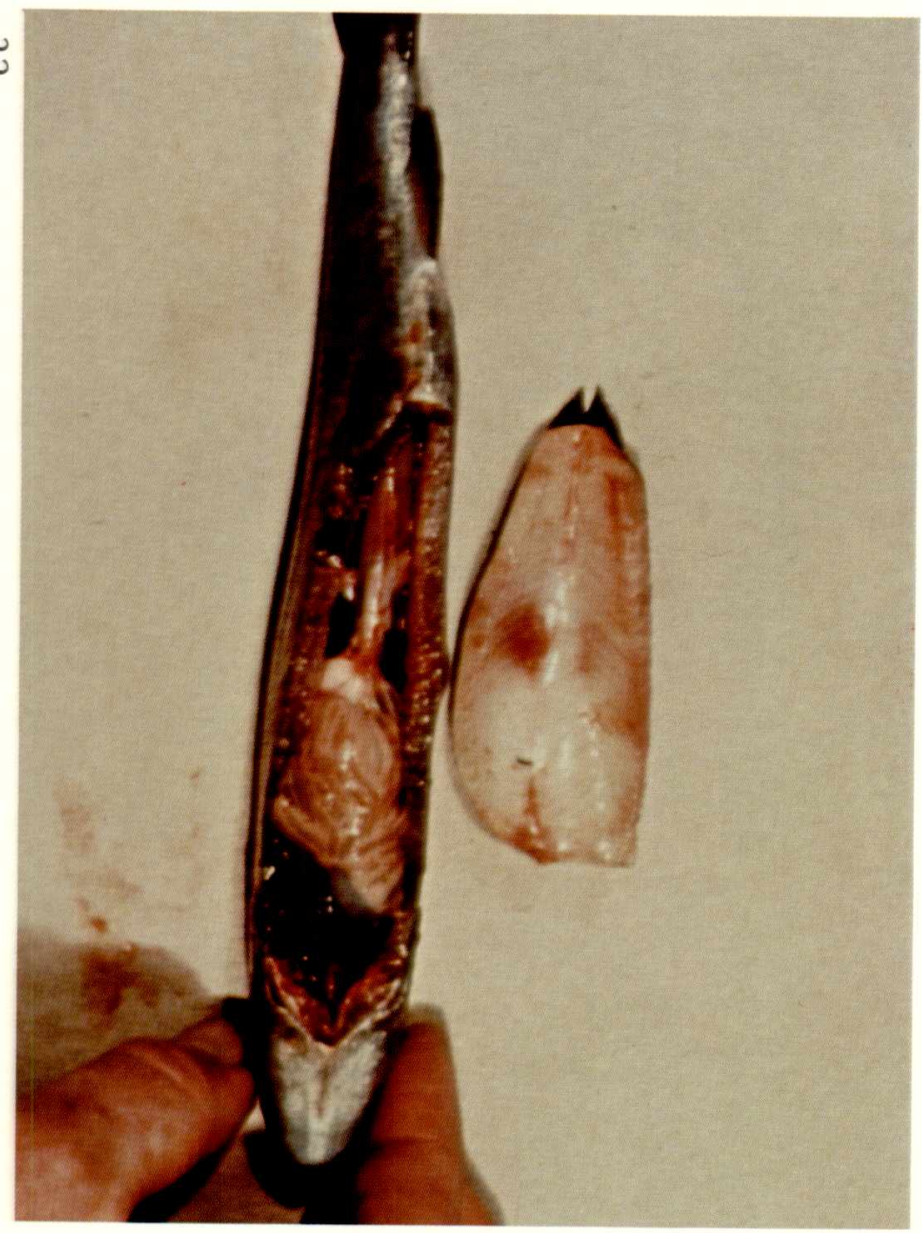

N

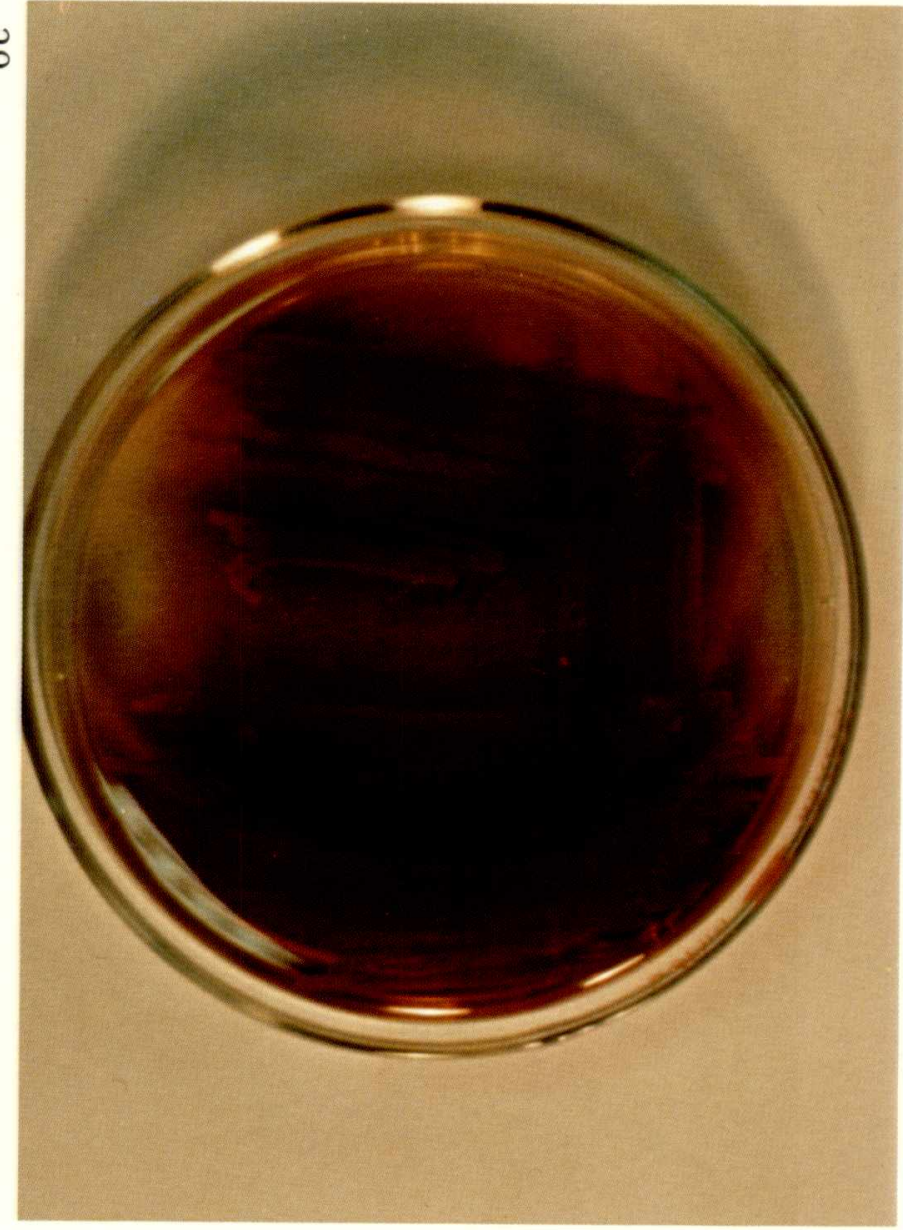

w

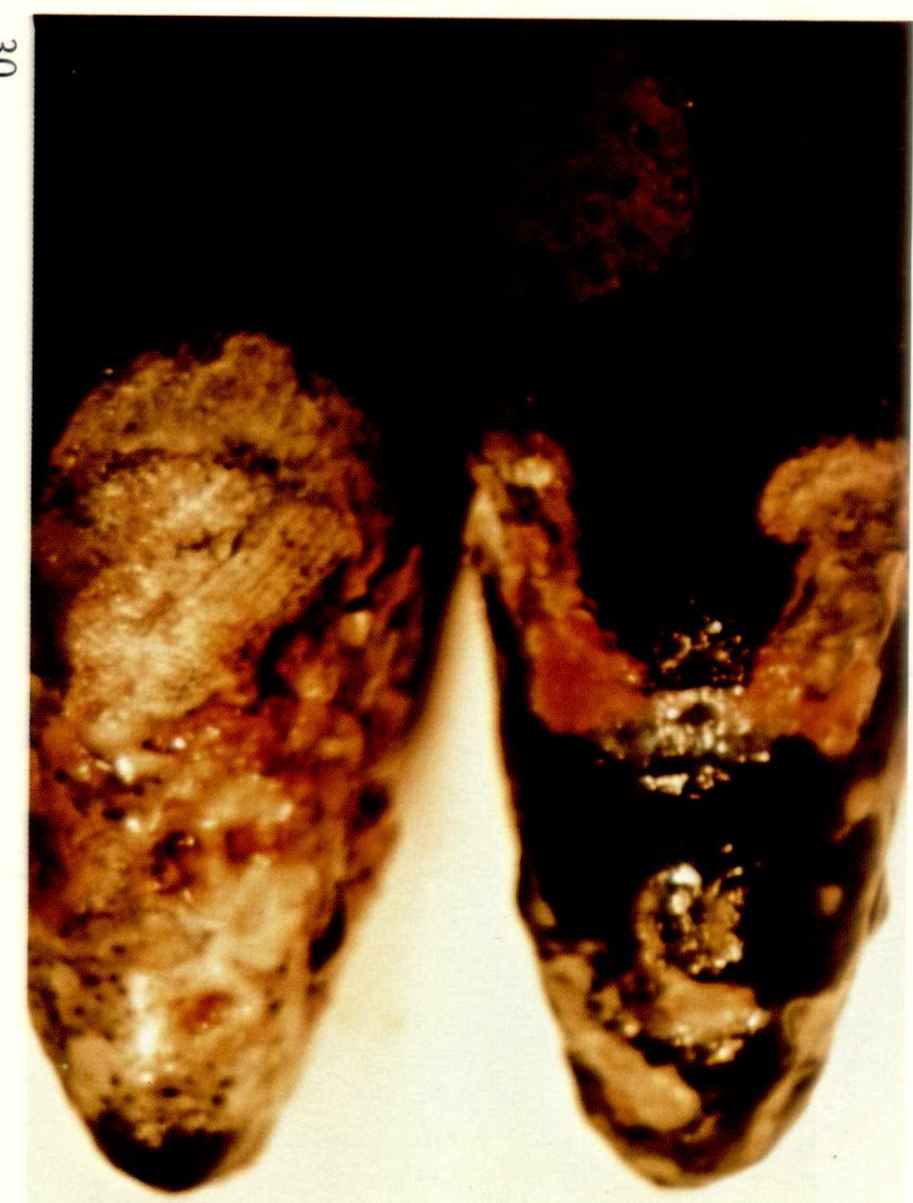


33. Chinook salmon (Oncorhynchus tshawytscha) with enteric redmouth. Note hemorrhagic fins without fraying and vent (J. Sullivan).

34. Chinook salmon (…tshawytscha) with enteric redmouth. Hemorrhaging in the visceral organs, particularly in the pancreatic tissue (J. Sullivan).

35. Chinook salmon (‥ tshawytscha) with enteric redmouth. Some inflammation of intestine and vent (J. Sullivan).

36. Chinook salmon (으. tschawytscha) with enteric redmouth. Exophthalmia, pale liver and hemorrhaging (J. Sullivan). 
$\omega$

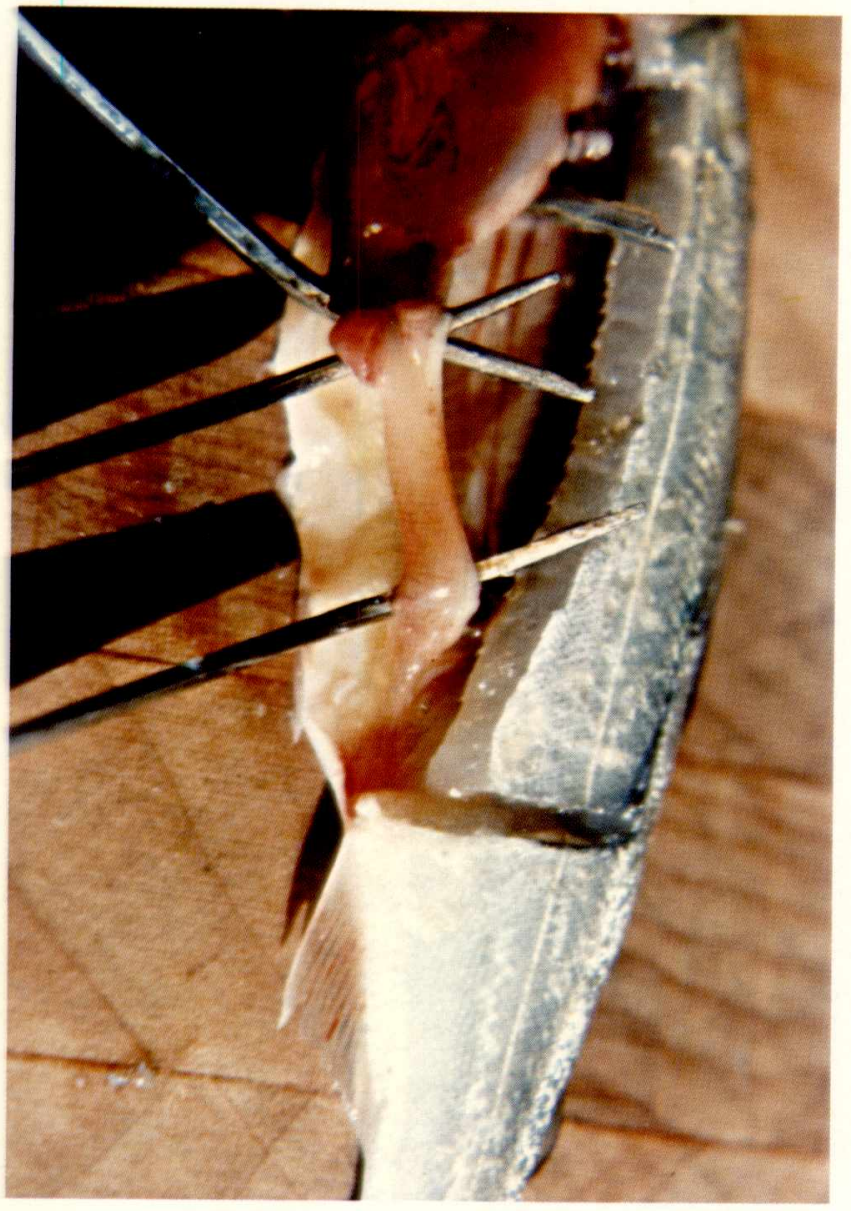

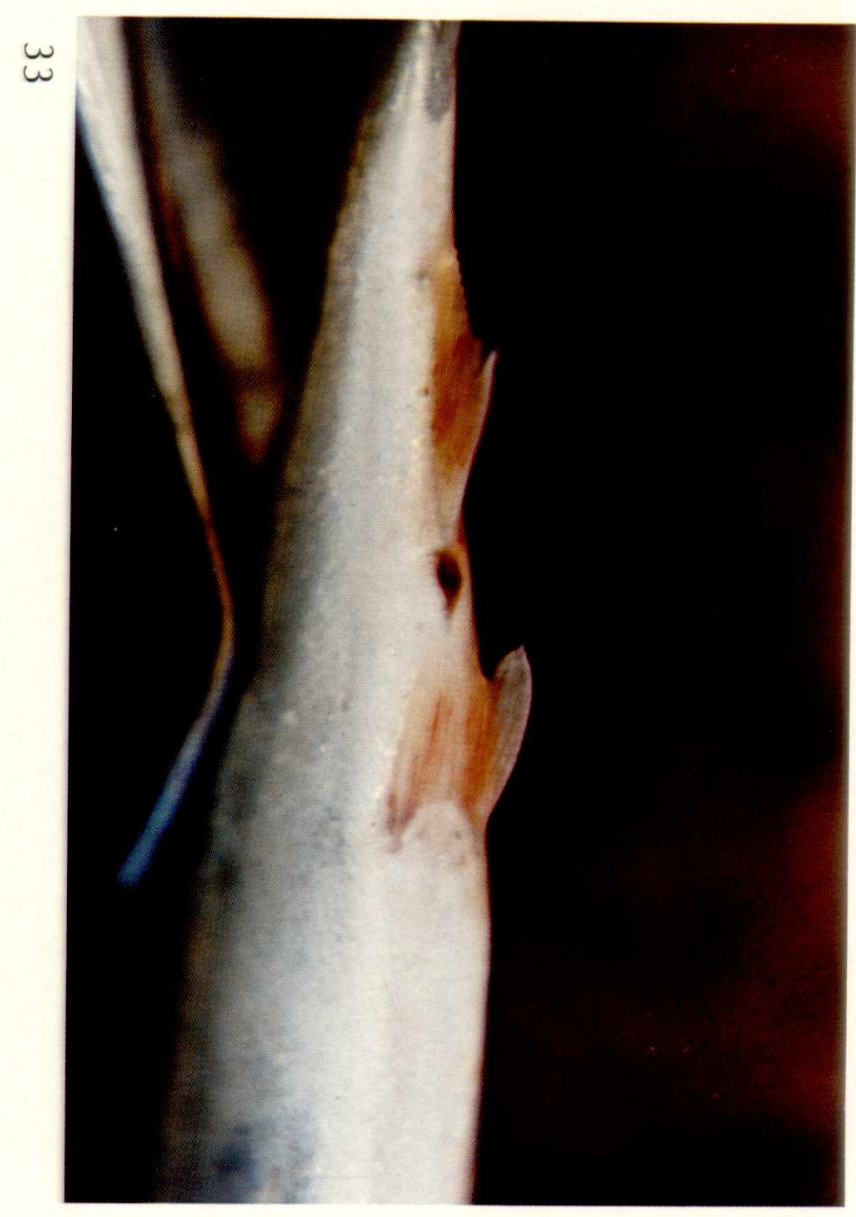

a

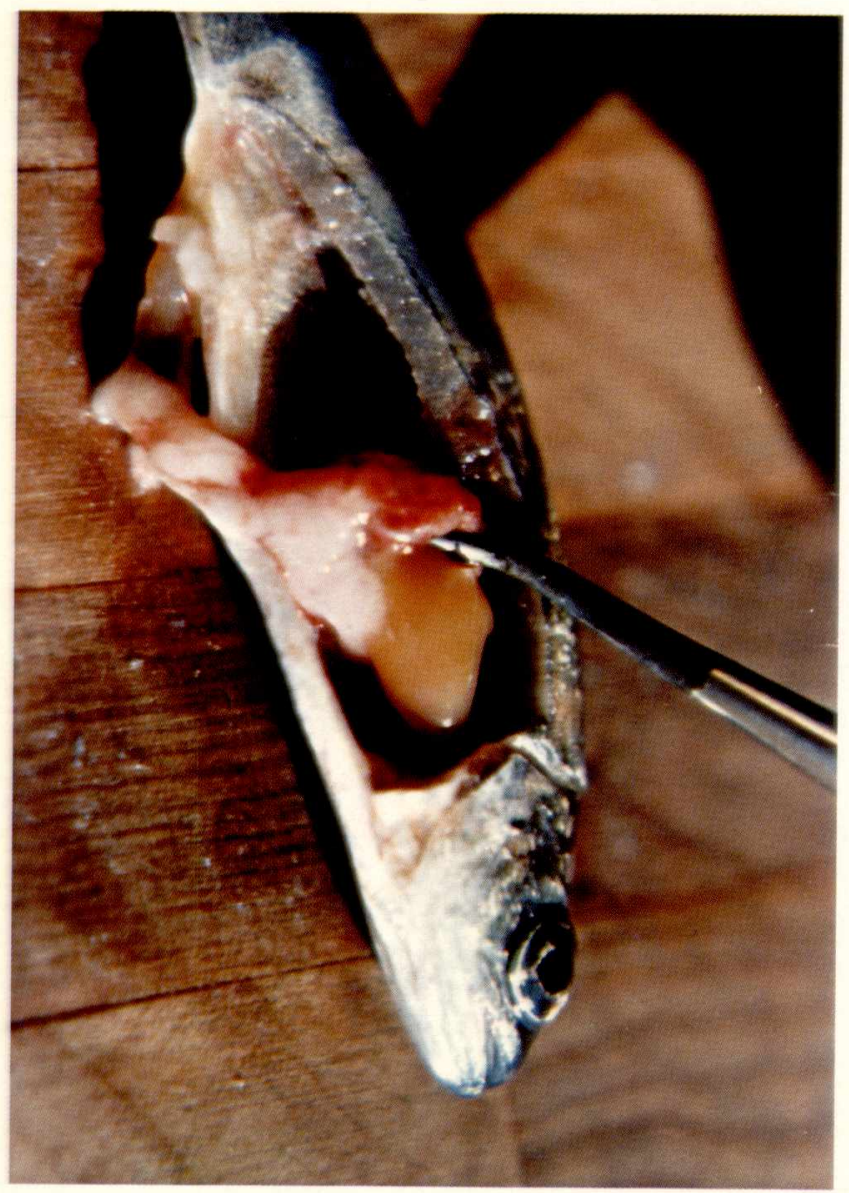


37. Kidney of a juvenile salmonid showing lesions and swelling produced by bacterial kidney disease (W.T. Yasutake).

38. Sockeye salmon (Oncorhynchus nerka) fry normal anterior kidney from Lake Nunavaugaluk Hatchery in North Bristol Bay, Alaska 1974. Hematoxylin and eosin (HEE) 100X (R. Grischkowsky).

39. Infectious hematopoietic necrosis virus epizootic in sockeye salmon ( ${ }_{0}$. nerka) alevins at Big Lake Hatchery in Alaska. This mortality, which occurred in 1979, claimed approximately $7 \mathrm{million}$ fish. Signs included listlessness, loss of equilibrium, erratic swimming, flashing and floating with little motion at the surface in low velocity water (M.J. Fallon).

40. Coho salmon ( 0 . kisutch) alevin with flecks of coagulated yolk in the yolk sac (arrow) at the Crystal Hatchery, Petersburg, Alaska (R. Zorich). 
w

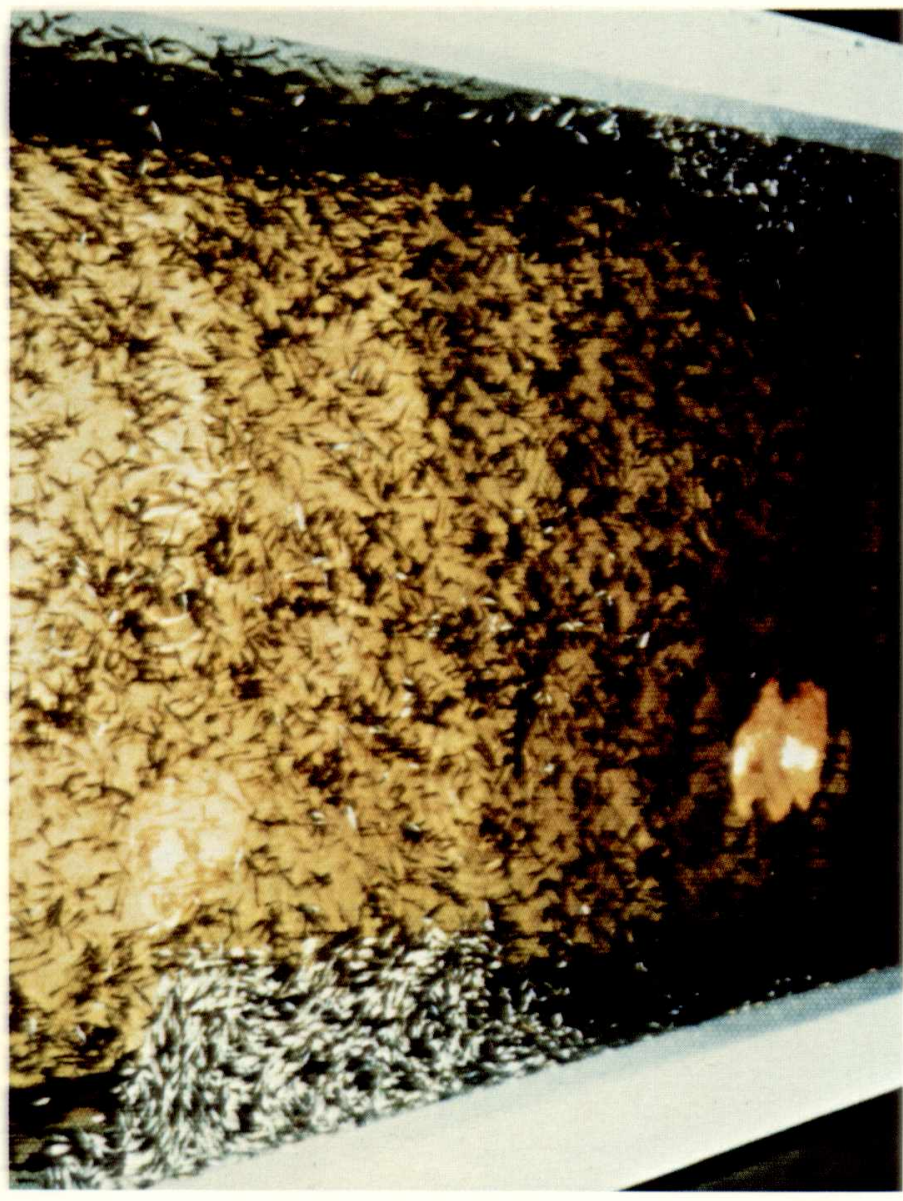

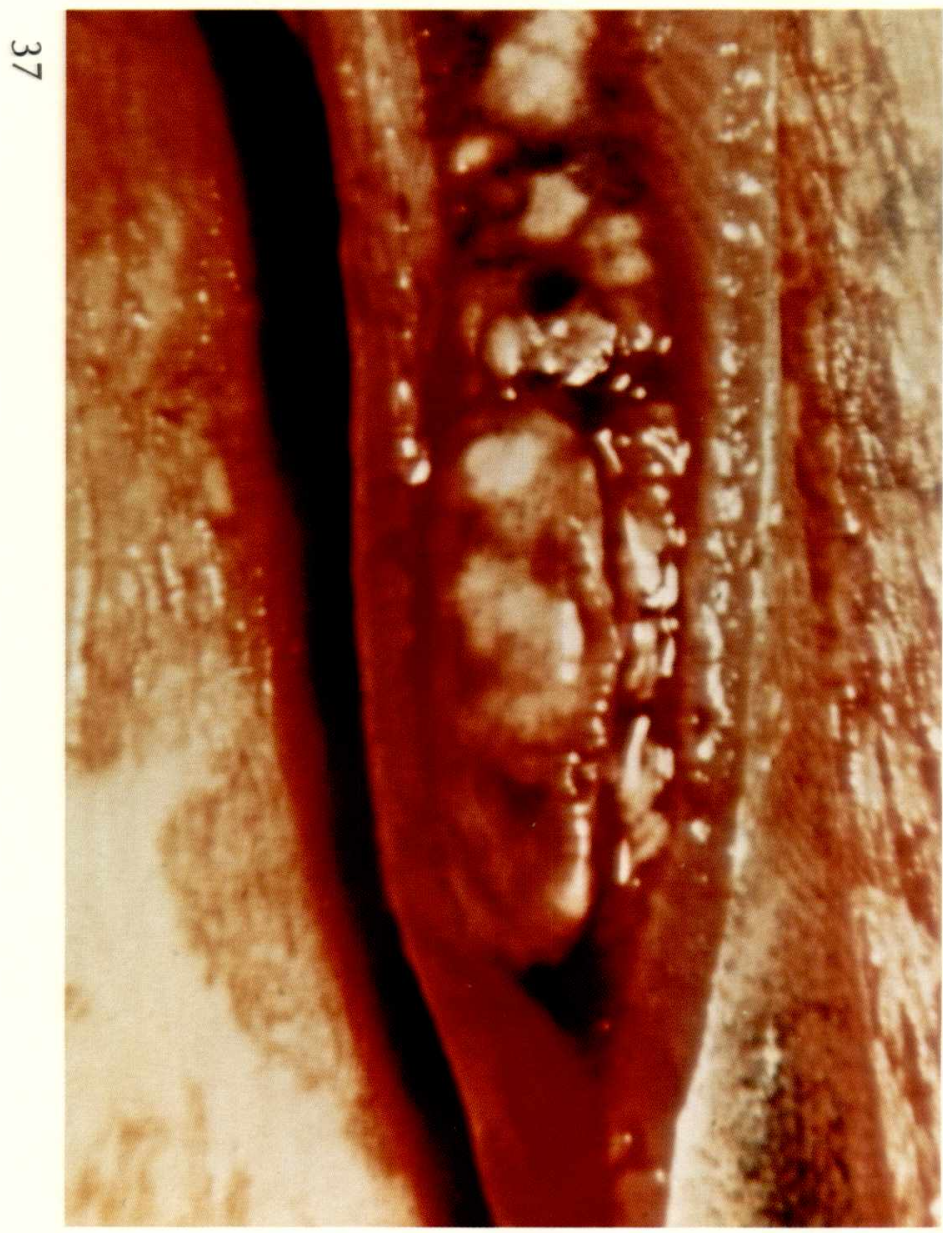

5

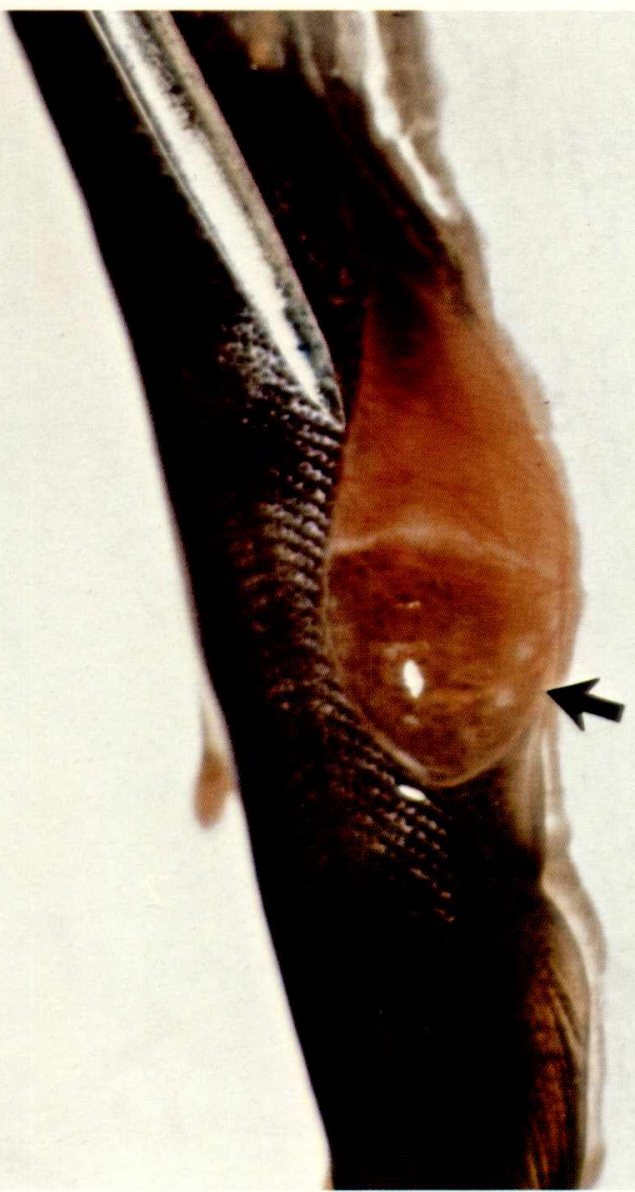

$\infty$

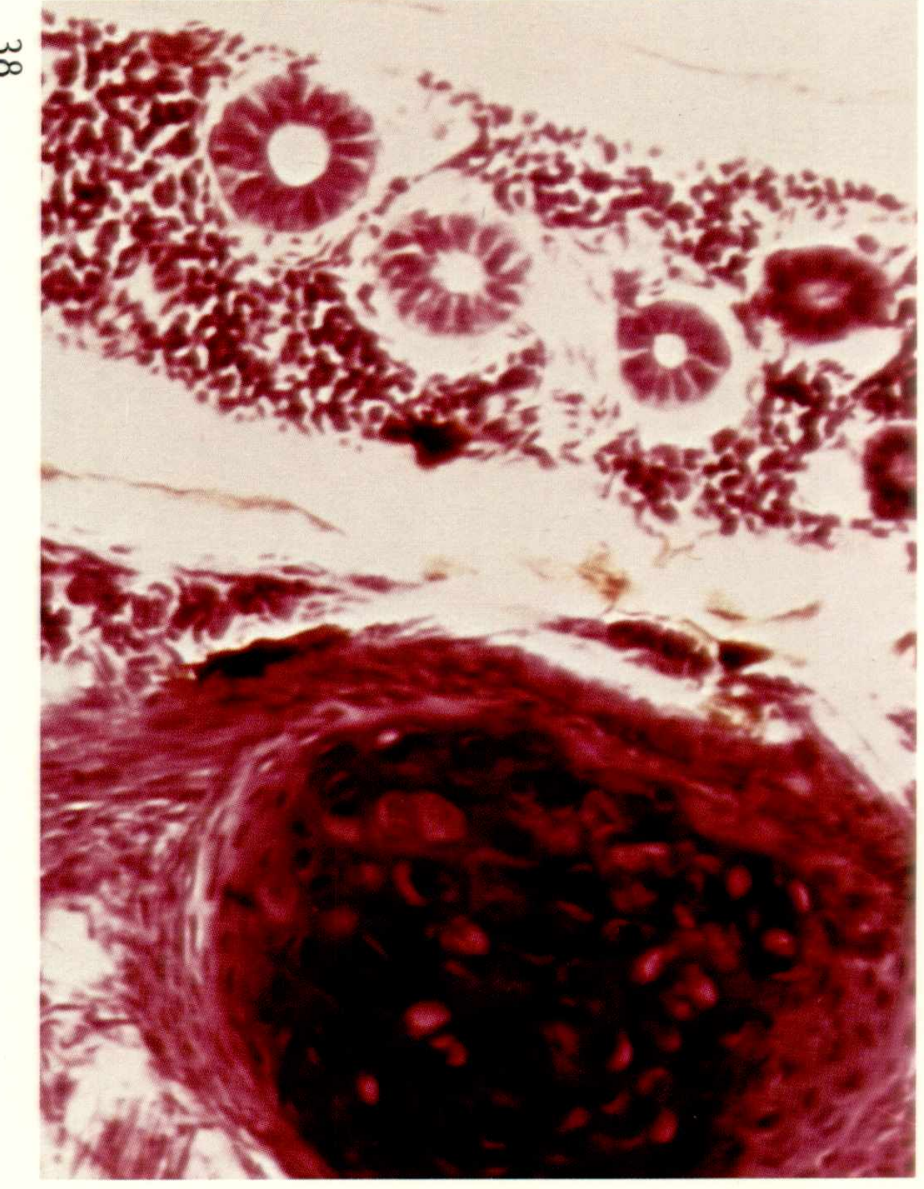


41. Hyperplasia and fusion of secondary gill lamellae of sockeye salmon (ㅁ․ nerka) caused by Ichtyobodo. Note the numerous Ichtyobodo flagellates (i) attached to gill tissues (200x, phase contrast). Hematoxylin and eosin (A.K. Hauck).

42. Pear-shaped 1. necator (i) attached to gill tissues of sockeye salmon ( $\underline{0}$. nerka). Note nucleus $(n)$ surrounded by lighter halo (200X). Hematoxylin and eosin (A.K. Hauck).

43. Ich trophozoite ( $t$ ) surrounded by hyperplastic (h) epidermis (e). Note dermis (d) and horseshoe-shaped nucleus ( $n$ ) (200X, phase contrast). Hematoxylin and eosin (A.K. Hauck).

44. Ichthyophthirius multifilis Fouquet. Note the horseshoe-shaped nucleaus (n) (400X, phase contrast) (R. Grischkowsky). 

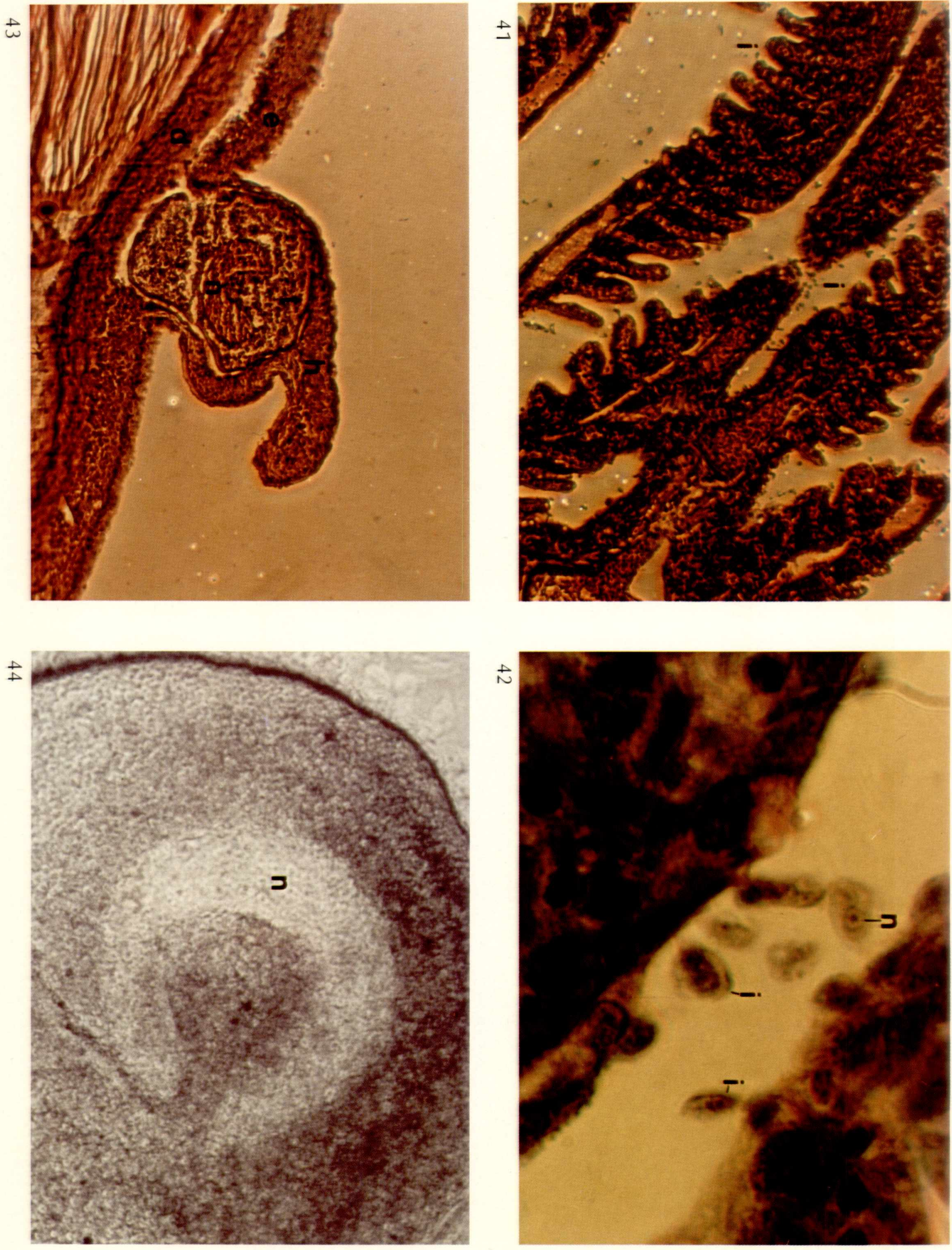
45. Pear-shaped body of Hexamita (h) adjacent to gut epithelium (e) of chinook salmon (‥ tshawytscha). Nuclei and flagella not visible in this preparation $(200 \overline{0} x)$. Hematoxyl in and eosin (A.K. Hauck).

46. Growth of hyphal filaments extending from nares and adjacent connective tissues ( $c$ ) into telencephalon $(t)$ of sockeye salmon ( 0 . nerka) (200X, phase contrast). Grocott's methenamine-silver nitrate (A.K. Hauck).

47. Minor hyperplasia produced in epidermis directly below laterally-viewed trichodinid $(t)$ parasitizing Alaskan sockeye salmon (ㅁ. nerka) fry (400X, phase contrast). Brown and Hopps new Gram stain (A.K. Hauck).

48. Aboral view of Trichodina spp. showing the denticulate ring (d) with broad outer hooks and slender pointed hooks projecting inward and the ciliary girdle (c) (2000x, phase contrast) (R. Grischkowsky). 
$\pm$

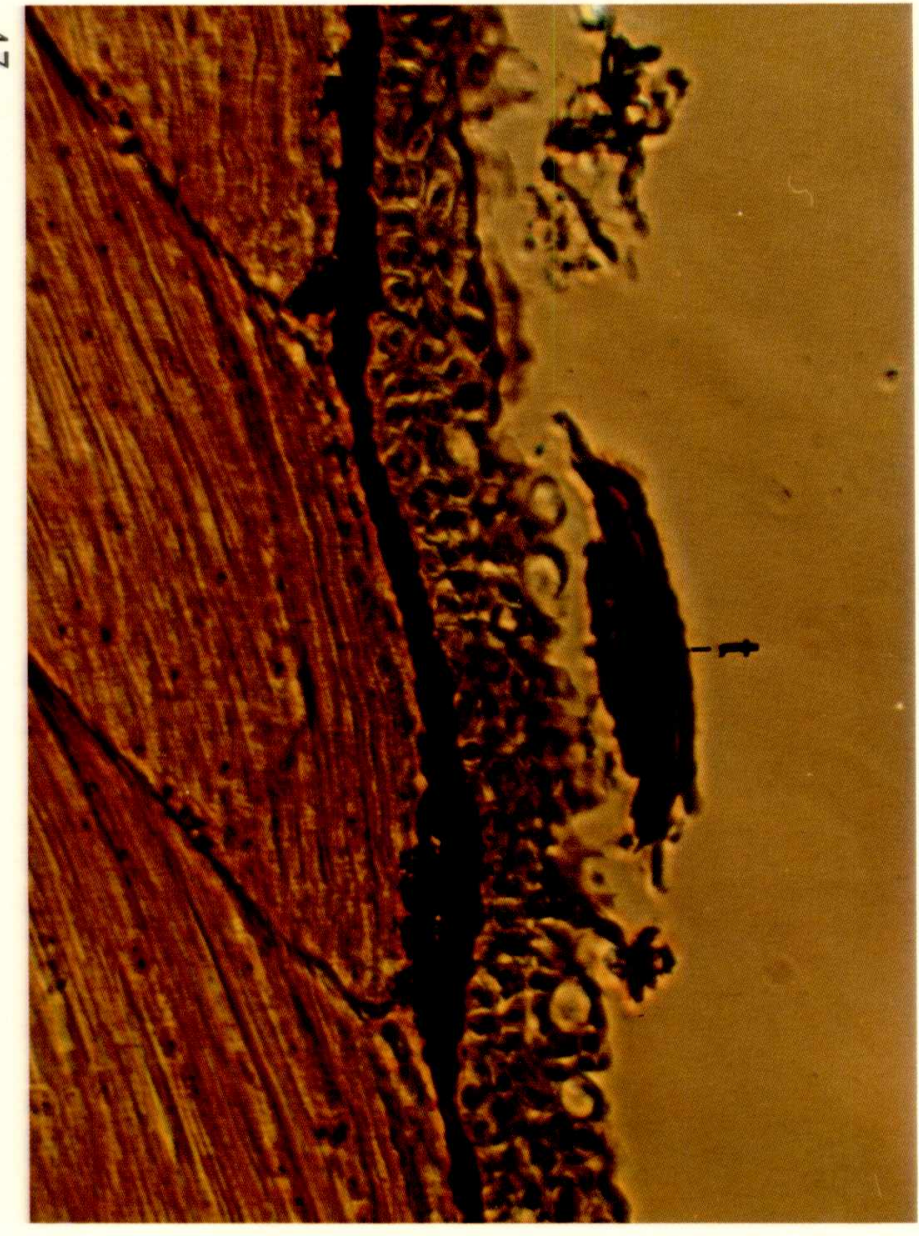

$\frac{1}{\infty}$

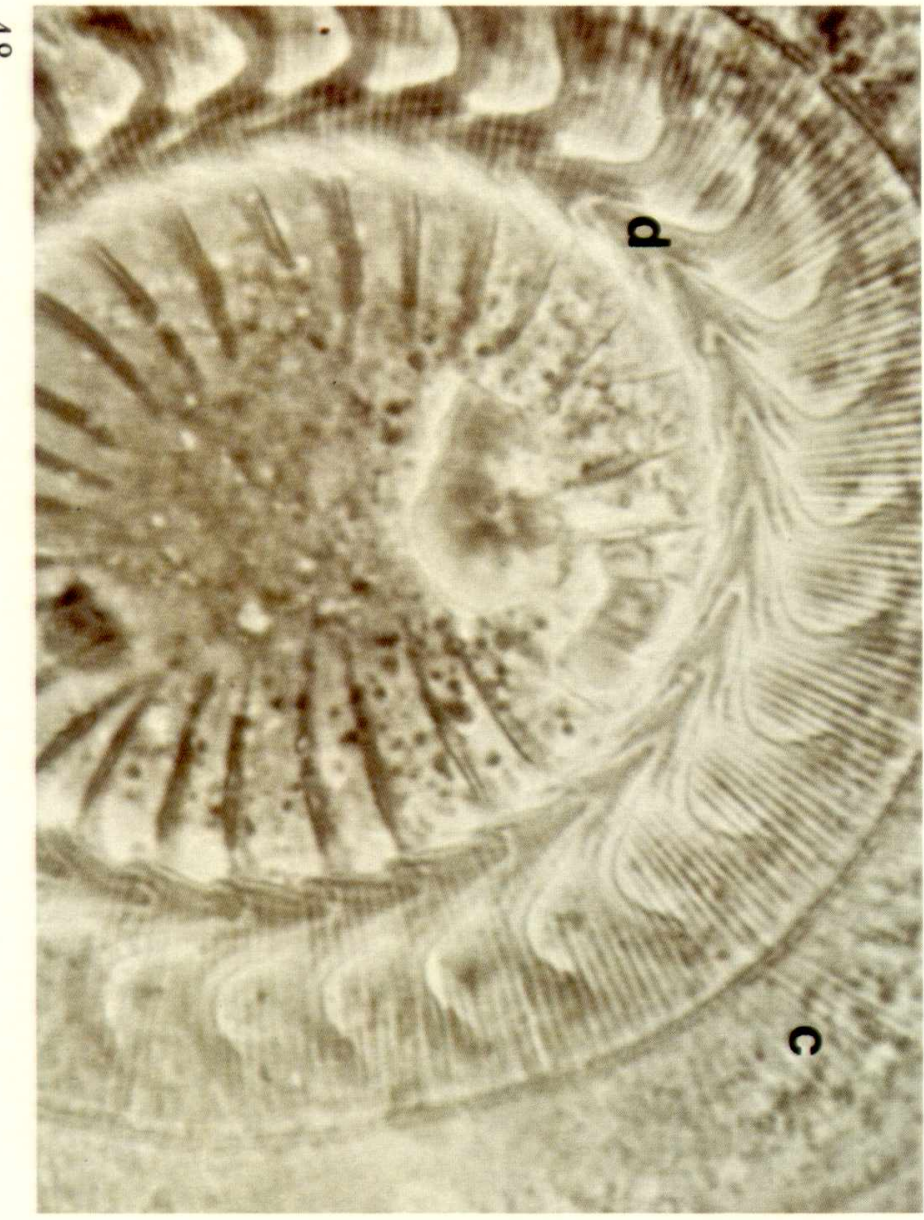

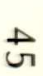
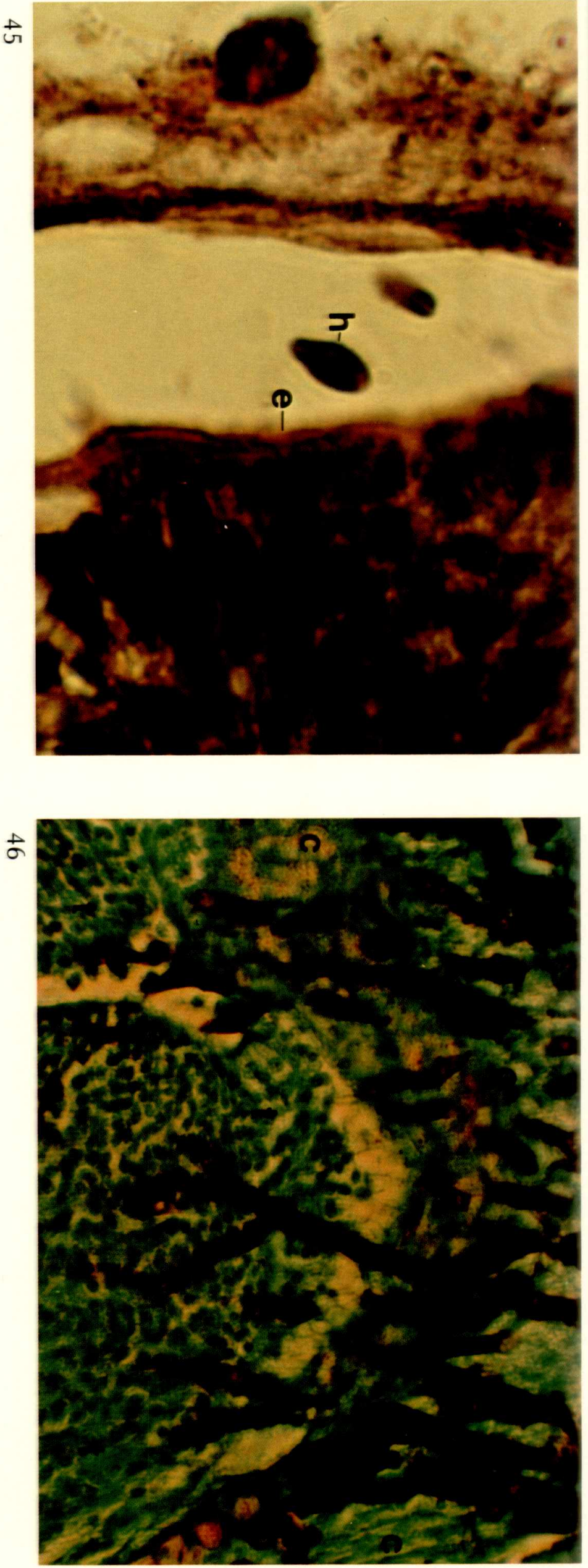
Part IV

INVERTEBRATES 


\title{
Shellfish: Public Health Significance and Diseases
}

\author{
Richard A. Neve'
}

The known outbreak of any disease in shellfish in Alaska is fortunately relatively rare. Any given disease could result though from the introduction of parasites or pathogens, from lack of food, even from chemical and physical alterations of the water. Also, the genetic makeup of any animal cannot be neglected. Perhaps the most important consideration of all is the interrelationship of 1 or all of the above factors in the environment.

In recent years it has been shown that many bacterial, parasitic, and other diseases become a problem only if the shellfish or finfish is held under environmental conditions unfavorable for the particular marine species. This concept has been very clearly explained by Snieszko (1973) and Wedemeyer (1974).

The relationship can be presented by an equation:

$$
H+P+S^{2}=D
$$

where " $H$ " is the host, "P"' is the pathogen, "S" is the stress caused by the environment and " $D$ " is the resulting disease.

While the stress factor, e.g. a rise in water temperature above $10 \mathrm{C}$ (this will trigger the appearance of "vibrio"), can be measured arithmetically, the stress produced by such a change is expressed exponentially. Thus, any change in the "S" factor will more easily shift the equation to the right with the resulting appearance of a particular disease.
Because of the increased fishing pressure on all species of $f i s h$ and shellfish in Alaska, diseases once considered rare may be encountered. This presents a grave problem in that advances in the understanding and control of disease in marine waters have come only in the last 10 to 15 years. The state is developing more hatcheries and more diseases will make their presence known. Shellfish culture--oysters, abalone, mussels, crabs, sea urchins and others--will very likely develop in the next few years. Diseases which economically impact the shellfish industry in the "lower 48" are known. These include viruses, bacteria, parasites and even some worms. In Alaskan waters these pathogenic factors could be lying in wait for the right environmental "stress" trigger to make their presence known.

In the absence to date of specific disease knowledge, emphasis in this portion of the compendium has been focused mainly on the long known toxin problems associated with Alaskan shellfish, and with some discussion of toxin problems occurring sporadically, but potentially very dangerous, in other animals encountered by commercial fishermen.

\section{Paralytic Shellfish Poisoning}

Shellfish diseases which endanger the lives of Alaskans are fortunately not numerous. The one we are most aware of, and fortunately most knowledgeable about, is the "Red Tide," which technically is not a disease. Rather it refers to the condition of coastal ocean waters in which the 
water color may range from Campbell's tomato soup orange to a bright red or magenta. The amount of color in the water is a measure of the number of small (microscopic in size) dinoflagellates. These tiny organisms under the right condition of temperature, salinity, nutrients, trace elements, and some as yet unknown factor(s) will increase and multiply their number rapidly. This growth occurs at certain times of the year, usually but not necessarily, in late August or September. The increased growth is referred to as a "bloom" and is similar to the normal plankton "bloom" which occur every spring in the Northern Hemisphere (March to April), with the important distinction that our "RED BLOOMS" or TIDES are frequently poisonous.

The name of the "disease" is Paralytic Shellfish Poisoning, frequently abbreviated as PSP. The animals affected in the order of importance are primarily molluscs and crustaceans, and include clams (Saxidomus gigantea), mussels (Mytilus edulis), oysters (Crassostrea gigas), cockles (Clinocardium nuttali), abalone (Haliotis $\frac{\text { kamt- }}{\text { sea cucumbers }}$ schatikana) and sea cucumbers (Stichopus $\frac{\text { californicus) }}{\text { St Pink }}$ shrimp (Pandalus $\frac{\text { borealis })}{\text { king and the }}$ (Paralithodes camtschatica), dungeness (Cancer magister), and tanner (Chionecetes bairdi) may contain the poison briefly. Historically, the butter clam (Saxidomus gigantea) has been the species most adversely affected-and then only because of the economic effect of beach closure to clam harvesting because of the occurrence of a red tide. In 1947 all clam areas were closed to commercial harvesting. In 1970, under the Department of Health and Social Services, 3 beach areas were approved for commercial harvesting. These are Polly Creek on the west side of Cook Inlet, Cordova Flats, adjacent to Prince William Sound and Swikshak, on the Alaska Peninsula northwest of Kodiak Island.

Recreational harvesting of razor clams (Siliqua patula) is popular on the Kenai Peninsula from Clam Gulch to Homer. These beaches are easy to reach, and therefore easy to monitor and to post warning signs if and when the need should arise.

Butter clams (Saxidomus gigantea) and littleneck clams (Prototheca semidecussata) are also popular bivalve edibles both to commercial and recreational fishermen. These species however, are potentially the most dangerous for human consumers. They have a black tipped siphon which contains up to $60 \%$ of the total amount of toxin found in any one clam. Historically, the butter clam is the most notorious for causing deaths of many who sampled Alaskan clams. There have been over 200 deaths since the late 1700 s along the coastline from Puget Sound to Bristol Bay attributed to toxic butter clams or mussels. The danger in butter clams is the very long time that they retain the toxin--up to 2 years. Recent studies by Alaskan researchers (Hall, Reichardt and Neve') have shown that clam areas with the highest toxin levels have not been exposed to the typical "Red Tide." Clams along the beaches of Porpoise Island (just outside of Glacier Bay National Monument) have been known by generations of local natives to be quite lethal--but no one remembers ever sighting a "Red Tide." The lack of such a sighting has been noted by fishing boat crews, tug boat operators, cruise ship and Alaska State Ferry personnel. There is an explanation for this unusual 
phenomenon of high PSP toxin levels and no red tides. Evidence now indicates that these high levels are caused by the dormant form of Gonyaulax catanella, the "Red Tide" dinoflagellate which exists in many areas of Southeast Alaska, Prince William Sound--all the way to Bristol Bay and the area between Port Mohler and Port Heiden. This toxin producing organism has been found from samples as far south as San Francisco bay. The one problem in Alaska is that the waters warm up too infrequently to always trigger a visible occurrence of toxic dinoflagellates. The wintering (cyst) form of the dinoflagellate can produce 10 times the amount of toxin found in the motile or swimming form and is therefore the real troublemaker.

\section{Symptoms}

A tingling sensation in the lips and tongue may develop within a few minutes after eating affected clams. A feeling of numbness in the legs, arms and neck follows. There may be a general muscular incoordination. There also may be dizziness, weakness, drowsiness, incoherent speech occasionally, headache, a firm rapid pulse of 80 to 100 per minute, some respiratory distress, vomiting, diarrhea and abdominal pain. The stricken person remains clear-headed until the later stages when muscular paralysis becomes severe and death (in severe cases) results from respiratory paralysis. The more poisonous shellfish eaten, the more severe will be the symptoms.

\section{Treatment}

There is no known antidote. Particular care should be given to avoid alcohol. Vomiting should be induced as soon as possible (to decrease the amount of toxin absorbed) followed by a rapid acting laxative. Artificial respiration should be administered if there are any respiratory difficulties, and may have to be continued for several hours. The critical period is 3 to 12 hours after ingestion of toxic clams. Recovery is usually complete within 24 hours if the critical period has passed.

A small percent of the population will be allergic to shellfish. There is an important distinction between PSP and an allergic response to eating shellfish. The typical signs are rashes, urticaria (hives) and swelling or intestinal disorders. Recovery requires several days and death nowadays is rare. Another name for allergenic poisoning is erythematous poisoning; it has been confused by many with paralytic shellfish poisoning. It is important to note that shellfish allergy may develop after years of normal shell$\mathrm{fish}$ consumption.

\section{Cones (Another Mollusk)}

One definite Alaskan cone, tentatively identified as Conus striatus, and 2 other species could be poisonous to commercial fishermen. They attach to crab nets or lines left on the bottom for any length of time. Of the 400 plus species known throughout the world, only 15 species are considered dangerous--and Alaska's north gulf has 1 of them.

The cone is dangerous because it can inflict a very serious or fatal puncture wound. The toxin is injected by a hypodermic-like tooth which is extended from the pointed head end of the shell. Therefore, handling a poisonous cone is very hazardous. 


\section{Coelenterates}

This phylum includes the jellyfish (Scyphozoa), hydroids (Hydrozoa) and sea anemones (Anthozoa). Many of the members of this phylum produce stinging cells (Nematocysts) such as the Portugese man-of-war (Physalia physalis). These species are not known to occur in Alaskan waters, but 2 species of large jellyfish may be brought up in nets, or stranded on sandy beaches along the north gulf. One, Chrysaora melanaster, is dark brown-and-purple striped. The other, Cyanea capillata, is often a shapeless mass of pale orange jelly with a lot of tangled tentacles. Both are potentially dangerous to anyone with allergic problems. Handling them may cause a severe rash, or an acute allergy shock, depending on the victim's susceptibility and past history of exposure.

\section{Echinoderms}

Members of the Echinoderm phylum are generally bottom-of-the-sea dwellers. At low tides, sea cucumbers (Holothuroidea) can be found and at very low tides sea urchins (Chinoidea) can also be obtained.

Sea urchins. Sea urchins are fairly abundant in Southeast Alaska and out the Aleutian Chain. If not handled properly, a puncture wound may occur, and secondary infection from marine bacteria will ensue. This can be painful and may last a long time. Sea urchins have commercial value in that the raw gonads are considered a delicacy and are in great demand in the orient. The hazard will be more to the commercial diver who handles them frequently and gets careless. Two species common in Alaska are (1) the big red urchin
(Strongylocentrotus franciscanus) and (2) the smaller 1 ight green urchin (Strongylocentrotus drobachiensis).

Sea cucumbers. These are the tube like animals which can discharge their internal organs if disturbed. The visceral fluid can be harmful to the eyes. The common edible species is Stickopus cal ifornicus.

\section{Crustaceans}

Recent studies (1978 and later) by Dr. Al Sparks, National Marine Fisheries Service, Seattle, have shown there are several diseases unknown until now in the dungeness crab (Cancer magister). One is a protozoan infection found throughout most of the host crabs' body which destroys much of the tissue that it invades. The appearance of tissue damage in crabs therefore, should alert the observer to the possible presence of a protozoan disease.

There is also an unidentified fungus which replaces gill tissue. An animal infected with either of these organisms could be nutritionally deprived and therefore, would look undernourished, and probably would not be appealing to a sport fisherman. Since crabs are not inspected that carefully by commercial processors, the meat would likely be mixed in with that from more wholesome crabs.

Another disease commonly thought to infest only the carapace of snow crabs is "black mat disease" or "black mat syndrome." The condition has been known for years--it consists of a black mass of fungal hyphae on the carapace of snow crabs. It is a nuisance in crab processing. The recent microscopic studies by Sparks and his group show that this disease 
may be systemic, and could be a serious disease in the snow crab family.

\section{Nematodes}

There is a worm disease found in dungeness crab eggs from Alaska to California. The worm belongs to the Nemertean phylum and the genus is Carcinomertes. Crabs with a carapace width greater than $10 \mathrm{~mm}$ show about $100 \%$ infection. The worm penetrates the egg casing, and eats the yolk by sticking its pharynx into it.

Clinical data on most of the Alaskan marine invertebrates is simply not available. Your cooperation in forwarding to the editor information on poisonings from any species will be appreciated.

\section{References}

HALL, S., R. A. NEVE' and P. B. REICHARDT. $1979 . \quad$ Chemical analysis of paralytic shellfish poisoning in Alaska, in Toxic Dinoflagellate Blooms. D. L. TAYLOR and H. H. SELIGER, eds. Elsevier, North Holland.

MIX, M. C. and A. K. SPARKS. 1980. Tanner crab Chionicetes bairdi rathbun Haemocyte classification and an evaluation of using differential counts to measure infection with a fungal disease. J. Fish Dis. 3:285-293.

SNIESZKO, S. F. Recent advances in scientific knowledge and developments pertaining to diseases of fishes. Adv. Vet. Sci. Comp. Med. $17: 291-314$.

WEDEMEYER, G. A. 1974. Stress as a predisposing factor in fish diseases. Fish and Wildlife Service, U.S. Dept. of the Interior. Fish Dis. Leaflet No. 38. 


\section{APPENDIX I}

SPECIMEN SUBMISSION

D. Ritter

Rabies diagnostic laboratory services are provided through the VirologyRabies Unit established in Fairbanks. To assure accurate and reliable reports, the directions listed below must be followed.

1. Specimens will be accepted from physicians, veterinarians, public health officials, health or sanitation aides, wildlife agents, authorized medical or physician assistants, or individuals designated by the Division of Public Health.

2. Rabies laboratory examinations will be limited to warm-blooded animals of any type in which there is a clear history of human or possible human exposure or animals deemed necessary by health or wildlife authorities.

3. Special instructions:

Virology-Rabies Unit must be notified prior to submission of rabies specimens. Call Fairbanks $479-7017$ or 7018 , or on weekends 456-5974. If no answer, dial 452-0890, listen for tone (Beep) and leave message. If busy, call 452-1166 and ask for Unit 890 .

4. Minimum information:

The following information of a completed Rabies Investigation Report (06-1272) must accompany each specimen. This information is essential for the evaluation of each case and expediting the transmission of results to the responsible physician.

A. Name and address of person(s) bitten and/or exposed.

B. Date of exposure.

C. Location of bite(s).

D. Severity of bite(s).

E. Date of first aid treatment and name of physician.

F. In the case of a dog or cat, was the animal vaccinated.

G. Provoked or unprovoked at tack.

H. Name and address of person sending specimen.

I. Name and address of person to receive the laboratory report.

5. Conditions of shipment:

A. No LIVING ANIMAL will be accepted for rabies diagnostic studies.

B. When killing the animal, DO NOT SHOOT IN THE HEAD or mutilate the head in any way.

C. Wear gloves when handling the animal, and send the head and part of the neck of large animals, such as dogs, foxes, wolves, lynx, etc. Sever the head at the neck and leave sufficient tissue attached to the region of the head to insure inclusion of the salivary glands. No other part of the animal should be submitted. 
D. Small animals, such as mice, voles and bats, may be sent intact if recently expired, and only after consultation with the Chief of the Virology-Rabies Unit or the State Medical Epidemiologist.

E. Specimens for rabies examination must be fresh. Decomposed specimens will be evaluated, and if unsuitable for assay, will be promptly incinerated and NO RABIES ASSAY will be attempted.

\section{Packing:}

A. Wear gloves when handling the animal.

B. Wrap the head in absorbent material, and place into two heavy, water-tight plastic bags. Tie-off the bags to prevent leakage.

c. Place the packaged head in a leak-proof container, and pack the bag next to a water-tight can of frozen water or freeze packs. An alternative is to use a second set of heavy plastic bags and fill these with ice cubes and seal the top tightly. Remote areas may use river ice, but this type of ice has rough edges and will cut the bag. This can be avoided by wrapping the ice in newspaper and then pack with absorbent material to prevent movement of the ice in the carton.

D. Remove the gloves and either burn the gloves or enclose them with the head for disposal, and then wash hands thoroughly with soap.

E. Check the carton or container for security and include the Rabies Investigation Report. Please include contact telephone numbers.

7. Labeling:

A. All rabies specimens must bear the special Rabies Biohazard label or the following statements:

MEDICAL MATERIALS
PACKAGING CONFORMS
WITH STANDARDS IN
49CFR 173.387 .42
CFR72.25(c), and
NIH GUIDE OF
FEBRUARY 10,1975

MEDICAL MATERIALS PACKAGING CONFORMS 49CFR 173.387 .42 NIH GUIDE OF FEBRUARY 10,1975 
8. Shipping:

A. Specimens may be brought directly to the Virology-Rabies Unit.

B. Ship specimens by AIR FREIGHT or AIR SPECIAL PACKAGE SERVICE. If mail service must be used, send via AIR MAIL--SPECIAL DELIVERY or EXPRESS MAIL, if available.

C. All shipments must be prepaid unless prior arrangements have been worked out with the Virology-Rabies Unit.

9. Reports:

A. A preliminary fluorescent microscopic report will be called on all positive animal heads and human exposure cases within 24 to 48 hours after specimen receipt, and confirmation copies of reports will be mailed to the submittors.

B. The final report will be forwarded 28 days after completion of the animal inoculation observation period.

10. Further information on rabies antiserum and vaccine for human use: Contact Alaska Division of Public Health Regional Health Officer of Laboratory serving your area.

Northern Regional Health Officer

Medical Dental Arts Building

Drawer 34, Suite 223

1919 Lathrop Street

Fairbanks, Alaska 99701

Phone: 452-1592

Southcentral Regional Health Officer

338 Denali Street

Room 222, Mackay Building

Anchorage, Alaska 99501

Phone: 274-1715

Southeast Regional Health Officer

210-A Ferry Way

Juneau, Alaska 99801

Phone: $586-1120$

Medical Epidemiologist

Section of Communicable Disease Control

338 Denali Street

Room 313 Mackay Building

Anchorage, Alaska 99501

Phone: 272-7534

Virology-Rabies Unit

Alaska Division of Public Health

Arctic Health Research Building, Room 233

University of Alaska Campus

Fairbanks, Alaska 99701

Phone: $479-7017$ or $479-7018$ 


\title{
SPECIMEN COLLECTION AND PRESERVATION
}

\author{
Jim Dau
}

\section{$\underline{\text { Introduction }}$}

Nearly everyone associated with wildlife in either a professional or recreational capacity, contacts diseased animals or their remains afield. Additonally, professional wildlife personnel receive specimens and/or queries from sportsmen concerned with the palatability of game animals that exhibit parasites or gross abnormalities. One purpose of this compendium has been to provide accurate descriptions of specific diseases to enable formulation of tentative diagnoses based on clinical symptoms or gross pathologic appearances. However, tentative diagnoses are not adequate substitutes for definitive diagnoses based on laboratory analyses of properly collected and preserved samples. The purposes of this appendix are: 1) to provide a procedural guideline for sample collection and preservation, and 2) to note the special aspects of collecting samples for the major wildlife disease categories.

The procedures listed are very general for 2 reasons. First, a detailed coverage of techniques for sampling each specific disease condition of mammals, birds, fish and invertebrates is beyond the scope of this publication. Second, asymptomatic acute diseases, conditions that manifest ambiguous symptoms, the tendency of disease to predispose free-living animals to other mortality sources, tissue deterioration, and an array of other factors of ten preclude establishment of even broad etiologic categories at the time of necropsy. Thus, a general procedural format that will hopefully not exclude any causative agent seems more useful to the wildlife field biologist than techniques written for specific etiologies.

\section{Necropsy Procedures and Techniques}

General comments. Before delving into the mechanics of what, where and how to collect samples for disease analysis, a few preliminary remarks are appropriate. Since many wildlife pathogens are infective for man, and considering that ubiquitous microorganisms normally maintained at subpathogenic levels by the host animal may proliferate to pathogenic levels during decomposition of the host, all specimens should be treated as potential reservoirs of human disease. Protection of personnel against infection should always be the primary concern of everyone involved in the sampling process. The essence of this is to "think clean," wear adequate protective clothing and follow aseptic techniques during the necropsy. Minimization of contamination results in better samples and reduced environmental contamination, while decreasing the probability of infection for personnel.

Documentation is an essential aspect of the sampling process. Complete, accurate, legible field notes specifying the circumstances of the animal's death, i.e. species, location, environmental characteristics, clinical symptoms and other pertinent observations, are necessary for generating "path. reports" that 
complement tissue samples. Photographs of parasites or lesions are extremely useful for determining diagnoses and indicating pathogenicity. A label showing the specimen number and some reference of scale (i.e. centimeter markings along 1 edge or simply a coin or knife) should appear in the photo. Exposure numbers with brief descriptions should be recorded in the field notes if many exposures are taken.

Specimen preparation. The procedure listed is for terrestrial mammals sampled under ideal conditions but can be applied to marine mammals, birds and fish with a little creative effort. Usually, conditions are somewhat less than ideal, and biologists must salvage what they can from a decomposing carcass under inclement weather conditions. Two people are ideally needed to conduct an efficient necropsy. This allows 1 person ("dirty") to actually remove tissues and 1 person ("clean") to take photographs, record notes, label and hold sample bags. For large animals, a third person may be desired to help disarticulate legs, move organs and aid in contaminating tasks.

1. Collect a blood sample. This will apply only when animals are living or can be sampled within minutes after death. The best sources for blood are the jugular vein, femoral artery or the heart. Avoid taking blood from open wounds as it will contain cellular debris. Blood should not be allowed to freeze or be exposed to excessive heat or agitation to prevent hemolysis. A suitable anticoagulant, i.e. E.D.T.A., should be added to whole blood samples to be used for measuring cell counts, packed cell volume, glucose assays (enzyme inhibitor also needed) or other parameters. Blood serum for antibody detection can be carefully pipetted or decanted into a sterile vial after allowing the blood to clot. The clot will form after standing about 12 hours at room temperature. Serum may be stored frozen, though repeated freezing and thawing should be avoided.

If the animal is found dead, serum many times can be collected from the heart clot. The whole clot or the yellow "chicken fat" portion if separation has occured, is placed in a plastic bag, allowed to stand for 12 hours, and then free serum is collected.

2. Visually inspect the carcass for external parasites, hair loss, traumatic injuries, discharges from the nose, mouth or vent and other abnormalities. Photographs, swabs of exudates, and external parasites should be taken at this time.

3. Position the animal right side down if it is important to collect a sterile sample of spleen tissue (i.e. for many bacterial surveys). Positioning the animal left side down exposes the liver and positions the rumen beneath the viscera and out of the way. Remove the skin from the upper side of the animal (Fig. 1). The testes or mammary glands should not be exposed or damaged during skinning if they are to be sampled. Making an incision through the skin along the dorsal surface of the animal and skinning the animal from its back towards its belly will reduce the possibility of accidentally harming testes or mammaries. This method of skinning also minimizes the possibility of opening the rumen in animals, i.e. caribou (Rangifer tarandus) that tend to bloat quickly after death. Bloating stretches the skin and muscles that cover the belly very tightly and makes it difficult to avoid punc- 


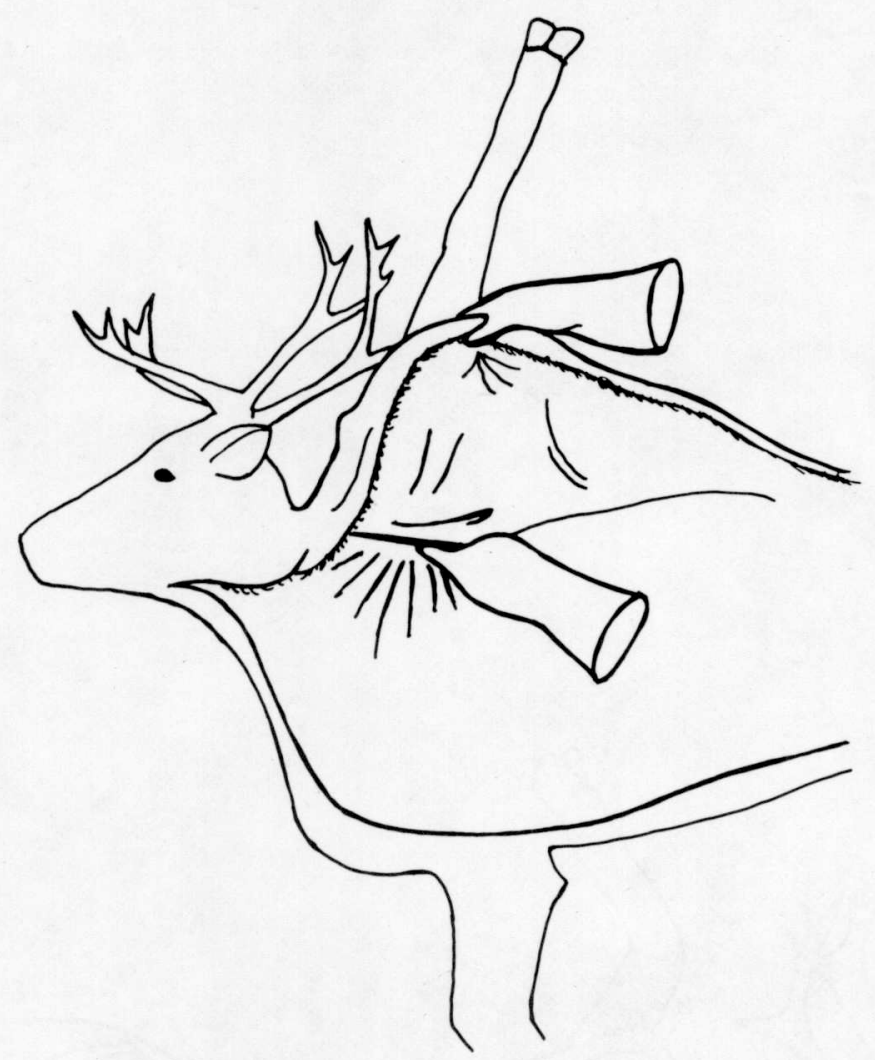

Figure I. Skinning the upper half of the body.

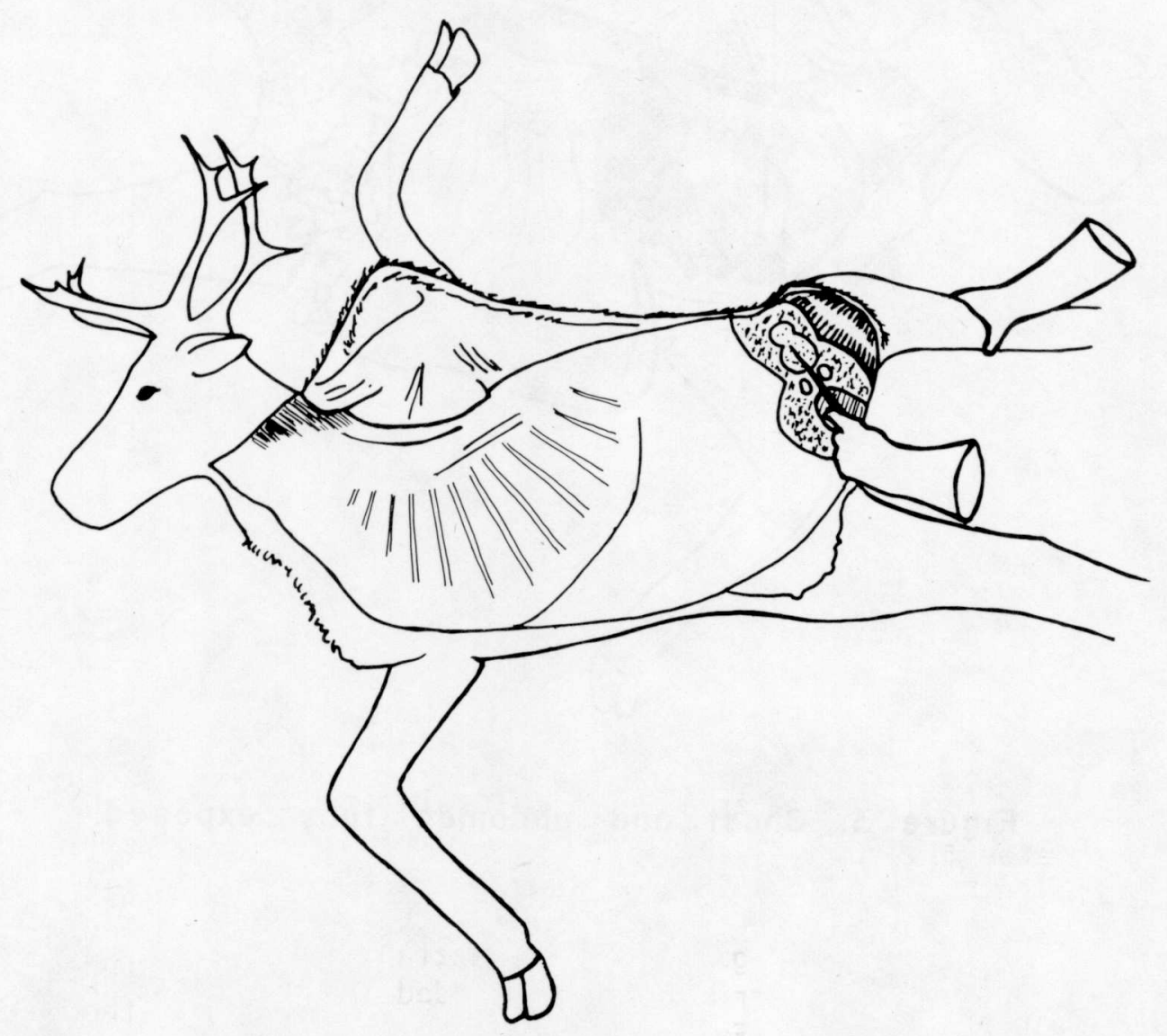

Figure 2. Disarticulating the left hip joint. 


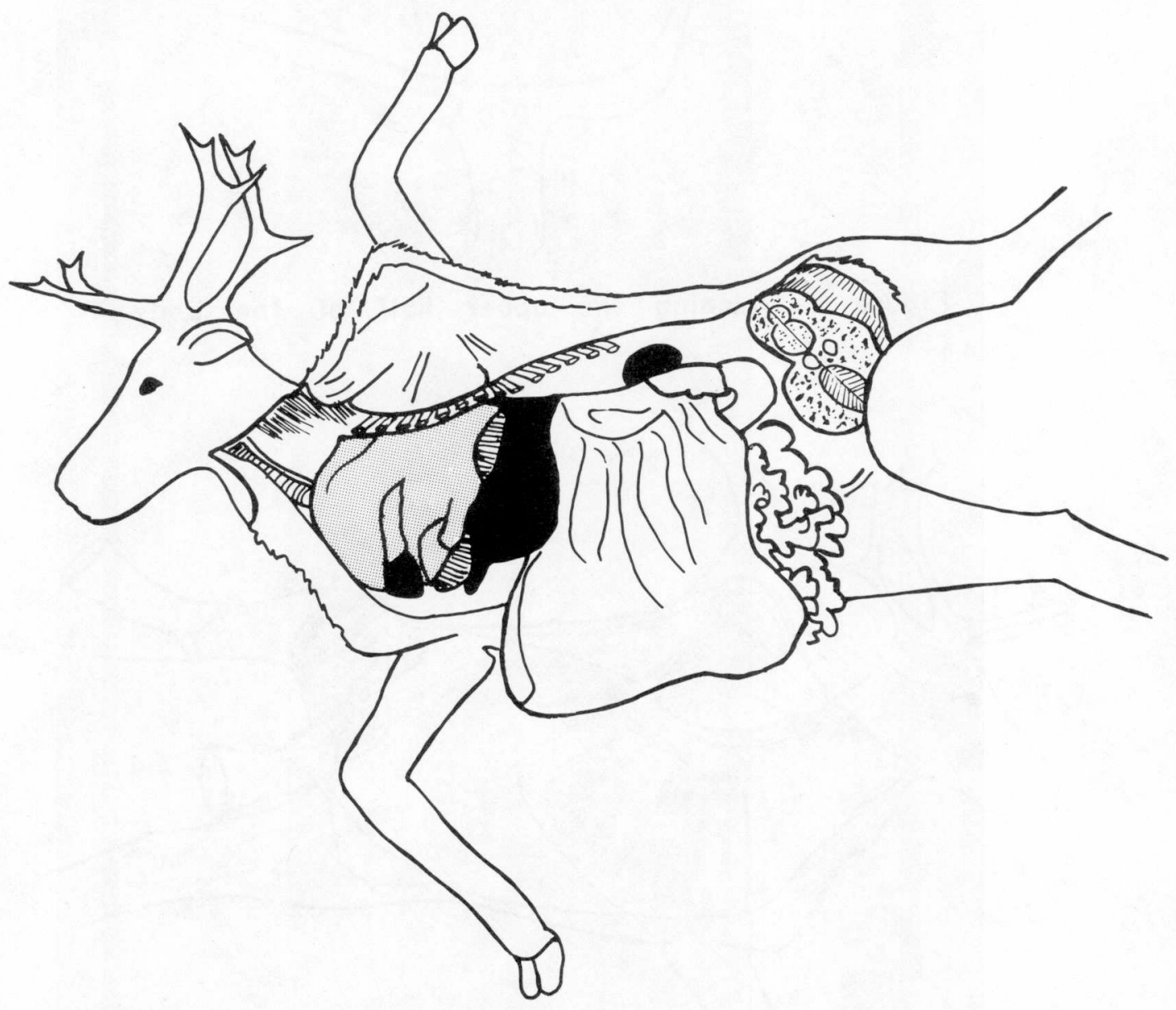

Figure 3. Chest and abdomen fully exposed. 
turing the rumen with the tip of the knife. Take care when skinning around subdermal lymph nodes, i.e. prescapular, prefemoral and mandibular lymph nodes, if these are to be sampled. Disinfect instruments each time before they are used to handle tissues. Tissues should not contact the sides of containers when placed inside. Remove the lymph nodes and testes or mammary glands after the upper surface of the animal has been skinned. To avoid contamination, remove 1 side of the animal before it is rolled over to be skinned on the other side. The external surface of the carcass can be kept fairly clean if the loosened skin is used as a surface to work on.

4. Raise the upper foreleg and cut beneath the scapula until the leg and shoulder can be laid back (Fig. 1). Disarticulate the upper rear leg at the pelvis by cutting the muscles through the coxo-femoral joint ( $F i g$. 2). If time is critical, the carcass can be temporarily left at this point as cooling will proceed. Sampling should resume as soon as possible to prevent tissue deterioration, especially in high ambient temperatures.

5. Open the abdomen to the sternum, taking care not to cut the intestines or rumen. Incising the upper surface of the abdominal wall as the animal lays on its side, instead of the ventral midline as is commonly practiced, will hold the viscera in the body cavity rather than allow these organs to spill out on the ground and become contaminated. Split the sternum with a knife or saw, being careful not to puncture the thoracic organs. Free the diaphragm from its upper costal attachment. Cut the intercostal muscles between every second or third rib and break them back at their points of articulation with the vertebrae. Split the pubis to expose the pelvic organs if desired. The specimen is now ready for removing tissue samples from the internal organs (Fig. 3).

Birds should be completely wetted with disinfectant before the skin covering the breast and abdomen is removed. Removal of the sternum and pectoral muscles at their points of articulation facilitates access to the internal organs.

Fish can usually be frozen or preserved whole. Large fish may require removal of the viscera and gill arches prior to preservation. A 3-5 $\mathrm{cm}$ kidney sample from large fish can be prepared by taking a cross section of the fish and dissecting away all other tissues except the kidney from the skeleton. The skeleton and kidney can then be preserved.

Tissue collection. Before disturbing the viscera, record an estimate of the time of death, gross internal abnormalities and the presence of visible internal parasites (e.g. Setaria). Aseptically collect samples of the major organs (heart, lung, liver, spleen and kidney) approximately 2 to 3 cubic centimeters in size, and place individually in containers labeled with specimen number and tissue type. (Note: whirl packs make very good containers as they are unbreakable, relatively fluid-tight and require a minimum amount of space). Collect internal lymph nodes if desired. Dissect out the reproductive organs and place in a separate container. These tissues should be frozen for subsequent culturing.

Samples of the major organs, skeletal muscle, diaphragm and tongue can now be collected for histology. These tissues can be placed in a single container with a suitable fixative (i.e. $10 \%$ buffered formalin). 
Tissues for histology should not be mutilated, since the ultimate analysis is based on the structure and organization of cells.

Examine the carcass for endoparasites after collecting tissue samples. Readers are encouraged to consult the parasitological literature for specific hosts and areas of parasite infestation. In general, the digestive tract, subcutaneous tissue, lungs, liver, heart and skeletal muscles may be required for a thorough parasitological examination.

\section{Causative Agents}

Bacterial, mycotic and viral diseases may be indicated by: 1) large epizootics involving many individuals of a population, 2) bloody mucous discharges from the mouth, nose or vent, 3) reddened and congested lungs, kidneys and intestines, 4) an enlarged, dark colored spleen, 5) an enlarged, off colored liver and 6) the presence of pus. Fish may exhibit external lesions or redness at the base of fins. Preserve both frozen and formalinfixed tissue samples and blood serum when possible if bacterial, mycotic or viral etiologies are suspected.

Ectoparasites (i.e. fleas, ticks and lice) are usually visible with the naked eye so require no further elaboration. Host reactions to endoparasites may result in cysts that obscure the parasitic organisms per se. Collect these cysts in their entirety by removing the affected block of host tissue. The best parasitological material, when dealing with the mature parasites and not cysts or eggs, results if the parasites are relaxed and cleansed in a warm saline solution and fixed in hot (not boiling) preservative. Endoparasites are often specific as to host species and anatomical region of attack. Therefore, it is important to note both of these aspects of the condition as well as the number of parasites present. Most parasites can be preserved in $70 \%$ alcohol with 5\% glycerol added.

Non-infectious diseases, i.e. toxicities and nutritional or mineral deficiencies are often indistinct. Hair bundles approximately $2 \mathrm{~cm}$ in diameter should be collected along with whole blood. Localized morbidities/mortalities may indicate a non-infectious etiology. Collect soil and water samples, in addition to tissue samples, and place them in inert containers (i.e. glass or plastic vials). These samples must be analyzed as quickly as possible as many toxins rapidly break down in moist conditions. Containers holding water should not contain any air space after being covered. Soil samples should extend a minimum of 10 $\mathrm{cm}$ deep. Plants for mineral assays or toxin analyses are collected in their entirety, quickly pressed and dried. Stomach samples from ruminants can be placed in porous cloth, and excess water squeezed out before placing in 10\% formalin. The entire stomach of monogastric animals can be placed directly in $10 \%$ formalin, if small enough, or portions of their contents removed and preserved. 
1. Nalgene bottles containing:

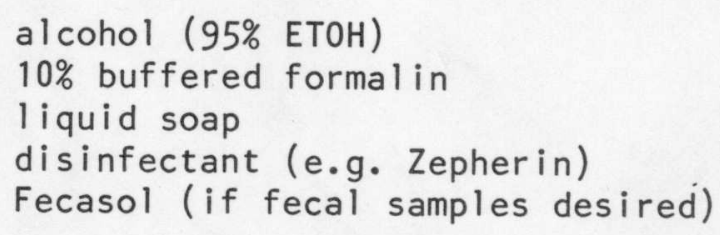

2. rubber gloves

3. knives \& sharpening stone

4. culturing material:

sterile swabs

transport media (e.g. Amies)

viral transport media

5. blood sampling material:

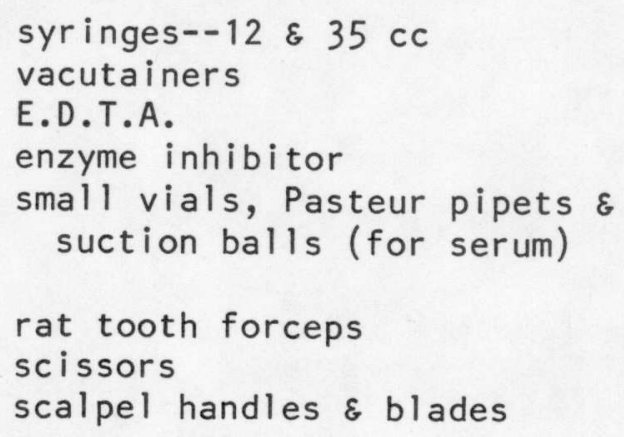

rat tooth forceps

scissors

scalpel handles \& blades

6. sampling instruments:

7. stainless steel pan for disinfectant

8. whirlpacks $(6,18 \& 36$ oz.)

9. labeling tape \& felt pen

10. miscellaneous equipment:

garbage bags

gauze

rope

tape measure

matches

coveralls

11. field book \& pencils 


\section{APPENDIX III}

MAMMALS

Common and Scientific Names of Species

Marsupialia

Didelphidae

Didelphis marsupialis virginiana Common opposum

Insectivora

Soricidae

Sorex pribilofensis

Sorex monticollis

Sorex cinereus

Sorex $\underline{\text { arcticus }}$

Pribilof shrew

Dusky shrew

Masked shrew

Arctic shrew

Primates

Cercopthecidae

Macaca mulatta Rhesus monkey

Pongidae

Homo sapiens

Man

\section{Edentata}

Dasypodidae

Dasypus novemcinctus

$\mathrm{Nine-banded} \mathrm{armadillo}$

\section{Lagomorpha}

Ochoton idae

Ochotona collaris Collard pika

Leporidae

Lepus americanus

Lepus europaeus

Lepus spp.

Sylvilagus floridanus

Oryctolagus cuniculus

Lepus arcticus

Snowshoe hare

European (Brown) hare

Jack rabbit

Eastern cottontail

0ld World, domestic; laboratory rabbit

Arctic hare

\section{Rodentia}

Sciuridae

Eutamias minimus

Sciurus carolinensis

Marmota monax

Marmota brower i

Marmota caligata

Spermophilus parryi i

Tamiasciurus hudsonicus

Glaucomys sabrinus

Castoridae

Castor canadensis

Least chipmunk

(Eastern) gray squirrel

Woodchuck

Alaska marmot

Hoary marmot

Arctic ground squirrel

Red squirrel

Northern flying squirrel

Beaver 
Cricetidae

Peromyscus maniculatus

Clethrionomys rutilis

Microtus pennsylvanicus

Microtus oeconomus

Microtus xanthognathus

Microtus miurus

Microtus abbreviatus

Ondatra zibethicus

Lemmus sibiricus

Synaptomys borealis

Dicrostonyx torquatus

Gerbillus spp.

Cricetus spp.

Muridae

Rattus norvegicus

Mus musculus

Rattus spp.

Caviidae

Cavia spp.

Erethizont idae

Erethizon dorsatum

\section{Cetacea}

Balaen idae

Balaena mysticetus

Eschrichtiidae

Eschrichtius robustus

Balaenopteridae

Balaenoptera physalus

Balaenoptera borealis

Physeteridae

Physeter catodon

Monodontidae

Delphinapterus leucas

Phocoenidae

Phocoena phocoena

\section{Carnivora}

Canidae

Canis latrans

Canis lupus

Vulpes vulpes

Urocyon cinereoargenteus

Alopex lagopus

Canis familiaris

Ursidae

Ursus americanus

Ursus arctos

Ursus maritimus

Procyonidae

Procyon lotor
Deer mouse

Northern red-backed vole

Meadow vole

Tundra vole

Yellow-cheeked vole

singing vole

Insular vole

Muskrat

Brown lemming

Northern bog lemming

Collared lemming

Gerbil

Hamster

Norway rat

House mouse

Rats

Guinea pig

Porcupine

Bowhead

Gray whale

Fin whale

Sei whale

Sperm whale

White whale, belukha

Harbor porpoise

Coyote

Wolf

Red (blue) fox

Gray fox

Arctic fox

Domestic dog

Black bear

Brown bear

Polar bear

Raccoon 
Mustel idae

Martes americana

Mustela erminea

Mustela nivalis

Mustela putorius

Mustela vison

Gulo gulo

Lutra canadensis

Enhydra lutris

Felidae

Fel is lynx

Felis $\frac{\text { rufus }}{\text { Felis }}$

Felis catus

Panthera leo

Otariidae

Eumetopias jubatus

Zalophus californianus

Callorhinus ursinus

Odobenidae

Odobenus rosmarus

Phocidae

Phoca vitulina

Erignathus barbatus

Mirounga angustirostris

Phoca hispida

Phoca fasciata

Perissodactyla

Equi dae

Equus caballus

Equus asinus

Artiodactyla

Suidae

Suş scrofa

Camelidae

Lama guan ico

Cervidae

Cervus elaphus

Odocoileus hemionus sitkensis

0docoileus hemionus columbianus

Odocoileus virginianus

Alces alces

Rangifer tarandus

Ant i locapridae

Antilocapra americana

Marten

Ermine

Least wease 1

Polecat (ferret)

Mink

Wolverine

River otter

Sea otter

Lynx

Bobcat

Domestic cat

Lion

Stellar's sea lion

California sea lion

Northern fur seal

Walrus

Harbor seal

Bearded seal

Northern elephant seal

Ringed seal

Ribbon seal

Domestic horse

Donkey

Domestic swine

Guanoco (Llama)

Wapiti (American elk); Red deer (European)

Black-tailed deer

Mule deer

White-tailed deer

Moose

Reindeer; Caribou

Pronghorn 


\section{Bovidae}

Bison bison

Bos taurus

Bos indicus

Ovibos moschatus

Ovis aries

Ovis dalli

Ovis canadensis

Oreamnos americanus

Capra hircus

Hemitragus jemlaicus

Rupicapra rupicapra
Bison

Domestic cattle, ox

Zebu

Muskox

Domestic (merino) sheep

Dall sheep

Bighorn sheep

Mountain goat

Domestic goat

(Himalayan) tahr

Chamois merriam 
Common and Scientific Names of Species

Sphen isciformes

Penguin

Gavi iformes

Gavi idae

Gavia immer

Common loon

Anseriformes (Waterfowl)

Anatidae

0lor columbianus

Cygnus olor

Branta canadensis

Anser albifrons

Chen caerulescens

Chen rossi i

Anas platyrhynchos

Anas acuta

$\overline{\text { Anas }}$ crecca carolinensis

Anas clypeata

Anthya sp.

Somateria mollissima dresseri

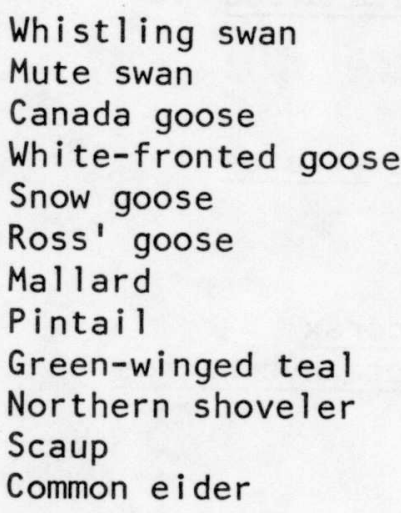

Falconiformes (Raptors)

Cathartidae

Falconidae

Falco peregrinus

Falco rusticolus

Falco sparverius

Vultures

Peregrine falcon

Gyrfalcon

American kestrel

Galliformes

Tetraonidae

Bonasa umbellus

Lagopus lagopus

Lagopus mutus

Phasianus colchicus

Canachites canadens is

Lagopus leucurus

\author{
Ruffed grouse \\ Willow ptarmigan \\ Rock ptarmigan \\ Ring-necked pheasant \\ Spruce grouse \\ White-tailed ptarmigan
}

Gruiformes

Gruidae

Grus canadensis

Rallidae

Fulica americana

Sandhill crane

American coot 
Charadri iformes

Laridae

Larus argentatus

Larus delawarensis

Larus atricilla

Alicidae

Uria sp.

Herring gull

Ring-billed gull

Laughing gull

Murre

Col umb i formes

Pigeons and Doves

Strigiformes

Strigidae

Bubo virginianus

Great horned owl

Nyctea scandiaca

Snowy owl

Piciformes

Picidae

Colaptes aurates

Yellow-shafted flicker

Passeriformes

Corvidae

Corvus corax

Corvus brachyryhnchos

Common raven

Common crow

Fringillidae

Serinus canarius

Finches

Canary 
FISH

Common and Scientific Names of Species

Petromyzont iformes

AGNATHA

Petromyzont idae

Ichthyomyzon castaneus

Chestnut lamprey

Rajiformes

CHONDR I CHTHYES

Rajidae

Baja ocellata

Winter skate

Raja radiata

Thorny skate

Clupe i formes

OSTEICHTHYES

Clupidae

Clupea harengus harengus

Clupea harengus pallasi

Atlantic herring

Pacific herring

Salmon iformes

Salmon idae

Oncorhynchus gorbuscha

Oncorhynchus keta

Oncorhynchus kisutch

Oncorhynchus masou

Oncorhynchus nerka

Oncorhynchus tshawytscha

Prosopium cyl indraceum

Prosopium williamsoni

Salmo clarki

Salmo gairdneri

Salmo salar

Salmo trutta

Salvel inus fontinal is

Salvelinus malma

Salvelinus namaycush

Stenodus leucichthys

Thymallus arcticus

Plecoglossidae

Plecoglossus altivel is

Esocidae

Esox lucius

Pink salmon

Chum salmon

Coho salmon

Cherry salmon (masou)

Sockeye salmon

Chinook salmon

Round whitefish

(Rocky) Mountain whitefish

Cutthroat trout

Rainbow trout (steelhead)

Atlantic salmon

Brown trout

Brook trout

Dolly varden

Lake trout

Inconnu (sheefish)

Arctic grayling

Ayu

Northern pike

Cypriniformes

Cyprinidae

Carassius auratus

Cyprinus carpio

Hybognathus hankinsoni

Leuciscus leuciscus

Nocomis biguttatus

Goldf ish

(Common) carp

Brassy minnow

Dace

Hornyhead chub 
Notropis cornutus

Notropis heterolepis

Phoxinus eos

Phoxinus phoxinus

Pimephales promelas

Semotilus atromaculatus

Catostomidae

Carpiodes cyprinus

Catostomus catostomus

Catostomus commerson i

Siluriformes

Ictaluridae

lctalurus nebulosus

Siluridae

Silurus glanis

Percopsiformes

Percopsidae

Percopsis omiscomaycus

Gadiformes

Gadi dae

Gadus macrocephalus

Gadus morhua

Lota lota

Pollachius virens

Zoarcidae

Lycodes lavalaei

Atheriniformes

Cyprinodont idae

Fundulus parvipinnis

Atherinidae

Atherinops affinis

Gasterosteiformes

Gasterosteidae

Culaea inconstans

Gasterosteus aculeatus Pungitius pungitius

Perciformes

Percichthyidae

Morone chrysops

Centrarchidae

Lepomis gibbosus

Micropterus dolomieui

Micropterus salmoides

Pomoxis annularis

Pomoxis nigromaculatus

Percidae

Perca flavescens

Percina caprodes
Common shiner

Blacknose shiner

Northern redbelly dace

Minnow

Fathead minnow

Creek chub

Quillback

Longnose sucker

White sucker

Brown bullhead

Catfish

Trout-perch

Pacific cod

Atlantic cod

Burbot

Pollock (coalfish; saithe)

Newfoundland eelpout

California killifish

Topsmelt

Brook stickleback

Threespine stickleback

$\mathrm{Ninespine} \mathrm{stickleback}$

White bass

Pumpkinseed (sunfish)

Smallmouth bass

Largemouth bass

White crappie

Black crappie

Yellow perch

Logperch 
Sciaen idae

Aplodinotus grunniens

Seriphus politus

Blenniidae

Hypsoblennius gilberti

St ichaeidae

Ulvaria subbifurcata

Gobi idae

Gillichthys mirabilis

Cottidae

Artedius fenestralis

Cottus bairdi

Cottus cognatus

Hemitripterus americanus

Myoxocephalus octodecemspinosus

Myoxocephalus scorpius

Triglops murrayi

Cichlidae

Symphysodon spp.

Cyclopteridae

Cyclopterus lumpus

Freshwater drum

Queenfish

Rockpool blenny

Radiated shanny

Longjaw mudsucker (goby)

Padded sculpin

Mottled sculpin

Slimy sculpin

Sea raven

Longhorn sculpin

Shorthorn sculpin

Moustache sculpin

Tropical discus fish

Lumpfish

Pleuronect iformes

Pleuronectidae

Limanda 1 imanda

Platichthys stellatus

Pleuronectes platessa

Dab

Starry flounder

European plaice

Tetraodont i formes

Tetraondont i dae

$\underline{\text { Shpoeroides testudineus }}$

Checkered puffer 


\section{APPENDIX IV}

The cross reference indices for mammals, birds and $f i s h$ have been provided as avenues of access and practical summations of the broad scope of information covered in this compendium. Diseases and pathogens appear in the left hand margin of the pages and are arranged in the format of the text. Each disease is listed by the name of the condition as it appears in the title or subtitle of the article rather than by its causative agent; e.g. Avian Cholera rather than Pasteurella multocida. Susceptible wildlife species are listed by common names in systematic groups across the top of the page. We regret that space limitations did not allow inclusion of scientific names with susceptible species; these can be found by referring to Appendix III.

The cross reference indices do not include every disease which may occur in Alaskan wildlife nor all wildlife species that may be affected by those diseases cited. It was designed to facilitate the efficient use of material contained within this compendium and to provide a general feeling for the scope of disease in Alaskan wildlife. The actual cross reference indices are located in a jacket on the inside of the back cover.

\section{Cross Reference Index}

in 


\section{GLOSSARY}

Acidosis - a condition of decreased alkalinity in the blood and other body
fluids.

Active immunity - disease resistance in an individual due to antibody production after exposure to antigen (i.e. through infection or inoculation).

Acute infection - an infection of short duration.

Adenocarcinoma - a malignant tumor originating in a glandular or ductal epithelium and tending to produce berry-like structures.

Adenovirus - one of a group of viruses causing upper respiratory disease; also present in latent infections.

Agglutination test - a diagnostic test utilizing the clumping of a particulate suspension of antigen by a reagent, usually an antibody.

Alkalosis - a condition of high blood alkalinity caused either by high intake of sodium bicarbonate, or by loss of hydrochloric acid or blood carbon dioxide.

Alveolar cyst - hydatid cyst formed by the larval stage of Echinococcus multilocularis.

Ampullae - flasklike dilatations of tubular structures, e.g. seminal vesicles.

Anemia - a condition marked by a significant decrease in hemoglobin concentration and in the number of circulating red blood cells.

Aneurysm - localized abnormal dilation of an artery due to weakening of the vessel wall.

Anorexia - lack of appetite.

Antiserum - any immune serum that contains antibodies that function chiefly to inactivate a specific infective virus or bacterium.

Aplasia - lack of embryonic development of an organ.

Arthrogryposis - permanent fixation of a joint in a fixed position.

Ataxia - inability to coordinate voluntary muscular movements.

Attenuated - having reduced density, strength or pathogenicity.

Autolytic bacteria - bacteria that spontaneously degenerate due to their own enzymatic activity.

Bacteremia - presence of bacteria in the blood.

Bacteria - extremely small, relatively simple procaryotic (lacking nuclear membrane) microorganisms traditionally classified with the fungi as Schizomycetes. 
Baermann technique - method of examining feces for nematode larvae rather than eggs; feces are contained in a screen or mesh in water; larvae swim out, settle to the bottom of a collecting tube, are transferred to a petri dish, and examined with a dissecting microscope.

Bleb - a localized collection of fluid, i.e. serum or blood, in the epidermis.

Butyrous - butterlike in appearance.

Caseous - resembling cheese or curd.

Caseous necrosis - tissue death involving loss of cellular integrity with the consequent conversion to a cheese-like substance, typical in tuberculosis.

Catabolism - metabolic breakdown by which complex substances are converted by living cells into more simple compounds.

Catarrh - an inflammation of mucous membranes, particularly of the respiratory tract.

Cercaria - free-swimming larvae of trematodes which may infect secondary intermediate hosts or which may encyst on vegetation or other surfaces to become a metacercaria.

Chlamydiae - similar to bacteria (having both DNA and RNA; sensitive to antibiotics; one-third the size of $\underline{E}$. coli) but differ in that they are obligate intracellular parasites.

Chlamydiosis - infection caused by chlamydia.

Chorion - the outermost of the embryonic membranes of the amniotes that encloses the embryo and all other membranes.

Chronic infection - an infection of long duration.

Clinical (overt) disease - a disease stage exhibiting gross signs apparent to direct observation.

Coccobacillus - a short, thick, oval bacillus, midway between the coccus (spherical) and bacillus (rod-shaped) forms in appearance.

Coenurus - an encysted tapeworm larva having a bladder with many daughter cysts arising from its walls.

Commensalism - an interspecific, symbiotic relationship wherein one species benefits while the other species is neither benefitted nor harmed.

Complement fixation test - diagnostic test to determine the presence of antigen or antibody in the blood by adding complement; complement binding is then indirectly assayed by adding an indicator system of antibodycoated erythrocytes. 
Congenital - refers to conditions present at, and usually prior to birth regardless of causation.

Conidiophore - branch of fungal mycelium which bears asexual spores.

Conidium - asexual spore formed by splitting off of conidiophore ( $p l$. conidia).

Conjunctivitis - inflammation of the conjunctiva, the membrane which lines the eyeball and covers the outer surface of the eyeball.

Corticosteroid - any steroid hormone secreted by the adrenal cortex of vertebrates.

Cretinism - a type of dwarfism caused by hypothyroidism and associated with generalized body changes, including mental deficiency.

Culturing - the process of growing living cells or microorganisms in a coltrolled, artificial environment.

Curettage - the scraping of the wall of a cavity or other surface to remove growths or other material.

Cyanosis - blue body color due to insufficient aeration of blood.

Cyst - any sac, normal or abnormal, containing liquid or semisolid material.

Cysticercus - an encysted tapeworm larva having a bladder with a single invaginated scolex.

Dark-field microscopy - a microscopic technique in which the specimen is placed at the concentration of an illuminating light cone and is seen with light scattered or diffracted by it; object appears white against a black background.

Desquammation - shedding, peeling and casting off, as of the superficial epithelium, mucous membranes, renal tubules or skin.

Differential diagnosis - distinguishing between diseases of similar character by comparing their signs and symptoms.

Dinoflagellata - an order of flagellate protozoans having fixed body shape determined by a thick covering of plates.

Dysplasia - abnormality of development.

Dyspnea - labored breathing.

Dystrophy - defective nutrition or abnormal development/degeneration.

Ecchymotic hemorrhage - sheetlike accumulation of blood under the skin giving the appearance of bruises.

Ectoparasite - a parasite that lives on the external surface of the host. 
Edema (dropsy) - the presence of abnormally large amounts of fluid in the intercellular tissue spaces of the body.

Egg capsules - fibrous envelopes formed by the parenchyma of cestodes which contain fertilized eggs.

Egg pouches - fibrous envelopes formed from parenchyma that receive eggs from uterine dilations.

Enanthematous - pertaining to an eruption upon a mucous surface.

Encephalitis - inflammation of the brain.

Endoparasite - a parasite that lives within the body of a host organism.

Enteritis - inflammation of the intestine, especially the small intestine.

Enzootic - a disease of low morbidity constantly present in an animal community.

Epididymis - elongated, cordlike structure along the posterior border of the testes in the ducts of which spermatozoans are stored ( $p l$. epididymides).

Epithelialization - the process of tissue becoming epithelial-like with cells close together and little intercellular substance.

Epizootic - an extensive outbreak of disease affecting many animals of one kind in one region simultaneously; a widely diffuse and spreading disease outbreak.

Epizootiology - the study of epizootics.

Epornitic - a disease of high morbidity only occasionally present in a bird population.

Erythema - localized redness of the skin in areas of variable size.

Etiology - demonstration of causes of disease.

Euthanatized - painlessly put to death.

Exophthalmos - abnormal protrusion of the eyeball from its orbit.

Exudate - proteinaceous, cellular material which passes through the blood vessel wall and is deposited in or on the tissue; usually due to inflammation.

Febrile disease - any disease associated with or characterized by a fever.

Fibroblast - a stellate connective tissue cell found in fibrous tissue that functions to support and bind tissues of all sorts. 
Flotation technique - method used to isolate the eggs and oocysts of endoparasites from feces; emulsification of feces in liquid of high specific gravity, whereby eggs and oocysts float and the top film can be microscopically examined.

Fluorescent antibody test - serologic test in which antibodies are stained with a fluorescent dye and the reaction observed microscopically with an ultraviolet light.

Fomite - an inanimate object that may harbor disease-causing microorganisms and transmit them from one place to another.

Friable - easily pulverized or crumbled.

Furuncle - small cutaneous abscess usually resulting from infection of a hair folicle by Staphylococcus aureus; a boil.

Gametocyte - an undifferentiated cell from which gametes are produced.

Gastritis - inflammation of the stomach.

Gram negative - losing the primary stain or decolorized by alcohol and subsequently stained red in Gram's staining method; a primary characteristic of certain microorganisms.

Gram positive - retaining the primary stain (blue) or resisting decolorization by alcohol in Gram's staining method, a primary characteristic of certain microorganisms.

Granulation tissue - a tissue that forms in wounds and consists of tiny, round, fleshy masses of capillaries and fibroblasts; a repair stage following inflammatory damage.

Granuloma - an accumulation of macrophages forming a small nodule or granule.

Halitosis - bad breath.

Halophilic - pertaining to an organism that requires high salt concentrations for growth and maintenance.

Hematocrit - the volume, after centrifugation, of red blood cells in relation to the total volume of the blood.

Hematopoiesis - the process by which the cellular elements of the blood are formed.

Hematopoietic - erythrocyte-producing.

Hematozoa - microorganisms living in the blood.

Hemodilution - increase in fluid content of blood with resulting decrease in the concentration of erythrocytes. 
Hemosiderin - insoluble form of stored iron that is visible microscopically without specific staining methods.

Hepatic - pertaining to the liver.

Hepatitis - inflammation of the liver.

Hepatization - conversion of tissue to liver-like substance.

Herpesvirus - a group of deoxyribonucleic acid (DNA)-containing animal viruses distinguished by a cubic capsid, enveloped virion, and having an affinity for the host nucleus as a site of maturation.

Histopathology - a branch of pathology that deals with microscopic tissue changes associated with disease.

Hydatid cyst - a cyst formed in tissues due to growth of larval Echinococcus granulosus.

Hydrocephalus - abnormal accumulation of fluid in the cranium accompanied by an enlargement of the head.

Hyperemia - an excess of blood within an organ or tissue caused by blood vessel dilation or impaired drainage, especially of the skin.

Hyperplasia - abnormal increase in the number of cells.

Hyperplastic - characterized by hyperplasia.

Hyperthermia - abnormally high body temperature; fever.

Hypha - one filament of the mycelium of a fungus ( $p l$. hyphae).

Hypoglycemia - low blood sugar.

Hypothermia - reduced body temperature; can be either induced or abnormal due to malfunction of central nervous sytem or endocrine system.

lcterus - jaundice; yellow coloration of the skin, mucous membranes, and secretions.

Immunodiffusion - a serological procedure in which antigen and antibody solutions diffuse toward each other through a gel matrix; interaction results in a precipitin line for each system.

Inferior brachygnathia - abnormal shortness of the under jaw.

Intranuclear inclusion body - any of the abnormal structures appearing within the host cell nucleus during the course of virus multiplication.

Isolate - a microorganism strain separated from a mixed-strain population. 
Jaundice - icterus; yellow coloration of the skin, mucous membranes and secretions.

Karyorrhexis - the process of a pyknotic nucleus undergoing fragmentation.

Keratinization - development of or conversion into hornlike tissue.

Lassitude - weakness; exhaustion.

Leukopenia - reduction in number of leukocytes.

Lordosis - vertical deformation of the vertebral column.

Lymphadenitis - inflammation of the lymph nodes. Lymphocytes - an agranular leukocyte functioning primarily in immune
processes.

Lymphosarcoma - a malignant tumor composed of lymphoid cells.

Macrophage - a large phagocyte (ameboid cell that engulfs foreign material) of the reticuloendothelial system.

Malaise - a general state of ill-being or the feeling of poor health. Malocclusion - a deviation in the normal meeting of the chewing surfaces of
the teeth.

Megaloschizont - a large schizont. from a trophozoite in a host cell that segments into merozoites.

Meningitis - inflammation of the three membranes (meninges) which envelop the brain and spinal cord.

Merozoite - an ameboid trophozoite in some sporozoans produced from the splitting up of the schizont.

Metacercaria - encysted stage of a trematode within or on tissues of an intermediate host or vegetation which is infective to final host.

Metacestode - an encysted tapeworm larva.

Metastasis - transfer of disease from 1 organ to another organ not directly connected with it.

Monocytes - large ( 12 micrometers), agranular leukocytes with a relatively small, eccentric, oval or kidney-shaped nucleus; develop into tissue macrophages.

Morbidity - ratio of diseased to non-diseased individuals in a population. 
Mouse inoculation test - a diagnostic test using groups of mice administered specific substances that may indicate an infective agent.

Mycoplasma - tiny, pleomorphic (more than one form) shapes; similar to bacteria but lacking a cell wall; requires special, enriched media for laboratory growth.

Mycotic - fungal; caused by growth of a parasitic fungi in any part of the body.

Myxobacteria - the slime bacteria. Vegetative cells are flexible, slender motile rods that tend to swarm and aggregate to form a fruiting body.

Myxosporidea - a class of protozoans having parasitic members in some fish, amphibians and certain invertebrates.

Myxovirus - a group of ribonucleic acid (RNA)-containing viruses characterized by hemagglutination and hemadosorption; includes influenza, fowl plaque and paramyxoviruses.

Necritic - of or pertaining to the region of shallow water adjoining the sea coast and extending from low tide mark to a depth of about 200 meters.

Necropsy - to perform an autopsy; examination of a body after death.

Necrosis - death of a cell or group of cells as a result of injury, disease or other pathologic state.

Necrotic foci - localized areas of dead tissue.

Neonatal ataxia - failure of muscular coordination or irregularity of muscular action in the newborn.

Nephritis - inflammation of the kidney; diffuse, progressive degenerative or proliferative lesion affecting renal parenchyma, the interstitial tissue, and renal vascular system.

Osteoarthritis - chronic multiple degeneration of joint tissue.

Osteodystrophy - any defective bone formation.

Osteomalacia - failure of bone to ossify due to a deficiency of calcium and phosphorus or vitamin D.

Osteomyelitis - inflammation of the bone caused by a pyogenic organism.

Osteoporosis - deossification with absolute decrease in bone tissue resulting in enlargement of marrow or Haversian spaces, decreased cortex thickness, and trabeculae and structural weakness.

Otarid seal - the sea lions, family Otariidae, superfamily Canoidea. 
Palpation - the act of feeling with the hand and fingers.

Parakeratosis - incomplete keratinization of epidermal cells characterized by retention of nuclei of cells attaining the level of the stratum corneum.

Paratenic host - secondary intermediate host which collects numerous parasite larvae in its tissues that are infective to a final host but which do not further develop in the paratenic host. Parenchyma - refers to the functioning units of an organ, as distinguished
from organ framework or stroma.

Paruterine organs - organs containing fertilized eggs of certain cestodes formed by parenchyma or uterine dilations.

Parvovirus (picodnavirus) - a group of very small, single-stranded deoxyribonucleic acid (DNA)-containing animal viruses that replicate in the nucleus of the infected cell; includes a hamster osteolytic virus, latent rat viruses, a parvovirus of swine and a canine parvovirus.

Passive immunity - antibodies administered (1) artificially through injection of serum or (2) naturally (i.e. maternal antibodies passed placentally or through colostrum) to provide short-lived immunity to disease.

Pathogenesis - origin and development of a pathological process or disease.

Pathognomonic - a distinctive characteristic of a specific disease that enables it to be distinguished from other diseases.

Peracute infection - an excessively acute infection.

Peritoneal dialysis - a process of selective diffusion through the serous membrane enveloping the abdominal viscera and lining the abdominal cavity.

Peritonitis - inflammation of the serous membrane enveloping the abdominal viscera and lining in the abdominal cavity.

Petechia - small hemorrhagic spot in the skin, membrane, etc. (pl. petechiae).

Petechial - characterized by petechia (small) hemorrhages.

Phagocyte - cell that ingests and often digests microorganisms, other cells or foreign material.

Phase contrast examination - microscopic technique whereby light is bent or refracted as it passes through different densities resulting in distinc$t$ ion of cellular structures.

Phycomycetes - a primitive class of true fungi belonging to the Eumycetes lacking regularly spaced septa in the actively growing portions of the plant body.

Pica - the tendency to eat undigestible material, i.e. wood. 
Pinheading - condition in fish where the head is disproportionately large with respect to rest of the body.

Plaque - (1) a patch or abnormal flat area on any internal or external body surface. (2) a clear area representing a colony of viruses on a plate culture formed by lysis of the host cell.

Plasmocyte - a plasma cell of the fluid portion of the blood in which the corpuscles are suspended; a fairly large ovoid cell with a small, eccentrically placed nucleus having chromatin adherent to nuclear membrane.

Pleomorphic - occurring in more than one distinct form during any one stage in the life cycle of an organism.

Polydactylism - presence of extra digits on feet.

Polymastigine - pertaining to invertebrates having flagella grouped in a tuft.

Polyvalent vaccine - a vaccine composed of mixtures of different organisms.

Porencephaly - a condition in which the cavity of a lateral ventricle extends to the surface of the cerebral hemisphere possibly from brain tissue destruction or maldevelopment.

Prepelagic seal pup - a seal that has never ventured to the open (pelagic) ocean.

Primary infection - original infection; term used to distinguish between a pioneering infectious agent (primary infection) and an opportunistic infectious agent (secondary infection).

Prolapse - the falling or sinking down of a part or organ.

Proteolytic - anything causing the breakdown of proteins.

Purulent - consisting of, containing, or forming pus.

Pyknosis - shrinkage of the nucleus.

Pyogenic - pus producing.

Redia - a larval stage of trematodes which develops in a snail host and gives rise to the next larval stage, the cercaria.

Reovirus - a group of ribonucleic acid-containing animal viruses including agents of encephalitis.

Rhabdovirus - a group of ribonucleic acid (RNA)-containing, bullet-shaped animal viruses which includes rabies virus and vesicular stomatitis.

Rickets - a disorder of calcium and phosphorus metabolism affecting bony structures due to vitamin $D$ deficiency. 
Rickettsiae - very small, rod-coccoid-pleomorphic microorganisms; obligately intracytoplasmic in human and animal cells; found in lumen of lice, fleas, ticks, mites and then transmitted to man and animals.

Rinderpest - an acute, contagious, and often fatal viral disease of cattle, sheep and goats characterized by fever and ulcerative lesions of the digestive tract.

Saprophytic - any vegetative organism growing on decomposing organic matter.

Schizogony - asexual reproduction of certain sporozoa by multiple fission of a trophozoite.

Schizont - a multinucleate cell in certain members of the Sporozoa that is produced from a trophozoite within a host cell that asexually gives rise
to merozoites.

Scolex - the head of certain tapeworms typically having a muscular pad with hooks and two pairs of lateral suckers.

Scoliosis - lateral deformation of the vertebral column.

Secondary infection - an infection by an opportunistic organism predisposed by an earlier (primary) infection.

Sedimentation technique - method used to concentrate trematode and acanthocephalan eggs which often sink to bottom in flotation solution; involves centrifugation of feces in formalin-triton-ether.

Septicemia - persistence of bacteria or their toxins in the blood. Serologic titer - reciprocal of the highest dilution of serum reacting in a
visible way with a specific antigen. Serology - the branch of science dealing with the properties and reactions of
blood sera.

Serotype - the type of a microorganism distinguished by the kinds and combinations of constituent antigens present in the cell.

Serous fluid - watery, albuminous fluid.

Serovar (serovariety) - serologic group.

Silver staining technique - used to stain mucopolysaccharides, diffuse proteins and glycoproteins in tissue; commonly used to stain Leptospira
bacteria.

Spina bifida - a congenital anomaly characterized by defective closure of the vertebral canal with herniation of the spinal cord meninges. 
Sporozoite - a motile, infective stage of certain sporozoans that results from sexual reproduction and gives rise to an asexual reproductive cycle in the host.

Symbiosis - an interspecific relationship that may be either beneficial or harmful to one organism.

Systemic infection - a generalized infection affecting no particular anatomic area.

Tetany - a state of increased neuromuscular irritability caused by a decrease of serum calcium, manifested by intermittent numbness, cramps or twitching of extremities, bizarre behavior, unconsciousness and convulsions.

Theront - infective form or stage of a parasite.

Thigmetactic - dense concentrations of marine mammals on certain hauling grounds.

Thrombus - a blood clot occurring on the wall of a blood vessel where the endothelium is damaged.

Tomite - progeny resulting from asexual fission of a mature ciliat.

Tonic spasm - a spasm which persists for some time without relaxation.

Torticollis - condition of persistent, involuntary contraction of neck muscles resulting in the head being twisted to an abnormal position.

Trophozoite - the active, motile, feeding stage of a protozoan as opposed to the non-motile, encysted stage.

Tubercle - a small, knoblike prominence caused by tuberculosis bacteria.

Tumor - a neoplasm; a mass of new tissue which persists and grows independently of its surrounding structures and has no functional use.

Urticaria - hives; transient skin eruption characterized by itching, red or pale, slightly raised areas caused by contact with an external agent.

Uterine prolapse - protrusion of the uterus through the vaginal orifice.

Vector - an agent, such as an insect or animal, capable of mechanical or biological transfer of a pathogen from one organism to another.

Vertical transmission - disease transmission between adult and offspring.

Vesicle - a small, thin-walled, bladder-like cavity usually filled with fluid. 

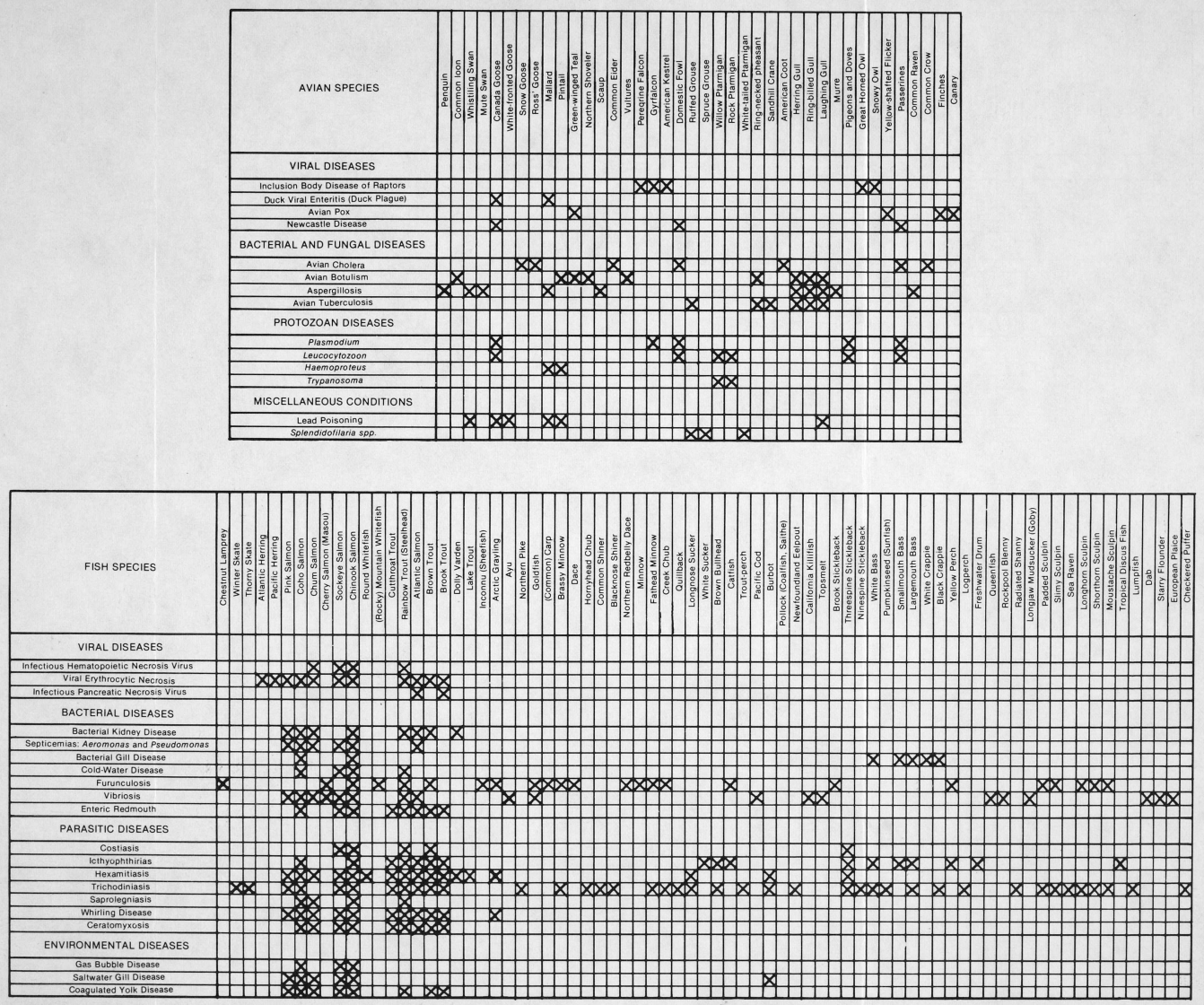


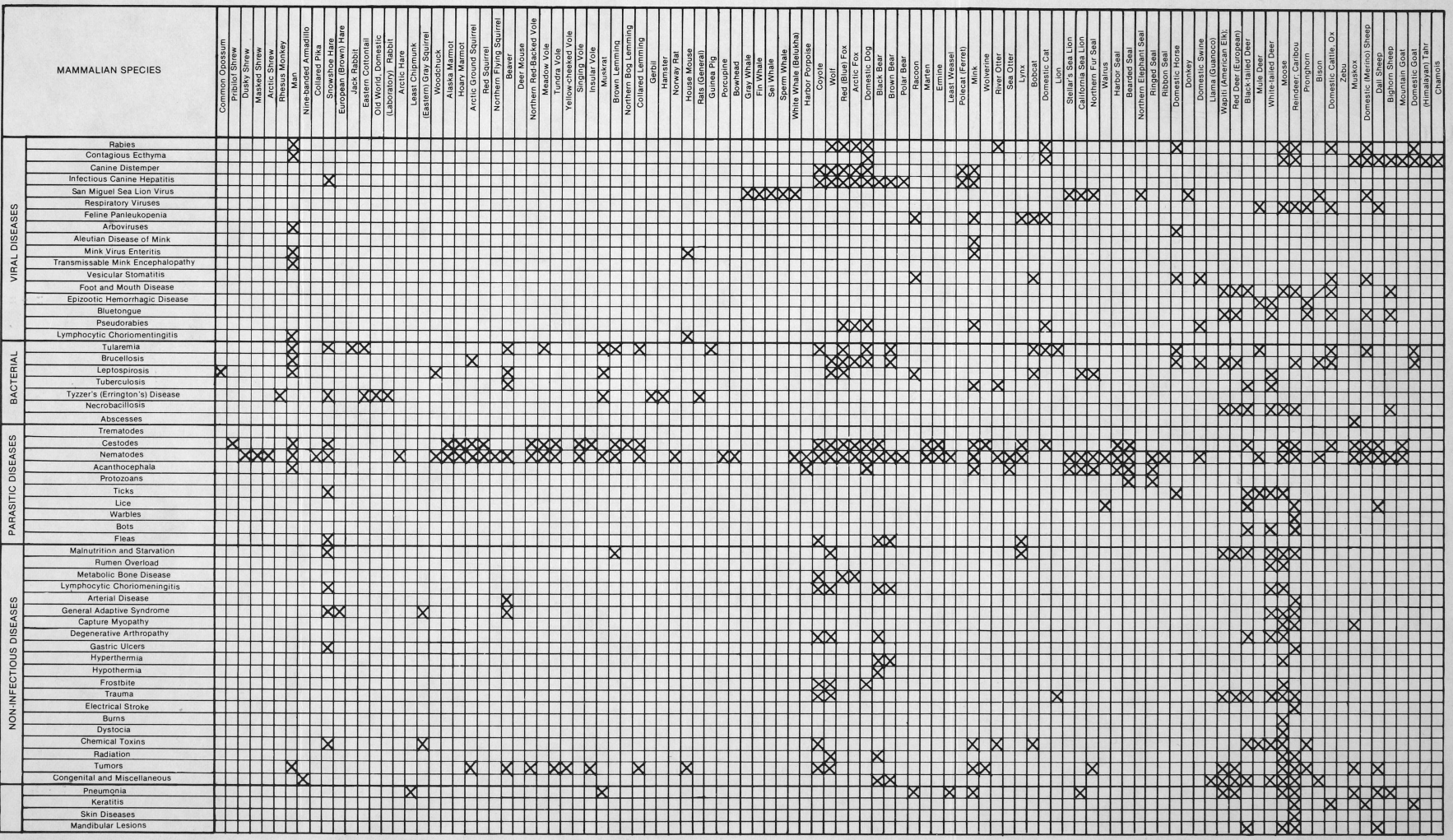

